Supplement of Adv. Geosci., 54, 137-147, 2020

https://doi.org/10.5194/adgeo-54-137-2020-supplement

(c) Author(s) 2020. This work is distributed under

the Creative Commons Attribution 4.0 License.

(c) (1)

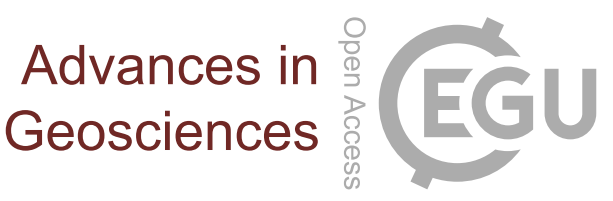

Supplement of

\title{
Storm Xaver over Europe in December 2013: Overview of energy impacts and North Sea events
}

\author{
Anthony James Kettle \\ Correspondence to: Anthony James Kettle (ake3358@gmail.com)
}

The copyright of individual parts of the supplement might differ from the CC BY 4.0 License. 


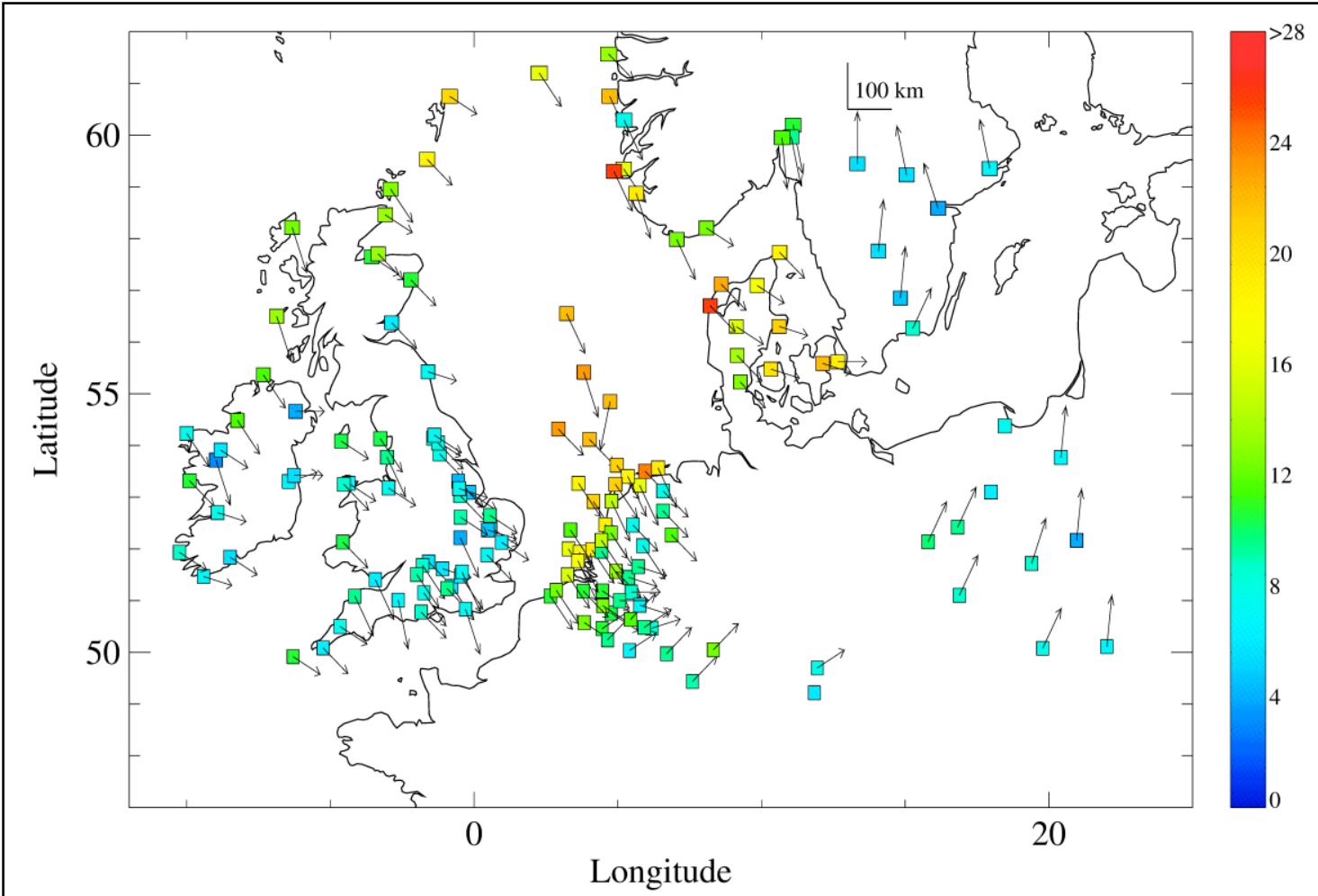

Figure S1. Wind speed (10 minute average, adjusted to $10 \mathrm{~m}$ height) and wind direction on 5 Dec. 2013 at 18:00 GMT for selected station records in the National Climate Data Center (NCDC) database. 


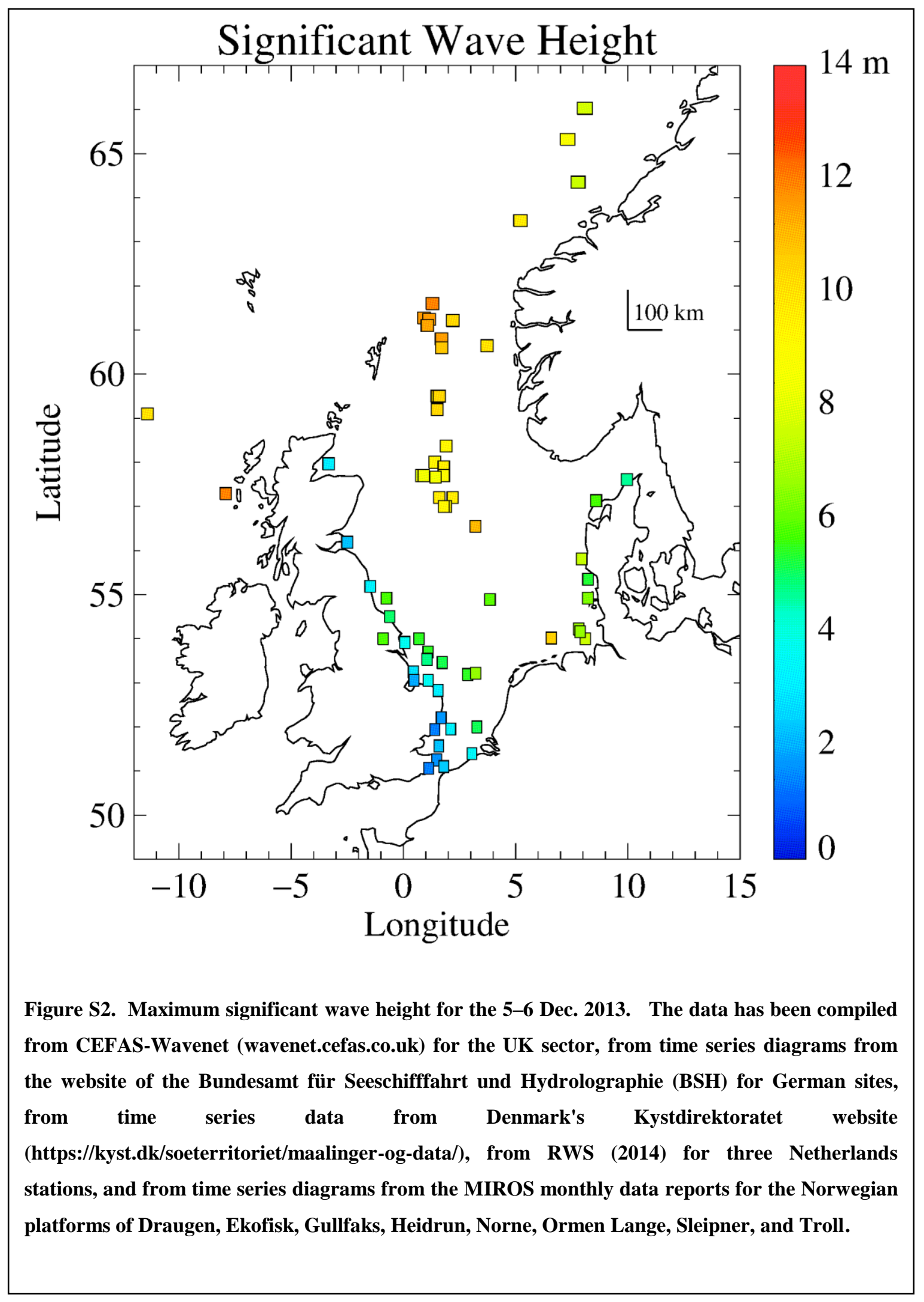




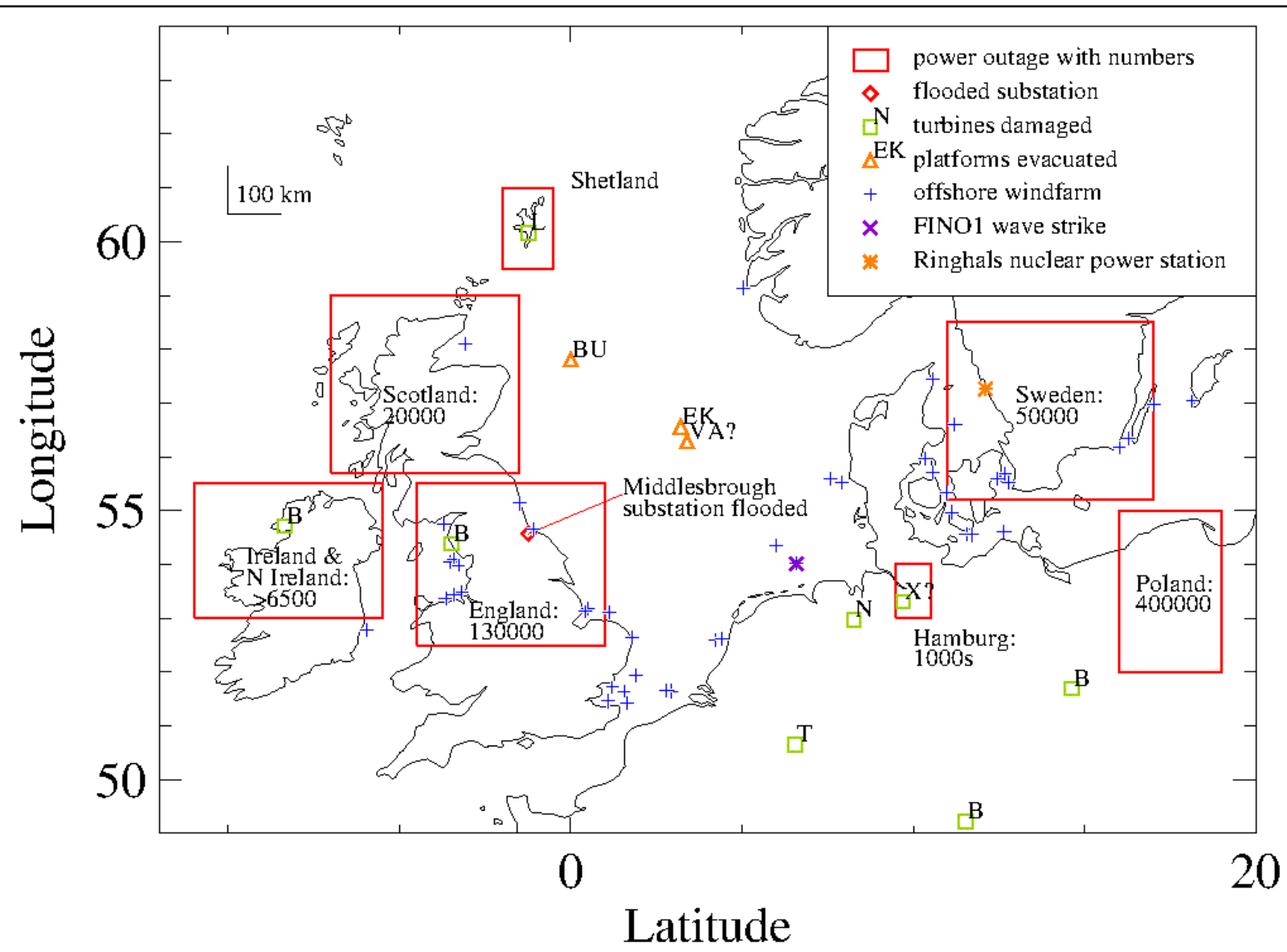

Figure S3. Thematic map of energy impacts by Storm Xaver on 5-6 Dec. 2013. The platform identifiers are: BU Buchan Alpha, EK Ekofisk, VA? Valhall, The wind turbine accident letter identifiers are: B blade damage, $L$ lightning strike, $T$ tower collapse, $X$ ? 'exploded'. The numbers are the number of customers (households and businesses) without power at some point during the storm. 


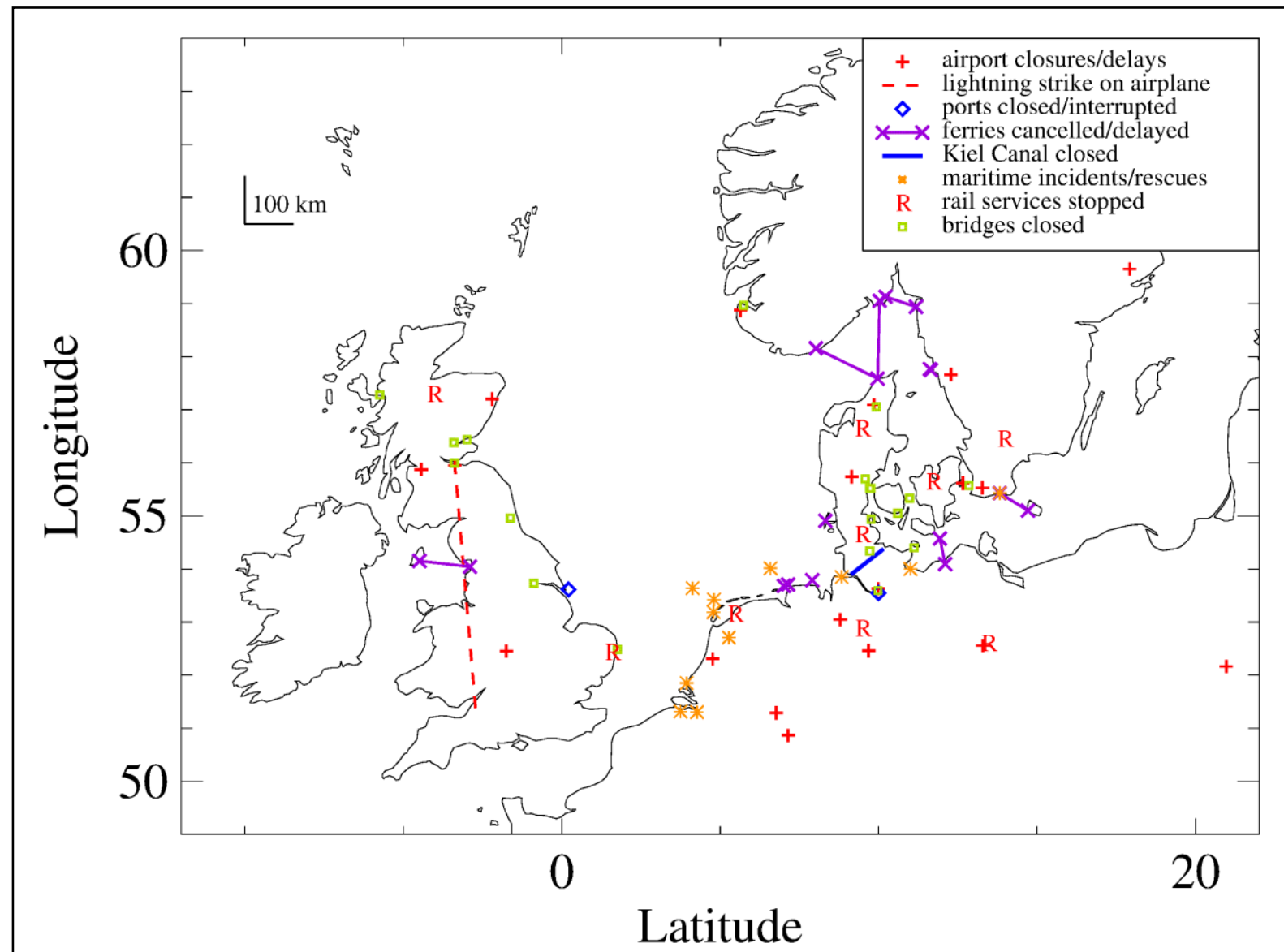

Figure S4. Thematic map of transportation impacts during Storm Xaver on 5-6 Dec. 2013. 


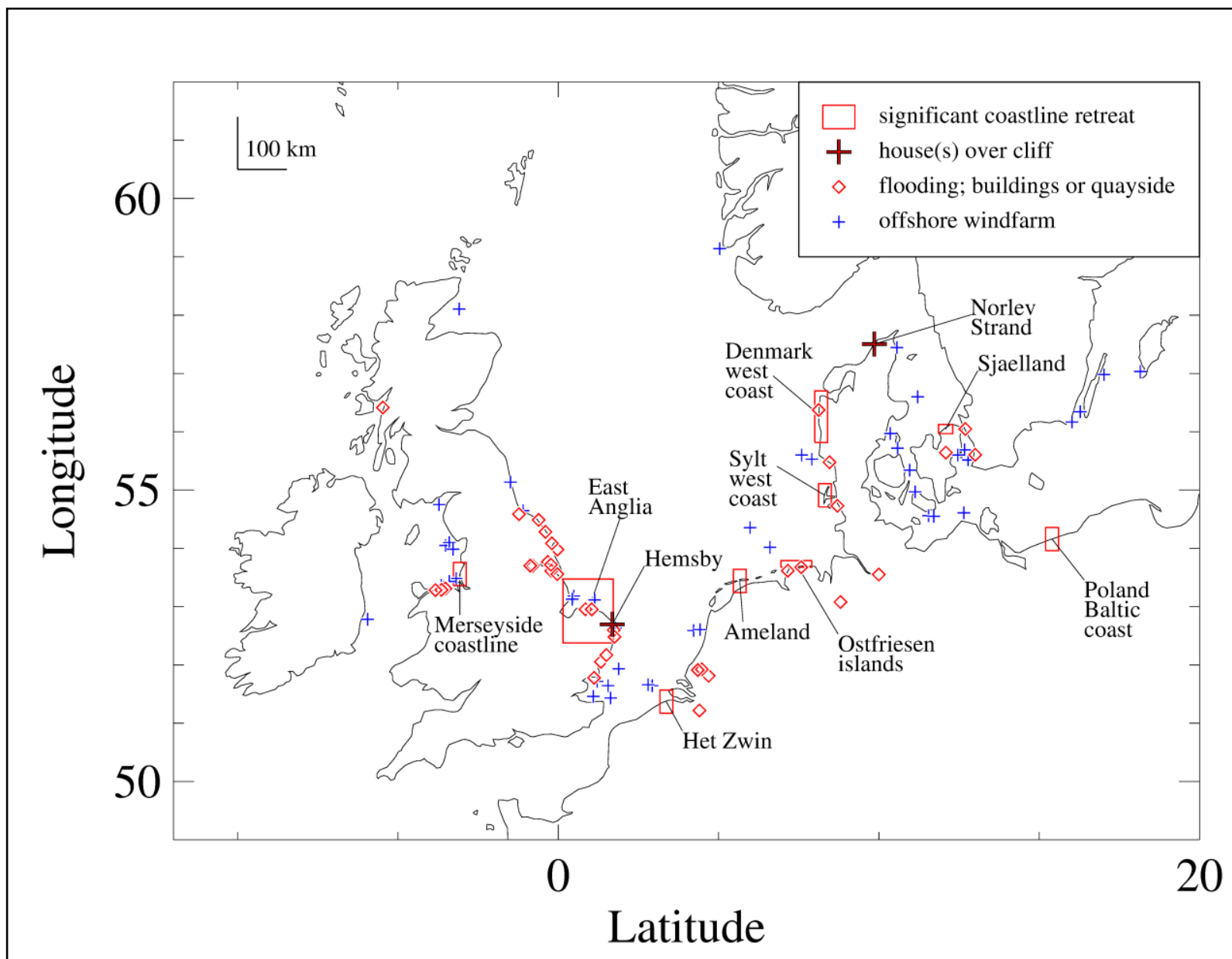

Figure S5. Thematic map of reported coastal damage and flooding during Storm Xaver on 5-6 Dec. 2013. 


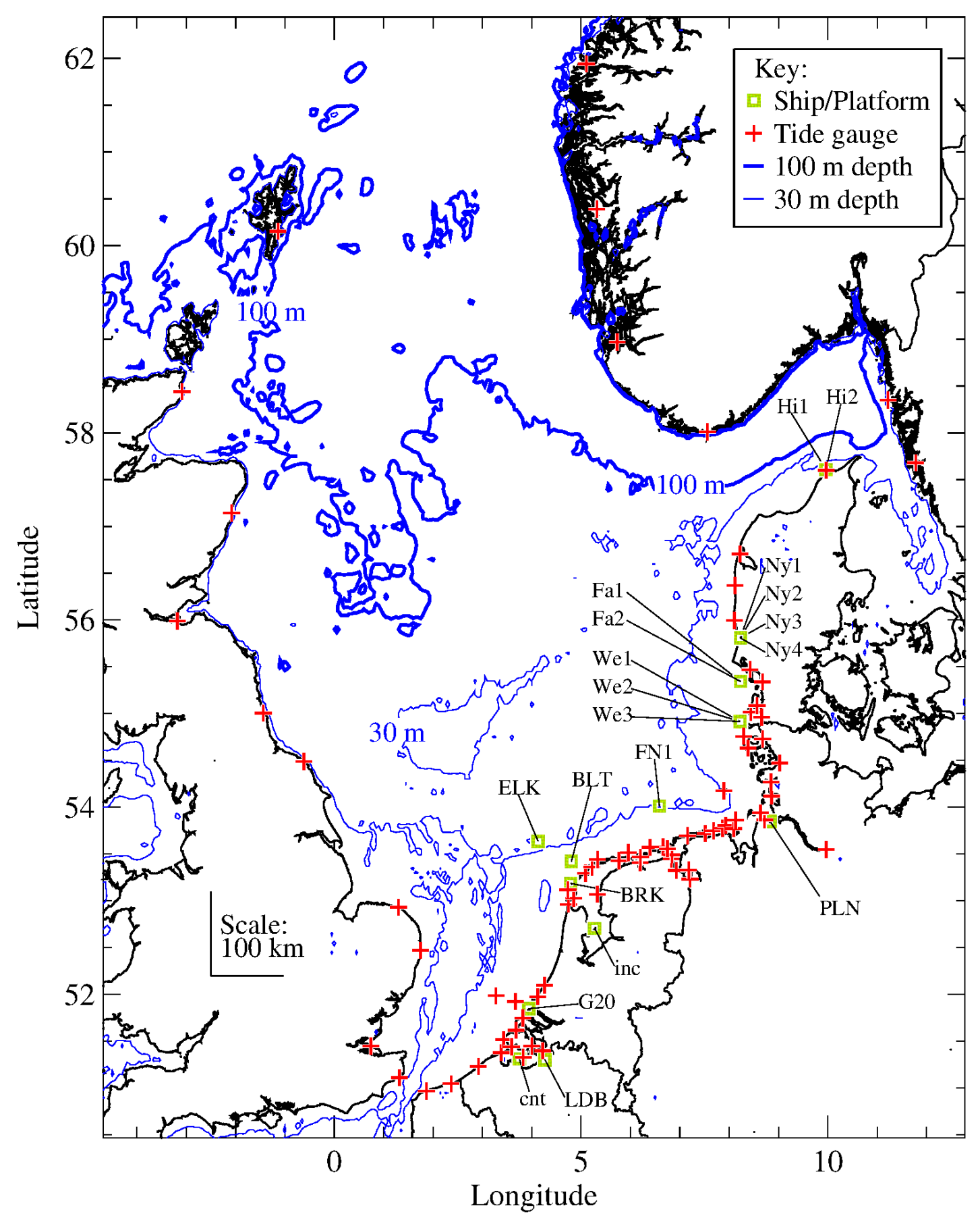

Figure S6. Map showing locations and identification of the North Sea offshore incidents during Storm Xaver of 5-6 Dec. 2013. 


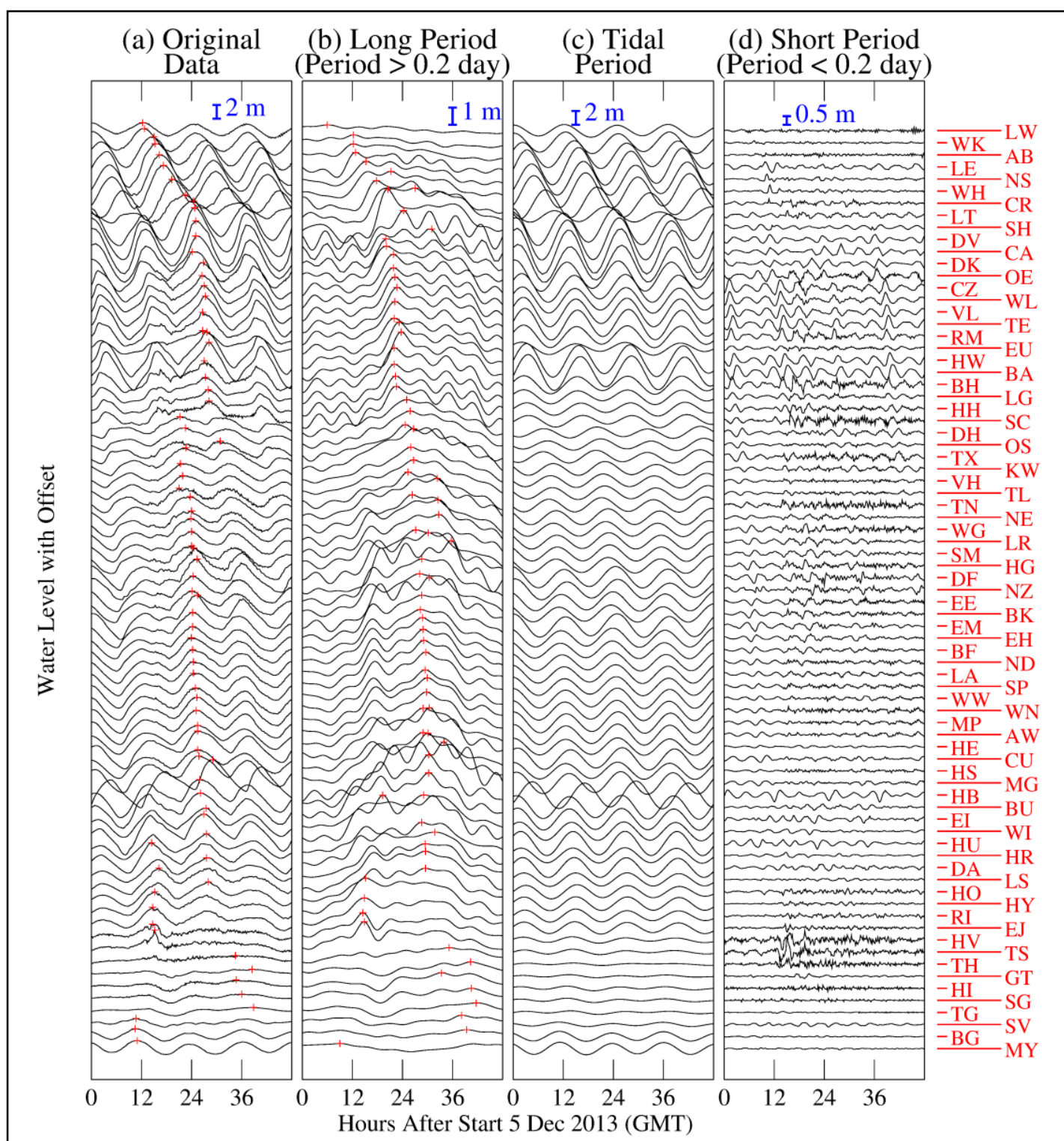

Figure S7. Time series of the (a) original water level data, and reconstructions of the (b) long period, (c) diurnal plus semi-diurnal tide, and (d) short period constructions of the original time series. The panels show all 77 water level stations that were analysed. The station identifications are given by two letter codes along right hand side of the panel, and the stations have been vertically offset according to counter-clockwise location around the North Sea starting from Lerwick in Scotland at the top and ending with Maløy on the Norwegian coast at the bottom. Red plus symbols in the first two panels indicate the maximum values during the two-day storm period. 


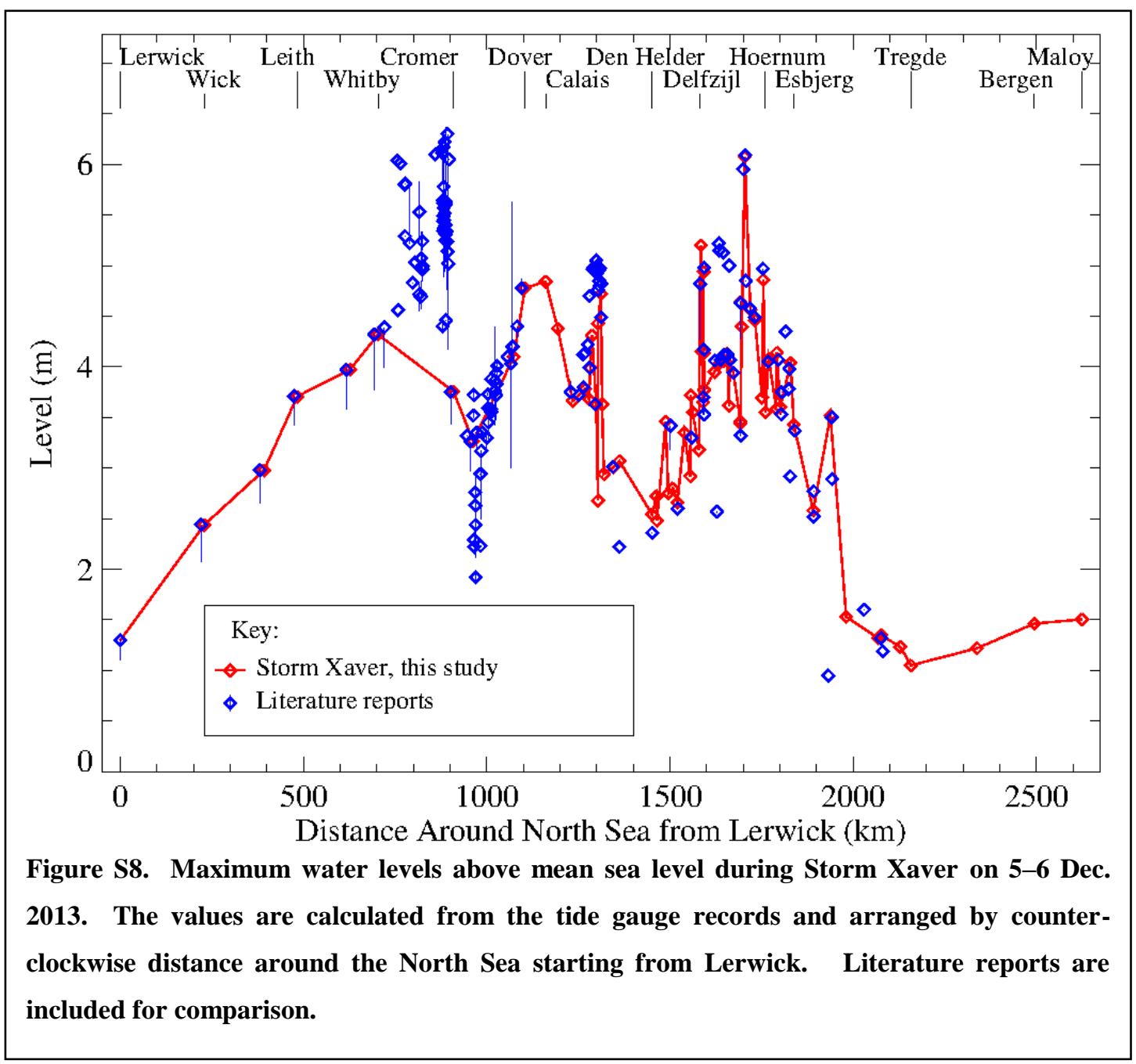




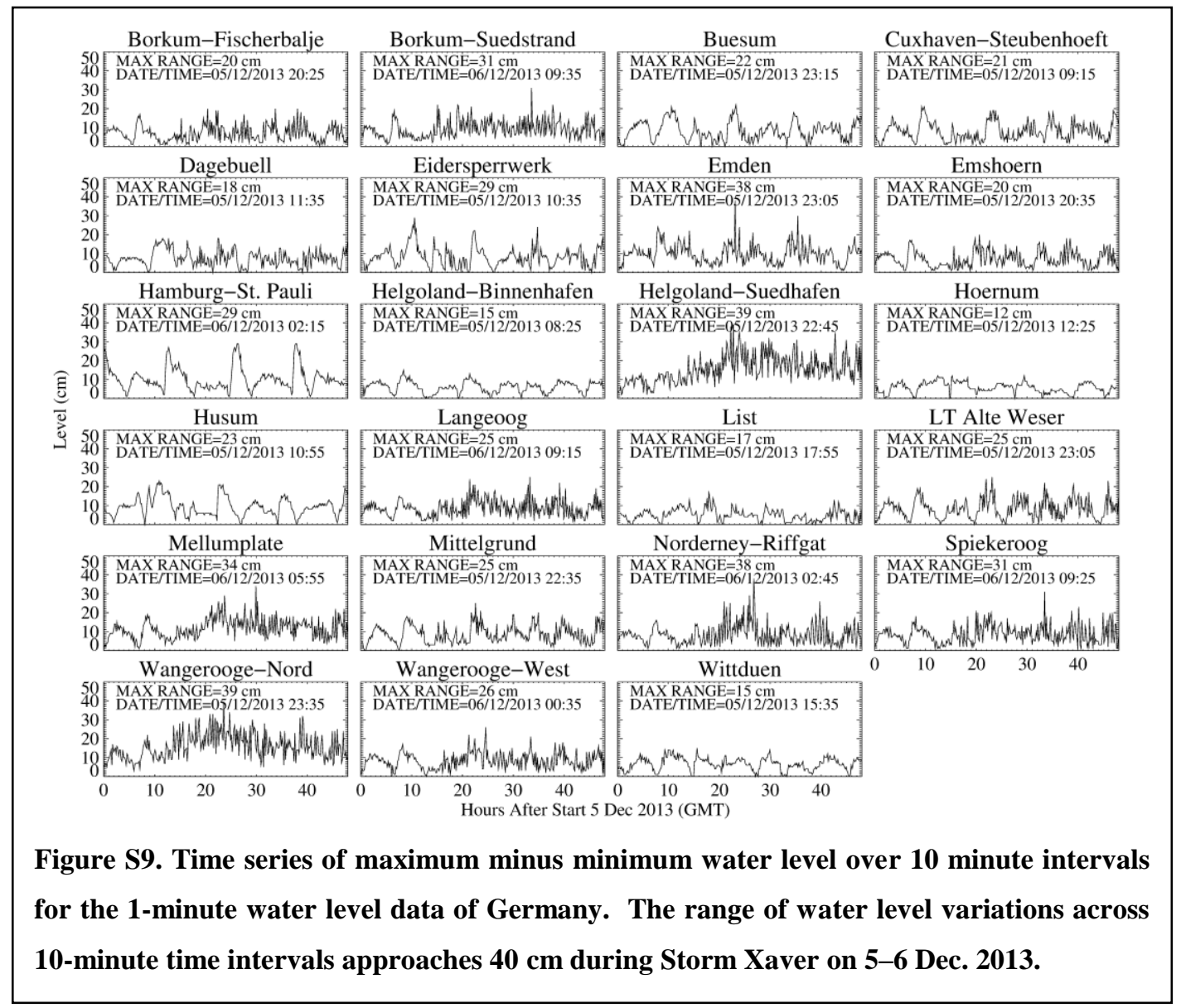




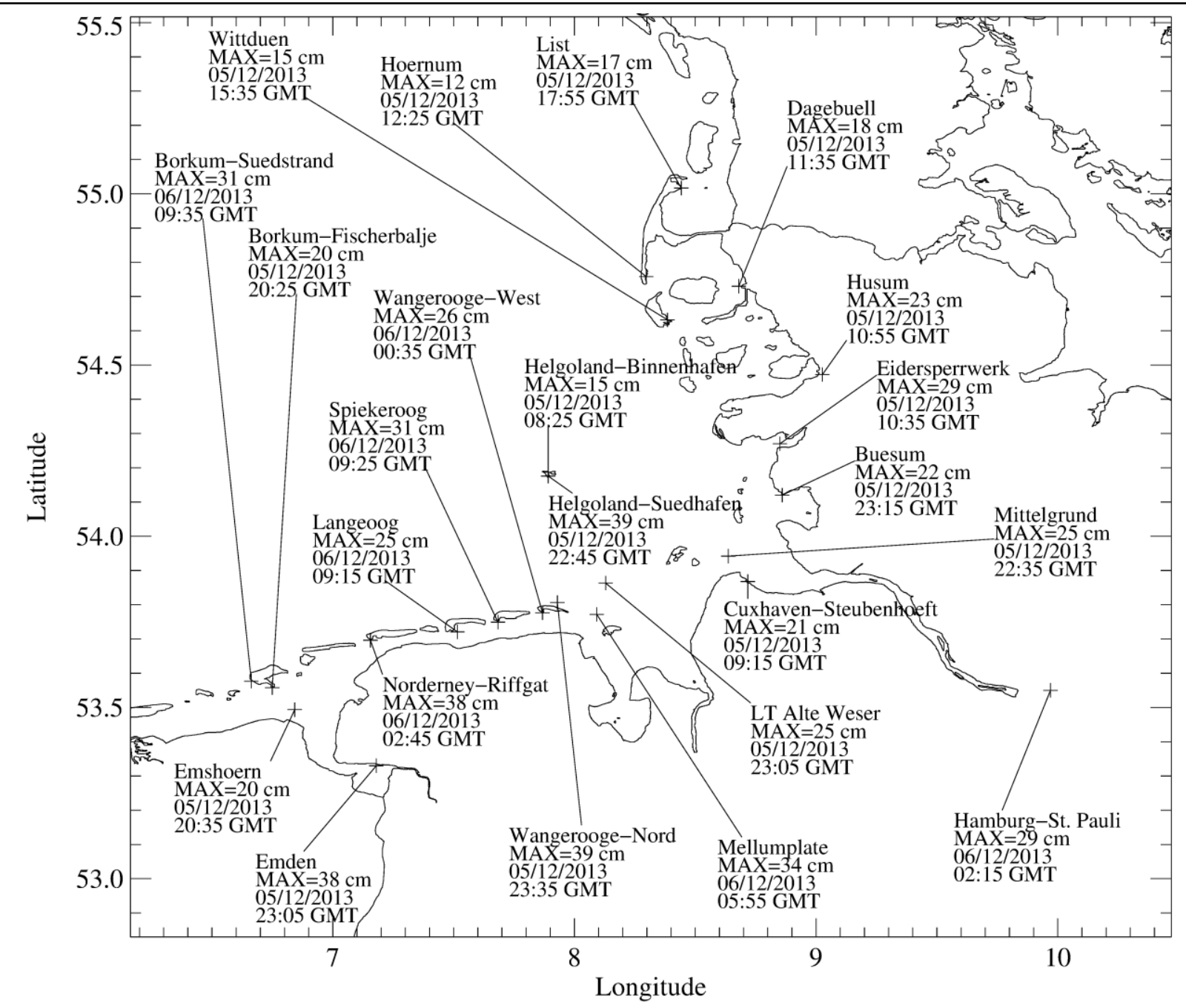

Figure S10. Map of the German tide gauge stations locations showing the maximum range of water level across 10 minute intervals from the original 1-minute time series data. The date and time of the maximum range is noted. 


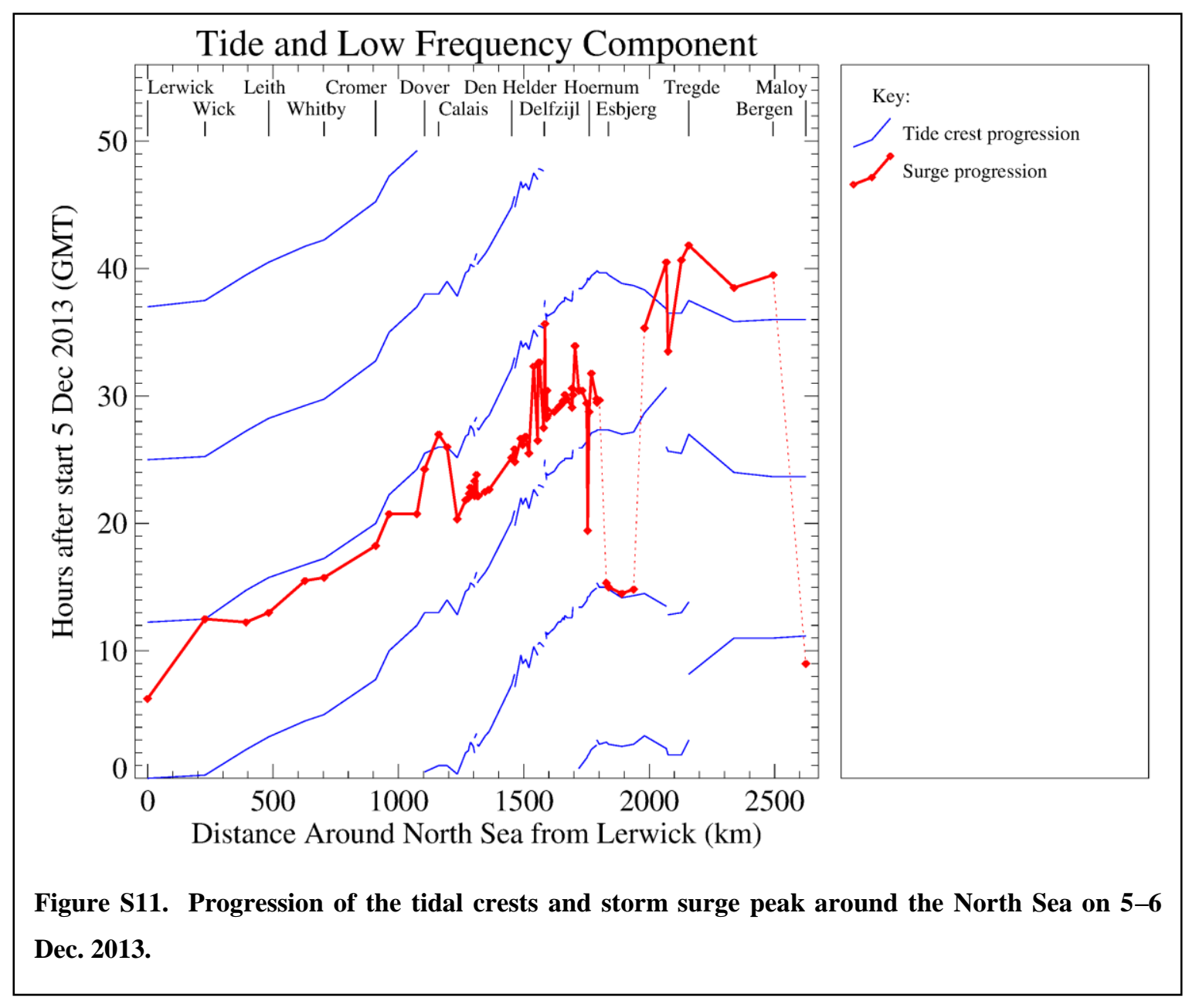




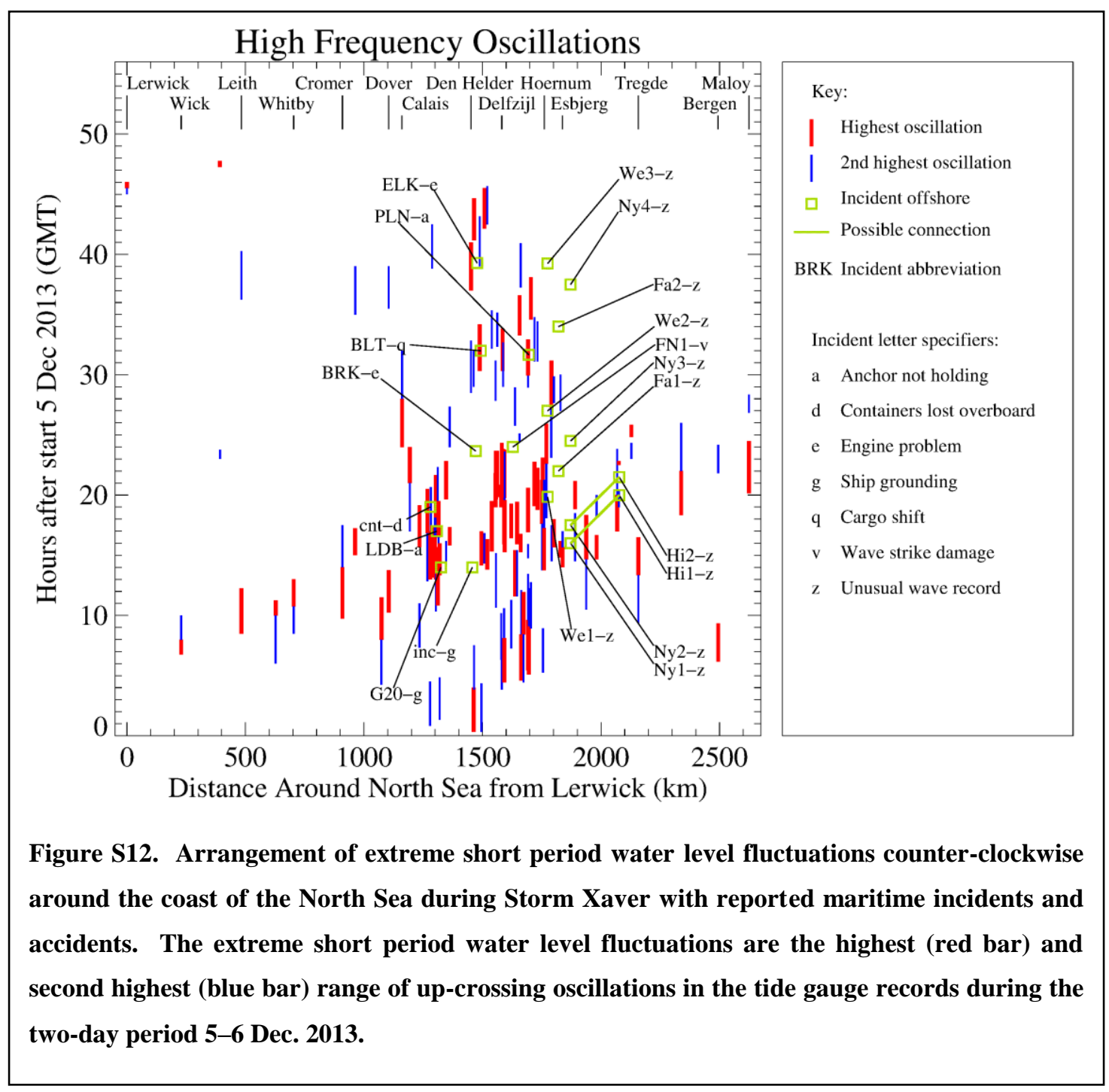


SECTION II. Supplementary tables

Table S1. Tide gauge information for 77 sites in the United Kingdom, France, Belgium, the Netherlands, Germany, Denmark, Sweden and Norway.

\begin{tabular}{|c|c|c|c|c|c|c|c|c|}
\hline$[1]$ & Station Name & $\mathrm{Abb}$ & $\begin{array}{l}\text { Coun } \\
\text { try } \\
{[4]}\end{array}$ & $\begin{array}{l}\text { Lati- } \\
\text { tude } \\
\text { (degree) } \\
{[5]}\end{array}$ & $\begin{array}{l}\text { Longi- } \\
\text { tude } \\
\text { (degree) } \\
{[6]}\end{array}$ & $\begin{array}{l}\Delta \mathrm{t} \\
\text { orig } \\
\text { (min) } \\
{[7]}\end{array}$ & $\begin{array}{l}\Delta \mathrm{t} \\
\text { use } \\
\text { (min) } \\
{[8]}\end{array}$ & $\begin{array}{l}\text { Source } \\
\text { [9] }\end{array}$ \\
\hline 1 & Lerwick & LW & UK & 60.15 & -1.14 & 15 & 15 & BODC \\
\hline 2 & Wick & WK & UK & 58.44 & -3.09 & 15 & 15 & BODC \\
\hline 3 & Aberdeen & $\mathrm{AB}$ & UK & 57.14 & -2.08 & 15 & 15 & BODC \\
\hline 4 & Leith & LE & UK & 55.99 & -3.18 & 15 & 15 & BODC \\
\hline 5 & North Shields & NS & UK & 55.01 & -1.44 & 15 & 15 & BODC \\
\hline 6 & Whitby & WH & UK & 54.49 & -0.61 & 15 & 15 & BODC \\
\hline 7 & Cromer & $\mathrm{CR}$ & UK & 52.93 & 1.30 & 15 & 15 & BODC \\
\hline 8 & Lowestoft & LT & UK & 52.47 & 1.75 & 15 & 15 & BODC \\
\hline 9 & Dover & DV & UK & 51.11 & 1.32 & 15 & 15 & BODC \\
\hline 10 & Calais & $\mathrm{CA}$ & FR & 50.97 & 1.87 & 60 & 60 & GESLA \\
\hline 11 & Dunkerque & DK & FR & 51.05 & 2.37 & 60 & 60 & GESLA \\
\hline 12 & Cadzand & $\mathrm{CZ}$ & $\mathrm{NE}$ & 51.38 & 3.38 & 10 & 10 & RWS \\
\hline 13 & Westkapelle & WL & $\mathrm{NE}$ & 51.52 & 3.43 & 10 & 10 & RWS \\
\hline 14 & Vlissingen & VL & $\mathrm{NE}$ & 51.44 & 3.60 & 10 & 10 & RWS \\
\hline 15 & Terneuzen & TE & $\mathrm{NE}$ & 51.33 & 3.83 & 10 & 10 & RWS \\
\hline 16 & Roompot buiten & $\mathrm{RM}$ & NE & 51.62 & 3.68 & 10 & 10 & RWS \\
\hline 17 & Hansweert & HW & $\mathrm{NE}$ & 51.45 & 4.00 & 10 & 10 & RWS \\
\hline 18 & Bath & BA & $\mathrm{NE}$ & 51.40 & 4.22 & 10 & 10 & RWS \\
\hline 19 & Euro platform & EU & NE & 51.99 & 3.28 & 10 & 10 & RWS \\
\hline 20 & Brouwershavensche Gat 08 & $\mathrm{BH}$ & $\mathrm{NE}$ & 51.75 & 3.82 & 10 & 10 & RWS \\
\hline 21 & Lichteiland Goeree & LG & NE & 51.93 & 3.67 & 10 & 10 & RWS \\
\hline 22 & Hoek van Holland & $\mathrm{HH}$ & $\mathrm{NE}$ & 51.98 & 4.12 & 10 & 10 & RWS \\
\hline 23 & Scheveningen & $\mathrm{SC}$ & $\mathrm{NE}$ & 52.10 & 4.26 & 10 & 10 & RWS \\
\hline 24 & Den Helder & $\mathrm{DH}$ & NE & 52.96 & 4.74 & 10 & 10 & RWS \\
\hline 25 & Oudeschild & OS & $\mathrm{NE}$ & 53.03 & 4.85 & 10 & 10 & RWS \\
\hline 26 & Texel Noordzee & TX & NE & 53.12 & 4.73 & 10 & 10 & RWS \\
\hline 27 & Kornwerderzand buiten & KW & $\mathrm{NE}$ & 53.07 & 5.33 & 10 & 10 & RWS \\
\hline 28 & Vlieland haven & $\mathrm{VH}$ & NE & 53.30 & 5.09 & 10 & 10 & RWS \\
\hline 29 & West-Terschelling & $\mathrm{TL}$ & $\mathrm{NE}$ & 53.36 & 5.22 & 10 & 10 & RWS \\
\hline 30 & Terschelling Noordzee & $\mathrm{TN}$ & $\mathrm{NE}$ & 53.44 & 5.33 & 10 & 10 & RWS \\
\hline 31 & Nes & $\mathrm{NE}$ & $\mathrm{NE}$ & 53.43 & 5.77 & 10 & 10 & RWS \\
\hline 32 & Wierumergronden & WG & NE & 53.52 & 5.96 & 10 & 10 & RWS \\
\hline 33 & Lauwersoog & LR & $\mathrm{NE}$ & 53.41 & 6.20 & 10 & 10 & RWS \\
\hline 34 & Schiermonnikoog & SM & NE & 53.47 & 6.20 & 10 & 10 & RWS \\
\hline 35 & Huibertgat & $\mathrm{HG}$ & NE & 53.57 & 6.40 & 10 & 10 & RWS \\
\hline 36 & Eemshaven & $\mathrm{EE}$ & $\mathrm{NE}$ & 53.45 & 6.83 & 10 & 10 & RWS \\
\hline 37 & Delfzijl & DF & NE & 53.33 & 6.93 & 10 & 10 & RWS \\
\hline 38 & Nieuwe Statenzijl & NZ & $\mathrm{NE}$ & 53.23 & 7.21 & 10 & 10 & RWS \\
\hline 39 & Borkum-Suedstrand & $\mathrm{BK}$ & $\mathrm{DE}$ & 53.58 & 6.66 & 1 & 10 & BAFG \\
\hline 40 & Emshoern & $\mathrm{EH}$ & $\mathrm{DE}$ & 53.49 & 6.84 & 1 & 10 & BAFG \\
\hline
\end{tabular}


Table S1 (continued)

\begin{tabular}{|c|c|c|c|c|c|c|c|c|}
\hline$[1]$ & Station Name & $\mathrm{Abb}$ & $\begin{array}{l}\text { Coun } \\
\text { try } \\
{[4]}\end{array}$ & $\begin{array}{l}\text { Lati- } \\
\text { tude } \\
\text { (degree) } \\
{[5]}\end{array}$ & $\begin{array}{l}\text { Longi- } \\
\text { tude } \\
\text { (degree) } \\
{[6]}\end{array}$ & $\begin{array}{l}\Delta \mathrm{t} \\
\text { orig } \\
\text { (min) } \\
{[7]}\end{array}$ & $\begin{array}{l}\Delta \mathrm{t} \\
\text { use } \\
\text { (min) } \\
{[8]}\end{array}$ & Source \\
\hline 41 & Borkum-Fischerbalje & $\mathrm{BF}$ & $\mathrm{DE}$ & 53.56 & 6.75 & 1 & 10 & BAFG \\
\hline 42 & Emden & EM & $\mathrm{DE}$ & 53.33 & 7.18 & 1 & 10 & BAFG \\
\hline 43 & Norderney-Riffgat & ND & $\mathrm{DE}$ & 53.70 & 7.16 & 1 & 10 & BAFG \\
\hline 44 & Langeoog & LA & $\mathrm{DE}$ & 53.72 & 7.52 & 1 & 10 & BAFG \\
\hline 45 & Spiekeroog & SP & $\mathrm{DE}$ & 53.75 & 7.68 & 1 & 10 & BAFG \\
\hline 46 & Wangerooge-West & WW & $\mathrm{DE}$ & 53.78 & 7.87 & 1 & 10 & BAFG \\
\hline 47 & Wangerooge-Nord & WN & $\mathrm{DE}$ & 53.81 & 7.93 & 1 & 10 & BAFG \\
\hline 48 & Mellumplate & MP & $\mathrm{DE}$ & 53.77 & 8.09 & 1 & 10 & BAFG \\
\hline 49 & LT Alte Weser & $\mathrm{AW}$ & $\mathrm{DE}$ & 53.86 & 8.13 & 1 & 10 & BAFG \\
\hline 50 & Helgoland-Suedhafen & $\mathrm{HS}$ & $\mathrm{DE}$ & 54.18 & 7.89 & 1 & 10 & BAFG \\
\hline 51 & Helgoland-Binnenhafen & $\mathrm{HE}$ & $\mathrm{DE}$ & 54.18 & 7.89 & 1 & 10 & BAFG \\
\hline 52 & Cuxhaven-Steubenhoeft & $\mathrm{CU}$ & $\mathrm{DE}$ & 53.87 & 8.72 & 1 & 10 & BAFG \\
\hline 53 & Mittelgrund & MG & $\mathrm{DE}$ & 53.94 & 8.64 & 1 & 10 & BAFG \\
\hline 54 & Hamburg-St. Pauli & $\mathrm{HB}$ & $\mathrm{DE}$ & 53.55 & 9.97 & 1 & 10 & BAFG \\
\hline 55 & Buesum & $\mathrm{BU}$ & $\mathrm{DE}$ & 54.12 & 8.86 & 1 & 10 & BAFG \\
\hline 56 & Eidersperrwerk & EI & $\mathrm{DE}$ & 54.27 & 8.85 & 1 & 10 & BAFG \\
\hline 57 & Husum & $\mathrm{HU}$ & $\mathrm{DE}$ & 54.47 & 9.03 & 1 & 10 & BAFG \\
\hline 58 & Wittduen & WI & $\mathrm{DE}$ & 54.63 & 8.39 & 1 & 10 & BAFG \\
\hline 59 & Dagebuell & DA & $\mathrm{DE}$ & 54.73 & 8.68 & 1 & 10 & BAFG \\
\hline 60 & Hoernum & $\mathrm{HR}$ & $\mathrm{DE}$ & 54.76 & 8.30 & 1 & 10 & BAFG \\
\hline 61 & List & $\mathrm{LS}$ & $\mathrm{DE}$ & 55.02 & 8.44 & 1 & 10 & BAFG \\
\hline 62 & Hojer & $\mathrm{HO}$ & DK & 54.96 & 8.66 & 10 & 10 & KDI \\
\hline 63 & Havneby & $\mathrm{HY}$ & DK & 55.09 & 8.57 & 10 & 10 & KDI \\
\hline 64 & Ribe & RI & DK & 55.34 & 8.68 & 10 & 10 & KDI \\
\hline 65 & Esbjerg & $\mathrm{EJ}$ & $\mathrm{DK}$ & 55.47 & 8.42 & 10 & 10 & KDI \\
\hline 66 & Hvide Sande (Havet) & $\mathrm{HV}$ & $\mathrm{DK}$ & 56.00 & 8.11 & 10 & 10 & KDI \\
\hline 67 & Thorsminde (Havn) & TS & DK & 56.37 & 8.12 & 10 & 10 & KDI \\
\hline 68 & Thyboron (Havn) & $\mathrm{TH}$ & $\mathrm{DK}$ & 56.71 & 8.22 & 10 & 10 & KDI \\
\hline 69 & Tregde & TG & NO & 58.01 & 7.56 & 10 & 10 & Kartv \\
\hline 70 & Stavanger & SV & NO & 58.97 & 5.73 & 10 & 10 & Kartv \\
\hline 71 & Bergen & $\mathrm{BG}$ & NO & 60.39 & 5.32 & 10 & 10 & Kartv \\
\hline 72 & Maloy & MY & NO & 61.94 & 5.11 & 10 & 10 & Kartv \\
\hline 73 & Sheerness & $\mathrm{SH}$ & UK & 51.45 & 0.74 & 15 & 15 & $\mathrm{IOC}$ \\
\hline 74 & Ostend & $\mathrm{OE}$ & $\mathrm{BE}$ & 51.23 & 2.92 & 5 & 10 & $\mathrm{IOC}$ \\
\hline 75 & Hirtshals & $\mathrm{HI}$ & DK & 57.60 & 9.97 & 10 & 10 & IOC \\
\hline 76 & GoteborgTorshamnen & GT & SW & 57.68 & 11.78 & 5 & 10 & IOC \\
\hline 77 & Smogen & SG & SW & 58.35 & 11.22 & 5 & 10 & IOC \\
\hline
\end{tabular}

Notes:

[1] Index

[2] Station name

[3] Station abbreviation used in the figures

[4] Country/Land abbreviations: UK United Kingdom, FR France, BE Belgium, NE Netherlands, DE Germany,

DK Denmark, SW Sweden, NO Norway

[5] Latitude

[6] Longitude

[7] Original data time interval in file from issuing source

[8] Data time interval used in the spectral analysis, averaging data from the issuing source where necessary 
[9] Issuing source abbreviations: BODC (British Oceanographic Data Centre) https://bodc.ac.uk/data/hosted_data_systems/sea_level/uk_tide_gauge_network/, GESLA (Global Extreme Sea Level Analysis) https://www.gesla.org, RWS (Rijkswatersaat Waterinfo) https://waterinfo.rws.nl/\#!/nav/expert/alle-groepen/ , BAFG (Bundesanstalt fuer Gewaesserkunde) email communication with Wilfried Wiechmann at Datenstelle-M1@bafg.de, KDI (Kystdirektoratet) https://kystatlas.kyst.dk/public2/data/vandstand/vandstand.html, Kartv (Kartverket) api.sehavniva.no/tideapi_en.html, IOC (Intergovernmental Oceanographic Commission, Sea level Monitoring Facility) www.ioc-sealevelmonitoring.org/index.php 
Table S2. Summary of maritime incidents in the North Sea area during Storm Xaver 5-6 Dec 2013.

Notes:

$\begin{array}{llllllll}\text { N } & \begin{array}{l}\text { Ship/Platform } \\ \begin{array}{l}\text { Name } \\ \text { or Incident } \\ {[2]}\end{array}\end{array} & \text { Abb } & \begin{array}{l}\text { Lati- } \\ \text { tude } \\ \text { [deg) } \\ {[4]}\end{array} & \begin{array}{l}\text { Longi- } \\ \text { tude } \\ \text { [deg }) \\ {[5]}\end{array} & \begin{array}{l}\text { Date } \\ \text { (GMT) } \\ \text { dddmm/yyyy } \\ {[6]}\end{array} & \begin{array}{l}\text { Time } \\ \text { GMT } \\ \text { hh:mm } \\ {[7]}\end{array} & \text { Source } \\ {[8]}\end{array}$

[1] Index

[2] Ship/platform name or incident identifier

[3] Abbreviation used in the figures

[4] Latitude

[5] Longitude

[6] Date of incident

[7] Time of incident

[8] Source:

- 24liveblog: Internet site: 24liveblog, Storm 5-6 december 2013 https://live.24liveblog.com/live/UYXaD, last access: 14 Apr 2020

- FINO1: Internet site: FINO1, 15-m wave damaged FINO1, 08Jan2014. http://www.fino1.de/meldungen/alle-meldungen/137-15-meter-welle-beschaedigt-fino1

- Fleet20131207: Internet site: Fleetmon, Container ship Burak Bayraktar in trouble off Texel, Netherlands, $\quad$ (correspondent: Mikhail Voytenko, 7Dec2013 06:31) https://www.fleetmon.com/maritime-news/2013/2810/container-ship-burak-bayraktar-trouble-texelnethe/

- Granneman: Email communication with Edwin Granneman, Netherlands Coastguard, 27 Feb. 2020

- GVA20131205: GVA, Vijf containers even op drift op de Schelde, 05/12/2013 20:57. https://www.gva.be/cnt/aid1500792/vijf-containers-op-drift-op-de-schelde-2

- Reemts: Email communication with Antke Reemts of the German Maritime Search and Rescue Service, 20 Sep. 2018 
- DMI: Analysis of data from Denmark's Kystdirektoratet website (https://kyst.dk/soeterritoriet/maalinger-og-data/

- LSH: Digitized time series data of significant wave height from Luecht, Fabian and Ove Peters, Bericht über die Sturmflut vom 05.-0.6.12.2013 an der Westküste Schleswig-Holsteins, Landesbetrieb für Küstenschutz, Nationalpark und Meeresschutz Schleswig-Holstein, Husum 26Feb2014, 19pp. [pdf document properties: title=Lfd; Author=Thorsten Nommensen; datestamp: 11Dec2018] 
Table S3. Sorted list of maximum ranges of up-crossing oscillations derived from the short period time series reconstructions for each North Sea tide gauge station of this analysis.

\begin{tabular}{|c|c|c|c|c|}
\hline $\mathrm{N}$ & Station Name & $\begin{array}{l}\text { Range } \\
(\mathrm{cm})\end{array}$ & $\begin{array}{l}\text { Midpoint of } \\
\text { Oscillation } \\
\text { (h after start } \\
5 \text { Dec 2013) }\end{array}$ & $\begin{array}{l}\text { Duration of } \\
\text { Oscillation } \\
\text { (h) }\end{array}$ \\
\hline 1 & Thorsminde (Havn) & 97.1 & 14.58 & 2.83 \\
\hline 2 & Hvide Sande (Havet) & 53.5 & 19.67 & 2.00 \\
\hline 3 & Brouwershavensche Gat 08 & 51.1 & 16.42 & 3.17 \\
\hline 4 & Terneuzen & 51.0 & 38.58 & 3.50 \\
\hline 5 & Cadzand & 50.7 & 37.67 & 3.67 \\
\hline 6 & Westkapelle & 50.3 & 12.92 & 3.50 \\
\hline 7 & Bath & 45.9 & 39.67 & 3.67 \\
\hline 8 & Roompot buiten & 45.7 & 13.08 & 3.17 \\
\hline 9 & Scheveningen & 45.3 & 15.92 & 1.50 \\
\hline 10 & Calais & 45.2 & 27.50 & 3.00 \\
\hline 11 & Hansweert & 44.9 & 39.00 & 3.67 \\
\hline 12 & Ostend & 43.1 & 35.83 & 3.33 \\
\hline 13 & Vlissingen & 42.2 & 13.17 & 3.67 \\
\hline 14 & Whitby & 36.9 & 10.38 & 2.25 \\
\hline 15 & Huibertgat & 34.7 & 18.75 & 3.17 \\
\hline 16 & Delfzijl & 34.3 & 32.67 & 2.00 \\
\hline 17 & Thyboron (Havn) & 34.0 & 14.83 & 2.00 \\
\hline 18 & Wierumergronden & 33.3 & 19.42 & 1.50 \\
\hline 19 & Texel Noordzee & 33.1 & 41.08 & 3.17 \\
\hline 20 & Hoek van Holland & 31.9 & 19.83 & 2.67 \\
\hline 21 & Dunkerque & 31.6 & 21.00 & 4.00 \\
\hline 22 & Terschelling Noordzee & 31.6 & 13.33 & 4.33 \\
\hline 23 & North Shields & 29.3 & 9.50 & 2.50 \\
\hline 24 & Dover & 29.2 & 35.38 & 3.25 \\
\hline 25 & Leith & 29.2 & 8.63 & 4.25 \\
\hline 26 & Hamburg-St. Pauli & 28.6 & 26.02 & 3.50 \\
\hline 27 & Nieuwe Statenzijl & 27.9 & 21.58 & 2.83 \\
\hline 28 & Lowestoft & 27.4 & 15.00 & 1.00 \\
\hline 29 & Borkum-Suedstrand & 27.1 & 14.43 & 2.33 \\
\hline 30 & Den Helder & 26.7 & 3.67 & 3.33 \\
\hline 31 & Cromer & 26.0 & 10.38 & 4.75 \\
\hline 32 & Eemshaven & 25.8 & 33.58 & 1.50 \\
\hline 33 & Schiermonnikoog & 24.7 & 19.67 & 4.00 \\
\hline 34 & Emshoern & 24.5 & 19.93 & 3.67 \\
\hline 35 & Lauwersoog & 24.0 & 19.58 & 4.17 \\
\hline 36 & Wangerooge-Nord & 23.7 & 15.35 & 1.50 \\
\hline 37 & Husum & 23.3 & 5.10 & 4.00 \\
\hline 38 & Emden & 22.7 & 8.35 & 3.83 \\
\hline 39 & Esbjerg & 21.9 & 14.33 & 1.00 \\
\hline 40 & Eidersperrwerk & 21.8 & 22.52 & 3.50 \\
\hline
\end{tabular}


Table S3a (continued)

\begin{tabular}{|c|c|c|c|c|}
\hline $\mathrm{N}$ & Station Name & $\begin{array}{l}\text { Range } \\
(\mathrm{cm})\end{array}$ & $\begin{array}{l}\text { Midpoint of } \\
\text { Oscillation } \\
\text { (h after start } \\
5 \text { Dec 2013) }\end{array}$ & $\begin{array}{l}\text { Duration of } \\
\text { Oscillation } \\
\text { (h) }\end{array}$ \\
\hline 41 & Kornwerderzand buiten & 21.7 & 30.17 & 4.00 \\
\hline 42 & Cuxhaven-Steubenhoeft & 20.2 & 9.52 & 3.50 \\
\hline 43 & Lerwick & 19.9 & 45.50 & 0.50 \\
\hline 44 & Hojer & 19.8 & 29.42 & 1.50 \\
\hline 45 & Dagebuell & 19.1 & 22.43 & 3.67 \\
\hline 46 & $\mathrm{Nes}$ & 19.1 & 24.33 & 2.00 \\
\hline 47 & Ribe & 18.9 & 27.17 & 1.67 \\
\hline 48 & Borkum-Fischerbalje & 18.9 & 7.02 & 3.50 \\
\hline 49 & Norderney-Riffgat & 18.7 & 7.27 & 3.33 \\
\hline 50 & Langeoog & 18.6 & 15.02 & 3.17 \\
\hline 51 & Hirtshals & 18.5 & 23.75 & 0.83 \\
\hline 52 & West-Terschelling & 18.5 & 41.92 & 3.83 \\
\hline 53 & Lichteiland Goeree & 18.5 & 13.25 & 3.50 \\
\hline 54 & Hoernum & 18.4 & 13.52 & 3.83 \\
\hline 55 & Buesum & 18.4 & 22.68 & 3.50 \\
\hline 56 & Mellumplate & 18.0 & 45.68 & 3.17 \\
\hline 57 & Spiekeroog & 17.8 & 16.43 & 1.33 \\
\hline 58 & Sheerness & 17.2 & 8.00 & 3.50 \\
\hline 59 & Oudeschild & 17.0 & 4.00 & 3.33 \\
\hline 60 & LT Alte Weser & 16.6 & 8.35 & 3.50 \\
\hline 61 & GoteborgTorshamnen & 15.0 & 19.75 & 3.17 \\
\hline 62 & Wangerooge-West & 14.4 & 15.43 & 3.33 \\
\hline 63 & Havneby & 14.1 & 15.58 & 2.83 \\
\hline 64 & Wittduen & 13.5 & 13.60 & 4.00 \\
\hline 65 & Euro platform & 13.5 & 26.83 & 1.67 \\
\hline 66 & Vlieland haven & 13.1 & 14.75 & 1.83 \\
\hline 67 & Smogen & 13.0 & 25.08 & 1.17 \\
\hline 68 & Wick & 12.9 & 6.62 & 2.25 \\
\hline 69 & Mittelgrund & 12.5 & 9.27 & 3.67 \\
\hline 70 & Aberdeen & 11.9 & 23.63 & 0.75 \\
\hline 71 & Stavanger & 11.3 & 9.67 & 3.33 \\
\hline 72 & Helgoland-Suedhafen & 10.3 & 30.27 & 1.33 \\
\hline 73 & List & 8.4 & 27.27 & 4.00 \\
\hline 74 & Helgoland-Binnenhafen & 7.9 & 8.10 & 3.67 \\
\hline 75 & Tregde & 5.7 & 13.25 & 3.50 \\
\hline 76 & Maloy & 5.6 & 8.92 & 2.50 \\
\hline 77 & Bergen & 4.9 & 6.08 & 3.50 \\
\hline
\end{tabular}


Table S4. Sorted list of return periods of water levels and references.

\begin{tabular}{|c|c|c|c|c|c|c|}
\hline $\mathrm{N}$ & $\begin{array}{l}\text { Location } \\
{[2]}\end{array}$ & $\begin{array}{l}\text { Coun } \\
\text { try } \\
{[3]}\end{array}$ & $\begin{array}{l}\text { Lati- } \\
\text { tude } \\
\text { (degree) } \\
{[4]}\end{array}$ & $\begin{array}{l}\text { Longi- } \\
\text { tude } \\
\text { (degree) } \\
{[5]}\end{array}$ & $\begin{array}{l}\text { Return } \\
\text { Period } \\
\text { (year) } \\
{[6]}\end{array}$ & $\begin{array}{l}\text { Source } \\
{[7]}\end{array}$ \\
\hline 1 & Thornham & UK & 52.96 & 0.58 & 4578 & WEA15 \\
\hline 2 & Blakeney & UK & 52.95 & 1.01 & 1876 & WEA15 \\
\hline 3 & Spurn Head & UK & 53.58 & 0.12 & 1800 & WEA15 \\
\hline 4 & Burnham Overy Staithe & UK & 52.96 & 0.75 & 1736 & WEA15 \\
\hline 5 & Scolt Head & UK & 52.98 & 0.71 & 1513 & WEA15 \\
\hline 6 & Stiffkey & UK & 52.95 & 0.93 & 1331 & WEA15 \\
\hline 7 & Hull & UK & 53.73 & -0.27 & 1285 & WEA15 \\
\hline 8 & Rye & UK & 50.95 & 0.74 & 1166 & WEA15 \\
\hline 9 & Roskilde Havn & DK & 55.70 & 12.10 & 976 & DEA19 \\
\hline 10 & Dover & UK & 51.11 & 1.32 & 843 & SW20 \\
\hline 11 & Bridlington & UK & 54.08 & 0.18 & 824 & WEA15 \\
\hline 12 & Kings Lynn & UK & 52.76 & 0.39 & 800 & WEA15 \\
\hline 13 & Immingham & UK & 53.63 & -0.19 & 787 & SW20 \\
\hline 14 & Boston & UK & 52.98 & -0.03 & 707 & WEA15 \\
\hline 15 & Wells-Next-The-Sea & UK & 52.95 & 0.85 & 692 & WEA15 \\
\hline 16 & Scarborough & UK & 54.28 & -0.39 & 626 & WEA15 \\
\hline 17 & Whitby & UK & 54.49 & -0.61 & 588 & WEA15 \\
\hline 18 & Pevensey Bay & UK & 50.82 & 0.34 & 449 & WEA15 \\
\hline 19 & North Shields & UK & 55.01 & -1.44 & 429 & WEA15 \\
\hline 20 & Thorsminde Havet & DK & 56.40 & 8.10 & 407 & DEA19 \\
\hline 21 & Kobenhavns Havn & DK & 55.70 & 12.60 & 285 & DEA19 \\
\hline 22 & Thorsminde Havn & DK & 56.40 & 8.10 & 243 & DEA19 \\
\hline 23 & Hornbaek Havn & DK & 56.10 & 12.50 & 220 & DEA19 \\
\hline 24 & Lowestoft & UK & 52.47 & 1.75 & 196 & WEA $15 b$ \\
\hline 25 & Holbaek Havn & DK & 55.70 & 11.70 & 187 & DEA19 \\
\hline 26 & Hals & DK & 57.00 & 10.30 & 169 & DEA19 \\
\hline 27 & Aldeburgh (Slaughden) & UK & 52.16 & 1.60 & 164 & WEA15 \\
\hline 28 & Great Yarmouth & UK & 52.57 & 1.73 & 146 & WEA15 \\
\hline 29 & Deal Pier & UK & 51.22 & 1.41 & 79 & WEA15 \\
\hline 30 & Arun Platform & UK & 50.77 & -0.49 & 75 & WEA15 \\
\hline 31 & Delfzijl & $\mathrm{NE}$ & 53.33 & 6.93 & 66 & RWS14b \\
\hline 32 & Brons Sluse Havet & DK & 55.20 & 8.70 & 63 & DEA19 \\
\hline 33 & Sandown & UK & 50.65 & -1.15 & 49 & WEA15 \\
\hline 34 & Liverpool & UK & 53.45 & -3.02 & 43 & SW20 \\
\hline 35 & Havnebyen/Sjaellands Odde & DK & 56.00 & 11.40 & 42 & DEA19 \\
\hline
\end{tabular}


Table S4 (continued)

\begin{tabular}{|c|c|c|c|c|c|c|}
\hline [1] & $\begin{array}{l}\text { Location } \\
{[2]}\end{array}$ & $\begin{array}{l}\text { Coun } \\
\text { try } \\
{[3]}\end{array}$ & $\begin{array}{l}\text { Lati- } \\
\text { tude } \\
\text { (degree) } \\
{[4]}\end{array}$ & $\begin{array}{l}\text { Longi- } \\
\text { tude } \\
\text { (degree) } \\
{[5]}\end{array}$ & $\begin{array}{l}\text { Return } \\
\text { Period } \\
\text { (year) } \\
{[6]}\end{array}$ & $\begin{array}{l}\text { Source } \\
{[7]}\end{array}$ \\
\hline 36 & Newhaven & UK & 50.78 & 0.06 & 42 & WEA15 \\
\hline 37 & Herne Bay & UK & 51.38 & 1.12 & 39 & WEA15 \\
\hline 38 & Southwold & UK & 52.33 & 1.68 & 39 & WEA15 \\
\hline 39 & Calshot & UK & 50.82 & -1.30 & 34 & WEA15 \\
\hline 40 & Odense Fjord (Gabet) & DK & 55.50 & 10.60 & 33 & DEA19 \\
\hline 41 & Nr. Sundby & DK & 57.10 & 9.90 & 31 & DEA19 \\
\hline 42 & Mando Havet & DK & 55.30 & 8.60 & 29 & DEA19 \\
\hline 43 & Clacton-on-Sea & UK & 51.79 & 1.16 & 29 & WEA15 \\
\hline 44 & Harwich & UK & 51.95 & 1.29 & 29 & WEA15 \\
\hline 45 & Tilbury & UK & 51.45 & 0.37 & 24 & WEA15 \\
\hline 46 & Slipshavn & DK & 55.30 & 10.80 & 23 & DEA19 \\
\hline 47 & Grenaa Havn & DK & 56.40 & 10.90 & 22 & DEA19 \\
\hline 48 & Ballen Havn & DK & 55.80 & 10.60 & 22 & DEA19 \\
\hline 49 & Juelsminde Havn & DK & 55.70 & 10.00 & 22 & DEA19 \\
\hline 50 & Leith & UK & 55.99 & -3.18 & 22 & WEA15 \\
\hline 51 & Aberdeen & UK & 57.14 & -2.08 & 20 & SW20 \\
\hline 52 & Cromer & UK & 52.93 & 1.30 & 20 & WEA15 \\
\hline 53 & Hanstholm & DK & 57.10 & 8.60 & 19 & DEA19 \\
\hline 54 & Aarhus Havn & DK & 56.10 & 10.20 & 19 & DEA19 \\
\hline 55 & Vlissingen & NE & 51.45 & 3.60 & 18 & RWS14b \\
\hline 56 & Shoreham & UK & 53.83 & -0.25 & 18 & WEA15 \\
\hline 57 & Southend-on-Sea & UK & 51.52 & 0.72 & 17 & WEA15 \\
\hline 58 & Kalundborg Havn & DK & 55.70 & 11.10 & 15 & DEA19 \\
\hline 59 & Lemvig Havn & DK & 56.60 & 8.30 & 15 & DEA19 \\
\hline 60 & Sheerness & UK & 51.45 & 0.74 & 15 & WEA 15 \\
\hline 61 & Llandudno & UK & 53.33 & -3.83 & 15 & SW20 \\
\hline 62 & Attrup & DK & 57.00 & 9.50 & 13 & DEA19 \\
\hline 63 & Roompot buiten & NE & 51.62 & 3.67 & 12 & RWS14b \\
\hline 64 & Vidaaslusen-Hojer I & DK & 55.00 & 8.70 & 12 & DEA19 \\
\hline 65 & Graadyb Barre & DK & 55.40 & 8.30 & 12 & DEA19 \\
\hline 66 & Portsmouth & UK & 50.80 & -1.11 & 12 & SW20 \\
\hline 67 & Bogense Havn & DK & 55.60 & 10.10 & 11 & DEA19 \\
\hline 68 & Korsor Havn & DK & 55.30 & 11.10 & 10 & DEA19 \\
\hline 69 & Kerteminde Havn & DK & 55.50 & 10.70 & 10 & DEA19 \\
\hline 70 & Southampton & UK & 50.88 & -1.40 & 10 & WEA15 \\
\hline
\end{tabular}


Table S4 (continued)

\begin{tabular}{|c|c|c|c|c|c|c|}
\hline [1] & $\begin{array}{l}\text { Location } \\
{[2]}\end{array}$ & $\begin{array}{l}\text { Coun } \\
\text { try } \\
{[3]}\end{array}$ & $\begin{array}{l}\text { Lati- } \\
\text { tude } \\
\text { (degree) } \\
{[4]}\end{array}$ & $\begin{array}{l}\text { Longi- } \\
\text { tude } \\
\text { (degree) } \\
{[5]}\end{array}$ & $\begin{array}{l}\text { Return } \\
\text { Period } \\
\text { (year) } \\
{[6]}\end{array}$ & $\begin{array}{l}\text { Source } \\
{[7]}\end{array}$ \\
\hline 71 & Ullapool & UK & 57.90 & -5.16 & 9.0 & SW20 \\
\hline 72 & Randers Havn & DK & 56.50 & 10.00 & 8.9 & DEA19 \\
\hline 73 & Hirtshals Havn & DK & 57.60 & 10.00 & 8.8 & DEA19 \\
\hline 74 & Skagen & DK & 57.70 & 10.60 & 8.6 & DEA19 \\
\hline 75 & Hoek van Holland & $\mathrm{NE}$ & 51.98 & 4.12 & 8.3 & RWS14b \\
\hline 76 & Dordrecht & $\mathrm{NE}$ & 51.82 & 4.67 & 7.7 & RWS14b \\
\hline 77 & Hvide Sande Havn & DK & 56.00 & 8.10 & 6.6 & DEA19 \\
\hline 78 & Fredericia Havn & DK & 55.60 & 9.80 & 5.3 & DEA19 \\
\hline 79 & Ribe Kammersluse Flyder & DK & 55.30 & 8.70 & 4.5 & DEA19 \\
\hline 80 & Antwerpen & $\mathrm{BE}$ & 51.22 & 4.40 & 4.5 & NEA14 \\
\hline 81 & Karrebaeksminde & DK & 55.20 & 11.60 & 4.4 & DEA19 \\
\hline 82 & Den Helder & $\mathrm{NE}$ & 52.97 & 4.75 & 4.2 & RWS14b \\
\hline 83 & Heysham & UK & 54.03 & -2.92 & 4.0 & WEA15 \\
\hline 84 & Tobermory & UK & 56.62 & -6.06 & 4.0 & WEA15 \\
\hline 85 & Esbjerg Havn & DK & 55.50 & 8.40 & 3.8 & DEA19 \\
\hline 86 & Skive Havn & DK & 56.60 & 9.10 & 3.1 & DEA19 \\
\hline 87 & Ballum Sluse & DK & 55.10 & 8.70 & 3.0 & DEA19 \\
\hline 88 & Holyhead & UK & 53.31 & -4.62 & 3.0 & WEA15 \\
\hline 89 & Yarmouth & UK & 52.57 & 1.73 & 3.0 & WEA15 \\
\hline 90 & Havneby Havn & DK & 55.10 & 8.60 & 2.9 & DEA19 \\
\hline 91 & Harlingen & $\mathrm{NE}$ & 53.17 & 5.42 & 2.9 & RWS14b \\
\hline 92 & Lymington & UK & 50.74 & -1.51 & 2.0 & WEA15 \\
\hline 93 & Hvide Sande Havet & DK & 56.00 & 8.10 & 1.8 & DEA19 \\
\hline 94 & Drogden Fyr & DK & 55.50 & 12.70 & 1.3 & DEA19 \\
\hline 95 & Logster & DK & 57.00 & 9.20 & 1.2 & DEA19 \\
\hline 96 & Koege Havn & DK & 55.50 & 12.20 & 1.0 & DEA19 \\
\hline 97 & Stornaway & UK & 58.21 & -6.39 & 1.0 & WEA15 \\
\hline 98 & Kinlochbervie & UK & 58.46 & -5.05 & 1.0 & WEA15 \\
\hline 99 & Wick & UK & 58.44 & -3.09 & 1.0 & WEA15 \\
\hline 100 & West Bay Harbour & UK & 50.71 & -2.76 & 1.0 & WEA15 \\
\hline 101 & Kloster Havn & DK & 56.30 & 8.30 & 0.25 & DEA19 \\
\hline 102 & Roedvig Havn & DK & 55.30 & 12.40 & 0.22 & DEA19 \\
\hline 103 & Hvalpsund & DK & 56.70 & 9.20 & 0.09 & DEA19 \\
\hline 104 & Kalvehave & DK & 55.00 & 12.20 & 0.06 & DEA19 \\
\hline
\end{tabular}

Notes:

[1] Index

[2] Location

[3] Country code

[4] Latitude

[5] Longitude

[6] Return period (years)

[8] Source:

- DEA19: Ditlevsen C, MM Ramos, C Sorensen, UR Ciocan, T Pionkowitz, Hojvandsstatistikker 2017, Miljo- og Foedevaremnisteriet, Kystdirektoratet, Lemvig, Februar, 2018 
- NEA14: Nossent J, L Boeckx, E Taverniers, M Deschamps, T Verwaest, F Mostaert, Sinterklaasstorm 6 december 2013. Beschrijving van de hydrometrische gebeurtenissen, Versie 4.0. WL Rapporten, 00-119, Waterbouwkundig Laboratorium, Antwerpen, Belgie. October 2014, WL2014R00_119_5

- RWS14b: RWS, Stormvloedrapport van 5 t/m 7 december (SR91) Sint-Nicolaasvloed 2013, Watermanagementcentrum Nederland, Rijkswaterstaat, prepared by Ing. J. Kroos, 19 Mar 2014, 48 $\mathrm{pp}$

- SW20: Surgewatch, Storm event 6th December 2013, https://www.surgewatch.org/events/1/ (last accessed 04Mar2020) TF20: www.tide-forecast.com

- WEA15: Wadey MP, ID Haigh, RJ Nichols et al, A comparison of the 31 January-1 February 1953 and 5-6 December 2013 coastal flood events around the UK, UK Frontiers in Marine Science, 2, 84, 2015.

- WEA15b: Wadey MP, JM Brown, ID Haigh, T Dolphin, P Wisse, Assessment and comparison of extreme sea levels and waves during the 2013/2014 storm season in two UK coastal regions, Nat. Hazards Earth Syst. Sci. Discuss., 3, 2665-2708, 2015. 
SECTION III. Working tables for literature survey

Table S0. Master list of tables in working notes

Table $\quad$ Content

\begin{tabular}{l|l}
\hline S0 & Master list of tables in working notes \\
\hline
\end{tabular}

\begin{tabular}{|l|l|}
\hline S1 & List of sources reviewed for project \\
\hline S2 & List of norma photos of event
\end{tabular}

S2 List of normal photos of event (arranged by year and then alphabetically)

S3 $\quad$ Ranking of storm among events; assessing importance of storm (arranged by year and then alphabetically)

S4 $\quad$ Severe forecast (arranged by year and then alphabetically)

S5 $\quad$ Storm not as bad as expected; not as bad as it could have been (arranged by year and then alphabetically)

S6 $\quad$ Storm worse than expected (arranged by year and then alphabetically)

S7 $\quad$ Extended period bad weather (arranged by year and then alphabetically)

S8 $\quad$ Names of the storm (arranged by year and then alphabetically)

S9 $\quad$ Satellite pictures and weather maps (arranged by year and then alphabetically)

S10 $\quad$ Satellite altimeter strip maps (arranged by year and then alphabetically)

S11 List meteorological data (arranged by year and then alphabetically)

S12 Significant wave height and sea state (arranged by year and then alphabetically)

S13 Wave period and other wave data (arranged by year and then alphabetically)

\begin{tabular}{|l|l}
\hline S14 & Surge reports and quantitative water levels (arranged by year and then alphabetically) \\
\hline
\end{tabular}

S15 $\quad$ Water current information (arranged by year and then alphabetically)

\begin{tabular}{l|l|}
\hline S16 & Return period of water level; ranking of water level \\
\hline S17 & Rerun period of wind speer rang of wind \\
\hline
\end{tabular}

S17 $\quad$ Return period of wind speed; ranking of wind speed

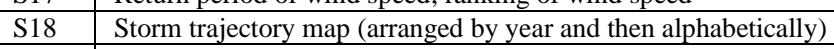

S19 Unusual pressure drop; time series central pressure; explosive characteristics (arranged by year and then alphabetically)

\begin{tabular}{lll}
\hline S20 & Squall line, convective thunderstorms, tornadoes (arranged by year and then alphabetically)
\end{tabular}

S21 Lightning (arranged by year and then alphabetically)

S22 1 Meso-vortex (arranged by year and then alphabetically)

S23 $\quad$ Meteotsunami (arranged by year and then alphabetically)

S24 1 Infragravity wave (arranged by year and then alphabetically)

S25 Wave dynamics and dike breaches; wave runup studies (arranged by year and then alphabetically)

S26 $\quad$ Precipitation, river level dike breaches (arranged by year and then alphabetically)

S27 $\quad$ Unusual peak of significant wave height in northern North Sea (arranged by year and then alphabetically)

S28 $\quad$ Double surge peak from wind and travelling wave (arranged by year and then alphabetically)

S29 $\quad$ Modelled turbulence kinetic energy in wave model (arranged by year and then alphabetically)

S30 $\quad$ Fatalities \& injuries

S31 Coastal flooding and evacuations (arranged by year and then alphabetically)

S32 $\quad$ Coastal dike heights and protection levels (arranged by year and then alphabetically)

S33 $\quad$ Surge barrier closures (arranged by year and then alphabetically)

S34 $\quad$ Beach damage and coastal issues (arranged by year and then alphabetically)

S35 $\quad$ Power interruptions (arranged by year and then alphabetically)

S36 List bridge closures, cancelled ferry crossings, port closures, airport cancel, rail interruptions, traffic accidents (arranged by year and then alphabetically)

S37 $\quad$ Structural damage to wind farms and wind energy impacts (arranged by year and then alphabetically)

S38 $\quad$ Hydropower impacts (arranged by year and then alphabetically)

S39 $\quad$ Structural damage to buildings, piers, and cultural monuments (arranged by year and then alphabetically)

S40 $\quad$ Forest damage and tree falls (arranged by year and then alphabetically)

S41 General ship/rig emergency reports/offshore incidents/platform evacuations (arranged by year and then alphabetically)

S42 $\quad$ Instrument failures during storm (arranged by year and then alphabetically)

S43 Model results and fields (arranged by year and then alphabetically)

S44 Climatological background of storm; unusual preceding weather events (arranged by year and then alphabetically)

S45 Xaver surge soon after spring tide; phase of surge and tide (arranged by year and then alphabetically)

S46 Tide analysis (arranged by year and then alphabetically)

S47 $\quad$ Data filtering and discretization issues (arranged by year and then alphabetically)

S48 $\quad$ Difficulties in modelling Storm Xaver water levels and surge (arranged by year and then alphabetically)

S49 $\quad$ Future sea level rise and flooding effects (arranged by year and then alphabetically)

S50 $\quad$ Storm event as manifestation of climate change (arranged by year and then alphabetically)

S51 $\quad$ Baltic Sea events (arranged by year and then alphabetically)

S52 $\quad$ Aftermath: new defenses \& new design criteria (arranged by year and then alphabetically)

\begin{tabular}{lll} 
S53 & Worst case storm surge situation (arranged by year and then alphabetically) \\
\hline
\end{tabular}

S54 Damage costs; insurance losses (arranged by year and then alphabetically)

S55 $\quad$ Online data sets (arranged by year and then alphabetically)

S56 Context and background informationwhere Strom Xaver not mentioned (arranged by year and then alphabetically)

S57 Climatological background for Storm Britta in Nov 2006; Storm Xaver not mentioned (arranged alphabetically)

S58 $\quad$ Errors in source reports for Xaver (arranged by year and then alphabetically)

S59 Abbreviations used in manuscript (arranged by year and then alphabetically)

S60 People contacted for information about Storm Xaver (arranged by year and then alphabetically)

Table S1. List of sources reviewed for project (arranged by year and then alphabetically) 


\begin{tabular}{|c|c|c|}
\hline Source & Type $^{1}$ & Full Reference and Notes \\
\hline Horner (1979) & 4 & Horner RW, The Thames Barrier Project, The Geographical Journal, 145, 242-253, 1979. \\
\hline $\begin{array}{l}\text { Sanders and Gyakum } \\
(1980)\end{array}$ & 4 & Sanders F and JR Gyakum, Synoptic-dynamic climatology of the 'Bomb', MWR, 108, 1589-1606, 1980 \\
\hline Stull (1988) & 4 & $\begin{array}{l}\text { Stull, R.B.: An Introduction to Boundary Layer Meteorology, Kluwer Academic Publishers, Dordrecht, } \\
\text { 1988. }\end{array}$ \\
\hline $\begin{array}{ll}\text { Winther-Jensen } & \text { and } \\
\text { Jorgensen (1999) }\end{array}$ & 4 & $\begin{array}{l}\text { Winther-Jensen, M and ER Jorgensen, When real life wind speed exceeds design wind assumptions, } 1999 \\
\text { European Wind Energy Conference, 1-5 March 1999, Nice, France pp.220-223. }\end{array}$ \\
\hline Goennert et al (2001) & 4 & $\begin{array}{l}\text { Goennert G,, SK Dube, T Murthy, W Siefert (2001): 7. Storm surges generated by extratropical cyclones - } \\
\text { case studies. In: Die Kueste } 63 \text { Sonderheft. Heide, Holstein: Boyens. pp 455-546 }\end{array}$ \\
\hline $\begin{array}{l}\text { Mueller-Navarra et al } \\
\text { (2003) }\end{array}$ & 4 & $\begin{array}{l}\text { Mueller-Navarra SH, W Lang, S Dick, KC Soetje, Ueber de Verfahren der Wasserstands- und } \\
\text { Stormflutvorhersage. Hydrodynamisch-numerische Modelle der Nord- und Ostsee und ein empirisch- } \\
\text { statistisches Verfahren fuer die Deutsche Bucht, promet. Jahrg. 29, Nr 1-4, 117-124, June } 2003\end{array}$ \\
\hline Gonnert et al (2004) & 4 & Gonnert G, K Isert, H Giese A Pluess, Charakterisierung der Tidekurve, Die Kueste, 68, 99-141, 2004 \\
\hline Ishihara et al (2005) & 4 & $\begin{array}{l}\text { Ishihara T, Tamaguchi, A, Takahara K, Mekaru T, Matsuura S, An analysis of damaged wind turbines by } \\
\text { Typhoon Maemi in 2003, The sixth Asia-Pacific Conference on Wind Engineering (APCWE-VI), Seoul, } \\
\text { Korea, September 12-14, } 2005\end{array}$ \\
\hline Eden (2006) & 4 & $\begin{array}{l}\text { Eden, P., Weather log. Consistently warm especially by night. Some heavy falls of rain. Weather Magazine, } \\
61, \text { i-iv, } 2006\end{array}$ \\
\hline Spiegel Online (2006c) & 4 & $\begin{array}{l}\text { Spiegel Online, Storm bashes northern Europe, one dead as freighter sinks in Baltic Storm, 02/11/2006c, } \\
\text { 13:00. }\end{array}$ \\
\hline Eden (2007) & 4 & Eden, P., Weather log, November, 2006, Weather Magazine, 62, i-iv, 2007. \\
\hline Cappelen (2007a) & 4 & $\begin{array}{l}\text { Cappelen, J., Vejret I } 2006 \text { - I Denmark, I Nuuk pa Gronland og I Torshavn pa faeroerne, Vejret, 110, 13-17, } \\
\text { 2007a. }\end{array}$ \\
\hline Cappelen (2007b) & 4 & $\begin{array}{l}\text { Cappelen, John, Teknisk rapport 07-01, Danmarks klima } 2006 \text { med Torshavn, Faeroerne og Nuuk, } \\
\text { Groenland - with English translations, DMI, Transport- og Energiministeriet, www.dmi.dk/dmi/tr07-01, } \\
\text { Kobenhavn, 2007b. }\end{array}$ \\
\hline NCIC (2007) & 4 & NCIC, National Climate Information Centre, UK annual weather summary, Weather, 62, p.42, 2007. \\
\hline Nielsen (2007) & 4 & Nielsen, Niels Woetmann, Om stormflod og eftersarsvejr I Danmark anno 2006, Vejret, 110, 24-33, 2007. \\
\hline Rosenorn (2007) & 4 & Rosenorn, Stig, Efterarsvejret 2006 (SON autumn quarter summary), Vejret, 110, pp.20-23, 2007 \\
\hline $\begin{array}{l}\text { Rosenthal and Lehner } \\
\text { (2007) }\end{array}$ & 4 & $\begin{array}{l}\text { Rosenthal, W. and S. Lehner, Individual wave height from SAR, Proc. 'Envisat Symposium 2007', } \\
\text { Montreux, Switzerland, 23-27 April } 2007 \quad \text { (ESA } \\
\text { https://www.semanticscholar.org/paper/INDIVIDUAL-WAVE-HEIGHT-FROM-SAR-Rosenthal- } \\
\text { Lehner/affabe337b05276112fca662375ec8a8b4bba4a6 }\end{array}$ \\
\hline $\begin{array}{l}\text { Cruz and Krausmann } \\
(2008)\end{array}$ & 4 & $\begin{array}{l}\text { Cruz AM and E Krausmann, Damage to offshore oil and gas facilities following hurricanes Katrina and Rita: } \\
\text { An overview, Journal of Loss Prevention in the Process Industries, 21, 620-626, } 2008 \text {. }\end{array}$ \\
\hline Lenton et al. (2008) & 4 & $\begin{array}{l}\text { Lenton TM, H Held, E Kiegler, JW Hall, W Lucht, S Rahmstorf, HJ Schellnhuber, Tipping elements in the } \\
\text { Earth's climate system, PNAS, 105, 1786-1793, 2008 www.pnas.org/cgi/doi/10.1073/pnas.0705414105 }\end{array}$ \\
\hline $\begin{array}{l}\text { Wihelmi and } \\
\text { Barjenbruch (2008) }\end{array}$ & 4 & $\begin{array}{l}\text { Wilhelmi J and U Barjenbruch, 2008, Application of radar gauges to measure the water level and the state of } \\
\text { the sea Proceedings of 31st international conference on coastal engineering, Hamburg, Germany }\end{array}$ \\
\hline Gonnert et al (2009) & 4 & $\begin{array}{l}\text { Gonnert G, J Jensen, H von Storch, S Thumm, T Wahl, R Weisse, Der Meeresspiegelansteig. Ursachen, } \\
\text { Tendezen, und Risikobwertung, Die Kueste, 76, FAK (2009), 225-256. }\end{array}$ \\
\hline Paskal (2009) & 4 & $\begin{array}{l}\text { Paskal, C., The vulnerability of energy infrastructure to environmental change, Energy, environment and } \\
\text { resource governance, Apr. 2009, EERG BP 2009/11 }\end{array}$ \\
\hline Diamond (2012) & 4 & $\begin{array}{l}\text { Diamond KE, Extreme weather impacts on offshore wind turbines: Lessons learned, Natural Resources and } \\
\text { Environment, 27, fall, pp.1-5, } 2012 \text {. }\end{array}$ \\
\hline De Jong (2012) & 4 & $\begin{array}{l}\text { de Jong, Matthijs S, Developing a parametric model for storm to determine the extreme surge level at the } \\
\text { Dutch coast, Delft University of Technology, June, } 2012\end{array}$ \\
\hline Goennert et al (2012) & 4 & $\begin{array}{l}\text { Goennert G, B Gerkensmeier, J-M Mueller, Ermittlung des Sturmflutbemesungswasserstandes fuer den } \\
\text { oeffentlichen Hochwasserschutz in Hamburg, Berichte des Landesbetriebes Strassen, Bruecken und } \\
\text { Gewaesser Nr 12/2012. }\end{array}$ \\
\hline Rose et al (2012) & 4 & $\begin{array}{l}\text { Rose S, P Jaramillo, MJ Small, I Grossmann, J Apt, Quantifying the hurricane risk to the offshore wind } \\
\text { turbines, PNAS, 109, 1-6, } 2012 \text {. }\end{array}$ \\
\hline 24liveblog (2013) & 1 & 24liveblog, Storm 5-6 december 2013, https://live.24liveblog.com/live/UYXaD \\
\hline $\begin{array}{l}\text { Aftenposten } \\
(20131205)\end{array}$ & 1 & $\begin{array}{l}\text { Aftenposten, Varsler full storm I Sor-Norge, 5Dec2013 15:03 (correspondent Per Annar Holm) } \\
\text { https://www.aftenposten.no/norge/i/bKEjl/varsler-full-storm-i-soer-norge }\end{array}$ \\
\hline $\begin{array}{ll}\text { Air } & \text { Worldwide } \\
(2013212) & \end{array}$ & 1 & $\begin{array}{l}\text { Air Worldwide, Press Release, Boston, 12Dec2013. https://www.air-worldwide.com/In-the-News/AIR- } \\
\text { Estimates-Losses-from-European-Windstorm-Xaver-at-Between-EUR-700-Million-and-EUR-1-4-Billion/ } \\
\text { (accessed 02Jan1990) }\end{array}$ \\
\hline BFG (2013) & 1 & BFG, Orkan Xaver: BfG beobachtet extreme Wellen in der Nordsee (06.12.2013) \\
\hline Bloomberg (2013) & 1 & $\begin{array}{l}\text { Bloomberg, Hamburg has worst flood in } 37 \text { year amid European storms, Nicholas Brautlecht, 6Dec2013, } \\
\text { 02:58PM GMT https://www.bloomberg.com/news/articles/2013-12-06/hamburg-has-worst-flood-in-37- } \\
\text { years-as-storms-rage-across-europe }\end{array}$ \\
\hline BBC (20131204) & 1 & BBC, Workers off Talisman Sinopec oil platform Buchan Alpha over bad weather forecast, 04Dec2013 \\
\hline BBC (20131205) & 1 & $\begin{array}{l}\text { BBC, In pictures: Winter storm hits UK, } 5 \text { December 2013, https://www.bbc.com/news/uk-scotland- } \\
25231224\end{array}$ \\
\hline BBC (20131206) & 1 & $\begin{array}{l}\text { BBC20131206, Deadly storm and tidal surge batter northern Europe, BBC } 6 \text { Dec2013 (report by Anna } \\
\text { Holligan) }\end{array}$ \\
\hline BODC (20131205) & 1 & $\begin{array}{cccccccc}\text { BODC, } & \text { Storm surge and coastal flooding, } & \text { News and Events, } & \text { 5Dec2013. } \\
\text { https://www.bodc.ac.uk/about/news } & \text { and events/storm surge } & \text { and coastal flooding.html } & \\
\end{array}$ \\
\hline BSH (2013) & 1 & $\begin{array}{l}\text { BSH, Die Nordseesturmfluten von 5. und 6.12.2013, 3pp, prepared by Stockmann,K. (title: Sturmflut } \\
\text { 2011_bm1101; author stamp: bm1101; document time stamp: 12/102013 12:5136PM) }\end{array}$ \\
\hline BT (20131208) & 1 & BT, Offer for Bodil: Koebte huset 15.august - nu er det vaek, 08Dec2013, 16:44, (contributor: Morten \\
\hline
\end{tabular}




\begin{tabular}{|c|c|c|}
\hline & & Eggert) https://www.bt.dk/danmark/offer-for-bodil-koebte-huset-15.-august-nu-er-det-vaek \\
\hline Cargolaw (2013) & 3 & Cargolaw 2013 [Tim Schwabedissen, 12Dec2013] \\
\hline Daily Mail (20131206) & 1 & $\begin{array}{l}\text { Daily Mail, Huge storm strikes Europe causing death and destruction and leading to cancellation of hundreds } \\
\text { of flights, 6Dec2013. (correspondent: Nick Enoch), https://www.dailymail.co.uk/news/article- } \\
2519517 / \text { Huge-storm-strikes-Europe-causing-death-destruction-leading-cancellation-hundreds- } \\
\text { flights.html }\end{array}$ \\
\hline $\begin{array}{l}\text { Deutschlander et al. } \\
\text { (2013) }\end{array}$ & 1 & $\begin{array}{l}\text { Deutschlander T, K Frierich, S Haeseler, C Lefebvre, Orkantief XAVER ueber Nordeuropea von 5. bis } 7 . \\
\text { Dezember 2013, Deutscher Wetterdienst DWD, Stand 30. Dezember 2013, 19pp. }\end{array}$ \\
\hline DW (20131205) & 1 & DW, Storm Xaver pummels Europe, flood surge feared, 05/12/2013 \\
\hline $\begin{array}{ll}\text { Energy } & \text { Voice } \\
(20131204) & \end{array}$ & 1 & $\begin{array}{l}\text { Energy Voice, Oil staff withdrawn from North Sea ahead of storm conditions, 04/12/2013 10:30AM, } \\
\text { https://www.energyvoice.com/other-news/healthandsafety/50140/oil-staff-withdrawn-north-sea-ahead- } \\
\text { storm-conditions/ }\end{array}$ \\
\hline Expressen (2013) & 1 & Expressen, Flera doda i Sverige efter stormen Sven, 06Dec2013, 17:34CET? \\
\hline Fleetmon (20131207) & 1 & $\begin{array}{l}\text { Fleetmon, Container ship Burak Bayraktar in trouble off Texel, Netherlands, (correspondent: Mikhail } \\
\text { Voytenko, 7Dec2013 06:31) https://www.fleetmon.com/maritime-news/2013/2810/container-ship-burak- } \\
\text { bayraktar-trouble-texel-nethe/ }\end{array}$ \\
\hline Fleetmon (20131208) & 1 & 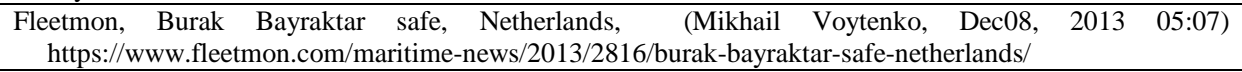 \\
\hline $\begin{array}{l}\text { Gandreassen } \\
(20131204)\end{array}$ & 1 & $\begin{array}{l}\text { Gandreassen, Oil workers moved from Ekofisk, 4Dec2013. https://gandreassen.com/oil-workers-moved- } \\
\text { from-ekofisk/ }\end{array}$ \\
\hline $\begin{array}{l}\text { Gccapitalideas } \\
(20131209)\end{array}$ & 1 & gccapitalideas, Windstorm Xaver, 9Dec2013, https://www.gccapitalideas.com/2013/12/09/windstorm-xaver/ \\
\hline GP (20131206) & 1 & $\begin{array}{l}\text { GP, Fortsatt risk for halka, 6Dec2013 (correspondent: D Henriksson, K Vikingsson, P Sydvik, TA } \\
\text { Akerblom) http://www.gp.se/nyheter/goteborg/1.2201325-fortsatt-risk-for-halka (accessed 1May2020) }\end{array}$ \\
\hline Gray (2013) & 1 & $\begin{array}{l}\text { Gray, Tom, Into the Wind, The AWEA Blog, Ireland, U.K., Germany set new wind generation records, } \\
\text { https://www.aweablog.org/ireland-u-k-germany-set-new-wind-generation-records/, 11Dec2013 }\end{array}$ \\
\hline GVA (20131205) & 1 & 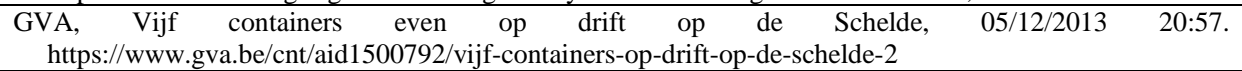 \\
\hline HBVL (20131205) & 1 & 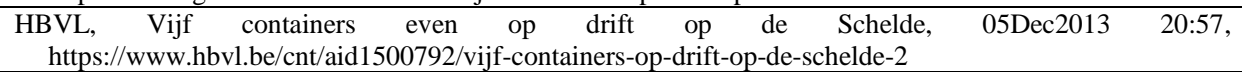 \\
\hline IWR (20131206) & 1 & $\begin{array}{l}\text { IWR, Orkantief 'Xaver' bringt neuen Rekord - Deutsche Windkraftanlagen produzieren erstmals Strom mit } \\
\text { ueber 26000 MW Leistung, 06Dec2013 09:31, https://www.iwr.de/news.php?id=25168 (accessed } \\
\text { 07May2020) }\end{array}$ \\
\hline KNMI (2013) & 1 & $\begin{array}{l}\text { KNMI, News report. De Zware storm van } 5 \text { december, } 06 \text { Dec 2013, https://www.knmi.nl/over-het- } \\
\text { knmi/nieuws/de-zware-storm-van-5-december }\end{array}$ \\
\hline $\begin{array}{ll}\text { Kristeligt } \\
(20131205)\end{array}$ Dagblad & 1 & $\begin{array}{l}\text { Kristeligt Dagblad, Stormen blaeser Skotland omkuld: Doodsfald og nebrud, (contributor: Ritzau), } \\
\text { 05Dec2013 12:50 https://www.kristeligt-dagblad.dk/udland/stormen-b1\%C3\%A6ser-skotland-omkuld- } \\
\text { d\%C3\%B8dsfald-og-nedbrud }\end{array}$ \\
\hline Kunz et al (20131206) & 1 & $\begin{array}{l}\text { Kunz M, B Muehr, K Schroeter, T Bessel, S Moehrle, T Muenzberg, S Brink, H-M Schmidt, Winterstorm } \\
\text { Xaver - Report. 06Dec2013 - Report No.1, Situation Report - 19:00CET, CEDIM Forensic Disaster } \\
\text { Analysis Group (FDA), Center for Disaster Management and Risk Reduction Technology. }\end{array}$ \\
\hline Li et al. (2013) & 4 & $\begin{array}{l}\text { Li Z-q, Chen S-j, Ma H, Feng T, Design defect of wind turbine operating in typhoon activity zone, } \\
\text { Engineering Failure Analysis, 27, 165-172, } 2013 \text {. }\end{array}$ \\
\hline $\begin{array}{l}\text { Liverpool } \\
(20131211)\end{array}$ & 1 & $\begin{array}{l}\text { Liverpool Echo, Formby sand dunes hit by stormy seas, 11Dec2013, (correspondent: Eleanor Barlow) } \\
\text { https://www.liverpoolecho.co.uk/news/liverpool-news/formby-sand-dunes-hit-stormy-6397344, accessed } \\
\text { 19Apr2020 }\end{array}$ \\
\hline $\begin{array}{l}\text { The Lowestoft Journal } \\
\text { (20131212) }\end{array}$ & 1 & $\begin{array}{l}\text { The Lowestoft Journal, "There is a strong sense Parliament has not yet considered properly this narrowly } \\
\text { averted national crisis" - MP secures Commons debate on flood, 12Dec2013, correspondent: Martin } \\
\text { George }\end{array}$ \\
\hline $\begin{array}{l}\text { The Lowestoft Journal } \\
\text { (20131214) }\end{array}$ & 1 & $\begin{array}{l}\text { The Lowestoft Journal, Suffolk MP hits out at environment secretary in statement about the floods, } \\
\text { 14Dec2013, correspondent: Annabelle Dickson. }\end{array}$ \\
\hline 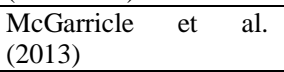 & 3 & $\begin{array}{l}\text { McGarricle P (ed), UK coastal monitoring and forecasting: Annual report for } 2013 \text { for the UK National Tide } \\
\text { Gauge Network, NERC 100017897, } 2013 \text { [pdf document properties: author=pamcg; created=16Apr2014] }\end{array}$ \\
\hline Mills et al. (20131206) & 1 & $\begin{array}{l}\text { Mills, Ian, Remko Scharoo, Luciana Fenoglio, Xaver affected much of northern Europe on } 5 \text { and } 6 \\
\text { December and caused worst storm surge for decades in the North Sea. } \\
\text { https://www.eumetsat.int/website/home/News/DAT_2087062.html } \\
\text { 28Jun2020) }\end{array}$ \\
\hline $\begin{array}{l}\text { National Wind Watch } \\
(20131210 \mathrm{a})\end{array}$ & 1 & $\begin{array}{l}\text { National Wind Watch, 10Dec2013a, Xaver zerstoert ein Windrad bei Vlatten, original source: Aachener } \\
\text { Zeitung, 6Dec2013 https://www.wind-watch.org/news/2013/12/10/xaver-zerstort-ein-windrad-bei-vlatten/ }\end{array}$ \\
\hline $\begin{array}{l}\text { National Wind Watch } \\
\text { (20131210b) }\end{array}$ & 1 & $\begin{array}{l}\text { National Wind Watch, Sturm 'Xaver': Windrad stuerzt auf Acker, 10Dec2013b, credit: von Manfred } \\
\text { Reinnarth, Koelnische Rundschau, 06/12/2013, https://www.wind-watch.org/news/2013/12/10/sturm- } \\
\text { xaver-windrad-sturzt-auf-acker/ }\end{array}$ \\
\hline $\begin{array}{l}\text { National Wnd Watch } \\
\text { (20131212) }\end{array}$ & 1 & $\begin{array}{l}\text { National Wind Watch, Sturm 'Xaver': Windrad stuerzt auf Acker, 10Dec2013b, credit: von Manfred } \\
\text { Reinnarth, Koelnische Rundschau, 06/12/2013, https://www.wind-watch.org/news/2013/12/10/sturm- } \\
\text { xaver-windrad-sturzt-auf-acker/ }\end{array}$ \\
\hline NLWKN (20131203) & 1 & $\begin{array}{l}\text { NLWKN, Sturmflutgefahr an der Kueste und auf den Inseln, Zwei meter ueber dem normalen Hochwasser in } \\
\text { der Nacht zum Freitag moeglich//pressinformation von } 29 \text { November 2013, (written by Herma Heyken, } \\
\text { Pressesprecherin), 03/12/2013 } \\
\text { https://www.nlwkn.niedersachsen.de/startseite/aktuelles/presse_und_offentlichkeitsarbeit/pressemitteilungen } \\
\text { /sturmflutgefahr-an-der-kueste-und-auf-den-inseln-120187.html }\end{array}$ \\
\hline NLWKN (20131206) & 1 & $\begin{array}{l}\text { NLWKN, Schwere Sturmflut gut Ueberstanden, Waserstaende zwischen Zweieinhale und Knapp vier metern } \\
\text { registriert // presseinformation von 6. Dezember 2013, (Ansprechpartnerin: Herma Heyken), 06/12/2013. }\end{array}$ \\
\hline $\begin{array}{l}\text { Nordbayern } \\
(20131207)\end{array}$ & 1 & $\begin{array}{l}\text { Nordbayern, Deining: 'Xaver' reisst Rotorblatt von Windrad ab. Windkraftanlage schleuderte Eisbrocken auf } \\
\text { die Strasse, 07/12/2013, 12:07. https://www.nordbayern.de/region/neumarkt/deining-xaver-reisst- }\end{array}$ \\
\hline
\end{tabular}




\begin{tabular}{|c|c|c|}
\hline & & rotorblatt-von-windrad-ab-1.3326492 \\
\hline NOS (20131206) & 1 & $\begin{array}{l}\text { NOS, Hoogste waterstand sinds 1953, 06/12/2013, 09:52, https://nos.nl/artikel/583218-hoogste-waterstand- } \\
\text { sinds-1953.html }\end{array}$ \\
\hline $\begin{array}{l}\text { Oceanografisch } \\
\text { Meteorologisch Station } \\
\text { (2013) }\end{array}$ & 1 & $\begin{array}{l}\text { Oceanografisch Meteorologisch Station, Stormverslag 05-06 december 2013, 26pp, } 2013 \text { [pdf document } \\
\text { properties: author=Myriam Sys; datestamp=15Dec2013] }\end{array}$ \\
\hline Reuters (20131205) & 1 & $\begin{array}{c}\begin{array}{c}\text { Reuters, ConocoPhillips cuts some output at Ekofisk field due } \\
\text { https://finance.yahoo.con/news/conocophillips-cuts-output-ekofisk-field... }\end{array} \\
\end{array}$ \\
\hline Rtv Oost (20131205) & 1 & $\begin{array}{l}\text { rtv Oost, Trainverkeer ten noorden van Zwolle stilgelegt vanwege storm 5Dec2013 14:02, } \\
\text { https://www.rtvoost.nl/nieuws/177568/Treinverkeer-ten-noorden-van-Zwolle-stilgelegd-vanwege- } \\
\text { storm }\end{array}$ \\
\hline SMHI (20131210) & 1 & $\begin{array}{l}\text { SMHI, Stormen Sven gav nya vattenstandsrekord i Oresund, https://www.smhi.se/nyhetsarkiv/stormen-sven- } \\
\text { gav-nya-vattenstandsrekord-i-oresund-1.34732, updated 20Mar2017; original datestamp 10Dec2013. }\end{array}$ \\
\hline $\begin{array}{l}\text { Spiegel International } \\
(20131206)\end{array}$ & 1 & $\begin{array}{l}\text { Spiegel International, Winter storm 'Xaver' batters northern Europe,6 Dec 2013, } 12: 56 \\
\text { https://www.spiegel.de/international/europe/tidal-surge-winter-storm-xaver-batters-northern-europe-a- } \\
\text { 937576.html\# } \\
\end{array}$ \\
\hline $\begin{array}{l}\text { Sueddeutsche Zeitung } \\
(20131215)\end{array}$ & 1 & $\begin{array}{c}\begin{array}{c}\text { Sueddeutsche } \\
\text { https://www.sueddeutsche.de/panorama/sturmschaeden-auf-sylt-xaver-holte-sich-land-1.1844100 }\end{array} \\
\end{array}$ \\
\hline $\begin{array}{l}\text { Sylter Rundschau } \\
(20131209)\end{array}$ & 1 & $\begin{array}{l}\text { Sylter Rundschau, Folgen des Orkans. Xaver 'knabberte' Sylt massiv an, 09Dec2013 06:00 (from Friederike } \\
\begin{array}{l}\text { Reussner) } \\
\text { id5098481.html }\end{array}\end{array}$ \\
\hline TheJournal (20131205) & 1 & $\begin{array}{l}\text { TheJournal, Homes remain without electricity after high winds batter power lines, 05Dec2013 08:20PM, } \\
\text { https://www.thejournal.ie/storm-ireland-1207783-Dec2013/ }\end{array}$ \\
\hline The Local (20131205) & 1 & $\begin{array}{ccccccc}\text { The } \begin{array}{c}\text { Local, } \\
\text { https://www.thelocal.se/20131205/storm-sven-sweeps-into-southern-sweden }\end{array} & \begin{array}{c}\text { Sven's strong } \\
\text { winds }\end{array} & \text { sweep } & \text { southern } & \text { Sweden. } & \text { 05Dec2013 } & \text { 15:57CET } \\
\end{array}$ \\
\hline $\begin{array}{l}\text { Unwetterzentrale } \\
(201312)\end{array}$ & 1 & $\begin{array}{l}\text { Unwetterzentrale, Orkantief XAVER - ein weiterer schwerer Wintersturm der letzten Jahrzehnte, Thomas } \\
\text { Savert and Stefan Laps, Dec. } 2013 \mathrm{http}: / / \text { www.unwetterzentrale.de/uwz/928.html }\end{array}$ \\
\hline Upstream (20131204) & 1 & Upstream, Talisman takes workers off Buchan Alpha, 4Dec2013 1710GMT (correspondent: Rob Watts) \\
\hline Upstream (20131205) & 1 & $\begin{array}{l}\text { Upstream, North Sea production curtailed over storm, 05Dec2013 (contributor: Bill Lehane and News } \\
\text { Wires) }\end{array}$ \\
\hline Upstream (20131206) & 1 & $\begin{array}{l}\text { Upstream, Buchan Alpha evacuated as North Sea storms loom, 6Dec2013 0000GMT (contributor Rob } \\
\text { Watts) }\end{array}$ \\
\hline Upstream (20131209) & 1 & Upstream, Workers return to Buchan A, 09Dec2013 12:58GMT (contributor: Rob Watts) \\
\hline ABPmer (2014) & 1 & $\begin{array}{l}\text { ABP mer, Ensuring Flood Resilience. An overview of the 5/6 December 2013, Associated British Ports, } \\
\text { Marine Environmental Research, July, } 2014\end{array}$ \\
\hline AON Benfield (2014) & 3 & $\begin{array}{l}\text { AON Benfield, Impact forecasting. December } 2013 \text { Global Catastrophe Recap, 2014. [document properties: } \\
\text { abrandt; date stamp: 10Jan2014] }\end{array}$ \\
\hline Badewien (2014) & 3 & $\begin{array}{l}\text { Badewien T., Long-term observatory @ Pile Spiekeroog ICBM,COSYNA Progress Report 2013, p24. } \\
\text { Helmholtz-Zentrum Geesthacht, June } 2014\end{array}$ \\
\hline Bancroft (2014) & 3 & $\begin{array}{l}\text { Bancroft, George P, Marine Weather Review - North Atlantic Area, September through December, Mariners } \\
\text { Weather Log, volume 58, No.1, April 2014, pp.33-40 }\end{array}$ \\
\hline BBC (20141205) & 1 & BBC, East coast surge: what happened next? (report by Richard Haugh), 5Dec2014 \\
\hline Blasi et al. (2014) & 3 & $\begin{array}{l}\text { Blasi C, S Mai, J Wilhelmi, T Zenz, U Barjenbruch, A powerful method of measuring sea wave spectra and } \\
\text { their direction, ICHE 2014, Hamburg - Lehfeldt and Kopmann (eds), Bundesanstalt fuer Wasserbau, } \\
\text { 2014. ISBN 978-3-939230-32-8 }\end{array}$ \\
\hline $\begin{array}{l}\text { CH2MHill Halcrow } \\
\text { (2014) }\end{array}$ & 3 & $\begin{array}{l}\text { CH2MHill Halcrow, Cell } 1 \text { Regional Coastal Monitoring Programme, Wave Data Analysis Report 2: 2013- } \\
\text { 2014, Final Report, March } 2014 \text { [document properties: author=Andy.Parson@ ch2m.com; datestamp; } \\
\text { 04/04/2014] }\end{array}$ \\
\hline Cipollini et al. (2014) & 2 & $\begin{array}{l}\text { Cipollini P, LJ West, HM Snaith, P Harwood, C Donlon, New altimetry products over shelf and coastal zone } \\
\text { from the eSurge processor, poster presentation, } 2014 \text { [document time stamp: 2014/10/22] }\end{array}$ \\
\hline Dunbar et al (2014) & 1 & $\begin{array}{l}\text { Dunbar I, N Phipps, M Szonyi, Risk Nexus. After the storm: how the UK's flood defences performed during } \\
\text { the surge following Xaver, Flood resilience review 09.14, Zurich Insurance Company Ltd., Mythenquai 2, } \\
\text { 8002, Zurich, Switzerland [document properties: date 28Aug2014; author= Zurich] }\end{array}$ \\
\hline Eden (201402) & 3 & Eden, Phillip, Weather Log December 2013, Weather, Feb 2014, pp.i-iv \\
\hline Eriksen (2014) & 1 & Eriksen J, Rekordvandstande i Isefjorden og Roskilde Fjord, Vejret, 138, 2 40-48, 2014 \\
\hline FINO1 (20140108) & 1 & $\begin{array}{l}\text { FINO1, 15-m wave damaged FINO1, 08Jan2014. http://www.fino1.de/meldungen/alle-meldungen/137-15- } \\
\text { meter-welle-beschaedigt-fino1 }\end{array}$ \\
\hline FUGRO GEOS (2014) & 3 & $\begin{array}{l}\text { FUGRO GEOS Ltd, Ormen Lange Monthly reports of wavescan data: December. Reporting period: } 1 \\
\text { December } 2013 \text { to } 31 \text { December 2013, Report Number: C70101/8177/R0, Issue date: } 23 \text { January 2014, } \\
\text { prepared by Heather Holt, Checked by Donald Brockie, approved by Mark Jones }\end{array}$ \\
\hline Gautier et al (2014) & 1 & $\begin{array}{l}\text { Gautier C, A Camarena, J van Nieuwkoop, SWAN hindcasts Wadden Sea, December 2013. Tidal inlet of } \\
\text { Ameland and eastern Wadden Sea. Deltares, 2014, 197pp. Project 1209433-007, Reference 1209433- } \\
007-\text { HYE-0005 }\end{array}$ \\
\hline Goennert et al. (2014) & 1 & $\begin{array}{l}\text { Goennert G, O Mueller, M Schaper, K Sossidi, Die Sturmflut nach dem Tief Xaver von 5. bis 7. Dezember } \\
\text { 2013. Berichte des Landesbetriebes Strassen, Bruecken und Gewaesser (LSBG), Freie und Hansestadt } \\
\text { Hamburg, Nr. 16/2014, 26pp. }\end{array}$ \\
\hline Hewson et al (2014) & 2 & $\begin{array}{l}\text { Hewson T, L Magnusson, O Breivik, F Prates, I Tsonevsky, HJW de Vries, Windstorms in northwest Europe } \\
\text { in late 2013, ECMWF Newsletter, No 139, pp 22-28, Spring } 2014 .\end{array}$ \\
\hline $\begin{array}{l}\text { Knaack and Heyken } \\
\text { (2014) }\end{array}$ & 1 & $\begin{array}{l}\text { Knaack H and H Heyken, Xaver hatte sehr schwere Stumflut im Gepaeck, Jahresbericht 2013. Der Zukunft } \\
\text { verplichtet, NLWKN, Niedersaechsischer Landesbetrieb fuer Waserwirtschaft, Kuesten- und } \\
\text { Naturschutz, pp.8-9, document date stamp 24Apr2014. }\end{array}$ \\
\hline Kristandt et al. (2014) & 3 & $\begin{array}{l}\text { Kristandt, J., B. Brecht, H. Frank, H. Knaack, Optimization of empirical storm surge forecast-modeling of } \\
\text { high resolution wind fields, Die Küste, } 81,301-348,2014\end{array}$ \\
\hline
\end{tabular}




\begin{tabular}{|c|c|c|}
\hline Leiding et al. (2014) & 1 & $\begin{array}{l}\text { Leiding T, B Tinz, G Rosenhagen, C Lefevre, S Haeseler, S Hagemann, I Bastigkeit, D Stein, P Schwenk, } \\
\text { S Mueller, O Outzen, K Herklotz, F Kinder, T Neumann, Meteorological and Oceanographic } \\
\text { Conditions at the FINO platforms during the severe storms Christian and Xaver, DEWI Magazin, } \\
\text { No.44, p16-25, 2014. }\end{array}$ \\
\hline $\begin{array}{lll}\text { Luecht } & \text { and } & \text { Peters } \\
(2014) & & \end{array}$ & 1 & $\begin{array}{l}\text { Luecht, Fabian and Ove Peters, Bericht ueber die Sturmflut vom 05.-0.6.12.2013 an der Westkueste } \\
\text { Schleswig-Holsteins, Landesbetrieb fuer Kuestenschutz, Nationalpark und Meeresschutz Schleswig- } \\
\text { Holstein, Husum 26Feb2014, 19pp. [pdf document properties: title=Lfd; Author=Thorsten } \\
\text { Nommensen; datestamp: 11Dec2018] }\end{array}$ \\
\hline Mai (2014) & 1 & $\begin{array}{l}\text { Mai, S., Sea state at the research platform FINO1 during the winter storm 'Xaver', BFG, Bundesamt fuer } \\
\text { Gewasserkunde, document properties: author=Mai, date=16May2014 }\end{array}$ \\
\hline Mai et al (2014) & 1 & $\begin{array}{l}\text { Mai S, J Wilhelmi, T Zenz, U Barjenbruch, Orkan 'Xaver' - Seegangsstatistik an den Stationen FINO1 und } \\
\text { Borkum-Suedstrand, 19. KFKI-Seminar, Bremerhaven 11.11.2014 (presentation slides) }\end{array}$ \\
\hline Matelski et al (2014) & 1 & $\begin{array}{l}\text { Matelski, Birgit, Frerk Jensen, Peter Voss, Jorg Peters, Maria Blumel, Auswertung zur Hydrologie des } \\
\text { Sturmtiefs Xaver 05.12.2013 bis 06.12.2013, Statusbericht vom 01.10.2014, Landesbetrieb fuer } \\
\text { Kuestenschutz, Nationalpark und Meeresschutz, Schleswig-Holstein, Husum, 01.10.2014, 25pp. }\end{array}$ \\
\hline $\begin{array}{l}\text { MIROS_draugen } \\
(2014)\end{array}$ & 3 & $\begin{array}{l}\text { MIROS, Monthly Report, Draugen, December 2013, Doc. No. ND/1022/13/12 (prepared by SRS, checked } \\
\text { by OO, approved by CNE) [pdf document properties: author=MIROS AS; datestamp=30Jan2014] }\end{array}$ \\
\hline $\begin{array}{l}\text { MIROS_ekofisk } \\
(2014)\end{array}$ & 3 & $\begin{array}{l}\text { MIROS, Monthly report, Ekofisk, December 2013, Doc. No. ND/1024/13/12, (prepared by SRS) 34pp, } \\
\text { 9Jan2014 [PDF document properties: author=Miros AS; datestamp=13Jan2014] }\end{array}$ \\
\hline $\begin{array}{l}\text { MIROS_gullfaks } \\
\text { (2014) }\end{array}$ & 3 & $\begin{array}{l}\text { MIROS, Manedsrapport Gullfaks C, Desember } 2013 \text {, Dok. Nr ND/1013/13/12, carried out by SRS, } \\
\text { controlled by CNE, approved by CO [pdf document properties: author=MIROS AS; date stamp: } \\
\text { 13Jan2014] }\end{array}$ \\
\hline $\begin{array}{l}\text { MIROS_heidrun } \\
\text { (2014) }\end{array}$ & 3 & $\begin{array}{l}\text { MIROS, Manedsrapport Heidrun, Desember } 2013 \text {, Dok. Nr. ND/1010/13/12, } 21 \mathrm{pp}, 07 \mathrm{Jan} 2014 \text {, carried out } \\
\text { by SRS, controlled by CNE, approved by OO [pdf properties: author=Miros AS; date stamp: } \\
08 / 01 / 2014 \text { ] }\end{array}$ \\
\hline $\begin{array}{l}\text { MIROS_heimdal } \\
\text { (2014) }\end{array}$ & 3 & $\begin{array}{l}\text { MIROS, Manedsrapport Heimdal, Desember 2013, Dok. Nr. ND/1047/13/12 (carried out be SRS, controlled } \\
\text { by CNE, approved by OO) [PDF document properties: author=Miros AS; datestamp=06Jan2014] }\end{array}$ \\
\hline MIROS_norne (2014) & 3 & $\begin{array}{l}\text { MIROS, Maanedsrapport Norne, Desember 2013, Dok. Nr. ND/1087/13/12, 21pp, carried out by SRS, } \\
\text { controlled by CNE, approved by OO [pdf properties: Author=Miros AS; datestamp: 06Jan2014] }\end{array}$ \\
\hline $\begin{array}{l}\text { MIROS_sleipner } \\
(2014)\end{array}$ & 3 & $\begin{array}{l}\text { MIROS, Manedsrapport Sleipner A, Desember 2013, Dok. Nr. ND/1017/13/12, 06Jan2014, prepared by } \\
\text { SRS, controlled by CNE, approved by OO. }\end{array}$ \\
\hline MIROS_troll (2014) & 3 & $\begin{array}{l}\text { MIROS, Manedsrapport, Troll A, Desember 2013, Doc. No. ND/1012/13/12, (prepared by SRS) 21pp, } \\
\text { 7Jan2014 [PDF document properties: author=Miros AS; datestamp=10Jan2014] }\end{array}$ \\
\hline Nossent et al (2014) & 1 & $\begin{array}{l}\text { Nossent J, L Boeckx, E Taverniers, M Deschamps, T Verwaest, F Mostaert, Sinterklaasstorm } 6 \text { december } \\
\text { 2013. Beschrijving van de hydrometrische gebeurtenissen, Versie 4.0. WL Rapporten, 00-119, } \\
\text { Waterbouwkundig Laboratorium, Antwerpen, Belgie. October 2014, WL2014R00_119_5 [document } \\
\text { date stamp: 15Oct2014; author= Deschamps, Maarten] }\end{array}$ \\
\hline NOZ (20140129) & 1 & $\begin{array}{l}\text { NOZ Nach Xaver: 9,4 Millionen Euro fuer Juist, Spiekeroog and Wangerooge, 29Jan2014 } \\
\text { https://www.noz.de/deutschland-welt/niedersachsen/artikel/446888/nach-xaver-9-4-millionen-euro-fur- } \\
\text { juist-spiekeroog-und-wangerooge-1 }\end{array}$ \\
\hline Pelt (2014) & 1 & Pelt AS, BODIL's stormflod i de indre dansk farvande, Vejret, 138, 24-29, 2014 \\
\hline RMS (2014) & 3 & $\begin{array}{l}\text { RMS, 2013-2014 Winter Storms in Europe. An Insurance and Catastrophe Modeling Perspective. RMS } \\
\text { White Paper. [PDF TIMESTAMP 11Mar2014] }\end{array}$ \\
\hline RWS (2014a) & 1 & $\begin{array}{l}\text { RWS, Watermanagementcentrum Nederland, Stormvloedflits } 2013-07 \text { van } 5 \mathrm{t} / \mathrm{m} 7 \text { december 2013, } \\
\text { Rijkswaterstaat (document time stamp: 07Jan2014), 2014a }\end{array}$ \\
\hline RWS (2014b) & 1 & $\begin{array}{cccccccccc}\text { RWS, } & \text { Stormvloedrapport } & \text { van } & 5 & \mathrm{t} / \mathrm{m} & 7 & \text { december } & \text { (SR91) } & \text { Sint-Nicolaasvloed } & 2013, \\
\text { Watermanagementcentrum Nederland, Rijkswaterstaat, prepared by Ing. J. Kroos, } 19 \text { Mar 2014b, } 48 \text { pp }\end{array}$ \\
\hline Slingo et al (2014) & 3 & $\begin{array}{l}\text { Slingo J, S Belcher, A Scaife, M McCarthy, A Saulter, K McBeath, A Jenkins, C Huntingford, T Marsh, J } \\
\text { Hannaford, S Parry, The Recent Storms and Floods in the UK, 29pp, Met Office, Fitzroy Road, Exeter, } \\
\text { Devon, EX1 3PB, UK, February, 2014 [pdf document properties: author=huw.lewis; date } \\
\text { stamp=11Feb2014] }\end{array}$ \\
\hline SMHI (20140703) & 3 & 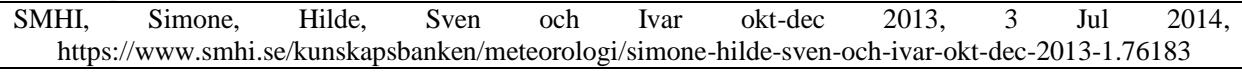 \\
\hline Spencer et al (2014) & 1 & $\begin{array}{l}\text { Spencer, T, S.M. Brooks, I. Moller, B.R. Evans, Where local matters: Impacts of a major North Sea storm } \\
\text { surge, EOS, 95, 269-270, 29July2014 }\end{array}$ \\
\hline Stanev et al. (2014) & 3 & $\begin{array}{l}\text { Stanev E, J Staneva, S Grayek, J Schulz-Stellenfleth, S. Grashorn, A Behrens, Numerical modelling and data } \\
\text { assimilation, COSYNA Progress Report 2013, pp.46-51, Helmholtz-Zentrum Geesthacht, June } 2014 .\end{array}$ \\
\hline Staneva et al (2014) & 2 & $\begin{array}{l}\text { Staneva J, K Wahle, E Stanev, Response of the German Bight Hydro and Sediment Dynamics to Wave, } \\
\text { Tidal and Atmospheric Forcing, 3rd GODAE OceanView Coastal Oceans and Shelf Seas Task Team } \\
\text { (COSS-TT) International Coordination Workshop, 21-24 January 2014, Rncon Beach Resort, Puerto } \\
\text { Rico, 36pp }\end{array}$ \\
\hline Thorne (2014) & 3 & Thorne, Colin, Geographies of UK flooding in 2013/4, The Geographical Journal, 180, 297-309, 2014. \\
\hline Van Dorland (2014) & 1 & van Dorland, R, Zware storm op 5 december, Zenit, p43, Januari 2014 \\
\hline $\begin{array}{l}\text { Van Rooijen and Oost } \\
\text { (2014) }\end{array}$ & 3 & $\begin{array}{l}\text { van Rooijen A, A Oost, Memo: Regionale advisering Ameland Noordwest, Deltares, 1209381-008-ZKS- } \\
\text { 0008, 43pp, 18Dec2014 [PDF document properties: titl=Regional advisering: Ameland NW; } \\
\text { author=Arnold van Rooijen; keywords: 1209381-008-ZKS-0008; date stamp: 18/12/2014] }\end{array}$ \\
\hline 4Coffshore (20151203) & 4 & 4Coffshore, Samso turbine collapse, Dec.03, 2015 \\
\hline Axer et al (2015) & 3 & $\begin{array}{l}\text { Axer T, T Bistry, M Klawa, M Mueller, M Suesser, Deutsche Ruck Sturm dokumentation } 2013 \text { Deutschland, } \\
\text { 2013, Deutsche Rueckversicherung Aktiengesellschaft, Hansaallee } 177,40549 \text { Duesseldorf, } \\
\text { www.deutscherueck.de [pdf document information: author=filiz; date stamp=07Aug2015] }\end{array}$ \\
\hline Carrion (2015) & 1 & $\begin{array}{l}\text { Carrion Aretxabala, BI, Morphological impact of the Sinterklaas storm at Het Zwin. Numerical modelling } \\
\text { with Xbeach, M.Sc. Civil Engineering, Delft University of Technology, } 2015 .\end{array}$ \\
\hline Cipollini et al (2015) & 1 & $\begin{array}{l}\text { Cipollini P, J Benveniste, H Bonekamp, L Miller, M Picot, P Ted Strub, D Vandemark, S Vignudelli, } \\
\text { Recovering more and better data from altimetry in the coastal zone: a community effort, Pilot ARCOM }\end{array}$ \\
\hline
\end{tabular}




\begin{tabular}{|c|c|c|}
\hline & & workshop Lisbon, 1/2 Sept 2015 \\
\hline Dan et al. (2015) & 1 & $\begin{array}{l}\text { Dan, Sebastian, Anne-Lise Montreuil, Rosalia Delgado, Tomas van Oyen, Large storm impact on a beach } \\
\text { under sand nourishments, The Proceedings of the Coastal Sediments 2015, edited by Ping Wang, Julie } \\
\text { D Rosati, and Jun Cheng, Coastal Sediments 2015, San Diego, USA, 11-15May2015 }\end{array}$ \\
\hline $\begin{array}{l}\text { Dissanayake and } \\
\text { Karunarathna (2015) }\end{array}$ & 2 & $\begin{array}{l}\text { Dissanayake P and H Karunarathna, Effect of storm clustering on beach/dune erosion, E-proceedings of the } \\
\text { 36th IAHR World Congress, 28June-3July, 2015, The Hague, The Netherlands }\end{array}$ \\
\hline $\begin{array}{l}\begin{array}{l}\text { Dissanayake et al. } \\
(2015)\end{array} \\
\end{array}$ & 2 & $\begin{array}{l}\text { Dissanayake P, J Brown, H Karunarathna, Impacts of storm chronology on the morphological changes of the } \\
\text { Formby beach and dune system, UK, Nat. Hazards Earth Syst. Sci., 15,1533-1543, } 2015 .\end{array}$ \\
\hline $\begin{array}{l}\text { Dissanayake et al } \\
(2015 b)\end{array}$ & 2 & $\begin{array}{l}\text { Dissanayake P, J Brown, P. Wisse, H Karunarathna, Comparison of storm cluster vs isolated event impacts } \\
\text { on beach.dune morphodynamics, Estuarine, Coastal, and Shelf Science, 164, 301-312, 2015b. }\end{array}$ \\
\hline $\begin{array}{l}\text { Fenoglio-Marc et al } \\
(2015 \mathrm{a})\end{array}$ & 1 & $\begin{array}{l}\text { Fenoglio-Marc L, R Scharroo, A Annuziato, L Mendoza, M Becker, J Lillibridge, Cyclone Xaver seen by } \\
\text { geodetic observations, Geophys Research Letters, 42, 9925-9932, 2015a }\end{array}$ \\
\hline $\begin{array}{l}\text { Fenoglio-Marc et al } \\
(2015 \mathrm{~b})\end{array}$ & 1 & $\begin{array}{c}\text { Fenoglio-Marc L, R Scharroo, A Annuziato, L Mendoza, M Becker, J Lillibridge, Cyclone Xaver seen by } \\
\text { geodetic observations, SUPPORTING MATERIAL, Geophys Research Letters, 42, 9925-9932, 2015b }\end{array}$ \\
\hline Fischer et al. (2015) & 3 & $\begin{array}{l}\text { Fischer, JG, C Senet, A Schneehorst, O Outzen, S Schirmel, K Herklotz, Sea state measurements in } \\
\text { Germanys first offshore wind farm "alpha ventus", in the south-eastern parts of the North Sea, } 2015 \\
\text { IEEE/OES Eleventh Current, Waves and Turbulence Measurement (CWTM), } 2015 \text { [PDF document } \\
\text { properties: datestamp: 14/01/2015] }\end{array}$ \\
\hline Gierlevsen et al. (2015) & 3 & $\begin{array}{l}\text { Gierlevsen T, H Lauridsen, F Langhans, J Bejdic, Met-ocean and wind resource related studies for nearshore } \\
\text { windfarms in Denmark, seminar at the Danish Energy Agency, } 27 \text { February } 2015 .\end{array}$ \\
\hline Gourgue et al (2015) & 3 & $\begin{array}{l}\text { Gourgue O, BB Sishah, J Vanlede, H Komijani, M Chen, Modelling tides and storm surges on the European } \\
\text { continental shelf, 22nd Telemac \& Mascaret User Club, STFC Daresbury Laboratory, UK, 13-16 Oct } \\
\text { 2015. [PDF document properties: datestamp: 05/10/2015] }\end{array}$ \\
\hline $\begin{array}{l}\text { Haigh and Bradshaw } \\
(2015)\end{array}$ & 3 & $\begin{array}{l}\text { Haigh I and E Bradshaw, A century of UK coastal flooding, Planet Earth, Winter 2015, (pdf document } \\
\text { properties: 13Jan2016) }\end{array}$ \\
\hline Jensen et al (2015) & 1 & $\begin{array}{l}\text { Jensen, J., A. Arns, T. Wahl, Yet another 100yr storm surge event: the role of individual storm surges on } \\
\text { design water levels, Journal of Marine Science and Technology, 23, 882-887, } 2015 .\end{array}$ \\
\hline $\begin{array}{ll}\text { Kendon } & \text { and } \\
\text { MarCarthy (2015) }\end{array}$ & 2 & Kendon M and M McCarthy, The UK's wet and stormy winter of 2013/2014, Weather, 70, 40-47, 2015 \\
\hline NLWKN (20151210) & 2 & $\begin{array}{l}\text { NLWKN20151210, Sturmflutwarndienst der Betriebstelle Norden-Norderney, NLWKN Niedersachsischer } \\
\text { Landesbetrieb fuer Wasserwirtschaft, Kuesten- und Naturschutz, Niedersachsen, document date stamp } \\
\text { 10Dec2015. }\end{array}$ \\
\hline Patzer (2015) & 3 & $\begin{array}{l}\text { Patzer, Marianne, Storm surge forecasting at DMI and perspectives on teh use of Earth Observations, ESA } \\
\text { eSurge Sympositum, Deltares, the Netherlands, (powerpoint presentation) January 21, 2015 }\end{array}$ \\
\hline Sibley et al (2015) & 2 & $\begin{array}{l}\text { Sibley A, D Cox, H Titley, Coastal flooding in England and Wales from Atlantic and North Sea storms } \\
\text { during the 2013/2014 winter, Weather, 70, 62-70, 2015 }\end{array}$ \\
\hline SMHI ( & 2 & $\begin{array}{l}\text { SMHI, Egon - saesongens foersta ordentliga storm, 12Jan2015. https://www.smhi.se/nyhetsarkiv/egon- } \\
\text { sasongens-forsta-ordentliga-storm- } 1.83408\end{array}$ \\
\hline Spencer et al (2015) & 1 & $\begin{array}{l}\text { Spencer T, SM Brooks, BR Evans, JA Tempest, I Moeller, Southern North Sea storm surge event of Dec.5, } \\
\text { 2013: Water levels, waves, and coastal impacts, Earth Science Reviews, 146, 120-145, } 2015 .\end{array}$ \\
\hline $\begin{array}{l}\text { Vanmass } \\
(2015)\end{array}$ & 2 & $\begin{array}{l}\text { Vanmassenhove, Niels, Storm surge measures ports Flemish coast, Blankenberge, Tuesday February 3rd, } \\
\text { Maritieme Sientverlening en Kust, Coastal Division, Flanders Hydraulic Research.[document properties: } \\
\text { title=Geintegreerd Kustveiligheidsplan; author=Maarten; datestamp=27/02/2015] }\end{array}$ \\
\hline Wadey et al (2015a) & 2 & $\begin{array}{l}\text { Wadey MP, ID Haigh, RJ Nichols, JM Brown, K Horsburgh, B Carroll, SL Gallop, T Mason, E Bradshaw, A } \\
\text { comparison of the } 31 \text { January-1 February } 1953 \text { and 5-6 December } 2013 \text { coastal flood events around the } \\
\text { UK, UK Frontiers in Marine Science, 2, 84, } 2015 .\end{array}$ \\
\hline Wadey et al (2015b) & 2 & $\begin{array}{l}\text { Wadey MP, JM Brown, ID Haigh, T Dolphin, P Wisse, Assessment and comparison of extreme sea levels } \\
\text { and waves during the 2013/2014 storm season in two UK coastal regions, Nat. Hazards Earth Syst. Sci. } \\
\text { Discuss., 3, 2665-2708, 2015b. }\end{array}$ \\
\hline Zijl et al (2015) & 1 & $\begin{array}{l}\text { Zijl F, J Sumihar, M Verlaan, Application of data assimilation for improved operational water level } \\
\text { forecasting on the northwest European shelf and North Sea, Ocean Dynamics, 65, 1699-1716, } 2015\end{array}$ \\
\hline Brooks et al (2016) & 2 & $\begin{array}{l}\text { Brooks SM, T Spencer, A McIvor, I Moller, Reconstructing and understanding the impacts of storms and } \\
\text { surges, southern North Sea, Earth Surface Processes and Landforms, 41, 855-864, } 2016 .\end{array}$ \\
\hline $\begin{array}{ll}\text { Caithness } & \text { Windfarm } \\
(20160105)\end{array}$ & 3 & $\begin{array}{l}\text { Caithness Windfarm, craigdr, Detailed accidents to } 31 \text { December 2015. Document time stamp 5Jan2016, } \\
175 \mathrm{pp}\end{array}$ \\
\hline Cheliotis et al. (2016) & 1 & $\begin{array}{l}\text { Cheliotis, I, G. Varlas, K. Christakos, The impact of cylone Xaver on hydropower potential in Norway, } \\
\text { conference paper, September } 2016 \text { In: T Karaostas, A Bais, PT Nastos (ed), Perspectives on Atmospheric } \\
\text { Sciences, Springer Atmospheric Sciences, Springer, Cham, } 2017 \text { https://doi.org/10.1007/978-3-319- } \\
\text { 35095-0_25, first online 10Sep2016 [pdf document properties: author=Konstantinos Christakos; } \\
\text { datestamp:07/09/2016] }\end{array}$ \\
\hline Chen and $\mathrm{Xu}(2016)$ & 4 & $\begin{array}{l}\text { Chen } \mathrm{X} \text { and J-Z Xu, Structural failure analysis of wind turbines impacted by super typhoon Usagi, } \\
\text { Engineering Failure Analysis, 60,391-404, } 2016\end{array}$ \\
\hline Christakos et al (2016) & 1 & $\begin{array}{l}\text { Christakos K, I Cheliotis, G Varlas, G-J Steeneveld, Offshore wind energy analysis of Cyclone Xaver over } \\
\text { North Europe, 13th Deep Sea Offshore Wind R\&D Conference, EERA DeepWind'2016, 20-22 January } \\
\text { 2016, Trondheim, Norway, Energy Procedia, 94, 37-44, } 2016 .\end{array}$ \\
\hline $\begin{array}{l}\text { Dangendorf et al } \\
(2016)\end{array}$ & 1 & $\begin{array}{l}\text { Dangendorf S, A Arns JG Pinto, P Ludwig, J Jensen, The exceptional influence of storm 'Xaver' on design } \\
\text { water levels in the German Bight, Environmental Research Letters, 11, 2016, 054001 }\end{array}$ \\
\hline ECMWF (20160316) & 1 & $\begin{array}{cccccc}\begin{array}{l}\text { ECMWF } \\
\text { https://confluence.ecmwf.int/pages/viewpage.action?pageId=28315390 }\end{array} & \text { (20160316), } 201312 & \text { Windstorm } & \text { Xaver/Bodil/Sven, } & \text { Northern } & \text { Europe } \\
\end{array}$ \\
\hline Gerber et al (2016) & 3 & $\begin{array}{l}\text { Gerber M, A Ganske, S Mueller-Navarra, G Rosenhagen, Categorization of meteorological conditions for } \\
\text { storm tide episodes in the German Bight, Meteorologische Zeitschrift, 25, 447-462, } 2016 .\end{array}$ \\
\hline $\begin{array}{l}\text { Mai and Barjenbruch } \\
\text { (2016) }\end{array}$ & 1 & $\begin{array}{l}\text { Mai S and U Barjenbruch, Water level measurements with radar gauges at the German North Sea coast, } \\
\text { [PDF document properties: author=IOC; subject: IOC/2016/MG/14 vol.5; datestamp: 18/04/2017] }\end{array}$ \\
\hline Matelski (2016) & 2 & Matelski, Birgit, Erfahrungen aus der Sturmflut Xaver von 5. und 6.12.2013 und dem Weihnachshochwasser \\
\hline
\end{tabular}




\begin{tabular}{|c|c|c|}
\hline & & $\begin{array}{l}2014 \text { in Schleswig-Holstein, IWASA } 2016 \text { Tagungsbeitrag, (46. IWASA, 7-8 Januar 2016; } \\
\text { Internationales Wasserbau-Symposium Aachen. [pdf document properties: autor=sonja; datestamp: } \\
\text { 26Apr2016] }\end{array}$ \\
\hline $\begin{array}{l}\text { Mawdsley and Haigh } \\
(2016)\end{array}$ & 3 & $\begin{array}{l}\text { Mawdsley RJ and ID Haigh, Spatial and temporal variability and long-term trends in skew surges globally, } \\
\text { Frontiers in Marine Science, 2016, doi: } 10.3389 / \text { fmars.2016.00029 }\end{array}$ \\
\hline Nederhoff et al. (2016) & 1 & $\begin{array}{l}\text { Nederhoff K, E Elias, T Vermaas, Erosie op Ameland Noordwest. Modelstudie: simulaties met Delft3D en } \\
\text { XBeach, Deltares, 117pp, July, } 2016\end{array}$ \\
\hline Sorensen (2016) & 3 & $\begin{array}{l}\text { Sorensen CS, Water NOT wanted - Coastal floods and flooding protection in Denmark, In RA Herrmann \& J } \\
\text { Jensen (eds), Sicherung von Daemmen, Deichen und Stauanlagen: Handbuch fuer Theorie und Praxis } \\
\text { (Vol V pp3-21). Siegen: Universitaet Siegen, } 2016\end{array}$ \\
\hline Sorensen et al (2016a) & 1 & $\begin{array}{l}\text { Sorensen CS, NK Dronen, P Knudsen, J Jensen, P Sorensen, An extreme event as a games changer in coastal } \\
\text { zone management, Journal of Coastal Research, (Special Issue, No 75), 700-704, 2016. Proceedings of } \\
\text { the 14th International Coastal Symposium (Sydney, Australia) ed by A Vila-Concejo, E Bruce, DM } \\
\text { Kennedy, RJ McCarroll, 2016a }\end{array}$ \\
\hline Soresent et al (2016b) & 3 & $\begin{array}{l}\text { Sorensen C, NH Broge, MR Molgaard, CS Schow, PThomsen, K Vognsen, P Knudsen, Assessing future } \\
\text { flood hazards for adaptation planning in a northern European Coastal Community, Frontiers in Marine } \\
\text { Science, 3:69, doi:10.3389/fmars.2016.00069, 2016b }\end{array}$ \\
\hline Staneva et al (2016a) & 2 & $\begin{array}{l}\text { Staneva J, K Wahle, H Guenther, E Stanev, Coupling of wave and circulation models in coastal-ocean } \\
\text { predicting systems: a case study for the German Bight, Ocean Sci., 12, 797-806, 2016a. }\end{array}$ \\
\hline Staneva et al (2016) & 1 & $\begin{array}{l}\text { Staneva J, K Wahle, W Koch, A Behrens, L Fenoglio-Marc, EV Stanev, Coastal flooding: impact of waves } \\
\text { on storm surge during extremes - a case study for the German Bight, Nat. Hazards Earth Syst. Sci., 16, } \\
\text { 2373-2389, } 2016\end{array}$ \\
\hline Brooks et al (2017) & 2 & $\begin{array}{l}\text { Brooks, SM, T Spencer, EK Christie, Storm impacts and shoreline recovery: Mechanisms and controls in the } \\
\text { southern North Sea, Geomorphology, 283, 48-60, } 2017 .\end{array}$ \\
\hline Cheliotis et al (2017) & 1 & $\begin{array}{l}\text { Cheliotis, I, G. Varlas, K. Christakos, The impact of cylone Xaver on hydropower potential in Norway, } \\
\text { conference paper, September } 2016 \text { In: T Karaostas, A Bais, PT Nastos (ed), Perspectives on Atmospheric } \\
\text { Sciences, Springer Atmospheric Sciences, Springer, Cham, 2017, https://doi.org/10.1007/978-3-319- } \\
\text { 35095-0_25 }\end{array}$ \\
\hline Evans (2017) & 3 & $\begin{array}{l}\text { Evans, Sun Yan, EA/2/2 Appendix 1, History of flooding sources, Boston Barrier Transport \& Works Act } \\
\begin{array}{l}\text { order application, Public Inquiry documents, [pdf document properties: author=BDB; } \\
\text { datestamp=16Mar2017] }\end{array} \\
\begin{array}{l}\text { agency.gov.uk/engagement/bostonbarriertwao/results/appendix-1---history-of-flooding-sources.pdf } \\
\text { hts://consult.environment- }\end{array}\end{array}$ \\
\hline $\begin{array}{lll}\text { Frohle } & \text { and } & \text { Dreier } \\
(2017)\end{array}$ & 3 & $\begin{array}{l}\text { Frohle, Peter and Norman Dreier, EarlyDike - Sensor - und risiko basiertes Fruhwarnsystem fuer Seedeiche, } \\
\text { Teilprojekt: Wellenmonitoring und Wellenbelastungssimulator (AP2), Hamburg, April } 2017\end{array}$ \\
\hline Jee (2017) & 3 & $\begin{array}{l}\text { Jee, Andrew, EA/13/2 Appendix 1. A summary of flooding events in Boston. [pdf document properties: } \\
\begin{array}{l}\text { author=Andrew } \begin{array}{c}\text { Jee; } \\
\text { agency.gov.uk/engagement/bostonbarriertwao/ }\end{array} \\
\text { stamp=17Mar2017] }\end{array} \\
\text { https://consult.environment- }\end{array}$ \\
\hline Jensen et al (2017) & 2 & $\begin{array}{l}\text { Jensen J, S Niehuser, A Arns, S Dangendorf, Sensor- und risikobasiertes Fruhwarn-system fuer Seedeiche } \\
\text { (EarlyDike), AP1 - Sturmflutmonitoring und Sturmflutssimulator - Fachbericht 2016, Siegen, April } 2017\end{array}$ \\
\hline Ribeiro et al (2017) & 1 & $\begin{array}{l}\text { Ribeiro R, R Rudge, D Rucinska, Analysis of physical factors of the windstorm Xaver in Poland: post- } \\
\text { hazard review, Weather, 72, 2017, pp.378-382 }\end{array}$ \\
\hline Staneva et al (2017) & 3 & $\begin{array}{l}\text { Staneva J, H Guenther, O Krueger, C Schrumm, V Alari, O Breivik, J-R Bidlot, K Mogensen, Impact of } \\
\text { wind waves on the air-sea momentum fluxes for different wind and sea state conditions and oceanic } \\
\text { responses, 1st International Workshop on waves, storm surges and coastal hazards, Liverpool, UK 10- } \\
\text { 15Sep2017 [pdf document properties: title=Anlass; author=Patrick Kalb-Anlass, datestamp=20/09/2017] }\end{array}$ \\
\hline Staneva et al (2017b) & 1 & $\begin{array}{l}\text { Staneva J, C Schrum, A Behrens, S Grayek, H Ho-Hagemann, V Alan, O Breivik, J-R. Bidlot, A North Sea- } \\
\text { Baltic Sea Regional Coupled Models: Atmosphere, wind waves and ocean, in Proceedings of the Eigth } \\
\text { EuroGOOS International Conference (Operational Oceanography. Serving Sustainable Marine } \\
\text { Development), 3-5 October 2017, Bergen, Norway, 2017b. }\end{array}$ \\
\hline Wahle et al. (2017) & 1 & $\begin{array}{l}\text { Wahle K, J Staneva, W Koch, L Fenoglio-Marc, HTM Ho-Hagemann, EV Stanev, An atmosphere-wave } \\
\text { regional coupled model: improving prediction of wave heights in the southern North Sea, Ocean Sci., 13, } \\
\text { 289-301, 2017.(doi:10.5194/os-13-289-2017). }\end{array}$ \\
\hline $\begin{array}{lll}\text { World } & \text { Bank } & \text { Group } \\
(2017) & & \\
\end{array}$ & 4 & $\begin{array}{l}\text { World Bank Group, Coastal Protection on the West Coast of Jutland, West Africa Coastal Areas } \\
\text { Management Program, Case Study 02 [PDF document date stamp: 14/11/2017] }\end{array}$ \\
\hline DEMA (2018) & 3 & Danish Emergency Management Agency DEMA, National Risk Profile for Denmark, April 2018 \\
\hline Ditlevsen et al (2018) & 3 & $\begin{array}{l}\text { Ditlevsen C, MM Ramos, C Sorensen, UR Ciocan, T Pionkowitz, Hojvandsstatistikker 2017, Miljo- og } \\
\text { Foedevaremnisteriet, Kystdirektoratet, Lemvig, Februar, } 2018\end{array}$ \\
\hline $\begin{array}{lll}\text { Dreier } & \text { and } & \text { Froehle } \\
(2018) & & \end{array}$ & & $\begin{array}{l}\text { Dreier, Norman and Peter Froehle, Operational wave forecast in the German Bight as part of a sensor- and } \\
\text { risk based early warning system, In: J-S Shim, I Chun, HS Lim (ed), Proceedings from the International } \\
\text { Coastal Symposium (ICS) } 2018 \text { (Busan, Republic of Korea), Journal of Coastal Research, Special Issue } \\
\text { No. 85, 1161-1165, 2018 }\end{array}$ \\
\hline $\begin{array}{ll}\begin{array}{l}\text { Environment } \\
(2018)\end{array} & \text { Agency } \\
\end{array}$ & 3 & Environment Agency, Thames Barrier Project Pack 2018, January, 2018 \\
\hline Fery et al. (2018) & 3 & $\begin{array}{l}\text { Fery, Natascha, Birger Tinz, Lydia Gates, Reproduction of storms over the North Sea and the Baltic with the } \\
\text { regional analysis COSMO-REA6 ISPR 2018, 17-19July2018, Bonn [pdf document properties: } \\
\text { datestamp=16/07/2018] }\end{array}$ \\
\hline Gosselin (2018) & 4 & $\begin{array}{l}\text { Gosselin, P., Massive damage ... Large Scale Engineering debacle threatens as North Sea wind turbine } \\
\text { breaks apart, 27April2018 https://notrickszone.com/2018/04/27/massive-damage-large-scale-engineering- } \\
\text { debacle-threatens-as-north-sea-wind-turbine-breaks-apart/ }\end{array}$ \\
\hline Kystdirektoratet (2018) & 3 & $\begin{array}{l}\text { Kystdirektoratet, Shoreface nourishment effects. An analysis of the } 2011 \text { nourishment performed at } \\
\text { Skodbjerge. Kystdirektoratet, Hojbovej 1, 7620 Lemvig, Dec } 2018 \text { [pdf document properties: } \\
\text { title=Shoreface nourishment effects_Skodbjerge_20.12.2018, datestamp=20/12/2018] }\end{array}$ \\
\hline $\mathrm{Li}(2018)$ & 1 & $\begin{array}{l}\text { Li, H., The Ameland Inlet during the Sinterklaas Storm: the role of flooding of watersheds, 2DH model } \\
\text { study in Delft3D-FLOW, M.Sc. Thesis, Utrecht University, 01May2018 }\end{array}$ \\
\hline
\end{tabular}




\begin{tabular}{|c|c|c|}
\hline Niehuser et al (2018) & 1 & $\begin{array}{l}\text { Niehuser S, S Dangendorf, A Arns, J Jensen, A high resolution storm surge forecast for the German Bight, } \\
\text { Conference: 9th Chinese-German Joint Symposium on Coastal and Ocean Engineering, Tainan, Taiwan, } \\
2018\end{array}$ \\
\hline $\begin{array}{l}\text { North Norfolk District } \\
\text { Council Coastal Team } \\
(2018)\end{array}$ & 1 & $\begin{array}{l}\text { North Norfolk District Council Coastal Team, Refurbishment of sea walls and groynes 2013-2015; Cromer } \\
\text { Coast Protection, (picture of plaque on wall taken 27Dec2018) www.northnorfolk.org/coastal }\end{array}$ \\
\hline Ulm et al. (2018) & 3 & $\begin{array}{l}\text { Ulm, Marius, Arne Arns, Juergen Jensen, Assessing consequences of extreme events for the German Bight, } \\
\text { 36th International Conference on Coastal Engineering } 2018 \text { - Coastal Protection and Risk - ID } 1527\end{array}$ \\
\hline $\begin{array}{ll}\text { Wind } & \text { Action } \\
(20180425) & \end{array}$ & 4 & $\begin{array}{l}\text { Wind Action, Massive damage in the wind farm - cause unclear, NDR.de, Christina Gerlach, } 25 \text { Apr2018 } \\
\text { http://www.windaction.org/posts/48258-massive-damage-in-the-wind-farm-cause- } \\
\text { unclear\#.XsD_dD17nIU }\end{array}$ \\
\hline BAM (2019) & 4 & $\begin{array}{l}\text { BAM, Safe arrival of Boston Barrier for tidal flood alleviation scheme, 15Nov2019 11:51, BAM Nuttall Ltd, } \\
\text { https://www.bam.com/en/press/press-releases/2019/11/safe-arrival-of-boston-barrier-for-tidal-flood- } \\
\text { alleviation-scheme }\end{array}$ \\
\hline $\begin{array}{l}\text { Buchana and McSharry } \\
\text { (2019) }\end{array}$ & 4 & $\begin{array}{l}\text { Buchana P, PR McSharry, Windstorm risk assessment for offshore wind farms in the North Sea, Wind } \\
\text { Energy, 22, 1219-1229, } 2019\end{array}$ \\
\hline $\begin{array}{l}\text { Giannopoulos et al } \\
(2019)\end{array}$ & 1 & $\begin{array}{l}\text { Giannopoulos G, L Peake, B Reid, J Andrews, A Grant, I Lorenzoni, M Goulden, J Waters, T Dolphin, J } \\
\text { Bremner, TJ Tolhurst, Environmental and social impacts of the } 2013 \text { storm surge on the North Norfolk } \\
\text { coast, powerpoint presentation date stamp 15May2019, unknown conference }\end{array}$ \\
\hline $\begin{array}{l}\text { North Norfolk District } \\
\text { Council Coastal Team } \\
(2019)\end{array}$ & 1 & $\begin{array}{l}\text { North Norfolk District Council Coastal Team, Sheringham Sea Defences; Repair and Recovery from the } \\
\text { December } 2013 \text { storm surge, www.northnorfolk.org/coastal, photo of plaque on wall taken 30Dec2019 }\end{array}$ \\
\hline Rucinska (2019) & 1 & $\begin{array}{l}\text { Rucinska D, Describing Storm Xaver in disaster terms, International Journal of Disaster Reduction, 34, 147- } \\
153,2019\end{array}$ \\
\hline $\begin{array}{l}\text { Schenk and Mueller- } \\
\text { Navarra (2019) }\end{array}$ & 2 & $\begin{array}{l}\text { Schenk, L and S Mueller-Navarra. 3.4.4. Windstaustatistiken und Haufigkeit von Sturmfluten 2012-2015 } \\
\text { https://www.bsh.de/DE/PUBLIKATIONEN/Nordseezustand_Aktuell/_Anlagen/Downloads/3_4_4_Wind } \\
\text { statistiken.pdf?_blob=publicationFile\&v=2 [pdf document properties: author=Ludwig Schenk; } \\
\text { datestamp=14Feb2019] }\end{array}$ \\
\hline Wikipedia (20191002) & 1 & Wikipedia, Stormen Bodil, https://da.wikipedia.org/wiki/Stormen_Bodil\#cite_note-39 (accessed 02Oct2019) \\
\hline Andrews (2020) & 2 & $\begin{array}{l}\text { Andrews, JE, Spit extension and barrier rollover at Blakeney Point and Salthouse: historic map and field } \\
\text { observations, Bull. geol. Soc. Norfolk, 69, 35-63, } 2020\end{array}$ \\
\hline $\begin{array}{l}\text { JBA Risk Management } \\
(2020)\end{array}$ & 1 & $\begin{array}{l}\text { JBA Risk Management, Storm Xaver 2013. Event Commentary, 2020. [PDF document properties: } \\
\text { author=Cameron Whitwham] }\end{array}$ \\
\hline $\begin{array}{l}\text { Surgewatch } \\
(20200304)\end{array}$ & 1 & $\begin{array}{l}\text { Surgewatch, Storm event 6th December 2013, https://www.surgewatch.org/events/1/ (last accessed } \\
\text { 04Mar2020) }\end{array}$ \\
\hline Thompson et al. (2020) & 4 & $\begin{array}{l}\text { Thompson F, E Renzl, A Sibley, DR Tappin, UK meteotsunamis: a revision and update on events and their } \\
\text { frequency, Weather, } 2000 \text {. }\end{array}$ \\
\hline Wikipedia (20200124) & 1 & WIKI, Cyclone Xaver, https://en.wikipedia.org/wiki/Cyclone_Xaver accessed 24Jan2020 \\
\hline Wikipedia (20200429) & 1 & Wikipedia, Stormen Sven, https://sv.wikipedia.org/wiki/Stormen_Sven\#cite_note-15, accessed 29Apr2020 \\
\hline Wikipedia (20200502) & 1 & Wikipedia, Orkan Xaver, https://de.wikipedia.org/wiki/Orkan_Xaver (accessed 2 May 2020) \\
\hline
\end{tabular}

${ }^{1}$ Type: $1=$ Xaver focus (or used as key example in general discussion); 2=1-4 case studies with Storm Xaver; 3=Storm Xaver is one of many case studies or mentioned only; 4=Xaver not mentioned; included for background information

Table S2. List of normal photos of event (arranged by year and then alphabetically)

\begin{tabular}{|c|c|}
\hline Source & Full Reference and Notes \\
\hline BBC (20131206) & $\begin{array}{l}\text { BBC, In pictures: Winter storm hits UK, } 5 \text { December 2013, https://www.bbc.com/news/uk-scotland-25231224 } \\
\text { FIG1. [PHOTO] Thousands evacuated from homes as storms hit much of UK. } \\
\text { Residents on this street in Rhyl, north Wales, rescued by RNLI (Reuters) } \\
\text { FIG2. [PHOTO] British Red Cross set up rescue centre in the Denbighshire town } \\
\text { but say } 500 \text { people could still be in need of help (Reuters). } \\
\text { FIG3. [PHOTO] Storm hit Welsh coastline about lunchtime (AP) } \\
\text { FIG4. [PHOTO] Earlier in West Lothian lorry driver dies when vehicle blown over } \\
\text { on to two cars (PA) } \\
\text { FIG5. [PHOTO] High winds brought down this lorry near Hamilton (PA). } \\
\text { FIG6. [PHOTO] Many roads have been closed across Scotland due to fallen trees (AP) } \\
\text { FIG7. [PHOTO] THis HGV overturned and came to rest in a precarious position on the } \\
\text { M90 Friarton Bridge near Perth (PA) } \\
\text { FIG8. [PHOTO] Meanwhile residents in Great Yarmouth Norfolk have been filling sandbags } \\
\text { in preparation for the stormy weather moving south (Getty Images) } \\
\text { FIG9. [PHOTO] It's a scene repeated in Gorlesston-on-Sea on the east coast of } \\
\text { England as residents prepare for the worst (Reuters). } \\
\text { FIG10.[PHOTO] Elsewhere in England, high tides followed the storm's tidal surges, } \\
\text { hitting coastal towns including Blackpool (AP) } \\
\text { FIG11.[PHOTO] The famous Blackpool beach was battered by the tide (AP) } \\
\text { FIG12.[PHOTO] In Merseyside the storm claimed a pirate ship build earlier this } \\
\text { year out of drift wood on New Brighton beach. (Bob Warwick) } \\
\text { FIG13.[PHOTO] This dramatic shot captures the scene on the Wirral as the storm } \\
\text { hit the north west of England (Laura Steen) } \\
\text { FIG14.[PHOTO] Further north the Cumbrian coastal village of Allonby has been } \\
\text { battered by heavy seas (PA) } \\
\text { FIG15.[PHOTO] Many homes in Northern Ireland have been left without power due to }\end{array}$ \\
\hline
\end{tabular}




\begin{tabular}{|c|c|}
\hline & damage done by the storm (PA) \\
\hline BBC (20131206) & $\begin{array}{l}\text { BBC20131206, Deadly storm and tidal surge batter northern Europe, BBC } 6 \text { Dec2013 (report by Anna Holligan) } \\
\text {-PHOTO: Residents of Hamburg woke up to find the historic fish market flooded } \\
\text {-PHOTO: Helsingor, southern Sweden: the coast road was flooded } \\
\text {-PHOTO: tidal surge battered Emden in northern Germany near Dutch border } \\
\text {-PHOTO: storm at Hemsby, eastern England, pushed several homes off the cliff } \\
\text {-PHOTO: in Scotland, a lorry driver was killed when his vehicle blew over } \\
\text {-PHOTO: heavy snow caused traffic chaos at Olpe, near Germany's industrial Ruhr region } \\
\text {-PHOTO: there were also traffic jams in snow parts of southern Sweden }\end{array}$ \\
\hline BT (20131208) & $\begin{array}{l}\text { BT, Offer for Bodil: Koebte huset 15.august - nu er det vaek, 08Dec2013, 16:44, (contributor: Morten Eggert) } \\
\text { https://www.bt.dk/danmark/offer-for-bodil-koebte-huset-15.-august-nu-er-det-vaek } \\
\text {-FIG. house tipped over cliff onto beach; family of } 3\end{array}$ \\
\hline $\begin{array}{l}\text { Daily } \\
\text { (20131206) }\end{array}$ & $\begin{array}{l}\text { Daily Mail, Huge storm strikes Europe causing death and destruction and leading to cancellation of hundreds of } \\
\text { flights, 6Dec2013. (correspondent: Nick Enoch), https://www.dailymail.co.uk/news/article-2519517/Huge-storm- } \\
\text { strikes-Europe-causing-death-destruction-leading-cancellation-hundreds-flights.html } \\
\text { FIG. [PHOTO] Firefighers stand at the site of a traffic accident } \\
\text { between the villages of Wicko and Poraj in the Pomerania region. } \\
\text { Three people killed and one injured when a tree fell on a car } \\
\text { due to heavy storms. } \\
\text { FIG. [PHOTO] Reception building of camping ground submerged in the } \\
\text { midday high tide on the beach at Bensersiel this morning. } \\
\text { FIG. [PHOTO] Hamburg's historic fish auction hall Fishmarkt is flooded } \\
\text { by the water of teh Elbe river today after the storm Xaver reached } \\
\text { the northern German city during the night } \\
\text { FIG. [PHOTO] A boat lies on its side today in Thorsminde Harbour } \\
\text { on the west coast of Jutland as Hurricane Xaver strikes }\end{array}$ \\
\hline Fleetmon (20131207) & $\begin{array}{l}\text { Fleetmon, Container ship Burak Bayraktar in trouble off Texel, Netherlands, (correspondent: Mikhail Voytenko, } \\
\text { 7Dec2013 06:31) https://www.fleetmon.com/maritime-news/2013/2810/container-ship-burak-bayraktar- } \\
\text { trouble-texel-nethe/ } \\
\text { FIG: map of ship positions } \\
\text { FIG: ship Burak Bayraktar in distress }\end{array}$ \\
\hline GP (20131206) & $\begin{array}{l}\text { GP, Fortsatt risk for halka, 6Dec2013 (correspondent: D Henriksson, K Vikingsson, P Sydvik, TA Akerblom) } \\
\text { http://www.gp.se/nyheter/goteborg/1.2201325-fortsatt-risk-for-halka (accessed 1May2020) } \\
\text { FIG. [PHOTO] Trafikproblem. Traffic jam on Riksvag } 40 \text { at Tolkabro west of Ulricehamn } \\
\text { (Adam Ihse) }\end{array}$ \\
\hline $\begin{array}{ll}\begin{array}{l}\text { Kristeligt } \\
(20131205)\end{array} & \text { Dagblad }\end{array}$ & $\begin{array}{l}\text { Kristeligt Dagblad, Stormen blaeser Skotland omkuld: Doodsfald og nebrud, (contributor: Ritzau), 05Dec2013 } \\
\text { 12:50 https://www.kristeligt-dagblad.dk/udland/stormen-bl\%C3\%A6ser-skotland-omkuld-d\%C3\%B8dsfald- } \\
\text { og-nedbrud } \\
\text { FIG. [PHOTO] Litter bins are washed along the promenade and waves bater the sea wall } \\
\text { in Blackpool, NW England on 5Dec2013 as high winds hit north of England and } \\
\text { Scotland. PHOTO: Paul Ellis }\end{array}$ \\
\hline $\begin{array}{l}\text { National Wind Watch } \\
(20131210 a)\end{array}$ & $\begin{array}{l}\text { National Wind Watch, 10Dec2013, Xaver zerstoert ein Windrad bei Vlatten, original source: Aachener Zeitung, } \\
\text { 6Dec2013 https://www.wind-watch.org/news/2013/12/10/xaver-zerstort-ein-windrad-bei-vlatten/ } \\
\text { FIG. [PHOTO] near Vlatten storm Xaver destroyed a wind turbine. Foto: Vietoris }\end{array}$ \\
\hline $\begin{array}{l}\text { National Wind Watch } \\
\text { (20131210b) }\end{array}$ & $\begin{array}{l}\text { National Wind Watch, Sturm 'Xaver': Windrad stuerzt auf Acker, 10Dec2013b, credit: von Manfred Reinnarth, } \\
\text { Koelnische Rundschau, 06/12/2013, https://www.wind-watch.org/news/2013/12/10/sturm-xaver-windrad- } \\
\text { sturzt-auf-acker/ } \\
\text {-PHOTO: turbine covering the field of Wilhelm-Josef Schaefer at Vlatten. } \\
\text {-PHOTO: hollow inside of turbine }\end{array}$ \\
\hline $\begin{array}{l}\text { National Wind Watch } \\
(20131212)\end{array}$ & $\begin{array}{l}\text { National Wind Watch, Sturm 'Xaver': Windrad stuerzt auf Acker, 10Dec2013b, credit: von Manfred Reinnarth, } \\
\text { Koelnische Rundschau, 06/12/2013, https://www.wind-watch.org/news/2013/12/10/sturm-xaver-windrad- } \\
\text { sturzt-auf-acker/ } \\
\text { FIG. [PHOTO] } 100 \text { meter colossus on the field by Mechernich (photo: Kuffner) } \\
\text { FIG. [PHOTO] wind turbine broken at } 25 \mathrm{~m} \text { height level and on field } \\
\text { FIG. [PHOTO] Amazingly hollow: wind turbine } 4 \text { investigated by experts }\end{array}$ \\
\hline $\begin{array}{l}\text { Nordbayern } \\
(20131207)\end{array}$ & $\begin{array}{l}\text { Nordbayern, Deining: 'Xaver' reisst Rotorblatt von Windrad ab. Windkraftanlage schleuderte Eisbrocken auf die } \\
\text { Strasse, 07/12/2013, 12:07. https://www.nordbayern.de/region/neumarkt/deining-xaver-reisst-rotorblatt-von- } \\
\text { windrad-ab-1.3326492 } \\
\text { FIG. wind blade sails } 150 \mathrm{~m} \text { from turbine Thurday-Friday night from Anlage } 7 \\
\text { of Deininger Windpark. Blade was damaged during mounting and was } \\
\text { to be exchanged. Area around site closed. [Gunter Distler] } \\
\text { FIG. Field covered with snow with woods in background }\end{array}$ \\
\hline $\begin{array}{l}\text { Spiegel International } \\
(20131206)\end{array}$ & $\begin{array}{l}\text { Spiegel International, Winter storm 'Xaver' batters northern Europe,6 Dec 2013, 12:56 } \\
\text { https://www.spiegel.de/international/europe/tidal-surge-winter-storm-xaver-batters-northern-europe-a- } \\
\text { 937576.html\# } \\
\text { FIG1. Parts of N Europe hit on Thursday and Friday with a tidal surge as } \\
\text { low-pressure system Xaver blows through the region, bringing icy } \\
\text { hurricane-force winds. Here, the North Sea rages near the German } \\
\text { town of Emden on Friday morning (Reuters). } \\
\text { FIG2. The storm brought rain, hail and snow with the tidal surge, and the } \\
\text { German port city of Hamburg seen its worst flooding in decades, } \\
\text { forcing authorities to close off parts of the city center on Thursday } \\
\text { night. Here, woman passes by the storm surge in central Hamburg (DPA) } \\
\text { FIG3. People stand on benches in Hamburg's historic fish market which } \\
\text { has been flooded by the Elbe River due to the storm (AFP) }\end{array}$ \\
\hline
\end{tabular}




\begin{tabular}{|c|c|}
\hline & $\begin{array}{l}\text { FIG4. Floodwaters in Hamburg early on Friday morning. City officials closed } \\
\text { off flooded areas and some people reportedly had trouble reaching } \\
\text { work in the morning (DPA) } \\
\text { FIG5. In Rostock fire fighters secure parts of a roof blown off by } \\
\text { hurricane-force winds (DPA) } \\
\text { FIG6. Streets also flooded in the northwestern German town of Norddeich on } \\
\text { Friday morning (Reuters) } \\
\text { FIG7. Water levels reached what was expected to be their high point in Hamburg } \\
\text { on Friday morning, some } 6 \mathrm{~m} \text { above level (Reuters) } \\
\text { FIG8. Waves overtake ferry station in the northern German city of Dagebuell } \\
\text { on Thursday night (DPA) } \\
\text { FIG9. The pier at the Bensersiel port of lower Saxony was also flooded by the } \\
\text { storm surge (DPA) } \\
\text { FIG10.Strong gusts of wind up to 155km/h reported across Germany, damaging roofs, } \\
\text { uprooting trees and causing traffic problems. That did not this man from } \\
\text { getting right in the thick of it on Thursday (DPA) } \\
\text { FIG11.The UK, Germany, Netherlands, Scandinavia most heavily affected by the severe } \\
\text { storm, but cancelled flights and tranins caused problems across Europe. } \\
\text { By Thursday night, officials reported three storm-related deaths in the } \\
\text { UK and Denmark. Here, planes await takeoff at the Hamburg airport on } \\
\text { Thursday (DPA) } \\
\text { FIG12.An uptrooted tree in the village of Sankt Peter Ording on the North Sea } \\
\text { coast on Thursday (Reuters) } \\
\text { FIG13.A rescue vessel patrols the North Sea island of Norderney on Thursday (DPA) } \\
\text { FIG14.Scotland also say high winds on Thursday. Here, rescue workers at the scene } \\
\text { a truck accident near Bathgate (AP) } \\
\text { FIG15.Snow from Xaver gnarled traffic near Olpe in the state of Nord Rhine Westphalia } \\
\text { on Friday morning (DPA) } \\
\text { FIG16.Here waves batter th promenade in Blackpool England on Thursday as the storm } \\
\text { hit the UK } \\
\text { FIG17.Homes on the tiny German island of Langeness as the North Sea rose on } \\
\text { Thursday (DPA) } \\
\text { FIG18.Traffic signs on the beach in the German village of Norddeich on Thursday } \\
\text { were nearly submerged by the storm surge. (Reuters) }\end{array}$ \\
\hline $\begin{array}{l}\text { Sueddeutsche Zeitung } \\
\text { (20131215) }\end{array}$ & $\begin{array}{c}\text { Sueddeutsche Zeitung, } \quad \text { Xaver } \\
\text { https://www.sueddeutsche.de/panorama/sturmschaeden-auf-sylt-xaver-holte-sich-land-1.1844100201315:36 } \\
\text { FIG: [PHOTO] part of a viewing platform and or stairs lying on the beach of Hoernum on a damaged dune }\end{array}$ \\
\hline $\begin{array}{l}\text { Sylter Rundschau } \\
(20131209)\end{array}$ & $\begin{array}{l}\text { Sylter Rundschau, Folgen des Orkans. Xaver 'knabberte' Sylt massiv an, 09Dec2013 06:00 (from Friederike } \\
\text { Reussner) https://www.shz.de/lokales/sylter-rundschau/xaver-knabberte-sylt-massiv-an-id5098481.html } \\
\text { FIG. [PHOTO] First floor of Wenningstedter Restaurant Wonnemayer damaged during Storm Xaver }\end{array}$ \\
\hline $\begin{array}{l}\text { TheJournal } \\
\text { (20131205) }\end{array}$ & $\begin{array}{l}\text { TheJournal, Homes remain without electricity after high winds batter power lines, 05Dec2013 08:20PM, } \\
\text { https://www.thejournal.ie/storm-ireland-1207783-Dec2013/ } \\
\text { PHOTO: coastal area Ireland [Sasko Lazarov, Photocall Ireland] }\end{array}$ \\
\hline The Local (20131205) & $\begin{array}{ccccc}\text { The Local, Sven's strong winds sweep southern Sweden. } & \text { 05Dec2013 } & \text { 15:57CET } \\
\text { https://www.thelocal.se/20131205/storm-sven-sweeps-into-southern-sweden } & & \\
\text {-FIG. [PHOTO] Storm Sven sweeps into Malmo [Joham Nilsson, TT] } & & \\
\end{array}$ \\
\hline Upstream (20131205) & $\begin{array}{l}\text { Upstream, North Sea production curtailed over storm, 05Dec2013 (contributor: Bill Lehane and News Wires) } \\
\text { FIG. [PHOTO] Weather impact. Platformscut output over storm, } \\
\text { seen here causing flooding in Wales. (Photo: Reuters, Scanpix) }\end{array}$ \\
\hline ABPmer (2014) & $\begin{array}{l}\text { ABP mer, Ensuring Flood Resilience. An overview of the 5/6 December 2013, Associated British Ports, Marine } \\
\text { Environmental Research, July, } 2014 \\
\text {-FIG_p3. [PHOTO] Weiring over Grimsby flood gate } \\
\text {-FIG_p3. [PHOTO] Coastal erosion at Hemsby [Albanpix] } \\
\text {-FIG_p3. [PHOTO] Wave damage to Cromer frontage [David Tipling] }\end{array}$ \\
\hline BBC (20141205) & $\begin{array}{l}\text { BBC, East coast surge: what happened next? (report by Richard Haugh), 5Dec2014 } \\
\text {-PHOTO: seven cliff top houses collapsed in Hemsby on the night of the worst storm surge for } 60 \text { years (beach } \\
\text { view of } 2 \text { collapsed bungalows) } \\
\text {-PHOTO: residents formed a human chain to help salvage items from homes in Hemsby (aerial view of } 4 \text { houses } \\
\text { over cliff edge) } \\
\text {-PHOTO: hundreds of homes in Lincolnshire were also evacuated during the tidal surge (ambulance crew } \\
\text { evacuating resident by boast) } \\
\text {-PHOTO: flats in Boston were flooded (woman on stairs of flat with } 50 \mathrm{~cm} \text { water) } \\
\text {-PHOTO: some families had to be rescued by boat on Boston High street (ambulance crew evacuating people in } \\
\text { zodiac) } \\
\text {-PHOTO: Hugh Drake lost } 25 \text { acres of his land and can not grow crops on a large section (picture of farmer in } \\
\text { front of coastal salt marsh) } \\
\text {-PHOTO: ironically, the Bizzaro restaurant in Boston was hosting a Venice themed night on } 5 \text { December } 2013 \text {. } \\
\text { The image on the right shows how the street outside the restaurant looks now (before and after photos of Boston } \\
\text { high street) } \\
\text {-PHOTO: Ray Mooney was still in his house in Hemsby when it started to be washed away (picture of Hemsby } \\
\text { house overhanging sand cliff) } \\
\text {-PHOTO: remains of the houses can still be seen on Hemsby Beach (steel trusses protruding from sand cliff) } \\
\text {-PHOTO: several pilot sea defence schemes have been placed along the beach with the help of residents (square } \\
\text { rock baskets on Hemsby beach) } \\
\text {-PHOTO: the Netherlands improved flood defences after } 1836 \text { people died in 1953. (archive photo of Dutch house }\end{array}$ \\
\hline
\end{tabular}




\begin{tabular}{|c|c|}
\hline & in $2 \mathrm{~m}$ of seawater from 1953 surge with ground floor washed away) \\
\hline Dunbar et al (2014) & $\begin{array}{l}\text { Dunbar I, N Phipps, M Szonyi, Risk Nexus. After the storm: how the UK's flood defences performed during the } \\
\text { surge following Xaver, Flood resilience review 09.14, Zurich Insurance Company Ltd., Mythenquai 2, } \\
\text { 8002, Zurich, Switzerland [document properties: date 28Aug2014; author= Zurich] } \\
\text {-FIG_p15. Scarborough beach front sustained significant storm damage, including damage to sea wall } \\
\text {-FIG_p16. Flooded building being dried out at a location in Lowestoft } \\
\text {-FIG_p17. Visible water line from the surge event at a location with protection installed. } \\
\text {-FIG_p20. Post-flood clean-up operation underway in Scarborough where sea front } \\
\text { arcades and cafes were flooded along the South Bay. The tidal level here } \\
\text { was estimated at approximately } 6 \mathrm{~m} \text { above normal levels. }\end{array}$ \\
\hline Eriksen (2014) & $\begin{array}{l}\text { Eriksen J, Rekordvandstande i Isefjorden og Roskilde Fjord, Vejret, 138, 2 40-48, } 2014 \\
\text {-FIG9. [PHOTO] Photo of level from the old rail bridge at Frederiksund } \\
\text { taken on a seith with still weather and normal water levels. } \\
\text { One can see a single bridge pilefrom the old rail bridge and in the } \\
\text { background railbed causeway (Jesper Eriksen) }\end{array}$ \\
\hline FINO1 (20140108) & $\begin{array}{l}\text { FINO1, 15-m wave damaged FINO1, 08Jan2014. http://www.fino1.de/meldungen/alle-meldungen/137-15-meter- } \\
\text { welle-beschaedigt-fino1 } \\
\text { FIG. [PHOTO] ripped } 15 \mathrm{~m} \text { deck grating on } 15 \mathrm{~m} \text { side of the FINO1 platform } \\
\text { FIG. [PHOTO] photo smashed small wooden deck at } 17 \mathrm{~m}\end{array}$ \\
\hline Goennert et al (2014) & $\begin{array}{l}\text { Goennert G, O Mueller, M Schaper, K Sossidi, Die Sturmflut nach dem Tief Xaver von 5. bis 7. Dezember } 2013 . \\
\text { Berichte des Landesbetriebes Strassen, Bruecken und Gewaesser (LSBG), Freie und Hansestadt Hamburg, } \\
\text { Nr. 16/2014, 26pp. } \\
\text {-FIG6. [PHOTO] Moorfleet main dike on morning of 6Dec2013 } \\
\text {-FIG8. [PHOTO] Storm flood at Landungsbrueckengebaude on 6Dec2013 0420 } \\
\text {-FIG9. [PHOTO] Qualmwasseraustritt on 6Dec2013 (ground water erupting out of ground) } \\
\text {-FIG11.[PHOTO] St. Pauli Fischmarkt on 6Dec2013 at 06:30 } \\
\text {-FIG12.[PHOTO] Am Sandtorkal (morning of 6Dec) } \\
\text {-FIG13.[PHOTO] Dalmannkai on evening of 6Dec2013 } \\
\text {-FIG14.[PHOTO] Dalmannkai on evening of 6Dec2013 } \\
\text {-FIG17. [PHOTO] Fischmarkt on monring 6Dec2013 }\end{array}$ \\
\hline $\begin{array}{l}\text { Knaack and Heyken } \\
(2014)\end{array}$ & $\begin{array}{l}\text { Knaack H and H Heyken, Xaver hatte sehr schwere Stumflut im Gepaeck, Jahresbericht 2013. Der Zukunft } \\
\text { verplichtet, NLWKN, Niedersaechsischer Landesbetrieb fuer Waserwirtschaft, Kuesten- und Naturschutz, } \\
\text { pp.8-9, document date stamp 24Apr2014. } \\
\text { FIG. [PHOTO] } 60000 \text { cubic meters sand will be brought to Juist to strengthen } \\
\text { the dunes }\end{array}$ \\
\hline Nossent et al (2014) & $\begin{array}{l}\text { Nossent J, L Boeckx, E Taverniers, M Deschamps, T Verwaest, F Mostaert, Sinterklaasstorm } 6 \text { december } 2013 . \\
\text { Beschrijving van de hydrometrische gebeurtenissen, Versie 4.0. WL Rapporten, 00-119, Waterbouwkundig } \\
\text { Laboratorium, Antwerpen, Belgie. October 2014, WL2014R00_119_5 [document date stamp: 15Oct2014; } \\
\text { author= Deschamps, Maarten] } \\
\text { FIG19. [PHOTO] Aerial oblique photo from 06/12/2013 of the height of Bergenmeersen and Paardeweide } \\
\text { (viewing direction from upstream to downstream) }\end{array}$ \\
\hline Pelt (2014) & $\begin{array}{l}\text { Pelt AS, BODIL's stormflod i de indre dansk farvande, Vejret, 138, 24-29, } 2014 \\
\text {-FIG4. [PHOTO] During the storm surge Nordsjaellands Kattegatkyst experienced } \\
\text { large erosion and damage. Photo of Rageleje Strandvej, which was } \\
\text { closed to through traffic because waves swept over the road. } \\
\text {-FIG. [PHOTO]. Backside photo of flooding in Lynaes Havn at Hundested in connection with } \\
\text { Bodil's record water level (photo Mikael Scharling) }\end{array}$ \\
\hline RMS (2014) & $\begin{array}{l}\text { RMS, 2013-2014 Winter Storms in Europe. An Insurance and Catastrophe Modeling Perspective. RMS White } \\
\text { Paper. [PDF TIMESTAMP 11Mar2014] } \\
\text { FIG8. [PHOTO] Repair work underway at the breached Boston Dike }\end{array}$ \\
\hline RWS (2014b) & $\begin{array}{l}\text { RWS, Stormvloedrapport van } 5 \mathrm{t} / \mathrm{m} 7 \text { december (SR91) Sint-Nicolaasvloed 2013, Watermanagementcentrum } \\
\text { Nederland, Rijkswaterstaat, prepared by Ing. J. Kroos, } 19 \text { Mar 2014b, } 48 \mathrm{pp} \\
\text { FIG8. [PHOTO] closed flood gate at Delfzij1 06Dec2013 } \\
\text { FIG11.[PHOTO] Seaweed flood line on dike at Emmapolder; west of Eemshaven }\end{array}$ \\
\hline Staneva et al (2014) & $\begin{array}{l}\text { Staneva J, K Wahle, E Stanev, Response of the German Bight Hydro and Sediment Dynamics to Wave, Tidal and } \\
\text { Atmospheric Forcing, 3rd GODAE OceanView Coastal Oceans and Shelf Seas Task Team (COSS-TT) } \\
\text { International Coordination Workshop, 21-24 January 2014, Rncon Beach Resort, Puerto Rico, 36pp } \\
\text {-FIG. [PHOTO] wave impact on pier in Germany } \\
\text {-FIG. [PHOTO] flooded fish market }\end{array}$ \\
\hline Thorne (2014) & $\begin{array}{l}\text { Thorne, Colin, Geographies of UK flooding in 2013/4, The Geographical Journal, 180, 297-309, } 2014 . \\
\text { FIG. [PHOTO] aerial view of the Thames Barrier closed during the surge }\end{array}$ \\
\hline $\begin{array}{l}\text { Van Rooijen and Oost } \\
\text { (2014) }\end{array}$ & $\begin{array}{l}\text { van Rooijen A, A Oost, Memo: Regionale advisering Ameland Noordwest, Deltares, 1209381-008-ZKS-0008, } \\
\text { 43pp, 18Dec2014 [PDF document properties: titl=Regional advisering: Ameland NW; author=Arnold van } \\
\text { Rooijen; keywords: 1209381-008-ZKS-0008; date stamp: 18/12/2014] } \\
\text { FIG2.4. [PHOTO] Impacts on the westkop of Ameland after the Sinterklaasstorm of 5-6Dec2013 } \\
\text { (exact location unknown) }\end{array}$ \\
\hline Axer et al (2015) & $\begin{array}{l}\text { Axer T, T Bistry, M Klawa, M Mueller, M Suesser, Deutsche Ruck Sturm dokumentation } 2013 \text { Deutschland, } \\
\text { 2013, Deutsche Rueckversicherung Aktiengesellschaft, Hansaallee 177, 40549 Duesseldorf, } \\
\text { www.deutscherueck.de [pdf document information: author=filiz; date stamp=07Aug2015] } \\
\text {-FIG_p39. [PHOTO] toppled Christmas tree in front of the Schloss Bellevue, residence of Bundespraesident } \\
\text { Joachim Gauck }\end{array}$ \\
\hline Carrion (2015) & $\begin{array}{l}\text { Carrion Aretxabala, BI, Morphological impact of the Sinterklaas storm at Het Zwin. Numerical modelling with } \\
\text { Xbeach, M.Sc. Civil Engineering, Delft University of Technology, } 2015 \text {. } \\
\text {-FIG2.10.[PHOTO] General view of the Dutch dunes at Het Zwin and records of }\end{array}$ \\
\hline
\end{tabular}




\begin{tabular}{|c|c|}
\hline & $\begin{array}{l}\text { large portion of dunes scarped by Sinterklaasstorm } \\
\text {-FIG2.11.[PHOTO] Record of the overwash event }\end{array}$ \\
\hline Fischer et al. (2015) & $\begin{array}{l}\text { Fischer, JG, C Senet, A Schneehorst, O Outzen, S Schirmel, K Herklotz, Sea state measurements in Germanys } \\
\text { first offshore wind farm "alpha ventus", in the south-eastern parts of the North Sea, } 2015 \text { IEEE/OES Eleventh } \\
\text { Current, Waves and Turbulence Measurement (CWTM), } 2015 \text { [PDF document properties: datestamp: } \\
\text { 14/01/2015] } \\
\text {-FIG2. [PHOTO] Extreme wave events at FINO1 in German Bight } \\
\text { (a) FINO1 research platform in German Bight } \\
\text { (b) Damage to lower hatchway of FINO1 (2013) }\end{array}$ \\
\hline NLWKN (20151210) & $\begin{array}{l}\text { NLWKN20151210, Sturmflutwarndienst der Betriebstelle Norden-Norderney, NLWKN Niedersachsischer } \\
\text { Landesbetrieb fuer Wasserwirtschaft, Kuesten- und Naturschutz, Niedersachsen, document date stamp } \\
\text { 10Dec2015. } \\
\text { FIG. [PHOTO] Harlingerland coast - Nikolausflut 2013; sea on dyke } \\
\text { FIG. [PHOTO] Neuharlingersiel - Nikolausflut 2013; coastal walkway flooded } \\
\text { FIG. [PHOTO] unidentified scene on German coast; sea on dyke }\end{array}$ \\
\hline Spencer et al (2015) & 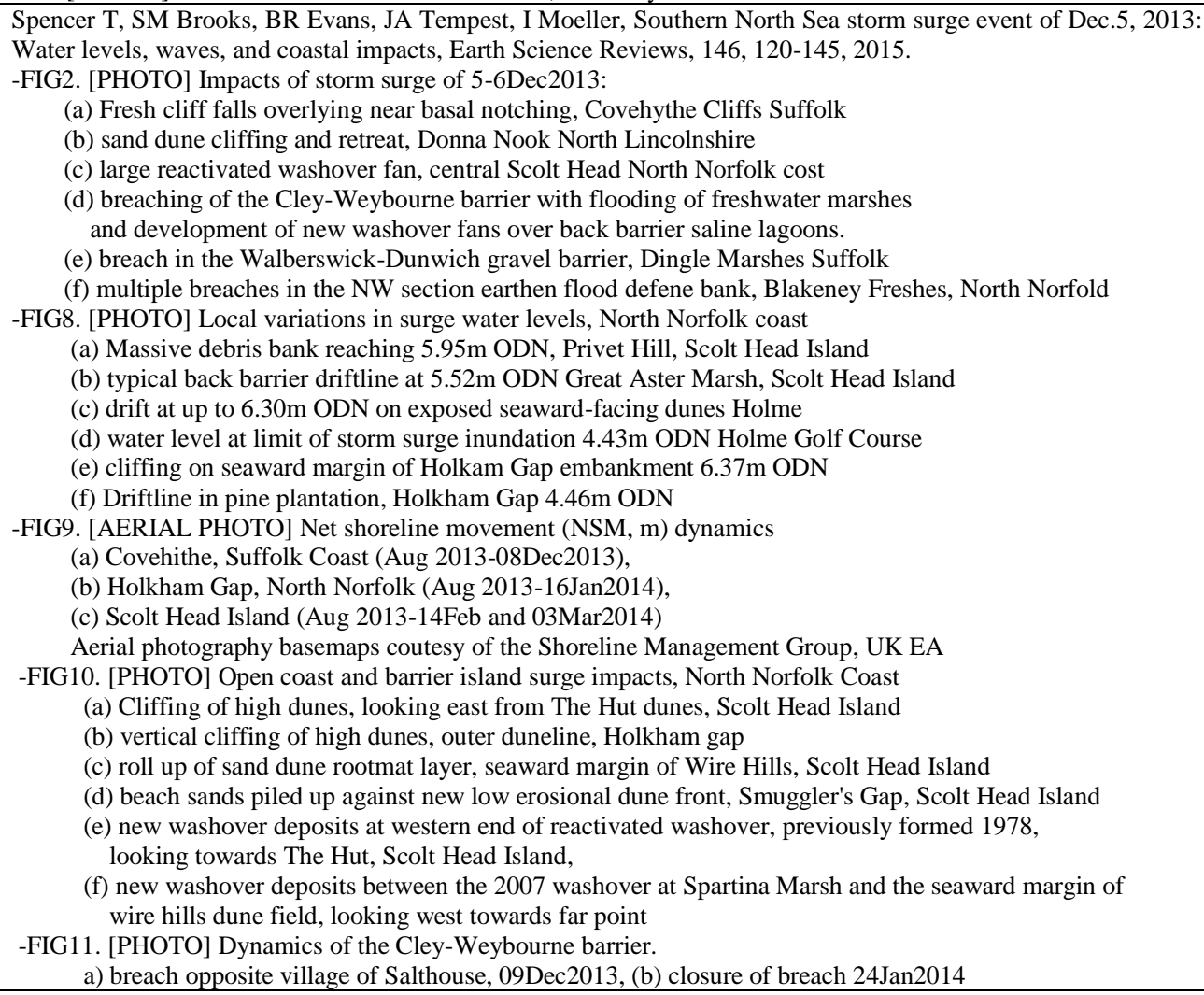 \\
\hline $\begin{array}{l}\text { Vanmas } \\
\text { (2015) }\end{array}$ & $\begin{array}{l}\text { Vanmassenhove, Niels, Storm surge measures ports Flemish coast, Blankenberge, Tuesday February 3rd, } \\
\text { Maritieme Sientverlening en Kust, Coastal Division, Flanders Hydraulic Research.[document properties: } \\
\text { title=Geintegreerd Kustveiligheidsplan; author=Maarten; datestamp=27/02/2015] } \\
\text {-FIG_S9: [PHOTO] view of one of the critical points in the harbour of Ostend } \\
\text { during the December } 2013 \text { storm } \\
\text {-FIG_S10: [PHOTO] emergency workers placing sandbags Ostend during Dec2013 flood } \\
\text {-FIG_S11: [PHOTO] harbour of Ostend during Dec } 2013 \text { storm }\end{array}$ \\
\hline Matelski (2016) & $\begin{array}{l}\text { Matelski, Birgit, Erfahrungen aus der Sturmflut Xaver von 5. und 6.12.2013 und dem Weihnachshochwasser } 2014 \\
\text { in Schleswig-Holstein, IWASA } 2016 \text { Tagungsbeitrag, (46. IWASA, 7-8 Januar 2016; Internationales } \\
\text { Wasserbau-Symposium Aachen. [pdf document properties: autor=sonja; datestamp: 26Apr2016] } \\
\text { FIG7. [PHOTO] Aerial photo showing retreat of southern tip of Sylt Island } \\
\text { (Hoernum Odde) } \\
\text { FIG8. [PHOTO] Photo of dike damage Nordstrand Alter Koog } \\
\text { FIG9. [PHOTO] Loose grass being gathered from inland dike }\end{array}$ \\
\hline Nederhoff et al (2016) & $\begin{array}{l}\text { Nederhoff K, E Elias, T Vermaas, Erosie op Ameland Noordwest. Modelstudie: simulaties met Delft3D en } \\
\text { XBeach, Deltares, 117pp, July, } 2016 \\
\text {-FIG2.4. [PHOTO] Impact on the westkop of Ameland after the Sinterklaasstorm 5-6Dec2013 }\end{array}$ \\
\hline Sorensen (2016) & $\begin{array}{l}\text { Sorensen CS, Water NOT wanted - Coastal floods and flooding protection in Denmark, In RA Herrmann \& J } \\
\text { Jensen (eds), Sicherung von Daemmen, Deichen und Stauanlagen: Handbuch fuer Theorie und Praxis (Vol V } \\
\text { pp3-21). Siegen: Universitaet Siegen, } 2016 \\
\text {-FIG12. [PHOTO] High water levels in the town of Lemvig, the Limfjord, during Storm Xaver 05Dec2013. } \\
\text { Note the flood protection wall winding along the harbour front. } \\
\text { (water level at 40cm of crest) } \\
\text {-FIG13. [PHOTO] Floods in identical locations in } 1921 \text { and during Xaver 6Dec2013 at } \\
\text { Frederikssund (left) and Helsinore (Sealand). } \\
\text { Sources: mx.dk (top left), helsingornetavis.dk (top right), and } \\
\text { Ministeriet for Offentlige Arbejder (1922) }\end{array}$ \\
\hline
\end{tabular}




\begin{tabular}{|c|c|}
\hline Brooks et al (2017) & $\begin{array}{l}\text { Brooks, SM, T Spencer, EK Christie, Storm impacts and shoreline recovery: Mechanisms and controls in the } \\
\text { southern North Sea, Geomorphology, 283, 48-60, } 2017 \text {. } \\
\text {-FIG2.[PHOTO] Superfrontage } 2 \text { on teh North Norfolk Coast. } \\
\text { (a) Brancaster Bay looking east towards Scolt Head Island in the far distance, } \\
\text { showing barrier cliffing following the 5Dec2013 storm (photo SM Brooks 12/01/2016) } \\
\text { (c) the barrier at Holkham Bay looking east towards Wells-next-the-Sea } \\
\text { shortly after the 5-6Dec2013 storm (photo T Spencer 16/01/2014) } \\
\text { (d) general setting of Scolt Head Island showing the barrier and back barrier marshes } \\
\text { following the 5Dec storm with Brancaster Bay in the far distance } \\
\text { (photo M. Page 09/12/2013) }\end{array}$ \\
\hline Evans (2017) & $\begin{array}{l}\text { Evans, Sun Yan, EA/2/2 Appendix 1, History of flooding sources, Boston Barrier Transport \& Works Act order } \\
\text { application, Public Inquiry documents, [pdf document properties: author=BDB; datestamp=16Mar2017] } \\
\text { https://consult.environment-agency.gov.uk/engagement/bostonbarriertwao/results/appendix-1---history-of- } \\
\text { flooding-sources.pdf } \\
\text {-FIG. [PHOTO] two night photographs showing flooding in streets of Boston 5Dec2013 }\end{array}$ \\
\hline Ribeiro et al (2017) & $\begin{array}{l}\text { Ribeiro R, R Rudge, D Rucinska, Analysis of physical factors of the windstorm Xaver in Poland: post-hazard } \\
\text { review, Weather, 72, 2017, pp.378-382 } \\
\text {-FIG6. [PHOTO] The island of Hooge on 5th December } 2013 \text { at two different times (a) 0850UTC and (b) } \\
\text { 1500UTC (Dagebuell, 2013) }\end{array}$ \\
\hline Staneva et al (2017) & $\begin{array}{l}\text { Staneva J, H Guenther, O Krueger, C Schrumm, V Alari, O Breivik, J-R Bidlot, K Mogensen, Impact of wind } \\
\text { waves on the air-sea momentum fluxes for different wind and sea state conditions and oceanic responses, 1st } \\
\text { International Workshop on waves, storm surges and coastal hazards, Liverpool, UK 10-15Sep2017 [pdf } \\
\text { document properties: title=Anlass; author=Patrick Kalb-Anlass, datestamp=20/09/2017] } \\
\text {-FIG. [PHOTO] Photo wave impact at unknown coastal location in German Bight } \\
\text {-FIG. [PHOTO] Photo of Hamburg fish market }\end{array}$ \\
\hline DEMA (2018) & $\begin{array}{l}\text { Danish Emergency Management Agency DEMA, National Risk Profile for Denmark, April } 2018 \\
\text { * -FIG. [PHOTO] A house in Roskilde is flooded from storm surge during Hurrican Bodil }\end{array}$ \\
\hline Fery et al (2018) & $\begin{array}{l}\text { Fery, Natascha, Birger Tinz, Lydia Gates, Reproduction of storms over the North Sea and the Baltic with the } \\
\text { regional analysis COSMO-REA6 ISPR 2018, 17-19July2018, Bonn [pdf document properties: } \\
\text { datestamp=16/07/2018] } \\
\text {-Photo of Hamburg Fishmarket flooded in early morning, probably during Storm Xaver (Birger Tinz) }\end{array}$ \\
\hline $\begin{array}{l}\text { North Norfolk District } \\
\text { Council Coastal Team } \\
\text { (2018) }\end{array}$ & $\begin{array}{l}\text { North Norfolk District Council Coastal Team, Refurbishment of sea walls and groynes 2013-2015; Cromer Coast } \\
\text { Protection, (picture of plaque on wall taken 27Dec2018) www.northnorfolk.org/coastal } \\
\text {-FIG6. [PHOTO] Photo damaged sea wall Cromer after Dec2013 storm Xaver }\end{array}$ \\
\hline $\begin{array}{l}\text { Giannopoulos et al } \\
\text { (2019) }\end{array}$ & $\begin{array}{l}\text { Giannopoulos G, L Peake, B Reid, J Andrews, A Grant, I Lorenzoni, M Goulden, J Waters, T Dolphin, J } \\
\text { Bremner, TJ Tolhurst, Environmental and social impacts of the } 2013 \text { storm surge on the North Norfolk coast, } \\
\text { powerpoint presentation date stamp 15May2019, unknown conference } \\
\text {-FIG1. (a) [MAP] schematic of the study area of the Storm Surge in the North Norfolk coast. } \\
\text { The Dec } 2013 \text { storm surge destroyed the coastal defence. spreading the sediments and } \\
\text { flooding with seawater the inland freshwater marshland ecosystem. UAV survey } \\
\text { (b) before and (c) after the storm surge } \\
\text {-FIG2. [PHOTO] Field photos showing physical alterations by the storm surge to the } \\
\text { coastal freshwater ecosystem } \\
\text { (a) protective dykes were eroded away and the sediments were dispersed, } \\
\text { (b) large areas were flooded with seawater that ponded for up to } 1 \text { months } \\
\text { (c) distinct patches of ponded seawater caused anoxic depressions. }\end{array}$ \\
\hline $\begin{array}{l}\text { North Norfolk District } \\
\text { Council Coastal Team } \\
\text { (2019) }\end{array}$ & $\begin{array}{l}\text { North Norfolk District Council Coastal Team, Sheringham Sea Defences; Repair and Recovery from the } \\
\text { December } 2013 \text { storm surge, www.northnorfolk.org/coastal, photo of plaque on wall taken 30Dec2019 } \\
\text {-FIG1. [PHOTO] damaged promenade Sheringham } \\
\text {-FIG2. [PHOTO] damaged promenade and earth bank Sheringham } \\
\text {-FIG3. [PHOTO] damaged sea wall and earth bank Sheringham } \\
\text {-FIG4. [PHOTO] tractor and cement truck repairing the damaged promenade Sheringham. }\end{array}$ \\
\hline Wikipedia (20191002) & $\begin{array}{l}\text { Wikipedia, Stormen Bodil, https://da.wikipedia.org/wiki/Stormen_Bodil\#cite_note-39 (accessed 02Oct2019) } \\
\text {-FIG1. [PHOTO] Flooding in Hamburg } \\
\text {-FIG3. [PHOTO] Tree fall in Denmark; Hadsund, north of Randers } \\
\text {-FIG4. [PHOTO] Fish market in Hamburg. 2h after the peak water level, } \\
\text { high water level continued at 7m above sea level }\end{array}$ \\
\hline Andrews (2020) & $\begin{array}{l}\text { Andrews, JE, Spit extension and barrier rollover at Blakeney Point and Salthouse: historic map and field } \\
\text { observations, Bull. geol. Soc. Norfolk, } 69,35-63,2020 \\
\text { *-FIG8. Flooded back barrier marshes at Salthouse (Dec2013) looking N. The emergent } \\
\text { islands in the floodwater are Little Eye (left) and the remains of Great Eye (right). } \\
\text { The area W of Littel Eye with teh row of posts is the site of the Little Eye } \\
\text { breach channel. Image from Mr. David North } \\
\text { *-FIG9. Landward edge of 5Dec2013 shingle washover fans spilling onto reclaimed saltmarsh } \\
\text { at Beach Road. The islands of brown sediment top left are the remnants of Great Eye } \\
\text { also visible in Fig8. Photograph taken 2Jan2014. } \\
\text { *-FIG11.Comparison of washover fans W of Little Eye in aerial photos (a) tken in June } 2008 \\
\text { after the Nov2007 surge and (b) taken in Sep2014 after the Dec2013 surge. } \\
\text { The shingle free re-entrant between the fans in (b) is centred on TG } 07675 \text { 44428. } \\
\text { Cley Eye pillbox is clear in the bottom right of both images. } \\
\text { *-FIG12.Little Eye breach channel (a) looking seaward (N) and (b) looking landward (S). } \\
\text { Note the pale grey Holocene muds exposed by channel bed erosion. The elevation } \\
\text { of these muds, just above mean sea level can be used to infer an age of around } \\
\text { 2000years BP based on the data in Funnell \& Boomer (1998, p55). Images from } \\
\text { Mr. David North 12Dec2013. }\end{array}$ \\
\hline
\end{tabular}




\begin{tabular}{|c|c|}
\hline & $\begin{array}{l}\text { *-FIG13.Transient ebb tidal delta formed at the sea end of the 'Little Eye breach channel'. } \\
\text { Image from Mr. David North 12Dec2013 } \\
\text { *-FIG14.Arnold's (Pope's) Marsh breach channel viewed (a) from the backbarrier looking E. } \\
\text { The artificially steepened bank east of the channel was overtopped in } 2013 \\
\text { retained some topography. (b) view SE along the barrier crest. } \\
\text { Image from Mr. David North 2Jan2014. }\end{array}$ \\
\hline WIKI (20200124) & $\begin{array}{l}\text { WIKI, Cyclone Xaver, https://en.wikipedia.org/wiki/Cyclone_Xaver accessed 24Jan2020 } \\
\text { FIG [PHOTO] Bremerhaven flooded by the waters of the Weser River } \\
\text { FIG [PHOTO] repairing electricity cables in the wake of Xaver, Baltasound, Unst, Shetland } \\
\text { FIG [PHOTO] Cyclone Xaver unleashing high waves near Warnemunde Germany } \\
\text { FIG [PHOTO] Defences overtopped at Victoria Dock, Hull } \\
\text { FIG [PHOTO] Homes undermined by the surge Hemsby Norfolk } \\
\text { FIG [PHOTO] Flooding in Hamburg's Hafencity } \\
\text { FIG [PHOTO] Breached sea dike at Burnham Norton, Norfolk, Seaward to left; flooding to right } \\
\text { FIG [PHOTO] December } 2013 \text { storm surge on Sheringham seafront }\end{array}$ \\
\hline Wikipedia (20200429) & $\begin{array}{l}\text { Wikipedia, Stormen Sven, https://sv.wikipedia.org/wiki/Stormen_Sven\#cite_note-15, accessed 29Apr2020 } \\
\text { FIG. Flooding in Hamburg due to Storm Sven, which was called Xaver there }\end{array}$ \\
\hline Wikipedia (20200502) & $\begin{array}{l}\text { Wikipedia, Orkan Xaver, https://de.wikipedia.org/wiki/Orkan_Xaver (accessed } 2 \text { May 2020) } \\
\text { FIG4. [PHOTO] On the German Baltic coast the main feature for a period was } \\
\text { the extreme minimum water level. Here Eckernfoerde } \\
\text { FIG5. [PHOTO] Hamburg HafenCity } \\
\text { FIG6. [PHOTO] Coast damage at Hemsby, Norfolk } \\
\text { FIG7. [PHOTO] Flooding of Grossen Elbstrasse in Hamburg-Altona } \\
\text { FIG8. [PHOTO] Impact on Handewitter Forst Apr } 2014\end{array}$ \\
\hline
\end{tabular}

Table S3. Ranking of storm among events; assessing importance of storm (arranged by year and then alphabetically)

\begin{tabular}{|c|c|}
\hline Source & ference and Notes \\
\hline $\begin{array}{l}\text { Aftenposten } \\
(20131205)\end{array}$ & 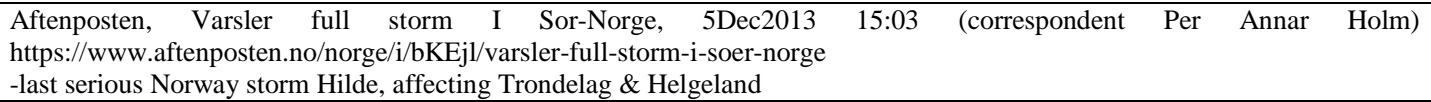 \\
\hline $\begin{array}{l}\text { Air Worldw } \\
\text { (20131212) }\end{array}$ & $\begin{array}{l}\text { Air Worldwide, Press Release, Boston, 12Dec2013. https://www.air-worldwide.com/In-the-News/AIR-Estimates-Losses- } \\
\text { from-European-Windstorm-Xaver-at-Between-EUR-700-Million-and-EUR-1-4-Billion/ (accessed 02Jan1990) } \\
\text {-comparisons with storm Christian Oct2013 and storm Anatol Dec1999 } \\
\text {-wind speeds for Xaver less than Christian and Xaver } \\
\text {-storm Xaver similar to the 1Feb1953 storm in terms of surge }\end{array}$ \\
\hline $\begin{array}{l}\text { BBC } \\
(20131206)\end{array}$ & $\begin{array}{l}\text { BBC20131206, Deadly storm and tidal surge batter northern Europe, BBC } 6 \text { Dec2013 (report by Anna Holligan) } \\
\text {-worst storm surge in eastern England in } 60 \text { years } \\
\text {-Hamburg experienced biggest storm surge since the 1960s }\end{array}$ \\
\hline $\begin{array}{l}\text { Bloomb } \\
(2013)\end{array}$ & $\begin{array}{l}\text { oomberg, Hamburg has worst flood in } 37 \text { year amid European storms, Nicholas Brautlecht, 6Dec2013, 02:58PM GMT } \\
\text { tps://www.bloomberg.com/news/articles/2013-12-06/hamburg-has-worst-flood-in-37-years-as-storms-rage-across-europe } \\
\text { econd highest water level on record (37y) } \\
\text { wer than storm surge 1976; higher than storm surge } 1962\end{array}$ \\
\hline BSH (2013) & $\begin{array}{l}\text { BSH, Die Nordseesturmfluten von 5. und 6.12.2013, 3pp, prepared by Stockmann,K. (title: Sturmflut 2011_bm1101; author } \\
\text { stamp: bm1101; document time stamp: 12/10/2013 12:5136PM) } \\
\text {-winds not so strong as Hurricane Christian 28Oct2013 } \\
\text {-water level progression similar to storm surge Feb } 1962 \\
\text {-Hamburg St Pauli skew surge Feb } 1962=4.03 \mathrm{~m} \text {; Dec } 2013=3.98 \mathrm{~cm}\end{array}$ \\
\hline $\begin{array}{l}\text { BODC } \\
(20131205)\end{array}$ & 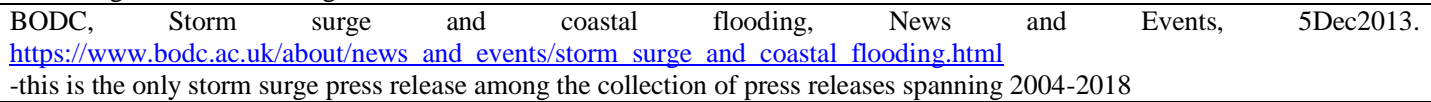 \\
\hline $\begin{array}{l}\text { De } \\
\text { et }:\end{array}$ & $\begin{array}{l}\text { Deutschlander T, K Frierich, S Haeseler, C Lefebvre, Orkantief XAVER ueber Nordeuropea von 5. bis 7. Dezember 2013, } \\
\text { Deutscher Wetterdienst DWD, Stand 30. Dezember 2013, 19pp. } \\
\text {-highest water level ever measured at Oresund Sweden } \\
\text {-UK Environment Agency says worst storm surge in } 60 \text { years } \\
\text {-Xaver storm surge at Hamburg at } 3.98 \mathrm{~m} \text { ueber MTHw was } 5^{\text {th }} \text { highest in } 100 \text { years after } 1976(4.67 \mathrm{~m}), 1962(4.03 \mathrm{~m}), 1994 \\
\quad(3.98 \mathrm{~m}), 1995(3.99 \mathrm{~m}) \\
\text {-Storm Xaver water levels on North Sea coast were highest since Storm Britta Nov. } 2006\end{array}$ \\
\hline $\begin{array}{l}\mathrm{G} \\
(2\end{array}$ & gccapitalideas.com/2013/12/09/windstorm-xaver/ \\
\hline Gray (2013) & $\begin{array}{l}\text { Gray, Tom, Into the Wind, The AWEA Blog, Ireland, U.K., Germany set new wind generation records, } \\
\text { https://www.aweablog.org/ireland-u-k-germany-set-new-wind-generation-records/, 11Dec2013 } \\
\text {-record wind energy production Germany 06Dec2013 }\end{array}$ \\
\hline $\begin{array}{l}\text { Kunz et } \\
(20131206)\end{array}$ & 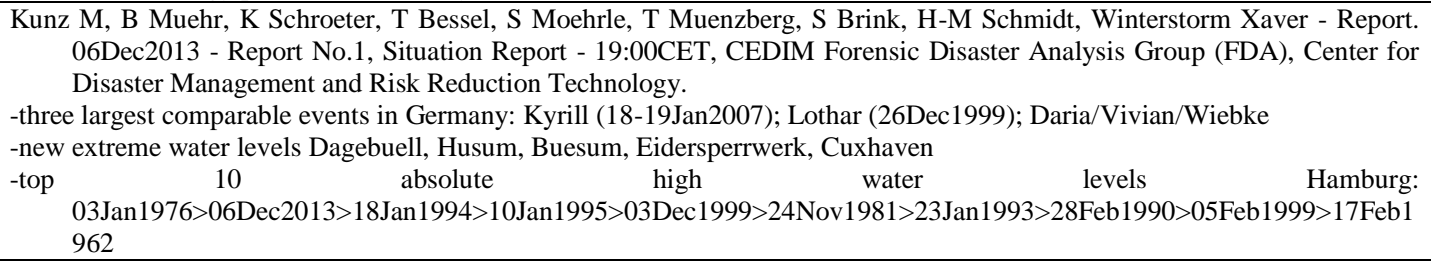 \\
\hline $\begin{array}{l}\text { The Lowestoft } \\
\text { Journal } \\
\text { (20131214) }\end{array}$ & $\begin{array}{l}\text { The Lowestoft Journal, Suffolk MP hits out at environment secretary in statement about the floods, 14Dec2013, } \\
\text { correspondent: Annabelle Dickson. } \\
\text {-conservative government minister wrote storm put defenses to greatest test in } 60 \text { years; for some locations water level was a } 1\end{array}$ \\
\hline
\end{tabular}




\begin{tabular}{|c|c|}
\hline & ent. \\
\hline $\begin{array}{l}\text { Mills et al } \\
(20131206)\end{array}$ & $\begin{array}{l}\text { Mills, Ian, Remko Scharoo, Luciana Fenoglio, Xaver affected much of northern Europe on } 5 \text { and } 6 \text { December and caused } \\
\text { worst storm surge for decades in the North Sea. https://www.eumetsat.int/website/home/News/DAT_2087062.html } \\
\text { (last accessed: } 19 \text { Nov2019,28Jun2020) } \\
\text {-record surges in Wadden Sea and barrier islands of the Netherlands and Germany } \\
\text {-comparison of altimeter strip information for Saral/AltiKa since launch in early2013; SWH and wind speeds highest of } \\
\text { previous } 9 \text { months }\end{array}$ \\
\hline $\begin{array}{l}\text { NLWKN } \\
(20131203)\end{array}$ & $\begin{array}{l}\text { NLWKN, Sturmflutgefahr an der Kueste und auf den Inseln, Zwei meter ueber dem normalen Hochwasser in der Nacht zum } \\
\text { Freitag moeglich//pressinformation von } 29 \text { November 2013, (written by Herma Heyken, Pressesprecherin), 03/12/2013 } \\
\text { https://www.nlwkn.niedersachsen.de/startseite/aktuelles/presse und offentlichkeitsarbeit/pressemitteilungen/sturmflutgefahr- } \\
\text { an-der-kueste-und-auf-den-inseln-120187.html } \\
\text {-last great storm surge on Niedersachsen coast was Hurricane Lennart Jan } 2013 \text { with } 12 \text { month quiet period before that } \\
\text {-storm surge for Britta } 1 \text { Nov2006 was a } 100 \text { year event for the Niedersachsen North Sea coast with Borkum water level of } \\
2.70 \mathrm{~m} \text { the same as the } 1962 \text { surge. }\end{array}$ \\
\hline $\begin{array}{l}\text { NLWKN } \\
(20131206)\end{array}$ & $\begin{array}{l}\text { NLWKN, Schwere Sturmflut gut Ueberstanden, Waserstaende zwischen Zweieinhale und Knapp vier metern registriert // } \\
\text { presseinformation von } 6 \text {. Dezember 2013, (Ansprechpartnerin: Herma Heyken), 06/12/2013. } \\
\text {-counts as worst storm surge of last 100y; } 2006 \text { record reached partly } \\
\text {-table comparing skew surge between Storm Xaver, Storm Britta, and } 1962 \text { storm surge; Storm Xaver was highest at most } \\
\text { stations. }\end{array}$ \\
\hline $\begin{array}{l}\text { NOS } \\
(20131206)\end{array}$ & $\begin{array}{l}\text { NOS, Hoogste waterstand sinds 1953, 06/12/2013, 09:52, https://nos.nl/artikel/583218-hoogste-waterstand-sinds-1953.html } \\
\text {-highest water level in Vlissingen (3.99m NAP) since } 1953 \text { surge (4.55m NAP) }\end{array}$ \\
\hline $\begin{array}{l}\text { Oceanografisch } \\
\text { Meteorologisch } \\
\text { Station (2013) }\end{array}$ & $\begin{array}{l}\text { Oceanografisch Meteorologisch Station, Stormverslag } 05-06 \text { december 2013, 26pp, } 2013 \text { [pdf document properties: } \\
\text { author=Myriam Sys; datestamp=15Dec2013] } \\
\text {-water levels in Oostende (633cm TAW) were the highest since } 1 \text { Feb1953 }(666 \mathrm{~cm} \text { TAW) }\end{array}$ \\
\hline SMHI & $\begin{array}{l}\text { SMHI, Stormen Sven gav nya vattenstandsrekord i Oresund, https://www.smhi.se/nyhetsarkiv/stormen-sven-gav-nya- } \\
\text { vattenstandsrekord-i-oresund-1.34732, updated 20Mar2017; original datestamp 10Dec2013. } \\
\text {-maximum trough-crest height Vaderoarna 10.7m; less than recordd of 13.9m during Adventstorm } 2011 \\
\text {-water level record maximum at Viken }(1.67 \mathrm{~m} ; 1976 \text { start) and Barseback }(1.59 \mathrm{~m} ; 1993 \mathrm{start}) \\
\text {-water level record minimum at Skanoor }(-1.58 \mathrm{~m}) \text {; old record -1.55m during Dec } 1999 \mathrm{storm}\end{array}$ \\
\hline $\begin{array}{l}\text { Spiegel } \\
\text { International } \\
(20131206)\end{array}$ & 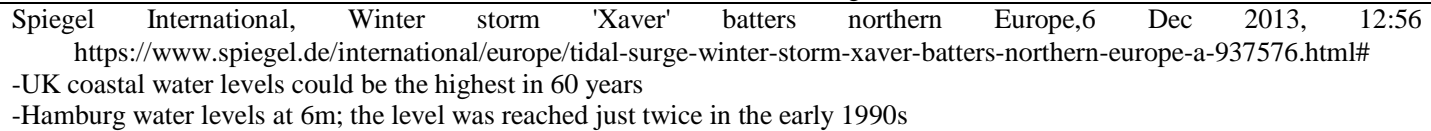 \\
\hline $\begin{array}{l}\text { Sueddeutsche } \\
\text { Zeitung } \\
(20131215)\end{array}$ & 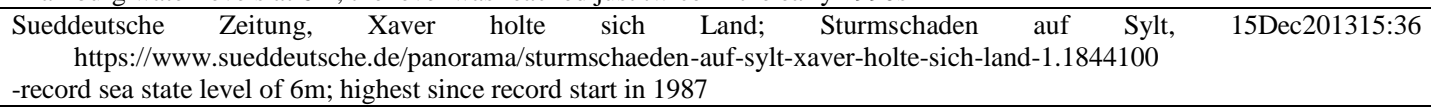 \\
\hline $\begin{array}{l}\text { Sylter } \\
\text { Rundschau } \\
(20131209)\end{array}$ & $\begin{array}{l}\text { Sylter Rundschau, Folgen des Orkans. Xaver 'knabberte' Sylt massiv an, 09Dec2013 06:00 (from Friederike Reussner) } \\
\text { https://www.shz.de/lokales/sylter-rundschau/xaver-knabberte-sylt-massiv-an-id5098481.html } \\
\text {-Arfst Hinrichsen, Landesamt fuer Kuestenschutz on Sylt beach damage: 'Das war schon heftig, solhe Schaeden habe ich } \\
\text { selten gesehen' }\end{array}$ \\
\hline $\begin{array}{l}\text { erzentra } \\
312)\end{array}$ & $\begin{array}{l}\text { Unwetterzentrale, Orkantief XAVER - ein weiterer schwerer Wintersturm der letzten Jahrzehnte, Thomas Savert and Stefan } \\
\text { Laps, Dec. } 2013 \text { http://www.unwetterzentrale.de/uwz/928.html } \\
\text {-rank } 2 \text { storm surge; Storm Xaver storm surge lower than 03Jan1976 } \\
\text {-squall lines observed for Storm Xaver (2013), Kyrill (2007) \& Emma (2008) } \\
\text {-Xaver was among the most powerfu storm of recent decades in Germany }\end{array}$ \\
\hline $\begin{array}{l}\text { AON Benfield } \\
(2014)\end{array}$ & $\begin{array}{l}\text { AON Benfield, Impact forecasting. December } 2013 \text { Global Catastrophe Recap, 2014. [document properties: abrandt; date } \\
\text { stamp: 10Jan2014] } \\
-1 \text { of } 4 \text { featured global catastrophe events for Dec } 2013 \\
\text {-other events are Storm Dirk 23-25Dec2013; winter storms US and Canada; floods SE Brazil worst in decades }\end{array}$ \\
\hline $\begin{array}{l}\text { ABPmer } \\
(2014)\end{array}$ & $\begin{array}{l}\text { ABPmer, Ensuring Flood Resilience. An overview of the 5/6 December 2013, Associated British Ports, Marine } \\
\text { Environmental Research, July, } 2014 \\
\text {-rank } 1 \text { water levels: Leith, North Shields, Immingham, Cromer, Dover } \\
\text {-rank } 2 \text { water levels: Aberdeen, Lowestoft } \\
\text {-Immingham flooding during } 2013 \text { surge but not } 1953\end{array}$ \\
\hline $\begin{array}{l}\text { BBC } \\
(2014\end{array}$ & $\begin{array}{l}\text { BBC, East coast surge: what happened next? (report by Richard Haugh), 5Dec2014 } \\
\text {-comparison with Storm Xaver } 2013 \text { to } 1953 \text { surge }\end{array}$ \\
\hline $\begin{array}{l}\text { CH2MHill } \\
\text { Halcrow (2014) }\end{array}$ & $\begin{array}{l}\text { CH2MHill Halcrow, Cell } 1 \text { Regional Coastal Monitoring Programme, Wave Data Analysis Report 2: 2013-2014, Final } \\
\text { Report, March } 2014 \text { [document properties: author=Andy.Parson @ ch2m.com; datestamp; 04/04/2014] } \\
\text {-Newbiggin wave buoy: storm analysis 2010-2014; Storm Xaver had highest energy at peak and wave period but not largest } \\
\text { peak height } \\
\text {-North Shields tide gauge: Storm Xaver had rank } 1 \text { water level that exceeded 31Jan1953 (rank2) } \\
\text {-Tyne Tees buoy: Storm Xaver did not have exceptional wave conditions } \\
\text {-Whitby waverider buoy: Storm Xaver had highest peak wave energy in record 2010-2014 } \\
\text {-Whitby tide gauge: Storm Xaver water level at rank 1; previous storm flood water level record 01Feb1982 (rank 2). } \\
\text {-Scarborough tide gauge: Storm Xaver was rank1 water level event; next highest water level Jan2005. }\end{array}$ \\
\hline $\begin{array}{l}\text { Dunbar } \\
(2014)\end{array}$ & $\begin{array}{l}\text { Dunbar I, N Phipps, M Szonyi, Risk Nexus. After the storm: how the UK's flood defences performed during the surge } \\
\text { following Xaver, Flood resilience review 09.14, Zurich Insurance Company Ltd., Mythenquai 2, 8002, Zurich, } \\
\text { Switzerland [document properties: date 28Aug2014; author= Zurich] } \\
\text {-comparable water levels between } 1953 \text { \& } 2013 \\
\text {-previous stormiest December Dec } 1969 \text { (gusts over threshold). } \\
\text {-Dover water level 4.7m OD; highest since } 1905 \\
\text {-new water level record Kingston upon Hull } 5.8 \mathrm{~m} \backslash \\
\text {-1953 surge event highest for at least } 250 \mathrm{y} \\
\text {-Southend water level } 4.1 \mathrm{~m} \text { or } 0.06 \mathrm{~m} \text { higher than previous record in } 2007\end{array}$ \\
\hline
\end{tabular}




\begin{tabular}{|c|c|}
\hline Eden (201402) & $\begin{array}{l}\text { Eden, Phillip, Weather Log December 2013, Weather, Feb 2014, pp.i-iv } \\
\text {-'a rapidly deepening depression tracked from just northwest of Scotland to the southern Baltic between the 5th and 7th, } \\
\text { bringing the greatest storm surge to the east coast since 1953' }\end{array}$ \\
\hline Eriksen (2014) & $\begin{array}{l}\text { Eriksen J, Rekordvandstande i Isefjorden og Roskilde Fjord, Vejret, } 138,240-48,2014 \\
\text {-Roskilde } 2.06 \mathrm{~m} \text { rank } 1 \text { storm surge in record back to 1992; previous record 1.36m 6Feb1999 } \\
\text {-Roskilde water level at 100-200y return level } \\
\text {-Holbaek } 1.95 \mathrm{~m} \text { rank } 1 \text { water level; time series start 1972; previous record } 1.73 \mathrm{~m} \text { 20Nov1973 } \\
\text {-Bodil similar to } 1973 \text { storm }\end{array}$ \\
\hline $\begin{array}{lll}\text { Gautier } & \text { et } \\
\text { (2014) }\end{array}$ & $\begin{array}{l}\text { Gautier C, A Camarena, J van Nieuwkoop, SWAN hindcasts Wadden Sea, December 2013. Tidal inlet of Ameland and } \\
\text { eastern Wadden Sea. Deltares, 2014, 197pp. Project 1209433-007, Reference 1209433-007-HYE-0005 } \\
\text {-storm Xaver assessed as having extreme water level and wave conditions at the } 1 \text { in } 100 \text { year level; similar to } 2006 \text { and } 2007 \\
\text {-water level at Delfzijl at level of } 1 \text { in } 50 \text { year to } 1 \text { in } 100 \text { year recurrence level. } \\
\text {-Storm Xaver asssessed as one of the most severe storms in terms of wind speed and water level for which there are proper } \\
\text { wave measurements in the Wadden Sea }\end{array}$ \\
\hline $\begin{array}{l}\text { Goennert et al } \\
\text { (2014) }\end{array}$ & $\begin{array}{l}\text { Goennert G, O Mueller, M Schaper, K Sossidi, Die Sturmflut nach dem Tief Xaver von 5. bis 7. Dezember 2013. Berichte des } \\
\text { Landesbetriebes Strassen, Bruecken und Gewaesser (LSBG), Freie und Hansestadt Hamburg, Nr. 16/2014, 26pp. } \\
\text {-Storm Xaver was rank2 storm surge after 03Jan1973 }\end{array}$ \\
\hline $\begin{array}{l}\text { Hewson et al } \\
(2014)\end{array}$ & $\begin{array}{l}\text { Hewson T, L Magnusson, O Breivik, F Prates, I Tsonevsky, HJW de Vries, Windstorms in northwest Europe in late 2013, } \\
\text { ECMWF Newsletter, No 139, pp 22-28, Spring 2014. } \\
\text {-FIG1. Areas exceeding the 5-year return period of 24-hour maximum wind gust for windstorms (a) Christian and (b) Xaver } \\
\text { as diagnosed using the ERA-Interim reanalysis as a proxy for observations. } \\
\text {-'Record surges were set up by the wind storm along the east coast of Britain, the coasts of the Netherlands and in the } \\
\text { Germany Bight' }\end{array}$ \\
\hline $\begin{array}{l}\text { Knaack and } \\
\text { Heyken (2014) }\end{array}$ & $\begin{array}{l}\text { Knaack H and H Heyken, Xaver hatte sehr schwere Stumflut im Gepaeck, Jahresbericht 2013. Der Zukunft verplichtet, } \\
\text { NLWKN, Niedersaechsischer Landesbetrieb fuer Waserwirtschaft, Kuesten- und Naturschutz, pp.8-9, document date } \\
\text { stamp 24Apr2014. } \\
\text {-Xaver water levels at Ems higher than } 1962 \text { and } 1976 \\
\text {-Xaver true surge was rank } 2 \text { event after } 1962 \\
\text {-Xaver water levels on Weser and Elbemuendung lower than } 19621976 \text { events (i.e., rank 3) }\end{array}$ \\
\hline $\begin{array}{l}\text { Kristandt et al } \\
\text { (2014) }\end{array}$ & $\begin{array}{l}\text { Kristandt, J., B. Brecht, H. Frank, H. Knaack, Optimization of empirical storm surge forecast-modeling of high resolution } \\
\text { wind fields, Die Küste, 81, 301-348, } 2014 \\
\text {-water levels at Norderney were 2.83 m above mean high water level; second after Fe } 1962 \text { surge }\end{array}$ \\
\hline $\begin{array}{l}\text { Leiding et al. } \\
\text { (2014) }\end{array}$ & $\begin{array}{l}\text { Leiding T, B Tinz, G Rosenhagen, C Lefevre, S Haeseler, S Hagemann, I Bastigkeit, D Stein, P Schwenk, S Mueller, O } \\
\text { Outzen, K Herklotz, F Kinder, T Neumann, Meteorological and Oceanographic Conditions at the FINO platforms during } \\
\text { the severe storms Christian and Xaver, DEWI Magazin, No.44, p16-25, 2014. } \\
\text {-FINO1: Storm Xaver had wind speed record since measurement start in 2002; previous wind speed record Storm Karla } \\
\text { 30Dec2006 23:50UTC with } 47.5 \mathrm{~m} / \mathrm{s} \text { gust at } 103 \mathrm{~m} \text { and } 37 \mathrm{~m} / \mathrm{s} 10 \mathrm{~min} \text { sustained wind speed } \\
\text {-FINO2: Storm Christian set wind speed record since measurement start in } 2007 \text {; Storm Xaver was remarkable for long } \\
\text { duration with wind speeds }>20 \mathrm{~m} / \mathrm{s} \text { for } 35 \mathrm{~h} \text {; wind turbine cutoff thresholds of } 25 \mathrm{~m} / \mathrm{s} \text { were exceeded for } 27 \mathrm{~h} \\
\text {-FINO3: Storm Christian set record for wind speed and gust since measurement start in 2009; for Storm Xaver wind speed } \\
\text { exceeded } 25 \mathrm{~m} / \mathrm{s} \text { for }>24 \mathrm{~h} \text {; 'FINO3 has weathered both storms without signifincant damage' }\end{array}$ \\
\hline $\begin{array}{l}\text { Luecht and } \\
\text { Peters (2014) }\end{array}$ & $\begin{array}{l}\text { Luecht, Fabian and Ove Peters, Bericht ueber die Sturmflut vom 05.-0.6.12.2013 an der Westkueste Schleswig-Holsteins, } \\
\text { Landesbetrieb fuer Kuestenschutz, Nationalpark und Meeresschutz Schleswig-Holstein, Husum 26Feb2014, 19pp. [pdf } \\
\text { document properties: title=Lfd; Author=Thorsten Nommensen; datestamp: 11Dec2018] } \\
\text {-water level Helgoland rank } 2 \text { during Storm Xaver; 3cm under highest level 1962 } \\
\text {-List tide gauge: storm surge duration at rank } 8 \\
\text {-damage on Sylt similar to Storm Anatol 3Dec1999 }\end{array}$ \\
\hline Mai (2014) & $\begin{array}{l}\text { Mai, S., Sea state at the research platform FINO1 during the winter storm 'Xaver', BFG, Bundesamt fuer Gewasserkunde, } \\
\text { document properties: author=Mai, date=16May2014 } \\
\text {-highest SWH ever measured at FINO1 by radar gauge sensors. }\end{array}$ \\
\hline $\begin{array}{l}\text { Matelski e } \\
(2014)\end{array}$ & $\begin{array}{l}\text { Matelski, Birgit, Frerk Jensen, Peter Voss, Jorg Peters, Maria Blumel, Auswertung zur Hydrologie des Sturmtiefs Xaver } \\
\text { 05.12.2013 bis 06.12.2013, Statusbericht vom 01.10.2014, Landesbetrieb fuer Kuestenschutz, Nationalpark und } \\
\text { Meeresschutz, Schleswig-Holstein, Husum, 01.10.2014, 25pp. } \\
\text {-rank } 5 \text { water level at List in series extending back to } 1900 \text {. } \\
\text {-water levels at Hamburg St Pauli } 39 \mathrm{~cm} \text { deeper than } 1962 \\
\text {-water level in Halgoland Binnenhaven } 21 \mathrm{~cm} \text { deeper than in Dec } 2013\end{array}$ \\
\hline $\begin{array}{l}\text { Nossent } \text { et al } \\
(2014)\end{array}$ & $\begin{array}{l}\text { Nossent J, L Boeckx, E Taverniers, M Deschamps, T Verwaest, F Mostaert, Sinterklaasstorm } 6 \text { december 2013. Beschrijving } \\
\text { van de hydrometrische gebeurtenissen, Versie 4.0. WL Rapporten, 00-119, Waterbouwkundig Laboratorium, } \\
\text { Antwerpen, Belgie. October 2014, WL2014R00_119_5 [document date stamp: 15Oct2014; author= Deschamps, } \\
\text { Maarten] } \\
\text {-Antwerp storm surge level ranked as a 4-5 year event } \\
\text {-Antwerp water levels exceeded during } 1954 \text { (7.77 mTAW), 03Jan1976 (7.31 mTAW), 27Feb1990 (7.52 mTAW), } \\
\text { 11Nov1992 (7.39 mTAW), 14Nov1993 (7.53 mTAW), 18Jan1994 (7.37 mTAW) }\end{array}$ \\
\hline Pelt (2014) & $\begin{array}{l}\text { Pelt AS, BODIL's stormflod i de indre dansk farvande, Vejret, 138, 24-29, } 2014 \\
\text {-mostly rank } 1 \text { water levels in measurement records of Roskilde Havn, Torsminde, Hornbaek, Klampenborg, Tolboden } \\
\text { Kobenhavn, Sjaellands Odde, Grenaa, Odense Fjord } \\
\text {-rank } 3 \text { wind speed at Odense } \\
\text {-Bodil storm surge was highest in Oresun and SE Kattegat since } 1862 \text {. }\end{array}$ \\
\hline RMS (2014) & $\begin{array}{l}\text { RMS, 2013-2014 Winter Storms in Europe. An Insurance and Catastrophe Modeling Perspective. RMS White Paper. [PDF } \\
\text { TIMESTAMP 11Mar2014] } \\
\text {-water levels on east coast at } 1953 \text { levels } \\
\text {-6.09m water level in Hamburg was same as } 1962 \text { event }\end{array}$ \\
\hline RWS (2014a) & $\begin{array}{l}\text { RWS, Watermanagementcentrum Nederland, Stormvloedflits 2013-07 van } 5 \mathrm{t} / \mathrm{m} 7 \text { december 2013, Rijkswaterstaat } \\
\text { (document time stamp: 07Jan2014), 2014a }\end{array}$ \\
\hline
\end{tabular}




\begin{tabular}{|c|c|}
\hline & $\begin{array}{l}\text {-Delfzijl: previous water level record exceeded by } 1 \mathrm{~cm} \text { during Storm Xaver; expected } 15 \text { times per } 1000 \text { years } \\
\text {-Vlissingen: highest water level since 1953; expected once in 20y } \\
\text {-Hoek van Holland: Storm Xaver water levels exceed by Storm Tilo 09Nov2007 and } 1953 \text { event. } \\
\text {-skew surge statistics: Delzijl twice per 100y; Vlissingen once per seven years. }\end{array}$ \\
\hline RWS (2014b) & $\begin{array}{l}\text { RWS, Stormvloedrapport van } 5 \mathrm{t} / \mathrm{m} 7 \text { december (SR91) Sint-Nicolaasvloed 2013, Watermanagementcentrum Nederland, } \\
\quad \text { Rijkswaterstaat, prepared by Ing. J. Kroos, } 19 \text { Mar 2014b, } 48 \text { pp } \\
\text {-previous storm surge report } 21 \text { Mar 2008; 5.5y previously } \\
\text {-Belgium: highest water level since } 1953 \\
\text {-UK: water level higher than } 1953 \\
\text {-Vlissingen: rank2 event } 1900-2013 \\
\text {-Hoek van Holland: rank } 3 \text { event } 1900-2013 \\
\text {-Den Helder: rank } 10 \text { event } 1900-2013 \\
\text {-Harlingen: rank } 10 \text { event } 1932-2013 \\
\text {-Delfzijl: rank } 2 \text { event } 1900-2013\end{array}$ \\
\hline $\begin{array}{lll}\text { Slingo } & \text { et } & \text { al } \\
(2014) & & \end{array}$ & $\begin{array}{l}\text { Slingo J, S Belcher, A Scaife, M McCarthy, A Saulter, K McBeath, A Jenkins, C Huntingford, T Marsh, J Hannaford, S Parry, } \\
\text { The Recent Storms and Floods in the UK, 29pp, Met Office, Fitzroy Road, Exeter, Devon, EX1 3PB, UK, February, } 2014 \\
\text { [pdf document properties: author=huw.lewis; date stamp=11Feb2014] } \\
\text {-East coast storm surge 5-6Dec2013 was worst event since 1953. Comparable damage averted because of improved sea } \\
\text { defences and advance forecasts } \\
\text {-surge event 5-6Dec2013 subordinate to later storms from end of Dec to Feb that caused inland flooding and coastal dame in } \\
\text { southwest } \\
\text {-Dec } 2013 \text { had highest number of stations recording gusts over a high threshold }\end{array}$ \\
\hline $\begin{array}{l}\text { SMHI } \\
\text { (20140703) }\end{array}$ & $\begin{array}{l}\text { SMHI, Simone, Hilde, Sven och Ivar okt-dec } 2013,3 \text { Jul 2014, https://www.smhi.se/kunskapsbanken/meteorologi/simone- } \\
\quad \text { hilde-sven-och-ivar-okt-dec-2013-1.76183 } \\
\text {-Sven } 1 \text { of } 4 \text { serious storms Oct-Dec } 2013 \\
\text {-Sven had least damge of } 4 \text { serious storms in terms of power outages \&forest damage } \\
\text {-return period of winds 5-10y; locally 10-20years }\end{array}$ \\
\hline $\begin{array}{l}\text { Spencer et al } \\
\text { (2014) }\end{array}$ & $\begin{array}{l}\text { Spencer, T, S.M. Brooks, I. Moller, B.R. Evans, Where local matters: Impacts of a major North Sea storm surge, EOS, } 95 \text {, } \\
\quad 269-270,29 J u l y 2014 \\
\text {-worst storm surge in } 60 \text { years } \\
\text {-water levels comparable to \& exceeded } 01 \text { Feb } 1953 \text { levels. }\end{array}$ \\
\hline $\begin{array}{l}\text { Staneva } \\
\text { (2014) }\end{array}$ & $\begin{array}{l}\text { Staneva J, K Wahle, E Stanev, Response of the German Bight Hydro and Sediment Dynamics to Wave, Tidal and } \\
\text { Atmospheric Forcing, 3rd GODAE OceanView Coastal Oceans and Shelf Seas Task Team (COSS-TT) International } \\
\text { Coordination Workshop, 21-24 January 2014, Rncon Beach Resort, Puerto Rico, 36pp } \\
\text {-coastal flooding during Storm Britta in } 2006 \text { worse than during Strom Xaver } 2013 \\
\text {-Storm Britta } 2006 \text { assumed one of the worst for 100y on the Niedersachsen coast }\end{array}$ \\
\hline Thorne (2014) & $\begin{array}{l}\text { Thorne, Colin, Geographies of UK flooding in 2013/4, The Geog } \\
\text {-Xaver storm surge highest ever recorded Humber \& Thames est }\end{array}$ \\
\hline $\begin{array}{l}\text { Van Dorland } \\
(2014)\end{array}$ & $\begin{array}{l}\text { van Dorland, R, Zware storm op } 5 \mathrm{c} \\
\text {-Storm Xaver is number } 60 \text { in KNN }\end{array}$ \\
\hline $\begin{array}{lll}\begin{array}{l}\text { Axer } \\
(2015)\end{array} & \text { et } & \text { al }\end{array}$ & $\begin{array}{l}\text { Axer T, T Bistry, M Klawa, M Mueller, M Suesser, Deutsche Ruck Sturm dokumentation } 2013 \text { Deutschland, 2013, Deutsche } \\
\text { Rueckversicherung Aktiengesellschaft, Hansaallee 177, } 40549 \text { Duesseldorf, www.deutscherueck.de [pdf document } \\
\text { information: author=filiz; date stamp=07Aug2015] } \\
\text {-Storm Xaver cause < half of damage of Storm Christian because wind field weaker } \\
\text {-more forest damage in dMechlenburg-Vorpommern during Xaver than Storm Christian. } \\
\text {-Storm Christian had a lot of toppled tree damage (late October when leaves were still on trees) } \\
\text {-absolute water level during Xaver rank } 2 \text { after Caplla } 1976 \\
\text {-skew surge dduring Xaver rank } 3 \text { after } 1976 \text { and } 1962 \text { events }\end{array}$ \\
\hline $\begin{array}{l}\text { Dan et al. } \\
(2015)\end{array}$ & $\begin{array}{l}\text { Dan, Sebastian, Anne-Lise Montreuil, Rosalia Delgado, Tomas van Oyen, Large storm impact on a beach under sand } \\
\text { nourishments, The Proceedings of the Coastal Sediments 2015, edited by Ping Wang, Julie D Rosati, and Jun Cheng, } \\
\text { Coastal Sediments 2015, San Diego, USA, 11-15May2015 } \\
\text {-storm Xaver had water levels that were the highest since the } 1953 \text { surge }\end{array}$ \\
\hline $\begin{array}{l}\text { Fenoglio-Marc } \\
\text { et al. (2015) }\end{array}$ & $\begin{array}{l}\text { Fenoglio-Marc L, R Scharroo, A Annuziato, L Mendoza, M Becker, J Lillibridge, Cyclone Xaver seen by geodetic } \\
\text { observations, Geophys Research Letters, 42, 9925-9932, 2015 } \\
\text {-'... the largest storm signal captured by satellite altimetry to date....' in the North Sea? }\end{array}$ \\
\hline $\begin{array}{l}\text { Gierlevsen et } \\
\text { al. (2015) }\end{array}$ & $\begin{array}{l}\text { Gierlevsen T, H Lauridsen, F Langhans, J Bejdic, Met-ocean and wind resource related studies for nearshore windfarms in } \\
\text { Denmark, seminar at the Danish Energy Agency, } 27 \text { February } 2015 . \\
\text {-Storm Bodil (2013) one of three important severe storms for offshore wind energy }\end{array}$ \\
\hline $\begin{array}{l}\text { Haigh and } \\
\text { Bradshaw } \\
(2015)\end{array}$ & $\begin{array}{l}\text { Haigh I and E Bradshaw, A century of UK coastal flooding, Planet Earth, Winter 2015, (pdf document properties: 13Jan2016) } \\
\text {-7 of } 96 \text { record surge events in the } 100 \text { year data base occurred in the winter of 2013/4 } \\
\text {-storms 5-6Dec2013 and 3Jan2014 were in the top } 10 \text { of sea level height. } \\
\text {-COBRA crisis committee briefed on several occasions on storms during 2013-2014 winter period }\end{array}$ \\
\hline $\begin{array}{lll}\text { Jensen } & \text { et } & \text { al } \\
(2015) & & \end{array}$ & $\begin{array}{l}\text { Jensen, J., A. Arns, T. Wahl, Yet another 100yr storm surge event: the role of individual storm surges on design water levels, } \\
\text { Journal of Marine Science and Technology, 23, 882-887, } 2015 . \\
\text {-highest water levels on record for some places } \\
\text {-media reported a century storm }\end{array}$ \\
\hline $\begin{array}{l}\text { Kendon and } \\
\text { McCarthy } \\
(2015)\end{array}$ & $\begin{array}{l}\text { Kendon M and M McCarthy, The UK's wet and stormy winter of 2013/2014, Weather, 70, 40-47, } 2015 \\
\text {-comparison of Dec } 2013 \text { surge with Jan-Feb } 1953 \\
\text {-UK Dec rainfall rank } 4 \text { in time series from } 1910\end{array}$ \\
\hline $\begin{array}{l}\text { NLWKN } \\
(20151210)\end{array}$ & $\begin{array}{l}\text { NLWKN20151210, Sturmflutwarndienst der Betriebstelle Norden-Norderney, NLWKN Niedersachsischer Landesbetrieb fuer } \\
\text { Wasserwirtschaft, Kuesten- und Naturschutz, Niedersachsen, document date stamp 10Dec2015. } \\
\text {-ranking of data since time series start (1951? or less likely 1901? } \\
\text { Emden surge height: } 2006>2013>1962>1976 \text {. } \\
\text { Norderney surge height: } 1962>2013>1976>2006 \text {. }\end{array}$ \\
\hline
\end{tabular}




\begin{tabular}{|c|c|}
\hline & Cuxhaven surge height: $1976>1962>2013>2006$ \\
\hline Patzer (2015) & $\begin{array}{l}\text { Patzer, Marianne, Storm surge forecasting at DMI and perspectives on teh use of Earth Observations, ESA eSurge } \\
\text { Sympositum, Deltares, the Netherlands, (powerpoint presentation) January } 21,2015 \\
-1000 \text { year event in Danish fjords and Sealand north coast }\end{array}$ \\
\hline $\begin{array}{l}\text { Spencer et al } \\
(2015)\end{array}$ & $\begin{array}{l}\text { Spencer T, SM Brooks, BR Evans, JA Tempest, I Moeller, Southern North Sea storm surge event of Dec.5, 2013: Water } \\
\text { levels, waves, and coastal impacts, Earth Science Reviews, 146, 120-145, } 2015 \\
\text {-'...water levels were higher than in the twentieth century benchmark surge event of } 31 \text { January-1 February 1953' } \\
\text {-North Shields, Whitby, Immingham: highest recorded water level in tide gauge record }\end{array}$ \\
\hline $\begin{array}{lll}\text { Wadey } & \text { et } & \text { al } \\
(2015 a) & & \end{array}$ & $\begin{array}{l}\text { Wadey MP, ID Haigh, RJ Nichols, JM Brown, K Horsburgh, B Carroll, SL Gallop, T Mason, E Bradshaw, A comparison of } \\
\text { the } 31 \text { January-1 February } 1953 \text { and 5-6 December } 2013 \text { coastal flood events around the UK, UK Frontiers in Marine Science, } \\
\text { 2, 84, 2015a. } \\
\text {-for some locations Xaver water level higher than } 1953 \text { flood; for other locations reverse true } \\
\text { - } 1953 \text { storm much slower propagation across the North Sea leading to higher surge in south } \\
\text {-1953 had large forest damage in Scotland that was not observed for Xaver } \\
\text {-Storm Xaver reported as 'biggest North Sea surge for } 60 \text { yaers' } \\
\text {-COBRA meeting briefing on 05Dec2013 like for Storm Tilo } 2007 \text { (Cabinet Office Briefing Room A); 'the highest level of } \\
\text { preparation in the UK for a potential regional or national disaster' }\end{array}$ \\
\hline $\begin{array}{l}\text { Wadey et al } \\
(2015 b)\end{array}$ & $\begin{array}{l}\text { Wadey MP, JM Brown, ID Haigh, T Dolphin, P Wisse, Assessment and comparison of extreme sea levels and waves during } \\
\text { the 2013/2014 storm season in two UK coastal regions, Nat. Hazards Earth Syst. Sci. Discuss., 3, 2665-2708, 2015b. } \\
\text {-Lowestoft: highest water level in the measurement record since } 1964 \text { start; } 1953 \text { surge was higher } \\
\text {-Liverpool: highest water level in record since start 1992; previous record 10Feb1997 } \\
\text {-LLandudno: rank } 4 \text { water level } \\
\text {-Heysham: rank } 3 \text { water level }\end{array}$ \\
\hline $\begin{array}{lll}\begin{array}{l}\text { Sibley } \\
(2015)\end{array} & \text { et } & \text { al } \\
\end{array}$ & $\begin{array}{l}\text { Sibley A, D Cox, H Titley, Coastal flooding in England and Wales from Atlantic and North Sea storms during the 2013/2014 } \\
\text { winter, Weather, 70, 62-70, } 2015 \\
\text {-rank } 1 \text { water levels reached in North Shields, Whitby, Immingham, Lowestoft, Dover, Sheerness, Liverpool, Portsmouth, } \\
\text { Newhaven, Ilfracombe, Hinkley Point, Newport, Mumbles, Milford Haven, Fishguard, Barmouth } \\
\text {-most significant storm surge event for over } 60 \text { years. } \\
\text {-comparable significant wave height in } 1953 \text { and } 2013 \\
\text {-return period of } 1953 \text { surge at Lowestoft estimated at 50y } \\
\text {-previous significant storm surges in history: } 13-14 J a n 1916 ; 3 \text { Feb1825, 14Dec1717 } \\
\text {-COBR briefings of storm surge on 5-6Dec2013 }\end{array}$ \\
\hline $\begin{array}{l}\text { Vanmassenhov } \\
\text { e (2015) }\end{array}$ & $\begin{array}{l}\text { Vanmassenhove, Niels, Storm surge measures ports Flemish coast, Blankenberge, Tuesday February 3rd, Maritieme } \\
\text { Sientverlening en Kust, Coastal Division, Flanders Hydraulic Research.[document properties: title=Geintegreerd } \\
\text { Kustveiligheidsplan; author=Maarten; datestamp=27/02/2015] } \\
\text {-predicted water level of Dec2013 storm corresponded with a 50y stormflood } \\
\text {-highest water level since } 1953\end{array}$ \\
\hline $\begin{array}{l}\begin{array}{l}\text { Brooks } \\
(2016)\end{array} \\
\text { et }\end{array}$ & $\begin{array}{l}\text { Brooks SM, T Spencer, A McIvor, I Moller, Reconstructing and understanding the impacts of storms and surges, southern } \\
\text { North Sea, Earth Surface Processes and Landforms, 41, 855-864, } 2016 \text {. } \\
\text {-water levels during Storm Xaver higher along North Norfolk coast than any storm in data base starting 11Mar1883 } \\
\text {-return period water levels } 188-787 \text { years; about an order of magnitude higher than any 20th century storm }\end{array}$ \\
\hline $\begin{array}{l}\text { Dangendorf et } \\
\text { al (2016) }\end{array}$ & $\begin{array}{l}\text { Dangendorf S, A Arns JG Pinto, P Ludwig, J Jensen, The exceptional influence of storm 'Xaver' on design water levels in the } \\
\text { German Bight, Environmental Research Letters, 11, 2016, } 054001 \\
\text {-Storm Xaver displaced Storm Capella } 1976 \text { as the observational maximum at many locations } \\
\text {-Storm Xaver had highest ever recorded extreme water levesl in parts of the German Bight }\end{array}$ \\
\hline $\begin{array}{l}\text { ECMWF } \\
(20160316)\end{array}$ & 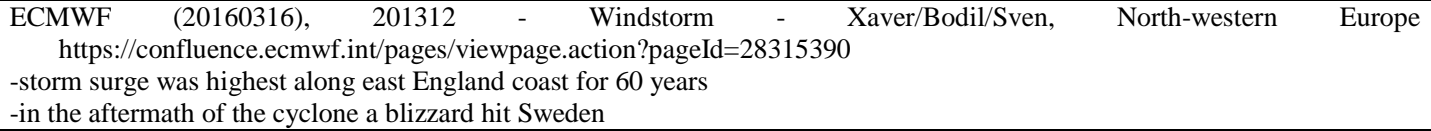 \\
\hline $\begin{array}{l}\text { Matelski } \\
\text { (2016) }\end{array}$ & $\begin{array}{l}\text { Matelski, Birgit, Erfahrungen aus der Sturmflut Xaver von 5. und 6.12.2013 und dem Weihnachshochwasser } 2014 \text { in } \\
\text { Shleswig-Holstein, IWASA } 2016 \text { Tagungsbeitrag, (46. IWASA, 7-8 Januar 2016; Internationales Wasserbau-Symposium } \\
\text { Aachen. [pdf document properties: autor=sonja; datestamp: 26Apr2016] } \\
\text {-for tide gauge on List auf Sylt highest water leve was rank } 5 \text { event in measurement time series since } 1900 \\
\text {-Hamburg St. Pauli: water level Xaver } 39 \mathrm{~cm} \text { higher than } 1962 \text { surge } \\
\text {-Helgoland Binnenhafen: water level Xaver } 38 \mathrm{~cm} \text { more than Storm Capella 03Jan1976 } \\
\text {-only a few tide gauges registered new records }\end{array}$ \\
\hline $\begin{array}{l}\text { Nederhoff et al } \\
\text { (2016) }\end{array}$ & $\begin{array}{l}\text { Nederhoff K, E Elias, T Vermaas, Erosie op Ameland Noordwest. Modelstudie: simulaties met Delft3D en XBeach, Deltares, } \\
\quad 117 \mathrm{pp} \text {, July, } 2016 \\
\text {-Sinterklaasstorm was a 50y event in terms of water level and wave height; damage to Amelander dunes }\end{array}$ \\
\hline $\begin{array}{l}\text { Sorensen } \\
(2016)\end{array}$ & $\begin{array}{l}\text { Sorensen CS, Water NOT wanted - Coastal floods and flooding protection in Denmark, In RA Herrmann \& J Jensen (eds), } \\
\text { Sicherung von Daemmen, Deichen und Stauanlagen: Handbuch fuer Theorie und Praxis (Vol V pp3-21). Siegen: } \\
\text { Universitaet Siegen, } 2016 \\
\text {-highest ever water levels at Hornbaek \& Copenhagen (series 1890-2015) } \\
\text {-many houses uninhabitable for some time } \\
\text {-other storms caused more fatalities \& dike breaches: 13-14Nov1872, 1921-1922, 1Nov2006 (inner seas) } \\
\text {-other surges North Sea side: } 1909,1911,30 \text { Sug1923, Jan 1976, 24Nov1981, 03Dec1999 }\end{array}$ \\
\hline $\begin{array}{l}\text { Sorensen et } \\
(2016 a)\end{array}$ & $\begin{array}{l}\text { Sorensen CS, NK Dronen, P Knudsen, J Jensen, P Sorensen, An extreme event as a games changer in coastal zone } \\
\text { management, Journal of Coastal Research, (Special Issue, No 75), 700-704, 2016. Proceedings of the 14th International } \\
\text { Coastal Symposium (Sydney, Australia) ed by A Vila-Concejo, E Bruce, DM Kennedy, RJ McCarroll, 2016a } \\
\text {-highest water level in Hornbaek tide gauge record 1890-2015 } \\
\text {-rank1 of highest Hornbaek storm surges: 01Jan1922, 18Dec1921, 7Nov1985, 27Nov2011, 5-6Dec2013 }\end{array}$ \\
\hline
\end{tabular}




\begin{tabular}{|c|c|}
\hline $\begin{array}{l}\text { Sorensen et al } \\
(2016 b)\end{array}$ & $\begin{array}{l}\text { Sorensen C, NH Broge, MR Molgaard, CS Schow, PThomsen, K Vognsen, P Knudsen, Assessing future flood hazards for } \\
\text { adaptation planning in a northern European Coastal Community, Frontiers in Marine Science, 3:69, } \\
\text { doi:10.3389/fmars.2016.00069, 2016b }\end{array}$ \\
\hline $\begin{array}{l}\text { Staneva et al } \\
(2016 a)\end{array}$ & $\begin{array}{l}\text { Staneva J, K Wahle, H Guenther, E Stanev, Coupling of wave and circulation models in coastal-ocean predicting systems: a } \\
\text { case study for the German Bight, Ocean Sci., 12, 797-806, 2016a. } \\
\text {-storm Britta } 2006 \text { water levels at } 100 \text { year return level } \\
\text {-two extreme storm case studies (Britta } 2006 \text { and Xaver 2013) to test coupled wave current model }\end{array}$ \\
\hline $\begin{array}{l}\text { Staneva et al } \\
(2016)\end{array}$ & $\begin{array}{l}\text { Staneva J, K Wahle, W Koch, A Behrens, L Fenoglio-Marc, EV Stanev, Coastal flooding: impact of waves on storm surge } \\
\text { during extremes - a case study for the German Bight, Nat. Hazards Earth Syst. Sci., 16, 2373-2389, } 2016 \\
\text {-DWD declares the storm to be the worst since the North Sea storm of } 1962 .\end{array}$ \\
\hline $\begin{array}{l}\text { Brooks et al. } \\
(2017)\end{array}$ & $\begin{array}{l}\text { Brooks, SM, T Spencer, EK Christie, Storm impacts and shoreline recovery: Mechanisms and controls in the southern North } \\
\text { Sea, Geomorphology, 283, 48-60, } 2017 \text {. } \\
\text {-for coastal cutback on the North Norfolk coast, Storm Xaver was far worse than Storm Britta, Storm Tilo or the Mar2007 } \\
\text { storm }\end{array}$ \\
\hline Evans (2017) & $\begin{array}{l}\text { Evans, Sun Yan, EA/2/2 Appendix 1, History of flooding sources, Boston Barrier Transport \& Works Act order application, } \\
\text { Public Inquiry documents, [pdf document properties: author=BDB; datestamp=16Mar2017] https://consult.environment- } \\
\text { agency.gov.uk/engagement/bostonbarriertwao/results/appendix-1---history-of-flooding-sources.pdf } \\
\text {-5.2 m surge (more than } 70 \mathrm{~cm} \text { above } 1953 \text { ) } \\
\text {-water was } 6.08 \mathrm{mODN} \text {; higher than } 1953 \& 1978\end{array}$ \\
\hline $\begin{array}{l}\text { Frohle and } \\
\text { Dreier (2017) }\end{array}$ & $\begin{array}{l}\text { Peter Frohle \& Norman Dreier, EarlyDike - Sensor - und risiko basiertes Fruhwarnsystem fuer Seedeiche, Teilprojekt: } \\
\text { Wellenmonitoring und Wellenbelastungssimulator (AP2), Hamburg, April } 2017 \\
\text {-target storms for wave runup analysis: Hurricane Tilo 8-9Nov2007, Hurricane Xaver 5-7 Dec 2013, Storm Elon and Felix 9- } \\
\text { 11 Jan2013, Hurricane Barbara 26Dec2016 }\end{array}$ \\
\hline Jee (2017) & $\begin{array}{l}\text { Jee, Andrew, EA/13/2 Appendix 1. A summary of flooding events in Boston. [pdf document properties: author=Andrew Jee; } \\
\text { Date stamp=17Mar2017] https://consult.environment-agency.gov.uk/engagement/bostonbarriertwao/ } \\
\text {-highest water levels at North Shields and Well among reported late 20th century storms. } \\
\text {-at Wick: rank } 2 \text { water level after } 1978\end{array}$ \\
\hline $\begin{array}{l}\text { Jensen et al } \\
(2017)\end{array}$ & $\begin{array}{l}\text { Jensen J, S Niehuser, A Arns, S Dangendorf, Sensor- und risikobasiertes Fruhwarn-system fuer Seedeiche (EarlyDike), AP1 - } \\
\text { Sturmflutmonitoring und Sturmflutssimulator - Fachbericht 2016, Siegen, April } 2017 \\
\text {-Storm Xaver is the focus storm for the development of a wave model; only Storm Britta } 2006 \text { and Storm Xaver } 2013 \\
\text { mentioned in the modelling exercise }\end{array}$ \\
\hline $\begin{array}{l}\text { Ribeiro et al } \\
(2017)\end{array}$ & $\begin{array}{l}\text { Ribeiro R, R Rudge, D Rucinska, Analysis of physical factors of the windstorm Xaver in Poland: post-hazard review, } \\
\text { Weather, 72, 2017, pp.378-382 } \\
\text {-Poland IMGW classifies level } 3 \text { storm: damage to buildings, power lines, forest; transport problems; threat to life } \\
\text {-Storm Xaver ranks with other severe European wind storms: Quimburga 1972, Wiebke 1990, Oratia (2000), Kyrill (2007) } \\
\text {-highest ever recorded storm surge water levels at some stations in England } \\
\text {-Stavoren meas wind speed 38m/s: highest ever recorded in Netherlands for any station since record start } 1910\end{array}$ \\
\hline $\begin{array}{l}\text { Staneva et al } \\
(2017)\end{array}$ & $\begin{array}{l}\text { Staneva J, H Guenther, O Krueger, C Schrumm, V Alari, O Breivik, J-R Bidlot, K Mogensen, Impact of wind waves on the } \\
\text { air-sea momentum fluxes for different wind and sea state conditions and oceanic responses, 1st International Workshop } \\
\text { on waves, storm surges and coastal hazards, Liverpool, UK 10-15Sep2017 [pdf document properties: title=Anlass; } \\
\text { author=Patrick Kalb-Anlass, datestamp=20/09/2017] } \\
\text {-Storm Christian had higher maximum wind speeds than Storm Xaver } \\
\text {-parameter wave breaking alpha was higher for Storm Christian than for Storm Xaver }\end{array}$ \\
\hline $\begin{array}{l}\text { Wahle et al } \\
(2017)\end{array}$ & $\begin{array}{l}\text { Wahle K, J Staneva, W Koch, L Fenoglio-Marc, HTM Ho-Hagemann, EV Stanev, An atmosphere-wave regional coupled } \\
\text { model: improving prediction of wave heights in the southern North Sea, Ocean Sci., 13, 289-301, 2017.(doi:10.5194/os- } \\
\text { 13-289-2017). } \\
\text {-one of most sever storms of the last decade }\end{array}$ \\
\hline $\begin{array}{l}\text { Ditlevsen et al } \\
(2018)\end{array}$ & $\begin{array}{l}\text { Ditlevsen C, MM Ramos, C Sorensen, UR Ciocan, T Pionkowitz, Hojvandsstatistikker 2017, Miljo- og Foedevaremnisteriet, } \\
\text { Kystdirektoratet, Lemvig, Februar, } 2018 \\
\text {-Storm Xaver had the highest storm surge in } 9 \text { of } 67 \text { stations; it was in the top } 20 \text { events for most stations. }\end{array}$ \\
\hline $\begin{array}{l}\text { Environment } \\
\text { Agency (2018) }\end{array}$ & $\begin{array}{l}\text { Environment Agency, Thames Barrier Project Pack 2018, January, } 2018 \\
\text {-largest recorded tide at Southend sind 1978, before Thames Barrier became operational; largestsurge along East Coast UK } \\
\text { since } 1953 .\end{array}$ \\
\hline DEMA (2018) & $\begin{array}{l}\text { Danish Emergency Management Agency DEMA, National Risk Profile for Denmark, April } 2018 \\
\text {-Storm Xaver was one of five Danish hurricane cases of last } 100 \text { years; others were 25-26Dec102, 23-24Oct1921, Oct 1967, } \\
\text { 24-26Nov1981, 3-4Dec1999; then strong storms 8Jan2005, 28Oct2013, 5-6Dec2013 }\end{array}$ \\
\hline $\begin{array}{l}\text { Fery et } \\
(2018)\end{array}$ & $\begin{array}{l}\text { Fery, Natascha, Birger Tinz, Lydia Gates, Reproduction of storms over the North Sea and the Baltic with the regional analysis } \\
\text { COSMO-REA6 ISPR 2018, 17-19July2018, Bonn [pdf document properties: datestamp=16/07/2018] } \\
\text {-Storrm Xaver one of five focus storms in DWD Extremeness project: Britta 2006, Kyrill 2007, Tilo 2007, Christian 2013, } \\
\text { Xaver } 2013\end{array}$ \\
\hline $\begin{array}{l}\text { Kystdirektorate } \\
\text { t (2018) }\end{array}$ & $\begin{array}{l}\text { Kystdirektoratet, Shoreface nourishment effects. An analysis of the } 2011 \text { nourishment performed at Skodbjerge. } \\
\text { Kystdirektoratet, Hojbovej 1, 7620 Lemvig, Dec 2018 [pdf document properties: title=Shoreface nourishment } \\
\text { effects_Skodbjerge_20.12.2018, datestamp=20/12/2018] } \\
\text {-storm with highest energy wave field in database 2006-2014; corresponds to period of highest ersional loss }\end{array}$ \\
\hline $\begin{array}{l}\text { North Norfolk } \\
\text { District } \\
\text { Council } \\
\text { Coastal Team } \\
\text { (2018) }\end{array}$ & $\begin{array}{l}\text { North Norfolk District Council Coastal Team, Refurbishment of sea walls and groynes 2013-2015; Cromer Coast Protection, } \\
\text { (picture of plaque on wall taken 27Dec2018) www.northnorfolk.org/coastal } \\
\text {-water levels along North Norfolk Coast higher in places than } 1953 \text { storm surge } \\
\text {-water levels at Thames Barrier highest ever since start of operations } 1982\end{array}$ \\
\hline $\begin{array}{l}\text { Ulm et al } \\
(2018)\end{array}$ & $\begin{array}{l}\text { Ulm, Marius, Arne Arns, Juergen Jensen, Assessing consequences of extreme events for the German Bight, 36th International } \\
\text { Conference on Coastal Engineering } 2018 \text { - Coastal Protection and Risk - ID } 1527 \\
\text {-Storm Britta } 2006 \text { regarded by local authorities as a close call for dike breach risk }\end{array}$ \\
\hline Giannopoulos & Giannopoulos G, L Peake, B Reid, J Andrews, A Grant, I Lorenzoni, M Goulden, J Waters, T Dolphin, J Bremner, TJ \\
\hline
\end{tabular}




\begin{tabular}{|c|c|}
\hline et al (2019) & $\begin{array}{l}\text { Tolhurst, Environmental and social impacts of the } 2013 \text { storm surge on the North Norfolk coast, powerpoint presentation } \\
\text { date stamp 15May2019, unknown conference } \\
\text {-'the biggest UK storm surge for } 60 \text { years' -UK Environment Agency } \\
\text {-tides along parts of N Norfolk coast reached higher levels than } 1953\end{array}$ \\
\hline $\begin{array}{l}\text { North Norfolk } \\
\text { District } \\
\text { Council } \\
\text { Coastal Team } \\
\text { (2019) }\end{array}$ & $\begin{array}{l}\text { North Norfolk District Council Coastal Team, Sheringham Sea Defences; Repair and Recovery from the December } 2013 \\
\text { storm surge, www.northnorfolk.org/coastal, photo of plaque on wall taken 30Dec } 2019 \\
\text {-for Storm Xaver water levels along parts of the Norfolk coast were higher than } 1953 \text { surge } \\
\text {-water levels at Thames Barrier highest ever since start of operations } 1982\end{array}$ \\
\hline $\begin{array}{l}\text { Rucinska } \\
\text { (2019) }\end{array}$ & $\begin{array}{l}\text { Rucinska D, Describing Storm Xaver in disaster terms, International Journal of Disaster Reduction, 34, 147-153, 2019 } \\
\text {-Hamburg had the second highest ever storm surge } \\
\text {-highest ever extreme ocean level was registered in northern Germany } \\
\text {-Netherlands had the highest ever measured winds ( } 38 \mathrm{~m} / \mathrm{s}) \text { since record start at Stavoren on the North Sea coast } \\
\text {-Netherlands wind speed was similar to } 1953 \\
\text {-Denmark had the highest ever extreme ocean level } \\
\text {-UK had the highest surge since } 1953\end{array}$ \\
\hline $\begin{array}{l}\text { Schenk and } \\
\text { Mueller- } \\
\text { Navarra (2019) }\end{array}$ & $\begin{array}{l}\text { Schenk, L and S Mueller-Navarra. 3.4.4. Windstaustatistiken und Haufigkeit von Sturmfluten } 2012-2015 \\
\text { https://www.bsh.de/DE/PUBLIKATIONEN/Nordseezustand_Aktuell/_Anlagen/Downloads/3_4_4_Windstatistiken.pdf? } \\
\text { _blob=publicationFile\&v=2 [pdf document properties: author=Ludwig Schenk; datestamp=14Feb2019] } \\
\text {-very severe storm surge with three consecutive storm tides } \\
\text {-Storm Xaver event resembled century surge of Feb1962 }\end{array}$ \\
\hline $\begin{array}{l}\text { Wikipedia } \\
(20191002)\end{array}$ & $\begin{array}{l}\text { Wikipedia, Stormen Bodil, https://da.wikipedia.org/wiki/Stormen_Bodil\#cite_note-39 (accessed 02Oct2019) } \\
\text {-Netherlands: highest water level since } 1953 \\
\text {-German authorities report waves up to } 6 \mathrm{~m} \text {; second highest since } 1825(?)\end{array}$ \\
\hline $\begin{array}{l}\text { Andrews } \\
(2020)\end{array}$ & $\begin{array}{l}\text { Andrews, JE, Spit extension and barrier rollover at Blakeney Point and Salthouse: historic map and field observations, Bull. } \\
\text { geol. Soc. Norfolk, 69, 35-63, } 2020 \\
\text {-comparable North Norfolk coastal damage for storm surge Nov2007, storm surge Dec2013, storm surge 12Feb1938, } \\
\text { storm surge 1Jan1995, storm surge } 1953\end{array}$ \\
\hline $\begin{array}{l}\text { JBA Risk } \\
\text { Management } \\
(2020)\end{array}$ & $\begin{array}{l}\text { JBA Risk Management, Storm Xaver 2013. Event Commentary, 2020. [PDF document properties: author=Cameron } \\
\text { Whitwham] } \\
\text {-COBRA meeting on 05Dec2013 beccause storm had potential to be regional/national disaster. } \\
\text {-new water level record at Kingston-Upon-Hull }\end{array}$ \\
\hline $\begin{array}{l}\text { Surgewatch } \\
(20200304)\end{array}$ & $\begin{array}{l}\text { Surgewatch, Storm event } 6 \text { th December } 2013 \text {, https://www.surgewatch.org/events/1/ (last accessed 04Mar2020) } \\
\text {-biggest event to impact UK east coast in more than half a century } \\
\text {-in many instances water level exceeded } 1953 \text { levels } \\
\text {-water levels exceeded the } 5 \text { year return period for } 12 \text { sites } \\
\text {-water level was highest on record for } 12 \text { sites } \\
\text {-Dover: return period } 843 y \\
\text {-Lowestoft return period: } 200 y \\
\text {-highest water level on record for Newhaven \& Portsmouth }\end{array}$ \\
\hline $\begin{array}{l}\text { WIKI } \\
(20200124)\end{array}$ & $\begin{array}{l}\text { WIKI, Cyclone Xaver, https://en.wikipedia.org/wiki/Cyclone_Xaver accessed 24Jan2020 } \\
\text {-AIR Worldwide estimated wind losses from storm 07-1.4 billion EUR; impact to be less than St. Jude storm } \\
\text {-UK forecast the worst storm surge in } 60 \text { years } \\
\text {-Germany liked the surge to the Hamburg surge of } 1962\end{array}$ \\
\hline $\begin{array}{l}\text { Wikipedia } \\
(20200502)\end{array}$ & $\begin{array}{l}\text { Wikipedia, Orkan Xaver, https://de.wikipedia.org/wiki/Orkan_Xaver (accessed } 2 \text { May 2020) } \\
\text {-weather pattern for Storm Xaver } 2013 \text { similar to } 1962 \text { Hamburg surge } \\
\text {-comparison with Capella 3-4Jan1976 }\end{array}$ \\
\hline
\end{tabular}

Table S4. Severe forecast (arranged by year and then alphabetically)

\begin{tabular}{|c|c|}
\hline Source & Full Reference and Notes \\
\hline $\begin{array}{l}\text { Aftenposten } \\
(20131205)\end{array}$ & 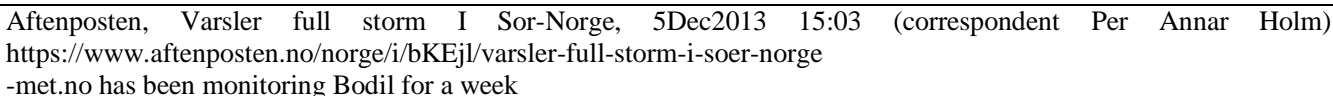 \\
\hline BBC (20131204) & $\begin{array}{l}\text { BBC, Workers off Talisman Sinopec oil platform Buchan Alpha over bad weather forecast,04Dec2013 } \\
\text {-severe weather forecast; } 85 \text { people taken off Buchan Alpha platform and production shut }\end{array}$ \\
\hline Bloomberg (2013) & $\begin{array}{l}\text { Bloomberg, Hamburg has worst flood in } 37 \text { year amid European storms, Nicholas Brautlecht, 6Dec2013, 02:58PM } \\
\text { GMT https://www.bloomberg.com/news/articles/2013-12-06/hamburg-has-worst-flood-in-37-years-as-storms-rage- } \\
\text { across-europe } \\
\text {-Thames Barrier closed with surge forecast to be worst for } 60 \text { years. }\end{array}$ \\
\hline BODC (20131205) & $\begin{array}{l}\text { BODC, Storm surge and coastal flooding, News and } \\
\text { https://www.bodc.ac.uk/about/news_and_events/storm_surge_and_coastal_flooding.html }\end{array}$ \\
\hline $\begin{array}{l}\text { Energy } \\
(20131204)\end{array}$ & $\begin{array}{l}\text { Energy Voice, Oil staff withdrawn from North Sea ahead of storm conditions, 04/12/2013 10:30AM, } \\
\text { https://www.energyvoice.com/other-news/healthandsafety/50140/oil-staff-withdrawn-north-sea-ahead-storm- } \\
\text { conditions/ } \\
\text {-North Sea forecast 04/12/2013 for 10m waves and storm force winds } \\
\text {-evacuation of Ekofisk, Valhall, Buchan Alpha }\end{array}$ \\
\hline DW (20131205) & $\begin{array}{l}\text { DW, Storm Xaver pummels Europe, flood surge feared, 05/12/2013 } \\
\text {-media report from late Thursday that surge water levels could rise }>3.5 \mathrm{~m} \text { above normal in the Bremen-Hamburg area }\end{array}$ \\
\hline $\begin{array}{l}\text { Gandreassen } \\
(20131204)\end{array}$ & $\begin{array}{l}\text { Gandreassen, Oil workers moved from Ekofisk, 4Dec2013. https://gandreassen.com/oil-workers-moved-from-ekofisk/ } \\
\text {-report of } 157 \text { workers evacuated from Ekofisk field } 1 \text { day in advance of Friday storm }\end{array}$ \\
\hline GVA (20131205) & GVA, Vijf containers even op drift op de Schelde, 05/12/2013 20:57. https://www.gva.be/cnt/aid1500792/vijf- \\
\hline
\end{tabular}




\begin{tabular}{|c|c|}
\hline & $\begin{array}{l}\text { containers-op-drift-op-de-schelde-2 } \\
\text {-warnings of storm gate closures in Antwerp for the following day (06/12/2013) }\end{array}$ \\
\hline Kunz et al (20131206) & $\begin{array}{l}\text { Kunz M, B Muehr, K Schroeter, T Bessel, S Moehrle, T Muenzberg, S Brink, H-M Schmidt, Winterstorm Xaver - } \\
\text { Report. 06Dec2013 - Report No.1, Situation Report - 19:00CET, CEDIM Forensic Disaster Analysis Group } \\
\text { (FDA), Center for Disaster Management and Risk Reduction Technology. } \\
\text {-northern federal states prepared long before surge arrives }\end{array}$ \\
\hline NLWKN (20131203) & $\begin{array}{l}\text { NLWKN, Sturmflutgefahr an der Kueste und auf den Inseln, Zwei meter ueber dem normalen Hochwasser in der } \\
\text { Nacht zum Freitag moeglich//pressinformation von } 29 \text { November 2013, (written by Herma Heyken, Pressesprecherin) } \\
\text {,03/12/2013 } \\
\text { https://www.nlwkn.niedersachsen.de/startseite/aktuelles/presse und offentlichkeitsarbeit/pressemitteilungen/sturmflut } \\
\text { gefahr-an-der-kueste-und-auf-den-inseln-120187.html } \\
\text {-storm surge danger for Thursday and Friday with water levels } 2 \mathrm{~m} \text { above normal } \\
\text {-water level information for Storm Britta , ranked as } 100 \mathrm{y} \text { event on Niedersachsen coast; Borkum water level } \\
\text { comparable with } 1962 \text { surge. } \\
\text {-storm surge predicted to be centered on Emsmundung and Jade }\end{array}$ \\
\hline $\begin{array}{l}\text { Oceanografisch } \\
\text { Meteorologisch Station } \\
\text { (2013) }\end{array}$ & $\begin{array}{l}\text { Oceanografisch Meteorologisch Station, Stormverslag } 05-06 \text { december 2013, 26pp, } 2013 \text { [pdf document properties: } \\
\text { author=Myriam Sys; datestamp=15Dec2013] } \\
\text {-wind forecasts available from 30Nov2013; storm conditions d=forecase starting from 01Dec2013 } \\
\text {-detailed wind and sea state forecasts from 04Dec2013 } \\
\text {-water level forecasts from different model from 01Dec2013 }\end{array}$ \\
\hline $\begin{array}{l}\text { Unwetterzentrale } \\
(201312)\end{array}$ & $\begin{array}{l}\text { Unwetterzentrale, Orkantief XAVER - ein weiterer schwerer Wintersturm der letzten Jahrzehnte, Thomas Savert and } \\
\text { Stefan Laps, Dec. } 2013 \mathrm{http}: / / \text { www.unwetterzentrale.de/uwz/928.html } \\
\text {-US GFS model made predictions from 29Nov of storm on Dec6. } \\
\text {-3-4 days before the storm most model predicted gusts to hurricane strength }\end{array}$ \\
\hline Upstream (20131205) & $\begin{array}{l}\text { Upstream, North Sea production curtailed over storm, 05Dec2013 (contributor: Bill Lehane and News Wires) } \\
\text {-North Sea platform production reduced and platforms de-manned 05Dec } 2013 \text { on basis of forecast of 10m waves }\end{array}$ \\
\hline Dunbar et al (2014) & $\begin{array}{l}\text { Dunbar I, N Phipps, M Szonyi, Risk Nexus. After the storm: how the UK's flood defences performed during the surge } \\
\text { following Xaver, Flood resilience review 09.14, Zurich Insurance Company Ltd., Mythenquai 2, 8002, Zurich, } \\
\text { Switzerland [document properties: date 28Aug2014; author= Zurich] } \\
\text {-4Dec2013: EA sent warning 'prepare for worst E coast tidal surge in 30y' }\end{array}$ \\
\hline Hewson et al (2014) & $\begin{array}{l}\text { Hewson T, L Magnusson, O Breivik, F Prates, I Tsonevsky, HJW de Vries, Windstorms in northwest Europe in late } \\
\text { 2013, ECMWF Newsletter, No 139, pp 22-28, Spring } 2014 \text {. } \\
\text {-ECMWF indication of strong winds 5-6 days in advance } \\
\text {-at longer lead times of 7-8days some of the ensemble runs predicted vigorous cyclones in the correct location but few } \\
\text { were sufficiently extreme. }\end{array}$ \\
\hline Nosen & $\begin{array}{l}\text { Nossent J, L Boeckx, E Taverniers, M Deschamps, T Verwaest, F Mostaert, Sinterklaasstorm } 6 \text { december } 2013 . \\
\text { Beschrijving van de hydrometrische gebeurtenissen, Versie 4.0. WL Rapporten, 00-119, Waterbouwkundig } \\
\text { Laboratorium, Antwerpen, Belgie. October 2014, WL2014R00_119_5 [document date stamp: 15Oct2014; } \\
\text { author= Deschamps, Maarten] } \\
\text {-storm surge moel predictions in the Schelde estuary for different lead times }\end{array}$ \\
\hline RWS (2014a) & $\begin{array}{l}\text { RWS, Watermanagementcentrum Nederland, Stormvloedflits } 2013-07 \text { van } 5 \mathrm{t} / \mathrm{m} 7 \text { december 2013, Rijkswaterstaat } \\
\text { (document time stamp: 07Jan2014), 2014a } \\
\text {-longterm forecast of storm surge on Netherlands coast from 30Nov2013 }\end{array}$ \\
\hline RWS (2014b) & $\begin{array}{l}\text { RWS, Stormvloedrapport van } 5 \mathrm{t} / \mathrm{m} 7 \text { december (SR91) Sint-Nicolaasvloed 2013, Watermanagementcentrum } \\
\text { Nederland, Rijkswaterstaat, prepared by Ing. J. Kroos, } 19 \mathrm{Mar} 2014 \mathrm{~b}, 48 \mathrm{pp} \\
\text {-advance forecast from } 27 \text { Nov2013 } \\
\text {-detailed } 12 \mathrm{~h} \text { forecasts before surge maximum at each reference station }\end{array}$ \\
\hline Van Dorland & $\begin{array}{l}\text { van Dorland, R, Zware storm op } 5 \text { december, Zenit, p43, Januari } 2014 \\
\text {-KNMI issued code orange severe weather warning for gusts in NW part of country on 04Dec2013 } \\
\text {-Upgraded to code red severe weather warning on 05Dec2013 }\end{array}$ \\
\hline Axer et al (2015) & $\begin{array}{l}\text { Axer T, T Bistry, M Klawa, M Mueller, M Suesser, Deutsche Ruck Sturm dokumentation } 2013 \text { Deutschland, 2013, } \\
\text { Deutsche Rueckversicherung Aktiengesellschaft, Hansaallee 177, } 40549 \text { Duesseldorf, www.deutscherueck.de } \\
\text { [pdf document information: author=filiz; date stamp=07Aug2015] } \\
\text {-damage low because of early warning of storm } \\
\text {-correct longrange forecast of development \& impact } \\
\text {-5-day advance forecast of significant storm surge potential } \\
\text {-1-day before height, weather service \& media broadcast hurricane gusts on coasts \& northern Germany }\end{array}$ \\
\hline Dan et al (2015) & $\begin{array}{l}\text { Dan, Sebastian, Anne-Lise Montreuil, Rosalia Delgado, Tomas van Oyen, Large storm impact on a beach under sand } \\
\text { nourishments, The Proceedings of the Coastal Sediments 2015, edited by Ping Wang, Julie D Rosati, and Jun } \\
\text { Cheng, Coastal Sediments 2015, San Diego, USA, 11-15May2015 } \\
\text {-pre-storm beach surveys were made on 3Dec2014 }\end{array}$ \\
\hline NLWKN (20151210) & 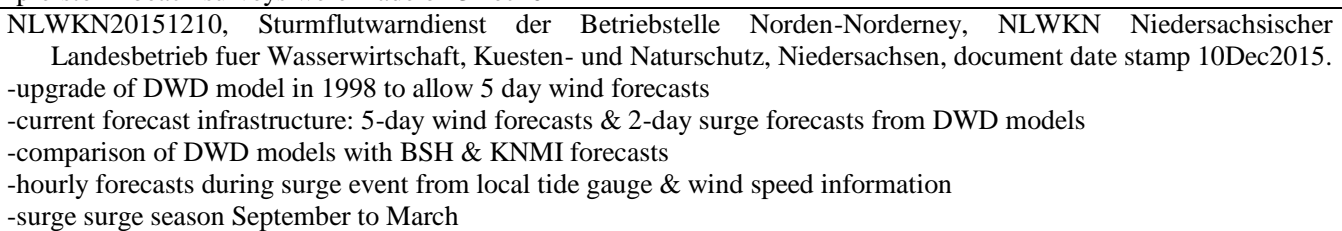 \\
\hline Wadey et al (2015) & $\begin{array}{l}\text { Wadey MP, ID Haigh, RJ Nichols, JM Brown, K Horsburgh, B Carroll, SL Gallop, T Mason, E Bradshaw, A } \\
\text { comparison of the } 31 \text { January-1 February } 1953 \text { and 5-6 December } 2013 \text { coastal flood events around the UK, UK } \\
\text { Frontiers in Marine Science, 2, 84, } 2015 \text {. } \\
\text {-noon } 31 \text { Jan } 1953 \text { forecast: 'All districts will have gale force winds, severe in many places, and squaly showers, mainly } \\
\text { of hail or snow. Considerable snowfall may occur over high ground. Thunderstorms will occur here and there. It } \\
\text { will be cold' (no mention coastal flooding) }\end{array}$ \\
\hline
\end{tabular}




\begin{tabular}{|c|c|}
\hline & -Storm Xaver surge forecast 7 days in advance \\
\hline Sibley et al. (2015) & $\begin{array}{l}\text { Sibley A, D Cox, H Titley, Coastal flooding in England and Wales from Atlantic and North Sea storms during the } \\
\text { 2013/2014 winter, Weather, 70, 62-70, } 2015 \\
\text {-ensemble forecast from 0600UTC 1Dec2013 indicates possible surge problem } \\
\text {-ensemble forecast from 0000UTC 5Dec2013 predicts surge height with small uncertainty range }\end{array}$ \\
\hline ECMWF (20160316) & $\begin{array}{l}\text { ECMWF (20160316), 201312 - Windstorm - Xaver/Bodil/Sven, } \\
\text { https://confluence.ecmwf.int/pages/viewpage.action?pageId=28315390 } \\
\text {-severe gusts forecast days in advance } \\
\text {-FIG4. [MAP] The series of figures above shows HRES forecasts of the } \\
\text { maximum wind gust during 5Dec and the MSLP valid 12UTC. The } \\
\text { color scale is the same as for the observationsin the previous } \\
\text { section. The first forecast is from 05Dec 00UTC and following } \\
\text { is } 04 \text { dec and so on (1day apart. Already the forecast from } \\
9 \text { days before had a cyclone in the area, but further west than } \\
\text { the latest forecast } \\
\text {-FIG7. [MAP] Probability of maximum wind gust >33m/s on 5Dec. } \\
\text { The first forecast is from 5 Dec 00UTC and the following are one } \\
\text { day apart. Up 3.5-4 days before the event, the probability } \\
\text { of hurricane wind gust were close to 100\% in western Scotland } \\
\text { and over the North Sea }\end{array}$ \\
\hline Ribeiro et al (2017) & $\begin{array}{l}\text { Ribeiro R, R Rudge, D Rucinska, Analysis of physical factors of the windstorm Xaver in Poland: post-hazard review, } \\
\text { Weather, 72, 2017, pp.378-382 } \\
\text {-forecast program European Storm issued } 18 \text { storm forecasts 3-11Dec } 2013 \\
\text {-ESTOFEX forecast very dangerous weather from 5Dec } 2013 \text { for Netherlands, Germany, Poland } \\
\text {-35 wrnings of strong winds 4-7Dec2013 } \\
\text {-FIG5. [MAP] Forecasts of expected significant weather threats issued on } \\
\text { (a) 4Dec 2249UTC, (b) 5Dec } 1139 \text { UTC, (c) 6Dec } 2215 \text { UTC, 8Dec } 0343 \text { UTC } \\
\text { (significant weather threats UK, Netherlands, Germany Sweden Poland; Norway, Czech republic, Austria marginal }\end{array}$ \\
\hline Wahle et al (2017) & $\begin{array}{l}\text { Wahle K, J Staneva, W Koch, L Fenoglio-Marc, HTM Ho-Hagemann, EV Stanev, An atmosphere-wave regional } \\
\text { coupled model: improving prediction of wave heights in the southern North Sea, Ocean Sci., 13, 289-301, 2017. } \\
\text { doi:10.5194/os-13-289-2017. } \\
\text {-'one of the most severe storms of the last decade' }\end{array}$ \\
\hline Wikipedia (20191002) & $\begin{array}{l}\text { Wikipedia, Stormen Bodil, https://da.wikipedia.org/wiki/Stormen_Bodil\#cite_note-39 (accessed 02Oct2019) } \\
\text {-DMI observed low P } 1 \text { week previously over Greenland; warnings } 5 \text { days in advance } \\
\text {-Denmark Strait NW Island reports 4Dec2013 of powerful low P; developed explos overnight } \\
\text {-UK Category } 2 \text { warning for Scotland \& N part of England; risk gust } 145 \mathrm{~km} / \mathrm{h} \\
\text {-EA warned of worst storm flood in 30y for English east coast } \\
\text {-DMI category } 2 \text { warning all Denmark, high water danger Vadehavet \& inner Danish farvand } \\
\text {-SMHI warned of high snowfall \& powerful gust } \\
\text {-NW Germany Niedersachsen, DWD warned gusts to } 140 \mathrm{~km} / \mathrm{h} \text { with repeat of } 1962 \text { storm surge }\end{array}$ \\
\hline WIKI (20200124) & $\begin{array}{l}\text { WIKI, Cyclone Xaver, https://en.wikipedia.org/wiki/Cyclone_Xaver accessed 24Jan2020 } \\
\text {-storm center only formed on } 4 \text { Dec } 2013 \text { off W coast of Iceland } \\
\text {-UK Met Office issued amber warning over Scotland \& N England with forecast gusts to reach } 145 \mathrm{kh} / \mathrm{m} \\
\text {-EA issued warning 4Dec2013 for E coast England for worst surge in } 60 \text { years in period 5-7 Dec } 2013 \\
\text {-Netherlands: Friesland, Groningen, North Holland place on Red Alert with winds force 9-11 forecast } \\
\text {-Denmark: force } 12 \text { gusts expected } \\
\text {-Sweden: SMHI gave level } 2 \text { warning for heavy snowfall \& snow drifts }\end{array}$ \\
\hline Wikipedia (20200429) & $\begin{array}{l}\text { Wikipedia, Stormen Sven, https://sv.wikipedia.org/wiki/Stormen_Sven\#cite_note-15, accessed 29Apr2020 } \\
\text {-SMHI issued class-2 warning 4Dec2013 for south Sweden } \\
\text {-warning upgraded to class-3 for Skane to Osterlen; extended to include Halland }\end{array}$ \\
\hline Wikipedia (20200502) & $\begin{array}{l}\text { Wikipedia, Orkan Xaver, https://de.wikipedia.org/wiki/Orkan_Xaver (accessed } 2 \text { May 2020) } \\
\text {-warnings of arctic storm starting from 1Dec2013 } \\
\text {-UK issued surge warning for } 30 \mathrm{y} \text { event; then upgraded to 50y event } \\
end{array}$ \\
\hline
\end{tabular}

Table S5. Storm not as bad as expected; not as bad as it could have been (arranged by year and then alphabetically)

\begin{tabular}{|c|c|}
\hline Source & Full Reference and Notes \\
\hline $\begin{array}{l}\text { Gccaptialideas } \\
(20131209)\end{array}$ & $\begin{array}{l}\text { gccapitalideas, Windstorm Xaver, 9Dec2013, https://www.gccapitalideas.com/2013/12/09/windstorm-xaver/ } \\
\text {-'despite some reports of roof damage and the widespread disruption to transportation, damage from the powerful } \\
\text { winds nevertheless appears to have been minimal and surge damage was limited by strengthened sea defences ...' } \\
\text {-'...improved flood defences protected } 800,000 \text { properties, meaning the impact was not as severe as } 1953 \text { ' }\end{array}$ \\
\hline $\begin{array}{l}\text { Nordbayern } \\
(20131207)\end{array}$ & $\begin{array}{l}\text { Nordbayern, Deining: 'Xaver' reisst Rotorblatt von Windrad ab. Windkraftanlage schleuderte Eisbrocken auf die } \\
\text { Strasse, 07/12/2013, 12:07. https://www.nordbayern.de/region/neumarkt/deining-xaver-reisst-rotorblatt-von- } \\
\text { windrad-ab-1.3326492 } \\
\text {-not much damage in northern Bavaria in spite of high winds }\end{array}$ \\
\hline $\begin{array}{l}\text { Oceanografisch } \\
\text { Meteorologisch } \\
\text { Station (2013) }\end{array}$ & $\begin{array}{l}\text { Oceanografisch Meteorologisch Station, Stormverslag 05-06 december 2013, 26pp, } 2013 \text { [pdf document } \\
\text { properties: author=Myriam Sys; datestamp=15Dec2013] } \\
\text {-discussion of worst case scenario } \\
\text {-air pressure only went down to } 1012 \mathrm{hPa} \text {; could have dropped to } 960 \mathrm{hPa} \\
\text {-surge would have been worst if wind were more northerly }\end{array}$ \\
\hline $\begin{array}{l}\text { Spiegel International } \\
(20131206)\end{array}$ & 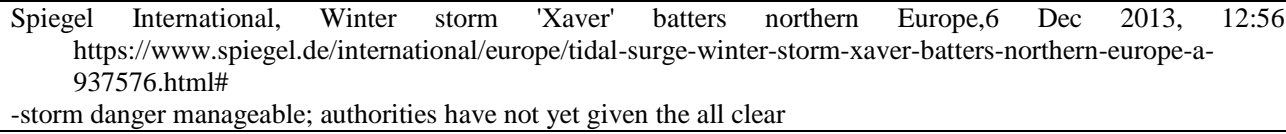 \\
\hline
\end{tabular}




\begin{tabular}{|c|c|}
\hline $\begin{array}{l}\text { Unwetterzentrale } \\
\text { (201312) }\end{array}$ & $\begin{array}{l}\text { Unwetterzentrale, Orkantief XAVER - ein weiterer schwerer Wintersturm der letzten Jahrzehnte, Thomas Savert } \\
\text { and Stefan Laps, Dec. } 2013 \text { http://www.unwetterzentrale.de/uwz/928.html } \\
\text {-early warning prevented much damage }\end{array}$ \\
\hline ABPmer (2014) & $\begin{array}{l}\text { ABPmer, Ensuring Flood Resilience. An overview of the 5/6 December 2013, Associated British Ports, Marine } \\
\text { Environmental Research, July, 2014 } \\
\text {-2013 surge much less than 1953: fatalities, flooded properties, flooded agricultural land } \\
\text {-aerial photo of London showing potential flooded area if Thames Barrier were absent. }\end{array}$ \\
\hline $\begin{array}{l}\text { CH2MHill Halcrow } \\
\text { (2014) }\end{array}$ & $\begin{array}{l}\text { CH2MHill Halcrow, Cell } 1 \text { Regional Coastal Monitoring Programme, Wave Data Analysis Report 2: 2013-2014, } \\
\text { Final Report, March } 2014 \text { [document properties: author=Andy.Parson @ ch2m.com; datestamp; 04/04/2014] } \\
\text {-'The storm surge that damaged many defences and received significant media attention } \\
\text { on 5th and 6th Decembr } 2013 \text { does not appear to have had exceptional wave conditions } \\
\text { at the Tyne Tees buoy, with a peak significant wave height of } 4.7 \mathrm{~m} \text { and storm duration } \\
\text { of } 38 \text { hours. However, the wave period was over } 14 \text { seconds, is unusual, and the } \\
\text { longest storm wave period recorded' }\end{array}$ \\
\hline Dunbar et al (2014) & $\begin{array}{l}\text { Dunbar I, N Phipps, M Szonyi, Risk Nexus. After the storm: how the UK's flood defences performed during the } \\
\text { surge following Xaver, Flood resilience review 09.14, Zurich Insurance Company Ltd., Mythenquai 2, 8002, } \\
\text { Zurich, Switzerland [document properties: date 28Aug2014; author= Zurich] } \\
\text {-EA aerial photo of London area flooed in absence of London Barrier } \\
\text {-cost of } 1953 \text { flood 500mill GBP; } 1983 \text { construction cost 535mill GBP } \\
\text {-EA: flood defenses in Hull river \& Humber estuary prevented flooding of } 100000 \text { homes \& 250mill GBP loss } \\
\text {-Warrington Water Scheme: flood protection scheme operated for first time during Xaver 2013; } 1400 \text { home } \\
\text { protected }\end{array}$ \\
\hline $\begin{array}{l}\text { Luecht and Peters } \\
\text { (2014) }\end{array}$ & $\begin{array}{l}\text { Luecht, Fabian and Ove Peters, Bericht ueber die Sturmflut vom 05.-0.6.12.2013 an der Westkueste Schleswig- } \\
\text { Holsteins, Landesbetrieb fuer Kuestenschutz, Nationalpark und Meeresschutz Schleswig-Holstein, Husum } \\
\text { 26Feb2014, 19pp. [pdf document properties: title=Lfd; Author=Thorsten Nommensen; datestamp: } \\
\text { 11Dec2018] } \\
\text {-in spite of extreme wind there was relatively little damage to coastal defences in Schleswig-Holstein } \\
\text {-exception was rock armour on Halligen, which had massive damage }\end{array}$ \\
\hline RMS (2014) & $\begin{array}{l}\text { RMS, 2013-2014 Winter Storms in Europe. An Insurance and Catastrophe Modeling Perspective. RMS White } \\
\text { Paper. [PDF TIMESTAMP 11Mar2014] } \\
\text {-wind damage in Europe minimal compares with UK events } \\
\text {-storm surge losses in winter 2013-2014 not exceptional } \\
\text {-flood defences have evolved; damage not as bad at } 1953 \text { storm } \\
\text {-storm surge losses for Germany were not as bad as } 1962 \text { or } 1976 \text { (Capella) events }\end{array}$ \\
\hline Thorne (2014) & $\begin{array}{l}\text { Thorne, Colin, Geographies of UK flooding in 2013/4, The Geographical Journal, 180, 297-309, } 2014 . \\
\text {-disruption and damage fom storm surge limited } \\
\text {-UK flooding damage for all 2013/2014 events } 290 \text { million GBP; } 2007 \text { summer floods cost } 3.2 \text { billion GBP }\end{array}$ \\
\hline Axer et al (2015) & $\begin{array}{l}\text { Axer T, T Bistry, M Klawa, M Mueller, M Suesser, Deutsche Ruck Sturm dokumentation } 2013 \text { Deutschland, } \\
\text { 2013, Deutsche Rueckversicherung Aktiengesellschaft, Hansaallee 177, 40549 Duesseldorf, } \\
\text { www.deutscherueck.de [pdf document information: author=filiz; date stamp=07Aug2015] } \\
\text {-limited damage because early warning of storm \& storm surge protection developed over previous decade } \\
\text {-no great damage because of early warning \& investment in coast protection after 1953,1962,1976 } \\
\text {-Baltic: storm surge lower than feared; sand bags made ready in Wismar } \\
\text {-'Im Vorfeld und waehrend des Ereignisses wurde ueber die potenzielle Katastrophe an der } \\
\text { Nordsee berichtet, die sich glucklicherweise nicht realisierte' (potential catastrophe on North Sea not realized) }\end{array}$ \\
\hline Dan et al (2015) & $\begin{array}{l}\text { Dan, Sebastian, Anne-Lise Montreuil, Rosalia Delgado, Tomas van Oyen, Large storm impact on a beach under } \\
\text { sand nourishments, The Proceedings of the Coastal Sediments 2015, edited by Ping Wang, Julie D Rosati, } \\
\text { and Jun Cheng, Coastal Sediments 2015, San Diego, USA, 11-15May2015 } \\
\text {-'The storm had a limited impact on teh study zone most probably due to the mild wave conditions and good } \\
\text { volumetric state of the beach' }\end{array}$ \\
\hline Jensen et al (2015) & $\begin{array}{l}\text { Jensen, J., A. Arns, T. Wahl, Yet another 100yr storm surge event: the role of individual storm surges on design } \\
\text { water levels, Journal of Marine Science and Technology, 23, 882-887, } 2015 \text {. } \\
\text {-calculated return water levels much less than 100y level reported in media }\end{array}$ \\
\hline $\begin{array}{l}\text { Dangendorf et al } \\
(2016)\end{array}$ & $\begin{array}{l}\text { Dangendorf S, A Arns JG Pinto, P Ludwig, J Jensen, The exceptional influence of storm 'Xaver' on design water } \\
\text { levels in the German Bight, Environmental Research Letters, 11, 2016, } 054001 \\
\text {-tide \& storm surge were out of phase; water levels could have been several decimeters higher }\end{array}$ \\
\hline Matelski (2016) & $\begin{array}{l}\text { Matelski, Birgit, Erfahrungen aus der Sturmflut Xaver von 5. und 6.12.2013 und dem Weihnachshochwasser } 2014 \\
\text { in Shleswig-Holstein, IWASA } 2016 \text { Tagungsbeitrag, (46. IWASA, 7-8 Januar 2016; Internationales } \\
\text { Wasserbau-Symposium Aachen. [pdf document properties: autor=sonja; datestamp: 26Apr2016] } \\
\text {-only a few stations registered new water level records }\end{array}$ \\
\hline $\begin{array}{l}\text { North Norfolk District } \\
\text { Council Coastal Team } \\
\text { (2018) }\end{array}$ & $\begin{array}{l}\text { North Norfolk District Council Coastal Team, Refurbishment of sea walls and groynes 2013-2015; Cromer Coast } \\
\text { Protection, (picture of plaque on wall taken 27Dec2018) www.northnorfolk.org/coastal } \\
\text {-no drowning fatalities in } 2013 \text { storm even though water levels similar to } 1953 \text { when }>2000 \text { drowned } \\
\text {-reason: effective sea defenses and huge advances in surge forecasting, modern communication, emergency } \\
\text { planning }\end{array}$ \\
\hline Ulm et al (2018) & $\begin{array}{l}\text { Ulm, Marius, Arne Arns, Juergen Jensen, Assessing consequences of extreme events for the German Bight, 36th } \\
\text { International Conference on Coastal Engineering } 2018 \text { - Coastal Protection and Risk - ID } 1527 \\
\text {-'earlier research suggests. Water levels may likely exceed historical events by at least } 1.40 \mathrm{~m} \text { ' }\end{array}$ \\
\hline $\begin{array}{l}\text { North Norfolk District } \\
\text { Council Coastal Team } \\
\text { (2019) }\end{array}$ & $\begin{array}{l}\text { North Norfolk District Council Coastal Team, Sheringham Sea Defences; Repair and Recovery from the } \\
\text { December } 2013 \text { storm surge, www.northnorfolk.org/coastal, photo of plaque on wall taken 30Dec2019 } \\
\text {-no drowning fataliies for } 2013 \text { storm in comparison to } 2013 \text { storm because of effective sea defences, advances in } \\
\text { weather/flood forecasting, modern communications, emergency planning }\end{array}$ \\
\hline Wikipedia (20191002) & $\begin{array}{l}\text { Wikipedia, Stormen Bodil, https://da.wikipedia.org/wiki/Stormen_Bodil\#cite_note-39 (accessed 02Oct2019) } \\
\text {-storm milder than expected on France's north coast near Denmark }\end{array}$ \\
\hline
\end{tabular}




\begin{tabular}{|l|l|}
\hline $\begin{array}{l}\text { JBA Risk Managment } \\
(2020)\end{array}$ & $\begin{array}{l}\text { JBA Risk Management, Storm Xaver 2013. Event Commentary, 2020. [PDF document properties: } \\
\text { author=Cameron Whitwham] } \\
\text {-UK flood defences avoided estimated 32 billion GBP in damage costs }\end{array}$ \\
\hline $\begin{array}{l}\text { Surgewatch } \\
(20200304)\end{array}$ & $\begin{array}{l}\text { Surgewatch, Storm event 6th December 2013, https://www.surgewatch.org/events/1/ (last accessed 04Mar2020) } \\
-800000 \text { properties protected by flood defences along 2800km coastline }\end{array}$ \\
\hline WIKI (20200124) & $\begin{array}{l}\text { WIKI, Cyclone Xaver, https://en.wikipedia.org/wiki/Cyclone_Xaver accessed 24Jan2020 } \\
\text {-Xaver storm surge weaker than expected on the north coast of France } \\
\text {-Peter Aldous MP Waveney: 'There is a strong sense in these communities that Parliament has not yet considered } \\
\text { properly this narrowly averted national crisis.' }\end{array}$ \\
\hline Wikipedia (20200502) & $\begin{array}{l}\text { Wikipedia, Orkan Xaver, https://de.wikipedia.org/wiki/Orkan_Xaver (accessed 2 May 2020) } \\
\text {-'In Deutschland waren de Schaeden weniger verheerend als zuvor befuerchtet.' }\end{array}$ \\
\hline
\end{tabular}

Table S6. Storm worse than expected (arranged by year and then alphabetically)

\begin{tabular}{|c|c|}
\hline Source & Full Reference and Notes \\
\hline $\begin{array}{l}\text { Sueddeautsche } \\
\text { Zeitung (20131215) }\end{array}$ & $\begin{array}{l}\text { Sueddeutsche Zeitung, Xaver holte sich Land; Sturmschaden auf Sylt, 15Dec201315:36 } \\
\text { https://www.sueddeutsche.de/panorama/sturmschaeden-auf-sylt-xaver-holte-sich-land-1.1844100 } \\
\text {-emergency sandbags placed at Keitum on east side of Sylt to prevent a water inbreak }\end{array}$ \\
\hline $\begin{array}{l}\text { Sylter Rundschau } \\
(20131209)\end{array}$ & $\begin{array}{l}\text { Sylter Rundschau, Folgen des Orkans. Xaver 'knabberte' Sylt massiv an, 09Dec2013 06:00 (from Friederike } \\
\text { Reussner) https://www.shz.de/lokales/sylter-rundschau/xaver-knabberte-sylt-massiv-an-id5098481.html } \\
\text {-storm damages Sylt boundary dunes and dikes }\end{array}$ \\
\hline $\begin{array}{l}\text { Unwetterzentrale } \\
(201312)\end{array}$ & $\begin{array}{l}\text { Unwetterzentrale, Orkantief XAVER - ein weiterer schwerer Wintersturm der letzten Jahrzehnte, Thomas Savert } \\
\text { and Stefan Laps, Dec. } 2013 \mathrm{http} / / \text { www.unwetterzentrale.de/uwz/928.html } \\
-10000 \text { sand bags for temporary dike at Sahlenburg }\end{array}$ \\
\hline BBC (20141205) & $\begin{array}{l}\text { BBC, East coast surge: what happened next? (report by Richard Haugh), } 5 \text { Dec } 2014 \\
\text {-house owners in Hemsby thought they would have } 30 y \text { before the sand cliff washed away } \\
\text {-photos show ambulance services evacuating people with zodiacs in the middle of the flood }\end{array}$ \\
\hline Dunbar et al (2014) & $\begin{array}{l}\text { Dunbar I, N Phipps, M Szonyi, Risk Nexus. After the storm: how the UK's flood defences performed during the } \\
\text { surge following Xaver, Flood resilience review 09.14, Zurich Insurance Company Ltd., Mythenquai } 2 \text {, } \\
\text { 8002, Zurich, Switzerland [document properties: date 28Aug2014; author= Zurich] } \\
\text {-2013 flood: } 100 \text { properties flooded \& flood protection structures damaged }\end{array}$ \\
\hline $\begin{array}{l}\text { Knaack and Heyken } \\
\text { (2014) }\end{array}$ & $\begin{array}{l}\text { Knaack H and H Heyken, Xaver hatte sehr schwere Stumflut im Gepaeck, Jahresbericht 2013. Der Zukunft } \\
\text { verplichtet, NLWKN, Niedersaechsischer Landesbetrieb fuer Waserwirtschaft, Kuesten- und Naturschutz, } \\
\text { pp.8-9, document date stamp 24Apr2014. } \\
\text {-winter of 2012-2013 had only one light storm surge at end of January; 2013-2014 had Orkan Christian and Xaver }\end{array}$ \\
\hline RWS (2014b) & $\begin{array}{l}\text { RWS, Stormvloedrapport van } 5 \mathrm{t} / \mathrm{m} 7 \text { december (SR91) Sint-Nicolaasvloed 2013, Watermanagementcentrum } \\
\text { Nederland, Rijkswaterstaat, prepared by Ing. J. Kroos, } 19 \mathrm{Mar} 2014 \mathrm{~b}, 48 \mathrm{pp} \\
\text {-Delfzijl water level } 52 \mathrm{~cm} \text { higher than forecast } \\
\text {-Roompot buiten second higher error at } 37 \mathrm{~cm} \text { higher than forecast }\end{array}$ \\
\hline $\begin{array}{l}\text { Haigh and Bradshaw } \\
\text { (2015) }\end{array}$ & $\begin{array}{l}\text { Haigh I and E Bradshaw, A century of UK coastal flooding, Planet Earth, Winter 2015, (pdf document properties: } \\
\text { 13Jan2016) } \\
\text {-COBRA crisis committee briefings for several storms }\end{array}$ \\
\hline Jensen et al (2015) & $\begin{array}{l}\text { Jensen, J., A. Arns, T. Wahl, Yet another 100yr storm surge event: the role of individual storm surges on design } \\
\text { water levels, Journal of Marine Science and Technology, 23, 882-887, } 2015 \text {. } \\
\text {-design water levels had to be revised upward 10-20cm for Emden, Wilhelshaven, Norderney }\end{array}$ \\
\hline $\begin{array}{l}\text { Vanmassenhove } \\
(2015)\end{array}$ & $\begin{array}{l}\text { Vanmassenhove, Niels, Storm surge measures ports Flemish coast, Blankenberge, Tuesday February 3rd, } \\
\text { Maritieme Sientverlening en Kust, Coastal Division, Flanders Hydraulic Research.[document properties: } \\
\text { title=Geintegreerd Kustveiligheidsplan; author=Maarten; datestamp=27/02/2015] } \\
\text {-emergency workers placed sandbags in Ostend Harbour }\end{array}$ \\
\hline Wadey et al (2015a) & $\begin{array}{l}\text { Wadey MP, ID Haigh, RJ Nichols, JM Brown, K Horsburgh, B Carroll, SL Gallop, T Mason, E Bradshaw, A } \\
\text { comparison of the } 31 \text { January-1 February } 1953 \text { and 5-6 December } 2013 \text { coastal flood events around the UK, UK } \\
\text { Frontiers in Marine Science, 2, 84, 2015a. } \\
\text {-large differences in local water levels; reasons not clear } \\
\text {-effect of wave field increasing the severity of the storm }\end{array}$ \\
\hline Sibley et al (2015a) & $\begin{array}{l}\text { Sibley A, D Cox, H Titley, Coastal flooding in England and Wales from Atlantic and North Sea storms during the } \\
\text { 2013/2014 winter, Weather, 70, 62-70, } 2015 \\
\text {-Whitby: '... power outages to the town making recovery difficult' } \\
\text {-River Haven at Boston south Lincolnshire: '....experienced breaches of sea defences with around } 200 \text { people } \\
\text { evacuated' } \\
\text {-'thousands of people were evacuated from homes in East Anglia as sea level rose' } \\
\text {-Rhyl and Conwy: 'four hundred people were evacuated as high tides and wave overtopped flood defences', }\end{array}$ \\
\hline Wadey et al (2015b) & $\begin{array}{l}\text { Wadey MP, JM Brown, ID Haigh, T Dolphin, P Wisse, Assessment and comparison of extreme sea levels and } \\
\text { waves during the 2013/2014 storm season in two UK coastal regions, Nat. Hazards Earth Syst. Sci. Discuss., } \\
\text { 3, 2665-2708, 2015b. } \\
\text {-breaching of water level defences in Suffolk }\end{array}$ \\
\hline Evans (2017) & $\begin{array}{l}\text { Evans, Sun Yan, EA/2/2 Appendix 1, History of flooding sources, Boston Barrier Transport \& Works Act order } \\
\text { application, Public Inquiry documents, [pdf document properties: author=BDB; datestamp=16Mar2017] } \\
\text { https://consult.environment-agency.gov.uk/engagement/bostonbarriertwao/results/appendix-1---history-of- } \\
\text { flooding-sources.pdf } \\
\text {-business and restaurants in norma operation until the flood hit }\end{array}$ \\
\hline WIKI (20200124) & $\begin{array}{l}\text { WIKI, Cyclone Xaver, https://en.wikipedia.org/wiki/Cyclone_Xaver accessed 24Jan2020 } \\
\text {-Sweden, Denmark higher water level than expected }\end{array}$ \\
\hline Wikipedia (20200429) & $\begin{array}{l}\text { Wikipedia, Stormen Sven, https://sv.wikipedia.org/wiki/Stormen_Sven\#cite_note-15, accessed 29Apr2020 } \\
\text {-Upgrade of SMHI weather warning from class-2 to class-3 during storm onThursday } 5 \text { Dec } 2013\end{array}$ \\
\hline
\end{tabular}


Table S7. Extended period bad weather (arranged by year and then alphabetically)

\begin{tabular}{|c|c|}
\hline Source & Full Reference and Notes \\
\hline BBC (20131206) & $\begin{array}{l}\text { BBC20131206, Deadly storm and tidal surge batter northern Europe, BBC } 6 \text { Dec2013 (report by Anna Holligan) } \\
\text {-flights cancelled at Schipol on 5Dec2013 but back to normal on 6Dec2013 }\end{array}$ \\
\hline BSH (2013) & $\begin{array}{l}\text { BSH, Die Nordseesturmfluten von 5. und 6.12.2013, 3pp, prepared by Stockmann,K. (title: Sturmflut 2011_bm1101; } \\
\text { author stamp: bm1101; document time stamp: 12/102013 12:5136PM) } \\
\text {-storm surge for } 4 \text { high tides from 5-7Dec2013 }\end{array}$ \\
\hline $\begin{array}{l}\text { Deutschlander et al. } \\
\text { (2013) }\end{array}$ & $\begin{array}{l}\text { Deutschlander T, K Frierich, S Haeseler, C Lefebvre, Orkantief XAVER ueber Nordeuropea von 5. bis 7. Dezember } \\
\text { 2013, Deutscher Wetterdienst DWD, Stand 30. Dezember 2013, 19pp. } \\
\text {-FIG7 [TIME SERIES] Time series of 10min gust for List/Sylt, Norderney, Rostock-Warnemuende shows high wind } \\
\text { duration 5-7Dec2013 } \\
\text {-FIG17. Time series of 10-min average wind speed for Brocken shows storm lasting >3 days starting on } 4 \text { Dec } 2013 \\
\text {-'Es wurde kurzzeitig winterlich mit Schnee bis in tiefere Lagen.' }\end{array}$ \\
\hline KNMI (2013) & $\begin{array}{l}\text { KNMI, News report. De Zware storm van } 5 \text { december, } 06 \text { Dec 2013, https://www.knmi.nl/over-het-knmi/nieuws/de- } \\
\text { zware-storm-van-5-december } \\
\text {-storm surge from long duration of northwest winds and long-fetch wind field }\end{array}$ \\
\hline SMHI (20131210) & $\begin{array}{l}\text { SMHI, Stormen Sven gav nya vattenstandsrekord i Oresund, https://www.smhi.se/nyhetsarkiv/stormen-sven-gav-nya- } \\
\text { vattenstandsrekord-i-oresund-1.34732, updated 20Mar2017; original datestamp 10Dec2013. } \\
\text {-short duration flood on west coast Sweden }\end{array}$ \\
\hline $\begin{array}{l}\text { Sylter Rundschau } \\
(20131209)\end{array}$ & $\begin{array}{l}\text { Sylter Rundschau, Folgen des Orkans. Xaver 'knabberte' Sylt massiv an, 09Dec2013 06:00 (from Friederike Reussner) } \\
\text { https://www.shz.de/lokales/sylter-rundschau/xaver-knabberte-sylt-massiv-an-id5098481.html } \\
\text {-storm damages Sylt dunes and coast over 2-day period }\end{array}$ \\
\hline $\begin{array}{l}\text { Unwetterzentrale } \\
\text { (201312) }\end{array}$ & $\begin{array}{l}\text { Unwetterzentrale, Orkantief XAVER - ein weiterer schwerer Wintersturm der letzten Jahrzehnte, Thomas Savert and } \\
\text { Stefan Laps, Dec. } 2013 \mathrm{http} / / / \mathrm{www} . \text { unwetterzentrale.de/uwz/928.html } \\
\text {-Xaver had exceptionally long duration } \\
\text {-Station Hiddensee-Dornbusch on Baltic Island had 41h of storm winds } \\
\text {-hurricane gusts for 32h from 5Dec2013 1610MEZ to 7Dec2013 0010MEZ } \\
\text {-storm gusts reported by Ellenbogen on north end of Sylt from 6Dec2013 07:10MEZ to 7Dec2013 03:10MEZ }\end{array}$ \\
\hline ABPmer (2014) & $\begin{array}{l}\text { ABPmer, Ensuring Flood Resilience. An overview of the 5/6 December 2013, Associated British Ports, Marine } \\
\text { Environmental Research, July, } 2014 \\
\text {-prolonged severe weather events Dec2013-Mar2014 } \\
\text {->50 closures of Thames Barrier during period Dec2013-Mar2014 }\end{array}$ \\
\hline $\begin{array}{l}\text { CH2MHill Halcrow } \\
(2014)\end{array}$ & $\begin{array}{l}\text { CH2MHill Halcrow, Cell } 1 \text { Regional Coastal Monitoring Programme, Wave Data Analysis Report 2: 2013-2014, Final } \\
\text { Report, March } 2014 \text { [document properties: author=Andy.Parson @ ch2m.com; datestamp; 04/04/2014] } \\
\text {-Newbiggin Ness wave buoy storm duration: } 20 \mathrm{~h} \\
\text {-Tyne Tees Wavenet buoy storm duration: } 38.5 \mathrm{~h} \\
\text {-Whitby waverider storm duration: } 26 \mathrm{~h}\end{array}$ \\
\hline Eden (201402) & $\begin{array}{l}\text { Eden, Phillip, Weather Log December 2013, Weather, Feb 2014, pp.i-iv } \\
-48 \text { hour cold snap }\end{array}$ \\
\hline Goennert et al (2014) & $\begin{array}{l}\text { Goennert G, O Mueller, M Schaper, K Sossidi, Die Sturmflut nach dem Tief Xaver von 5. bis 7. Dezember } 2013 . \\
\text { Berichte des Landesbetriebes Strassen, Bruecken und Gewaesser (LSBG), Freie und Hansestadt Hamburg, Nr. } \\
\text { 16/2014, 26pp. } \\
\text {-wind speed over the North Sea over } 20 \mathrm{~m} / \mathrm{s} \text { for } 19 \mathrm{~h} \text { in the direction } 270-300 \mathrm{deg} \text { as measured at Scharhoern }\end{array}$ \\
\hline $\begin{array}{l}\text { Knaack and Heyken } \\
(2014)\end{array}$ & $\begin{array}{l}\text { Knaack H and H Heyken, Xaver hatte sehr schwere Stumflut im Gepaeck, Jahresbericht 2013. Der Zukunft } \\
\text { verplichtet, NLWKN, Niedersaechsischer Landesbetrieb fuer Waserwirtschaft, Kuesten- und Naturschutz, pp.8-9, } \\
\text { document date stamp 24Apr2014. } \\
\text {-Xaver low pressure center moved slowly across North Sea and brought } 4 \text { storm surges }\end{array}$ \\
\hline Kristandt et at. (2014) & $\begin{array}{l}\text { Kristandt, J., B. Brecht, H. Frank, H. Knaack, Optimization of empirical storm surge forecast-modeling of high } \\
\text { resolution wind fields, Die Küste, } 81,301-348,2014 \\
\text {-surge flooding continued over } 4 \text { tide tide cycles }\end{array}$ \\
\hline Leiding et al (2014) & $\begin{array}{l}\text { Leiding T, B Tinz, G Rosenhagen, C Lefevre, S Haeseler, S Hagemann, I Bastigkeit, D Stein, P Schwenk, S Mueller, } \\
\text { O Outzen, K Herklotz, F Kinder, T Neumann, Meteorological and Oceanographic Conditions at the FINO } \\
\text { platforms during the severe storms Christian and Xaver, DEWI Magazin, No.44, p16-25, } 2014 \text {. } \\
\text {-Storm Xaver raged in German Bight for } 2.5 \text { days; several storm tides. } \\
\text {-FINO2: wind speed }>20 \mathrm{~m} / \mathrm{s} \text { for } 35 \mathrm{~h} \\
\text {-FINO3: wind speed }>25 \mathrm{~m} / \mathrm{s} \text { for }>24 \mathrm{~h}\end{array}$ \\
\hline $\begin{array}{l}\begin{array}{l}\text { Luecht } \\
(2014)\end{array} \\
\text { and }\end{array}$ & $\begin{array}{l}\text { Luecht, Fabian and Ove Peters, Bericht ueber die Sturmflut vom 05.-0.6.12.2013 an der Westkueste Schleswig- } \\
\text { Holsteins, Landesbetrieb fuer Kuestenschutz, Nationalpark und Meeresschutz Schleswig-Holstein, Husum } \\
\text { 26Feb2014, 19pp. [pdf document properties: title=Lfd; Author=Thorsten Nommensen; datestamp: 11Dec2018] } \\
\text {-storm flood levels on } 3 \text { consecutive tides } \\
\text {-storm flood duration was rank } 8 \text { in List tide gauge record going back to } 1900 \\
\text {-for worst storm tide water levels at NHN+2m for } 532 \text { min }\end{array}$ \\
\hline Pelt (2014) & $\begin{array}{l}\text { Pelt AS, BODIL's stormflod i de indre dansk farvande, Vejret, 138, 24-29, } 2014 \\
\text {-water levels above normal DVR90 from Dec 3, } 2013 \\
\text {-unusually long storm duration surge at Hornbaek } 11 \mathrm{~h} \\
\text {-long duration } 15 \mathrm{~h} \text { high water Kobenhavn }\end{array}$ \\
\hline RMS (2014) & $\begin{array}{l}\text { RMS, 2013-2014 Winter Storms in Europe. An Insurance and Catastrophe Modeling Perspective. RMS White Paper. } \\
\text { [PDF TIMESTAMP 11Mar2014] } \\
\text {-storm surge lasted 40h across } 3 \text { tidal cycles }\end{array}$ \\
\hline Spencer et al (2014) & $\begin{array}{l}\text { Spencer, T, S.M. Brooks, I. Moller, B.R. Evans, Where local matters: Impacts of a major North Sea storm surge, EOS, } \\
\text { 95, 269-270, 29July2014 } \\
\text {-2 month period of storms Dec2013-Jan2014 of which Xaver was the first }\end{array}$ \\
\hline
\end{tabular}




\begin{tabular}{|c|c|}
\hline Thorne (2014) & $\begin{array}{l}\text { Thorne, Colin, Geographies of UK flooding in 2013/4, The Geographical Journal, 180, 297-309, } 2014 . \\
\text {-listing of all 2013/2014 winter storms that caused flooding and coast damge }\end{array}$ \\
\hline Axer et al (2015) & $\begin{array}{l}\text { Axer T, T Bistry, M Klawa, M Mueller, M Suesser, Deutsche Ruck Sturm dokumentation } 2013 \text { Deutschland, 2013, } \\
\text { Deutsche Rueckversicherung Aktiengesellschaft, Hansaallee 177, } 40549 \text { Duesseldorf, www.deutscherueck.de } \\
\text { [pdf document information: author=filiz; date stamp=07Aug2015] } \\
\text {-slow path Xaver from S Sweden into Baltic caused long duration >1day NW wind over Nsea \& Baltic } \\
\text {-consequence was unusually high sea state }>8 \mathrm{~m} \text { in German Bight \& Polish Baltic coast (DWD2013c) }\end{array}$ \\
\hline $\begin{array}{l}\text { Haigh and Bradshaw } \\
(2015)\end{array}$ & $\begin{array}{l}\text { Haigh I and E Bradshaw, A century of UK coastal flooding, Planet Earth, Winter 2015, (pdf document properties: } \\
\text { 13Jan2016) } \\
\text {-cluster of winter storms during winter 2013-2014 }\end{array}$ \\
\hline $\begin{array}{l}\text { Kendon and McCarthy } \\
(2015)\end{array}$ & $\begin{array}{l}\text { Kendon M and M McCarthy, The UK's wet and stormy winter of 2013/2014, Weather, 70, 40-47, } 2015 \\
\text {-wet \& stormy conditions persisted through Dec } 2013 \text { to Feb } 2014\end{array}$ \\
\hline Matelski (2016) & $\begin{array}{l}\text { Matelski, Birgit, Erfahrungen aus der Sturmflut Xaver von 5. und 6.12.2013 und dem Weihnachshochwasser } 2014 \text { in } \\
\text { Shleswig-Holstein, IWASA } 2016 \text { Tagungsbeitrag, (46. IWASA, 7-8 Januar 2016; Internationales Wasserbau- } \\
\text { Symposium Aachen. [pdf document properties: autor=sonja; datestamp: 26Apr2016] } \\
\text {-three storm surge peaks } \\
\text {-long duration flood conditions: water on sand fo 9h; dunes subjected to wave attack } \\
\text {-on List auf Sylt water level }>700 \mathrm{~cm} \text { PN for } 532 \mathrm{~min}\end{array}$ \\
\hline Wadey et al (2015a) & $\begin{array}{l}\text { Wadey MP, ID Haigh, RJ Nichols, JM Brown, K Horsburgh, B Carroll, SL Gallop, T Mason, E Bradshaw, A } \\
\text { comparison of the } 31 \text { January-1 February } 1953 \text { and 5-6 December } 2013 \text { coastal flood events around the UK, UK } \\
\text { Frontiers in Marine Science, 2, 84, } 2015 \text { a. } \\
\text {-Storm Xaver propagation across the North Sea very fast in comparison with } 31 \text { Jan1953 storm }\end{array}$ \\
\hline Wadey et al (2015b) & $\begin{array}{l}\text { Wadey MP, JM Brown, ID Haigh, T Dolphin, P Wisse, Assessment and comparison of extreme sea levels and waves } \\
\text { during the 2013/2014 storm season in two UK coastal regions, Nat. Hazards Earth Syst. Sci. Discuss., 3, 2665- } \\
2708,2015 \text { b. } \\
\text {-surge continued over } 2 \text { high tides }\end{array}$ \\
\hline Brooks et al (2016) & $\begin{array}{l}\text { Brooks SM, T Spencer, A McIvor, I Moller, Reconstructing and understanding the impacts of storms and surges, } \\
\text { southern North Sea, Earth Surface Processes and Landforms, } 41,855-864,2016 \text {. } \\
\text {-14h period when surge residual }>1 \mathrm{~m} \text {; exceeded only by Storm Britta 01Nov2006 with } 18.75 \mathrm{~h} \text { time interval }\end{array}$ \\
\hline Sorensen et al (2016) & $\begin{array}{l}\text { Sorensen CS, NK Dronen, P Knudsen, J Jensen, P Sorensen, An extreme event as a games changer in coastal zone } \\
\text { management, Journal of Coastal Research, (Special Issue, No 75), 700-704, 2016. Proceedings of the 14th } \\
\text { International Coastal Symposium (Sydney, Australia) ed by A Vila-Concejo, E Bruce, DM Kennedy, RJ } \\
\text { McCarroll }\end{array}$ \\
\hline Staneva et al (2016) & $\begin{array}{l}\text { Staneva J, K Wahle, W Koch, A Behrens, L Fenoglio-Marc, EV Stanev, Coastal flooding: impact of waves on storm } \\
\text { surge during extremes - a case study for the German Bight, Nat. Hazards Earth Syst. Sci., 16, 2373-2389, 2016 } \\
\text {-'During } 4 \text { to } 7 \text { December, the storm depression Xaver moved from thesouth of Iceland over the Faroe Islands to } \\
\text { Norway and southern Sweden and further over the Baltic to Lithuania, Latvia, and Estonia' }\end{array}$ \\
\hline $\begin{array}{l}\text { Frohle and Dreier } \\
(2017)\end{array}$ & $\begin{array}{l}\text { Peter Frohle \& Norman Dreier, EarlyDike - Sensor - und risiko basiertes Fruhwarnsystem fuer Seedeiche, Teilprojekt: } \\
\text { Wellenmonitoring und Wellenbelastungssimulator (AP2), Hamburg, April } 2017 \\
\text {-FIG13. Time series graph of } 60 \mathrm{~m} \text { wind speed at FINO1; wind speed above } 5 \mathrm{~m} / \mathrm{s} \text { threshold 3-9Dec2013 } \\
\text {-FIG14. Time series graph of } 14 \mathrm{~m} \text { wind speed at Helgoland; wind speed above } 5 \mathrm{~m} / \mathrm{s} \text { threshold 3-9Dec2013 }\end{array}$ \\
\hline Wahle et al (2017) & $\begin{array}{l}\text { Wahle K, J Staneva, W Koch, L Fenoglio-Marc, HTM Ho-Hagemann, EV Stanev, An atmosphere-wave regional } \\
\text { coupled model: improving prediction of wave heights in the southern North Sea, Ocean Sci., 13, 289-301, } \\
\text { 2017.(doi:10.5194/os-13-289-2017). } \\
\text {-storm exceptional because of long duration of nearly two days }\end{array}$ \\
\hline $\begin{array}{l}\text { Environment Agency } \\
(2018)\end{array}$ & $\begin{array}{l}\text { Environment Agency, Thames Barrier Project Pack 2018, January, } 2018 \\
\text {-record number of Thames Barrier closures in Jan \& Feb } 2014\end{array}$ \\
\hline Kystdirektoratet (2018) & $\begin{array}{l}\text { Kystdirektoratet, Shoreface nourishment effects. An analysis of the } 2011 \text { nourishment performed at Skodbjerge. } \\
\text { Kystdirektoratet, Hojbovej } 1,7620 \text { Lemvig, Dec } 2018 \text { [pdf document properties: title=Shoreface nourishment } \\
\text { effects_Skodbjerge_20.12.2018, datestamp=20/12/2018] } \\
\text {-unusually long duration of high wave fields: } 36 \mathrm{~h} \text { with } \mathrm{Hm} 0>5 \mathrm{~m}\end{array}$ \\
\hline $\begin{array}{l}\text { Schenk and Mueller- } \\
\text { Navarra (2019) }\end{array}$ & $\begin{array}{l}\text { Schenk, L and S Mueller-Navarra. 3.4.4. Windstaustatistiken und Haufigkeit von Sturmfluten 2012-2015 } \\
\text { https://www.bsh.de/DE/PUBLIKATIONEN/Nordseezustand_Aktuell/_Anlagen/Downloads/3_4_4_Windstatistik } \\
\text { en.pdf?__blob=publicationFile\&v=2 [pdf document properties: author=Ludwig Schenk; datestamp=14Feb2019] } \\
\text {-3 consecutive storm surges at Hamburg }\end{array}$ \\
\hline Wikipedia (20191002) & $\begin{array}{l}\text { Wikipedia, Stormen Bodil, https://da.wikipedia.org/wiki/Stormen_Bodil\#cite_note-39 (accessed 02Oct2019) } \\
\text {-flooding in low-lying parts of Denmark for a week after the storm }\end{array}$ \\
\hline WIKI (20200124) & $\begin{array}{l}\text { WIKI, Cyclone Xaver, https://en.wikipedia.org/wiki/Cyclone_Xaver accessed 24Jan2020 } \\
\text {-3 storms to UK around Christmas: Cyclone Dirk, Anne, Christina with heavy rains and flooding } \\
\text {-parts of England had wettest January from record start more than } 100 \text { years previously } \\
\text {-Feb } 2014200 \text { properties evacuated in Somerset \& Devon } \\
\text {-Europe February: flooding in France, large snowfalls Austria and Slovenia; large waves Portugal and Spain }\end{array}$ \\
\hline
\end{tabular}

Table S8. Names of the storm (arranged by year and then alphabetically)

\begin{tabular}{|l|l|l|}
\hline Name & Full Reference and Notes \\
\hline Bodil & (Norway, & $\begin{array}{c}\text { Aftenposten, Varsler full storm I Sor-Norge, 5Dec2013 15:03 (correspondent Per Annar Holm) } \\
\text { https://www.aftenposten.no/norge/i/bKEj1/varsler-full-storm-i-soer-norge } \\
\text { Deutschlander T, K Frierich, S Haeseler, C Lefebvre, Orkantief XAVER ueber Nordeuropea von 5. bis 7. } \\
\text { Dezember 2013, Deutscher Wetterdienst DWD, Stand 30. Dezember 2013, 19pp. } \\
\text { Cipollini P, LJ West, HM Snaith, P Harwood, C Donlon, New altimetry products over shelf and coastal zone from } \\
\text { the eSurge processor, poster presentation, 2014 [document time stamp: 2014/10/22] } \\
\text { Hewson T, L Magnusson, O Breivik, F Prates, I Tsonevsky, HJW de Vries, Windstorms in northwest Europe in }\end{array}$ \\
\hline
\end{tabular}




\begin{tabular}{|c|c|}
\hline & 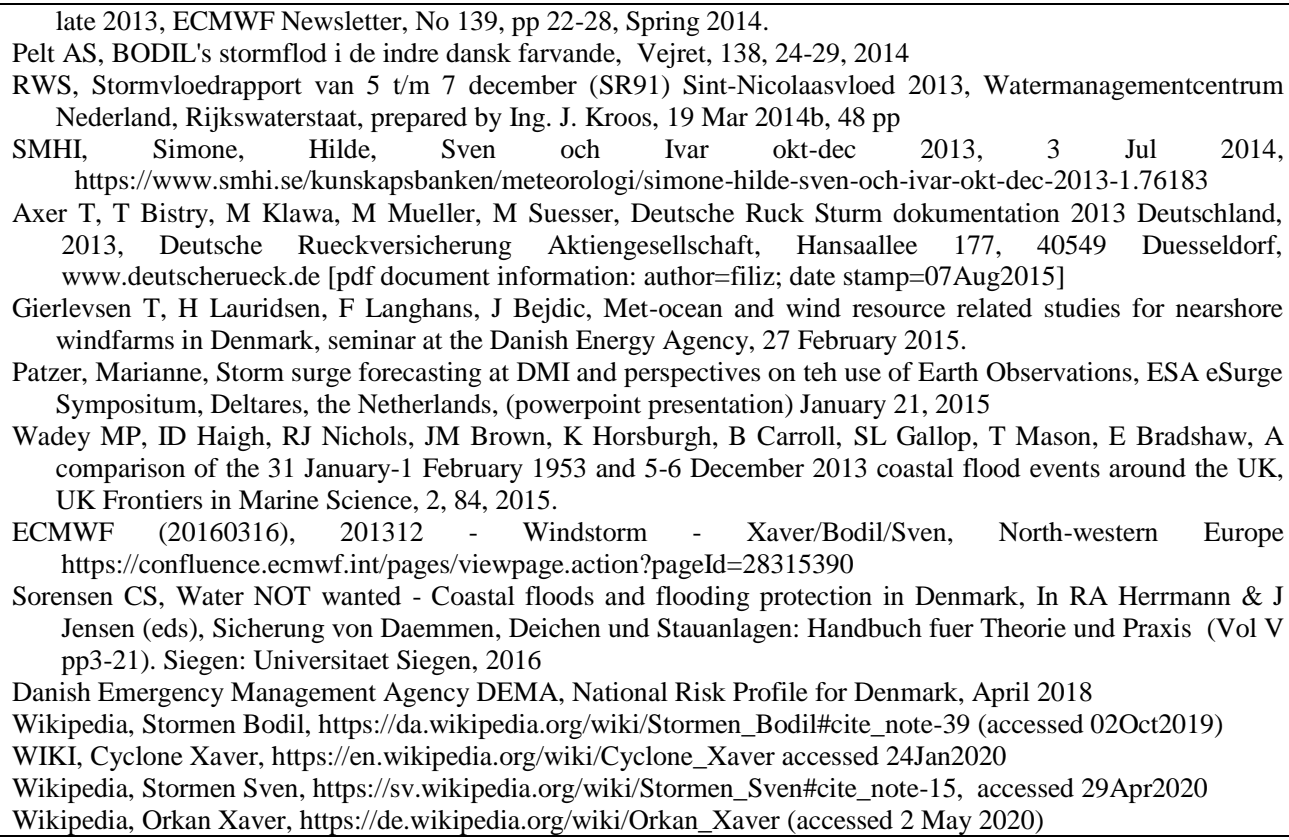 \\
\hline Cameron & WIKI, Cyclone Xaver, https://en.wikipedia.org/wiki/Cyclone_Xaver accessed 24Jan2020 \\
\hline Ksavery (Poland) & $\begin{array}{l}\text { Deutschlander T, K Frierich, S Haeseler, C Lefebvre, Orkantief XAVER ueber Nordeuropea von 5. bis } 7 . \\
\text { Dezember 2013, Deutscher Wetterdienst DWD, Stand 30. Dezember 2013, 19pp. } \\
\text { Wikipedia, Stormen Bodil, https://da.wikipedia.org/wiki/Stormen_Bodil\#cite_note-39 (accessed 02Oct2019) } \\
\text { Wikipedia, Orkan Xaver, https://de.wikipedia.org/wiki/Orkan_Xaver (accessed } 2 \text { May 2020) }\end{array}$ \\
\hline Nikolausflut & $\begin{array}{l}\text { NLWKN20151210, Sturmflutwarndienst der Betriebstelle Norden-Norderney, NLWKN } \\
\text { WIKI, Cyclone Xaver, https://en.wikipedia.org/wiki/Cyclone_Xaver accessed 24Jan2020 } \\
\text { Niedersachsischer Landesbetrieb fuer Wasserwirtschaft, Kuesten- und Naturschutz, Niedersachsen, document } \\
\text { date stamp 10Dec2015. }\end{array}$ \\
\hline Nikolaus-Orkan & Wikipedia, Orkan Xaver, https://de.wikipedia.org/wiki/Orkan_Xaver (accessed 2 May 2020) \\
\hline $\begin{array}{l}\text { Nordseesturmfluten } \\
\text { von 5. und 6.12.2013 }\end{array}$ & $\begin{array}{l}\text { BSH, Die Nordseesturmfluten von 5. und } 6.12 .2013 \text {, 3pp, prepared by Stockmann,K. (title: Sturmflut } \\
\text { 2011_bm1101; author stamp: bm1101; document time stamp: 12/102013 12:5136PM) }\end{array}$ \\
\hline St Nicholas & $\begin{array}{l}\text { Hewson T, L Magnusson, O Breivik, F Prates, I Tsonevsky, HJW de Vries, Windstorms in northwest Europe in } \\
\text { late 2013, ECMWF Newsletter, No 139, pp 22-28, Spring } 2014 .\end{array}$ \\
\hline Sint-Nicolaasvloed & $\begin{array}{l}\text { RWS, Stormvloedrapport van } 5 \mathrm{t} / \mathrm{m} 7 \text { december (SR91) Sint-Nicolaasvloed 2013, Watermanagementcentrum } \\
\text { Nederland, Rijkswaterstaat, prepared by Ing. J. Kroos, } 19 \text { Mar 2014b, } 48 \mathrm{pp}\end{array}$ \\
\hline Sinterklaasstorm & $\begin{array}{l}\text { Oceanografisch Meteorologisch Station, Stormverslag 05-06 december 2013, 26pp, } 2013 \text { [pdf document } \\
\text { properties: author=Myriam Sys; datestamp=15Dec2013] } \\
\text { RWS, Watermanagementcentrum Nederland, Stormvloedflits 2013-07 van } 5 \text { t/m } 7 \text { december 2013, } \\
\text { Rijkswaterstaat (document time stamp: 07Jan2014), 2014a } \\
\text { Nossent J, L Boeckx, E Taverniers, M Deschamps, T Verwaest, F Mostaert, Sinterklaasstorm } 6 \text { december } 2013 . \\
\text { Beschrijving van de hydrometrische gebeurtenissen, Versie 4.0. WL Rapporten, 00-119, Waterbouwkundig } \\
\text { Laboratorium, Antwerpen, Belgie. October 2014, WL2014R00_119_5 [document date stamp: 15Oct2014; } \\
\text { author= Deschamps, Maarten] } \\
\text { van Rooijen A, A Oost, Memo: Regionale advisering Ameland Noordwest, Deltares, 1209381-008-ZKS-0008, } \\
\text { 43pp, 18Dec2014 [PDF document properties: titl=Regional advisering: Ameland NW; author=Arnold van } \\
\text { Rooijen; keywords: 1209381-008-ZKS-0008; date stamp: 18/12/2014] } \\
\text { Axer T, T Bistry, M Klawa, M Mueller, M Suesser, Deutsche Ruck Sturm dokumentation } 2013 \text { Deutschland, } \\
\text { 2013, Deutsche Rueckversicherung Aktiengesellschaft, Hansaallee 177, 40549 Duesseldorf, } \\
\text { www.deutscherueck.de [pdf document information: author=filiz; date stamp=07Aug2015] } \\
\text { Carrion Aretxabala, BI, Morphological impact of the Sinterklaas storm at Het Zwin. Numerical modelling with } \\
\text { Xbeach, M.Sc. Civil Engineering, Delft University of Technology, 2015. } \\
\text { Dan, Sebastian, Anne-Lise Montreuil, Rosalia Delgado, Tomas van Oyen, Large storm impact on a beach under } \\
\text { sand nourishments, The Proceedings of the Coastal Sediments 2015, edited by Ping Wang, Julie D Rosati, } \\
\text { and Jun Cheng, Coastal Sediments 2015, San Diego, USA, 11-15May2015 } \\
\text { Nederhoff K, E Elias, T Vermaas, Erosie op Ameland Noordwest. Modelstudie: simulaties met Delft3D en } \\
\text { XBeach, Deltares, 117pp, July, 2016 } \\
\text { Li, H., The Ameland Inlet during the Sinterklaas Storm: the role of flooding of watersheds, 2DH model study in } \\
\text { Delft3D-FLOW, M.Sc. Thesis, Utrecht University, 01May2018 } \\
\text { Wikipedia, Stormen Bodil, https://da.wikipedia.org/wiki/Stormen_Bodil\#cite_note-39 (accessed 02Oct2019) } \\
\text { WIKI, Cyclone Xaver, https://en.wikipedia.org/wiki/Cyclone_Xaver accessed 24Jan2020 } \\
\text { Wikipedia, Orkan Xaver, https://de.wikipedia.org/wiki/Orkan_Xaver (accessed 2 May 2020) }\end{array}$ \\
\hline $\begin{array}{l}\text { Storm surge of } 5 \\
2013\end{array}$ & $\begin{array}{l}\text { Spencer, T, S.M. Brooks, I. Moller, B.R. Evans, Where local matters: Impacts of a major North Sea storm surge, } \\
\text { EOS, 95, 269-270, 29July2014 }\end{array}$ \\
\hline Sven (Sweden) & $\begin{array}{l}\text { Aftenposten, Varsler full storm I Sor-Norge, 5Dec2013 15:03 (correspondent Per Annar Holm) } \\
\text { https://www.aftenposten.no/norge/i/bKEjl/varsler-full-storm-i-soer-norge } \\
\text { Deutschlander T, K Frierich, S Haeseler, C Lefebvre, Orkantief XAVER ueber Nordeuropea von 5. bis } 7 . \\
\text { Dezember 2013, Deutscher Wetterdienst DWD, Stand 30. Dezember 2013, 19pp. } \\
\text { Expressen, Flera doda i Sverige efter stormen Sven, 06Dec2013,17:34CET? }\end{array}$ \\
\hline
\end{tabular}


GP, Fortsatt risk for halka, 6Dec2013 (correspondent: D Henriksson, K Vikingsson, P Sydvik, TA Akerblom) http://www.gp.se/nyheter/goteborg/1.2201325-fortsatt-risk-for-halka (accessed 1May2020)

The Local, Sven's strong winds sweep southern Sweden. 05Dec2013 15:57CET https://www.thelocal.se/20131205/storm-sven-sweeps-into-southern-sweden

SMHI, Stormen Sven gav nya vattenstandsrekord i Oresund, https://www.smhi.se/nyhetsarkiv/stormen-sven-gavnya-vattenstandsrekord-i-oresund-1.34732, updated 20Mar2017; original datestamp 10Dec2013.

Hewson T, L Magnusson, O Breivik, F Prates, I Tsonevsky, HJW de Vries, Windstorms in northwest Europe in late 2013, ECMWF Newsletter, No 139, pp 22-28, Spring 2014.

RWS, Stormvloedrapport van $5 \mathrm{t} / \mathrm{m} 7$ december (SR91) Sint-Nicolaasvloed 2013, Watermanagementcentrum Nederland, Rijkswaterstaat, prepared by Ing. J. Kroos, 19 Mar 2014b, 48 pp

SMHI, Simone, Hilde, Sven och Ivar okt-dec 2013, 3 Jul 2014, https://www.smhi.se/kunskapsbanken/meteorologi/simone-hilde-sven-och-ivar-okt-dec-2013-1.76183

Axer T, T Bistry, M Klawa, M Mueller, M Suesser, Deutsche Ruck Sturm dokumentation 2013 Deutschland, 2013, Deutsche Rueckversicherung Aktiengesellschaft, Hansaallee 177, 40549 Duesseldorf, www.deutscherueck.de [pdf document information: author=filiz; date stamp=07Aug2015]

Wadey MP, ID Haigh, RJ Nichols, JM Brown, K Horsburgh, B Carroll, SL Gallop, T Mason, E Bradshaw, A comparison of the 31 January-1 February 1953 and 5-6 December 2013 coastal flood events around the UK, UK Frontiers in Marine Science, 2, 84, 2015.

ECMWF (20160316), 201312 - Windstorm - Xaver/Bodil/Sven, North-western Europe https://confluence.ecmwf.int/pages/viewpage.action?pageId=28315390

Wikipedia, Stormen Bodil, https://da.wikipedia.org/wiki/Stormen_Bodil\#cite_note-39 (accessed 02Oct2019)

WIKI, Cyclone Xaver, https://en.wikipedia.org/wiki/Cyclone_Xaver accessed 24Jan2020

Wikipedia, Stormen Sven, https://sv.wikipedia.org/wiki/Stormen_Sven\#cite_note-15, accessed 29Apr2020

Wikipedia, Orkan Xaver, https://de.wikipedia.org/wiki/Orkan_Xaver (accessed 2 May 2020)

Aftenposten, Varsler full storm I Sor-Norge, 5Dec2013 15:03 (correspondent Per Annar Holm) https://www.aftenposten.no/norge/i/bKEjl/varsler-full-storm-i-soer-norge

Deutschlander T, K Frierich, S Haeseler, C Lefebvre, Orkantief XAVER ueber Nordeuropea von 5. bis 7. Dezember 2013, Deutscher Wetterdienst DWD, Stand 30. Dezember 2013, 19pp.

Kunz M, B Muehr, K Schroeter, T Bessel, S Moehrle, T Muenzberg, S Brink, H-M Schmidt, Winterstorm Xaver Report. 06Dec2013 - Report No.1, Situation Report - 19:00CET, CEDIM Forensic Disaster Analysis Group (FDA), Center for Disaster Management and Risk Reduction Technology.

Sueddeutsche Zeitung, Xaver holte sich Land; Sturmschaden auf Sylt, 15Dec201315:36 https://www.sueddeutsche.de/panorama/sturmschaeden-auf-sylt-xaver-holte-sich-land-1.1844100

Unwetterzentrale, Orkantief XAVER - ein weiterer schwerer Wintersturm der letzten Jahrzehnte, Thomas Savert and Stefan Laps, Dec. 2013 http://www.unwetterzentrale.de/uwz/928.html

AON Benfield, Impact forecasting. December 2013 Global Catastrophe Recap, 2014. [document properties: abrandt; date stamp: 10Jan2014]

Blasi C, S Mai, J Wilhelmi, T Zenz, U Barjenbruch, A powerful method of measuring sea wave spectra and their direction, ICHE 2014, Hamburg - Lehfeldt and Kopmann (eds), Bundesanstalt fuer Wasserbau, 2014. ISBN 978-3-939230-32-8

Cipollini P, LJ West, HM Snaith, P Harwood, C Donlon, New altimetry products over shelf and coastal zone from the eSurge processor, poster presentation, 2014 [document time stamp: 2014/10/22]

Dunbar I, N Phipps, M Szonyi, Risk Nexus. After the storm: how the UK's flood defences performed during the surge following Xaver, Flood resilience review 09.14, Zurich Insurance Company Ltd., Mythenquai 2, 8002, Zurich, Switzerland [document properties: date 28Aug2014; author= Zurich]

Hewson T, L Magnusson, O Breivik, F Prates, I Tsonevsky, HJW de Vries, Windstorms in northwest Europe in late 2013, ECMWF Newsletter, No 139, pp 22-28, Spring 2014. (from Berlin's Free University)

Knaack H and H Heyken, Xaver hatte sehr schwere Stumflut im Gepaeck, Jahresbericht 2013. Der Zukunft verplichtet, NLWKN, Niedersaechsischer Landesbetrieb fuer Waserwirtschaft, Kuesten- und Naturschutz, pp.8-9, document date stamp 24Apr2014.

Kristandt, J., B. Brecht, H. Frank, H. Knaack, Optimization of empirical storm surge forecast-modeling of high resolution wind fields, Die Küste, 81, 301-348, 2014

Leiding T, B Tinz, G Rosenhagen, C Lefevre, S Haeseler, S Hagemann, I Bastigkeit, D Stein, P Schwenk, S Mueller, O Outzen, K Herklotz, F Kinder, T Neumann, Meteorological and Oceanographic Conditions at the FINO platforms during the severe storms Christian and Xaver, DEWI Magazin, No.44, p16-25, 2014.

Mai, S., Sea state at the research platform FINO1 during the winter storm 'Xaver', BFG, Bundesamt fuer Gewasserkunde, document properties: author=Mai, date $=16$ May2014

RMS, 2013-2014 Winter Storms in Europe. An Insurance and Catastrophe Modeling Perspective. RMS White Paper. [PDF TIMESTAMP 11Mar2014]

RWS, Stormvloedrapport van $5 \mathrm{t} / \mathrm{m} 7$ december (SR91) Sint-Nicolaasvloed 2013, Watermanagementcentrum Nederland, Rijkswaterstaat, prepared by Ing. J. Kroos, 19 Mar 2014b, 48 pp

Axer T, T Bistry, M Klawa, M Mueller, M Suesser, Deutsche Ruck Sturm dokumentation 2013 Deutschland, 2013, Deutsche Rueckversicherung Aktiengesellschaft, Hansaallee 177, 40549 Duesseldorf, www.deutscherueck.de [pdf document information: author=filiz; date stamp=07Aug2015]

Dan, Sebastian, Anne-Lise Montreuil, Rosalia Delgado, Tomas van Oyen, Large storm impact on a beach under sand nourishments, The Proceedings of the Coastal Sediments 2015, edited by Ping Wang, Julie D Rosati, and Jun Cheng, Coastal Sediments 2015, San Diego, USA, 11-15May2015

Fenoglio-Marc L, R Scharroo, A Annuziato, L Mendoza, M Becker, J Lillibridge, Cyclone Xaver seen by geodetic observations, Geophys Research Letters, 42, 9925-9932, 2015

Jensen, J., A. Arns, T. Wahl, Yet another 100yr storm surge event: the role of individual storm surges on design water levels, Journal of Marine Science and Technology, 23, 882-887, 2015.

NLWKN20151210, Sturmflutwarndienst der Betriebstelle Norden-Norderney, NLWKN Niedersachsischer Landesbetrieb fuer Wasserwirtschaft, Kuesten- und Naturschutz, Niedersachsen, document date stamp 10Dec2015.

Patzer, Marianne, Storm surge forecasting at DMI and perspectives on teh use of Earth Observations, ESA eSurge 


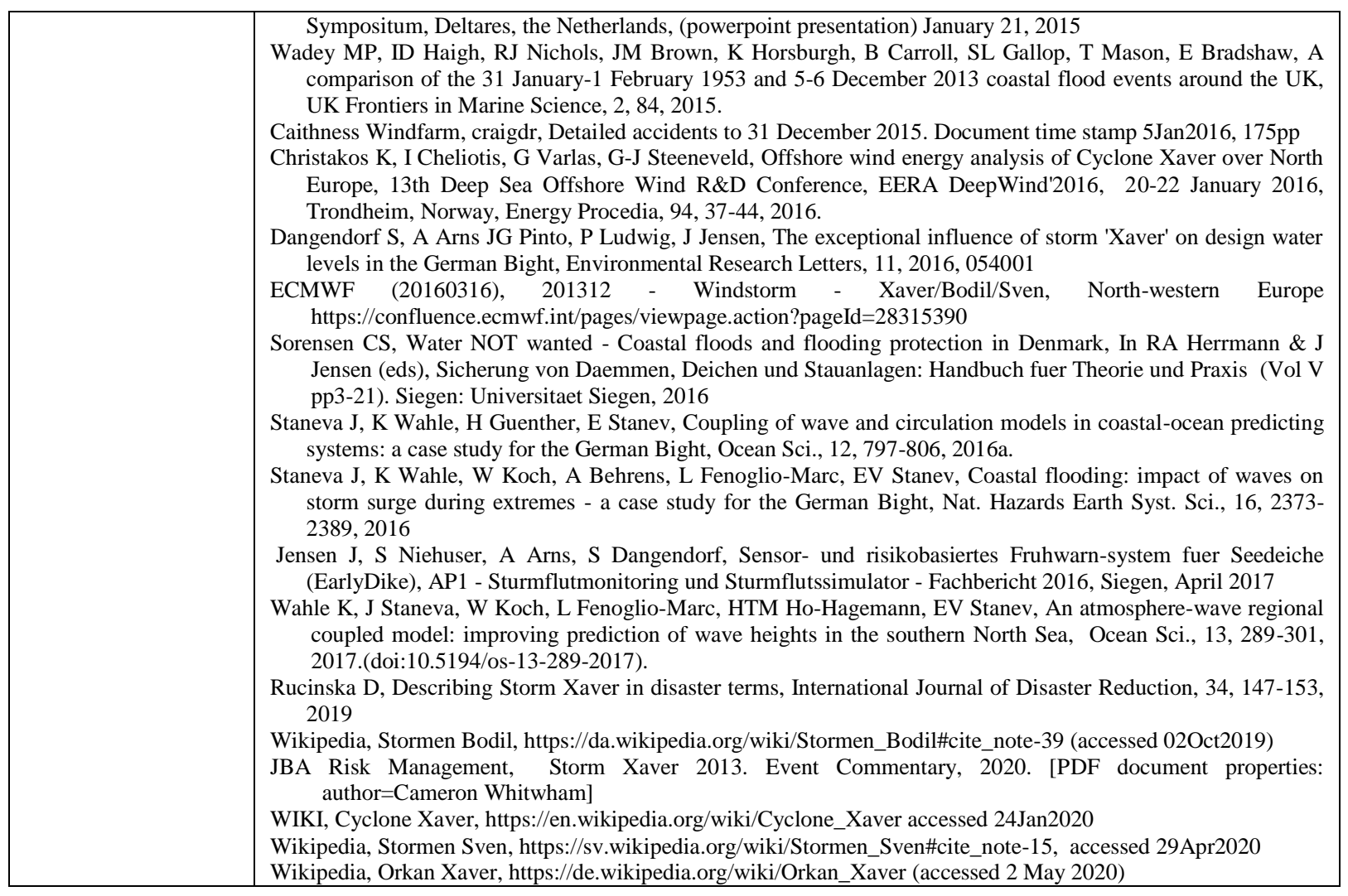

Table S9. Satellite pictures and weather maps (arranged by year and then alphabetically)

\begin{tabular}{|c|c|}
\hline Source & Full Reference and Notes \\
\hline $\begin{array}{l}\text { Deutschlander et al } \\
\text { (2013) }\end{array}$ & $\begin{array}{l}\text { Deutschlander T, K Frierich, S Haeseler, C Lefebvre, Orkantief XAVER ueber Nordeuropea von 5. bis } 7 . \\
\text { Dezember 2013, Deutscher Wetterdienst DWD, Stand 30. Dezember 2013, 19pp. } \\
\text {-ABB2a.[MAP] Bodenanalyse Nordatlantik/Europa von 5.Dezember 2013, 00UTC } \\
\text {-ABB2b.[MAP] Bodenanalyse Nordatlantik/Europa von 5.Dezember 2013, 18UTC } \\
\text {-ABB2c.[MAP] Bodenanalyse Nordatlantik/Europa von 6.Dezember 2013, 00UTC } \\
\text {-ABB3. [MAP] Satellitenbild mit Luftdruck (in hPa) und Wind (Richtung und Geschwindigkeit) } \\
\text { am 6.Dezember } 2013 \text { 00UTC, ueber dem nordlichen Europea (Quelle: DWD) } \\
\text { Photo of cloud field with superimposed surface air pressure \& wind arrows } \\
\text {-ABB4. [MAP] Satellitenbild von Europa con 6.Dezember 2013, 06UTC [Quelle: DWD] } \\
\text { Photo of cloud field MET10 IR100-STC }\end{array}$ \\
\hline Mills (20131206) & $\begin{array}{l}\text { Mills, Ian, Remko Scharoo, Luciana Fenoglio, Xaver affected much of northern Europe on } 5 \text { and } 6 \text { December and } \\
\text { caused worst storm } \quad \text { surge for decades in the North } \\
\text { https://www.eumetsat.int/website/home/News/DAT_2087062.html (last accessed: 19Nov2019, 28Jun2020) } \\
\text {-FIG1. Met-10 and Jason, 05Dec2013 1800UTC; channel IR 10.8um; source EUMETrain } \\
\text {-FIG2. Met-10, 05Dec2013, 1800UTC; Airmass with the MSLP (pressure lines) }\end{array}$ \\
\hline $\begin{array}{l}\text { Unwetterzentrale } \\
\text { (201312) }\end{array}$ & $\begin{array}{l}\text { Unwetterzentrale, Orkantief XAVER - ein weiterer schwerer Wintersturm der letzten Jahrzehnte, Thomas Savert } \\
\text { and Stefan Laps, Dec. } 2013 \text { http://www.unwetterzentrale.de/uwz/928.html } \\
\text {-FIG1. [MAP] UKMO weather maps E Atlantic \& Europe 04Dec2013 0600UTC to 07Dec2013 1200UTC } \\
\text {-FIG2. [MAP] IR? cloud image Europe and eastern Atlantic for 4Dec2013 21MEZ as base image for storm } \\
\text { trajectory } \\
\text {-FIG3. [MAP] IR satellite picture 05Dec2013 0800MEZ shows Xaver with it center over N North Sea } \\
\text {-FIG15. [MAP] visible satellite image with lightning band in front of the Netherlands coast }\end{array}$ \\
\hline Eden (201402) & $\begin{array}{l}\text { Eden, Phillip, Weather Log December 2013, Weather, Feb 2014, pp.i-iv } \\
\text {-series of daily weather maps of surface pressure for Europe }\end{array}$ \\
\hline Eriksen (2014) & $\begin{array}{l}\text { Eriksen J, Rekordvandstande i Isefjorden og Roskilde Fjord, Vejret, 138, 2 40-48, } 2014 \\
\text {-FIG1. Measured winds and surface air pressure torsdag kl 18utc. } \\
\text { Bodil is marked with a red L. } \\
\text {-FIG3. Measured winds and surface air pressure Friday kl 00utc. } \\
\text { Bodil is marked with a red L. Central P 962.5hPa } \\
\text {-FIG4. Measured winds and surface air pressure Friday kl 06utc. } \\
\text { Bodil is marked with a red L. Central P 962.6hPa } \\
\text {-FIG5. Measured winds and surface air pressure Friday kl 18utc. } \\
\text { Bodil is marked with a red L. Central P 970.7hPa } \\
\text {-FIG6. Measured winds and surface air pressure Saturday kl 00utc. } \\
\text { Bodil is marked with a red L } \\
\text {-FIG7. Measured winds and surface air pressure Saturday kl 06utc. } \\
\text { Bodil is marked with a red L }\end{array}$ \\
\hline Goennert et al. (2014) & Goennert G, O Mueller, M Schaper, K Sossidi, Die Sturmflut nach dem Tief Xaver von 5. bis 7. Dezember 2013. \\
\hline
\end{tabular}




\begin{tabular}{|c|c|}
\hline & $\begin{array}{l}\text { Berichte des Landesbetriebes Strassen, Bruecken und Gewaesser (LSBG), Freie und Hansestadt Hamburg, } \\
\text { Nr. 16/2014, 26pp. } \\
\text {-FIG3. [MAP] Satellite cloud image with air pressure and wind direction on 6Dec2013 0000UTC }\end{array}$ \\
\hline Matelski et al. (2014) & $\begin{array}{l}\text { Matelski, Birgit, Frerk Jensen, Peter Voss, Jorg Peters, Maria Blumel, Auswertung zur Hydrologie des Sturmtiefs } \\
\text { Xaver 05.12.2013 bis 06.12.2013, Statusbericht vom 01.10.2014, Landesbetrieb fuer Kuestenschutz, } \\
\text { Nationalpark und Meeresschutz, Schleswig-Holstein, Husum, 01.10.2014, 25pp. } \\
\text {-FIG3. Satellite image with air pressure (hPa) and wind (direction and speed) on } \\
\text { 6Dec2013 00UTC (source: DWD) }\end{array}$ \\
\hline Nossent et al (2014) & $\begin{array}{l}\text { Nossent J, L Boeckx, E Taverniers, M Deschamps, T Verwaest, F Mostaert, Sinterklaasstorm } 6 \text { december } 2013 . \\
\text { Beschrijving van de hydrometrische gebeurtenissen, Versie 4.0. WL Rapporten, 00-119, Waterbouwkundig } \\
\text { Laboratorium, Antwerpen, Belgie. October 2014, WL2014R00_119_5 [document date stamp: 15Oct2014; } \\
\text { author= Deschamps, Maarten] } \\
\text {-FIG2. [MAP] weather map 05Dec2015 0000UTC, 0600UTC, 1200UTC (KNMI2013b, Kroos 2013) } \\
\text {-FIG3. [MAP] Model-derived wind fields at 05Dec2013 0700/1300/1900CET } \\
\text {-FIG4. [MAP] Model-derived wind field at 05Dec2013 1900CET; max in German Bight }\end{array}$ \\
\hline RWS (2014a) & $\begin{array}{l}\begin{array}{l}\text { RWS, Watermanagementcentrum Nederland, } \\
\text { Rijkswaterstaat (document time stamp: 07Jan2014), 2014a }\end{array} \\
\text {-FIG. Surface pressure weather map of Europe and eastern North Atlantic 05Dec2013 } \\
\quad \text { cold fronts marked } \\
\text {-FIG. Visible satellite image 06Dec2013 1200??? From EUMETSAT }\end{array}$ \\
\hline RWS (2014b) & $\begin{array}{l}\text { RWS, Stormvloedrapport van } 5 \mathrm{t} / \mathrm{m} 7 \text { december (SR91) Sint-Nicolaasvloed 2013, Watermanagementcentrum } \\
\text { Nederland, Rijkswaterstaat, prepared by Ing. J. Kroos, } 19 \text { Mar 2014b, } 48 \mathrm{pp} \\
\text {-FIG_p0: Visible satellite image of storm over Europe } \\
\text {-FIG2. [MAP] Weather map from 05Dec2013 0100MEZ } \\
\text {-FIG3. [MAP] Weather map from 05Dec2013 0700MEZ } \\
\text {-FIG4. [MAP] Weather map from 05Dec2013 1300MEZ } \\
\text {-FIG5. [MAP] Thunderstorm radar picture 05Dec2013 1510MEZ }\end{array}$ \\
\hline$(2015)$ & $\begin{array}{l}\text { Axer T, T Bistry, M Klawa, M Mueller, M Suesser, Deutsche Ruck Sturm dokumentation } 2013 \text { Deutschland, } \\
\text { 2013, Deutsche Rueckversicherung Aktiengesellschaft, Hansaallee 177, 40549 } \\
\text { www.deutscherueck.de [pdf document information: author=filiz; date stamp=07Aug2015] } \\
\text {-FIG_p48. [MAP] Hurricane Xaver 05Dec2013 0100MEZ } \\
\text {-FIG_p48. [MAP] Hurricane Xaver 06Dec2013 0100MEZ } \\
\end{array}$ \\
\hline $\begin{array}{l}\text { Kendon and McCarthy } \\
\text { (2015) }\end{array}$ & $\begin{array}{l}\text { Kendon M and M McCarthy, The UK's wet and stormy winter of 2013/2014, Weather, 70, 40-47, } 2015 \\
\text { FIG1a. UK Met Office analysis map for 1200UTC 05Dec2019 showing surface pressure and fronts }\end{array}$ \\
\hline Sibley et al. (2015) & $\begin{array}{l}\text { Sibley A, D Cox, H Titley, Coastal flooding in England and Wales from Atlantic and North Sea storms during the } \\
\text { 2013/2014 winter, Weather, 70, 62-70, 2015 } \\
\text {-FIG1. Surface pressure with fronts marked: 04Dec2013 1200UTC, 05Dec2013 0000UTC; 05Dec2013 1200UTC }\end{array}$ \\
\hline Spencer et al (2015) & $\begin{array}{l}\text { Spencer T, SM Brooks, BR Evans, JA Tempest, I Moeller, Southern North Sea storm surge event of Dec.5, 2013: } \\
\text { Water levels, waves, and coastal impacts, Earth Science Reviews, 146, 120-145, } 2015 \\
\text {-FIG4.(map) Met analysis chart at 1200UTC } 5 \text { Dec } 2013\end{array}$ \\
\hline Christakos et al (2016) & $\begin{array}{l}\text { Christakos K, I Cheliotis, G Varlas, G-J Steeneveld, Offshore wind energy analysis of Cyclone Xaver over North } \\
\text { Europe, 13th Deep Sea Offshore Wind R\&D Conference, EERA DeepWind'2016, 20-22 January 2016, } \\
\text { Trondheim, Norway, Energy Procedia, 94, 37-44, 2016. } \\
\text { FIG1. (a) Surface pressure analysis map on 5Dec2013 1200UTC from UKMO, Xaver P=967hPa } \\
\text { (b) Regional SatRep over North Sea 5Dec2013 0900UTC showing fronts \& thorms }\end{array}$ \\
\hline $\begin{array}{l}\text { Dangendorf et al } \\
(2016)\end{array}$ & $\begin{array}{l}\text { Dangendorf S, A Arns JG Pinto, P Ludwig, J Jensen, The exceptional influence of storm 'Xaver' on design water } \\
\text { levels in the German Bight, Environmental Research Letters, 11, 2016, 054001 } \\
\text {-FIG3. German weather maps for 05Dec2013 1200GMT and 06Dec2013 1200UTC }\end{array}$ \\
\hline ECMWF (20160316) & $\begin{array}{lllll}\text { ECMWF } \quad \text { (20160316), 201312 }-\quad \text { Windstorm }-\quad \text { Xaver/Bodil/Sven, } & \text { North-western } & \text { Europe } \\
\text { https://confluence.ecmwf.int/pages/viewpage.action?pageId=28315390 } & & \\
\text {-FIG2. [MAP] Satellite images (Meteosat from yr.no) from 5Dec 0000-2100UTC } & & \\
\text { every 3h. } & & \\
\end{array}$ \\
\hline$(2017)$ & $\begin{array}{l}\text { Cheliotis, I, G. Varlas, K. Christakos, The impact of cylone Xaver on hydropower potential in Norway, } \\
\text { conference paper, September } 2016 \text { In: T Karaostas, A Bais, PT Nastos (ed), Perspectives on Atmospheric } \\
\text { Sciences, Springer Atmospheric Sciences, Springer, Cham, 2017, https://doi.org/10.1007/978-3-319-35095-0_25 } \\
\text { FIG1. [MAP] Surface pressure analysis map hPa on 5Dec2013 at 12:00UTC from UK Met office sfc analysis } \\
\text { archive }\end{array}$ \\
\hline Ribeiro et al (2017) & $\begin{array}{l}\text { Ribeiro R, R Rudge, D Rucinska, Analysis of physical factors of the windstorm Xaver in Poland: post-hazard } \\
\text { review, Weather, 72, 2017, pp.378-382 } \\
\text {-FIG1. [MAP] Pressure lines in Poland on 5Dec2013 1800UTC (adapted from Eumetsat 2013) } \\
\text {-FIG2. [MAP] Clouds of storm Xaver in Europe on 5Dec2013 at 2330UTC }\end{array}$ \\
\hline Fery et al (2018) & $\begin{array}{l}\text { Fery, Natascha, Birger Tinz, Lydia Gates, Reproduction of storms over the North Sea and the Baltic with the } \\
\text { regional analysis COSMO-REA6 ISPR 2018, 17-19July2018, Bonn [pdf document properties: } \\
\text { datestamp }=16 / 07 / 2018 \text { : } \\
\text {-satellite cloud image of Storm Xaver }\end{array}$ \\
\hline Wikipedia (20191002) & $\begin{array}{l}\text { Wikipedia, Stormen Bodil, https://da.wikipedia.org/wiki/Stormen_Bodil\#cite_note-39 (accessed 02Oct2019) } \\
\text {-FIG2. [MAP] Meteorological development of storm }\end{array}$ \\
\hline WIKI (20200124) & $\begin{array}{l}\text { WIKI, Cyclone Xaver, https://en.wikipedia.org/wiki/Cyclone_Xaver accessed 24Jan2020 } \\
\text { FIG [SATELLITE] Xaver making landfall over Norway and Denmark on 05Dec2013 } \\
\text { FIG [MAP] weather map loop eastern Atlantic \& Europe } \\
\text { FIG [MAP] surface pressure chart 12:00 UTC 05Dec2013 }\end{array}$ \\
\hline Wikipedia (20200502) & $\begin{array}{l}\text { Wikipedia, Orkan Xaver, https://de.wikipedia.org/wiki/Orkan_Xaver (accessed } 2 \text { May 2020) } \\
\text { FIG1. [MAP] Surface pressure chart of NOAA on 5Dec2013 12:00UTC } \\
\text { FIG2. [MAP] Weather map of Orkan Xaver 5Dec2013 00:00UTC-9Dec 12:00UTC }\end{array}$ \\
\hline
\end{tabular}


Table S10. Satellite altimeter strip maps (arranged by year and then alphabetically)

\begin{tabular}{|c|c|}
\hline Source & Full Reference and Notes \\
\hline Mills et al (20131206) & $\begin{array}{l}\text { Mills, Ian, Remko Scharoo, Luciana Fenoglio, Xaver affected much of northern Europe on } 5 \text { and } 6 \text { December and } \\
\text { caused worst storm furge for decades in the North } \\
\text { https://www.eumetsat.int/website/home/News/DAT_2087062.html (last accessed: 19Nov2019, 28Jun2020) } \\
\text {-Jason strip map } \\
\text {-FIG3. SARAL/AltiKa compared with tidal gauges: wind speed, wave height, sea level anomaly. 06Dec2013 } \\
\text { 0447UTC } \\
\text { Helgoland Suedhaven, Luchtturm Alte Weser, Norderney Riffgat, Borkum Fischerbalje } \\
\text { (a) time series water level Helgoland Suedhaven, Leuchtturm Alte Weser } \\
\text { (b) time series wind speed Norderney Riffgat and Borkum Fischerbalje }\end{array}$ \\
\hline Cipollini et al (2015) & $\begin{array}{l}\text { Cipollini P, LJ West, HM Snaith, P Harwood, C Donlon, New altimetry products over shelf and coastal zone from } \\
\text { the eSurge processor, poster presentation, } 2014 \text { [document time stamp: 2014/10/22] } \\
\text {-Cryosat-2 sea level strip map along axis of Kattegat and Danish Belt } \\
\text {-surge increases from } 0.1 \mathrm{~m} \text { at Swedish coast to } 1.2 \mathrm{~m} \text { at Danish islands } \\
\text {-additional } 0.9-1.0 \mathrm{~m} \text { increase within } 10 \mathrm{~km} \text { of coast }\end{array}$ \\
\hline Cipollini et al (2015) & $\begin{array}{l}\text { Cipollini P, J Benveniste, H Bonekamp, L Miller, M Picot, P Ted Strub, D Vandemark, S Vignudelli, Recovering } \\
\text { more and better data from altimetry in the coastal zone: a community effort, Pilot ARCOM workshop Lisbon, } \\
\text { 1/2 Sept } 2015 \\
\text {-FIG. [MAP] } 3 \text { panels of E North Sea at different times during Storm Xaver surge } \\
\text {-FIG. [PROFILE] latitude profiles from SARAL of U10, SWH, SLA during storm Xaver } \\
\text {-FIG. [MAP] DMI model of storm Xaver sea level Friday 06Dec2013 23:05 (Madsen etal) } \\
\text {-FIG. [PROFILE] Cryosat-2 profile of relative total water level along Kattegat 23:05GMT 06Dec2013 } \\
\text {-FIG. [MAP] map of Cryosat-2 total water level along Kattegat. }\end{array}$ \\
\hline $\begin{array}{l}\text { Fenoglio-Marc et al } \\
(2015)\end{array}$ & $\begin{array}{l}\text { Fenoglio-Marc L, R Scharroo, A Annuziato, L Mendoza, M Becker, J Lillibridge, Cyclone Xaver seen by geodetic } \\
\text { observations, Geophys Research Letters, 42, 9925-9932, } 2015 \\
\text {-ascending pass } 629 \text { of SARAL mission crosses North Sea at 04:47UTC 6Dec2013 } \\
\text {-FIG1. Study area: (right) German Bight and (left) enlarged to North Sea with SARAL/ALtika altimeter ground } \\
\text { track } \\
\text { and in situ stations with GPS (circle), sea level (triangle), wave height (inverted triangle) \& wind speed } \\
\text { (square) data } \\
\text {-FIG3. (left) Surge at the time of the overflight predicted by BSHmod and HyFlux2 simulations with various } \\
\text { wind forcing and derived from in situ data. Profiles at the SARAL/Altika overflight of wind speed, } \\
\text { significant wave height, and surge height derived from altimeter observations and from models. } \\
\text { Gray lines correspond to observations before and after Cyclone Xaver. }\end{array}$ \\
\hline Patzer (2015) & $\begin{array}{l}\text { Patzer, Marianne, Storm surge forecasting at DMI and perspectives on teh use of Earth Observations, ESA eSurge } \\
\text { Sympositum, Deltares, the Netherlands, (powerpoint presentation) January } 21,2015 \\
\text {-FIG. [MAP] map of storm surge water level, altimeter strip water level \& } 3 \text { Zeeland tide gauges } \\
\text { 07Dec2013 00:00 }\end{array}$ \\
\hline Staneva et al (2016) & $\begin{array}{l}\text { Staneva J, K Wahle, W Koch, A Behrens, L Fenoglio-Marc, EV Stanev, Coastal flooding: impact of waves on } \\
\text { storm surge during extremes - a case study for the German Bight, Nat. Hazards Earth Syst. Sci., 16, 2373- } \\
\text { 2389, } 2016 \\
\text {-RADS Radar Altimeter Database System: Jason-2, Cryosat-2, SARAL/AltikKa } \\
\text {-FIG6. Along track observed and modelled significant wave height (m): } \\
\text { (a) SARAL/ALtiKa ground track for overflight in calm conditions 03Dec2013 1800UTC; } \\
\text { (b) during storm XAVER 06Dec2013 0400UTC; } \\
\text { (c) observed \& modelled significant wave height on 03Dec2013 } 1800 \mathrm{UTC} \text {, } \\
\text { (d) during storm XAVER 06Dec2013 } \\
\text {-FIG8. (a) Surge at the time of SARAL over in calm conditions on 03Dec2013 1800UTC } \\
\text { (b) Surge at the time of storm Xaver 06Dec2013 0400UTC } \\
\text { (c) profiles of SARAL/Altika overflight of surge height derived from the altimeter observations } \\
\text { and GETM mdoel on 03Dec2013 1800UTC } \\
\text { (d) profiles of SARAL/Altika overflight of surge height derived from altimeter observations } \\
\text { and GETM model 06Dec2013 at 0400UTC. }\end{array}$ \\
\hline Wahle et al (2017) & $\begin{array}{l}\text { Wahle K, J Staneva, W Koch, L Fenoglio-Marc, HTM Ho-Hagemann, EV Stanev, An atmosphere-wave regional } \\
\text { coupled model: improving prediction of wave heights in the southern North Sea, Ocean Sci., 13, 289-301, } \\
\text { 2017.(doi:10.5194/os-13-289-2017). } \\
\text {-FIG3. Tracks of all satellites during the study period (1Oct2013 to 31Dec2013) } \\
\text {-FIG6. [STRIPMAP] Latitude profile of wave height (m) and wind speed (m/s) from the Saral/AltiKa } \\
\text { data and as modelled by WAM-NS under calm weather conditions on 13Nov2013. } \\
\text { The track of the satellite (white line) is shown together with the } \\
\text { model significant wave height at the time of the passage. } \\
\text { * -FIG7. [STRIPMAP] As Fig6 but for Storm Xaver on 6Dec2013 }\end{array}$ \\
\hline
\end{tabular}

Table S11. List meteorological data (arranged by year and then alphabetically)

\begin{tabular}{|c|c|c|c|}
\hline Data type & Location & Time Interval & Full Reference and Notes \\
\hline $\begin{array}{l}\text { [MAP] ABB5. map with } \\
\text { data listed for maximum } 3 \\
\text { second wind gusts over } 6 \\
\text { hour time intervals }\end{array}$ & $\begin{array}{l}\text { Northern Germany, southern } \\
\text { North Sea, western Baltic } \\
\text { Sea and neighboring } \\
\text { countries }\end{array}$ & $\begin{array}{l}5 \text { Dec } 2013 \\
12-18 \mathrm{UTC} \& \\
6 \text { Dec } 2013 \\
\text { 00-06UTC }\end{array}$ & $\begin{array}{l}\text { Deutschlander T, K Frierich, S Haeseler, C Lefebvre, } \\
\text { Orkantief XAVER ueber Nordeuropea von 5. bis } 7 . \\
\text { Dezember 2013, Deutscher Wetterdienst DWD, Stand } 30 . \\
\text { Dezember 2013, 19pp. }\end{array}$ \\
\hline [MAP] ABB6. Map with the & Northern Germany, southern & 5Dec2913 & Deutschlander $\mathrm{T}, \mathrm{K}$ Frierich, $\mathrm{S}$ Haeseler, $\mathrm{C}$ Lefebvre, \\
\hline
\end{tabular}




\begin{tabular}{|c|c|c|c|}
\hline $\begin{array}{l}\text { data listed for } 10-\mathrm{min} \\
\text { average wind }\end{array}$ & $\begin{array}{l}\text { North Sea, western Baltic } \\
\text { Sea and neighboring } \\
\text { countries }\end{array}$ & 19:00UTC & $\begin{array}{l}\text { Orkantief XAVER ueber Nordeuropea von 5. bis } 7 . \\
\text { Dezember 2013, Deutscher Wetterdienst DWD, Stand } 30 . \\
\text { Dezember 2013, 19pp. }\end{array}$ \\
\hline $\begin{array}{l}\text { [TABLE] Highest daily peak } \\
\text { gusts and } 10 \text {-min mean wind } \\
\text { speed }\end{array}$ & $\begin{array}{l}\text { Stations in Germany ranked } \\
\text { in descending order of the } \\
\text { peak gust on } 5 \text { Dec } 2013\end{array}$ & $\begin{array}{l}5 \text { Dec } 2013,6 \\
\text { Dec 2013, } 7 \\
\text { Dec } 2013\end{array}$ & $\begin{array}{l}\text { Deutschlander T, K Frierich, S Haeseler, C Lefebvre, } \\
\text { Orkantief XAVER ueber Nordeuropea von 5. bis } 7 . \\
\text { Dezember 2013, Deutscher Wetterdienst DWD, Stand } 30 . \\
\text { Dezember 2013, 19pp. }\end{array}$ \\
\hline $\begin{array}{l}\text { [FIG] ABB7. Time series of } \\
\text { peak gust over 10-min } \\
\text { intervals and } 10 \text {-min average } \\
\text { wind speed }\end{array}$ & $\begin{array}{l}\text { List/Sylt, Norderney, } \\
\text { Rostock/Warnemunde }\end{array}$ & 5-8 Dec 2013 & $\begin{array}{l}\text { Deutschlander T, K Frierich, S Haeseler, C Lefebvre, } \\
\text { Orkantief XAVER ueber Nordeuropea von 5. bis } 7 . \\
\text { Dezember 2013, Deutscher Wetterdienst DWD, Stand } 30 . \\
\text { Dezember 2013, 19pp. }\end{array}$ \\
\hline $\begin{array}{l}\text { [FIG] ABB8. Time series of } \\
1 \text {-min average wind speed, } \\
\text { peak gust and air pressure }\end{array}$ & Hamburg St. Pauli & 5 Dec2013 & $\begin{array}{l}\text { Deutschlander T, K Frierich, S Haeseler, C Lefebvre, } \\
\text { Orkantief XAVER ueber Nordeuropea von 5. bis } 7 . \\
\text { Dezember 2013, Deutscher Wetterdienst DWD, Stand } 30 . \\
\text { Dezember 2013, 19pp. }\end{array}$ \\
\hline $\begin{array}{l}{[\mathrm{FIG}] \mathrm{ABB} 9 . \text { Time series of }} \\
\text { wind direction }\end{array}$ & $\begin{array}{l}\text { List/Sylt, Norderney, } \\
\text { Rostock/Warnemunde }\end{array}$ & 5-8 Dec 2013 & $\begin{array}{l}\text { Deutschlander T, K Frierich, S Haeseler, C Lefebvre, } \\
\text { Orkantief XAVER ueber Nordeuropea von 5. bis } 7 . \\
\text { Dezember 2013, Deutscher Wetterdienst DWD, Stand } 30 . \\
\text { Dezember 2013, 19pp. }\end{array}$ \\
\hline $\begin{array}{l}{[\mathrm{MAP}] \quad \mathrm{ABB} 15 .} \\
\text { precipitation in } \mathrm{mm} \text {; initial } \\
\text { values, unverified }\end{array}$ & Germany & 5 Dec 2013 & $\begin{array}{l}\text { Deutschlander T, K Frierich, S Haeseler, C Lefebvre, } \\
\text { Orkantief XAVER ueber Nordeuropea von 5. bis } 7 . \\
\text { Dezember 2013, Deutscher Wetterdienst DWD, Stand } 30 . \\
\text { Dezember 2013, 19pp. }\end{array}$ \\
\hline $\begin{array}{l}{[\text { MAP] ABB16. Total snow }} \\
\text { height over one day }\end{array}$ & Northern Germany & $\begin{array}{lrr}6 & \text { Dec } & 2013 \\
\text { and } & 7 & \text { Dec } \\
2013 & \end{array}$ & $\begin{array}{l}\text { Deutschlander T, K Frierich, S Haeseler, C Lefebvre, } \\
\text { Orkantief XAVER ueber Nordeuropea von 5. bis } 7 . \\
\text { Dezember 2013, Deutscher Wetterdienst DWD, Stand } 30 . \\
\text { Dezember 2013, 19pp. }\end{array}$ \\
\hline $\begin{array}{l}{[\mathrm{FIG}] \text { ABB8. Time series }} \\
\text { of } 10 \text {-min average wind } \\
\text { speed }\end{array}$ & Brocken & $\begin{array}{l}3 \text { day interval } \\
4-6 \text { Dec } 2013\end{array}$ & $\begin{array}{l}\text { Deutschlander T, K Frierich, S Haeseler, C Lefebvre, } \\
\text { Orkantief XAVER ueber Nordeuropea von 5. bis } 7 . \\
\text { Dezember 2013, Deutscher Wetterdienst DWD, Stand } 30 . \\
\text { Dezember 2013, 19pp. }\end{array}$ \\
\hline $\begin{array}{l}\text { [TEXT] statements } \text { of } \\
\text { maximum } 10 \text { min wind } \\
\text { speed and gust }\end{array}$ & $\begin{array}{lr}\text { Stavoren } & \text { (Netherlands); } \\
\text { Nissum Fjord } \quad \begin{array}{r}\text { (Denmark); } \\
\text { Eieroya }\end{array} \\
\text { Nidingen, Vaaderooarna } \\
\text { (Sweden); } \\
\text { Snieszka/Schneekoppe, } \\
\text { Ustka (Poland) }\end{array}$ & $\begin{array}{l}\text { Point } \\
\text { measurements }\end{array}$ & $\begin{array}{l}\text { Deutschlander T, K Frierich, S Haeseler, C Lefebvre, } \\
\text { Orkantief XAVER ueber Nordeuropea von 5. bis } 7 . \\
\text { Dezember 2013, Deutscher Wetterdienst DWD, Stand } 30 . \\
\text { Dezember 2013, 19pp. }\end{array}$ \\
\hline $\begin{array}{l}\text { [TEXT] Maximum wind } \\
\text { gust }\end{array}$ & Stavoren, Vlieland & 5-6 Dec 2013 & $\begin{array}{l}\text { KNMI, News report. De Zware storm van } 5 \text { december, } 06 \\
\text { Dec 2013, https://www.knmi.nl/over-het-knmi/nieuws/de- } \\
\text { zware-storm-van-5-december }\end{array}$ \\
\hline [TEXT] peak gusts & $\begin{array}{l}\text { UK: Aonach Mor; Germany: } \\
\text { Sylt, Kiel Leuchtturm, } \\
\text { Rostock-Warnemuende, } \\
\text { List, Norderney, Brocken, } \\
\text { Feldberg/Black Forest }\end{array}$ & 6 Dec 2013 & $\begin{array}{l}\text { Kunz M, B Muehr, K Schroeter, T Bessel, S Moehrle, T } \\
\text { Muenzberg, S Brink, H-M Schmidt, Winterstorm Xaver - } \\
\text { Report. 06Dec2013 - Report No.1, Situation Report - } \\
\text { 19:00CET, CEDIM Forensic Disaster Analysis Group } \\
\text { (FDA), Center for Disaster Management and Risk } \\
\text { Reduction Technology. }\end{array}$ \\
\hline $\begin{array}{l}{[\text { FIG] Time series wind }} \\
\text { speed }\end{array}$ & $\begin{array}{l}\text { Norderney Riffgat, Borkum } \\
\text { Fischerbalje }\end{array}$ & 4-6 Dec 2013 & $\begin{array}{l}\text { Mills, Ian, Remko Scharoo, Luciana Fenoglio, Xaver affected } \\
\text { much of northern Europe on } 5 \text { and } 6 \text { December and caused } \\
\text { worst storm surge for decades in the North Sea. } \\
\text { https://www.eumetsat.int/website/home/News/DAT_20870 } \\
\text { 62.html (last accessed: 19Nov2019, 28Jun2020) }\end{array}$ \\
\hline $\begin{array}{l}\text { [FIG] Time series of wind } \\
\text { speed and direction and gust }\end{array}$ & $\begin{array}{l}\text { Zeebrugge Meteopark, } \\
\text { Zeebrugge } \\
\text { Daminstrumentatie } \\
\text { (Westelijke Dam) }\end{array}$ & 5-6 Dec 2013 & $\begin{array}{l}\text { Oceanografisch Meteorologisch Station, Stormverslag 05-06 } \\
\text { december 2013, 26pp, } 2013 \text { [pdf document properties: } \\
\text { author=Myriam Sys; datestamp=15Dec2013] }\end{array}$ \\
\hline $\begin{array}{l}\text { [TABLE] Tabulated peak } \\
\text { gusts }\end{array}$ & Many stations in Germany & 5-6 Dec 2013 & $\begin{array}{l}\text { Unwetterzentrale, Orkantief XAVER - ein weiterer schwerer } \\
\text { Wintersturm der letzten Jahrzehnte, Thomas Savert and } \\
\text { Stefan } \\
\text { http://www.unwetterzentrale.de/uwz/928.html }\end{array}$ \\
\hline [MAP] 1 hour peak gusts & $\begin{array}{l}\text { Ireland, UK, Netherlands, } \\
\text { northern Germany, } \\
\text { Denmark, southern Norway }\end{array}$ & $\begin{array}{l}5 \quad \text { Dec } 2013 \\
0700-0800 \\
\text { MEZ }\end{array}$ & $\begin{array}{l}\text { Unwetterzentrale, Orkantief XAVER - ein weiterer schwerer } \\
\text { Wintersturm der letzten Jahrzehnte, Thomas Savert and } \\
\text { Stefan } \\
\text { http://www.unwetterzentrale.de/uwz/928.html }\end{array}$ \\
\hline [MAP] 24 h peak gusts & Germany & $\begin{array}{l}5 \text { Dec } 2013 \\
0700 \text { MEZ to } \\
6 \text { Dec } 2013 \\
0700 \mathrm{MEZ}\end{array}$ & $\begin{array}{l}\text { Unwetterzentrale, Orkantief XAVER - ein weiterer schwerer } \\
\text { Wintersturm der letzten Jahrzehnte, Thomas Savert and } \\
\text { Stefan } \\
\text { http://www.unwetterzentrale.de/uwz/928.html }\end{array}$ \\
\hline [MAP] 24 h peak gusts & Germany & $\begin{array}{l}6 \text { Dec } 2013 \\
0700 \text { MEZ to } \\
7 \text { Dec } 2013 \\
0700 \mathrm{MEZ}\end{array}$ & $\begin{array}{l}\text { Unwetterzentrale, Orkantief XAVER - ein weiterer schwerer } \\
\text { Wintersturm der letzten Jahrzehnte, Thomas Savert and } \\
\text { Stefan } \\
\text { http://www.unwetterzentrale.de/uwz/928.html }\end{array}$ \\
\hline $\begin{array}{lccc}{[\mathrm{MAP}]} & 24 & \mathrm{~h} & \text { snowfall } \\
\text { accumulations } & & \\
\end{array}$ & Germany & $\begin{array}{l}5 \text { Dec } 2013 \\
0700 \text { MEZ to } \\
\end{array}$ & $\begin{array}{l}\text { Unwetterzentrale, Orkantief XAVER - ein weiterer schwerer } \\
\text { Wintersturm der letzten Jahrzehnte, Thomas Savert and }\end{array}$ \\
\hline
\end{tabular}




\begin{tabular}{|c|c|c|c|}
\hline & & $\begin{array}{l}6 \text { Dec } 2013 \\
0700 \mathrm{MEZ}\end{array}$ & $\begin{array}{lcc}\text { Stefan } & \text { Laps, } & \text { Dec. } \\
\text { http://www.unwetterzentrale.de/uwz/928.html }\end{array}$ \\
\hline $\begin{array}{lccc}\text { [MAP] } & 24 & \mathrm{~h} & \text { snowfall } \\
\text { accumulations } & & \end{array}$ & Germany & $\begin{array}{l}6 \text { Dec } 2013 \\
0700 \text { MEZ to } \\
7 \text { Dec } 2013 \\
0700 \mathrm{MEZ}\end{array}$ & 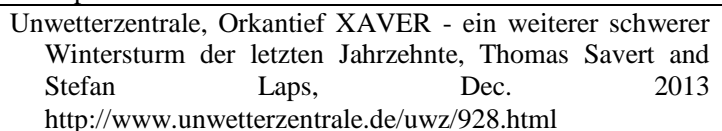 \\
\hline $\begin{array}{lr}{[\mathrm{TEXT}]} & \text { mention of met- } \\
\text { ocean } & \text { measurements } \\
\text { available through COSYNA } \\
\text { project }\end{array}$ & $\begin{array}{l}\text { Station Watt } \quad \text { (Pile at } \\
\text { Spiekeroog) }\end{array}$ & $?$ & $\begin{array}{l}\text { Badewien T., Long-term observatory @ Pile Spiekeroog } \\
\text { ICBM,COSYNA Progress Report 2013, p24. Helmholtz- } \\
\text { Zentrum Geesthacht, June } 2014\end{array}$ \\
\hline $\begin{array}{l}\text { [TABLE] Wind speed and } \\
\text { gust. }\end{array}$ & $\begin{array}{lll}\text { Northeast } & \text { Atlantic } & \text { and } \\
\text { North Sea } & & \end{array}$ & 5 Dec 2013 & $\begin{array}{l}\text { Bancroft, George P, Marine Weather Review - North Atlantic } \\
\text { Area, September through December, Mariners Weather } \\
\text { Log, volume 58, No.1, April 2014, pp.33-40 }\end{array}$ \\
\hline $\begin{array}{l}{[\text { TABLE] list of monthly }} \\
\text { minimum } \text { temperatures } \\
\text { during cold air outbreak }\end{array}$ & UK and Europe & Month list & $\begin{array}{l}\text { Eden, Phillip, Weather Log December 2013, Weather, Feb } \\
\text { 2014, pp.i-iv }\end{array}$ \\
\hline $\begin{array}{l}\text { [FIG] Time series of wind } \\
\text { gust, wind speed, wind } \\
\text { direction, air temperature, } \\
\text { atmospheric pressure }\end{array}$ & $\begin{array}{l}\text { Ormen Lange Norwegian } \\
\text { Sea platform }\end{array}$ & 1-31 Dec 2013 & $\begin{array}{l}\text { FUGRO GEOS Ltd, Ormen Lange Monthly reports of } \\
\text { wavescan data: December. Reporting period: } 1 \text { December } \\
2013 \text { to 31 December 2013, Report Number: } \\
\text { C70101/8177/R0, Issue date: 23 January 2014, prepared by } \\
\text { Heather Holt, Checked by Donald Brockie, approved by } \\
\text { Mark Jones }\end{array}$ \\
\hline $\begin{array}{l}\text { [FIG] Time series of wind } \\
\text { speed and wind direction }\end{array}$ & AWG platfrom & 5-7 Dec 2013 & $\begin{array}{l}\text { Gautier C, A Camarena, J van Nieuwkoop, SWAN hindcasts } \\
\text { Wadden Sea, December 2013. Tidal inlet of Ameland and } \\
\text { eastern Wadden Sea. Deltares, 2014, 197pp. Project } \\
\text { 1209433-007, Reference 1209433-007-HYE-0005 }\end{array}$ \\
\hline $\begin{array}{l}\text { [FIG] Time series of wind } \\
\text { speed and direction }\end{array}$ & UITHZWD & 5-7 Dec 2013 & $\begin{array}{l}\text { Gautier C, A Camarena, J van Nieuwkoop, SWAN hindcasts } \\
\text { Wadden Sea, December 2013. Tidal inlet of Ameland and } \\
\text { eastern Wadden Sea. Deltares, 2014, 197pp. Project } \\
\text { 1209433-007, Reference 1209433-007-HYE-0005 }\end{array}$ \\
\hline $\begin{array}{l}\text { [FIG] Time series of wind } \\
\text { speed and direction at } \\
\text { Scharhoern }\end{array}$ & Scharhoern & 4-8 Dec 2013 & $\begin{array}{l}\text { Goennert G, O Mueller, M Schaper, K Sossidi, Die Sturmflut } \\
\text { nach dem Tief Xaver von 5. bis 7. Dezember } 2013 . \\
\text { Berichte des Landesbetriebes Strassen, Bruecken und } \\
\text { Gewaesser (LSBG), Freie und Hansestadt Hamburg, Nr. } \\
\text { 16/2014, 26pp. }\end{array}$ \\
\hline $\begin{array}{l}\text { [MAP] } 24 \text { hour maximum } \\
\text { gusts }\end{array}$ & Northwest Europe & $\begin{array}{l}5 \text { Dec2013 } \\
0000-2400 \\
\text { UTC }\end{array}$ & $\begin{array}{l}\text { Hewson T, L Magnusson, O Breivik, F Prates, I Tsonevsky, } \\
\text { HJW de Vries, Windstorms in northwest Europe in late } \\
\text { 2013, ECMWF Newsletter, No 139, pp 22-28, Spring } \\
\text { 2014. }\end{array}$ \\
\hline $\begin{array}{l}{[\text { TEXT] Maximum average }} \\
\text { wind speed } 118 \mathrm{~km} / \mathrm{h}\end{array}$ & Norderney & 5-6 Dec 2013 & $\begin{array}{l}\text { Knaack H and H Heyken, Xaver hatte sehr schwere Stumflut } \\
\text { im Gepaeck, Jahresbericht 2013. Der Zukunft verplichtet, } \\
\text { NLWKN, Niedersaechsischer Landesbetrieb fuer } \\
\text { Waserwirtschaft, Kuesten- und Naturschutz, pp.8-9, } \\
\text { document date stamp 24Apr2014. }\end{array}$ \\
\hline $\begin{array}{l}\text { [MAP] maximum gusts at } \\
10 \mathrm{~m} ; \text { FINO1 max gust at } \\
100 \mathrm{~m} 49.0 \mathrm{~m} / \mathrm{s} ; \mathrm{FINO} 2 \mathrm{max} \\
\text { gust at } 100 \mathrm{~m} 38.6 \mathrm{~m} / \mathrm{s} \text {; } \\
\text { FINO3 max gust at } \\
100 \mathrm{~m} 43.2 \mathrm{~m} / \mathrm{s}\end{array}$ & $\begin{array}{l}\text { northern Germany, North } \\
\text { Sea and Baltic Sea }\end{array}$ & $\begin{array}{l}(5-6 \quad \text { Dec } \\
2013)^{-}\end{array}$ & $\begin{array}{l}\text { Leiding T, B Tinz, G Rosenhagen, C Lefevre, S Haeseler, S } \\
\text { Hagemann, I Bastigkeit, D Stein, P Schwenk, S Mueller, O } \\
\text { Outzen, K Herklotz, F Kinder, T Neumann, Meteorological } \\
\text { and Oceanographic Conditions at the FINO platforms } \\
\text { during the severe storms Christian and Xaver, DEWI } \\
\text { Magazin, No.44, p16-25, 2014. }\end{array}$ \\
\hline $\begin{array}{l}\text { [FIG] Time series graph of } \\
10 \mathrm{~min} \text { wind speed and wind } \\
\text { direction and atmospheric } \\
\text { pressure at } 100 \mathrm{~m} \text { height }\end{array}$ & FINO1, FINO2, FINO3 & 5-7 Dec 2013 & $\begin{array}{l}\text { Leiding T, B Tinz, G Rosenhagen, C Lefevre, S Haeseler, S } \\
\text { Hagemann, I Bastigkeit, D Stein, P Schwenk, S Mueller, O } \\
\text { Outzen, K Herklotz, F Kinder, T Neumann, Meteorological } \\
\text { and Oceanographic Conditions at the FINO platforms } \\
\text { during the severe storms Christian and Xaver, DEWI } \\
\text { Magazin, No.44, p16-25, 2014. }\end{array}$ \\
\hline $\begin{array}{l}\text { [FIG] Time series graph of } \\
\text { wind speed, wind gust, wind } \\
\text { direction }\end{array}$ & Messpfahl Westerland & 1-9Dec2013 & $\begin{array}{l}\text { Luecht, Fabian and Ove Peters, Bericht ueber die Sturmflut } \\
\text { vom 05.-0.6.12.2013 an der Westkueste Schleswig- } \\
\text { Holsteins, Landesbetrieb fuer Kuestenschutz, Nationalpark } \\
\text { und Meeresschutz Schleswig-Holstein, Husum 26Feb2014, } \\
\text { 19pp. [pdf document properties: title=Lfd; } \\
\text { Author=Thorsten Nommensen; datestamp: 11Dec2018] }\end{array}$ \\
\hline $\begin{array}{l}\text { [TEXT] highest wind speed } \\
\text { at FINO1 platform }(30 \mathrm{~m} / \mathrm{s})\end{array}$ & FINO1 & 4-7 Dec 2013 & $\begin{array}{l}\text { Mai, S., Sea state at the research platform FINO1 during the } \\
\text { winter storm 'Xaver', BFG, Bundesamt fuer } \\
\begin{array}{l}\text { Gewasserkunde, document properties: author=Mai, } \\
\text { date=16May2014 }\end{array}\end{array}$ \\
\hline $\begin{array}{l}\text { [FIG] Time series of wind } \\
\text { speed and wind direction } \\
\text { with gust indicated }\end{array}$ & $\begin{array}{l}\text { Hallig Hooge, Buesum, } \\
\text { Strucklahnungshoern }\end{array}$ & 4-7 Dec 2013 & $\begin{array}{l}\text { Matelski, Birgit, Frerk Jensen, Peter Voss, Jorg Peters, Maria } \\
\text { Blumel, Auswertung zur Hydrologie des Sturmtiefs Xaver } \\
05.12 .2013 \text { bis 06.12.2013, Statusbericht vom 01.10.2014, } \\
\text { Landesbetrieb fuer Kuestenschutz, Nationalpark und } \\
\text { Meeresschutz, Schleswig-Holstein, Husum, 01.10.2014, } \\
\text { 25pp. }\end{array}$ \\
\hline $\begin{array}{l}\text { [FIG] Time series of } 10 \mathrm{~min} \\
\text { wind speed reduced to } 10 \mathrm{~m} \text {, }\end{array}$ & Draugen & 1-31 Dec 2013 & $\begin{array}{l}\text { MIROS, Monthly Report, Draugen, December } 2013 \text {, Doc. No. } \\
\text { ND/1022/13/12 (prepared by SRS, checked by OO, }\end{array}$ \\
\hline
\end{tabular}




\begin{tabular}{|c|c|c|c|}
\hline $\begin{array}{l}\text { 3second gust reduced to } \\
10 \mathrm{~m}, \text { air temperature, } \\
\text { relative humidity, air } \\
\text { pressure, cloud height, } \\
\text { visibility, precipitation }\end{array}$ & & & $\begin{array}{l}\text { approved by CNE) [pdf document properties: } \\
\text { author=MIROS AS; datestamp=30Jan2014] }\end{array}$ \\
\hline $\begin{array}{l}{[\mathrm{FIG}] \text { Time series of } 10 \mathrm{~min}} \\
\text { wind speed reduced to } 10 \mathrm{~m} \text {, } \\
3 \text { second gust reduced to } \\
10 \mathrm{~m} \text {, air temperature, } \\
\text { relative humidity, air } \\
\text { pressure, sea temperature, } \\
\text { cloud height, visibility }\end{array}$ & Ekofisk & 1-31 Dec 2013 & $\begin{array}{l}\text { MIROS, Monthly report, Ekofisk, December } 2013 \text {, Doc. No. } \\
\begin{array}{l}\text { ND/1024/13/12, (prepared by SRS) 34pp, 9Jan2014 [PDF } \\
\text { document } \quad \text { properties: } \\
\text { datestamp=13Jan2014] }\end{array}\end{array}$ \\
\hline $\begin{array}{l}\text { [FIG] Time series of } 10 \mathrm{~min} \\
\text { wind speed reduced to } 10 \mathrm{~m} \text {, } \\
3 \text { second gust reduced to } \\
10 \mathrm{~m} \text {, air temperature, } \\
\text { relative humidity, air } \\
\text { pressure, sea temperature, } \\
\text { cloud height, visibility }\end{array}$ & Gullfaks & 1-31 Dec 2013 & $\begin{array}{l}\text { MIROS, Manedsrapport Gullfaks C, Desember 2013, Dok. Nr } \\
\text { ND/1013/13/12, carried out by SRS, controlled by CNE, } \\
\text { approved by CO [pdf document properties: author=MIROS } \\
\text { AS; date stamp: 13Jan2014] }\end{array}$ \\
\hline $\begin{array}{l}\text { [FIG] Time series of } 10 \mathrm{~min} \\
\text { wind speed reduced to } 10 \mathrm{~m} \text {, } \\
3 \text { second gust reduced to } \\
10 \mathrm{~m}, \text { air temperature, } \\
\text { relative humidity, air } \\
\text { pressure, sea temperature, } \\
\text { cloud height, visibility }\end{array}$ & Heidrun & 1-31 Dec 2013 & $\begin{array}{l}\text { MIROS, Manedsrapport Heidrun, Desember } 2013 \text {, Dok. Nr. } \\
\text { ND/1010/13/12, } 21 \text { pp, 07Jan2014, carried out by SRS, } \\
\text { controlled by CNE, approved by OO [pdf properties: } \\
\text { author=Miros AS; date stamp: 08/01/2014] }\end{array}$ \\
\hline $\begin{array}{l}\text { [FIG] Time series of } 10 \mathrm{~min} \\
\text { wind speed reduced to } 10 \mathrm{~m} \text {, } \\
3 \text { second gust reduced to } \\
10 \mathrm{~m}, \text { air temperature, } \\
\text { relative humidity, air } \\
\text { pressure, sea temperature, } \\
\text { cloud height, visibility }\end{array}$ & Heimdal & 1-31 Dec 2013 & $\begin{array}{l}\text { MIROS, Manedsrapport Heimdal, Desember 2013, Dok. Nr. } \\
\text { ND/1047/13/12 (carried out be SRS, controlled by CNE, } \\
\text { approved by OO) [PDF document properties: author=Miros } \\
\text { AS; datestamp=06Jan2014] }\end{array}$ \\
\hline $\begin{array}{l}\text { FIG] Time series of } 10 \mathrm{~min} \\
\text { wind speed reduced to } 10 \mathrm{~m} \text {, } \\
3 \text { second gust reduced to } \\
10 \mathrm{~m}, \text { air temperature, } \\
\text { relative humidity, air } \\
\text { pressure, sea temperature, } \\
\text { cloud height, visibility }\end{array}$ & Norne & 1-31 Dec 2013 & $\begin{array}{l}\text { MIROS, Maanedsrapport Norne, Desember } 2013 \text {, Dok. Nr. } \\
\text { ND/1087/13/12, 21pp, carried out by SRS, controlled by } \\
\text { CNE, approved by OO [pdf properties: Author=Miros AS; } \\
\text { datestamp: 06Jan2014] }\end{array}$ \\
\hline $\begin{array}{l}\text { [FIG] Time series of } 10 \mathrm{~min} \\
\text { wind speed reduced to } 10 \mathrm{~m} \text {, } \\
3 \text { second gust reduced to } \\
10 \mathrm{~m}, \text { air temperature, } \\
\text { relative humidity, air } \\
\text { pressure, sea temperature, } \\
\text { cloud height, visibility }\end{array}$ & Sleipner A & 1-31 Dec 2013 & $\begin{array}{l}\text { MIROS, Manedsrapport Sleipner A, Desember 2013, Dok. Nr. } \\
\text { ND/1017/13/12, 06Jan2014, prepared by SRS, controlled } \\
\text { by CNE, approved by OO. }\end{array}$ \\
\hline $\begin{array}{l}\text { [FIG] Time series of } 10 \mathrm{~min} \\
\text { wind speed reduced to } 10 \mathrm{~m} \text {, } \\
3 \text { second gust reduced to } \\
10 \mathrm{~m}, \text { air temperature, } \\
\text { relative humidity, air } \\
\text { pressure, sea temperature, } \\
\text { cloud height, visibility }\end{array}$ & Troll A & 1-31 Dec 2013 & 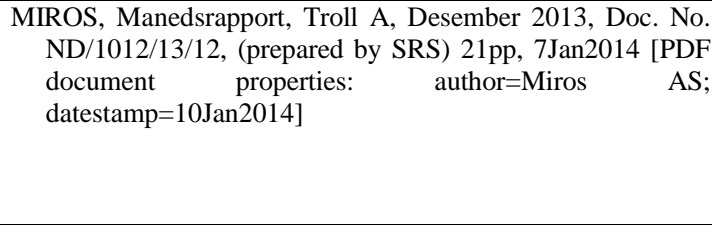 \\
\hline $\begin{array}{l}\text { [FIG] Time serie } \mathrm{s} \text { wind } \\
\text { speed and direction }\end{array}$ & Terneuzen, Hansweert & 5 Dec 2013 & $\begin{array}{l}\text { Nossent J, L Boeckx, E Taverniers, M Deschamps, T } \\
\text { Verwaest, F Mostaert, Sinterklaasstorm } 6 \text { december } 2013 . \\
\text { Beschrijving van de hydrometrische gebeurtenissen, Versie } \\
\text { 4.0. WL Rapporten, 00-119, Waterbouwkundig } \\
\text { Laboratorium, Antwerpen, Belgie. October 2014, } \\
\text { WL2014R00_119_5 [document date stamp: 15Oct2014; } \\
\text { author= Deschamps, Maarten] }\end{array}$ \\
\hline $\begin{array}{l}{[\mathrm{TEXT}] \text { Highest average }} \\
\text { wind and gust }\end{array}$ & Torsminde & 5Dec2013 & $\begin{array}{l}\text { Pelt AS, BODIL's stormflod i de indre dansk farvande, } \\
\text { Vejret, 138, 24-29, } 2014\end{array}$ \\
\hline $\begin{array}{l}\text { [FIG] Time series graphs of } \\
\text { wind speed and direction }\end{array}$ & $\begin{array}{lr}\text { Europlatform, } & \text { Platform } \\
\text { K13a, } & \text { IJmuiden, } \\
\text { Lauwersoog, Lichteiland } & \text { Loeree, Hoek van Holland } \\
\text { Goer }\end{array}$ & 5-6 Dec 2013 & $\begin{array}{l}\text { RWS, Stormvloedrapport van } 5 \text { t/m } 7 \text { december (SR91) Sint- } \\
\text { Nicolaasvloed 2013, Watermanagementcentrum } \\
\text { Nederland, Rijkswaterstaat, prepared by Ing. J. Kroos, } 19 \\
\text { Mar 2014b, } 48 \text { pp }\end{array}$ \\
\hline $\begin{array}{l}\text { [TEXT] Highest wind and } \\
\text { highest gust }\end{array}$ & Vaderoarna & 5-6 Dec 2013 & $\begin{array}{l}\text { SMHI, Simone, Hilde, Sven och Ivar okt-dec 2013, } 3 \text { Jul } \\
\text { 2014, } \\
\text { https://www.smhi.se/kunskapsbanken/meteorologi/simon } \\
\text { e-hilde-sven-och-ivar-okt-dec-2013-1.76183 }\end{array}$ \\
\hline $\begin{array}{l}{[\mathrm{TEXT}] \text { Highest hourly }} \\
\text { average wind speed }\end{array}$ & Vlieland & 5-6Dec2013 & $\begin{array}{l}\text { van Dorland, R, Zware storm op } 5 \text { december, Zenit, p43, } \\
\text { Januari } 2014\end{array}$ \\
\hline $\begin{array}{l}\text { [FIG] Map with highest } \\
\text { gusts values written }\end{array}$ & $\begin{array}{l}\text { Station in the Netherlands } \\
\text { and offshore }\end{array}$ & 5 Dec 2013 & $\begin{array}{l}\text { van Dorland, R, Zware storm op } 5 \text { december, Zenit, p43, } \\
\text { Januari } 2014\end{array}$ \\
\hline
\end{tabular}




\begin{tabular}{|c|c|c|c|}
\hline [TEXT] Highest gusts & $\begin{array}{l}\text { Edinburgh, Drumalbin, } \\
\text { Scotland mountains, List auf } \\
\text { Sylt (Ellenbogen), } \\
\text { Glucksburg-Meierwik, } \\
\text { Borkum, Wangerooge, } \\
\text { Rostock-Warnemuende, } \\
\text { Dresden/Klotzsche, } \\
\text { Chieming in Bayern }\end{array}$ & 5Dec2013 & $\begin{array}{llr}\text { Axer T, T Bistry, M Klawa, M Mueller, M Suesser, Deutsche } \\
\text { Ruck Sturm dokumentation 2013 Deutschland, 2013, } \\
\begin{array}{l}\text { Deutsche } \\
\text { Rueckversicherung }\end{array} \text { Aktiengesellschaft, } \\
\text { Hansaallee } & 177, \quad 40549 & \text { Duesseldorf, } \\
\text { www.deutscherueck.de [pdf document } & \text { information: } \\
\text { author=filiz; date stamp=07Aug2015] } & \end{array}$ \\
\hline $\begin{array}{l}\text { [FIG] Time series average } \\
10 \mathrm{~m} \text { wind speed }\end{array}$ & $\begin{array}{l}\text { Cadzand, Vlakte van de } \\
\text { Raan }\end{array}$ & 5-7 Dec 2013 & $\begin{array}{l}\text { Carrion Aretxabala, BI, Morphological impact of the } \\
\text { Sinterklaas storm at Het Zwin. Numerical modelling with } \\
\text { Xbeach, M.Sc. Civil Engineering, Delft University of } \\
\text { Technology, 2015. }\end{array}$ \\
\hline $\begin{array}{l}\text { [FIG] Time series of wind } \\
\text { speed and wind direction }\end{array}$ & Wirral Peninsula & $\begin{array}{l}1 \text { Dec } 2013- \\
31 \text { Jan } 2014\end{array}$ & $\begin{array}{l}\text { Dissanayake P, J Brown, P. Wisse, H Karunarathna, } \\
\text { Comparison of storm cluster vs isolated event impacts on } \\
\text { beach.dune morphodynamics, Estuarine, Coastal, and } \\
\text { Shelf Science, 164, 301-312, 2015b. }\end{array}$ \\
\hline $\begin{array}{l}\text { [STRIP PROFILE] SARAL- } \\
\text { AltiKa satellite } \\
\text { wind speed }\end{array}$ & $\begin{array}{l}\text { Strip profile through North } \\
\text { Sea }\end{array}$ & $\begin{array}{l}6 \text { Dec } 2013 \\
\text { 0447UTC }\end{array}$ & $\begin{array}{l}\text { Fenoglio-Marc L, R Scharroo, A Annuziato, L Mendoza, M } \\
\text { Becker, J Lillibridge, Cyclone Xaver seen by geodetic } \\
\text { observations, Geophys Research Letters, 42, 9925-9932, } \\
2015\end{array}$ \\
\hline $\begin{array}{l}\text { [FIG] Time series of wind } \\
\text { speed }\end{array}$ & FINO1 & 3-7Dec2013 & $\begin{array}{l}\text { Fenoglio-Marc L, R Scharroo, A Annuziato, L Mendoza, M } \\
\text { Becker, J Lillibridge, Cyclone Xaver seen by geodetic } \\
\text { observations, Geophys Research Letters, 42, 9925-9932, } \\
2015\end{array}$ \\
\hline $\begin{array}{l}\text { [FIG] Time series of wind } \\
\text { speed and wind direction; } \\
10 \mathrm{~m} ; \text { maximum value } 22 \mathrm{~m} / \mathrm{s}\end{array}$ & Bornholm Airport & $\begin{array}{l}1-13 \\
2013\end{array}$ & $\begin{array}{l}\text { Gierlevsen T, H Lauridsen, F Langhans, J Bejdic, Met-ocean } \\
\text { and wind resource related studies for nearshore windfarms } \\
\text { in Denmark, seminar at the Danish Energy Agency, } 27 \\
\text { February } 2015 \text {. }\end{array}$ \\
\hline $\begin{array}{l}\text { [FIG] Time series of wind } \\
\text { speed and wind direction; } \\
10 \mathrm{~m} \text {; maximum value } 27 \mathrm{~m} / \mathrm{s}\end{array}$ & Arkona offshore site & $\begin{array}{l}1-13 \\
2013\end{array}$ & $\begin{array}{l}\text { Gierlevsen T, H Lauridsen, F Langhans, J Bejdic, Met-ocean } \\
\text { and wind resource related studies for nearshore windfarms } \\
\text { in Denmark, seminar at the Danish Energy Agency, } 27 \\
\text { February 2015. }\end{array}$ \\
\hline $\begin{array}{l}\text { [TEXT] Maximum gusts in } \\
\text { UK }\end{array}$ & 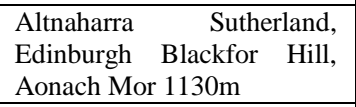 & 5-6 Dec 2013 & $\begin{array}{l}\text { Kendon M and M McCarthy, The UK's wet and stormy winter } \\
\text { of 2013/2014, Weather, 70, 40-47, } 2015\end{array}$ \\
\hline $\begin{array}{l}\text { [TEXT] Maximum gusts in } \\
\text { UK }\end{array}$ & $\begin{array}{l}\text { Aonanach Mor near Fort } \\
\text { William, } \\
\text { Cairngormsm Cairnwell } \\
\text { Boulmer Northumburgh, } \\
\text { Weybourne Norfolk }\end{array}$ & 5-6 Dec 2013 & $\begin{array}{l}\text { Sibley A, D Cox, H Titley, Coastal flooding in England and } \\
\text { Wales from Atlantic and North Sea storms during the } \\
\text { 2013/2014 winter, Weather, 70, 62-70, } 2015\end{array}$ \\
\hline $\begin{array}{l}\text { [TABLE] Maximum wind } \\
\text { gust }\end{array}$ & $\begin{array}{l}\text { Skillinge, Malmo, Horby, } \\
\text { Hano, Helsingborg, Olands } \\
\text { sodra udde, Hallands } \\
\text { Vadero, Vaxjo, Ljungby, } \\
\text { Nidingen, Olands norra } \\
\text { udde,Tomtebacken, } \\
\text { Goteborg, Farosund-Ar, } \\
\text { Malexander, Masekar, } \\
\text { Visingso, Harstena, Hallum, } \\
\text { Gotska Sandon, Vaderoarna, } \\
\text { Nordkoster, Naven, } \\
\text { Palgrunden, Landsort }\end{array}$ & 5-6 Dec 2013 & $\begin{array}{c}\text { SMHI, Egon - saesongens foersta ordentliga storm, } \\
\text { 12Jan2015. } \\
\text { https://www.smhi.se/nyhetsarkiv/egon- } \\
\text { sasongens-forsta-ordentliga-storm-1.83408 }\end{array}$ \\
\hline $\begin{array}{lll}{[\mathrm{TEXT}]} & \text { Maximum } & \text { gust } \\
110 \mathrm{~km} / \mathrm{h} & & \end{array}$ & Wattisham & 5 Dec 2013 & $\begin{array}{l}\text { Spencer T, SM Brooks, BR Evans, JA Tempest, I Moeller, } \\
\text { Southern North Sea storm surge event of Dec.5, 2013: } \\
\text { Water levels, waves, and coastal impacts, Earth Science } \\
\text { Reviews, } 146,120-145,2015\end{array}$ \\
\hline $\begin{array}{l}\text { [FIG] Time series of hourly- } \\
\text { average wind speed and } \\
\text { wind direction at } 100 \mathrm{~m} \\
\text { height }\end{array}$ & FINO1, FINO2, FINO3 & 4-7 Dec 2013 & $\begin{array}{l}\text { Christakos K, I Cheliotis, G Varlas, G-J Steeneveld, Offshore } \\
\text { wind energy analysis of Cyclone Xaver over North Europe, } \\
\text { 13th Deep Sea Offshore Wind R\&D Conference, EERA } \\
\text { DeepWind'2016, 20-22 January 2016, Trondheim, } \\
\text { Norway, Energy Procedia, 94, 37-44, 2016. }\end{array}$ \\
\hline $\begin{array}{l}\text { [FIG] Time series of wind } \\
\text { speed, wind direction, } \\
\text { surface atmospheric pressure }\end{array}$ & Norderney & 5-6 Dec 2013 & $\begin{array}{l}\text { Dangendorf S, A Arns JG Pinto, P Ludwig, J Jensen, The } \\
\text { exceptional influence of storm 'Xaver' on design water } \\
\text { levels in the German Bight, Environmental Research } \\
\text { Letters, 11, 2016,054001 }\end{array}$ \\
\hline $\begin{array}{l}\text { [FIG] Map of maximum } \\
\text { obserbed wind gusts and } \\
\text { mean wind during } 24 \mathrm{~h} \\
\text { period }\end{array}$ & Northwest Europe & 5 Dec 2013 & $\begin{array}{l}\text { ECMWF }(20160316), \quad 201312-\quad \text { Windstorm - } \\
\text { Xaver/Bodil/Sven, } \\
\text { https://confluence.ecmwf.int/pages/viewpage.action?page } \\
\text { Id=28315390 }\end{array}$ \\
\hline $\begin{array}{l}{[\text { FIG] Time series of } 10} \\
\text { meter wind speed for } \\
\text { Ekofisk and Thorsminde }\end{array}$ & est Europe & 4-6 Dec 2013 & $\begin{array}{l}\text { ECMWF (20160316), } 201312-\quad \text { Windstorm - } \\
\text { Xaver/Bodil/Sven, } \\
\text { https://confluence.ecmwf.int/pages/viewpage.action?page } \\
\text { Id=28315390 }\end{array}$ \\
\hline [FIG] Time series of wind & Messstation & 4-7 Dec 2013 & Matelski, Birgit, Erfahrungen aus der Sturmflut Xaver von 5. \\
\hline
\end{tabular}




\begin{tabular}{|c|c|c|c|}
\hline speed and direction & $\begin{array}{l}\text { Strucklahnungshoern } \\
\text { (Nordstrand) }\end{array}$ & & $\begin{array}{l}\text { und 6.12.2013 und dem Weihnachshochwasser } 2014 \text { in } \\
\text { Shleswig-Holstein, IWASA } 2016 \text { Tagungsbeitrag, (46. } \\
\text { IWASA, 7-8 Januar 2016; Internationales Wasserbau- } \\
\text { Symposium Aachen. [pdf document properties: } \\
\text { autor=sonja; datestamp: 26Apr2016] }\end{array}$ \\
\hline [TEXT] maximum gusts & $\begin{array}{l}\text { Strucklahnungshoern, Hallig } \\
\text { Hooge, Buesum }\end{array}$ & 5-6 Dec 2013 & $\begin{array}{l}\text { Matelski, Birgit, Erfahrungen aus der Sturmflut Xaver von } 5 . \\
\text { und 6.12.2013 und dem Weihnachshochwasser } 2014 \text { in } \\
\text { Shleswig-Holstein, IWASA } 2016 \text { Tagungsbeitrag, (46. } \\
\text { IWASA, 7-8 Januar 2016; Internationales Wasserbau- } \\
\text { Symposium Aachen. [pdf document properties: } \\
\text { autor=sonja; datestamp: 26Apr2016] }\end{array}$ \\
\hline $\begin{array}{l}\text { [FIG] Time series graph of } \\
\text { wind magnitude, } r \text { wind } \\
\text { direction and sea level } \\
\text { pressure }\end{array}$ & Elbe station & 1-8 Dec 2013 & $\begin{array}{l}\text { Staneva J, K Wahle, W Koch, A Behrens, L Fenoglio-Marc, } \\
\text { EV Stanev, Coastal flooding: impact of waves on storm } \\
\text { surge during extremes - a case study for the German Bight, } \\
\text { Nat. Hazards Earth Syst. Sci., 16, 2373-2389, } 2016\end{array}$ \\
\hline 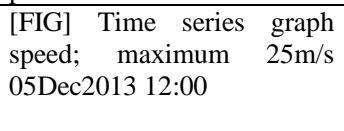 & FINO1 at $60 \mathrm{~m}$ & 2-9 Dec 2013 & $\begin{array}{lcr}\text { Peter Frohle \& Norman Dreier, EarlyDike - Sensor - und } \\
\text { risiko basiertes } & \text { Fruhwarnsystem fuer } & \text { Seedeiche, } \\
\text { Teilprojekt: } & \text { Wellenmonitoring } & \text { und } \\
\text { Wellenbelastungssimulator (AP2), Hamburg, April } 2017\end{array}$ \\
\hline $\begin{array}{l}\text { [FIG] Time series graph } \\
\text { wind speed; maximum } \\
21 \mathrm{~m} / \mathrm{s} \text { 05Dec2013 } 12: 00\end{array}$ & Helgoland at $14 \mathrm{~m}$ & 2-9Dec 2013 & $\begin{array}{l}\text { Frohle P \& N Dreier, EarlyDike - Sensor - und risiko } \\
\text { basiertes Fruhwarnsystem fuer Seedeiche, Teilprojekt: } \\
\text { Wellenmonitoring und Wellenbelastungssimulator (AP2), } \\
\text { Hamburg, April } 2017\end{array}$ \\
\hline $\begin{array}{l}{[\mathrm{TEXT}] \text { Maximum wind }} \\
\text { speed and gust }\end{array}$ & $\begin{array}{l}\text { Kolo, Leszno, Kalisz, Leba, } \\
\text { Kasprowy Wierch, Ustka, } \\
\text { Sniezka }\end{array}$ & 6-10 Dec 2013 & $\begin{array}{l}\text { Ribeiro R, R Rudge, D Rucinska, Analysis of physical factors } \\
\text { of the windstorm Xaver in Poland: post-hazard review, } \\
\text { Weather, 72, 2017, pp.378-382 }\end{array}$ \\
\hline $\begin{array}{l}\text { [FIG] Time series of wind } \\
\text { magnitude and wind } \\
\text { direction }\end{array}$ & FINO1 & $\begin{array}{l}1 \text { Oct 2013-31 } \\
\text { Dec 2013, } 1-8 \\
\text { Dec } 2013\end{array}$ & $\begin{array}{l}\text { Staneva J, H Guenther, O Krueger, C Schrumm, V Alari, O } \\
\text { Breivik, J-R Bidlot, K Mogensen, Impact of wind waves } \\
\text { on the air-sea momentum fluxes for different wind and } \\
\text { sea state conditions and oceanic responses, 1st } \\
\text { International Workshop on waves, storm surges and } \\
\text { coastal hazards, Liverpool, UK 10-15Sep2017 [pdf } \\
\text { document properties: title=Anlass; author=Patrick Kalb- } \\
\text { Anlass, datestamp=20/09/2017] }\end{array}$ \\
\hline $\begin{array}{l}\text { [FIG] Time series of wind } \\
\text { speed }\end{array}$ & Helgoland & 3-8 Dec 2013 & $\begin{array}{l}\text { Staneva J, C Schrum, A Behrens, S Grayek, H Ho-Hagemann, } \\
\text { V Alan, O Breivik, J-R. Bidlot, A North Sea-Baltic Sea } \\
\text { Regional Coupled Models: Atmosphere, wind waves and } \\
\text { ocean, in Proceedings of the Eigth EuroGOOS } \\
\text { International Conference (Operational Oceanography. } \\
\text { Serving Sustainable Marine Development), 3-5 October } \\
\text { 2017, Bergen, Norway, 2017b. }\end{array}$ \\
\hline $\begin{array}{l}\text { [FIG] Time series graph of } \\
\text { wind speed at } 100 \mathrm{~m} \text { height }\end{array}$ & FINO1 & 3-7Dec2013 & $\begin{array}{l}\text { Wahle K, J Staneva, W Koch, L Fenoglio-Marc, HTM Ho- } \\
\text { Hagemann, EV Stanev, An atmosphere-wave regional } \\
\text { coupled model: improving prediction of wave heights in } \\
\text { the southern North Sea, Ocean Sci., 13, 289-301, } \\
\text { 2017.(doi:10.5194/os-13-289-2017). }\end{array}$ \\
\hline $\begin{array}{l}\text { [FIG] Latitude profile graph } \\
\text { of altimeter-derived wind } \\
\text { speed from SARAL-AltiKa }\end{array}$ & North Sea axis $53-60 \mathrm{~N}$ & $\begin{array}{l}6 \text { Dec } 2013 \\
04: 46: 55 \text { UTC }\end{array}$ & $\begin{array}{l}\text { Wahle K, J Staneva, W Koch, L Fenoglio-Marc, HTM Ho- } \\
\text { Hagemann, EV Stanev, An atmosphere-wave regional } \\
\text { coupled model: improving prediction of wave heights in } \\
\text { the southern North Sea, Ocean Sci., 13, 289-301, } \\
\text { 2017.(doi:10.5194/os-13-289-2017). }\end{array}$ \\
\hline $\begin{array}{l}\text { [FIG] Time series graph of } \\
\text { measured wind speed }\end{array}$ & Helgoland and Westerland & 3-8 Dec 2013 & $\begin{array}{l}\text { Wahle K, J Staneva, W Koch, L Fenoglio-Marc, HTM Ho- } \\
\text { Hagemann, EV Stanev, An atmosphere-wave regional } \\
\text { coupled model: improving prediction of wave heights in } \\
\text { the southern North Sea, Ocean Sci., 13, 289-301, } \\
\text { 2017.(doi:10.5194/os-13-289-2017). }\end{array}$ \\
\hline $\begin{array}{l}\text { [FIG] Time series graph of } \\
10 \mathrm{~m} \text { wind speed }\end{array}$ & FINO1 & $\begin{array}{lr}2 & \text { weeks } \\
\text { across } & \text { storm } \\
\text { period } & \end{array}$ & $\begin{array}{l}\text { Wahle K, J Staneva, W Koch, L Fenoglio-Marc, HTM Ho- } \\
\text { Hagemann, EV Stanev, An atmosphere-wave regional } \\
\text { coupled model: improving prediction of wave heights in } \\
\text { the southern North Sea, Ocean Sci., 13, 289-301, } \\
\text { 2017.(doi:10.5194/os-13-289-2017). }\end{array}$ \\
\hline $\begin{array}{l}{[\text { TEXT] highest average }} \\
\text { wind speed and highest gust }\end{array}$ & Nissum Fjord & 5-6 Dec 2013 & $\begin{array}{l}\text { Danish Emergency Management Agency DEMA, National } \\
\text { Risk Profile for Denmark, April } 2018\end{array}$ \\
\hline $\begin{array}{l}\text { [FIG] Time series of } 10 \mathrm{~m} \\
\text { wind speed }\end{array}$ & Hallig Hooge & 5-6 Dec 2013 & $\begin{array}{l}\text { Dreier, Norman and Peter Froehle, Operational wave forecast } \\
\text { in the German Bight as part of a sensor- and risk based } \\
\text { early warning system, In: J-S Shim, I Chun, HS Lim (ed), } \\
\text { Proceedings from the International Coastal Symposium } \\
\text { (ICS) } 2018 \text { (Busan, Republic of Korea), Journal of } \\
\text { Coastal Research, Special Issue No. 85, 1161-1165, } 2018\end{array}$ \\
\hline $\begin{array}{l}\text { [FIG] Time series of wind } \\
\text { speed and wind direction }\end{array}$ & FINO1 & 1-9 Dec 2013 & $\begin{array}{l}\text { Fery, Natascha, Birger Tinz, Lydia Gates, Reproduction of } \\
\text { storms over the North Sea and the Baltic with the regional } \\
\text { analysis COSMO-REA6 ISPR 2018, 17-19July2018, } \\
\text { Bonn [pdf document properties: datestamp=16/07/2018] }\end{array}$ \\
\hline
\end{tabular}


Table S12. Significant wave height and sea state (arranged by year and then alphabetically)

\begin{tabular}{|c|c|c|c|}
\hline Data type & Location & Time Interval & Full Reference and Notes \\
\hline 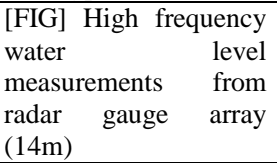 & FINO1 & $\begin{array}{l}\text { 6Dec2013 } \\
00: 09-00: 11\end{array}$ & $\begin{array}{l}\text { BFG, Orkan Xaver: BfG beobachtet extreme Wellen in der } \\
\text { Nordsee }(06.12 .2013)\end{array}$ \\
\hline \begin{tabular}{lrr} 
MAP] & \multicolumn{2}{r}{ ABB10. } \\
Gridded & significant \\
wave & height & of \\
combined & waves and \\
swell & &
\end{tabular} & $\begin{array}{l}\text { North Sea and Western } \\
\text { Approaches; Baltic Sea }\end{array}$ & $\begin{array}{l}6 \text { Dec } 2013 \\
\text { 00:00UTC }\end{array}$ & $\begin{array}{l}\text { Deutschlander T, K Frierich, S Haeseler, C Lefebvre, Orkantief } \\
\text { XAVER ueber Nordeuropea von 5. bis 7. Dezember 2013, } \\
\text { Deutscher Wetterdienst DWD, Stand 30. Dezember 2013, } \\
\text { 19pp. }\end{array}$ \\
\hline $\begin{array}{l}{[\mathrm{FIG}] \text { ABB11. Time }} \\
\text { series of significant } \\
\text { wave height, period, } \\
\text { wave direction, and sea } \\
\text { temperature }\end{array}$ & Station Elbe & $\begin{array}{l}2 \text { Dec } 2013- \\
9 \text { Dec } 2013 \\
09: 59 U T C\end{array}$ & $\begin{array}{l}\text { Deutschlander T, K Frierich, S Haeseler, C Lefebvre, Orkantief } \\
\text { XAVER ueber Nordeuropea von 5. bis 7. Dezember 2013, } \\
\text { Deutscher Wetterdienst DWD, Stand 30. Dezember 2013, } \\
\text { 19pp. }\end{array}$ \\
\hline $\begin{array}{l}\text { [FIG] Saral-AltiKa } \\
\text { strip map of signficant } \\
\text { wave height }\end{array}$ & $\begin{array}{l}\text { North-south transect of } \\
\text { North Sea }\end{array}$ & $\begin{array}{l}\text { 06Dec2013 } \\
04: 47 U T C\end{array}$ & $\begin{array}{l}\text { Mills, Ian, Remko Scharoo, Luciana Fenoglio, Xaver affected } \\
\text { much of northern Europe on } 5 \text { and } 6 \text { December and caused } \\
\text { worst storm surge for decades in the North Sea. } \\
\text { https://www.eumetsat.int/website/home/News/DAT_2087062.h } \\
\text { tml (last accessed: 19Nov2019, 28Jun2020) }\end{array}$ \\
\hline $\begin{array}{l}{[\mathrm{FIG}] \text { Time series of }} \\
\text { significant wave } \\
\text { height, height of } \\
\text { highest } 1 \% \text { and } 10 \% \text { of } \\
\text { waves, wave direction } \\
\text { (period } 2-5 \mathrm{~s} \text { and }>10 \mathrm{~s} \text { ), } \\
\text { wave low frequency } \\
\text { energy, equivalent } \\
\text { wave height for period } \\
>10 \mathrm{~s}\end{array}$ & $\begin{array}{l}\text { Westhinder, Akkaert, } \\
\text { Bol van Heist, Oostende, } \\
\text { Scheur Wielingen }\end{array}$ & 5-6 Dec 2013 & $\begin{array}{l}\text { Oceanografisch Meteorologisch Station, Stormverslag 05-06 } \\
\text { december 2013, 26pp, 2013 [pdf document properties: } \\
\text { author=Myriam Sys; datestamp=15Dec2013] }\end{array}$ \\
\hline $\begin{array}{l}{[\text { TEXT] Maximum }} \\
\text { significant wave height } \\
\text { and maximum wave } \\
\text { height }\end{array}$ & Waverider at Vaderoarna & 5 Dec2013 & $\begin{array}{l}\text { SMHI, Stormen Sven gav nya vattenstandsrekord i Oresund, } \\
\text { https://www.smhi.se/nyhetsarkiv/stormen-sven-gav-nya- } \\
\text { vattenstandsrekord-i-oresund-1.34732, updated 20Mar2017; } \\
\text { original datestamp 10Dec2013. }\end{array}$ \\
\hline $\begin{array}{l}\text { [TEXT] record sea } \\
\text { state of } 6 \mathrm{~m}\end{array}$ & North Sea area of Sylt & 5-6 Dec 2013 & $\begin{array}{l}\text { Sueddeutsche Zeitung, Xaver holte sich Land; Sturmschaden auf } \\
\text { Sylt, } \\
\text { 15Dec201315:36 } \\
\text { https://www.sueddeutsche.de/panorama/sturmschaeden-auf- } \\
\text { sylt-xaver-holte-sich-land-1.1844100 }\end{array}$ \\
\hline $\begin{array}{l}\text { [TABLE] Significant } \\
\text { wave height }\end{array}$ & $\begin{array}{l}\text { Northeast Atlantic and } \\
\text { North Sea }\end{array}$ & 5 Dec 2013 & $\begin{array}{l}\text { Bancroft, George P, Marine Weather Review - North Atlantic } \\
\text { Area, September through December, Mariners Weather Log, } \\
\text { volume 58, No.1, April 2014, pp.33-40 }\end{array}$ \\
\hline $\begin{array}{l}\text { [FIG] Time } \\
\text { significant } \\
\text { height; wave } \\
\text { significant wave height } \\
9.04 \mathrm{~m} \text {; maximum } \\
\text { trough to crest wave } \\
\text { height } 15.5 \mathrm{~m}\end{array}$ & FINO1 & 4-9 Dec 2013 & $\begin{array}{l}\text { Blasi C, S Mai, J Wilhelmi, T Zenz, U Barjenbruch, A powerful } \\
\text { method of measuring sea wave spectra and their direction, } \\
\text { ICHE 2014, Hamburg - Lehfeldt and Kopmann (eds), } \\
\text { Bundesanstalt fuer Wasserbau, 2014. ISBN 978-3-939230-32-8 }\end{array}$ \\
\hline $\begin{array}{l}\text { [TABLE] Significant } \\
\text { wave wave height at } \\
\text { peak }\end{array}$ & $\begin{array}{l}\text { Newbiggin Ness, Tyne } \\
\text { Tees, Whitby waverider }\end{array}$ & 5-6 Dec 2013 & 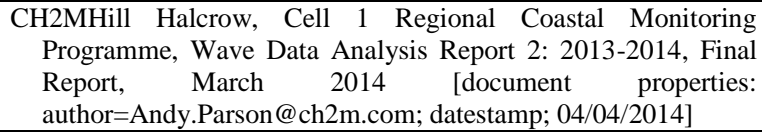 \\
\hline $\begin{array}{l}\text { [FIG] Time series of } \\
\text { significant wave height } \\
\text { and maximum wave } \\
\text { height; tables of } \\
\text { maximum values }\end{array}$ & $\begin{array}{l}\text { Ormen Lange Norwegian } \\
\text { Sea Platform }\end{array}$ & $\begin{array}{ll}1-31 & \text { Dec } \\
2013 & \end{array}$ & $\begin{array}{l}\text { FUGRO GEOS Ltd, Ormen Lange Monthly reports of wavescan } \\
\text { data: December. Reporting period: } 1 \text { December } 2013 \text { to } 31 \\
\text { December 2013, Report Number: C70101/8177/R0, Issue date: } \\
23 \text { January 2014, prepared by Heather Holt, Checked by } \\
\text { Donald Brockie, approved by Mark Jones }\end{array}$ \\
\hline $\begin{array}{l}\text { [FIG] Time series of } \\
\text { significant wave height }\end{array}$ & $\begin{array}{l}\text { Buoy array in Ameland } \\
\text { inlet area }\end{array}$ & 5-7 Dec 2013 & $\begin{array}{l}\text { Gautier C, A Camarena, J van Nieuwkoop, SWAN hindcasts } \\
\text { Wadden Sea, December 2013. Tidal inlet of Ameland and } \\
\text { eastern Wadden Sea. Deltares, 2014, 197pp. Project 1209433- } \\
\text { 007, Reference 1209433-007-HYE-0005 }\end{array}$ \\
\hline $\begin{array}{l}\text { [FIG] Time series of } \\
\text { significant wave height }\end{array}$ & $\begin{array}{l}\text { Buoy array in eastern } \\
\text { Wadden Sea }\end{array}$ & 5-7 Dec 2013 & $\begin{array}{l}\text { Gautier C, A Camarena, J van Nieuwkoop, SWAN hindcasts } \\
\text { Wadden Sea, December 2013. Tidal inlet of Ameland and } \\
\text { eastern Wadden Sea. Deltares, 2014, 197pp. Project 1209433- } \\
\text { 007, Reference 1209433-007-HYE-0005 }\end{array}$ \\
\hline $\begin{array}{lr}{[\mathrm{FIG}] \text { Time series of }} \\
\text { significant wave height } \\
\text { and maximum wave } \\
\text { height; } \\
\text { Hs }=10 \mathrm{~m} \quad \text { FINO1 } \\
\mathrm{Hmax}=16 \mathrm{~m} ; & \text { FINO3 } \\
\mathrm{Hs}=9.1 \mathrm{~m} & \text { and }\end{array}$ & FINO1, FINO2, FINO3 & 5-7 Dec 2013 & $\begin{array}{l}\text { Leiding T, B Tinz, G Rosenhagen, C Lefevre, S Haeseler, S } \\
\text { Hagemann, I Bastigkeit, D Stein, P Schwenk, S Mueller, O } \\
\text { Outzen, K Herklotz, F Kinder, T Neumann, Meteorological and } \\
\text { Oceanographic Conditions at the FINO platforms during the } \\
\text { severe storms Christian and Xaver, DEWI Magazin, No.44, } \\
\text { p16-25, 2014. }\end{array}$ \\
\hline
\end{tabular}




\begin{tabular}{|c|c|c|c|}
\hline \multicolumn{4}{|l|}{$\mathrm{Hmax}=13.5 \mathrm{~m}$} \\
\hline $\begin{array}{l}\text { [FIG] Time series of } \\
\text { signficant wave height }\end{array}$ & $\begin{array}{l}\text { wave measurement buoy } \\
\text { Westerland }\end{array}$ & 2-9 Dec 2013 & $\begin{array}{l}\text { Luecht, Fabian and Ove Peters, Bericht ueber die Sturmflut vom } \\
\text { 05.-0.6.12.2013 an der Westkueste Schleswig-Holsteins, } \\
\text { Landesbetrieb fuer Kuestenschutz, Nationalpark und } \\
\text { Meeresschutz Schleswig-Holstein, Husum 26Feb2014, 19pp. } \\
\text { [pdf document properties: title=Lfd; Author=Thorsten } \\
\text { Nommensen; datestamp: 11Dec2018] }\end{array}$ \\
\hline $\begin{array}{l}{[\mathrm{FIG}] \text { Radar gauge }} \\
\text { half-hour time series of } \\
\text { Hs, mean period, } \\
\text { direction, spread; max } \\
\text { Hs }=9.04 \mathrm{~m} \text {; max wave } \\
\text { height } 15.5 \mathrm{~m}\end{array}$ & FINO1 & 4-7 Dec2013 & $\begin{array}{l}\text { Mai, S., Sea state at the research platform FINO1 during the winter } \\
\text { storm 'Xaver', BFG, Bundesamt fuer Gewasserkunde, } \\
\text { document properties: author=Mai, date }=16 \text { May } 2014\end{array}$ \\
\hline $\begin{array}{l}\text { [FIG] Time series of } \\
\text { significant wave height } \\
\text { and maximum wave } \\
\text { height. }\end{array}$ & $\begin{array}{l}\text { FINO1, } \\
\text { Suedstrand }\end{array}$ & 4-9 Dec 2013 & 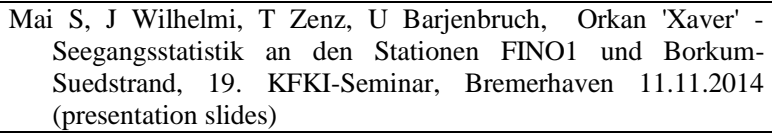 \\
\hline $\begin{array}{l}\text { [FIG] Time series of } \\
\text { significant wave height }\end{array}$ & Westerland & 4-8 Dec 2013 & $\begin{array}{l}\text { Matelski, Birgit, Frerk Jensen, Peter Voss, Jorg Peters, Maria } \\
\text { Blumel, Auswertung zur Hydrologie des Sturmtiefs Xaver } \\
\text { 05.12.2013 bis 06.12.2013, Statusbericht vom 01.10.2014, } \\
\text { Landesbetrieb fuer Kuestenschutz, Nationalpark und } \\
\text { Meeresschutz, Schleswig-Holstein, Husum, 01.10.2014, 25pp. }\end{array}$ \\
\hline $\begin{array}{l}\text { [FIG] Time series of } \\
\text { signficant wave height } \\
\text { and maximum wave } \\
\text { height }\end{array}$ & Draugen & $\begin{array}{ll}1-31 & \text { Dec } \\
2013 & \end{array}$ & $\begin{array}{l}\text { MIROS, Monthly Report, Draugen, December 2013, Doc. No. } \\
\text { ND/1022/13/12 (prepared by SRS, checked by OO, approved } \\
\text { by CNE) [pdf document properties: author=MIROS AS; } \\
\text { datestamp=30Jan2014] }\end{array}$ \\
\hline $\begin{array}{l}\text { [FIG] Time series of } \\
\text { significant wave height } \\
\text { and maximum wave } \\
\text { height from spectral } \\
\text { analysis and time } \\
\text { series data; waverider } \\
\text { and altimeter }\end{array}$ & Ekofisk & $\begin{array}{ll}1-31 & \text { Dec } \\
2013 & \end{array}$ & $\begin{array}{l}\text { MIROS, Monthly report, Ekofisk, December 2013, Doc. No. } \\
\text { ND/1024/13/12, (prepared by SRS) 34pp, 9Jan2014 [PDF } \\
\text { document properties: author=Miros AS; datestamp=13Jan2014] }\end{array}$ \\
\hline $\begin{array}{l}\text { FIG] Time series of } \\
\text { significant wave height } \\
\text { and maximum wave } \\
\text { height }\end{array}$ & Heidrun & $\begin{array}{ll}1-31 & \text { Dec } \\
2013 & \end{array}$ & $\begin{array}{l}\text { MIROS, Manedsrapport Heidrun, Desember 2013, Dok. Nr. } \\
\text { ND/1010/13/12, } 21 \text { pp, 07Jan2014, carried out by SRS, } \\
\text { controlled by CNE, approved by OO [pdf properties: } \\
\text { author=Miros AS; date stamp: } 08 / 01 / 2014]\end{array}$ \\
\hline $\begin{array}{l}\text { FIG] Time series of } \\
\text { significant wave height } \\
\text { and maximum wave } \\
\text { height }\end{array}$ & Norne & $\begin{array}{ll}1-31 & \text { Dec } \\
2013 & \end{array}$ & $\begin{array}{l}\text { MIROS, Maanedsrapport Norne, Desember 2013, Dok. Nr. } \\
\text { ND/1087/13/12, 21pp, carried out by SRS, controlled by CNE, } \\
\text { approved by OO [pdf properties: Author=Miros AS; datestamp: } \\
\text { 06Jan2014] }\end{array}$ \\
\hline $\begin{array}{l}\text { [FIG] Time series of } \\
\text { significant wave height } \\
\text { and maximum wave } \\
\text { height from spectral } \\
\text { analysis and time } \\
\text { series data; waverider } \\
\text { and altimeter }\end{array}$ & Sleipner A & $\begin{array}{ll}1-31 & \text { Dec } \\
2013 & \end{array}$ & $\begin{array}{l}\text { MIROS, Manedsrapport Sleipner A, Desember } 2013 \text {, Dok. Nr. } \\
\text { ND/1017/13/12, 06Jan2014, prepared by SRS, controlled by } \\
\text { CNE, approved by OO. }\end{array}$ \\
\hline $\begin{array}{l}\text { [FIG] Time series of } \\
\text { significant wave height }\end{array}$ & $\begin{array}{l}\text { Scheur West Wandelaar, } \\
\text { Europlatform, Platform } \\
\text { K13a, IJ muiden }\end{array}$ & 5-6 Dec 2013 & $\begin{array}{l}\text { RWS, Stormvloedrapport van } 5 \mathrm{t} / \mathrm{m} 7 \text { december (SR91) Sint- } \\
\text { Nicolaasvloed 2013, Watermanagementcentrum Nederland, } \\
\text { Rijkswaterstaat, prepared by Ing. J. Kroos, } 19 \text { Mar 2014b, } 48 \\
\text { pp }\end{array}$ \\
\hline $\begin{array}{l}\text { [FIG] Time series of } \\
\text { maximum wave height }\end{array}$ & $\begin{array}{l}\text { Scheur West Wandelaar, } \\
\text { Europlatform, Platform } \\
\text { K13a, IJ muiden }\end{array}$ & 5-6 Dec 2013 & $\begin{array}{l}\text { RWS, Stormvloedrapport van } 5 \mathrm{t} / \mathrm{m} 7 \text { december (SR91) Sint- } \\
\text { Nicolaasvloed 2013, Watermanagementcentrum Nederland, } \\
\text { Rijkswaterstaat, prepared by Ing. J. Kroos, } 19 \text { Mar 2014b, } 48 \\
\text { pp }\end{array}$ \\
\hline $\begin{array}{l}\text { [TABLE] Table of } \\
\text { maximum 'globally } \\
\text { corrected' significant } \\
\text { wave height in ranked } \\
\text { storm list } \\
\end{array}$ & $\begin{array}{l}\text { Scheur west Wandelaar, } \\
\text { Euro platform, IJmuiden } \\
\text { munitiestortplaats, } \\
\text { Eierlandse Gat, } \\
\text { (Schiermonnikoog Nord }\end{array}$ & 5-6 Dec 2013 & $\begin{array}{l}\text { RWS, Stormvloedrapport van } 5 \mathrm{t} / \mathrm{m} 7 \text { december (SR91) Sint- } \\
\text { Nicolaasvloed 2013, Watermanagementcentrum Nederland, } \\
\text { Rijkswaterstaat, prepared by Ing. J. Kroos, } 19 \text { Mar 2014b, } 48 \\
\text { pp }\end{array}$ \\
\hline $\begin{array}{l}\text { [TEXT] Maximum } \\
\text { wave height }\end{array}$ & Vaderoarna & 5-6 Dec 2013 & $\begin{array}{l}\text { SMHI, Simone, Hilde, Sven och Ivar okt-dec 2013, } 3 \text { Jul 2014, } \\
\text { https://www.smhi.se/kunskapsbanken/meteorologi/simone- } \\
\text { hilde-sven-och-ivar-okt-dec-2013-1.76183 }\end{array}$ \\
\hline $\begin{array}{l}\text { TEXT] Maximum } \\
\text { significant wave height } \\
3.8 \mathrm{~m}\end{array}$ & $\begin{array}{ll}\text { Blakeney } & \text { Overfalls } \\
\text { Waverider } & \end{array}$ & 5-6 Dec 2013 & $\begin{array}{l}\text { Spencer, T, S.M. Brooks, I. Moller, B.R. Evans, Where local } \\
\text { matters: Impacts of a major North Sea storm surge, EOS, 95, } \\
\text { 269-270, 29July2014 }\end{array}$ \\
\hline $\begin{array}{l}\text { [FIG] Time series } \\
\text { signifcant wave height }\end{array}$ & $\begin{array}{l}\text { Wave buoy } 1 \_1 \\
\text { Amelander Zeegat }\end{array}$ & 5-7 Dec 2013 & $\begin{array}{c}\text { van Rooijen A, A Oost, Memo: Regionale advisering Ameland } \\
\text { Noordwest, Deltares, 1209381-008-ZKS-0008, 43pp, } \\
\text { 18Dec2014 [PDF document properties: titl=Regional } \\
\text { advisering: Ameland NW; author=Arnold van Rooijen; } \\
\text { keywords: 1209381-008-ZKS-0008; date stamp: 18/12/2014] }\end{array}$ \\
\hline $\begin{array}{l}\text { [FIG] Time series } \\
\text { significant wave height }\end{array}$ & SCHW, SCHO, WIEL & 5-7 Dec 2013 & $\begin{array}{l}\text { Carrion Aretxabala, BI, Morphological impact of the Sinterklaas } \\
\text { storm at Het Zwin. Numerical modelling with Xbeach, M.Sc. }\end{array}$ \\
\hline
\end{tabular}




\begin{tabular}{|c|c|c|c|}
\hline & & & Civil Engineering, Delft University of Technology, 2015. \\
\hline $\begin{array}{l}{[\mathrm{FIG]} \mathrm{Time} \mathrm{series} \mathrm{of}} \\
\text { significant wave height } \\
\text { and maximum wave } \\
\text { height from Nortek } \\
\text { AWAC instrument } \\
600 \mathrm{~m} \text { from shore }\end{array}$ & $\begin{array}{l}\text { Beach profile } 104 \text { south } \\
\text { of Ostend Belgium }\end{array}$ & 4-8 Dec 2013 & $\begin{array}{l}\text { Dan, Sebastian, Anne-Lise Montreuil, Rosalia Delgado, Tomas } \\
\text { van Oyen, Large storm impact on a beach under sand } \\
\text { nourishments, The Proceedings of the Coastal Sediments } \\
\text { 2015, edited by Ping Wang, Julie D Rosati, and Jun Cheng, } \\
\text { Coastal Sediments 2015, San Diego, USA, 11-15May2015 }\end{array}$ \\
\hline $\begin{array}{l}\text { [FIG] Time series of } \\
\text { significant wave height }\end{array}$ & $\begin{array}{l}\text { [FIG] Time series of } \\
\text { significant wave height }\end{array}$ & 5 Dec 2013 & $\begin{array}{l}\text { Dissanayake } \mathrm{P} \text { and } \mathrm{H} \text { Karunarathna, Effect of storm clustering on } \\
\text { beach/dune erosion, E-proceedings of the 36th IAHR World } \\
\text { Congress, 28June-3July, 2015, The Hague, The Netherlands }\end{array}$ \\
\hline $\begin{array}{l}\text { [FIG] Time series of } \\
\text { significant wave height }\end{array}$ & $\begin{array}{l}\text { Liverpool Bay Wavenet } \\
\text { waverider }\end{array}$ & 5 Dec 2013 & $\begin{array}{l}\text { Dissanayake P, J Brown, H Karunarathna, Impacts of storm } \\
\text { chronology on the morphological changes of the Formby } \\
\text { beach and dune system, UK, Nat. Hazards Earth Syst. Sci., } \\
15,1533-1543,2015 \text {. }\end{array}$ \\
\hline $\begin{array}{l}\text { [FIG] Time series of } \\
\text { significant wave height }\end{array}$ & $\begin{array}{l}\text { Liverpool Bay Wavenet } \\
\text { waverider }\end{array}$ & $\begin{array}{l}1 \text { Dec 2013- } \\
\text { 31 Jan } 2014\end{array}$ & $\begin{array}{l}\text { Dissanayake P, J Brown, P. Wisse, H Karunarathna, Comparison } \\
\text { of storm cluster vs isolated event impacts on beach.dune } \\
\text { morphodynamics, Estuarine, Coastal, and Shelf Science, 164, } \\
\text { 301-312, 2015b. }\end{array}$ \\
\hline $\begin{array}{lr}\text { STRIP } & \text { PROFILE] } \\
\text { AltiKa } & \text { satellite } \\
\text { altimetry } & \text { significant } \\
\text { wave height } & \\
\end{array}$ & $\begin{array}{l}\text { Strip profile through } \\
\text { North Sea }\end{array}$ & $\begin{array}{l}6 \text { Dec } 2013 \\
\text { 0447UTC }\end{array}$ & $\begin{array}{l}\text { Fenoglio-Marc L, R Scharroo, A Annuziato, L Mendoza, M } \\
\text { Becker, J Lillibridge, Cyclone Xaver seen by geodetic } \\
\text { observations, Geophys Research Letters, 42, 9925-9932, } 2015\end{array}$ \\
\hline [FIG] Time series data & FINO1 & 3-7 Dec 2013 & $\begin{array}{l}\text { Fenoglio-Marc L, R Scharroo, A Annuziato, L Mendoza, M } \\
\text { Becker, J Lillibridge, Cyclone Xaver seen by geodetic } \\
\text { observations, Geophys Research Letters, 42, 9925-9932, } 2015\end{array}$ \\
\hline $\begin{array}{l}\text { [FIG] Time series of } \\
\text { significant wave height } \\
\text { from RADAC, } \\
\text { Waverider buoy, } \\
\text { AWAC }\end{array}$ & FINO1 & 4-7 Dec 2013 & $\begin{array}{l}\text { Fischer, JG, C Senet, A Schneehorst, O Outzen, S Schirmel, K } \\
\text { Herklotz, Sea state measurements in Germanys first offshore } \\
\text { wind farm "alpha ventus", in the south-eastern parts of the } \\
\text { North Sea, } 2015 \text { IEEE/OES Eleventh Current, Waves and } \\
\text { Turbulence Measurement (CWTM), } 2015 \text { [PDF document } \\
\text { properties: datestamp: 14/01/2015] }\end{array}$ \\
\hline $\begin{array}{l}\text { [FIG] Significant wave } \\
\text { height, wave period } \\
\text { and wave direction; } \\
\text { maximum Hs } 4.5 \mathrm{~m} \\
\text { (instrument failure) }\end{array}$ & Fjaltring & $\begin{array}{l}7 \text { Nov } 2013- \\
7 \text { Dec } 2013\end{array}$ & $\begin{array}{l}\text { Gierlevsen T, H Lauridsen, F Langhans, J Bejdic, Met-ocean and } \\
\text { wind resource related studies for nearshore windfarms in } \\
\text { Denmark, seminar at the Danish Energy Agency, } 27 \text { February } \\
2015 .\end{array}$ \\
\hline $\begin{array}{l}\text { [FIG] Significant wave } \\
\text { height, wave period } \\
\text { and wave direction; } \\
\text { maximum Hs } 4.5 \mathrm{~m} \\
\text { (instrument failure) }\end{array}$ & Bornholm, Baltic Sea & $\begin{array}{l}7 \text { Nov } 2013- \\
7 \text { Dec } 2013\end{array}$ & $\begin{array}{l}\text { Gierlevsen T, H Lauridsen, F Langhans, J Bejdic, Met-ocean and } \\
\text { wind resource related studies for nearshore windfarms in } \\
\text { Denmark, seminar at the Danish Energy Agency, } 27 \text { February } \\
2015 .\end{array}$ \\
\hline $\begin{array}{l}{[\text { TEXT] combined }} \\
\text { wave height } 8-10 \mathrm{~m}\end{array}$ & $\begin{array}{l}\text { North Sea buoy or } \\
\text { platform at } 56.3 \mathrm{~N}, 02.4 \mathrm{E}\end{array}$ & 5-6 Dec 2013 & $\begin{array}{l}\text { Sibley A, D Cox, H Titley, Coastal flooding in England and Wales } \\
\text { from Atlantic and North Sea storms during the 2013/2014 } \\
\text { winter, Weather, 70, 62-70, 2015 }\end{array}$ \\
\hline $\begin{array}{l}{[\mathrm{TEXT}] \text { maximum }} \\
\text { significant wave height } \\
\text { of } 4.7 \mathrm{~m} \text { and period of } \\
14 \mathrm{~s}\end{array}$ & $\begin{array}{lll}\begin{array}{l}\text { Tyne } \\
\text { buoy }\end{array} & \text { Tees } & \text { WaveNet }\end{array}$ & 5-6 Dec 2013 & $\begin{array}{l}\text { Sibley A, D Cox, H Titley, Coastal flooding in England and Wales } \\
\text { from Atlantic and North Sea storms during the 2013/2014 } \\
\text { winter, Weather, 70, 62-70, } 2015\end{array}$ \\
\hline $\begin{array}{l}\text { [FIG] Time series of } \\
\text { significant wave height }\end{array}$ & $\begin{array}{lr}\text { Blakeney } & \text { Overfalls, } \\
\text { Chapel Point, North } \\
\text { Well, } \quad \text { Happisburgh, } \\
\text { Felixstower } \quad \text { Sizewell, } \\
\text { South Knock }\end{array}$ & 5-6 Dec 2013 & $\begin{array}{l}\text { Spencer T, SM Brooks, BR Evans, JA Tempest, I Moeller, } \\
\text { Southern North Sea storm surge event of Dec.5, 2013: Water } \\
\text { levels, waves, and coastal impacts, Earth Science Reviews, } \\
\text { 146, 120-145, } 2015\end{array}$ \\
\hline $\begin{array}{l}{[\mathrm{FIG]} \text { Time series of }} \\
\text { significant wave height }\end{array}$ & Liverpool, Sizewell & 4-6 Dec 2013 & $\begin{array}{l}\text { Wadey MP, JM Brown, ID Haigh, T Dolphin, P Wisse, } \\
\text { Assessment and comparison of extreme sea levels and waves } \\
\text { during the 2013/2014 storm season in two UK coastal regions, } \\
\text { Nat. Hazards Earth Syst. Sci. Discuss., 3, 2665-2708, 2015b. }\end{array}$ \\
\hline $\begin{array}{lcr}{[\mathrm{FIG}]} & \text { Time } & \text { series } \\
\text { significant wave height }\end{array}$ & Blakeney & 2-7 Dec 2013 & $\begin{array}{l}\text { Brooks SM, T Spencer, A McIvor, I Moller, Reconstructing and } \\
\text { understanding the impacts of storms and surges, southern North } \\
\text { Sea, Earth Surface Processes and Landforms, 41, 855-864, } \\
2016 \text {. }\end{array}$ \\
\hline $\begin{array}{l}\text { [FIG] Time series of } \\
\text { Hmax and significant } \\
\text { wave height }\end{array}$ & $\begin{array}{l}\text { Lighthouse Alte Weser, } \\
\text { Borkum Suedstrand }\end{array}$ & 5-8 Dec 2013 & $\begin{array}{l}\text { Mai S and U Barjenbruch, Water level measurements with radar } \\
\text { gauges at the German North Sea coast, [PDF document } \\
\text { properties: author=IOC; subject: IOC/2016/MG/14 vol.5; } \\
\text { datestamp: 18/04/2017] }\end{array}$ \\
\hline $\begin{array}{l}{[\mathrm{FIG]} \text { Time series of }} \\
\text { significant wave height }\end{array}$ & $\begin{array}{l}\text { Seegangmessstation } \\
\text { Westerland }\end{array}$ & 4-8 Dec 2013 & $\begin{array}{l}\text { Matelski, Birgit, Erfahrungen aus der Sturmflut Xaver von 5. und } \\
\text { 6.12.2013 und dem Weihnachshochwasser 2014 in Shleswig- } \\
\text { Holstein, IWASA } 2016 \text { Tagungsbeitrag, (46. IWASA, 7-8 } \\
\text { Januar 2016; Internationales Wasserbau-Symposium Aachen. } \\
\text { [pdf document properties: autor=sonja; datestamp: 26Apr2016] }\end{array}$ \\
\hline $\begin{array}{l}\text { [FIG] Time series of } \\
\text { measured wave } \\
\text { conditions in } 20 \mathrm{~m} \text { deep } \\
\text { water }\end{array}$ & $\begin{array}{l}\text { Amelander Zeegat buoy } \\
1 \_1\end{array}$ & 5-8 Dec 2013 & $\begin{array}{l}\text { Nederhoff K, E Elias, T Vermaas, Erosie op Ameland Noordwest. } \\
\text { Modelstudie: simulaties met Delft3D en XBeach, Deltares, } \\
117 \mathrm{pp} \text {, July, } 2016\end{array}$ \\
\hline
\end{tabular}




\begin{tabular}{|c|c|c|c|}
\hline $\begin{array}{l}{[\mathrm{FIG} \text { Time series }} \\
\text { siggnificant wave } \\
\text { height from } \mathrm{ADCP}\end{array}$ & Hornbaek & 4-6 Dec 2013 & $\begin{array}{l}\text { Sorensen CS, NK Dronen, P Knudsen, J Jensen, P Sorensen, An } \\
\text { extreme event as a games changer in coastal zone management, } \\
\text { Journal of Coastal Research, (Special Issue, No 75), 700-704, } \\
2016 \text {. Proceedings of the 14th International Coastal } \\
\text { Symposium (Sydney, Australia) ed by A Vila-Concejo, E } \\
\text { Bruce, DM Kennedy, RJ McCarroll }\end{array}$ \\
\hline $\begin{array}{l}\text { [FIG] Time series of } \\
\text { significant wave height }\end{array}$ & $\begin{array}{l}\text { Elbe station, Westerland } \\
\text { station }\end{array}$ & 2-8 Dec 2013 & $\begin{array}{l}\text { Staneva J, K Wahle, W Koch, A Behrens, L Fenoglio-Marc, EV } \\
\text { Stanev, Coastal flooding: impact of waves on storm surge } \\
\text { during extremes - a case study for the German Bight, Nat. } \\
\text { Hazards Earth Syst. Sci., 16, 2373-2389, } 2016\end{array}$ \\
\hline $\begin{array}{l}{[\mathrm{FIG} \text { Time } \quad \text { series }} \\
\text { significant } \quad \text { wave } \\
\text { height; maximum } 9.5 \mathrm{~m} \\
\text { 05Dec2013 23:30 }\end{array}$ & $\begin{array}{ll}\text { FINO1 } & \text { waverider } \\
\text { checked } & \end{array}$ & 2-9 Dec 2013 & $\begin{array}{l}\text { Peter Frohle \& Norman Dreier, EarlyDike - Sensor - und risiko } \\
\text { basiertes Fruhwarnsystem fuer Seedeiche, Teilprojekt: } \\
\text { Wellenmonitoring und Wellenbelastungssimulator (AP2), } \\
\text { Hamburg, April } 2017\end{array}$ \\
\hline $\begin{array}{l}{[\text { FIG] Time series }} \\
\text { significant } \quad \text { wave } \\
\text { height; maximum } 7.9 \mathrm{~m} \\
\text { 06Dec2013 02:00 }\end{array}$ & $\begin{array}{l}\text { Aussen Elbe buoy } \\
\text { checked }\end{array}$ & 2-9 Dec 2013 & $\begin{array}{l}\text { Peter Frohle \& Norman Dreier, EarlyDike - Sensor - und risiko } \\
\text { basiertes Fruhwarnsystem fuer Seedeiche, Teilprojekt: } \\
\text { Wellenmonitoring und Wellenbelastungssimulator (AP2), } \\
\text { Hamburg, April } 2017\end{array}$ \\
\hline $\begin{array}{l}\text { [FIG] Spectrum of } \\
\text { wave energy; wind } \\
\text { waves at } 11 \mathrm{~s} \text {, swell at } \\
13 \mathrm{~s} \text { (highest), highest } \\
\text { observable energy at } \\
20 \text { s }\end{array}$ & FINO1 waverider buoy & $\begin{array}{l}\text { Dec2013 } \\
22: 44 U T C\end{array}$ & $\begin{array}{l}\text { Peter Frohle \& Norman Dreier, EarlyDike - Sensor - und risiko } \\
\text { basiertes Fruhwarnsystem fuer Seedeiche, Teilprojekt: } \\
\text { Wellenmonitoring und Wellenbelastungssimulator (AP2), } \\
\text { Hamburg, April } 2017\end{array}$ \\
\hline $\begin{array}{l}\text { [FIG] Time series of } \\
\text { signficant wave height }\end{array}$ & Helgoland wave buoys & 3-8 Dec 2013 & $\begin{array}{l}\text { Staneva J, C Schrum, A Behrens, S Grayek, H Ho-Hagemann, V } \\
\text { Alan, O Breivik, J-R. Bidlot, A North Sea-Baltic Sea Regional } \\
\text { Coupled Models: Atmosphere, wind waves and ocean, in } \\
\text { Proceedings of the Eigth EuroGOOS International Conference } \\
\text { (Operational Oceanography. Serving Sustainable Marine } \\
\text { Development), 3-5 October 2017, Bergen, Norway, 2017b. }\end{array}$ \\
\hline $\begin{array}{l}\text { [FIG] Time series } \\
\text { graph of signficant } \\
\text { wave height }\end{array}$ & FINO1 & 3-7Dec2013 & $\begin{array}{l}\text { Wahle K, J Staneva, W Koch, L Fenoglio-Marc, HTM Ho- } \\
\text { Hagemann, EV Stanev, An atmosphere-wave regional } \\
\text { coupled model: improving prediction of wave heights in the } \\
\text { southern North Sea, Ocean Sci., 13, 289-301, } \\
\text { 2017.(doi:10.5194/os-13-289-2017). }\end{array}$ \\
\hline $\begin{array}{l}\text { [FIG] Latitude profile } \\
\text { graph of altimeter- } \\
\text { derived significant } \\
\text { wave height from } \\
\text { SARAL-AltiKa }\end{array}$ & North Sea axis $53-60 \mathrm{~N}$ & $\begin{array}{lll}6 & \text { Dec } 2013 \\
04: 46: 55 \text { UTC }\end{array}$ & $\begin{array}{l}\text { Wahle K, J Staneva, W Koch, L Fenoglio-Marc, HTM Ho- } \\
\text { Hagemann, EV Stanev, An atmosphere-wave regional } \\
\text { coupled model: improving prediction of wave heights in the } \\
\text { southern North Sea, Ocean Sci., 13, 289-301, } \\
\text { 2017.(doi:10.5194/os-13-289-2017). }\end{array}$ \\
\hline $\begin{array}{l}\text { [FIG] Time series } \\
\text { graph of measured } \\
\text { significant wave height }\end{array}$ & $\begin{array}{l}\text { Helgoland } \\
\text { Westerland }\end{array}$ & 3-8 Dec 2013 & $\begin{array}{l}\text { Wahle K, J Staneva, W Koch, L Fenoglio-Marc, HTM Ho- } \\
\text { Hagemann, EV Stanev, An atmosphere-wave regional } \\
\text { coupled model: improving prediction of wave heights in the } \\
\text { southern North Sea, Ocean Sci., 13, 289-301, } \\
\text { 2017.(doi:10.5194/os-13-289-2017). }\end{array}$ \\
\hline $\begin{array}{l}\text { [FIG] Time series } \\
\text { graph of signficant } \\
\text { wave height }\end{array}$ & FINO1 & $\begin{array}{lr}2 & \text { weeks } \\
\text { across } & \text { storm } \\
\text { period } & \end{array}$ & $\begin{array}{l}\text { Wahle K, J Staneva, W Koch, L Fenoglio-Marc, HTM Ho- } \\
\text { Hagemann, EV Stanev, An atmosphere-wave regional } \\
\text { coupled model: improving prediction of wave heights in the } \\
\text { southern North Sea, Ocean Sci., 13, 289-301, } \\
\text { 2017.(doi:10.5194/os-13-289-2017). }\end{array}$ \\
\hline $\begin{array}{l}\text { [FIG] Time series of } \\
\text { significant wave height }\end{array}$ & Untjehoern wave gauge & 5-6 Dec 2013 & $\begin{array}{l}\text { Dreier, Norman and Peter Froehle, Operational wave forecast in } \\
\text { the German Bight as part of a sensor- and risk based early } \\
\text { warning system, In: J-S Shim, I Chun, HS Lim (ed), } \\
\text { Proceedings from the International Coastal Symposium (ICS) } \\
2018 \text { (Busan, Republic of Korea), Journal of Coastal } \\
\text { Research, Special Issue No. 85, 1161-1165, } 2018\end{array}$ \\
\hline $\begin{array}{l}\text { [TABLE] Highest } \\
\text { significant wave height } \\
8.6 \mathrm{~m}\end{array}$ & Nymindegab & 5-9Dec2013 & $\begin{array}{l}\text { Kystdirektoratet, Shoreface nourishment effects. An analysis of } \\
\text { the 2011 nourishment performed at Skodbjerge. } \\
\text { Kystdirektoratet, Hojbovej } 1,7620 \text { Lemvig, Dec } 2018 \text { [pdf } \\
\text { document properties: title=Shoreface nourishment } \\
\text { effects_Skodbjerge_20.12.2018, datestamp=20/12/2018] }\end{array}$ \\
\hline
\end{tabular}

Table S13. Wave period and other wave data (arranged by year and then alphabetically)

\begin{tabular}{|c|c|c|c|}
\hline Data type & Location & Time Interval & Full Reference and Notes \\
\hline $\begin{array}{l}{[\mathrm{FIG}] \text { Wave period }(15 \mathrm{~s})} \\
\text { from high frequency radar } \\
\text { gauge trace }\end{array}$ & FINO1 & $\begin{array}{l}6 \text { Dec } 2013 \\
00: 09-00: 11\end{array}$ & $\begin{array}{l}\text { BFG, Orkan Xaver: BfG beobachtet extreme Wellen in der } \\
\text { Nordsee (06.12.2013) }\end{array}$ \\
\hline $\begin{array}{l}\text { [FIG] Time series of } \\
\text { average wave period and } \\
\text { peak period }\end{array}$ & $\begin{array}{l}\text { Westhinder, Akkaert, Bol } \\
\text { van Heist, Oostende, } \\
\text { Scheur Wielingen }\end{array}$ & 5-6 Dec 2013 & $\begin{array}{l}\text { Oceanografisch Meteorologisch Station, Stormverslag 05- } \\
06 \text { december 2013, 26pp, 2013 [pdf document } \\
\text { properties: author=Myriam Sys; datestamp=15Dec2013] }\end{array}$ \\
\hline $\begin{array}{l}\text { [FIG] Time series of } \\
\text { average wave period; } \\
\text { maximum of } 30 \text { minute }\end{array}$ & FINO1 & 4-9 Dec 2013 & $\begin{array}{l}\text { Blasi C, S Mai, J Wilhelmi, T Zenz, U Barjenbruch, A } \\
\text { powerful method of measuring sea wave spectra and } \\
\text { their direction, ICHE 2014, Hamburg - Lehfeldt and }\end{array}$ \\
\hline
\end{tabular}




\begin{tabular}{|c|c|c|c|}
\hline average wave period $11.1 \mathrm{~s}$ & & & $\begin{array}{l}\text { Kopmann (eds), Bundesanstalt fuer Wasserbau, } 2014 . \\
\text { ISBN 978-3-939230-32-8 }\end{array}$ \\
\hline $\begin{array}{l}\text { [TABLE] Wave } \\
\text { period and energy }\end{array}$ & $\begin{array}{l}\text { Newbiggin Ness, Tyne } \\
\text { Tees, Whitby waverider }\end{array}$ & 5-6 Dec 2013 & $\begin{array}{l}\text { CH2MHill Halcrow, Cell 1 Regional Coastal Monitoring } \\
\text { Programme, Wave Data Analysis Report 2: } 2013-2014, \\
\text { Final Report, March 2014 [document properties: } \\
\text { author=Andy.Parson@ch2m.com; } \\
\text { 04/04/2014] }\end{array}$ \\
\hline $\begin{array}{l}{[\mathrm{FIG}] \text { Time series of mean }} \\
\text { period, peak period, and } \\
\text { wave direction }\end{array}$ & $\begin{array}{l}\text { Ormen Lange Norwegian } \\
\text { Sea Platform }\end{array}$ & $\begin{array}{l}1-31 \\
2013\end{array}$ & $\begin{array}{l}\text { FUGRO GEOS Ltd, Ormen Lange Monthly reports of } \\
\text { wavescan data: December. Reporting period: } 1 \\
\text { December } 2013 \text { to } 31 \text { December 2013, Report Number: } \\
\text { C70101/8177/R0, Issue date: } 23 \text { January 2014, prepared } \\
\text { by Heather Holt, Checked by Donald Brockie, approved } \\
\text { by Mark Jones }\end{array}$ \\
\hline \begin{tabular}{lrrr} 
FIG] & \multicolumn{2}{c}{ Wave } & spectra \\
grapshs & at & $2-5$ & hour \\
intervals & & &
\end{tabular} & Buoys near Ameland inlet & 5-6 Dec 2013 & $\begin{array}{l}\text { Gautier C, A Camarena, J van Nieuwkoop, SWAN } \\
\text { hindcasts Wadden Sea, December 2013. Tidal inlet of } \\
\text { Ameland and eastern Wadden Sea. Deltares, 2014, } \\
\text { 197pp. Project 1209433-007, Reference 1209433-007- } \\
\text { HYE-0005 }\end{array}$ \\
\hline $\begin{array}{l}\text { [FIG] Wave spectra graphs } \\
\text { at 2-5 hour intervals }\end{array}$ & $\begin{array}{l}\text { Buoys in eastern Wadden } \\
\text { Sea }\end{array}$ & $5-6$ Dec 2013 & $\begin{array}{l}\text { Gautier C, A Camarena, J van Nieuwkoop, SWAN } \\
\text { hindcasts Wadden Sea, December 2013. Tidal inlet of } \\
\text { Ameland and eastern Wadden Sea. Deltares, 2014, } \\
\text { 197pp. Project 1209433-007, Reference 1209433-007- } \\
\text { HYE-0005 }\end{array}$ \\
\hline $\begin{array}{l}\text { [FIG] Time series of wave } \\
\text { peak period and wave } \\
\text { direction }\end{array}$ & $\begin{array}{l}\text { Wave measurement buoy } \\
\text { Westerland }\end{array}$ & 2-9Dec2013 & $\begin{array}{l}\text { Luecht, Fabian and Ove Peters, Bericht ueber die Sturmflut } \\
\text { vom 05.-0.6.12.2013 an der Westkueste Schleswig- } \\
\text { Holsteins, Landesbetrieb fuer Kuestenschutz, } \\
\text { Nationalpark und Meeresschutz Schleswig-Holstein, } \\
\text { Husum 26Feb2014, 19pp. [pdf document properties: } \\
\text { title=Lfd; Author=Thorsten Nommensen; datestamp: } \\
\text { 11Dec2018] }\end{array}$ \\
\hline $\begin{array}{l}{[\mathrm{FIG}] \text { Time series of }} \\
\text { average wave period and } \\
\text { wave peak period }\end{array}$ & $\begin{array}{l}\text { FINO1, } \\
\text { Suedstrand }\end{array}$ & 4-9 Dec 2013 & $\begin{array}{l}\text { Mai S, J Wilhelmi, T Zenz, U Barjenbruch, Orkan 'Xaver' - } \\
\text { Seegangsstatistik an den Stationen FINO1 und Borkum- } \\
\text { Suedstrand, 19. KFKI-Seminar, Bremerhaven } \\
\text { 11.11.2014 (presentation slides) }\end{array}$ \\
\hline $\begin{array}{l}\text { [FIG] Spectrograms of } \\
\text { wave energy on axes of } \\
\text { frequency versus time }\end{array}$ & $\begin{array}{l}\text { FINO1, } \\
\text { Suedstrand }\end{array}$ & 4-9 Dec 2013 & $\begin{array}{l}\text { Mai S, J Wilhelmi, T Zenz, U Barjenbruch, Orkan 'Xaver' - } \\
\text { Seegangsstatistik an den Stationen FINO1 und Borkum- } \\
\text { Suedstrand, 19. KFKI-Seminar, Bremerhaven } \\
\text { 11.11.2014 (presentation slides) }\end{array}$ \\
\hline $\begin{array}{l}\text { [FIG] Time series of wave } \\
\text { period }\end{array}$ & Westerland & 4-8 Dec 2013 & $\begin{array}{l}\text { Matelski, Birgit, Frerk Jensen, Peter Voss, Jorg Peters, } \\
\text { Maria Blumel, Auswertung zur Hydrologie des } \\
\text { Sturmtiefs Xaver 05.12.2013 bis 06.12.2013, } \\
\text { Statusbericht vom 01.10.2014, Landesbetrieb fuer } \\
\text { Kuestenschutz, Nationalpark und Meeresschutz, } \\
\text { Schleswig-Holstein, Husum, 01.10.2014, 25pp. }\end{array}$ \\
\hline $\begin{array}{l}{[\mathrm{FIG}] \text { Time series of }} \\
\text { average upcrossing period } \\
\text { and wave peak period }\end{array}$ & Draugen & $\begin{array}{l}1-31 \\
2013\end{array}$ & $\begin{array}{l}\text { MIROS, Monthly Report, Draugen, December } 2013 \text {, Doc. } \\
\text { No. ND/1022/13/12 (prepared by SRS, checked by OO, } \\
\text { approved by CNE) [pdf document properties: } \\
\text { author=MIROS AS; datestamp=30Jan2014] }\end{array}$ \\
\hline $\begin{array}{l}{[\mathrm{FIG} \text { Time series of }} \\
\text { average wave period from } \\
\text { spectral analysis and time } \\
\text { series data; wave rider and } \\
\text { altimeter }\end{array}$ & Ekofisk & $\begin{array}{l}1-31 \\
2013\end{array}$ & $\begin{array}{l}\text { MIROS, Monthly report, Ekofisk, December 2013, Doc. } \\
\text { No. ND/1024/13/12, (prepared by SRS) 34pp, 9Jan2014 } \\
\text { [PDF document properties: author=Miros AS; } \\
\text { datestamp=13Jan2014] }\end{array}$ \\
\hline $\begin{array}{l}\text { [FIG] Average upcrossing } \\
\text { period and primary wave } \\
\text { peak period }\end{array}$ & Heidrun & $\begin{array}{l}1-31 \\
2013\end{array}$ & $\begin{array}{l}\text { MIROS, Manedsrapport Heidrun, Desember 2013, Dok. Nr. } \\
\text { ND/1010/13/12, } 21 \text { pp, 07Jan2014, carried out by SRS, } \\
\text { controlled by CNE, approved by OO [pdf properties: } \\
\text { author=Miros AS; date stamp: } 08 / 01 / 2014 \text { ] }\end{array}$ \\
\hline $\begin{array}{l}\text { [FIG] Average upcrossing } \\
\text { period and primary wave } \\
\text { peak period }\end{array}$ & Norne & $\begin{array}{l}1-31 \\
2013\end{array}$ & $\begin{array}{l}\text { MIROS, Maanedsrapport Norne, Desember } 2013 \text {, Dok. Nr. } \\
\text { ND/1087/13/12, 21pp, carried out by SRS, controlled by } \\
\text { CNE, approved by OO [pdf properties: Author=Miros } \\
\text { AS; datestamp: 06Jan2014] }\end{array}$ \\
\hline $\begin{array}{l}{[\mathrm{FIG} \text { Time series of }} \\
\text { average wave period from } \\
\text { spectral analysis and time } \\
\text { series data; wave rider and } \\
\text { altimeter }\end{array}$ & Sleipner A & $\begin{array}{l}1-31 \\
2013\end{array}$ & $\begin{array}{l}\text { MIROS, Manedsrapport Sleipner A, Desember 2013, Dok. } \\
\text { Nr. ND/1017/13/12, 06Jan2014, prepared by SRS, } \\
\text { controlled by CNE, approved by OO. }\end{array}$ \\
\hline $\begin{array}{l}{[\mathrm{FIG}] \text { Time series of }} \\
\text { average upcrossing wave } \\
\text { period, peak period of } \\
\text { primary wave, average } \\
\text { wave direction }\end{array}$ & Troll A & $\begin{array}{l}1-31 \\
2013\end{array}$ & $\begin{array}{l}\text { MIROS, Manedsrapport, Troll A, Desember 2013, Doc. No. } \\
\text { ND/1012/13/12, (prepared by SRS) 21pp, 7Jan2014 } \\
\text { [PDF document properties: author=Miros AS; } \\
\text { datestamp=10Jan2014] }\end{array}$ \\
\hline $\begin{array}{l}\text { [FIG] Time series of waver } \\
\text { period }\end{array}$ & $\begin{array}{l}\text { Scheur west Wandelaar, } \\
\text { Euro platform, IJmuiden, } \\
\text { Platform K13a }\end{array}$ & 5-6 Dec 2013 & $\begin{array}{l}\text { RWS, Stormvloedrapport van } 5 \mathrm{t} / \mathrm{m} 7 \text { december (SR91) } \\
\text { Sint-Nicolaasvloed 2013, Watermanagementcentrum } \\
\text { Nederland, Rijkswaterstaat, prepared by Ing. J. Kroos, }\end{array}$ \\
\hline
\end{tabular}




\begin{tabular}{|c|c|c|c|}
\hline & & & 19 Mar 2014b, 48 pp \\
\hline $\begin{array}{l}\text { [TABLE] Table of } \\
\text { maximum 'globally } \\
\text { corrected' wave periods in } \\
\text { ranked storm list }\end{array}$ & $\begin{array}{l}\text { Scheur west Wandelaar, } \\
\text { Euro platform, IJmuiden } \\
\text { munitiestortplaats, } \\
\text { Eierlandse Gat, } \\
\text { (Schiermonnikoog Nord }\end{array}$ & 5-6 Dec 2013 & $\begin{array}{l}\text { RWS, Stormvloedrapport van } 5 \mathrm{t} / \mathrm{m} 7 \text { december (SR91) } \\
\text { Sint-Nicolaasvloed 2013, Watermanagementcentrum } \\
\text { Nederland, Rijkswaterstaat, prepared by Ing. J. Kroos, } \\
19 \text { Mar 2014b, } 48 \text { pp }\end{array}$ \\
\hline $\begin{array}{l}\text { [FIG] Time series of wave } \\
\text { peak period }\end{array}$ & $\begin{array}{l}\text { Wave buoy 1_1 Amelander } \\
\text { Zeegat }\end{array}$ & 5-7Dec2013 & $\begin{array}{l}\text { van Rooijen A, A Oost, Memo: Regionale advisering } \\
\text { Ameland Noordwest, Deltares, 1209381-008-ZKS-0008, } \\
\text { 43pp, 18Dec2014 [PDF document properties: } \\
\text { titl=Regional advisering: Ameland NW; author=Arnold } \\
\text { van Rooijen; keywords: 1209381-008-ZKS-0008; date } \\
\text { stamp: 18/12/2014] }\end{array}$ \\
\hline $\begin{array}{l}\text { [FIG] Time series of peak } \\
\text { period and wave direction }\end{array}$ & SCHW, SCHO, WIEL & 5-7 Dec 2013 & $\begin{array}{l}\text { Carrion Aretxabala, BI, Morphological impact of the } \\
\text { Sinterklaas storm at Het Zwin. Numerical modelling } \\
\text { with Xbeach, M.Sc. Civil Engineering, Delft University } \\
\text { of Technology, 2015. }\end{array}$ \\
\hline $\begin{array}{l}\text { [FIG] Time series of wave } \\
\text { period and wave direction } \\
\text { from Nortek AWAK } \\
\text { instrument } 600 \mathrm{~m} \text { from } \\
\text { shore }\end{array}$ & $\begin{array}{l}\text { Beach profile } 104 \text { south of } \\
\text { Ostend }\end{array}$ & 4-8 Dec 2013 & $\begin{array}{l}\text { Dan, Sebastian, Anne-Lise Montreuil, Rosalia Delgado, } \\
\text { Tomas van Oyen, Large storm impact on a beach under } \\
\text { sand nourishments, The Proceedings of the Coastal } \\
\text { Sediments 2015, edited by Ping Wang, Julie D Rosati, } \\
\text { and Jun Cheng, Coastal Sediments 2015, San Diego, } \\
\text { USA, 11-15May2015 }\end{array}$ \\
\hline $\begin{array}{l}\text { [TEXT] reference to peak } \\
\text { period and wave direction } \\
\text { information for } 2012 \text { to } \\
2013 \text { across storm period }\end{array}$ & FINO1 & ? & $\begin{array}{l}\text { Fischer, JG, C Senet, A Schneehorst, O Outzen, S Schirmel, } \\
\text { K Herklotz, Sea state measurements in Germanys first } \\
\text { offshore wind farm "alpha ventus", in the south-eastern } \\
\text { parts of the North Sea, } 2015 \text { IEEE/OES Eleventh } \\
\text { Current, Waves and Turbulence Measurement } \\
\text { (CWTM), 2015 [PDF document properties: datestamp: } \\
\text { 14/01/2015] }\end{array}$ \\
\hline $\begin{array}{l}{[\mathrm{FIG}] \text { Time series of wave }} \\
\text { period }\end{array}$ & Liverpool, Sizewell & 4-6 Dec 2013 & $\begin{array}{l}\text { Wadey MP, JM Brown, ID Haigh, T Dolphin, P Wisse, } \\
\text { Assessment and comparison of extreme sea levels and } \\
\text { waves during the 2013/2014 storm season in two UK } \\
\text { coastal regions, Nat. Hazards Earth Syst. Sci. Discuss., } \\
\text { 3, 2665-2708, 2015b. }\end{array}$ \\
\hline $\begin{array}{l}{[\mathrm{FIG}] \text { Time series of }} \\
\text { average wave period }\end{array}$ & $\begin{array}{l}\text { Lighthouse Alte Weser, } \\
\text { Borkum Suedstrand }\end{array}$ & 4-8 Dec 2013 & $\begin{array}{l}\text { Mai S and U Barjenbruch, Water level measurements with } \\
\text { radar gauges at the German North Sea coast, [PDF } \\
\text { document properties: author=IOC; subject: } \\
\text { IOC/2016/MG/14 vol.5; datestamp: } 18 / 04 / 2017 \text { ] }\end{array}$ \\
\hline $\begin{array}{l}\text { [FIG] Time series of wave } \\
\text { period }\end{array}$ & $\begin{array}{l}\text { Seeganssmessstation } \\
\text { Westerland }\end{array}$ & 4-8 Dec 2013 & $\begin{array}{l}\text { Matelski, Birgit, Erfahrungen aus der Sturmflut Xaver von } \\
\text { 5. und 6.12.2013 und dem Weihnachshochwasser } 2014 \\
\text { in Shleswig-Holstein, IWASA 2016 Tagungsbeitrag, } \\
\text { (46. IWASA, 7-8 Januar 2016; Internationales } \\
\text { Wasserbau-Symposium Aachen. [pdf document } \\
\text { properties: autor=sonja; datestamp: 26Apr2016] }\end{array}$ \\
\hline $\begin{array}{l}\text { [FIG] Wave period and } \\
\text { direction }\end{array}$ & Amelander Zeegat buoy & 5-8 Dec 2013 & $\begin{array}{l}\text { Nederhoff K, E Elias, T Vermaas, Erosie op Ameland } \\
\text { Noordwest. Modelstudie: simulaties met Delft3D en } \\
\text { XBeach, Deltares, 117pp, July, 2016 }\end{array}$ \\
\hline $\begin{array}{l}\text { [TEXT] wave peak period } \\
10 \text { s from ADCP }\end{array}$ & Hornbaek & 5Dec2013 & $\begin{array}{l}\text { Sorensen CS, NK Dronen, P Knudsen, J Jensen, P } \\
\text { Sorensen, An extreme event as a games changer in } \\
\text { coastal zone management, Journal of Coastal Research, } \\
\text { (Special Issue, No 75), 700-704, 2016. Proceedings of } \\
\text { the 14th International Coastal Symposium (Sydney, } \\
\text { Australia) ed by A Vila-Concejo, E Bruce, DM } \\
\text { Kennedy, RJ McCarroll }\end{array}$ \\
\hline $\begin{array}{l}\text { [FIG] Two dimensional } \\
\text { graph of wave spectral } \\
\text { density versus frequency } \\
\text { and time }\end{array}$ & Elbe buoy and FINO1 buoy & 2-8 Dec 2013 & $\begin{array}{l}\text { Staneva J, K Wahle, W Koch, A Behrens, L Fenoglio-Marc, } \\
\text { EV Stanev, Coastal flooding: impact of waves on storm } \\
\text { surge during extremes - a case study for the German } \\
\text { Bight, Nat. Hazards Earth Syst. Sci., 16, 2373-2389, } \\
2016\end{array}$ \\
\hline $\begin{array}{l}\text { [FIG] Two dimensional } \\
\text { graph of wave spectral } \\
\text { density versus frequency } \\
\text { and time }\end{array}$ & Elbe buoy & 1-8 Dec 2013 & 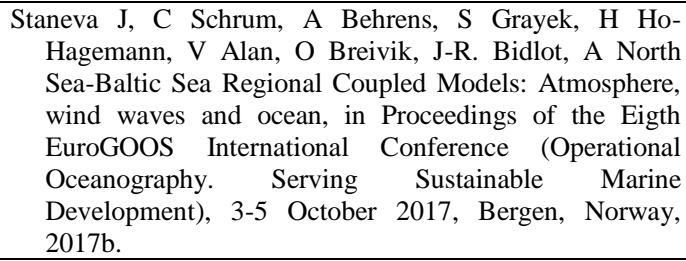 \\
\hline $\begin{array}{l}{[\mathrm{FIG} \text { Energy spectrum }} \\
\text { from wave buoy for } \\
\text { different directions }\end{array}$ & FINO1 & $\begin{array}{l}5 \text { Dec } 2013 \\
\text { 22:44UTC }\end{array}$ & $\begin{array}{l}\text { J Jensen, S Niehuser, A Arns, S Dangendorf, Sensor- und } \\
\text { risikobasiertes Fruhwarn-system fuer Seedeiche } \\
\text { (EarlyDike), AP1 - Sturmflutmonitoring und } \\
\text { Sturmflutssimulator - Fachbericht 2016, Siegen, April } \\
2017\end{array}$ \\
\hline [TABLE] peak period & Nymindegab & 5-9 Dec 2013 & $\begin{array}{l}\text { Kystdirektoratet, Shoreface } \begin{array}{c}\text { nourishment } \\
\text { effects. }\end{array} \\
\text { analysis of the } 2011 \text { nourishment performed } \\
\text { at }\end{array}$ \\
\hline
\end{tabular}




\begin{tabular}{|l|l|l|l|}
\hline & & $\begin{array}{l}\text { Skodbjerge. Kystdirektoratet, Hojbovej 1, 7620 Lemvig, } \\
\text { Dec 2018 [pdf document properties: title=Shoreface } \\
\text { nourishment effects_Skodbjerge_20.12.2018, } \\
\text { datestamp=20/12/2018] }\end{array}$ \\
\hline
\end{tabular}

Table S14. Surge reports and quantitative water levels (arranged by year and then alphabetically)

\begin{tabular}{|c|c|c|c|}
\hline Data type & Location & Time Interval & Full Reference and Notes \\
\hline $\begin{array}{l}\text { [TABLE] MHW, true surge, } \\
\text { skew surge on each } \\
\text { semidiurnal tidal cycle }\end{array}$ & $\begin{array}{l}\text { Helgoland, Borkum, Emden, } \\
\text { Wilhelmshaven, } \\
\text { Bremerhaven, Bremen, } \\
\text { Cuxhaven, Brunsbuettel, } \\
\text { Hamburg, Zollenspieker, } \\
\text { Buesum, Eidersperrwerk, } \\
\text { Husum, Dagebuell }\end{array}$ & 5-7Dec2013 & $\begin{array}{l}\text { BSH, Die Nordseesturmfluten von } 5 \text {. und } 6.12 .2013 \text {, 3pp, } \\
\text { prepared by Stockmann,K. (title: Sturmflut 2011_bm1101; } \\
\text { author stamp: bm1101; document time stamp: } 12 / 102013 \\
\text { 12:5136PM) }\end{array}$ \\
\hline $\begin{array}{l}\text { [FIG] ABB12. Time series } \\
\text { of water level with lines for } \\
\text { MThw and MTnw }\end{array}$ & $\begin{array}{l}\text { Husum and Norderney- } \\
\text { Riffgat }\end{array}$ & 5-8 Dec 2013 & $\begin{array}{l}\text { Deutschlander T, K Frierich, S Haeseler, C Lefebvre, } \\
\text { Orkantief XAVER ueber Nordeuropea von 5. bis } 7 . \\
\text { Dezember 2013, Deutscher Wetterdienst DWD, Stand } 30 . \\
\text { Dezember 2013, 19pp. }\end{array}$ \\
\hline $\begin{array}{l}\text { [FIG] ABB13. Time series } \\
\text { of water level with lines for } \\
\text { MThw and MTnw }\end{array}$ & $\begin{array}{l}\text { Flensburg and } \\
\text { Warnemuende }\end{array}$ & 5-8 Dec 2013 & $\begin{array}{l}\text { Deutschlander T, K Frierich, S Haeseler, C Lefebvre, } \\
\text { Orkantief XAVER ueber Nordeuropea von 5. bis } 7 . \\
\text { Dezember 2013, Deutscher Wetterdienst DWD, Stand } 30 . \\
\text { Dezember 2013, 19pp. }\end{array}$ \\
\hline $\begin{array}{l}\text { [FIG] ABB13. Time series } \\
\text { of water level with lines for } \\
\text { MThw and MTnw }\end{array}$ & Hamburg St. Pauli & 5-8 Dec 2013 & $\begin{array}{l}\text { Deutschlander T, K Frierich, S Haeseler, C Lefebvre, } \\
\text { Orkantief XAVER ueber Nordeuropea von 5. bis } 7 . \\
\text { Dezember 2013, Deutscher Wetterdienst DWD, Stand } 30 . \\
\text { Dezember 2013, 19pp. }\end{array}$ \\
\hline $\begin{array}{l}\text { [TEXT] Maximum water } \\
\text { levels }\end{array}$ & $\begin{array}{lr}\text { Hamburg, } & \text { Dagebuell, } \\
\text { Husum, } & \text { Buesum, } \\
\text { Eidersperrwerk, } & \text { Cuxhaven }\end{array}$ & 5-6 Dec 2013 & $\begin{array}{l}\text { Kunz M, B Muehr, K Schroeter, T Bessel, S Moehrle, T } \\
\text { Muenzberg, S Brink, H-M Schmidt, Winterstorm Xaver - } \\
\text { Report. 06Dec2013 - Report No.1, Situation Report - } \\
\text { 19:00CET, CEDIM Forensic Disaster Analysis Group } \\
\text { (FDA), Center for Disaster Management and Risk } \\
\text { Reduction Technology. }\end{array}$ \\
\hline $\begin{array}{l}\text { [TABLE] Water level } \\
\text { maximum and minimum; } \\
\text { true surge maximum and } \\
\text { minimum }\end{array}$ & $\begin{array}{l}\text { Aberdeen, Cromer, Dover, } \\
\text { Harwich, Immingham, } \\
\text { Leith, Lerwick, Lowestoft, } \\
\text { North Shields, Sheerness, } \\
\text { Whitby, Wick }\end{array}$ & 5-7 Dec 2013 & $\begin{array}{l}\text { McGarricle P (ed), UK coastal monitoring and forecasting: } \\
\text { Annual report for } 2013 \text { for the UK National Tide Gauge } \\
\text { Network, NERC } 100017897, \quad 2013 \quad \text { [pdf document } \\
\text { properties: author=pamcg; created=16Apr2014] }\end{array}$ \\
\hline $\begin{array}{l}\text { [FIG] Saral-AltiKa strip map } \\
\text { of water level }\end{array}$ & $\begin{array}{l}\text { North-south transect of } \\
\text { North Sea }\end{array}$ & $\begin{array}{l}\text { 06Dec2013 } \\
\text { 04:47UTC }\end{array}$ & $\begin{array}{l}\text { Mills, Ian, Remko Scharoo, Luciana Fenoglio, Xaver affected } \\
\text { much of northern Europe on } 5 \text { and } 6 \text { December and caused } \\
\text { worst storm surge for decades in the North Sea. } \\
\text { https://www.eumetsat.int/website/home/News/DAT_20870 } \\
\text { 62.html (last accessed: 19Nov2019, 28Jun2020) }\end{array}$ \\
\hline $\begin{array}{l}\text { [FIG] TIme series of water } \\
\text { level anomaly }\end{array}$ & $\begin{array}{l}\text { Helgoland Suedhaven, } \\
\text { Leuchtturm Alte Weser }\end{array}$ & 4-6 Dec 2013 & $\begin{array}{l}\text { Mills, Ian, Remko Scharoo, Luciana Fenoglio, Xaver affected } \\
\text { much of northern Europe on } 5 \text { and } 6 \text { December and caused } \\
\text { worst storm surge for decades in the North Sea. } \\
\text { https://www.eumetsat.int/website/home/News/DAT_20870 } \\
\text { 62.html (last accessed: 19Nov2019, 28Jun2020) }\end{array}$ \\
\hline $\begin{array}{l}{[\text { TEXT] Maximum water }} \\
\text { level NAP }\end{array}$ & Vlissingen & $\begin{array}{l}\text { 6Dec2013 } \\
04: 00 \mathrm{MEZ}\end{array}$ & $\begin{array}{l}\text { NOS, Hoogste waterstand sinds } 1953,06 / 12 / 2013,09: 52 \text {, } \\
\text { https://nos.nl/artikel/583218-hoogste-waterstand-sinds- } \\
\text { 1953.html }\end{array}$ \\
\hline $\begin{array}{l}\text { [FIG] Time series of water } \\
\text { level, astronomical tide and } \\
\text { true surge residual }\end{array}$ & Oostende, Zeebrugge & 5-6 Dec 2013 & $\begin{array}{l}\text { Oceanografisch Meteorologisch Station, Stormverslag 05-06 } \\
\text { december 2013, 26pp, } 2013 \text { [pdf document properties: } \\
\text { author=Myriam Sys; datestamp=15Dec2013] }\end{array}$ \\
\hline $\begin{array}{l}\text { [TABLE] water level of the } \\
\text { midnight flood of } 6 \text { Dec } 2013 \\
\text { (values over average high } \\
\text { water) }\end{array}$ & $\begin{array}{l}\text { Borkum, Norderney, } \\
\text { Langeoog, Spiekeroog, } \\
\text { Wangeroog West, Leyhoern, } \\
\text { Bensersiel, Emssperrwerk, } \\
\text { Knock,Emden, Vareler } \\
\text { Schleuse, Fedderwardersiel, } \\
\text { Wilhelmshaven, Cuxhaven, } \\
\text { Huntesperrwerk, } \\
\text { Ochtumsperrwerk, Spieka } \\
\text { Neufeld, Otterndorf, Stader } \\
\text { Sand }\end{array}$ & $\begin{array}{l}\text { Midnight } \\
\text { flood, } 6 \text { Dec } \\
2013\end{array}$ & $\begin{array}{l}\text { NLWKN, Schwere Sturmflut gut Ueberstanden, Waserstaende } \\
\text { zwischen Zweieinhale und Knapp vier metern registriert // } \\
\text { presseinformation von 6. Dezember 2013, } \\
\text { (Ansprechpartnerin: Herma Heyken), 06/12/2013. }\end{array}$ \\
\hline $\begin{array}{l}\text { [TEXT] Maximum water } \\
\text { levels }\end{array}$ & $\begin{array}{l}\text { Wiken, Barseback, } \\
\text { Halmstad, Malmo }\end{array}$ & 5-6 Dec 2013 & $\begin{array}{l}\text { SMHI, Stormen Sven gav nya vattenstandsrekord i Oresund, } \\
\text { https://www.smhi.se/nyhetsarkiv/stormen-sven-gav-nya- } \\
\text { vattenstandsrekord-i-oresund-1.34732, } \\
\text { 20Mar2017; original datestamp 10Dec2013. }\end{array}$ \\
\hline $\begin{array}{l}\text { [TEXT] Minimum water } \\
\text { level }\end{array}$ & Skanoor & 5-6 Dec 2013 & $\begin{array}{l}\text { SMHI, Stormen Sven gav nya vattenstandsrekord i Oresund, } \\
\text { https://www.smhi.se/nyhetsarkiv/stormen-sven-gav-nya- } \\
\text { vattenstandsrekord-i-oresund-1.34732, } \\
\text { 20Mar2017; original datestamp 10Dec2013. }\end{array}$ \\
\hline [TEXT] Maximum flood & Hamburg-St Pauli & 6 Dec 2013 & Unwetterzentrale, Orkantief XAVER - ein weiterer schwerer \\
\hline
\end{tabular}




\begin{tabular}{|c|c|c|c|}
\hline $\begin{array}{l}\text { water level at } 6.09 \mathrm{~m} \mathrm{NN} \text { and } \\
\text { skew surge at } 3.98 \mathrm{~m} \mathrm{uMH}\end{array}$ & & & $\begin{array}{l}\text { Wintersturm der letzten Jahrzehnte, Thomas Savert and } \\
\text { Stefan } \\
\text { http://www.unwetterzentrale.de/uwz/928.html }\end{array}$ \\
\hline $\begin{array}{l}\text { [TEXT] Skew surge } 3.54 \mathrm{~m} \\
\text { uMH }\end{array}$ & Cuxhaven & 6 Dec 2013 & $\begin{array}{l}\text { Unwetterzentrale, Orkantief XAVER - ein weiterer schwerer } \\
\text { Wintersturm der letzten Jahrzehnte, Thomas Savert and } \\
\text { Stefan } \\
\text { http://www.unwetterzentrale.de/uwz/928.html }\end{array}$ \\
\hline $\begin{array}{l}\text { [TABLE] water level, } \\
\text { expected, tide, residual surge }\end{array}$ & $\begin{array}{l}\text { Lerwick, Wick, Aberdeen, } \\
\text { Leith, North } \begin{array}{r}\text { Shields, } \\
\text { Immingham, }\end{array} \text { Cromer, } \\
\text { Lowestoft, Dover. }\end{array}$ & 5-6 Dec 2013 & $\begin{array}{l}\text { ABP mer, Ensuring Flood Resilience. An overview of the 5/6 } \\
\text { December 2013, Associated British Ports, Marine } \\
\text { Environmental Research, July, 2014 }\end{array}$ \\
\hline $\begin{array}{l}\text { [TEXT] base of water } 20 \\
\text { feet above normal with } 15 \\
\text { foot above that. }\end{array}$ & Hemsby UK & $\begin{array}{l}\text { Night 5-6 Dec } \\
2013\end{array}$ & $\begin{array}{l}\text { BBC, East coast surge: what happened next? (report by } \\
\text { Richard Haugh), 5Dec } 2014\end{array}$ \\
\hline $\begin{array}{l}\text { [TEXT] Highest storm flood } \\
\text { water levels }\end{array}$ & $\begin{array}{l}\text { North Shields, Whitby, } \\
\text { Scarborough }\end{array}$ & 5-6 Dec 2013 & $\begin{array}{l}\text { CH2MHill Halcrow, Cell 1 Regional Coastal Monitoring } \\
\text { Programme, Wave Data Analysis Report 2: } 2013-2014 \text {, } \\
\text { Final Report, March } 2014 \text { [document properties: } \\
\text { author=Andy.Parson@ @ ch2m.com; datestamp; 04/04/2014] }\end{array}$ \\
\hline $\begin{array}{l}\text { [FIGURE] Time series tide } \\
\text { gauge water lvel }\end{array}$ & $\begin{array}{l}\text { North Shields, Whitby, } \\
\text { Scarborough }\end{array}$ & 5-6 Dec 2013 & $\begin{array}{l}\text { CH2MHill Halcrow, Cell 1 Regional Coastal Monitoring } \\
\text { Programme, Wave Data Analysis Report 2: 2013-2014, } \\
\text { Final Report, March } 2014 \text { [document properties: } \\
\text { author=Andy.Parson@ @ ch2m.com; datestamp; 04/04/2014] }\end{array}$ \\
\hline $\begin{array}{l}\text { [FIGURE] Time series surge } \\
\text { residual }\end{array}$ & $\begin{array}{l}\text { North Shields, Whitby, } \\
\text { Scarborough }\end{array}$ & 5-6 Dec 2013 & $\begin{array}{l}\text { CH2MHill Halcrow, Cell 1 Regional Coastal Monitoring } \\
\text { Programme, Wave Data Analysis Report 2: } 2013-2014 \text {, } \\
\text { Final Report, March 2014 [document properties: } \\
\text { author=Andy.Parson@ @ ch2m.com; datestamp; 04/04/2014] }\end{array}$ \\
\hline $\begin{array}{l}{[\text { MAP } \quad \text { WITH } \quad \text { DATA }]} \\
\text { Maximum water levels }\end{array}$ & $\begin{array}{ll}\text { Immingham, } & \text { Cromer, } \\
\text { Lowestoft, } & \text { Harwich, } \\
\text { Sheernes, Dover, } & \text { Kingston } \\
\text { Upon Hull } & \end{array}$ & 5-6 Dec 2013 & $\begin{array}{l}\text { Dunbar I, N Phipps, M Szonyi, Risk Nexus. After the storm: } \\
\text { how the UK's flood defences performed during the surge } \\
\text { following Xaver, Flood resilience review 09.14, Zurich } \\
\text { Insurance Company Ltd., Mythenquai 2, 8002, Zurich, } \\
\text { Switzerland [document properties: date 28Aug2014; } \\
\text { author=Zurich] }\end{array}$ \\
\hline [TIMESERIES] Water level & Holbaek and Roskilde & 6-7 Dec 2013 & $\begin{array}{l}\text { Eriksen J, Rekordvandstande i Isefjorden og Roskilde Fjord, } \\
\text { Vejret, } 138,240-48,2014\end{array}$ \\
\hline $\begin{array}{l}\text { [TEXT] Highest surge water } \\
\text { level }\end{array}$ & Borkum & 6 Dec 2013 & $\begin{array}{l}\text { FINO1, 15-m wave damaged FINO1, 08Jan2014. } \\
\text { http://www.fino1.de/meldungen/alle-meldungen/137-15- } \\
\text { meter-welle-beschaedigt-fino1 }\end{array}$ \\
\hline $\begin{array}{l}{[\text { FIG] Time series of }} \\
\text { measured water level }\end{array}$ & $\begin{array}{l}\text { Terschelling Noordzee, Nes, } \\
\text { Wierumerwad, Lauwersoog, } \\
\text { Eemshaven, Nieuw } \\
\text { Statenzijl }\end{array}$ & 5-7 Dec 2013 & $\begin{array}{l}\text { Gautier C, A Camarena, J van Nieuwkoop, SWAN hindcasts } \\
\text { Wadden Sea, December 2013. Tidal inlet of Ameland and } \\
\text { eastern Wadden Sea. Deltares, 2014, 197pp. Project } \\
\text { 1209433-007, Reference 1209433-007-HYE-0005 }\end{array}$ \\
\hline $\begin{array}{l}\text { [TIMESERIES] Observed } \\
\text { water level and astronomical } \\
\text { tide }\end{array}$ & Vlissingen & $\begin{array}{l}\text { 2Dec2013 } \\
\text { 0000UTC to } \\
\text { 12Dec2013 } \\
\text { 0000UTC }\end{array}$ & $\begin{array}{l}\text { Hewson T, L Magnusson, O Breivik, F Prates, I Tsonevsky, } \\
\text { HJW de Vries, Windstorms in northwest Europe in late } \\
2013 \text {, ECMWF Newsletter, No 139, pp 22-28, Spring } \\
\text { 2014. }\end{array}$ \\
\hline $\begin{array}{l}\text { [TEXT] Maximum water } \\
\text { level over average high } \\
\text { water } 2.83 \mathrm{~m}\end{array}$ & Norderney & 5-6 Dec 2013 & $\begin{array}{l}\text { Knaack H and H Heyken, Xaver hatte sehr schwere Stumflut } \\
\text { im Gepaeck, Jahresbericht 2013. Der Zukunft verplichtet, } \\
\text { NLWKN, Niedersaechsischer Landesbetrieb fuer } \\
\text { Waserwirtschaft, Kuesten- und Naturschutz, pp.8-9, } \\
\text { document date stamp 24Apr2014. }\end{array}$ \\
\hline $\begin{array}{l}\text { [TEXT] Maximum water } \\
\text { level over average high } \\
\text { water } 2.83 \mathrm{~m}\end{array}$ & Norderney & 5-6 Dec 2013 & $\begin{array}{l}\text { Kristandt, J., B. Brecht, H. Frank, H. Knaack, Optimization of } \\
\text { empirical storm surge forecast-modeling of high resolution } \\
\text { wind fields, Die Küste, } 81,301-348,2014\end{array}$ \\
\hline $\begin{array}{l}\text { [TABLE] Highest absolute } \\
\text { water levels and MTHW } \\
\text { skew surges }\end{array}$ & $\begin{array}{l}\text { List, Hoernum, Wittduen, } \\
\text { Dagebuell, Hooge Anleger, } \\
\text { Husum, Eidersperrwerk, } \\
\text { Cuxhaven, } \\
\text { Stoersperrwerk, } \\
\text { Glueckstadt, Schulau, St. } \\
\text { Pauli }\end{array}$ & $5-6$ Dec 2013 & $\begin{array}{l}\text { Luecht, Fabian and Ove Peters, Bericht ueber die Sturmflut } \\
\text { vom 05.-0.6.12.2013 an der Westkueste Schleswig- } \\
\text { Holsteins, Landesbetrieb fuer Kuestenschutz, Nationalpark } \\
\text { und Meeresschutz Schleswig-Holstein, Husum 26Feb2014, } \\
\text { 19pp. [pdf document properties: title=Lfd; } \\
\text { Author=Thorsten Nommensen; datestamp: 11Dec2018] }\end{array}$ \\
\hline $\begin{array}{l}\text { [TIMESERIES] Water level } \\
\text { data }\end{array}$ & FINO1, FINO2, FINO3 & 1-8 Dec 2013 & $\begin{array}{l}\text { Leiding T, B Tinz, G Rosenhagen, C Lefevre, S Haeseler, S } \\
\text { Hagemann, I Bastigkeit, D Stein, P Schwenk, S Mueller, O } \\
\text { Outzen, K Herklotz, F Kinder, T Neumann, Meteorological } \\
\text { and Oceanographic Conditions at the FINO platforms } \\
\text { during the severe storms Christian and Xaver, DEWI } \\
\text { Magazin, No.44, p16-25, 2014. }\end{array}$ \\
\hline $\begin{array}{l}\text { [FIG] Time series of water } \\
\text { level }\end{array}$ & FINO1, Borkum-Suedstrand & 4-9 Dec 2013 & $\begin{array}{l}\text { Mai S, J Wilhelmi, T Zenz, U Barjenbruch, Orkan 'Xaver' - } \\
\text { Seegangsstatistik an den Stationen FINO1 und Borkum- } \\
\text { Suedstrand, 19. KFKI-Seminar, Bremerhaven 11.11.2014 } \\
\text { (presentation slides) }\end{array}$ \\
\hline $\begin{array}{l}{[\mathrm{FIG}] \text { Time series of the }} \\
\text { measured water level, } \\
\text { modelled tide, and surge } \\
\text { residual }\end{array}$ & $\begin{array}{l}\text { Wyk, Hamburg St. Pauli, } \\
\text { Husum }\end{array}$ & 5-6 Dec 2013 & $\begin{array}{l}\text { Matelski, Birgit, Frerk Jensen, Peter Voss, Jorg Peters, Maria } \\
\text { Blumel, Auswertung zur Hydrologie des Sturmtiefs Xaver } \\
\text { 05.12.2013 bis 06.12.2013, Statusbericht vom 01.10.2014, } \\
\text { Landesbetrieb fuer Kuestenschutz, Nationalpark und }\end{array}$ \\
\hline
\end{tabular}




\begin{tabular}{|c|c|c|c|}
\hline & & & $\begin{array}{l}\text { Meeresschutz, Schleswig-Holstein, Husum, 01.10.2014, } \\
\text { 25pp. }\end{array}$ \\
\hline $\begin{array}{l}\text { [TABLE] Maximum water } \\
\text { levels, MThw levels }\end{array}$ & $\begin{array}{l}62 \text { stations in Schleswig- } \\
\text { Holstein }\end{array}$ & 5-6 Dec 2013 & $\begin{array}{l}\text { Matelski, Birgit, Frerk Jensen, Peter Voss, Jorg Peters, Maria } \\
\text { Blumel, Auswertung zur Hydrologie des Sturmtiefs Xaver } \\
\text { 05.12.2013 bis 06.12.2013, Statusbericht vom 01.10.2014, } \\
\text { Landesbetrieb fuer Kuestenschutz, Nationalpark und } \\
\text { Meeresschutz, Schleswig-Holstein, Husum, 01.10.2014, } \\
\text { 25pp. }\end{array}$ \\
\hline $\begin{array}{l}\text { [FIG] Time series of water } \\
\text { level }\end{array}$ & Draugen & 1-31 Dec 2013 & $\begin{array}{l}\text { MIROS, Monthly Report, Draugen, December } 2013 \text {, Doc. No. } \\
\text { ND/1022/13/12 (prepared by SRS, checked by OO, } \\
\text { approved by CNE) [pdf document properties: } \\
\text { author=MIROS AS; datestamp=30Jan2014] }\end{array}$ \\
\hline $\begin{array}{l}\text { [FIG] Time series of water } \\
\text { level from altimeter }\end{array}$ & Ekofisk & 1-31 Dec 2013 & 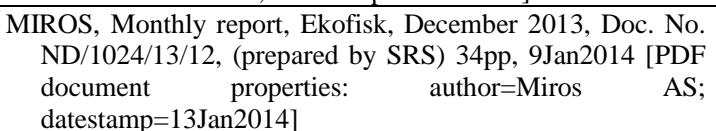 \\
\hline $\begin{array}{l}\text { FIG] Time series of water } \\
\text { level from altimeter }\end{array}$ & Heidrun & 1-31 Dec 2013 & $\begin{array}{l}\text { MIROS, Manedsrapport Heidrun, Desember } 2013 \text {, Dok. Nr. } \\
\text { ND/1010/13/12, } 21 \text { pp, 07Jan2014, carried out by SRS, } \\
\text { controlled by CNE, approved by OO [pdf properties: } \\
\text { author=Miros AS; date stamp: 08/01/2014] }\end{array}$ \\
\hline $\begin{array}{l}\text { [FIG] Time series of water } \\
\text { level from altimeter }\end{array}$ & Sleipner A & 1-31 Dec 2013 & $\begin{array}{l}\text { MIROS, Manedsrapport Sleipner A, Desember 2013, Dok. Nr. } \\
\text { ND/1017/13/12, 06Jan2014, prepared by SRS, controlled } \\
\text { by CNE, approved by OO. }\end{array}$ \\
\hline $\begin{array}{l}\text { TABLE] Highest } \text { storm } \\
\text { surge water levels with times }\end{array}$ & $\begin{array}{l}\text { Vlissingen, Zandvliet, Kallo, } \\
\text { Prosperpolder, } \\
\text { Liefkenshoek, Antwerpen, } \\
\text { Boom, Hemiksem, Tielrode, } \\
\text { Sint-Amands, Walem, } \\
\text { Mechelin Benedinsluis, } \\
\text { Dendemonde, Hombeek, } \\
\text { Duffel, Lier Molbrug, } \\
\text { Schoonarde, Waasmunster } \\
\text { Manta, Emblem, Kessel, } \\
\text { Wetteren, Melle }\end{array}$ & 6 Dec 2013 & $\begin{array}{l}\text { Nossent J, L Boeckx, E Taverniers, M Deschamps, T } \\
\text { Verwaest, F Mostaert, Sinterklaasstorm } 6 \text { december 2013. } \\
\text { Beschrijving van de hydrometrische gebeurtenissen, Versie } \\
\text { 4.0. WL Rapporten, 00-119, Waterbouwkundig } \\
\text { Laboratorium, Antwerpen, Belgie. October 2014, } \\
\text { WL2014R00_119_5 [document date stamp: 15Oct2014; } \\
\text { author= Deschamps, Maarten] }\end{array}$ \\
\hline $\begin{array}{l}\text { [FIG] Time series of } \\
\text { observed water level, } \\
\text { astronomical tide, true surge } \\
\text { residual }\end{array}$ & Vlissingen & 4-8 Dec 2013 & $\begin{array}{l}\text { Nossent J, L Boeckx, E Taverniers, M Deschamps, T } \\
\text { Verwaest, F Mostaert, Sinterklaasstorm } 6 \text { december } 2013 \text {. } \\
\text { Beschrijving van de hydrometrische gebeurtenissen, Versie } \\
\text { 4.0. WL Rapporten, 00-119, Waterbouwkundig } \\
\text { Laboratorium, Antwerpen, Belgie. October 2014, } \\
\text { WL2014R00_119_5 [document date stamp: 15Oct2014; } \\
\text { author= Deschamps, Maarten] }\end{array}$ \\
\hline [TEXT] Highest water levels & $\begin{array}{l}\text { Roskilde Haven, Torminde, } \\
\text { Harnbaek, Klampenborg, } \\
\text { Tolboden Kobenhavn, } \\
\text { Sjaellands Odde, Grenaa, } \\
\text { Odense Fjjord }\end{array}$ & $\begin{array}{l}\text { 5-6Dec2013 } \\
\text { Various times }\end{array}$ & $\begin{array}{l}\text { Pelt AS, BODIL's stormflod i de indre dansk farvande, } \\
\text { Vejret, 138, 24-29, } 2014\end{array}$ \\
\hline $\begin{array}{l}\text { [TEXT] Extreme low water } \\
\text { levels }\end{array}$ & $\begin{array}{l}\text { Koge Bugt, Flensborg, } \\
\text { Rodby, Ronne og Tegn }\end{array}$ & 5-6Dec2013 & $\begin{array}{l}\text { Pelt AS, BODIL's stormflod i de indre dansk farvande, } \\
\text { Vejret, 138, 24-29, } 2014\end{array}$ \\
\hline $\begin{array}{lc}{[\text { MAP }} & \text { TEXT] } \\
\text { coastal water levels }\end{array}$ & $\begin{array}{lr}\text { Lerwick, Wick, } & \text { Aberdeen, } \\
\text { Leith, North Shields, } \\
\text { Whitby, Immingham, } \\
\text { Cromer, } \\
\text { Harwich, Herne Bayestoft, } \\
\text { Sheerness, Dover, Ostend, } \\
\text { Borkum, } \\
\begin{array}{l}\text { Binnenhafen, Heligoland } \\
\text { Hirtshals, Cuxhaven, } \\
\text { Torshamnen }\end{array}\end{array}$ & 5-6 Dec 2013 & $\begin{array}{l}\text { RMS, 2013-2014 Winter Storms in Europe. An Insurance and } \\
\text { Catastrophe Modeling Perspective. RMS White Paper. } \\
\text { [PDF TIMESTAMP 11Mar2014] }\end{array}$ \\
\hline $\begin{array}{l}\text { [TABLE] Maximum water } \\
\text { level, astronomical tide, } \\
\text { skew surge for successive } \\
\text { storm tides }\end{array}$ & \begin{tabular}{l}
\multicolumn{3}{l}{ Vlissingen, Roompot buiten, } \\
Hoek van Holland, \\
Dordrecht, Den Helder, \\
Harlingen, Delfzijl
\end{tabular} & 5-7 Dec2013 & $\begin{array}{l}\text { RWS, Watermanagementcentrum Nederland, Stormvloedflits } \\
\text { 2013-07 van } 5 \mathrm{t} / \mathrm{m} 7 \text { december 2013, Rijkswaterstaat } \\
\text { (document time stamp: 07Jan2014), 2014a }\end{array}$ \\
\hline $\begin{array}{l}\text { [TABLE] Maximum water } \\
\text { level, astronomical tide, } \\
\text { skew surge for successive } \\
\text { storm tides }\end{array}$ & $\begin{array}{l}\text { Vlissingen, Roompot buiten, } \\
\text { Hoek van Holland, Den } \\
\text { Helder, Harlingen, Delfzijl }\end{array}$ & 5-6 Dec 2013 & $\begin{array}{l}\text { RWS, Stormvloedrapport van } 5 \mathrm{t} / \mathrm{m} 7 \text { december (SR91) Sint- } \\
\text { Nicolaasvloed 2013, Watermanagementcentrum } \\
\text { Nederland, Rijkswaterstaat, prepared by Ing. J. Kroos, } 19 \\
\text { Mar 2014b, } 48 \text { pp }\end{array}$ \\
\hline $\begin{array}{l}\text { [FIG] Time series of } \\
\text { measured water level, } \\
\text { modelled astronomical tide, } \\
\text { residual }\end{array}$ & \begin{tabular}{l}
\multicolumn{3}{l}{ Vlissingen, Roompot buiten, } \\
Hoek van Holland, \\
Dordrecht, Den Helder, \\
Harlingen, Delfzijl
\end{tabular} & 5-7 Dec 2013 & 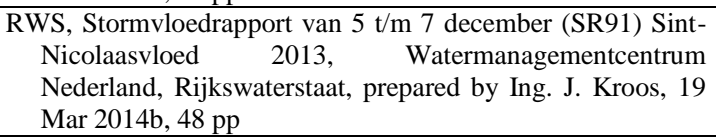 \\
\hline [TEXT] Highest water levels & Viken, Barseback, Halmstad & 6-7 Dec 2013 & $\begin{array}{l}\text { SMHI, Simone, Hilde, Sven och Ivar okt-dec 2013, } 3 \text { Jul } \\
\text { 2014, } \\
\text { https://www.smhi.se/kunskapsbanken/meteorologi/simone- } \\
\text { hilde-sven-och-ivar-okt-dec-2013-1.76183 }\end{array}$ \\
\hline
\end{tabular}




\begin{tabular}{|c|c|c|c|}
\hline [TEXT] Lowest water level & Skanoor & 6-7 Dec 2013 & $\begin{array}{l}\text { SMHI, Simone, Hilde, Sven och Ivar okt-dec 2013, } 3 \text { Jul } \\
\text { 2014, } \\
\text { https://www.smhi.se/kunskapsbanken/meteorologi/simone- } \\
\text { hilde-sven-och-ivar-okt-dec-2013-1.76183 }\end{array}$ \\
\hline $\begin{array}{l}{[\text { TEXT] Maximum water }} \\
\text { levels \& skew surge from } \\
\text { beach survey }\end{array}$ & Morston, Blakeney, Cley & 5-6 Dec 2013 & $\begin{array}{l}\text { Spencer, T, S.M. Brooks, I. Moller, B.R. Evans, Where local } \\
\text { matters: Impacts of a major North Sea storm surge, EOS, } \\
\text { 95, 269-270, 29July } 2014\end{array}$ \\
\hline $\begin{array}{l}{[\mathrm{TEXT}] \text { Maximum water }} \\
\text { levels from beach survey }\end{array}$ & Wells-next-the-Sea & 5-6 Dec 2013 & $\begin{array}{l}\text { Spencer, T, S.M. Brooks, I. Moller, B.R. Evans, Where local } \\
\text { matters: Impacts of a major North Sea storm surge, EOS, } \\
\text { 95, 269-270, 29July2014 }\end{array}$ \\
\hline $\begin{array}{l}\text { [PROFILE] Maximum water } \\
\text { levels from beach survey }\end{array}$ & $\begin{array}{l}\text { Holme-next-the-Sea, } \\
\text { Thornham, Titchwell, } \\
\text { Brancaster Beach, } \\
\text { Brancaster Staithe, Burnham } \\
\text { Deepdale, Burnham Overy } \\
\text { Staithe, Holkham Gap, Well } \\
\text { Stiffkey, Morston, } \\
\text { Blakeney, Cley, Salthouse }\end{array}$ & 5-6 Dec 2013 & $\begin{array}{l}\text { Spencer, T, S.M. Brooks, I. Moller, B.R. Evans, Where local } \\
\text { matters: Impacts of a major North Sea storm surge, EOS, } \\
\text { 95, 269-270, 29July2014 }\end{array}$ \\
\hline $\begin{array}{l}\text { [FIG] Time series of surface } \\
\text { elevation }\end{array}$ & Sylt, Grena, Sassnitz & 4-10 Dec 2013 & $\begin{array}{l}\text { Stanev E, J Staneva, S Grayek, J Schulz-Stellenfleth, S. } \\
\text { Grashorn, A Behrens, Numerical modelling and data } \\
\text { assimilation, COSYNA Progress Report 2013, pp.46-51, } \\
\text { Helmholtz-Zentrum Geesthacht, June 2014. }\end{array}$ \\
\hline $\begin{array}{l}\text { [FIG] Time series of sea } \\
\text { surface elevation }\end{array}$ & $\begin{array}{l}4 \text { unlabelled stations in } \\
\text { German Bight identified on } \\
\text { map only }\end{array}$ & 3-9 Dec 2013 & $\begin{array}{l}\text { Staneva J, K Wahle, E Stanev, Response of the German Bight } \\
\text { Hydro and Sediment Dynamics to Wave, Tidal and } \\
\text { Atmospheric Forcing, 3rd GODAE OceanView Coastal } \\
\text { Oceans and Shelf Seas Task Team (COSS-TT) } \\
\text { International Coordination Workshop, 21-24 January } \\
\text { 2014, Rncon Beach Resort, Puerto Rico, 36pp }\end{array}$ \\
\hline $\begin{array}{l}{[\text { FIG] Time series of }} \\
\text { measured water level and } \\
\text { modelled astronomical tide. } \\
\text { Highest water level } 3.8 \mathrm{~m} \text {; } \\
\text { highest corresponding tide } \\
1.4 \mathrm{~m}\end{array}$ & Lauwerzoog & 4-9 Dec 2013 & $\begin{array}{l}\text { van Rooijen A, A Oost, Memo: Regionale advisering } \\
\text { Ameland Noordwest, Deltares, 1209381-008-ZKS-0008, } \\
\text { 43pp, 18Dec2014 [PDF document properties: } \\
\text { titl=Regional advisering: Ameland NW; author=Arnold } \\
\text { van Rooijen; keywords: 1209381-008-ZKS-0008; date } \\
\text { stamp: 18/12/2014] }\end{array}$ \\
\hline $\begin{array}{l}\text { [FIG] Time series of water } \\
\text { leve in } 20 \mathrm{~m} \text { depth. } \\
\text { Maximum water level } 2.6 \mathrm{~m} \\
\text { NAP on } 5 \text { Dec } 2013 \\
\text { 21:00GMT }\end{array}$ & Terschelling Noordzee & 4-7 Dec 2013 & $\begin{array}{l}\text { van Rooijen A, A Oost, Memo: Regionale advisering } \\
\text { Ameland Noordwest, Deltares, 1209381-008-ZKS-0008, } \\
\text { 43pp, 18Dec2014 [PDF document properties: } \\
\text { titl=Regional advisering: Ameland NW; author=Arnold } \\
\text { van Rooijen; keywords: 1209381-008-ZKS-0008; date } \\
\text { stamp: 18/12/2014] }\end{array}$ \\
\hline $\begin{array}{lcc}{[\text { TEXT] Absolute }} & \text { water } \\
\text { level and skew surge } & \end{array}$ & Hamburg-St.Pauli & 5-6 Dec 2013 & 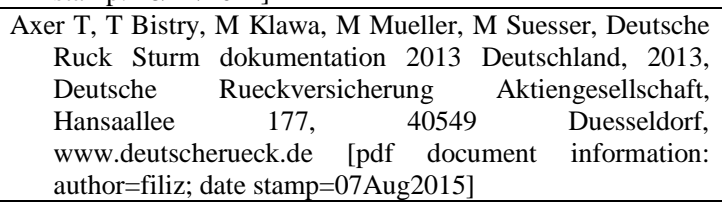 \\
\hline $\begin{array}{l}\text { [FIG] Time series measured } \\
\text { water level, astronomical } \\
\text { tide, surge residual }\end{array}$ & Cadzand & 5-7 Dec 2013 & $\begin{array}{l}\text { Carrion Aretxabala, BI, Morphological impact of the } \\
\text { Sinterklaas storm at Het Zwin. Numerical modelling with } \\
\text { Xbeach, M.Sc. Civil Engineering, Delft University of } \\
\text { Technology, 2015. }\end{array}$ \\
\hline $\begin{array}{l}\text { [FIG] Water level from tide } \\
\text { gauge }\end{array}$ & Ostend & 4-8 Dec 2013 & $\begin{array}{l}\text { Dan, Sebastian, Anne-Lise Montreuil, Rosalia Delgado, } \\
\text { Tomas van Oyen, Large storm impact on a beach under } \\
\text { sand nourishments, The Proceedings of the Coastal } \\
\text { Sediments 2015, edited by Ping Wang, Julie D Rosati, and } \\
\text { Jun Cheng, Coastal Sediments 2015, San Diego, USA, 11- } \\
\text { 15May2015 }\end{array}$ \\
\hline $\begin{array}{l}\text { [FIG] Time series of water } \\
\text { level from tide gauge }\end{array}$ & Liverpool Gladstone Dock & 5 Dec 2015 & $\begin{array}{l}\text { Dissanayake P and H Karunarathna, Effect of storm clustering } \\
\text { on beach/dune erosion, E-proceedings of the 36th IAHR } \\
\text { World Congress, 28June-3July, 2015, The Hague, The } \\
\text { Netherlands }\end{array}$ \\
\hline $\begin{array}{l}\text { [FIG] Time series of water } \\
\text { level from tide gauge }\end{array}$ & Liverpool Gladstone Dock & 5 Dec 2013 & $\begin{array}{l}\text { Dissanayake P, J Brown, H Karunarathna, Impacts of storm } \\
\text { chronology on the morphological changes of the Formby } \\
\text { beach and dune system, UK, Nat. Hazards Earth Syst. Sci., } \\
15,1533-1543,2015 \text {. }\end{array}$ \\
\hline $\begin{array}{l}\text { [FIG] Time series of water } \\
\text { level from tide gauge }\end{array}$ & Liverpool Gladstone Dock & $\begin{array}{l}\text { 1 Dec } 2013- \\
\text { 31 Jan } 2014\end{array}$ & $\begin{array}{l}\text { Dissanayake P, J Brown, P. Wisse, H Karunarathna, } \\
\text { Comparison of storm cluster vs isolated event impacts on } \\
\text { beach.dune morphodynamics, Estuarine, Coastal, and } \\
\text { Shelf Science, 164, 301-312, 2015b. }\end{array}$ \\
\hline $\begin{array}{l}\text { [STRIP } \text { PROFILE] AltiKa } \\
\text { satellite altimetry detided } \\
\text { water level height }\end{array}$ & $\begin{array}{l}\text { Strip profile through North } \\
\text { Sea }\end{array}$ & $\begin{array}{l}6 \text { Dec } 2013 \\
\text { 0447UTC }\end{array}$ & $\begin{array}{l}\text { Fenoglio-Marc L, R Scharroo, A Annuziato, L Mendoza, M } \\
\text { Becker, J Lillibridge, Cyclone Xaver seen by geodetic } \\
\text { observations, Geophys Research Letters, 42, 9925-9932, } \\
2015\end{array}$ \\
\hline [FIG] True & $\begin{array}{l}\text { Lowestoft, } \\
\text { Suedstrand, }\end{array}$ & $5-6 \operatorname{Dec} 2013$ & $\begin{array}{l}\text { Fenoglio-Marc L, R Scharroo, A Annuziato, L Mendoza, M } \\
\text { Becker, J Lillibridge, Cyclone Xaver seen by geodetic }\end{array}$ \\
\hline
\end{tabular}




\begin{tabular}{|c|c|c|c|}
\hline & $\begin{array}{l}\text { Borkum Fischerbalje, } \\
\text { Emden, } \\
\text { Helgoland, Mellumplate, } \\
\text { Leuchtturm Alte Weser, } \\
\text { Wilhelmshaven, Dwarsgat, } \\
\text { Hoernum/Sylt, } \\
\text { Bremerhaven, Dagebuell, } \\
\text { Cuxhaven, Busum }\end{array}$ & & $\begin{array}{l}\text { observations, Geophys Research Letters, 42, 9925-9932, } \\
2015\end{array}$ \\
\hline $\begin{array}{l}\text { [FIG] Time series water } \\
\text { level data }\end{array}$ & FINO1 & 3-7Dec2013 & $\begin{array}{l}\text { Fenoglio-Marc L, R Scharroo, A Annuziato, L Mendoza, M } \\
\text { Becker, J Lillibridge, Cyclone Xaver seen by geodetic } \\
\text { observations, Geophys Research Letters, 42, 9925-9932, } \\
2015\end{array}$ \\
\hline $\begin{array}{l}\text { [FIG] Time series of water } \\
\text { level; maximum } 1.3 \mathrm{~m} \text { where } \\
\text { normal high tide } 0.2 \mathrm{~m}\end{array}$ & Saeby/ Fredrikshavn & $\begin{array}{l}1 \text { Nov } 2013- \\
31 \text { Dec } 2013\end{array}$ & $\begin{array}{l}\text { Gierlevsen T, H Lauridsen, F Langhans, J Bejdic, Met-ocean } \\
\text { and wind resource related studies for nearshore windfarms } \\
\text { in Denmark, seminar at the Danish Energy Agency, } 27 \\
\text { February 2015. }\end{array}$ \\
\hline $\begin{array}{l}\text { [FIG] Time series of water } \\
\text { level }\end{array}$ & Wandelaar & 1-31 Dec 2013 & $\begin{array}{l}\text { Gourgue O, BB Sishah, J Vanlede, H Komijani, M Chen, } \\
\text { Modelling tides and storm surges on the European } \\
\text { continental shelf, 22nd Telemac \& Mascaret User Club, } \\
\text { STFC Daresbury Laboratory, UK, } 13-16 \text { Oct 2015. [PDF } \\
\text { document properties: datestamp: 05/10/2015] }\end{array}$ \\
\hline $\begin{array}{l}\text { [FIG] Maximum water level } \\
\text { in cm Pegel Null PN }\end{array}$ & $\begin{array}{l}\text { List, Hoernum, Wittduen, } \\
\text { Wyk, Dagebuell, Buesum, } \\
\text { Helgoland, Cuxhaven, LT } \\
\text { Alte Weser, Wilhelmshaven, } \\
\text { Norderney, Emden }\end{array}$ & 5-6 Dec 2013 & $\begin{array}{l}\text { Jensen, J., A. Arns, T. Wahl, Yet another 100yr storm surge } \\
\text { event: the role of individual storm surges on design water } \\
\text { levels, Journal of Marine Science and Technology, 23, 882- } \\
\text { 887, } 2015 \text {. }\end{array}$ \\
\hline $\begin{array}{lll}\text { [FIG] } & \text { Maximum water } \\
\text { levels } & & \end{array}$ & $\begin{array}{ll}\text { Emden, } & \text { Norderney, } \\
\text { Cuxhaven } & \end{array}$ & 5-6 Dec 2013 & $\begin{array}{lllr}\text { NLWKN, Sturmflutdienst der } & \text { Betriebstelle } & \text { Norden- } \\
\text { Norderney, Niedersaechischer } & \text { Landesbetrieb fuer } \\
\text { Wasserwirtschaft, Kuesten- } & \text { und Naturschutz, } \\
\text { Niedersachsen, 12/2015 [document date stamp 10Dec2015] }\end{array}$ \\
\hline $\begin{array}{l}\text { [TABLE] Maximum water } \\
\text { level, maximum true surge, } \\
\text { maximum astronomical tide, } \\
\text { skew surge }\end{array}$ & 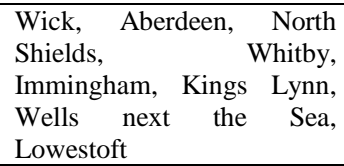 & 5 Dec 2013 & $\begin{array}{l}\text { Spencer T, SM Brooks, BR Evans, JA Tempest, I Moeller, } \\
\text { Southern North Sea storm surge event of Dec.5, 2013: } \\
\text { Water levels, waves, and coastal impacts, Earth Science } \\
\text { Reviews, 146, 120-145, } 2015\end{array}$ \\
\hline $\begin{array}{l}\text { [TABLE] Highest water } \\
\text { level referenced to ODN; } \\
\text { skew surge }\end{array}$ & $\begin{array}{l}\text { All class A stations around } \\
\text { the UK; other stations from } \\
\text { Channel } \quad \text { Coastal } \\
\text { Observatory and Associated } \\
\text { British Ports }\end{array}$ & 5-6 Dec 2013 & $\begin{array}{l}\text { Wadey MP, ID Haigh, RJ Nichols, JM Brown, K Horsburgh, } \\
\text { B Carroll, SL Gallop, T Mason, E Bradshaw, A } \\
\text { comparison of the } 31 \text { January-1 February } 1953 \text { and 5-6 } \\
\text { December } 2013 \text { coastal flood events around the UK, UK } \\
\text { Frontiers in Marine Science, 2, 84, 2015a. }\end{array}$ \\
\hline $\begin{array}{l}\text { [FIG] Time series of gauge } \\
\text { water level, calculated } \\
\text { astronomical tide, and true } \\
\text { surge residual }\end{array}$ & Liverpool, Lowestoft & 4-6 Dec 2013 & $\begin{array}{l}\text { Wadey MP, JM Brown, ID Haigh, T Dolphin, P Wisse, } \\
\text { Assessment and comparison of extreme sea levels and } \\
\text { waves during the } 2013 / 2014 \text { storm season in two UK } \\
\text { coastal regions, Nat. Hazards Earth Syst. Sci. Discuss., } 3 \text {, } \\
2665-2708,2015 \text { b. }\end{array}$ \\
\hline $\begin{array}{l}\text { [TABLE] High water, } \\
\text { calculated astronomical tide, } \\
\text { skew surge }\end{array}$ & Lowestoft & 5 Dec 2013 & $\begin{array}{l}\text { Wadey MP, JM Brown, ID Haigh, T Dolphin, P Wisse, } \\
\text { Assessment and comparison of extreme sea levels and } \\
\text { waves during the 2013/2014 storm season in two UK } \\
\text { coastal regions, Nat. Hazards Earth Syst. Sci. Discuss., 3, } \\
2665-2708,2015 \text { b. }\end{array}$ \\
\hline $\begin{array}{l}\text { [TABLE] Absolute water } \\
\text { levels referenced Ordnance } \\
\text { Datum Newlyn ODN }\end{array}$ & UK tide gauge stations & 5-6 Dec 2013 & $\begin{array}{l}\text { Sibley A, D Cox, H Titley, Coastal flooding in England and } \\
\text { Wales from Atlantic and North Sea storms during the } \\
\text { 2013/2014 winter, Weather, 70,62-70, 2015 }\end{array}$ \\
\hline $\begin{array}{l}\text { [FIG] Time series of true } \\
\text { surge }\end{array}$ & Hoek van Holland & 4-8 Dec 2013 & $\begin{array}{l}\text { F Zijl, J Sumihar, M Verlaan, Application of data } \\
\text { assimilation for improved operational water level } \\
\text { forecasting on the northwest European shelf and North Sea, } \\
\text { Ocean Dynamics, } 65,1699-1716,2015\end{array}$ \\
\hline $\begin{array}{l}\text { [FIG] Time series of still } \\
\text { water levels }\end{array}$ & Immingham and Cromer & 5-7 Dec 2013 & $\begin{array}{l}\text { Brooks SM, T Spencer, A McIvor, I Moller, Reconstructing } \\
\text { and understanding the impacts of storms and surges, } \\
\text { southern North Sea, Earth Surface Processes and } \\
\text { Landforms, 41, 855-864, 2016. }\end{array}$ \\
\hline $\begin{array}{l}\text { [FIG] Time series of water } \\
\text { level, astronomical tide, true } \\
\text { surge residual }\end{array}$ & Norderney & 5-6 Dec 2013 & $\begin{array}{l}\text { Dangendorf S, A Arns JG Pinto, P Ludwig, J Jensen, The } \\
\text { exceptional influence of storm 'Xaver' on design water } \\
\text { levels in the German Bight, Environmental Research } \\
\text { Letters, 11, 2016,054001 }\end{array}$ \\
\hline $\begin{array}{l}\text { [FIG] Time series of water } \\
\text { level }\end{array}$ & $\begin{array}{l}\text { Lighthouse Alte Weser, } \\
\text { Borkum Suedstrand }\end{array}$ & 5-8 Dec 2013 & $\begin{array}{l}\text { Mai S and U Barjenbruch, Water level measurements with } \\
\text { radar gauges at the German North Sea coast, [PDF } \\
\text { document properties: author=IOC; } \quad \text { subject: } \\
\text { IOC/2016/MG/14 vol.5; datestamp: } 18 / 04 / 2017] \\
\end{array}$ \\
\hline $\begin{array}{l}\text { [FIG] Time series of water } \\
\text { level, astronomical tide, and } \\
\text { surge residual }\end{array}$ & List auf Sylt & 5-7 Dec 2013 & $\begin{array}{l}\text { Matelski, Birgit, Erfahrungen aus der Sturmflut Xaver von } 5 . \\
\text { und 6.12.2013 und dem Weihnachshochwasser } 2014 \text { in } \\
\text { Shleswig-Holstein, IWASA } 2016 \text { Tagungsbeitrag, (46. } \\
\text { IWASA, 7-8 Januar 2016; Internationales Wasserbau- } \\
\text { Symposium Aachen. [pdf document properties: }\end{array}$ \\
\hline
\end{tabular}




\begin{tabular}{|c|c|c|c|}
\hline & & & autor=sonja; datestamp: 26Apr2016] \\
\hline $\begin{array}{l}\text { [FIG] Time series of water } \\
\text { level, astronomical tide, and } \\
\text { surge residual }\end{array}$ & Husum & 5-6 Dec 2013 & $\begin{array}{l}\text { Matelski, Birgit, Erfahrungen aus der Sturmflut Xaver von } 5 . \\
\text { und 6.12.2013 und dem Weihnachshochwasser } 2014 \text { in } \\
\text { Shleswig-Holstein, IWASA } 2016 \text { Tagungsbeitrag, (46. } \\
\text { IWASA, 7-8 Januar 2016; Internationales Wasserbau- } \\
\text { Symposium Aachen. [pdf document properties: } \\
\text { autor=sonja; datestamp: 26Apr2016] }\end{array}$ \\
\hline $\begin{array}{l}\text { [FIG] Time series of water } \\
\text { level, astronomical tide, and } \\
\text { surge residual }\end{array}$ & $\begin{array}{l}\text { Holmersiel, } \\
\text { Nordstrandischmoor-Hallig, } \\
\text { Nordstrandischmoor }\end{array}$ & 4-8 Dec 2013 & $\begin{array}{l}\text { Matelski, Birgit, Erfahrungen aus der Sturmflut Xaver von } 5 . \\
\text { und 6.12.2013 und dem Weihnachshochwasser } 2014 \text { in } \\
\text { Shleswig-Holstein, IWASA } 2016 \text { Tagungsbeitrag, (46. } \\
\text { IWASA, 7-8 Januar 2016; Internationales Wasserbau- } \\
\text { Symposium Aachen. [pdf document properties: } \\
\text { autor=sonja; datestamp: 26Apr2016] }\end{array}$ \\
\hline $\begin{array}{l}\text { [FIG] Maximum nontidal } \\
\text { residual and skew surge as } \\
\text { point on scatterplot }\end{array}$ & Immingham & 5-6 Dec 2013 & $\begin{array}{l}\text { Mawdsley RJ and ID Haigh, Spatial and temporal variability } \\
\text { and long-term trends in skew surges globally, Frontiers in } \\
\text { Marine Science, 2016, doi: } 10.3389 / \text { fmars. } 2016.00029\end{array}$ \\
\hline $\begin{array}{l}\text { [FIG] Time series of water } \\
\text { level }\end{array}$ & $\begin{array}{l}\text { Terschelling, } \\
\text { Wierummergronden, Nes }\end{array}$ & 5-8 Dec 2013 & $\begin{array}{l}\text { Nederhoff K, E Elias, T Vermaas, Erosie op Ameland } \\
\text { Noordwest. Modelstudie: simulaties met Delft3D en } \\
\text { XBeach, Deltares, 117pp, July, 2016 }\end{array}$ \\
\hline $\begin{array}{l}\text { [FIG] Time series of water } \\
\text { levels with text indicating } \\
\text { highest water level } 1.96 \mathrm{~m} \\
\text { DVR90 }\end{array}$ & Hornbaek & 4-6Dec2013 & $\begin{array}{l}\text { Sorensen CS, NK Dronen, P Knudsen, J Jensen, P Sorensen, } \\
\text { An extreme event as a games changer in coastal zone } \\
\text { management, Journal of Coastal Research, (Special Issue, } \\
\text { No 75), 700-704, 2016. Proceedings of the 14th } \\
\text { International Coastal Symposium (Sydney, Australia) ed by } \\
\text { A Vila-Concejo, E Bruce, DM Kennedy, RJ McCarroll }\end{array}$ \\
\hline $\begin{array}{l}\text { [FIG] Time series of tide } \\
\text { gauge water levels }\end{array}$ & $\begin{array}{l}4 \text { German Bight stations, } \\
\text { unidentified but marked on } \\
\text { map }\end{array}$ & 4-9 Dec 2013 & $\begin{array}{l}\text { Staneva J, K Wahle, H Guenther, E Stanev, Coupling of wave } \\
\text { and circulation models in coastal-ocean predicting systems: } \\
\text { a case study for the German Bight, Ocean Sci., 12, 797- } \\
\text { 806, 2016a. }\end{array}$ \\
\hline $\begin{array}{l}\text { [FIG] Time series of water } \\
\text { level and computed surge }\end{array}$ & Helgoland & 4-8 Dec 2013 & $\begin{array}{l}\text { Staneva J, K Wahle, W Koch, A Behrens, L Fenoglio-Marc, } \\
\text { EV Stanev, Coastal flooding: impact of waves on storm } \\
\text { surge during extremes - a case study for the German Bight, } \\
\text { Nat. Hazards Earth Syst. Sci., 16, 2373-2389, } 2016\end{array}$ \\
\hline $\begin{array}{l}\text { [TEXT] } 5.2 \mathrm{~m} \text { surge; water } \\
\text { level } 6.08 \mathrm{~m} \text { ODN }\end{array}$ & Boston UK & 5-6 Dec 2013 & $\begin{array}{l}\text { Evans, Sun Yan, EA/2/2 Appendix 1, History of flooding } \\
\text { sources, Boston Barrier Transport \& Works Act order } \\
\text { application, Public Inquiry documents, [pdf document } \\
\text { properties: author=BDB; datestamp=16Mar2017] } \\
\text { https://consult.environment- } \\
\text { agency.gov.uk/engagement/bostonbarriertwao/results/appe } \\
\text { ndix-1---history-of-flooding-sources.pdf }\end{array}$ \\
\hline $\begin{array}{l}\text { [TABLE] Highest flood } \\
\text { water levels }\end{array}$ & $\begin{array}{l}\text { Wick, North Shields, } \\
\text { Immingham, Boston, Wells, } \\
\text { Lowestoft, Sheerness }\end{array}$ & 5-6 Dec 2013 & $\begin{array}{l}\text { Jee, Andrew, EA/13/2 Appendix 1. A summary of flooding } \\
\text { events in Boston. [pdf document properties: } \\
\text { author=Andrew Jee; Date stamp=17Mar2017] } \\
\text { https://consult.environment- } \\
\text { agency.gov.uk/engagement/bostonbarriertwao/ }\end{array}$ \\
\hline $\begin{array}{l}\text { [FIG] Time series of the } \\
\text { surge residual from } \mathrm{ADCP}\end{array}$ & FINO1 & 4-7 Dec 2013 & $\begin{array}{l}\text { Staneva J, H Guenther, O Krueger, C Schrumm, V Alari, O } \\
\text { Breivik, J-R Bidlot, K Mogensen, Impact of wind waves on } \\
\text { the air-sea momentum fluxes for different wind and sea } \\
\text { state conditions and oceanic responses, 1st International } \\
\text { Workshop on waves, storm surges and coastal hazards, } \\
\text { Liverpool, UK 10-15Sep2017 [pdf document properties: } \\
\text { title=Anlass; author=Patrick Kalb-Anlass, } \\
\text { datestamp=20/09/2017] }\end{array}$ \\
\hline $\begin{array}{l}\text { [FIG] Time series of surge } \\
\text { residual }\end{array}$ & $\begin{array}{l}\text { Helgoland? (uncertain if is } \\
\text { from ADCP data at } \\
\text { FINO1?) }\end{array}$ & 4-7 Dec 2013 & $\begin{array}{l}\text { Staneva J, C Schrum, A Behrens, S Grayek, H Ho- } \\
\text { Hagemann, V Alan, O Breivik, J-R. Bidlot, A North Sea- } \\
\text { Baltic Sea Regional Coupled Models: Atmosphere, wind } \\
\text { waves and ocean, in Proceedings of the Eigth EuroGOOS } \\
\text { International Conference (Operational Oceanography. } \\
\text { Serving Sustainable Marine Development), 3-5 October } \\
\text { 2017, Bergen, Norway, 2017b. }\end{array}$ \\
\hline $\begin{array}{l}\text { [FIG] Time series of water } \\
\text { level }\end{array}$ & Pellworm Anleger & 5-6 Dec 2013 & $\begin{array}{l}\text { Dreier, Norman and Peter Froehle, Operational wave forecast } \\
\text { in the German Bight as part of a sensor- and risk based } \\
\text { early warning system, In: J-S Shim, I Chun, HS Lim (ed), } \\
\text { Proceedings from the International Coastal Symposium } \\
\text { (ICS) } 2018 \text { (Busan, Republic of Korea), Journal of Coastal } \\
\text { Research, Special Issue No. } 85,1161-1165,2018\end{array}$ \\
\hline $\begin{array}{l}{[\mathrm{TEXT}] \text { Water level and }} \\
\text { surge at Southend on two } \\
\text { tidal cycles }\end{array}$ & Southend & 5-6 Dec 2013 & $\begin{array}{l}\text { Environment Agency, Thames Barrier Project Pack 2018, } \\
\text { January, } 2018\end{array}$ \\
\hline $\begin{array}{l}\text { [TABLE] Maximum water } \\
\text { level } 2.1 \mathrm{~m}\end{array}$ & Hvide Sande & 5-9 Dec 2013 & $\begin{array}{l}\text { Kystdirektoratet, Shoreface nourishment effects. An analysis } \\
\text { of the } 2011 \text { nourishment performed at Skodbjerge. } \\
\text { Kystdirektoratet, Hojbovej } 1,7620 \text { Lemvig, Dec } 2018 \text { [pdf } \\
\text { document properties: title=Shoreface nourishment } \\
\text { effects_Skodbjerge_20.12.2018, datestamp }=20 / 12 / 2018 \text { ] }\end{array}$ \\
\hline
\end{tabular}




\begin{tabular}{|c|c|c|c|}
\hline $\begin{array}{l}{[\text { FIG] Time series of true }} \\
\text { surge and tide }\end{array}$ & $\begin{array}{l}\text { Dagebuell, Norderney, } \\
\text { Husum, LT Alte Weser, } \\
\text { Wyk, Helgoland, Wittduen, } \\
\text { Emden, Hoernum, } \\
\text { Wilhelmshaven, Cuxhaven, } \\
\text { List }\end{array}$ & 5-7 Dec 2013 & $\begin{array}{l}\text { Niehuser S, S Dangendorf, A Arns, J Jensen, A high } \\
\text { resolution storm surge forecast for the German Bight, } \\
\text { Conference: 9th Chinese-German Joint Symposium on } \\
\text { Coastal and Ocean Engineering, Tainan, Taiwan, } 2018\end{array}$ \\
\hline $\begin{array}{l}\text { [FIG] Time series of water } \\
\text { level, astronomical tide, } \\
\text { surge residual }\end{array}$ & Hamburg St Pauli & 5-6 Dec 2013 & $\begin{array}{l}\text { Schenk, L and S Mueller-Navarra. 3.4.4. Windstaustatistiken } \\
\text { und Haufigkeit von Sturmfluten 2012-2015 } \\
\text { https://www.bsh.de/DE/PUBLIKATIONEN/Nordseezustan } \\
\text { d_Aktuell/_Anlagen/Downloads/3_4_4_Windstatistiken.pd } \\
\text { f?_blob=publicationFile\&v=2 [pdf document properties: } \\
\text { author=Ludwig Schenk; datestamp=14Feb2019] }\end{array}$ \\
\hline $\begin{array}{l}{[\text { TEXT] Maximum water }} \\
\text { level }\end{array}$ & $\begin{array}{l}\text { Ostend Belgium and Viken } \\
\text { Hoganas Kommune }\end{array}$ & 5-6 Dec 2013 & $\begin{array}{l}\text { Wikipedia, Stormen } \\
\text { https://da.wikipedia.org/wiki/Stormen_Bodil\#cite_note-39 } \\
\text { (accessed 02Oct2019) }\end{array}$ \\
\hline $\begin{array}{l}{[\mathrm{FIG}] \text { Map with maximum }} \\
\text { water levels printed }\end{array}$ & $\begin{array}{l}\text { Kingston-upon-Hull, } \\
\text { Immingham, Cromer, } \\
\text { Lowestoft, } \\
\text { Sheerness, Dover }\end{array}$ & 5-6 Dec 2013 & $\begin{array}{l}\text { JBA Risk Management, Storm Xaver 2013. Event } \\
\text { Commentary, 2020. [PDF document properties: } \\
\text { author=Cameron Whitwham] }\end{array}$ \\
\hline $\begin{array}{l}\text { [TABLE] Maximum water } \\
\text { levels with times }\end{array}$ & 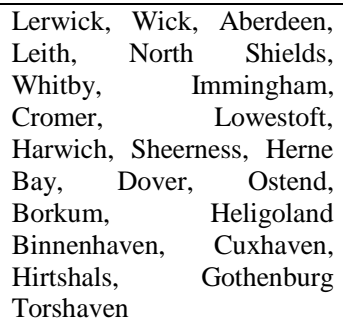 & 5-6 Dec 2013 & 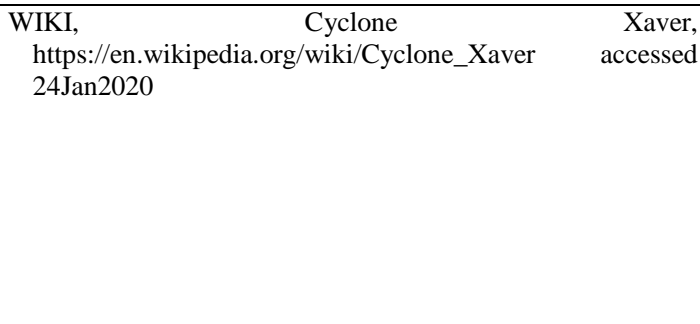 \\
\hline $\begin{array}{l}\text { [TABLE] Maximum water } \\
\text { levels with astronomical } \\
\text { tide, skew surge, and return } \\
\text { period }\end{array}$ & $\begin{array}{l}\text { Dover, Immingham, } \\
\text { Whitby, North Shields, } \\
\text { Lowestoft, } \\
\text { Newhaven, Leith, Aberdeen, } \\
\text { Llandudno, Portsmouth, } \\
\text { Ullapool }\end{array}$ & 5-6 Dec 2013 & $\begin{array}{l}\begin{array}{l}\text { Surgewatch, Storm event 6th } \\
\text { https://www.surgewatch.org/events/1/ }\end{array} \\
\text { 04Mar2020) }\end{array}$ \\
\hline $\begin{array}{l}\text { [TEXT] Maximum water } \\
\text { level } 1.2 \mathrm{~m} \text { over normal }\end{array}$ & Goteborg & $\begin{array}{lrl}6 & \text { Dec } & 2013 \\
1800 \mathrm{M} & \end{array}$ & $\begin{array}{l}\text { Wikipedia, } \\
\text { https://sv.wikipedia.org/wiki/Stormen_Sven\#cite_note-15, } \\
\text { accessed 29Apr2020 }\end{array}$ \\
\hline
\end{tabular}

Table S15. Water current information (arranged by year and then alphabetically)

\begin{tabular}{|l|l|l|l|}
\hline Data type & Location & Time Interval & Full Reference and Notes \\
\hline $\begin{array}{l}\text { [FIG] Time series of the } \\
\text { ADCP water currents at 4,6, }\end{array}$ & FINO1 & $1-7$ Dec 2013 & $\begin{array}{l}\text { Staneva J, H Guenther, O Krueger, C Schrumm, V Alari, O } \\
\text { Breivik, J-R Bidlot, K Mogensen, Impact of wind waves on } \\
\text { the air-sea momentum fluxes for different wind and sea state } \\
\text { conditions and oceanic responses, 1st International Workshop } \\
\text { on waves, storm surges and coastal hazards, Liverpool, UK } \\
\text { 10-15Sep2017 [pdf document properties: title=Anlass; } \\
\text { author=Patrick Kalb-Anlass, datestamp=20/09/2017] }\end{array}$ \\
\hline
\end{tabular}

Table S16. Return period of water level; ranking of water level

\begin{tabular}{|c|c|}
\hline Source & ull Reference and Notes \\
\hline Bloomberg (2013) & $\begin{array}{l}\text { Bloomberg, Hamburg has worst flood in } 37 \text { year amid European storms, Nicholas Brautlecht, 6Dec2013, } \\
\text { 02:58PM GMT https://www.bloomberg.com/news/articles/2013-12-06/hamburg-has-worst-flood-in-37-years-as- } \\
\text { storms-rage-across-europe } \\
\text {-Hamberg water levels 1976>2013>1962 }\end{array}$ \\
\hline $\begin{array}{l}\text { Gccapitalideas } \\
(20131209)\end{array}$ & $\begin{array}{l}\text { gccapitalideas, Windstorm Xaver, 9Dec2013, https://www.gccapitalideas.com/2013/12/09/windstorm-xaver/ } \\
\text {-worst flooding in Dover Kent in more than } 100 \text { years }\end{array}$ \\
\hline Kunz et al (20131206) & $\begin{array}{l}\text { Kunz M, B Muehr, K Schroeter, T Bessel, S Moehrle, T Muenzberg, S Brink, H-M Schmidt, Winterstorm Xaver - } \\
\text { Report. 06Dec2013 - Report No.1, Situation Report - 19:00CET, CEDIM Forensic Disaster Analysis Group } \\
\text { (FDA), Center for Disaster Management and Risk Reduction Technology. } \\
\text {-Rank } 2 \text { water level Hamburg St Pauli after 03 Jan 1976; measurement start } 1825 \\
\text {-New records: Dagebuell, Husum, Buesum, Eidersperrwerk, Cuxhaven }\end{array}$ \\
\hline NLWKN (2013) & $\begin{array}{l}\text { NLWKN, Schwere Sturmflut gut Ueberstanden, Waserstaende zwischen Zweieinhale und Knapp vier metern } \\
\text { registriert // presseinformation von 6. Dezember 2013, (Ansprechpartnerin: Herma Heyken), 06/12/2013. } \\
\text {-list of skew surges for station on Niedersachsen coast with comparison ranking with } 2006 \text { and } 1962 \text { events. }\end{array}$ \\
\hline NOS (20131206) & $\begin{array}{l}\text { NOS, Hoogste waterstand sinds } 1953,06 / 12 / 2013,09: 52 \text {, https://nos.nl/artikel/583218-hoogste-waterstand-sinds- } \\
\text { 1953.html } \\
\text {-water level Vlissingen highest since } 1953\end{array}$ \\
\hline $\begin{array}{l}\text { Oceanografisch } \\
\text { Meteorologisch } \\
\text { Station (2013) }\end{array}$ & $\begin{array}{l}\text { Oceanografisch Meteorologisch Station, Stormverslag 05-06 december 2013, 26pp, } 2013 \text { [pdf document } \\
\text { properties: author=Myriam Sys; datestamp=15Dec2013] } \\
\text {-water level in Oostende was the highest since } 1 \text { Feb } 2013\end{array}$ \\
\hline SMHI (20131210) & SMHI, Stormen Sven gav nya vattenstandsrekord i Oresund, https://www.smhi.se/nyhetsarkiv/stormen-sven-gav- \\
\hline
\end{tabular}




\begin{tabular}{|c|c|}
\hline & $\begin{array}{l}\text { nya-vattenstandsrekord-i-oresund-1.34732, updated 20Mar2017; original datestamp 10Dec2013. } \\
\text {-Viken: rank } 1 \text { water level }(1.67 \mathrm{~m}) \text { since record start } 1976 \\
\text {-Barseback: rank } 1 \text { water level since record start } 1993 \\
\text {-Skanoor: rank } 1 \text { minimum water level }-1.58 \mathrm{~m} \text {; previous record -1.55m from Dec } 1999\end{array}$ \\
\hline $\begin{array}{l}\text { Unwetterzentrale } \\
(201312)\end{array}$ & $\begin{array}{l}\text { Unwetterzentrale, Orkantief XAVER - ein weiterer schwerer Wintersturm der letzten Jahrzehnte, Thomas Savert } \\
\text { and Stefan Laps, Dec. } 2013 \text { http://www.unwetterzentrale.de/uwz/928.html } \\
\text {-Flood level Hamburg-St Pauli was second highest since } 3 \text { Jan } 1976 \\
\text {-Storm Xaver belongs to the strongest storm occurrence in the past decades }\end{array}$ \\
\hline $\begin{array}{l}\text { The Lowestoft Journal } \\
\text { (20131212) }\end{array}$ & $\begin{array}{l}\text { The Lowestoft Journal, Suffolk MP hits out at environment secretary in statement about the floods, 14Dec2013, } \\
\text { correspondent: Annabelle Dickson. } \\
\text {-UK environment minsiter Owen Patterson claims surge was freak event with } 1 \text { in } 500 \mathrm{y} \text { return period } \\
\text {-MP Peter Aldous points out last surge was } 6 \text { years previously }\end{array}$ \\
\hline ABPmer (2014) & $\begin{array}{l}\text { ABP mer, Ensuring Flood Resilience. An overview of the 5/6 December 2013, Associated British Ports, Marine } \\
\text { Environmental Research, July, } 2014 \\
\text {-water level ranking: Lerwick, Wick, Aberdeen, Leith, North Shields, Immingham, Cromer, Lowestoft, Dover. } \\
\text {-return period: Immingham, Lowestoft }\end{array}$ \\
\hline $\begin{array}{l}\text { CH2MHill Halcrow } \\
\text { (2014) }\end{array}$ & $\begin{array}{l}\text { CH2MHill Halcrow, Cell } 1 \text { Regional Coastal Monitoring Programme, Wave Data Analysis Report 2: 2013-2014, } \\
\text { Final Report, March } 2014 \text { [document properties: author=Andy.Parson @ ch2m.com; datestamp; 04/04/2014] } \\
\text {-North Shields tde gauge water level: return period } 1 \text { in } 200 \mathrm{y} \text { to } 1 \text { in } 500 \mathrm{y} \\
\text {-Whitby tide gauge water level: } 1 \text { in } 100 \mathrm{y} \text { to } 1 \text { in } 500 \mathrm{y} \\
\text {-Scarborough tide gauge water level: } 1 \text { in } 150 \mathrm{y} \text { to } 1 \text { in } 500 \mathrm{y}\end{array}$ \\
\hline Dunbar et al. (2014) & $\begin{array}{l}\text { Dunbar I, N Phipps, M Szonyi, Risk Nexus. After the storm: how the UK's flood defences performed during the } \\
\text { surge following Xaver, Flood resilience review 09.14, Zurich Insurance Company Ltd., Mythenquai 2, 8002, } \\
\text { Zurich, Switzerland [document properties: date 28Aug2014; author= Zurich] } \\
\text {-1953 surge was most serious for at least } 250 \text { years. } \\
\text {-Thames Barrier designed to handle } 1 \text { in } 1000 y \text { event taking climate change and sea level rise into account } \\
-400000 \text { people in Hull area no protected for } 1 \text { in } 100 \text { year standard } \\
\text {-Warrington Water Scheme designed to provide protection at } 1 \text { in } 100 \text { year standard. }\end{array}$ \\
\hline Eriksen (2014) & $\begin{array}{l}\text { Eriksen J, Rekordvandstande i Isefjorden og Roskilde Fjord, Vejret, 138, } 2 \text { 40-48, } 2014 \\
\text {-Roskilde water level at 100-200y return level }\end{array}$ \\
\hline Gautier et al (2014) & $\begin{array}{l}\text { Gautier C, A Camarena, J van Nieuwkoop, SWAN hindcasts Wadden Sea, December 2013. Tidal inlet of } \\
\text { Ameland and eastern Wadden Sea. Deltares, 2014, 197pp. Project 1209433-007, Reference 1209433-007- } \\
\text { HYE-0005 } \\
\text {-maximum water level Delfzijl assessed at } 1 \text { in } 50 \text { year to } 1 \text { in } 100 \text { year recurrence interval }\end{array}$ \\
\hline $\begin{array}{l}\text { Knaack and Heyken } \\
(2014)\end{array}$ & $\begin{array}{l}\text { Knaack H and H Heyken, Xaver hatte sehr schwere Stumflut im Gepaeck, Jahresbericht 2013. Der Zukunft } \\
\text { verplichtet, NLWKN, Niedersaechsischer Landesbetrieb fuer Waserwirtschaft, Kuesten- und Naturschutz, } \\
\text { pp.8-9, document date stamp 24Apr2014. } \\
\text {-Xaver rank } 2 \text { event at Ems; rank } 3 \text { event at Weser- and Elbemuendung }\end{array}$ \\
\hline Nossent et al (2014) & $\begin{array}{l}\text { Nossent J, L Boeckx, E Taverniers, M Deschamps, T Verwaest, F Mostaert, Sinterklaasstorm } 6 \text { december } 2013 . \\
\text { Beschrijving van de hydrometrische gebeurtenissen, Versie 4.0. WL Rapporten, 00-119, Waterbouwkundig } \\
\text { Laboratorium, Antwerpen, Belgie. October 2014, WL2014R00_119_5 [document date stamp: 15Oct2014; } \\
\text { author= Deschamps, Maarten] } \\
\text {-Antwerp water level at 4-5 year return level }\end{array}$ \\
\hline Pelt (2014) & $\begin{array}{l}\text { Pelt AS, BODIL's stormflod i de indre dansk farvande, Vejret, 138, 24-29, } 2014 \\
\text {-rank } 1 \text { water levels: Roskilde Havn, Hornbaek, Klampenborg, Tolboden, Sjaellands Odde, Odense Fjord } \\
\text {-rank } 2 \text { water level: Grenaa } \\
-5 \text { surge events in Kobenhavn at } 1.7 \mathrm{~m} \text { level of Bodil since } 1600\end{array}$ \\
\hline RMS (2014) & $\begin{array}{l}\text { RMS, 2013-2014 Winter Storms in Europe. An Insurance and Catastrophe Modeling Perspective. RMS White } \\
\text { Paper. [PDF TIMESTAMP 11Mar2014] } \\
\text {-overtopping of defences indicates that East Coast defences could not withstand a stronger storm at } 1 \text { in } 1000 \text { year } \\
\text { level }\end{array}$ \\
\hline RWS (2014a) & $\begin{array}{l}\text { RWS, Watermanagementcentrum Nederland, } \begin{array}{c}\text { Stormvloedflits } \\
\text { Rijkswaterstaat (document time stamp: 07Jan2014), 2014a }\end{array} \\
\text {-Delfzijl: previous water level record exceeded by } 1 \mathrm{~cm} \text { during Storm Xaver; expected } 15 \text { times per } 1000 \text { years } \\
\text {-Vlissingen: highest water level since 1953; expected once in 20y } \\
\text {-Hoek van Holland: Storm Xaver water levels exceed by Storm Tilo 09Nov2007 and } 1953 \text { event. } \\
\text {-skew surge statistics: Delzijl twice per 100y; Vlissingen once per seven years. }\end{array}$ \\
\hline RWS (2014b) & $\begin{array}{l}\text { RWS, Stormvloedrapport van } 5 \mathrm{t} / \mathrm{m} 7 \text { december (SR91) Sint-Nicolaasvloed 2013, Watermanagementcentrum } \\
\text { Nederland, Rijkswaterstaat, prepared by Ing. J. Kroos, } 19 \text { Mar 2014b, } 48 \text { pp } \\
\text {-high water per } 1000 \text { years: Den Helder 240, Harlingen 130, Delfzijl 15, Vlissingen 53, Roompot buiten 79, Hoek } \\
\text { van Holland 120, Dordrecht } 130\end{array}$ \\
\hline $\begin{array}{l}\text { Van Rooijen and Oost } \\
\text { (2014) }\end{array}$ & $\begin{array}{l}\text { van Rooijen A, A Oost, Memo: Regionale advisering Ameland Noordwest, Deltares, } 1209381-008-Z K S-0008 \text {, } \\
\text { 43pp, 18Dec2014 [PDF document properties: titl=Regional advisering: Ameland NW; author=Arnold van } \\
\text { Rooijen; keywords: 1209381-008-ZKS-0008; date stamp: 18/12/2014] } \\
\text {-Xbeach model study of beach erosion for water levels at different return periods to } 1 \text { in } 1000 \text { year event } \\
\text {-Storm Xaver was a } 10 \text { year storm in the these simulations. }\end{array}$ \\
\hline NLWKN (20151210) & $\begin{array}{l}\text { NLWKN, Sturmflutdienst der Betriebstelle Norden-Norderney, Niedersaechischer Landesbetrieb fuer } \\
\quad \text { Wasserwirtschaft, Kuesten- und Naturschutz, Niedersachsen, 12/2015 [document date stamp 10Dec2015] } \\
\text {-Norderney return period }>20 \mathrm{y} \\
\text {-Emden return period }>20 \mathrm{y} \\
\text {-Cuxhaven return period 2-20 years }\end{array}$ \\
\hline Patzer (2015) & $\begin{array}{l}\text { Patzer, Marianne, Storm surge forecasting at DMI and perspectives on teh use of Earth Observations, ESA eSurge } \\
\text { Sympositum, Deltares, the Netherlands, (powerpoint presentation) January } 21,2015 \\
-1000 y e a r \text { water level event in Danish fjords and Sealand north coast }\end{array}$ \\
\hline
\end{tabular}




\begin{tabular}{|c|c|}
\hline Sibley et al. (2015) & $\begin{array}{l}\text { Sibley A, D Cox, H Titley, Coastal flooding in England and Wales from Atlantic and North Sea storms during the } \\
\text { 2013/2014 winter, Weather, 70, 62-70, } 2015 \\
\text {-Storm Xaver water level rank } 1 \text { even in most tide gauge stations in UK }\end{array}$ \\
\hline $\begin{array}{l}\text { Haigh and Bradshaw } \\
(2015)\end{array}$ & $\begin{array}{l}\text { Haigh I and E Bradshaw, A century of UK coastal flooding, Planet Earth, Winter 2015, (pdf document properties: } \\
\text { 13Jan2016) } \\
\text {-sea level for 5-6Dec2013 and 3Jan2014 were in top } 10 \text { water levels of } 96 \text { surge events in data base for last } \\
\text { century }\end{array}$ \\
\hline Jensen et al (2015) & $\begin{array}{l}\text { Jensen, J., A. Arns, T. Wahl, Yet another 100yr storm surge event: the role of individual storm surges on design } \\
\text { water levels, Journal of Marine Science and Technology, 23, 882-887, } 2015 \text {. } \\
\text {-calculated return period of absolute water level }\end{array}$ \\
\hline Spencer et al (2015) & $\begin{array}{l}\text { Spencer T, SM Brooks, BR Evans, JA Tempest, I Moeller, Southern North Sea storm surge event of Dec.5, 2013: } \\
\text { Water levels, waves, and coastal impacts, Earth Science Reviews, 146, 120-145, } 2015 \\
\text {-FIG3. [MAP] Variations in tidal ratio with stations plotted (right), Grimsby to Southend on Sea } \\
\text { and extreme water level (from Environment Agency 2011b). Return periods of extreme water levels } \\
\text { Inset top: tides in the North Sea } \\
\text { as derived from observations. Red lines are phase line of the M2 tide, labelled in } \\
\text { hours after the moon's transit through the Greenwich meridion. Blue line give } \\
\text { the mean tidal range at spring tide. } \\
\text {-FIG15. (return period) Statistical analysis of return periods of extreme water levels at } \\
\text { (a) Immingham and (b) Lowestoft. Analysis based on one maximum annual sea level value } \\
\text { AMAX (black squares) or skew surge joint probability method (green squares). } \\
\text { Open circles show } 10 \text { highest water levels on record } \\
\text { (documented at http://www.ntlsf.org/data/uk-network-real-time). } \\
\text { Central point in circle indicates reported landscape change and/or significant coastal flooding. } \\
1953 \text { and } 2013 \text { storm surge maximum water levels indicated by a red circle } \\
\text {-Wick, Aberdeen: } 1 \text { in } 25 y \text { event } \\
\text {-Whitby to Lowestoft: return periods } 1 \text { in } 200 y \text { to } 1 \text { in } 1000 y \\
\text {-Whitby, Immingham: } ~ 1000 y \text { flooding evnts }\end{array}$ \\
\hline $\begin{array}{ll}\text { Van } & \text { massenhove } \\
(2015) & \end{array}$ & $\begin{array}{l}\text { Vanmassenhove, Niels, Storm surge measures ports Flemish coast, Blankenberge, Tuesday February 3rd, } \\
\text { Maritieme Sientverlening en Kust, Coastal Division, Flanders Hydraulic Research.[document properties: } \\
\text { title=Geintegreerd Kustveiligheidsplan; author=Maarten; datestamp=27/02/2015] } \\
\text {-water level for Dec } 2013 \text { corresponded to } 50 \text { year flood event } \\
\text {-map of Belgiam flooding for } 1000 \text { year flood event ( } 7 \mathrm{~m} \mathrm{TAW} \text { ) }\end{array}$ \\
\hline Wadey et al. (2015a) & $\begin{array}{l}\text { Wadey MP, ID Haigh, RJ Nichols, JM Brown, K Horsburgh, B Carroll, SL Gallop, T Mason, E Bradshaw, A } \\
\text { comparison of the } 31 \text { January-1 February } 1953 \text { and 5-6 December } 2013 \text { coastal flood events around the UK, } \\
\text { UK Frontiers in Marine Science, 2, 84, 2015a. } \\
\text {-return period of waters referenced to } 2008 \text { (because of significant sea level rise; } 2008 \text { sea level } 0.11 \mathrm{~m} \text { higher than } \\
1953 \text { levels) }\end{array}$ \\
\hline Wadey et al (2015b) & $\begin{array}{l}\text { Wadey MP, JM Brown, ID Haigh, T Dolphin, P Wisse, Assessment and comparison of extreme sea levels and } \\
\text { waves during the 2013/2014 storm season in two UK coastal regions, Nat. Hazards Earth Syst. Sci. Discuss., } \\
3,2665-2708,2015 \mathrm{~b} \text {. } \\
\text {-Lowestoft: return period water level } 196 \text { years } \\
\text {-Sizewell: return period waves <1 year } \\
\text {-Liverpool: return period water level } 44 \text { years } \\
\text {-Liverpool: return period waves } 5 \text { years } \\
\text {-Tabulated curve for return periods of water level (Liverpool, Lowestoft) and waves (Sizewell, Lowestoft) }\end{array}$ \\
\hline Brooks et al (2016) & $\begin{array}{l}\text { Brooks SM, T Spencer, A McIvor, I Moller, Reconstructing and understanding the impacts of storms and surges, } \\
\text { southern North Sea, Earth Surface Processes and Landforms, 41, 855-864, } 2016 . \\
\text {-return period Immingham=787 years } \\
\text {-return period Lowestoft=188 years }\end{array}$ \\
\hline $\begin{array}{l}\begin{array}{l}\text { Dangendorf et } \\
\text { (2016) }\end{array} \\
\text { al }\end{array}$ & $\begin{array}{l}\text { Dangendorf S, A Arns JG Pinto, P Ludwig, J Jensen, The exceptional influence of storm 'Xaver' on design water } \\
\text { levels in the German Bight, Environmental Research Letters, 11, 2016, 054001 } \\
\text {-Coastal protection structures designed for return period events } 100-10000 \text { years depending on national standards } \\
\text {-return period models including Storm Xaver resulted in } 40 \mathrm{~cm} \text { increase in } 200 \text { year design water levels for } \\
\text { stations in Lower Saxony; station in Schleswig-Holstein had slight decrease in design water levels. } \\
\text {-important to reassess return water levels during lifetime of coastal protection structure. }\end{array}$ \\
\hline ECMWF (20160316) & $\begin{array}{ccccc}\text { ECMWF (20160316), 201312 - Windstorm - Xaver/Bodil/Sven, } & \text { North-western } & \text { Europe } \\
\text { https://confluence.ecmwf.int/pages/viewpage.action?pageId=28315390 } & & \\
\text {-highest water levels on east coast of England for 60 years. } & & \\
\end{array}$ \\
\hline Matelski (2016) & $\begin{array}{l}\text { Matelski, Birgit, Erfahrungen aus der Sturmflut Xaver von 5. und 6.12.2013 und dem Weihnachshochwasser } 2014 \\
\text { in Shleswig-Holstein, IWASA 2016 Tagungsbeitrag, (46. IWASA, 7-8 Januar 2016; Internationales } \\
\text { Wasserbau-Symposium Aachen. [pdf document properties: autor=sonja; datestamp: 26Apr2016] } \\
\text {-highest water level on List auf Sylt reached rank } 5 \text { in record extending back to } 1900\end{array}$ \\
\hline Sorensen (2016) & $\begin{array}{l}\text { Sorensen CS, Water NOT wanted - Coastal floods and flooding protection in Denmark, In RA Herrmann \& J } \\
\text { Jensen (eds), Sicherung von Daemmen, Deichen und Stauanlagen: Handbuch fuer Theorie und Praxis (Vol V } \\
\text { pp3-21). Siegen: Universitaet Siegen, } 2016 \\
\text {-highest ever water levels at Hornbaek \& Copenhagen (series 1890-2015) }\end{array}$ \\
\hline Sorensen et al (2016) & $\begin{array}{l}\text { Sorensen CS, NK Dronen, P Knudsen, J Jensen, P Sorensen, An extreme event as a games changer in coastal zone } \\
\text { management, Journal of Coastal Research, (Special Issue, No 75), 700-704, 2016. Proceedings of the 14th } \\
\text { International Coastal Symposium (Sydney, Australia) ed by A Vila-Concejo, E Bruce, DM Kennedy, RJ } \\
\text { McCarroll } \\
\text {-water level at Hornbaek at 1000y level during storm Xaver }\end{array}$ \\
\hline Ditlevsen et al (2018) & $\begin{array}{l}\text { Ditlevsen C, MM Ramos, C Sorensen, UR Ciocan, T Pionkowitz, Hojvandsstatistikker 2017, Miljo- og } \\
\text { Foedevaremnisteriet, Kystdirektoratet, Lemvig, Februar, } 2018 \\
\text {-Storm Xaver water levels presented with return period analysis curves. }\end{array}$ \\
\hline
\end{tabular}




\begin{tabular}{|l|l|}
\hline $\begin{array}{l}\text { JBA Risk } \\
\text { Management (2020) }\end{array}$ & $\begin{array}{r}\text { JBA Risk Management, Storm Xaver 2013. Event Commentary, 2020. [PDF document properties: } \\
\text { author=Cameron Whitwham] } \\
\text {-record water level at Kingston-upon-Hull at 5.8m } \\
\text {-map of return periods of Xaver water level at stations around UK }\end{array}$ \\
\hline $\begin{array}{l}\text { Surgewave } \\
\text { (20200304) }\end{array}$ & $\begin{array}{l}\text { Surgewatch, Storm event 6th December 2013, https://www.surgewatch.org/events/1/ (last accessed 04Mar2020) } \\
\text {-list of return periods for: Dover, Immingham, Whitby, North Shields, Lowestoft, Liverpool, Newhaven, Leith, } \\
\text { Aberdeen, Llandudno, Portsmouth, Ullapool }\end{array}$ \\
\hline Wikipedia (20200502) & $\begin{array}{l}\text { Wikipedia, Orkan Xaver, https://de.wikipedia.org/wiki/Orkan_Xaver (accessed 2 May 2020) } \\
\text {-Xaver had 2nd highest water in Hamburg record starting in 1825; highest level during Hurrican Capella 1976 }\end{array}$ \\
\hline
\end{tabular}

Table S17. Return period of wind speed; ranking of wind speed

\begin{tabular}{|c|c|}
\hline Source & Full Reference and Notes \\
\hline $\begin{array}{l}\text { Winther-Jensen and } \\
\text { Jorgensen (1999) }\end{array}$ & $\begin{array}{l}\text { Winther-Jensen, M and ER Jorgensen, When real life wind speed exceeds design wind assumptions, } 1999 \\
\text { European Wind Energy Conference, } 1-5 \text { March 1999, Nice, France pp.220-223. } \\
\text {-bridges designed for 50y return wind speed } \\
\text {-survivability in 100y or } 10000 \text { y event (accidental loading) }\end{array}$ \\
\hline $\begin{array}{l}\text { Cruz and Krausmann } \\
(2008)\end{array}$ & $\begin{array}{l}\text { Cruz AM and E Krausmann, Damage to offshore oil and gas facilities following hurricanes Katrina and Rita: An } \\
\text { overview, Journal of Loss Prevention in the Process Industries, 21, 620-626, } 2008 . \\
\text {-offshore petroleum platforms design standard } 100 \text { y event }\end{array}$ \\
\hline Kunz et al (20131206) & $\begin{array}{l}\text { Kunz M, B Muehr, K Schroeter, T Bessel, S Moehrle, T Muenzberg, S Brink, H-M Schmidt, Winterstorm Xaver - } \\
\text { Report. 06Dec2013 - Report No.1, Situation Report - 19:00CET, CEDIM Forensic Disaster Analysis Group } \\
\text { (FDA), Center for Disaster Management and Risk Reduction Technology. } \\
\text {-storm Xaver wind speed reached the 2-10 year level }\end{array}$ \\
\hline Pelt (2014) & $\begin{array}{l}\text { Pelt AS, BODIL's stormflod i de indre dansk farvande, Vejret, 138, 24-29, } 2014 \\
\text {-Denmarks rank } 3 \text { for Torsminde average wind speed }\end{array}$ \\
\hline SMHI (20140703) & 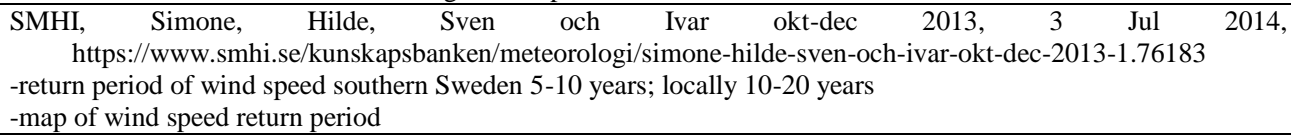 \\
\hline Ribeiro et al (2017) & $\begin{array}{l}\text { Ribeiro R, R Rudge, D Rucinska, Analysis of physical factors of the windstorm Xaver in Poland: post-hazard } \\
\text { review, Weather, 72, 2017, pp.378-382 } \\
\text {-highest ever Netherlands wind speed since } 1910 \text { recorded at Stavorean }(38 \mathrm{~m} / \mathrm{s})\end{array}$ \\
\hline
\end{tabular}

Table S18. Storm trajectory map (arranged by year and then alphabetically)

\begin{tabular}{|c|c|}
\hline Source & Full Reference and Notes \\
\hline $\begin{array}{l}\text { Deutschlander et al. } \\
\text { (2013) }\end{array}$ & $\begin{array}{l}\text { Deutschlander T, K Frierich, S Haeseler, C Lefebvre, Orkantief XAVER ueber Nordeuropea von 5. bis } 7 . \\
\text { Dezember 2013, Deutscher Wetterdienst DWD, Stand 30. Dezember 2013, 19pp. } \\
\text {-trajectory of low pressure centre between } 4 \text { Dec } 2013 \text { 18:00UTC and } 7 \text { Dec2013 00:00UTC }\end{array}$ \\
\hline KNMI (2013) & $\begin{array}{l}\text { KNMI, News report. De Zware storm van } 5 \text { december, } 06 \text { Dec 2013, https://www.knmi.nl/over-het- } \\
\text { knmi/nieuws/de-zware-storm-van-5-december } \\
\text {-path of low pressure center on snapshot map of sea level pressure field }\end{array}$ \\
\hline $\begin{array}{l}\text { Oceanografisch } \\
\text { Meteorologisch } \\
\text { Station (2013) }\end{array}$ & $\begin{array}{l}\text { Oceanografisch Meteorologisch Station, Stormverslag 05-06 december 2013, 26pp, } 2013 \text { [pdf document } \\
\text { properties: author=Myriam Sys; datestamp=15Dec2013] } \\
\text {-FIG1. [MAP] storm track at the 12h intervals }\end{array}$ \\
\hline $\begin{array}{l}\text { Unwetterzentrale } \\
\text { (201312) }\end{array}$ & $\begin{array}{l}\text { Unwetterzentrale, Orkantief XAVER - ein weiterer schwerer Wintersturm der letzten Jahrzehnte, Thomas Savert } \\
\text { and Stefan Laps, Dec. } 2013 \text { http://www.unwetterzentrale.de/uwz/928.html } \\
\text {-FIG2. [MAP] Situation of the developing low on Wedesday 04Dec2013 2100MEZ with the expected trajectory }\end{array}$ \\
\hline Goennert et al (2014) & $\begin{array}{l}\text { Goennert G, O Mueller, M Schaper, K Sossidi, Die Sturmflut nach dem Tief Xaver von 5. bis 7. Dezember } 2013 . \\
\text { Berichte des Landesbetriebes Strassen, Bruecken und Gewaesser (LSBG), Freie und Hansestadt Hamburg, } \\
\text { Nr. 16/2014, 26pp. } \\
\text {-FIG2.Trajectory of Storm Xaver and other storms that caused storm surges }\end{array}$ \\
\hline RMS (2014) & $\begin{array}{l}\text { RMS, 2013-2014 Winter Storms in Europe. An Insurance and Catastrophe Modeling Perspective. RMS White } \\
\text { Paper. [PDF TIMESTAMP 11Mar2014] } \\
\text {-FIG2. storm trajectory Xaver \& other storms during winter 2013-2014 }\end{array}$ \\
\hline RWS (2014b) & $\begin{array}{l}\text { RWS, Stormvloedrapport van } 5 \text { t/m } 7 \text { december (SR91) Sint-Nicolaasvloed 2013, Watermanagementcentrum } \\
\text { Nederland, Rijkswaterstaat, prepared by Ing. J. Kroos, } 19 \text { Mar 2014b, } 48 \text { pp } \\
\text {-Bijlage 3. Surface pressure } 6 \text { Dec } 2013 \text { 0000UTC with low pressure track } 4 \text { Dec } 2013 \text { 1300MEZ to } 6 \text { Dec } 2013 \\
\text { 0100 MEZ }\end{array}$ \\
\hline Carrion (2015) & $\begin{array}{l}\text { Carrion Aretxabala, BI, Morphological impact of the Sinterklaas storm at Het Zwin. Numerical modelling with } \\
\text { Xbeach, M.Sc. Civil Engineering, Delft University of Technology, } 2015 . \\
\text {-FIG2.5. [MAP] European Commission forecast and first assessment of the } \\
\text { of the Sinterklaas storm } \\
\text { (a) Forecast of the path of the storm issued 5Dec. } \\
\text { High wind and storm surge alerts issued for England, Germany, Denmark, Ne } \\
\text { (b) on 6Dec preliminary assessment issued stating estimated storm levels; } \\
\text { flood location and type of damage }\end{array}$ \\
\hline $\begin{array}{l}\text { Fenoglio-Marc et al } \\
(2015)\end{array}$ & $\begin{array}{l}\text { Fenoglio-Marc L, R Scharroo, A Annuziato, L Mendoza, M Becker, J Lillibridge, Cyclone Xaver seen by } \\
\text { geodetic observations, SUPPORTING MATERIAL, Geophys Research Letters, 42, 9925-9932, } 2015 \\
\text {-FIG. storm pressure centre trajectory on rectimlinear coordinates }\end{array}$ \\
\hline Wadey et al. (2015) & $\begin{array}{l}\text { Wadey MP, ID Haigh, RJ Nichols, JM Brown, K Horsburgh, B Carroll, SL Gallop, T Mason, E Bradshaw, A } \\
\text { comparison of the } 31 \text { January-1 February } 1953 \text { and 5-6 December } 2013 \text { coastal flood events around the UK, } \\
\text { UK Frontiers in Marine Science, 2, 84, } 2015 \text {. }\end{array}$ \\
\hline
\end{tabular}




\begin{tabular}{|c|c|}
\hline & -FIG1b: Storm Xaver storm track on map with rectilinear latitude-longitude coordinates. \\
\hline Cheliotis et al (2016) & $\begin{array}{l}\text { Cheliotis, I, G. Varlas, K. Christakos, The impact of cylone Xaver on hydropower potential in Norway, } \\
\text { conference paper, September } 2016 \text { In: T Karaostas, A Bais, PT Nastos (ed), Perspectives on Atmospheric } \\
\text { Sciences, Springer Atmospheric Sciences, Springer, Cham, } 2017 \text { https://doi.org/10.1007/978-3-319-35095- } \\
\text { 0_25, first online 10Sep2016 [pdf document properties: author=Konstantinos Christakos; datestamp: } \\
\text { 07/09/2016] } \\
\text { - FIG3. Mean sea level pressure track of cyclone Xaver as simulated by WRF }\end{array}$ \\
\hline Christakos et al (2016) & $\begin{array}{l}\text { Christakos K, I Cheliotis, G Varlas, G-J Steeneveld, Offshore wind energy analysis of Cyclone Xaver over North } \\
\text { Europe, 13th Deep Sea Offshore Wind R\&D Conference, EERA DeepWind'2016, 20-22 January 2016, } \\
\text { Trondheim, Norway, Energy Procedia, 94, 37-44, } 2016 . \\
\text {-FIG4. Mean sea level pressure in hPa and maximum WPD in kW/m2 at 100m tracks } \\
\text { for cyclone Xaver as simulated by the WRF model }\end{array}$ \\
\hline $\begin{array}{l}\text { Dangendorf et al } \\
(2016)\end{array}$ & $\begin{array}{l}\text { Dangendorf S, A Arns JG Pinto, P Ludwig, J Jensen, The exceptional influence of storm 'Xaver' on design water } \\
\text { levels in the German Bight, Environmental Research Letters, 11, 2016, } 054001 \\
\text {-FIG3. Storm Xaver trajectory from CCLM model and ERA-Interim at } 6 \mathrm{~h} \text { intervals on rectilinear coordinates }\end{array}$ \\
\hline
\end{tabular}

Table S19. Unusual pressure drop; time series central pressure; explosive characteristics (arranged by year and then alphabetically)

\begin{tabular}{|c|c|}
\hline Source & ull Reference and Notes \\
\hline $\begin{array}{l}\text { Air } \text { Worldwide } \\
(20131212)\end{array}$ & $\begin{array}{l}\text { Air Worldwide, Press Release, Boston, 12Dec2013. https://www.air-worldwide.com/In-the-News/AIR- } \\
\text { Estimates-Losses-from-European-Windstorm-Xaver-at-Between-EUR-700-Million-and-EUR-1-4-Billion/ } \\
\text { (accessed 02Jan1990) } \\
\text {-developed into powerful extratropical cyclone as it passed N of Scotland } \\
\text {-explosive cyclogenesis: central pressure decrease } 1010 \mathrm{mb} \text { to } 975 \mathrm{mb} \text { in } 24 \mathrm{~h} \text { from late Thurs }\end{array}$ \\
\hline Kunz et al (20131206) & $\begin{array}{l}\text { Kunz M, B Muehr, K Schroeter, T Bessel, S Moehrle, T Muenzberg, S Brink, H-M Schmidt, Winterstorm Xaver - } \\
\text { Report. 06Dec2013 - Report No.1, Situation Report - 19:00CET, CEDIM Forensic Disaster Analysis Group } \\
\text { (FDA), Center for Disaster Management and Risk Reduction Technology. } \\
\text {-Xaver started between Newfoundland and Greenland with pressure } 1015 \mathrm{hPa} \\
\text {-presure } 975 \mathrm{hPa} 5 \mathrm{Dec} 20130700 \mathrm{CET} \text {; decrease }>25 \mathrm{hPa} \text { in } 24 \mathrm{~h} ; 17.1 \mathrm{hPa} \text { in } 3 \mathrm{~h}\end{array}$ \\
\hline $\begin{array}{l}\text { Oceanografisch } \\
\text { Meteorologisch } \\
\text { Station (2013) }\end{array}$ & $\begin{array}{l}\text { Oceanografisch Meteorologisch Station, Stormverslag 05-06 december 2013, 26pp, } 2013 \text { [pdf document } \\
\text { properties: author=Myriam Sys; datestamp=15Dec2013] } \\
\text {-description of central pressure evolution }\end{array}$ \\
\hline Bancroft (2014) & $\begin{array}{l}\text { Bancroft, George P, Marine Weather Review - North Atlantic Area, September through December, Mariners } \\
\text { Weather Log, volume 58, No.1, April 2014, pp.33-40 } \\
\text {-low pressure deepened by } 44 \mathrm{hPa} \text { in } 24 \mathrm{~h} \\
\text {-maximum intensity of } 960 \mathrm{hPa} \text { inland near 59N 12E at 1800UTC 5Dec2013 }\end{array}$ \\
\hline Hewson et al (2014) & $\begin{array}{l}\text { Hewson T, L Magnusson, O Breivik, F Prates, I Tsonevsky, HJW de Vries, Windstorms in northwest Europe in } \\
\text { late 2013, ECMWF Newsletter, No 139, pp 22-28, Spring } 2014 . \\
\text {-6 h deepeing of } 13 \mathrm{hPa} 5 \text { Dec2013 0000-0600UTC } \\
\text {-maximum } 24 \mathrm{~h} \text { deepening about } 44 \mathrm{hPa} \text {, which is extreme } \\
\text {-minimum central pressure } 961 \mathrm{hPa} 1800 \mathrm{UTC} 5 \mathrm{Dec} 2013\end{array}$ \\
\hline RMS (2014) & $\begin{array}{l}\text { RMS, 2013-2014 Winter Storms in Europe. An Insurance and Catastrophe Modeling Perspective. RMS White } \\
\text { Paper. [PDF TIMESTAMP 11Mar2014] } \\
\text {-Storms Xaver and Christian had low pressure center to east of UK; they were intensifying storms. }\end{array}$ \\
\hline RWS (2014b) & $\begin{array}{l}\text { RWS, Stormvloedrapport van } 5 \mathrm{t} / \mathrm{m} 7 \text { december (SR91) Sint-Nicolaasvloed 2013, Watermanagementcentrum } \\
\text { Nederland, Rijkswaterstaat, prepared by Ing. J. Kroos, } 19 \mathrm{Mar} 2014 \mathrm{~b}, 48 \mathrm{pp} \\
\text {-unusually fast drop of the central pressure by more than } 25 \mathrm{hPa} \text { in } 24 \mathrm{~h}\end{array}$ \\
\hline (2015) & $\begin{array}{l}\text { Axer T, T Bistry, M Klawa, M Mueller, M Suesser, Deutsche Ruck Sturm dokumentation } 2013 \text { Deutschland, } \\
\text { 2013, Deutsche Rueckversicherung Aktiengesellschaft, Hansaallee 177, 40549 Duesseldorf, } \\
\text { www.deutscherueck.de [pdf document information: author=filiz; date stamp=07Aug2015] } \\
\text {-description of rapid pressure drop during Xaver }\end{array}$ \\
\hline Dan et al (2015) & $\begin{array}{l}\text { Dan, Sebastian, Anne-Lise Montreuil, Rosalia Delgado, Tomas van Oyen, Large storm impact on a beach under } \\
\text { sand nourishments, The Proceedings of the Coastal Sediments 2015, edited by Ping Wang, Julie D Rosati, and } \\
\text { Jun Cheng, Coastal Sediments } 2015 \text {, San Diego, USA, 11-15May2015 } \\
\text {-atmospheric pressure on 5Dec dropped to } 1012 \mathrm{hPa} \text { from } 1030 \mathrm{hPa} \text { one day before }\end{array}$ \\
\hline $\begin{array}{l}\text { Fenoglio-Marc et al } \\
(2015 b)\end{array}$ & $\begin{array}{l}\text { Fenoglio-Marc L, R Scharroo, A Annuziato, L Mendoza, M Becker, J Lillibridge, Cyclone Xaver seen by } \\
\text { geodetic observations, SUPPORTING MATERIAL, Geophys Research Letters, 42, 9925-9932, 2015b } \\
\text {-map of trajectory of low pressure center with minimum pressures indicated }\end{array}$ \\
\hline $\begin{array}{l}\text { Dangendorf et al. } \\
(2016)\end{array}$ & $\begin{array}{l}\text { Dangendorf S, A Arns JG Pinto, P Ludwig, J Jensen, The exceptional influence of storm 'Xaver' on design water } \\
\text { levels in the German Bight, Environmental Research Letters, 11, 2016, } 054001 \\
\text {-core pressure decreased from the } 999 \mathrm{hPa} \text { to } 961 \mathrm{hPa} \text { in } 24 \mathrm{~h} \text {; explosive characteristics (Sanders and Gyakum, } \\
1980 \text { ) }\end{array}$ \\
\hline $\begin{array}{l}\text { JBA } \\
\text { Management (2020) }\end{array}$ & $\begin{array}{l}\text { JBA Risk Management, Storm Xaver 2013. Event Commentary, 2020. [PDF document properties: } \\
\text { author=Cameron Whitwham] } \\
\text {-reference to explosive cyclogenesis }\end{array}$ \\
\hline
\end{tabular}

Table S20. Squall line, convective thunderstorms, tornadoes (arranged by year and then alphabetically)

\begin{tabular}{|l|l|}
\hline Source & Full Reference and Notes \\
\hline Unwetterzentrale & $\begin{array}{l}\text { Unwetterzentrale, Orkantief XAVER - ein weiterer schwerer Wintersturm der letzten Jahrzehnte, Thomas Savert } \\
\text { (201312) }\end{array}$ \\
& and Stefan Laps, Dec. 2013 http://www.unwetterzentrale.de/uwz/928.html \\
& -lighting activity in the squall line with extremely strong high $(850 \mathrm{hPa}) 130-170 \mathrm{~km} / \mathrm{h}$ winds to the surface \\
& -already on morning Thursday 5Dec2013 convection front with strong lightning activity \\
& -05Dec2013 17:00 convection front on line Netherlands to Munsterland \& Weserbergland; only few lightning \\
\hline
\end{tabular}




\begin{tabular}{|c|c|}
\hline & $\begin{array}{l}\text { flashes in E } \\
\text {-2 suspected tornado cases: Niedersachsen \& Mecklenburg-Vorpommern }\end{array}$ \\
\hline FINO1 (20140108) & $\begin{array}{l}\text { FINO1, } 15 \text {-m wave damaged FINO1, 08Jan2014. http://www.fino1.de/meldungen/alle-meldungen/137-15-meter- } \\
\text { welle-beschaedigt-fino1 } \\
\text {-unusual wave event that damaged } 15 \mathrm{~m} \text { working deck } \\
\text {-additional damage to } 17 \mathrm{~m} \text { access platform from swinging counterweight set in motion by waves? or wind }\end{array}$ \\
\hline RWS (2014b) & $\begin{array}{l}\text { RWS, Stormvloedrapport van } 5 \mathrm{t} / \mathrm{m} 7 \text { december (SR91) Sint-Nicolaasvloed 2013, Watermanagementcentrum } \\
\text { Nederland, Rijkswaterstaat, prepared by Ing. J. Kroos, } 19 \text { Mar 2014b, } 48 \mathrm{pp} \\
\text {-map showing convective thunderstorm line in front of Netherlands coast }\end{array}$ \\
\hline Van Doreland (2014) & $\begin{array}{l}\text { van Dorland, R, Zware storm op } 5 \text { december, Zenit, p43, Januari } 2014 \\
\text {-squall line passage with hail }\end{array}$ \\
\hline ECMWF (20160316) & $\begin{array}{l}\text { ECMWF (20160316), 201312- Windstorm - Xaver/Bodil/Sven, } \\
\text { https://confluence.ecmwf.int/pages/viewpage.action?pageId=28315390 } \\
\text {-FIG5. [TIMESERIES] These figures show 10m wind speed for Ekofisk in } \\
\text { the North Sea and Torsminde in western Denmark. The HRES } \\
\text { forecast in in red and observations in blue. The forecast is } \\
\text { initialized 04Dec 00UTC. One interesting figure in the model } \\
\text { for both sites, is the large variations from hour to hour close } \\
\text { to the peak of the storm. }\end{array}$ \\
\hline Ribeiro et al (2017) & $\begin{array}{l}\text { Ribeiro R, R Rudge, D Rucinska, Analysis of physical factors of the windstorm Xaver in Poland: post-hazard } \\
\text { review, Weather, 72, 2017, pp.378-382 } \\
\text {-reference to tornado threat in Poland; worst ever tornado Lublin } 20 \mathrm{July} 1931 \text { with } 111 \mathrm{~m} / \mathrm{s}\end{array}$ \\
\hline
\end{tabular}

Table S21. Lightning (arranged by year and then alphabetically)

\begin{tabular}{|l|l|}
\hline Source & Full Reference and Notes \\
\hline Van Dorland (2014) & $\begin{array}{l}\text { van Dorland, R, Zware storm op 5 december, Zenit, p43, Januari 2014 } \\
\text {-map showing time of passage of lightning systems across the Netherlands from the KNMI lightning detection } \\
\text { network }\end{array}$ \\
\hline $\begin{array}{l}\text { Caithness Windfarm } \\
\text { 20160105 }\end{array}$ & $\begin{array}{l}\text { Caithness Windfarm, craigdr, Detailed accidents to 31 December 2015. Document time stamp 5Jan2016, 175pp } \\
\text {-Burradale Wind Farm Shetland, UK: lightning caused fist-sized hole }\end{array}$ \\
\hline WIKI (20200124) & $\begin{array}{l}\text { WIKI, Cyclone Xaver, https://en.wikipedia.org/wiki/Cyclone_Xaver accessed 24Jan2020 } \\
\text {-easyjet flight Bristol to Edinburgh hit by lightning and diverted to Newcastle }\end{array}$ \\
\hline Wikipedia (20200502) & $\begin{array}{l}\text { Wikipedia, Orkan Xaver, https://de.wikipedia.org/wiki/Orkan_Xaver (accessed 2 May 2020) } \\
\text { - FIG3. [MAP] Trajectory of hurricane front anhand der Blitzentladungen in the area of the Netherlands }\end{array}$ \\
\hline
\end{tabular}

Table S22. Meso-vortex (arranged by year and then alphabetically)

\begin{tabular}{|l|l|}
\hline Source & Full Reference and Notes \\
\hline Hewson et al. (2014) & $\begin{array}{r}\text { Hewson T, L Magnusson, O Breivik, F Prates, I Tsonevsky, HJW de Vries, Windstorms in northwest Europe in } \\
\text { late 2013, ECMWF Newsletter, No 139, pp 22-28, Spring 2014. } \\
\text {-'...intense meso-vortex hanging back to the west of the main low for a time, and this enhanced the strong wind } \\
\text { swath running into western Scotland' }\end{array}$ \\
\hline
\end{tabular}

Table S23. Meteotsunami (arranged by year and then alphabetically)

\begin{tabular}{|c|c|}
\hline Source & Full Reference and Notes \\
\hline Goennert et al (2001) & $\begin{array}{l}\text { Goennert G,, SK Dube, T Murthy, W Siefert (2001): 7. Storm surges generated by extratropical cyclones - case } \\
\text { studies. In: Die Kueste } 63 \text { Sonderheft. Heide, Holstein: Boyens. pp 455-546 } \\
\text {-Timmerman (1971): cold fronts over S part of North Sea can produce 'gust bumps' } \\
\text {-water level increases only occur for propagation speed 29-36kt (54-67 km/h) } \\
\text {-numerical simulation oof event 13Dec1956 } \\
\text {-FIG7.18.Sudden water level changes (gust bumps) during Mar27 } 1966 \text { at several locations } \\
\text { on the Netherlands coast (Timmerman, 1971) } \\
\text { maximum amplitude 1m } \\
\text {-FIG7.20.Sudden water level changes (gust bumps) at several locations on the Netherlands coast } \\
\text { on 13Dec1956 (Timmerman 1971) } \\
\text { maximum amplitude 56cm at Katwuk aan Zee }\end{array}$ \\
\hline $\begin{array}{l}\text { McGarricle et al } \\
(2013)\end{array}$ & 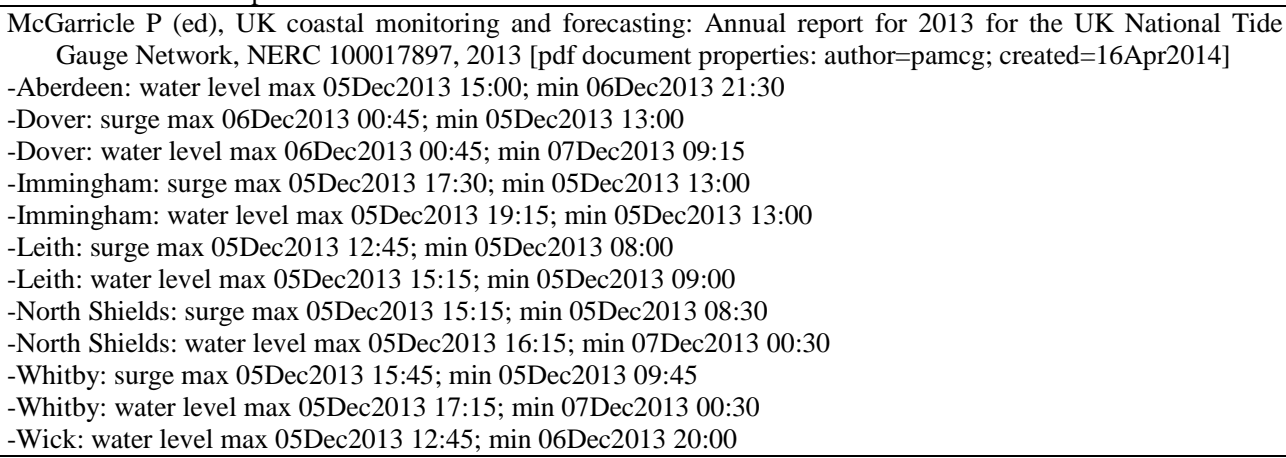 \\
\hline Pelt (2014) & $\begin{array}{l}\text { Pelt AS, BODIL's stormflod i de indre dansk farvande, Vejret, 138, 24-29, } 2014 \\
\text {-water rose suddenly with character of little flood wave at Torsminde }\end{array}$ \\
\hline
\end{tabular}




\begin{tabular}{|l|l|}
\hline & -seiches or skvulper; water masses in motion between Kattegat and Nord Sjaeland \\
\hline RWS (2014b) & $\begin{array}{l}\text { RWS, Stormvloedrapport van } 5 \mathrm{t} / \mathrm{m} 7 \text { december (SR91) Sint-Nicolaasvloed 2013, Watermanagementcentrum } \\
\text { Nederland, Rijkswaterstaat, prepared by Ing. J. Kroos, 19 Mar 2014b, 48 pp } \\
\text {-buistoot/meteotsunami assessed for Hoek van Holland soon after cold front passage; 70 cm amplitude, 1 h } \\
\text { FWHM }\end{array}$ \\
\hline DEMA (2018) & $\begin{array}{l}\text { Danish Emergency Management Agency DEMA, National Risk Profile for Denmark, April 2018 } \\
\text {-mention of general meteotsunami threat for Denmark }\end{array}$ \\
\hline Wikipedia (20200429) & $\begin{array}{l}\text { Wikipedia, Stormen Sven, https://sv.wikipedia.org/wiki/Stormen_Sven\#cite_note-15, accessed 29Apr2020 } \\
\text {-restaurante in Helsingborg with windows stove in by wave and flooded }\end{array}$ \\
\hline
\end{tabular}

Table S24. Infragravity wave (arranged by year and then alphabetically)

\begin{tabular}{|l|l|}
\hline Source & Full Reference and Notes \\
\hline $\begin{array}{l}\text { Oceanografisch } \\
\text { Meteorologisch }\end{array}$ & $\begin{array}{c}\text { Oceanografisch Meteorologisch Station, Stormverslag 05-06 december 2013, 26pp, 2013 [pdf document } \\
\text { properties: author=Myriam Sys; datestamp=15Dec2013] } \\
\text {-analysis of long period waves: energy, equivalent wave height, spectra }\end{array}$ \\
\hline Gautier et al (2014) & $\begin{array}{c}\text { Gautier C, A Camarena, J van Nieuwkoop, SWAN hindcasts Wadden Sea, December 2013. Tidal inlet of } \\
\text { Ameland and eastern Wadden Sea. Deltares, 2014, 197pp. Project 1209433-007, Reference 1209433-007- } \\
\text { HYE-0005 } \\
\text {-one of key objectives of SWAN model study to assess penetration of low frequency waves from North Sea into } \\
\text { Wadden Sea } \\
\text {-spectra graphs show indication of high energy 30s waves in front of Delfzijl }\end{array}$ \\
\hline $\begin{array}{l}\text { Carrion Aretxabala, BI, Morphological impact of the Sinterklaas storm at Het Zwin. Numerical modelling with } \\
\text { Xbeach, M.Sc. Civil Engineering, Delft University of Technology, 2015. } \\
\text {-mention of infragravity waves of 35s period as important mechanism for dune damage }\end{array}$ \\
\hline $\begin{array}{l}\text { Wahle K, J Staneva, W Koch, L Fenoglio-Marc, HTM Ho-Hagemann, EV Stanev, An atmosphere-wave regional } \\
\text { coupled model: improving prediction of wave heights in the southern North Sea, Ocean Sci., 13, 289-301, } \\
\text { 2017.(doi:10.5194/os-13-289-2017). } \\
\text {-waverider 2D spectra indicating short episodes of infragravity waves at the Elbe and FINO1 buoys with wave } \\
\text { periods }>25 s\end{array}$ \\
\hline
\end{tabular}

Table S25. Wave dynamics and dike breaches; wave runup studies (arranged by year and then alphabetically)

\begin{tabular}{|c|c|}
\hline Source & Full Reference and Notes \\
\hline $\begin{array}{l}\text { Sueddeutsche Zeitung } \\
\text { (20131215) }\end{array}$ & $\begin{array}{l}\text { Sueddeutsche Zeitung, Xaver holte sich Land; Sturmschaden auf Sylt, 15Dec201315:36 } \\
\text { https://www.sueddeutsche.de/panorama/sturmschaeden-auf-sylt-xaver-holte-sich-land-1.1844100 } \\
-40 \mathrm{~m} \text { of coast line retreat at Hoernum Odde } \\
-57 \% \text { of } 21 \mathrm{~km} \text { stretch of Sylt west coast with dune collapse damage } \\
\text {-line of the border dunes broken through in 5 places } \\
\text {-Keitum on east side of Sylt: water inbreak after cliff collapse requiring sand bag }\end{array}$ \\
\hline Carrion (2015) & $\begin{array}{l}\text { Carrion Aretxabala, BI, Morphological impact of the Sinterklaas storm at Het Zwin. Numerical modelling with } \\
\text { Xbeach, M.Sc. Civil Engineering, Delft University of Technology, } 2015 \text {. } \\
\text {-gap in dune line at Het Zwin and overwash phenomenon }\end{array}$ \\
\hline Brooks et al. (2016) & $\begin{array}{l}\text { Brooks SM, T Spencer, A McIvor, I Moller, Reconstructing and understanding the impacts of storms and surges, } \\
\text { southern North Sea, Earth Surface Processes and Landforms, } 41,855-864,2016 \text {. } \\
\text {-FIG 4. aerial photo interpretation shows large along shore differences with characteristic spatial scale 100- } \\
1000 \mathrm{~m}\end{array}$ \\
\hline $\begin{array}{l}\text { Dreier and Foehle } \\
(2018)\end{array}$ & $\begin{array}{l}\text { Dreier, Norman and Peter Froehle, Operational wave forecast in the German Bight as part of a sensor- and risk } \\
\text { based early warning system, In: J-S Shim, I Chun, HS Lim (ed), Proceedings from the International Coastal } \\
\text { Symposium (ICS) } 2018 \text { (Busan, Republic of Korea), Journal of Coastal Research, Special Issue No. 85, 1161- } \\
1165,2018 \\
\text {-measurements of water level, significant wave height, wave runup at Untjehoern } \\
\text {-wave runup 2m above waver level and 1m above significant wave height }\end{array}$ \\
\hline $\begin{array}{l}\text { Surgewatch } \\
(20200304)\end{array}$ & $\begin{array}{l}\text { Surgewatch, Storm event 6th December 2013, https://www.surgewatch.org/events/1/ (last accessed 04Mar2020) } \\
\text {-50 instances of breasched (manmade and natural) defences across UK coastline; multiple overtopping } \\
\text {-'flooded from wave overtopping': Boston, Great Yarmouth, Lowestoft, } \\
\text { North Berwick, Jaywick, North Wales, Blackpool, Cleveleys, Walcott, } \\
\text { Cromer, Whitstable, Portgordon, New Brighton (JBA, 2014) }\end{array}$ \\
\hline
\end{tabular}

Table S26. Precipitation, river level dike breaches (arranged by year and then alphabetically)

\begin{tabular}{|c|c|}
\hline Source & Full Reference and Notes \\
\hline Goennert et al (2014) & $\begin{array}{l}\text { Goennert G, O Mueller, M Schaper, K Sossidi, Die Sturmflut nach dem Tief Xaver von 5. bis 7. Dezember } 2013 . \\
\text { Berichte des Landesbetriebes Strassen, Bruecken und Gewaesser (LSBG), Freie und Hansestadt Hamburg, } \\
\text { Nr. 16/2014, 26pp. } \\
\text {-precip Hamburg-Fuhlsbuettel 5Dec2013 } 11.9 \mathrm{~mm} \text {; no information that this contributed to Elbe water levels at } \\
\text { Hamburg } \\
\text {-reference to flooding in Elbe and Danube in June } 2013 \text {. }\end{array}$ \\
\hline Noosent et al (2014) & $\begin{array}{l}\text { Nossent J, L Boeckx, E Taverniers, M Deschamps, T Verwaest, F Mostaert, Sinterklaasstorm } 6 \text { december } 2013 . \\
\text { Beschrijving van de hydrometrische gebeurtenissen, Versie 4.0. WL Rapporten, 00-119, Waterbouwkundig } \\
\text { Laboratorium, Antwerpen, Belgie. October 2014, WL2014R00_119_5 [document date stamp: 15Oct2014; } \\
\text { author= Deschamps, Maarten] } \\
\text {-focus on Belgian precipiation and river heights/flow in Antwerp estuary during Storm Xaver }\end{array}$ \\
\hline
\end{tabular}




\begin{tabular}{|l|l|}
\hline Wadey et al (2015b) & $\begin{array}{l}\text { Wadey MP, JM Brown, ID Haigh, T Dolphin, P Wisse, Assessment and comparison of extreme sea levels and } \\
\text { waves during the 2013/2014 storm season in two UK coastal regions, Nat. Hazards Earth Syst. Sci. Discuss., } \\
3,2665-2708,2015 \mathrm{~b} .\end{array}$ \\
& $\begin{array}{l}\text {-dike breaches in Suffolk occurred along rivers in two areas and not on the open coast } \\
\text {-river levels high because of heavy precipitation during period } \\
\text {-1953 flooding would have been worse if there had additonally been alot of rain. }\end{array}$ \\
\hline
\end{tabular}

Table S27. Unusual peak of significant wave height in northern North Sea (arranged by year and then alphabetically)

\begin{tabular}{|l|l|}
\hline Source & Full Reference and Notes \\
\hline Wahle et al (2017) & Wahle K, J Staneva, W Koch, L Fenoglio-Marc, HTM Ho-Hagemann, EV Stanev, An atmosphere-wave regional \\
& coupled model: improving prediction of wave heights in the southern North Sea, Ocean Sci., 13, 289-301, \\
& 2017.(doi:10.5194/os-13-289-2017). \\
& -unusual peak in altimeter-derived significant wave height 58-59N in northern North Sea \\
\hline
\end{tabular}

Table S28. Double surge peak from wind and travelling wave (arranged by year and then alphabetically)

\begin{tabular}{|l|l|}
\hline Source & Full Reference and Notes \\
\hline Wahle et al (2017) & $\begin{array}{l}\text { Wahle K, J Staneva, W Koch, L Fenoglio-Marc, HTM Ho-Hagemann, EV Stanev, An atmosphere-wave regional } \\
\text { coupled model: improving prediction of wave heights in the southern North Sea, Ocean Sci., 13, 289-301, } \\
\text { 2017.(doi:10.5194/os-13-289-2017). } \\
\text {-external surge from Aberdeen and Lowestoft caused second storm surge maximum in the German Bight } \\
\text {-'as demonstrated by Staneva et al (2016), the wave induced mechanisms contributed to a persistent increase in the } \\
\text { surge after the first maximum...' }\end{array}$ \\
\hline $\begin{array}{l}\text { Niehuser S, S Dangendorf, A Arns, J Jensen, A high resolution storm surge forecast for the German Bight, } \\
\text { Conference: 9th Chinese-German Joint Symposium on Coastal and Ocean Engineering, Tainan, Taiwan, 2018 } \\
\text {-double peak of true surge from wind effect and travelling surge wave }\end{array}$ \\
\hline
\end{tabular}

Table S29. Modelled turbulence kinetic energy in wave model (arranged by year and then alphabetically)

\begin{tabular}{|l|l|}
\hline Source & Full Reference and Notes \\
\hline Gautier et al (2014) & $\begin{array}{c}\text { Gautier C, A Camarena, J van Nieuwkoop, SWAN hindcasts Wadden Sea, December 2013. Tidal inlet of } \\
\text { Ameland and eastern Wadden Sea. Deltares, 2014, 197pp. Project 1209433-007, Reference 1209433-007- } \\
\text { HYE-0005 } \\
\text {-wave dissipation in the SWAN model of the Wadden Sea }\end{array}$ \\
\hline $\begin{array}{c}\text { Staneva J, K Wahle, H Guenther, E Stanev, Coupling of wave and circulation models in coastal-ocean predicting } \\
\text { systems: a case study for the German Bight, Ocean Sci., 12, 797-806, 2016a. } \\
\text {-map modelled turbulence kinetic energy produced for Storm Britta with high values along coast }\end{array}$ \\
\hline
\end{tabular}

Table S30. Fatalities \& injuries

\begin{tabular}{|c|c|}
\hline Source & Full Reference and Notes \\
\hline BBC (20131205) & $\begin{array}{l}\text { BBC, In pictures: Winter storm hits UK, } 5 \text { December 2013, https://www.bbc.com/news/uk-scotland-25231224 } \\
-2 \text { men killed in UK durign the storm } \\
\text { FIG4. [PHOTO] Earlier in West Lothian lorry driver dies when vehicle blown over } \\
\text { on to two cars (PA) }\end{array}$ \\
\hline BBC (20131206) & $\begin{array}{l}\text { BBC20131206, Deadly storm and tidal surge batter northern Europe, BBC } 6 \text { Dec2013 (report by Anna Holligan) } \\
\text {-at least } 7 \text { people killed } \\
\text {-Poland, Poraj: tree blown onto car \& } 3 \text { people killed } \\
\text {-UK: } 2 \text { deaths; lorry driver killed when truck overturned near Edinburgh, man crushed by falling tree } \\
\text { Nottinghamshire } \\
\text {-Denmark: } 1 \text { death; woman died after lorry blown over } \\
\text {-Sweden: } 1 \text { death; } 2 \text { sailors swept off ship in southern Sweden, not found by rescue services }\end{array}$ \\
\hline Bloomberg (2013) & $\begin{array}{l}\text { Bloomberg, Hamburg has worst flood in } 37 \text { year amid European storms, Nicholas Brautlecht, 6Dec2013, } \\
\text { 02:58PM GMT https://www.bloomberg.com/news/articles/2013-12-06/hamburg-has-worst-flood-in-37-years-as- } \\
\text { storms-rage-across-europe } \\
-3 \text { people killed Poland }\end{array}$ \\
\hline $\begin{array}{l}\text { Daily } \\
(20131206)\end{array}$ & $\begin{array}{l}\text { Daily Mail, Huge storm strikes Europe causing death and destruction and leading to cancellation of hundreds of } \\
\text { flights, 6Dec2013. (correspondent: Nick Enoch), https://www.dailymail.co.uk/news/article-2519517/Huge-storm- } \\
\text { strikes-Europe-causing-death-destruction-leading-cancellation-hundreds-flights.html } \\
-7 \text { people killed Europe: } 3 \text { in N Poland car accident, } 1 \text { in truck overturn Denmark }\end{array}$ \\
\hline $\begin{array}{l}\text { Deutschlander et al } \\
(2013)\end{array}$ & $\begin{array}{l}\text { Deutschlander T, K Frierich, S Haeseler, C Lefebvre, Orkantief XAVER ueber Nordeuropea von } 5 . \text { bis } 7 . \\
\text { Dezember 2013, Deutscher Wetterdienst DWD, Stand 30. Dezember 2013, 19pp. } \\
-10 \text { fatalities across Europe }\end{array}$ \\
\hline DW (20131205) & $\begin{array}{l}\text { DW, Storm Xaver pummels Europe, flood surge feared, 05/12/2013 } \\
\text {-driver killed Scotland when truck overturned } \\
\text {-scooter rider killed England by falling tree } \\
\text {-72 year old woman killed Denmark when vehicle blown off road }\end{array}$ \\
\hline Expressen (2013) & $\begin{array}{l}\text { Expressen, Flera doda i Sverige efter stormen Sven, 06Dec2013, 17:34CET? } \\
\text {-5 killed in Sweden, mostly by falling trees and branches } \\
\text {-two seamen swept off Dutch cargo ship Marietje Andrea off Ystad on Thursday morning; search stopped after } 6 \\
\text { h. }\end{array}$ \\
\hline $\begin{array}{l}\text { Gccapitalideas } \\
\text { (20131209) }\end{array}$ & $\begin{array}{l}\text { gccapitalideas, Windstorm Xaver, 9Dec2013, https://www.gccapitalideas.com/2013/12/09/windstorm-xaver/ } \\
-8 \text { people killed in storm }\end{array}$ \\
\hline
\end{tabular}




\begin{tabular}{|c|c|}
\hline $\begin{array}{l}\text { Kristeligt Dagblad } \\
(20131205)\end{array}$ & $\begin{array}{l}\text { Kristeligt Dagblad, Stormen blaeser Skotland omkuld: Doodsfald og nebrud, (contributor: Ritzau), 05Dec2013 } \\
\text { 12:50 https://www.kristeligt-dagblad.dk/udland/stormen-bl\%C3\%A6ser-skotland-omkuld-d\%C3\%B8dsfald- } \\
\text { og-nedbrud } \\
\text {-1 transport truck driver killed when truck overturned in Wesst Lothian near Edinburgh } \\
\text {-at least two other injuries as consequence of fallen trees }\end{array}$ \\
\hline Kunz et al (2013) & $\begin{array}{l}\text { Kunz M, B Muehr, K Schroeter, T Bessel, S Moehrle, T Muenzberg, S Brink, H-M Schmidt, Winterstorm Xaver - } \\
\text { Report. 06Dec2013 - Report No.1, Situation Report - 19:00CET, CEDIM Forensic Disaster Analysis Group } \\
\text { (FDA), Center for Disaster Management and Risk Reduction Technology. } \\
-6 \text { deaths }\end{array}$ \\
\hline $\begin{array}{l}\text { National Wind Watch } \\
\text { (20131210) }\end{array}$ & $\begin{array}{l}\text { National Wind Watch, 10Dec2013, Xaver zerstoert ein Windrad bei Vlatten, original source: Aachener Zeitung, } \\
\text { 6Dec2013 https://www.wind-watch.org/news/2013/12/10/xaver-zerstort-ein-windrad-bei-vlatten/ } \\
\text {-woman injured }\end{array}$ \\
\hline $\begin{array}{l}\text { Nordbayern } \\
(20131207)\end{array}$ & $\begin{array}{l}\text { Nordbayern, Deining: 'Xaver' reisst Rotorblatt von Windrad ab. Windkraftanlage schleuderte Eisbrocken auf die } \\
\text { Strasse, 07/12/2013, 12:07. https://www.nordbayern.de/region/neumarkt/deining-xaver-reisst-rotorblatt-von- } \\
\text { windrad-ab-1.3326492 } \\
\text {-three people lightly injured northern Bavaria }\end{array}$ \\
\hline $\begin{array}{l}\text { Spiegel International } \\
(20131206)\end{array}$ & $\begin{array}{l}\text { Spiegel International, Winter storm 'Xaver' batters northern Europe,6 Dec } 2013,12: 56 \\
\text { https://www.spiegel.de/international/europe/tidal-surge-winter-storm-xaver-batters-northern-europe-a- } \\
\text { 937576.html\# } \\
\text {-3 storm-related deaths in UK and Denmark } \\
\text { FIG14.Scotland also say high winds on Thursday. Here, rescue workers at the scene } \\
\text { a truck accident near Bathgate (AP) }\end{array}$ \\
\hline The Local (20131205) & 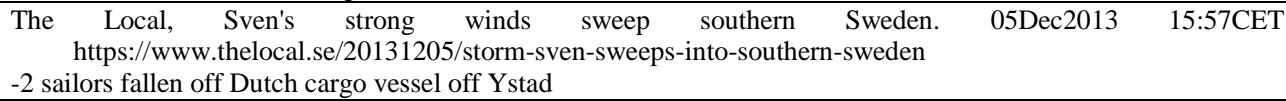 \\
\hline $\begin{array}{l}\text { Unwetterzentrale } \\
(201312)\end{array}$ & $\begin{array}{l}\text { Unwetterzentrale, Orkantief XAVER - ein weiterer schwerer Wintersturm der letzten Jahrzehnte, Thomas Savert } \\
\text { and Stefan Laps, Dec. } 2013 \text { http://www.unwetterzentrale.de/uwz/928.html } \\
\text {-10 people killed across Europe: } 5 \text { in Poland, } 4 \text { in Germany, } 1 \text { in UK }\end{array}$ \\
\hline ABPmer (2014) & $\begin{array}{l}\text { ABP mer, Ensuring Flood Resilience. An overview of the 5/6 December 2013, Associated British Ports, Marine } \\
\text { Environmental Research, July, } 2014 \\
\text {-no fatalities on coast; } 2 \text { fatalities inland from high winds }\end{array}$ \\
\hline AON Benfield (2014) & $\begin{array}{l}\text { AON Benfield, Impact forecasting. December } 2013 \text { Global Catastrophe Recap, 2014. [document properties: } \\
\text { abrandt; date stamp: 10Jan2014] } \\
-15 \text { fatalities Europe }\end{array}$ \\
\hline RWS (2014b) & $\begin{array}{l}\text { RWS, Stormvloedrapport van } 5 \text { t/m } 7 \text { december (SR91) Sint-Nicolaasvloed 2013, Watermanagementcentrum } \\
\text { Nederland, Rijkswaterstaat, prepared by Ing. J. Kroos, } 19 \text { Mar 2014b, } 48 \text { pp } \\
-15 \text { fatalities across Europe }\end{array}$ \\
\hline Carrion (2015) & $\begin{array}{l}\text { Carrion Aretxabala, BI, Morphological impact of the Sinterklaas storm at Het Zwin. Numerical modelling with } \\
\text { Xbeach, M.Sc. Civil Engineering, Delft University of Technology, } 2015 . \\
\text {-FIG2.5. European commissiion info sheet with } 7 \text { fatalities }\end{array}$ \\
\hline Wadey et al (2015) & $\begin{array}{l}\text { Wadey MP, ID Haigh, RJ Nichols, JM Brown, K Horsburgh, B Carroll, SL Gallop, T Mason, E Bradshaw, A } \\
\text { comparison of the } 31 \text { January-1 February } 1953 \text { and 5-6 December } 2013 \text { coastal flood events around the UK, } \\
\text { UK Frontiers in Marine Science, } 2,84,2015 \text {. } \\
-15 \text { fatalities in NW Europe but non from coastal flooding }\end{array}$ \\
\hline Ribeiro et al (2017) & $\begin{array}{l}\text { Ribeiro R, R Rudge, D Rucinska, Analysis of physical factors of the windstorm Xaver in Poland: post-hazard } \\
\text { review, Weather, 72, 2017, pp.378-382 } \\
\text {-Poland: } 4 \text { fatalities \& } 53 \text { injured }\end{array}$ \\
\hline DEMA (2018) & $\begin{array}{l}\text { Danish Emergency Management Agency DEMA, National Risk Profile for Denmark, April } 2018 \\
\text {-one fatality Denmark }\end{array}$ \\
\hline Rucinska (2019) & $\begin{array}{l}\text { Rucinska D, Describing Storm Xaver in disaster terms, International Journal of Disaster Reduction, 34, 147-153, } \\
2019 \\
\text {-Poland 4, Germany 7, UK 2, Netherlands } 3 \text { (including people swept out to sea) }\end{array}$ \\
\hline Wikipedia (20191002) & $\begin{array}{l}\text { Wikipedia, Stormen Bodil, https://da.wikipedia.org/wiki/Stormen_Bodil\#cite_note-39 (accessed 02Oct2019) } \\
-15 \text { fatalities across Europe with description of circumstances }\end{array}$ \\
\hline $\begin{array}{l}\text { JBA Risk } \\
\text { Management (2020) }\end{array}$ & $\begin{array}{l}\text { JBA Risk Management, Storm Xaver 2013. Event Commentary, 2020. [PDF document properties: } \\
\text { author=Cameron Whitwham] } \\
-15 \text { fatalities across Europe; } 2 \text { fatalities UK }\end{array}$ \\
\hline WIKI (20200124) & $\begin{array}{l}\text { WIKI, Cyclone Xaver, https://en.wikipedia.org/wiki/Cyclone_Xaver accessed 24Jan2020 } \\
\text {-5 Poland, } 2 \text { UK, } 1 \text { Denmark, } 7 \text { Sweden }\end{array}$ \\
\hline Wikipedia (20200429) & $\begin{array}{l}\text { Wikipedia, Stormen Sven, https://sv.wikipedia.org/wiki/Stormen_Sven\#cite_note-15, accessed 29Apr2020 } \\
\text {-listing and description of fatalities in Sweden }\end{array}$ \\
\hline Wikipedia (20200502) & $\begin{array}{l}\text { Wikipedia, Orkan Xaver, https://de.wikipedia.org/wiki/Orkan_Xaver (accessed } 2 \text { May 2020) } \\
\text {-listing fatalities across Europe, with some brief descriptions } \\
\text {-Germany: } 82 \text { y old woman died in ambulance accident during storm } \\
\text {-Denmark: } 72 \text { y old woman died in truck traffic accident } \\
\text {-Austria: truck driver killed when gusts blew truck into head-on collision with bus. } \\
\text {-UK: man killed by falling branch Nottinghamshire }\end{array}$ \\
\hline
\end{tabular}

Table S31. Coastal flooding and evacuations (arranged by year and then alphabetically)

\begin{tabular}{|l|l|}
\hline Source & Full Reference and Notes \\
\hline BBC (20131205) & $\begin{array}{l}\text { BBC, In pictures: Winter storm hits UK, 5 December 2013, https://www.bbc.com/news/uk-scotland-25231224 } \\
\text { FIG1. [PHOTO] Thousands evacuated from homes as storms hit much of UK. } \\
\text { Residents on this street in Rhyl, north Wales, rescued by RNLI (Reuters) }\end{array}$ \\
\hline
\end{tabular}




\begin{tabular}{|c|c|}
\hline & $\begin{array}{l}\text { FIG2. [PHOTO] British Red Cross set up rescue centre in the Denbighshire town } \\
\text { but say } 500 \text { people could still be in need of help (Reuters). } \\
\text { FIG10.[PHOTO] Elsewhere in England, high tides followed the storm's tidal surges, } \\
\text { hitting coastal towns including Blackpool (AP) } \\
\text { FIG11.[PHOTO] The famous Blackpool beach was battered by the tide (AP) } \\
\text { FIG12.[PHOTO] In Merseyside the storm claimed a pirate ship build earlier this } \\
\text { year out of drift wood on New Brighton beach. (Bob Warwick) } \\
\text { FIG13.[PHOTO] This dramatic shot captures the scene on the Wirral as the storm } \\
\text { hit the north west of England (Laura Steen) }\end{array}$ \\
\hline BBC (20131206) & $\begin{array}{l}\text { BBC20131206, Deadly storm and tidal surge batter northern Europe, BBC } 6 \text { Dec2013 (report by Anna Holligan) } \\
\text {-eastern England: } 10000 \text { homes evacuated Norfolk \& Suffolk; houses collapse Hemsby } \\
\text {-north Wales: residents rescued by lifeboat crews } \\
\text {-Hamburg: Fish Market and some streets near river flooded } \\
\text {-Netherlands: limited flooding, sea dykes held; some flooding Dordrecht and Rotterdam }\end{array}$ \\
\hline Bloomberg (2013) & $\begin{array}{l}\text { Bloomberg, Hamburg has worst flood in } 37 \text { year amid European storms, Nicholas Brautlecht, 6Dec2013, 02:58PM } \\
\text { GMT https://www.bloomberg.com/news/articles/2013-12-06/hamburg-has-worst-flood-in-37-years-as-storms-rage- } \\
\text { across-europe } \\
\text {-thousands evacuated from coastal areas of UK during night 5-6Dec2013 } \\
\text {-port flooded plus quayside streets \& squares }\end{array}$ \\
\hline Daily Mail (20131206) & $\begin{array}{l}\text { Daily Mail, Huge storm strikes Europe causing death and destruction and leading to cancellation of hundreds of } \\
\text { flights, 6Dec2013. (correspondent: Nick Enoch), https://www.dailymail.co.uk/news/article-2519517/Huge-storm- } \\
\text { strikes-Europe-causing-death-destruction-leading-cancellation-hundreds-flights.html } \\
\text {-flooding Thorsminde, Hamburg Fischmarkt, Bensersiel }\end{array}$ \\
\hline $\begin{array}{l}\text { Deutschlander et al } \\
(2013)\end{array}$ & $\begin{array}{l}\text { Deutschlander T, K Frierich, S Haeseler, C Lefebvre, Orkantief XAVER ueber Nordeuropea von 5. bis 7. Dezember } \\
\text { 2013, Deutscher Wetterdienst DWD, Stand 30. Dezember 2013, 19pp. } \\
\text {-flooding in Hallig area of Germany }\end{array}$ \\
\hline $\begin{array}{l}\text { Gccapitalideas } \\
(20131209)\end{array}$ & $\begin{array}{l}\text { gccapitalideas, Windstorm Xaver, 9Dec2013, https://www.gccapitalideas.com/2013/12/09/windstorm-xaver/ } \\
\text {-UK: } 1400 \text { properties flooded in UK: } 400 \text { Humber region, } 300 \text { Rhyl North Wales, } 300 \text { Boston Lincolnshire, } 200 \\
\text { Whitby; also Scarborough, Great Yarmouth, Lowestoft } \\
\text {-Germany: damage from coast flooding in Hamburg; historic fish market flooded } \\
\text {-Netherlands: some flooding in Dordrecht, Rotterdam, Vlaardingen }\end{array}$ \\
\hline Kunz (20131206) & $\begin{array}{l}\text { Kunz M, B Muehr, K Schroeter, T Bessel, S Moehrle, T Muenzberg, S Brink, H-M Schmidt, Winterstorm Xaver - } \\
\text { Report. 06Dec2013 - Report No.1, Situation Report - 19:00CET, CEDIM Forensic Disaster Analysis Group } \\
\text { (FDA), Center for Disaster Management and Risk Reduction Technology. } \\
\text {-thousands evacuated from lowland areas in UK } \\
\text {-Fish Market and Elbstrasse in Hamburg completely flooded } \\
\text {-pollutant release hazard } \\
\text {-Borkum: evacuation of residents of the roadsted prepared }\end{array}$ \\
\hline $\begin{array}{l}\text { Spiegel International } \\
(20131206)\end{array}$ & $\begin{array}{l}\text { Spiegel International, Winter storm 'Xaver' batters northern Europe, } 6 \text { Dec } 2013, \\
\text { https://www.spiegel.de/international/europe/tidal-surge-winter-storm-xaver-batters-northern-europe-a- } \\
\text { 937576.html\# } \\
\text { FIG2. The storm brought rain, hail and snow with the tidal surge, and the } \\
\text { German port city of Hamburg seen its worst flooding in decades, } \\
\text { forcing authorities to close off parts of the city center on Thursday } \\
\text { night. Here, woman passes by the storm surge in central Hamburg (DPA) } \\
\text { FIG3. People stand on benches in Hamburg's historic fish market which } \\
\text { has been flooded by the Elbe River due to the storm (AFP) } \\
\text { FIG4. Floodwaters in Hamburg early on Friday morning. City officials closed } \\
\text { off flooded areas and some people reportedly had trouble reaching } \\
\text { work in the morning (DPA) } \\
\text { FIG6. Streets also flooded in the northwestern German town of Norddeich on } \\
\text { Friday morning (Reuters) } \\
\text { FIG7. Water levels reached what was expected to be their high point in Hamburg } \\
\text { on Friday morning, some } 6 \mathrm{~m} \text { above level (Reuters) } \\
\text { FIG8. Waves overtake ferry station in the northern German city of Dagebuell } \\
\text { on Thursday night (DPA) } \\
\text { FIG9. The pier at the Bensersiel port of lower Saxony was also flooded by the } \\
\text { storm surge (DPA) } \\
\text { FIG16.Here waves batter the promenade in Blackpool England on Thursday as the storm } \\
\text { hit the UK } \\
\text { FIG17.Homes on the tiny German island of Langeness as the North Sea rose on } \\
\text { Thursday (DPA) } \\
\text { FIG18. Traffic signs on the beach in the German village of Norddeich on Thursday } \\
\text { were nearly submerged by the storm surge. (Reuters) }\end{array}$ \\
\hline $\begin{array}{l}\text { Unwetterzentrale } \\
(201312)\end{array}$ & $\begin{array}{l}\text { Unwetterzentrale, Orkantief XAVER - ein weiterer schwerer Wintersturm der letzten Jahrzehnte, Thomas Savert and } \\
\text { Stefan Laps, Dec. } 2013 \text { http://www.unwetterzentrale.de/uwz/928.html } \\
\text {-Hamburg hafen: streets \& quays flooded }\end{array}$ \\
\hline ABPmer (2014) & $\begin{array}{l}\text { ABP mer, Ensuring Flood Resilience. An overview of the 5/6 December 2013, Associated British Ports, Marine } \\
\text { Environmental Research, July, } 2014 \\
\text {-Immingham port flooded } \\
\text {-Lowestoft flood damage } \\
\text {-Hull frontage flooded; } 115 \text { businesses \& } 149 \text { residential properties; water level 5.8mODN, 0.4m below crest of Hull } \\
\quad \text { Tidal Surge Barrier. }\end{array}$ \\
\hline BBC (20141205) & $\begin{array}{l}\text { BBC, East coast surge: what happened next? (report by Richard Haugh), 5Dec2014 } \\
\text {-home evacuations:Kent: } 500 \text { homes; Lincolnshire: 600homes; Humber: 400homes; Jaywick, Essex: } 2500 \text { homes }\end{array}$ \\
\hline
\end{tabular}




\begin{tabular}{|c|c|}
\hline & $\begin{array}{l}\text { evacuated; Lowestoft, Suffolk: town center flooded } \\
\text {-Hugh Drake, farmer, lost } 25 \text { acres of farmland; } 2 \text { years before crops can grow because of salt }\end{array}$ \\
\hline $\begin{array}{l}\text { CH2MHill Halcrow } \\
\text { (2014) }\end{array}$ & $\begin{array}{l}\text { CH2MHill Halcrow, Cell } 1 \text { Regional Coastal Monitoring Programme, Wave Data Analysis Report 2: 2013-2014, Final } \\
\text { Report, March } 2014 \text { [document properties: author=Andy.Parson@ch2m.com; datestamp; 04/04/2014] } \\
\text {-extensive areas of Whitby town centre flooded }\end{array}$ \\
\hline Dunbar et al (2014) & $\begin{array}{l}\text { Dunbar I, N Phipps, M Szonyi, Risk Nexus. After the storm: how the UK's flood defences performed during the surge } \\
\text { following Xaver, Flood resilience review 09.14, Zurich Insurance Company Ltd., Mythenquai 2, 8002, Zurich, } \\
\text { Switzerland [document properties: date 28Aug2014; author= Zurich] } \\
\text {-thousands of people evacuated } \\
\text {-XAVER: } 1400 \text { flooded homes, } 1000 \text { businesses, } 3200 \text { ha farmland } \\
\text {-Kingston-upon-Hull new record: } 5.8 \mathrm{~m} \text {; significant flooding } \\
\text {-2013 flood: } 100 \text { properties flooded \& flood protection structures damaged }\end{array}$ \\
\hline Eden (201402) & $\begin{array}{l}\text { Eden, Phillip, Weather Log December 2013, Weather, Feb 2014, pp.i-iv } \\
\text {-coastal flooding at Boston (Lincs), Jaywick (Essex), Rhyl (Denbyshire), }\end{array}$ \\
\hline Eriksen (2014) & $\begin{array}{l}\text { Eriksen J, Rekordvandstande i Isefjorden og Roskilde Fjord, Vejret, 138, } 240-48,2014 \\
\text {-map of coastal flooding Roskilde }\end{array}$ \\
\hline Goennert et al. (2014) & $\begin{array}{l}\text { Goennert G, O Mueller, M Schaper, K Sossidi, Die Sturmflut nach dem Tief Xaver von 5. bis 7. Dezember } 2013 . \\
\text { Berichte des Landesbetriebes Strassen, Bruecken und Gewaesser (LSBG), Freie und Hansestadt Hamburg, Nr. } \\
\text { 16/2014, 26pp. } \\
\text {-flooding in Hamburg }\end{array}$ \\
\hline Leiding et al (2014) & $\begin{array}{l}\text { Leiding T, B Tinz, G Rosenhagen, C Lefevre, S Haeseler, S Hagemann, I Bastigkeit, D Stein, P Schwenk, S Mueller, } \\
\text { O Outzen, K Herklotz, F Kinder, T Neumann, Meteorological and Oceanographic Conditions at the FINO } \\
\text { platforms during the severe storms Christian and Xaver, DEWI Magazin, No.44, p16-25, } 2014 . \\
\text {-'Hallig lowlands were flooded' }\end{array}$ \\
\hline Nossent et al (2014) & $\begin{array}{l}\text { Nossent J, L Boeckx, E Taverniers, M Deschamps, T Verwaest, F Mostaert, Sinterklaasstorm } 6 \text { december } 2013 . \\
\text { Beschrijving van de hydrometrische gebeurtenissen, Versie 4.0. WL Rapporten, 00-119, Waterbouwkundig } \\
\text { Laboratorium, Antwerpen, Belgie. October 2014, WL2014R00_119_5 [document date stamp: 15Oct2014; } \\
\text { author= Deschamps, Maarten] } \\
\text {-Antwerp quayside flooded }\end{array}$ \\
\hline RMS (2014) & $\begin{array}{l}\text { RMS, 2013-2014 Winter Storms in Europe. An Insurance and Catastrophe Modeling Perspective. RMS White Paper. } \\
\text { [PDF TIMESTAMP 11Mar2014] } \\
\text {-1400 properties flooded in England and Wales } \\
\text {-flooding on low-lying North Sea islands of Langeness and Hooge near Denmark }\end{array}$ \\
\hline Thorne (2014) & $\begin{array}{l}\text { Thorne, Colin, Geographies of UK flooding in 2013/4, The Geographical Journal, 180, 297-309, } 2014 . \\
\text {-brief outline of east coast areas flooded during storm surge }\end{array}$ \\
\hline Axer et al (2015) & $\begin{array}{l}\text { Axer T, T Bistry, M Klawa, M Mueller, M Suesser, Deutsche Ruck Sturm dokumentation } 2013 \text { Deutschland, 2013, } \\
\text { Deutsche Rueckversicherung Aktiengesellschaft, Hansaallee 177, } 40549 \text { Duesseldorf, www.deutscherueck.de } \\
\text { [pdf document information: author=filiz; date stamp=07Aug2015] } \\
\text {-UK: } 10000 \text { houses evacuated } \\
\text {-Hamburg: Fischmarkt and Elbstrasse flooded }\end{array}$ \\
\hline $\begin{array}{l}\text { Haigh and Bradshaw } \\
(2015)\end{array}$ & $\begin{array}{l}\text { Haigh I and E Bradshaw, A century of UK coastal flooding, Planet Earth, Winter 2015, (pdf document properties: } \\
\text { 13Jan2016) } \\
\text {-flooded } 2800 \text { homes and } 1000 \text { businesses }\end{array}$ \\
\hline $\begin{array}{l}\text { Kendon and McCarthy } \\
\text { (2015) }\end{array}$ & $\begin{array}{l}\text { Kendon M and M McCarthy, The UK's wet and stormy winter of 2013/2014, Weather, 70, 40-47, } 2015 \\
\text {-'several hundred properties on the coasts of eastern England and North Wales were inundated' }\end{array}$ \\
\hline Sibley et al (2015) & $\begin{array}{l}\text { Sibley A, D Cox, H Titley, Coastal flooding in England and Wales from Atlantic and North Sea storms during the } \\
\text { 2013/2014 winter, Weather, 70, 62-70, } 2015 \\
\text {-Newcastle quayside under water at high tide } \\
\text {-Scarborough, Whitby: seafront properties flooded } \\
\text { * -power outages to Whitby making recovery difficult } \\
\text {-Humber estuary towns flooded: Reedness \& Goole } \\
\text { * -River Haven at Boston Linolnshire had breaches of sea defences with } 200 \text { people evacuated } \\
\text {-thousands of people evacuated East Anglia } \\
\text {-flooding: Great Yarmouth, Lowestoft, Wells-next-the Sea, Blakeney, Cley } \\
\text {-Hemsby: cliff collapse \& lifeboat station destroyed } \\
\text {-several hundred homes evacuated Kent } \\
\text {-risk of flooding Sandwich \& Seasalter }\end{array}$ \\
\hline Wadey et al (2015a) & $\begin{array}{l}\text { Wadey MP, ID Haigh, RJ Nichols, JM Brown, K Horsburgh, B Carroll, SL Gallop, T Mason, E Bradshaw, A } \\
\text { comparison of the } 31 \text { January-1 February } 1953 \text { and 5-6 December } 2013 \text { coastal flood events around the UK, UK } \\
\text { Frontiers in Marine Science, 2, 84, 2015a. } \\
\text {-listing of flooded areas and evacuations in Wales, Liverpool area, eastern England, Sussex/Kent }\end{array}$ \\
\hline Wadey et al (2015b) & $\begin{array}{l}\text { Wadey MP, JM Brown, ID Haigh, T Dolphin, P Wisse, Assessment and comparison of extreme sea levels and waves } \\
\text { during the 2013/2014 storm season in two UK coastal regions, Nat. Hazards Earth Syst. Sci. Discuss., 3, 2665- } \\
2708,2015 \text { b. } \\
\text {->2800 properties across UK flooded including > } 800 \text { in Boston, Lincolnshire } \\
\text {-Suffolk: total of } 22 \text { breaches reported across county causing severe flooding; failure of sea defences at Blythburgh } \\
\text {-floods in Mersea estuary: } 19 \text { businesses flooded and } 4 \text { domestic properties } \\
\text {-Lowestoft: } 143 \text { commercial properties, } 90 \text { residential properties flooded } \\
\text {-Waveney discrict: } 170 \text { properties flooded }\end{array}$ \\
\hline Sorensen (2016) & $\begin{array}{l}\text { Sorensen CS, Water NOT wanted - Coastal floods and flooding protection in Denmark, In RA Herrmann \& J Jensen } \\
\text { (eds), Sicherung von Daemmen, Deichen und Stauanlagen: Handbuch fuer Theorie und Praxis (Vol V pp3-21). } \\
\text { Siegen: Universitaet Siegen, } 2016 \\
\text {-Xaver impacted other parts of Denmark compared with Britta } 2006 \\
\text {-some houses uninhabitable for some time and still are }\end{array}$ \\
\hline
\end{tabular}




\begin{tabular}{|c|c|}
\hline Sorensen et al (2016b) & $\begin{array}{l}\text { Sorensen C, NH Broge, MR Molgaard, CS Schow, PThomsen, K Vognsen, P Knudsen, Assessing future flood hazards } \\
\text { for adaptation planning in a northern European Coastal Community, Frontiers in Marine Science, 3:69, } \\
\text { doi: } 10.3389 / \text { fmars.2016.00069, 2016b } \\
\text {-mention of flooding in Thyboron during Dec } 2013 \text { flood }\end{array}$ \\
\hline Evans (2017) & $\begin{array}{l}\text { Evans, Sun Yan, EA/2/2 Appendix 1, History of flooding sources, Boston Barrier Transport \& Works Act order } \\
\text { application, Public Inquiry documents, [pdf document properties: author=BDB; datestamp=16Mar2017] } \\
\text { https://consult.environment-agency.gov.uk/engagement/bostonbarriertwao/results/appendix-1---history-of- } \\
\text { flooding-sources.pdf } \\
\text {-desription of flood in Boston UK }\end{array}$ \\
\hline DEMA (2018) & $\begin{array}{l}\text { Danish Emergency Management Agency DEMA, National Risk Profile for Denmark, April } 2018 \\
\text {-600 homes destroyed by flooding Denmark } \\
\text {-water } 2 \mathrm{~m} \text { above normal Roskilde Fjord } \\
\text {-major flooding and destruction Holbaek Fjord, Odense Fjord, Isefjord, Copenhagen }\end{array}$ \\
\hline $\begin{array}{l}\text { Giannopoulos et al. } \\
(2019)\end{array}$ & $\begin{array}{l}\text { Giannopoulos G, L Peake, B Reid, J Andrews, A Grant, I Lorenzoni, M Goulden, J Waters, T Dolphin, J Bremner, TJ } \\
\text { Tolhurst, Environmental and social impacts of the } 2013 \text { storm surge on the North Norfolk coast, powerpoint } \\
\text { presentation date stamp 15May2019, unknown conference } \\
\text {-coastal floodign and ecosystem impacts }\end{array}$ \\
\hline Rucinska (2019) & $\begin{array}{l}\text { Rucinska D, Describing Storm Xaver in disaster terms, International Journal of Disaster Reduction, 34, 147-153, } 2019 \\
-10000 \text { people evacuated in UK }\end{array}$ \\
\hline Wikipedia (20191002) & $\begin{array}{l}\text { Wikipedia, Stormen Bodil, https://da.wikipedia.org/wiki/Stormen_Bodil\#cite_note-39 (accessed 02Oct2019) } \\
\text {-flooding of Viking ship museum in Roskilde } \\
\text {-Frederikssum \& Jyllinge Nordmark } 600 \text { families lost their home } \\
\text {-in low lying areas water was being fought up to a week after the storm } \\
\text {-Belgium: evacuation of } 2083 \text { people from Bredene kommune between Sas Slijkens \& Spuikeon } \\
\text {-flooding: Rotterdam, Dordrecht, Vlaardingen } \\
\text {-Tyne flooded its banks } \\
\text {-flooding at Port Clarence on Tees } \\
\text {-dike break at Greatham Creek report with CH-47 Chinook from RAF } \\
\text {-parts of Hamburg flooded }\end{array}$ \\
\hline $\begin{array}{l}\text { JBA Risk Management } \\
(2020)\end{array}$ & $\begin{array}{l}\text { JBA Risk Management, Storm Xaver 2013. Event Commentary, 2020. [PDF document properties: author=Cameron } \\
\text { Whitwham] } \\
\text {-Map of number of houses flooded in different areas of UK; Boston worst and \& then Humber } \\
\text {-1000 businesses \& } 1400 \text { houses (4200) people flooded in UK }\end{array}$ \\
\hline $\begin{array}{l}\text { Surgewatch } \\
(20200304)\end{array}$ & $\begin{array}{l}\text { Surgewatch, Storm event } 6 \text { th December } 2013 \text {, https://www.surgewatch.org/events/1/ (last accessed 04Mar2020) } \\
\text {-seawater inundation } 1400 \text { homes \& } 1000 \text { businesses, 3200ha farmland } \\
\text {-10000 people evacuated alond coast }\end{array}$ \\
\hline WIKI (20200124) & $\begin{array}{l}\text { WIKI, Cyclone Xaver, https://en.wikipedia.org/wiki/Cyclone_Xaver accessed 24Jan2020 } \\
\text {-ecavuations: Rhyll Wales; Belgium: Bredene evacuated } 2083 \text { residents along Bruges-Ostend canal, people in Great } \\
\text { Yarmouth told to prepare to evacuate in case River Yare flooded } \\
\text {-flood: Rhyl Wales, Kinmel Bay, Llanddulas, Conwy, Oban, } \\
\text { Port Clarence, Whitby, Scarborough, Bridlington, Cleethorpes, Hull, Paull, N Lincolnshire, } \\
\text { Boston, Wells-Next-the-Sea, Lowestoft, Snape on Alde-Ore estuary; Waldringfield on River Deben, } \\
\text { Rotterdam, Dordrecht, Vlaardingen }\end{array}$ \\
\hline Wikipedia (20200429) & $\begin{array}{l}\text { Wikipedia, Stormen Sven, https://sv.wikipedia.org/wiki/Stormen_Sven\#cite_note-15, accessed 29Apr2020 } \\
\text {-worst coastal flooding in Malmo and Helsingborg }\end{array}$ \\
\hline Wikipedia (20200502) & $\begin{array}{l}\text { Wikipedia, Orkan Xaver, https://de.wikipedia.org/wiki/Orkan_Xaver (accessed } 2 \text { May 2020) } \\
\text {-parts of Bremen susceiptable to surge flooding evacuated } \\
\text {-police closed off parts of Hamburg } \\
\text {-'Mehrere Halligen meldeten Land unter' } \\
\text {-offshore winds Baltic cause low water levels in Flensburger Foerde and Schleimundung } \\
\text {-Badewanneeffekt later caused high water levels of 140m on southern Baltic coast }\end{array}$ \\
\hline
\end{tabular}

Table S32. Coastal dike heights and protection levels (arranged by year and then alphabetically)

\begin{tabular}{|c|c|}
\hline Source & Full Reference and Notes \\
\hline Horner (1979) & $\begin{array}{l}\text { Horner RW, The Thames Barrier Project, The Geographical Journal, 145, 242-253, } 1979 . \\
\text {-Thames Barrier height 7.2 mODN; designed to protect at 1000year return period }\end{array}$ \\
\hline Goennert et al. (2012) & $\begin{array}{l}\text { Goennert G, B Gerkensmeier, J-M Mueller, Ermittlung des Sturmflutbemesungswasserstandes fuer den } \\
\text { oeffentlichen Hochwasserschutz in Hamburg, Berichte des Landesbetriebes Strassen, Bruecken und Gewaesser } \\
\text { Nr } 12 / 2012 \text {. } \\
\text {-maps of surge flooding protection levels around the North Sea expressed in terms of return period: present and } \\
\text { future } \\
\text {-London at } 1000 y \text { level; Netherlandsmajor cities at } 10000 \mathrm{y} \text { level, Belgium at } 1000 \mathrm{y} \text {, UK unknown, Germany } \\
\text { unknown, Denmark at } 2.5-300 \text { year except Thyboron (1000 year) }\end{array}$ \\
\hline Gautier et al (2014) & $\begin{array}{l}\text { Gautier C, A Camarena, J van Nieuwkoop, SWAN hindcasts Wadden Sea, December 2013. Tidal inlet of } \\
\text { Ameland and eastern Wadden Sea. Deltares, 2014, 197pp. Project 1209433-007, Reference 1209433-007- } \\
\text { HYE-0005 } \\
\text {-map of the Netherlands showing protections levels expressed as recurrence interval }\end{array}$ \\
\hline $\begin{array}{lll}\text { Van } & \text { Rooijen and } \\
\text { Oosten }(2014)\end{array}$ & $\begin{array}{l}\text { van Rooijen A, A Oost, Memo: Regionale advisering Ameland Noordwest, Deltares, 1209381-008-ZKS-0008, } \\
\text { 43pp, 18Dec2014 [PDF document properties: titl=Regional advisering: Ameland NW; author=Arnold van } \\
\text { Rooijen; keywords: } 1209381-008-Z K S-0008 \text {; date stamp: } 18 / 12 / 2014 \text { ] } \\
\text {-lowest level in protective dune line in NW Ameland at } 6.5 \mathrm{~m} ; 7 \mathrm{~m} \text { stuifdijk at east end of dunes }\end{array}$ \\
\hline Carrion (2015) & $\begin{array}{l}\text { Carrion Aretxabala, BI, Morphological impact of the Sinterklaas storm at Het Zwin. Numerical modelling with } \\
\text { Xbeach, M.Sc. Civil Engineering, Delft University of Technology, } 2015 \text {. }\end{array}$ \\
\hline
\end{tabular}




\begin{tabular}{|c|c|}
\hline & $\begin{array}{l}\text {-'The safety assessment against flooding in the Netherlands requires all } \\
\text { component of the so-called ring dike (i.e. dikes, dunes, levels, etc) } \\
\text { to be able to cope with the hydraulic conditions associated to a } \\
\text { return period of } 10000 \text { years.' }\end{array}$ \\
\hline $\begin{array}{ll}\text { Van } & \text { Massenhove } \\
(2015) & \end{array}$ & $\begin{array}{l}\text { Vanmassenhove, Niels, Storm surge measures ports Flemish coast, Blankenberge, Tuesday February 3rd, } \\
\text { Maritieme Sientverlening en Kust, Coastal Division, Flanders Hydraulic Research.[document properties: } \\
\text { title=Geintegreerd Kustveiligheidsplan; author=Maarten; datestamp=27/02/2015] } \\
\text {-current quayside height Zeebrugge } 6.68 \mathrm{~m}, 7.31 \mathrm{~m} \text { TAW; proposed surge barrier height } 8.0 \mathrm{~m}, 9.0 \mathrm{~m} \text { TAW } \\
\text {-map of Belgian coast flooding for a } 1000 \mathrm{y} \text { water level event } 7 \mathrm{~m} \text { TAW }\end{array}$ \\
\hline Wadey et al (2015b) & $\begin{array}{l}\text { Wadey MP, JM Brown, ID Haigh, T Dolphin, P Wisse, Assessment and comparison of extreme sea levels and } \\
\text { waves during the 2013/2014 storm season in two UK coastal regions, Nat. Hazards Earth Syst. Sci. Discuss., } \\
3,2665-2708,2015 \mathrm{~b} \text {. } \\
\text { * -after Waverley report from } 1953 \text { floods; flood defences to withstand 100y still water level } \\
\text {-joint sea level \& wave condition method; standard 50-100 years } \\
\text {-more recent risk management \& insurance policy; defences should not breach with } 1 \text { in } 200 \mathrm{y} \text { event } \\
\text {-wave overtopping of promenades \& seawalls accepted } \\
\text { * -locally more stringent design criterion: } 1000 \mathrm{y} \text { for London; } 10000 \mathrm{y} \text { for power stations } \\
\text {-Munich Re will pay out on reinsurance claims to limit of } 1 \text { in } 200 \mathrm{y} \text { level }\end{array}$ \\
\hline Nederhoff et al (2016) & $\begin{array}{l}\text { Nederhoff K, E Elias, T Vermaas, Erosie op Ameland Noordwest. Modelstudie: simulaties met Delft3D en } \\
\text { XBeach, Deltares, } 117 \mathrm{pp} \text {, July, } 2016 \\
\text {-height of protection dunes in northwest Ameland: lowest } 6.5 \mathrm{~m} \text {; variation } 7-25 \mathrm{~m} \text { NAP }\end{array}$ \\
\hline Sorensen (2016) & $\begin{array}{l}\text { Sorensen CS, Water NOT wanted - Coastal floods and flooding protection in Denmark, In RA Herrmann \& J } \\
\text { Jensen (eds), Sicherung von Daemmen, Deichen und Stauanlagen: Handbuch fuer Theorie und Praxis (Vol V } \\
\text { pp3-21). Siegen: Universitaet Siegen, } 2016 \\
\text {-all Denmark underlain by soft sediments except Bornholm } \\
\text {-Waddensee dikes: dike materials and profile; surge protection at } 20-500 \text { year extreme water level } \\
\text {-Jutland west coast: Thyboron dike protects at } 1000 \mathrm{y} \text { level; rest of coast at } 100 \mathrm{y} \text { level } \\
\text {-Jutland west coast: dune protection minimum } 40 \mathrm{~m} \text { wide \& } 5 \mathrm{~m} \text { high; dune safety depends on wave runup } \\
\text {--inner Danaish seas: } 1872 \text { surge ( } 4000 \text { year event) used as basis of design criterion for modern dikes }\end{array}$ \\
\hline Sorensen et al (2016b) & $\begin{array}{l}\text { Sorensen C, NH Broge, MR Molgaard, CS Schow, PThomsen, K Vognsen, P Knudsen, Assessing future flood } \\
\text { hazards for adaptation planning in a northern European Coastal Community, Frontiers in Marine Science, } \\
\text { 3:69, doi:10.3389/fmars. } 2016.00069,2016 \mathrm{~b} \\
\text {-Thyboron protected by dikes at the } 1000 \text { year level. }\end{array}$ \\
\hline $\begin{array}{l}\text { Kystdirektoratet } \\
\text { (2018) }\end{array}$ & $\begin{array}{l}\text { Kystdirektoratet, Shoreface nourishment effects. An analysis of the } 2011 \text { nourishment performed at Skodbjerge. } \\
\text { Kystdirektoratet, Hojbovej } 1,7620 \text { Lemvig, Dec } 2018 \text { [pdf document properties: title=Shoreface nourishment } \\
\text { effects_Skodbjerge_20.12.2018, datestamp=20/12/2018] } \\
\text {-Denmark policy to protect from sea flooding on the North Sea west coast at the } 100 \mathrm{y} \text { return period except for } \\
\text { Thyboron (1000y return period) }\end{array}$ \\
\hline Ulm et al (2018) & $\begin{array}{l}\text { Ulm, Marius, Arne Arns, Juergen Jensen, Assessing consequences of extreme events for the German Bight, 36th } \\
\text { International Conference on Coastal Engineering } 2018 \text { - Coastal Protection and Risk - ID } 1527 \\
\text {-dike heights 6.50-8.40 m ASL }\end{array}$ \\
\hline
\end{tabular}

Table S33. Surge barrier closures (arranged by year and then alphabetically)

\begin{tabular}{|l|l|}
\hline Source & Full Reference and Notes \\
\hline $\begin{array}{l}\text { Air Worldwide } \\
(20131212)\end{array}$ & $\begin{array}{l}\text { Air Worldwide, Press Release, Boston, 12Dec2013. https://www.air-worldwide.com/In-the-News/AIR- } \\
\text { Estimates-Losses-from-European-Windstorm-Xaver-at-Between-EUR-700-Million-and-EUR-1-4-Billion/ } \\
\text { (accessed 02Jan1990) }\end{array}$ \\
& $\begin{array}{l}\text {-Thames Barrier closed for 2 days } \\
\text {-2m water level difference between front and back of Thames barrier } \\
\text {-Hamburg closes all 38 flood gates }\end{array}$ \\
\hline BBC (20131210) & $\begin{array}{l}\text { BBC20131206, Deadly storm and tidal surge batter northern Europe, BBC 6 Dec2013 (report by Anna Holligan) } \\
\text {-Thames Barrier closed for second day to protect London } \\
\text {-eastern Scheldt storm surge barrier closed }\end{array}$ \\
\hline $\begin{array}{l}\text { Gcapticalideas } \\
\text { (20131209) }\end{array}$ & $\begin{array}{l}\text { gccapitalideas, Windstorm Xaver, 9Dec2013, https://www.gccapitalideas.com/2013/12/09/windstorm-xaver/ } \\
\text {-Thames Barrier closed } \\
\text {-all 38 flood-gates in Hamburg closed } \\
\text {-several barriers closed in the Netherlands including the Eastern Scheldt Barrier }\end{array}$ \\
\hline DW (20131205) & $\begin{array}{l}\text { DW, Storm Xaver pummels Europe, flood surge feared, 05/12/2013 } \\
\text {-eastern Scheldt barrier closed for the first time in 6 years }\end{array}$ \\
\hline GVA (20131205) & $\begin{array}{l}\text { GVA, Vijf containers even op drift op de Schelde, 05/12/2013 20:57. https://www.gva.be/cnt/aid1500792/vijf- } \\
\text { containers-op-drift-op-de-schelde-2 } \\
\text {-warnings that storm gates will be closed in Antwerp }\end{array}$ \\
\hline Kunz et al (20131206) & $\begin{array}{l}\text { Kunz M, B Muehr, K Schroeter, T Bessel, S Moehrle, T Muenzberg, S Brink, H-M Schmidt, Winterstorm Xaver - } \\
\text { Report. 06Dec2013 - Report No.1, Situation Report - 19:00CET, CEDIM Forensic Disaster Analysis Group } \\
\text { (FDA), Center for Disaster Management and Risk Reduction Technology. }\end{array}$ \\
& $\begin{array}{l}\text {-Bremen: 50 gates and dike openings closed and flood barrages at Kennedy Bridge; dike being monitored; 28000 } \\
\text { sandbags prepared; new Weser dike recently enhanced } \\
\text {-Lower Saxony: 9 barrages at Ems, Hunte, Weser, Unterelbe closed; since 2008 >400 million EUR for coast } \\
\text { protection } \\
\text {-East Frisian Islands: all dike openings closed } \\
\text {-Hamburg 38 flood gates closed }\end{array}$ \\
\hline NLWKN, Schwere Sturmflut gut Ueberstanden, Waserstaende zwischen Zweieinhale und Knapp vier metern \\
\hline NLWKN (20131206)
\end{tabular}




\begin{tabular}{|c|c|}
\hline & $\begin{array}{l}\text { registriert // presseinformation von 6. Dezember 2013, (Ansprechpartnerin: Herma Heyken), 06/12/2013. } \\
-9 \text { barriers operated by NLWKN closed: Ems, Hunte, Weser, Unterelbe } \\
\text {-Emssperrwerke bei Gandersum in Landkreis Leer closed } \\
\text {-6 barriers on the Elbe closed } \\
\text {-Hunte and Ochtumsperrwerke close } \\
\text {-many barriers to remain closed for next high tide or to be closed again (Emssperrwerke) }\end{array}$ \\
\hline NOS (20131206) & $\begin{array}{l}\text { NOS, Hoogste waterstand sinds } 1953,06 / 12 / 2013,09: 52 \text {, https://nos.nl/artikel/583218-hoogste-waterstand-sinds- } \\
\text { 1953.html } \\
\text {-closure of Oosterscheldekering with its } 62 \text { gates for first time since } 2007\end{array}$ \\
\hline $\begin{array}{l}\text { Spiegel International } \\
(20131206)\end{array}$ & $\begin{array}{l}\text { Spiegel International, Winter storm } \\
\text { https://www.spiegel.de/international/europe/tidal-surge-winter-storm-xaver-batters-northern-europe-a- } \\
\text { 937576.html\# } \\
\text {-Thames Barrier closed late Thursday }\end{array}$ \\
\hline ABPmer (2014) & $\begin{array}{l}\text { ABP mer, Ensuring Flood Resilience. An overview of the 5/6 December 2013, Associated British Ports, Marine } \\
\text { Environmental Research, July, } 2014 \\
\text {-Thames Barrier protected } 380,000 \text { properties from surge } \\
\text {-Thames Barrier closed }>50 \text { times during period Dec } 2013-\text { Mar } 2014 \text { due to prolonged stormy weather. } \\
\text {-tidal defenses at Hull protected } 19,000 \text { properties } \\
\text {-'water levels at Hull were recorded as reaching 5.8mODN, } 0.4 \mathrm{~m} \text { below crest of the Hull Tidal Surge Barrier' }\end{array}$ \\
\hline Dunbar et al (2014) & $\begin{array}{l}\text { Dunbar I, N Phipps, M Szonyi, Risk Nexus. After the storm: how the UK's flood defences performed during the } \\
\text { surge following Xaver, Flood resilience review 09.14, Zurich Insurance Company Ltd., Mythenquai 2, 8002, } \\
\text { Zurich, Switzerland [document properties: date 28Aug2014; author= Zurich] } \\
\text {-Thames Barrier closed several times during Xaver; } 2 \mathrm{~m} \text { water level differnce across barrier } \\
\text {-Hull Barrier: water level at } 5.8 \mathrm{~m} \text {; top of barrier at } 6.3 \mathrm{~m}\end{array}$ \\
\hline Nossent et al (2014) & $\begin{array}{l}\text { Nossent J, L Boeckx, E Taverniers, M Deschamps, T Verwaest, F Mostaert, Sinterklaasstorm } 6 \text { december } 2013 . \\
\text { Beschrijving van de hydrometrische gebeurtenissen, Versie 4.0. WL Rapporten, 00-119, Waterbouwkundig } \\
\text { Laboratorium, Antwerpen, Belgie. October 2014, WL2014R00_119_5 [document date stamp: 15Oct2014; } \\
\text { author= Deschamps, Maarten] } \\
\text {-water level threshold exceeded for Antwerp flood gates to close }\end{array}$ \\
\hline RWS (2014b) & $\begin{array}{l}\text { RWS, Stormvloedrapport van } 5 \mathrm{t} / \mathrm{m} 7 \text { december (SR91) Sint-Nicolaasvloed 2013, Watermanagementcentrum } \\
\quad \text { Nederland, Rijkswaterstaat, prepared by Ing. J. Kroos, } 19 \text { Mar 2014b, } 48 \text { pp } \\
\text {-water level threshold for Maeslant- \& Hartelkering for Dordrecht \& Rotterdam not met } \\
\text {-Hollandse IJssel Stormvloedkering closed } \\
\text {-Emssperrwerk closed } \\
\text {-Oosterscheldkering }\end{array}$ \\
\hline Slingo et al (2014) & $\begin{array}{l}\text { Slingo J, S Belcher, A Scaife, M McCarthy, A Saulter, K McBeath, A Jenkins, C Huntingford, T Marsh, J } \\
\text { Hannaford, S Parry, The Recent Storms and Floods in the UK, 29pp, Met Office, Fitzroy Road, Exeter, } \\
\text { Devon, EX1 3PB, UK, February, } 2014 \text { [pdf document properties: author=huw.lewis; date stamp=11Feb2014] } \\
\text {-Thames barrier closed during surge 5-6 Dec } 2013\end{array}$ \\
\hline Thorne (2014) & $\begin{array}{l}\text { Thorne, Colin, Geographies of UK flooding in 2013/4, The Geographical Journal, 180, 297-309, } 2014 . \\
\text {-Thames and Hull barriers closed during surge; } 800000 \text { properties protected from flooding }\end{array}$ \\
\hline Axer et al (2015) & $\begin{array}{l}\text { Axer T, T Bistry, M Klawa, M Mueller, M Suesser, Deutsche Ruck Sturm dokumentation } 2013 \text { Deutschland, } \\
\text { 2013, Deutsche Rueckversicherung Aktiengesellschaft, Hansaallee 177, 40549 Duesseldorf, } \\
\text { www.deutscherueck.de [pdf document information: author=filiz; date stamp=07Aug2015] } \\
\text {-Thames barrier closed } \\
\text {-Oostersheldt barrier closed } \\
\text {-Niedersachsen: all barriers closed: Ems, Hunte, Weser, Underelbe }\end{array}$ \\
\hline $\begin{array}{l}\text { Kendon and McCarthy } \\
\text { (2015) }\end{array}$ & $\begin{array}{l}\text { Kendon M and M McCarthy, The UK's wet and stormy winter of 2013/2014, Weather, 70, 40-47, } 2015 \\
\text {-Thames Barrier closed }\end{array}$ \\
\hline Sibley et al. (2015) & $\begin{array}{l}\text { Sibley A, D Cox, H Titley, Coastal flooding in England and Wales from Atlantic and North Sea storms during the } \\
\text { 2013/2014 winter, Weather, } 70,62-70,2015 \\
\text {-'hundreds of thousands of properties and around } 2000 \mathrm{~km} 2 \text { of agricultural land are now protected, including by } \\
\text { the Hull and Thames Barriers' }\end{array}$ \\
\hline Wadey et al (2015) & $\begin{array}{l}\text { Wadey MP, ID Haigh, RJ Nichols, JM Brown, K Horsburgh, B Carroll, SL Gallop, T Mason, E Bradshaw, A } \\
\text { comparison of the } 31 \text { January-1 February } 1953 \text { and 5-6 December } 2013 \text { coastal flood events around the UK, } \\
\text { UK Frontiers in Marine Science, 2, 84, } 2015 \text {. } \\
\text {-Thames barrier closed for } 2 \text { days with } 2 \mathrm{~m} \text { water level difference between ront and back. }\end{array}$ \\
\hline $\begin{array}{l}\text { Environment Agency } \\
(2018)\end{array}$ & $\begin{array}{l}\text { Environment Agency, Thames Barrier Project Pack 2018, January, } 2018 \\
\text {-Thames Barrier, Dartford Creek Barrier, Barking Barrier, Royal Docks }\end{array}$ \\
\hline $\begin{array}{l}\text { North Norfolk District } \\
\text { Council Coastal Team } \\
\text { (2018) }\end{array}$ & $\begin{array}{l}\text { North Norfolk District Council Coastal Team, Refurbishment of sea walls and groynes 2013-2015; Cromer Coast } \\
\text { Protection, (picture of plaque on wall taken 27Dec2018) www.northnorfolk.org/coastal } \\
\text {-highest water level at Thames Barrier since start of operation in } 1982\end{array}$ \\
\hline $\begin{array}{l}\text { North Norfolk District } \\
\text { Council Coastal Team } \\
\text { (2019) }\end{array}$ & $\begin{array}{l}\text { North Norfolk District Council Coastal Team, Sheringham Sea Defences; Repair and Recovery from the } \\
\text { December } 2013 \text { storm surge, www.northnorfolk.org/coastal, photo of plaque on wall taken 30Dec2019 } \\
\text {-highest water level at Thames Barrier since start of operation in } 1982\end{array}$ \\
\hline Wikipedia (20201002) & $\begin{array}{l}\text { Wikipedia, Stormen Bodil, https://da.wikipedia.org/wiki/Stormen_Bodil\#cite_note-39 (accessed 02Oct2019) } \\
\text {-62 gates of Oosterscheldekering, Thames Barrier, Hamburg } 38 \text { flood gates }\end{array}$ \\
\hline WIKI (20200124) & $\begin{array}{l}\text { WIKI, Cyclone Xaver, https://en.wikipedia.org/wiki/Cyclone_Xaver accessed 24Jan2020 } \\
\text {-Thames Barrier, } \\
\text {-Eastern Scheldt storm surge barrier closed 62locks Thursday night, } \\
\text {-Hamburg closed all } 38 \text { flood gates }\end{array}$ \\
\hline Wikipedia (20200502) & $\begin{array}{l}\text { Wikipedia, Orkan Xaver, https://de.wikipedia.org/wiki/Orkan_Xaver (accessed } 2 \text { May 2020) } \\
\text {-Thames Barrier closed } \\
\text {-Deltawerke closed }\end{array}$ \\
\hline
\end{tabular}


Table S34. Beach damage and coastal issues; salt water contamination of groundwater; sewer systems (arranged by year and then alphabetically)

\begin{tabular}{|c|c|}
\hline Source & Full Reference and Notes \\
\hline BBC (20131206) & $\begin{array}{l}\text { BBC20131206, Deadly storm and tidal surge batter northern Europe, BBC } 6 \text { Dec2013 (report by Anna Holligan) } \\
\text {-houses fall off sand cliff at east Hemsby UK }\end{array}$ \\
\hline BT (20131208) & $\begin{array}{l}\text { BT, Offer for Bodil: Koebte huset 15.august - nu er det vaek, 08Dec2013, 16:44, (contributor: Morten Eggert) } \\
\text { https://www.bt.dk/danmark/offer-for-bodil-koebte-huset-15.-august-nu-er-det-vaek } \\
\text {-summer house over sand cliff at Norlev Strand in Denmark on 06Dec2013 during Storm Bodil }\end{array}$ \\
\hline $\begin{array}{l}\text { Deutschlander et al } \\
\text { (2013) }\end{array}$ & $\begin{array}{l}\text { Deutschlander T, K Frierich, S Haeseler, C Lefebvre, Orkantief XAVER ueber Nordeuropea von 5. bis } 7 . \\
\text { Dezember 2013, Deutscher Wetterdienst DWD, Stand 30. Dezember 2013, 19pp. } \\
\text {-Auf den Inseln gab es Duenenabbruuche und Sandabtragungen' } \\
\text {-houses in Hemsby toppled over cliff due to coast erosion }\end{array}$ \\
\hline $\begin{array}{l}\text { Gccapitalideas } \\
\text { (20131209) }\end{array}$ & $\begin{array}{l}\text { gccapitalideas, Windstorm Xaver, 9Dec2013, https://www.gccapitalideas.com/2013/12/09/windstorm-xaver/ } \\
\text {-waves erodes cliffs at Hemsby }\end{array}$ \\
\hline Kunz et al (20131206) & $\begin{array}{l}\text { Kunz M, B Muehr, K Schroeter, T Bessel, S Moehrle, T Muenzberg, S Brink, H-M Schmidt, Winterstorm Xaver - } \\
\text { Report. 06Dec2013 - Report No.1, Situation Report - 19:00CET, CEDIM Forensic Disaster Analysis Group } \\
\text { (FDA), Center for Disaster Management and Risk Reduction Technology. } \\
\text {-dune destruction expected at Juist, Spiekeroog, Wangeroog, Norderney }\end{array}$ \\
\hline $\begin{array}{l}\text { Liverpool } \\
(20131211)\end{array}$ & $\begin{array}{l}\begin{array}{l}\text { Liverpool Echo, Formby sand dunes hit by stormy seas, 11Dec2013, (correspondent: Eleanor Barlow) } \\
\text { https://www.liverpoolecho.co.uk/news/liverpool-news/formby-sand-dunes-hit-stormy-6397344, accessed } \\
19 \text { Apr } 2020\end{array} \\
\text {-10m of sand cliff cutback at Formby, Merseyside coast, Irish Sea } \\
\text {-last worst storm Feb2002 with } 12 \mathrm{~m} \text { of sand cliff cutback }\end{array}$ \\
\hline NLWKN (20131206) & $\begin{array}{l}\text { NLWKN, Schwere Sturmflut gut Ueberstanden, Waserstaende zwischen Zweieinhale und Knapp vier metern } \\
\text { registriert // presseinformation von 6. Dezember 2013, (Ansprechpartnerin: Herma Heyken), 06/12/2013. } \\
\text {-on the islands first estimates of NLWKN leaders of significant dune collapse } \\
\text {-Hammersee auf Juist and Zeltplatzduenen auf Spiekeroog } \\
\text {-Harlehoern dune on Wangerooge hit } \\
\text {-Westinnengroden was under water; seawater streaming over the deckwork } \\
\text {-precise damage not yet finally assessed; water levels still too high to survey damage } \\
\text {-detailed measurements of the beach and dunes to be carried out in the coming weeks } \\
\text {-Spiekeroog and Wangerooge protection dunes strengthened in } 2012 \\
\text {-Langeoog dunes strengthened at Pirotal in } 2013 \\
\text {-no great damage to the mainland coastal protection structures } \\
\text {-Niedersachsen spent } 400 \text { millionen Euro in coastal protection since 2008. (Stefan Wenzel minister) }\end{array}$ \\
\hline NOS (20131206) & $\begin{array}{l}\text { NOS, Hoogste waterstand sinds } 1953,06 / 12 / 2013,09: 52 \text {, https://nos.nl/artikel/583218-hoogste-waterstand-sinds- } \\
\text { 1953.html } \\
\text {-dune damage in North Sea }\end{array}$ \\
\hline $\begin{array}{l}\text { Sueddeutsche Zeitung } \\
\text { (20131215) }\end{array}$ & $\begin{array}{l}\text { Sueddeutsche Zeitung, Xaver holte sich Land; Sturmschaden auf Sylt, 15Dec201315:36 } \\
\text { https://www.sueddeutsche.de/panorama/sturmschaeden-auf-sylt-xaver-holte-sich-land-1.1844100 } \\
\text {-40m of coastline retreat at Hoernum Odde } \\
\text {-dune line broken through in } 5 \text { places } \\
\text {-dune collapse at } 57 \% 21 \mathrm{~km} \text { stretch at west side of Sylt }\end{array}$ \\
\hline $\begin{array}{l}\text { Sylter Rundschau } \\
(20131209)\end{array}$ & $\begin{array}{l}\text { Sylter Rundschau, Folgen des Orkans. Xaver 'knabberte' Sylt massiv an, 09Dec2013 06:00 (from Friederike } \\
\text { Reussner) https://www.shz.de/lokales/sylter-rundschau/xaver-knabberte-sylt-massiv-an-id5098481.html } \\
\text {-Sylt west coast beaten on } 23 \mathrm{~km} \text { stretch; } 11.5 \mathrm{~km} \text { of coast without damage } \\
\text {-worst stretch } 1.2 \mathrm{~km} \text { at Hoernumer Odde; } 20 \mathrm{~m} \text { lost in places } \\
\text {-average coastline loss } 2-3 \mathrm{~m} \\
\text {-wave breathrough in one location with water staning in dune valley. } \\
\text {-Dikjen Deel: dunes broken } \\
\text {-List Moevenberg Dike: some damage to concrete plate armour that started several months previously. }\end{array}$ \\
\hline $\begin{array}{l}\text { Unwetterzentrale } \\
\text { (201312) }\end{array}$ & $\begin{array}{l}\text { Unwetterzentrale, Orkantief XAVER - ein weiterer schwerer Wintersturm der letzten Jahrzehnte, Thomas Savert } \\
\text { and Stefan Laps, Dec. } 2013 \text { http://www.unwetterzentrale.de/uwz/928.html } \\
\text {-Sudspitze on Sylt Island devastated \& Unterfeuer destroyed }\end{array}$ \\
\hline ABPmer (2014) & $\begin{array}{l}\text { ABP mer, Ensuring Flood Resilience. An overview of the 5/6 December 2013, Associated British Ports, Marine } \\
\text { Environmental Research, July, } 2014 \\
\text {-'the storm surge caused considerable cliff and dune retreats (e.g., Hemsby and Covehythe) and barrier breaches } \\
\text { (e.g., Benacre), whilst waves battered the shoreline causing damage to sea defences and other coastal } \\
\text { infrastructure, } \\
\text {-FIG_p3. [PHOTO] Coastal erosion at Hemsby [Albanpix] } \\
\text {-FIG_p3. [PHOTO] Wave damage to Cromer frontage [David Tipling] }\end{array}$ \\
\hline BBC (20141205) & $\begin{array}{l}\text { BBC, East coast surge: what happened next? (report by Richard Haugh), 5Dec2014 } \\
\text {-houses over cliff edge at Hemsby }\end{array}$ \\
\hline $\begin{array}{l}\text { Knaack and Heyken } \\
(2014)\end{array}$ & $\begin{array}{l}\text { Knaack H and H Heyken, Xaver hatte sehr schwere Stumflut im Gepaeck, Jahresbericht 2013. Der Zukunft } \\
\text { verplichtet, NLWKN, Niedersaechsischer Landesbetrieb fuer Waserwirtschaft, Kuesten- und Naturschutz, } \\
\text { pp.8-9, document date stamp 24Apr2014. } \\
\text {-signficant dune collapse: Wangerooge, Spiekeroog, Juist } \\
\text {-no significant damage to mainland coast } \\
\text {-response: summer 2.5million Euro \& } 180000 \mathrm{~m} 3 \text { sand for strengthening }\end{array}$ \\
\hline $\begin{array}{l}\text { Luecht and Peters } \\
(2014)\end{array}$ & $\begin{array}{l}\text { Luecht, Fabian and Ove Peters, Bericht ueber die Sturmflut vom 05.-0.6.12.2013 an der Westkueste Schleswig- } \\
\text { Holsteins, Landesbetrieb fuer Kuestenschutz, Nationalpark und Meeresschutz Schleswig-Holstein, Husum } \\
\text { 26Feb2014, 19pp. [pdf document properties: title=Lfd; Author=Thorsten Nommensen; datestamp: }\end{array}$ \\
\hline
\end{tabular}




\begin{tabular}{|c|c|}
\hline & $\begin{array}{l}\text { 11Dec2018] } \\
\text {-catalog of coastal damage Schleswig-Holstein } \\
\text {-two places of wave overtopping of main land protection dike in Helgoland } \\
\text {-loss of sand nourishment put in place in } 2000 \\
\text {-erosion of sand cliffs on west coast of Sylt; more in south than north } \\
\text {-list of costs for damage repair and clear treibsel }\end{array}$ \\
\hline NOZ (20140129) & $\begin{array}{l}\text { NOZ Nach Xaver: 9,4 Millionen Euro fuer Juist, Spiekeroog and Wangerooge, 29Jan2014 } \\
\text { https://www.noz.de/deutschland-welt/niedersachsen/artikel/446888/nach-xaver-9-4-millionen-euro-fur-juist- } \\
\text { spiekeroog-und-wangerooge-1 } \\
\text {-Hammersee on Juist, coast retreat 7m } \\
\text {-Wangerooge: coast retreat of } 13 \mathrm{~m} \text { at Harlehoernduenen } \\
\text {-Spiekeroog: } 10 \mathrm{~m} \text { of coastline retreat at Zeltplatz }\end{array}$ \\
\hline Pelt (2014) & $\begin{array}{l}\text { Pelt AS, BODIL's stormflod i de indre dansk farvande, Vejret, } 138,24-29,2014 \\
\text {-FIG4. [PHOTO] During the storm surge Nordsjaellands Kattegatkyst experienced } \\
\text { large erosion and damage. Photo of Rageleje Strandvej, which was } \\
\text { closed to through traffic because waves swept over the road. }\end{array}$ \\
\hline $\begin{array}{l}\text { Van Rooijen and Oost } \\
\text { (2014) }\end{array}$ & $\begin{array}{l}\text { van Rooijen A, A Oost, Memo: Regionale advisering Ameland Noordwest, Deltares, 1209381-008-ZKS-0008, } \\
\text { 43pp, 18Dec2014 [PDF document properties: titl=Regional advisering: Ameland NW; author=Arnold van } \\
\text { Rooijen; keywords: } 1209381-008-Z K S-0008 \text {; date stamp: 18/12/2014] } \\
\text {-cutback of steep dune face 10-20m for some profiles } \\
\text {-loss of height of dune line at these points } 9-7 \mathrm{~m} \text { and } 11-9 \mathrm{~m} \\
\text {-water recharge area of Ameland groundwater reservoir threatened. }\end{array}$ \\
\hline Thorne (2014) & $\begin{array}{l}\text { Thorne, Colin, Geographies of UK flooding in 2013/4, The Geographical Journal, 180, 297-309, } 2014 . \\
\text {-map of damaged coastal defences on East Coast and Irish sea that required repair. } \\
\text {-2800km of linear defences required repair }\end{array}$ \\
\hline Axer et al (2015) & $\begin{array}{l}\text { Axer T, T Bistry, M Klawa, M Mueller, M Suesser, Deutsche Ruck Sturm dokumentation } 2013 \text { Deutschland, } \\
\begin{array}{l}\text { 2013, Deutsche Rueckversicherung Aktiengesellschaft, Hansaallee 177, 40549 } \\
\text { www.deutscherueck.de [pdf document information: author=filiz; date stamp=07Aug2015] }\end{array} \\
\text {-significant dune losses Juist \& Spiekeroog; Wangerooge beach part washed away } \\
\text {-significant sand loss from Sylt, Fohr, Amrun } \\
\text {-dike damaged at a few places: Buesum }\end{array}$ \\
\hline Carrion (2015) & $\begin{array}{l}\text { Carrion Aretxabala, BI, Morphological impact of the Sinterklaas storm at Het Zwin. Numerical modelling with } \\
\text { Xbeach, M.Sc. Civil Engineering, Delft University of Technology, } 2015 . \\
\text {-dune damage and overwash at Het Zwin on Belgium Netherlands border } \\
\text {-belgian dunes cut back 4-10m; Netherlands dunes 2-20m }\end{array}$ \\
\hline Dan et al (2015) & $\begin{array}{l}\text { Dan, Sebastian, Anne-Lise Montreuil, Rosalia Delgado, Tomas van Oyen, Large storm impact on a beach under } \\
\text { sand nourishments, The Proceedings of the Coastal Sediments 2015, edited by Ping Wang, Julie D Rosati, and } \\
\text { Jun Cheng, Coastal Sediments 2015, San Diego, USA, 11-15May2015 } \\
\text {-dune scarp cut back } 8.9 \mathrm{~m} \text { at beach profile } 100 \text { and } 13.5 \mathrm{~m} \text { at beach profile } 104 \text { near Ostend }\end{array}$ \\
\hline $\begin{array}{l}\text { Dissanayake and } \\
\text { Karunarathna (2015) }\end{array}$ & $\begin{array}{l}\text { Dissanayake P and H Karunarathna, Effect of storm clustering on beach/dune erosion, E-proceedings of the 36th } \\
\text { IAHR World Congress, 28June-3July, 2015, The Hague, The Netherlands } \\
\text {-Xbeach model of dune damage at Formby Point }\end{array}$ \\
\hline 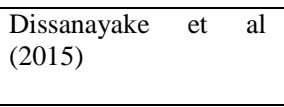 & $\begin{array}{l}\text { Dissanayake P, J Brown, H Karunarathna, Impacts of storm chronology on the morphological changes of the } \\
\text { Formby beach and dune system, UK, Nat. Hazards Earth Syst. Sci., 15,1533-1543, } 2015 . \\
-4 \mathrm{~m} \text { dune retreat at Formby Point in Liverpool Bay }\end{array}$ \\
\hline $\begin{array}{l}\text { Dissanayake et al } \\
(2015 b)\end{array}$ & $\begin{array}{l}\text { Dissanayake P, J Brown, P. Wisse, H Karunarathna, Comparison of storm cluster vs isolated event impacts on } \\
\text { beach.dune morphodynamics, Estuarine, Coastal, and Shelf Science, 164, 301-312, 2015b. } \\
\text {-Xbeach model study of beach damage at Sefton coast on Liverpool Bay }\end{array}$ \\
\hline Sibley et al (2015) & $\begin{array}{l}\text { Sibley A, D Cox, H Titley, Coastal flooding in England and Wales from Atlantic and North Sea storms during the } \\
2013 / 2014 \text { winter, Weather, } 70,62-70,2015 \\
\text {-'with erosion to coastal cliffs a number of dwellings were undermined as cliffs collapsed, for instance near } \\
\text { Hemsby, Norfolk where a lifeboat station was destroyed.' }\end{array}$ \\
\hline Spencer et al (2015) & $\begin{array}{l}\text { Spencer T, SM Brooks, BR Evans, JA Tempest, I Moeller, Southern North Sea storm surge event of Dec.5, 2013: } \\
\text { Water levels, waves, and coastal impacts, Earth Science Reviews, 146, 120-145, } 2015 \\
\text {-10 y of shoreline retreat during storm } \\
\text {-cliff top edge cut back by } 12 \mathrm{~m} \text { at some places. } \\
\text {-large differences in wave runup measured in some places } \\
\text {-cliff collapse/promenade damage Weybourne to Happisburgh; } \\
\text {-Bacton-Walcott } 72 \text { cliftop homes damaged/destroyed, washover aprons, } \\
\text {-breaching Benacre-Easton Bavents \& Walberswick-Dunwich, } \\
\text {-cliff front notch between BenacreBroad Suffolk to Covehithe, Suffolk } \\
\text {-two major breaches at Blakeney-Cley-Salthouse; } \\
\text {-TAB3. Short term (Net Shoreline Movement NSM) and longer term (End Point Rate) } \\
\text { rates of shoreline change on the Suffolk, North Lincolnshirem and } \\
\text { North Norfolk coasts as determined by Digital Shoreline Analysis } \\
\text { Covehithe, 5.87; Donna Nook, 13.59; Holkham Gap west, 19.37; Holkham Gap east, 11.52; Scolt Head Is, } \\
\text { 5.49 }\end{array}$ \\
\hline Wadey et al (2015a) & $\begin{array}{l}\text { Wadey MP, ID Haigh, RJ Nichols, JM Brown, K Horsburgh, B Carroll, SL Gallop, T Mason, E Bradshaw, A } \\
\text { comparison of the } 31 \text { January-1 February } 1953 \text { and 5-6 December } 2013 \text { coastal flood events around the UK, UK } \\
\text { Frontiers in Marine Science, 2, 84, 2015a. } \\
\text {-'substantial coastal erosion' in the UK } \\
\text {-1953 storm was worse than Storm Xaver } 2013 \text { for erosion } \\
\text {-2013 storm created new desolated landscape at Spurn Head }\end{array}$ \\
\hline Wadey et al. (2015b) & Wadey MP, JM Brown, ID Haigh, T Dolphin, P Wisse, Assessment and comparison of extreme sea levels and \\
\hline
\end{tabular}




\begin{tabular}{|c|c|}
\hline & $\begin{array}{l}\text { waves during the 2013/2014 storm season in two UK coastal regions, Nat. Hazards Earth Syst. Sci. Discuss., } \\
\text { 3, 2665-2708, 2015b. } \\
\text {-Formby (Sefton) with notoriously fast eroding dune system 4m/year; } 13 \mathrm{~m} \text { of dune lost during 2013-2014 storms } \\
\text {-previous bad case of dune loss at Sefton, } 13.6 \mathrm{~m} \text { during Feb } 1990 \text { storm }\end{array}$ \\
\hline Brooks et al (2016) & $\begin{array}{l}\text { Brooks SM, T Spencer, A McIvor, I Moller, Reconstructing and understanding the impacts of storms and surges, } \\
\text { southern North Sea, Earth Surface Processes and Landforms, } 41,855-864,2016 \text {. } \\
\text {-average shoreline retreat Scolt Head Island } 8.14 \pm 0.39 \mathrm{~m} \text {; maximum retreat } 13 \mathrm{~m} \\
\text {-FIG } 4 \text {. aerial photo interpretation shows large along shore differences with characteristic spatial scale 100- } \\
1000 \mathrm{~m}\end{array}$ \\
\hline Matelski (2016) & $\begin{array}{l}\text { Matelski, Birgit, Erfahrungen aus der Sturmflut Xaver von 5. und 6.12.2013 und dem Weihnachshochwasser } 2014 \\
\text { in Schleswig-Holstein, IWASA } 2016 \text { Tagungsbeitrag, (46. IWASA, 7-8 Januar 2016; Internationales } \\
\text { Wasserbau-Symposium Aachen. [pdf document properties: autor=sonja; datestamp: 26Apr2016] } \\
\text {-coastline retreat up to 40m at Hoernum Odde on southern point of Sylt } \\
\text {-FIG7. [PHOTO] Aerial photo showing retreat of southern tip of Sylt Island } \\
\text { (Hoernum Odde) } \\
\text {-dunes subject to wave damage during 9h period when the water covered the sand }\end{array}$ \\
\hline Nederhoff et al (2016) & $\begin{array}{l}\text { Nederhoff K, E Elias, T Vermaas, Erosie op Ameland Noordwest. Modelstudie: simulaties met Delft3D en } \\
\text { XBeach, Deltares, } 117 \mathrm{pp} \text {, July, } 2016 \\
\text {-Delft3D and XBeach model study of threat of seawater contamination of Amelander water catchment area }\end{array}$ \\
\hline Sorensen (2016) & $\begin{array}{l}\text { Sorensen CS, Water NOT wanted - Coastal floods and flooding protection in Denmark, In RA Herrmann \& J } \\
\text { Jensen (eds), Sicherung von Daemmen, Deichen und Stauanlagen: Handbuch fuer Theorie und Praxis (Vol V } \\
\text { pp3-21). Siegen: Universitaet Siegen, } 2016 \\
\text {-Jutland west coast: for centuries people moved inland as coast receded; harbours from late 19C } \\
\text {-Jutland west coast: erosion stopped by sand nourishment } 2-3 \text { million m3/year along 110km coastline since 1980s }\end{array}$ \\
\hline Sorensen et al (2016a) & $\begin{array}{l}\text { Sorensen CS, NK Dronen, P Knudsen, J Jensen, P Sorensen, An extreme event as a games changer in coastal zone } \\
\text { management, Journal of Coastal Research, (Special Issue, No 75), 700-704, 2016. Proceedings of the 14th } \\
\text { International Coastal Symposium (Sydney, Australia) ed by A Vila-Concejo, E Bruce, DM Kennedy, RJ } \\
\text { McCarroll, 2016a } \\
\text {-Storm Xaver caused as much coastal erosion damage in the NE Zeeland, Denmark as all the storm events of the } \\
\text { past 100y. }\end{array}$ \\
\hline $\begin{array}{l}\text { Sorensent } \\
(2016 b)\end{array}$ & $\begin{array}{l}\text { Sorensen C, NH Broge, MR Molgaard, CS Schow, PThomsen, K Vognsen, P Knudsen, Assessing future flood } \\
\text { hazards for adaptation planning in a northern European Coastal Community, Frontiers in Marine Science, } \\
\text { 3:69, doi:10.3389/fmars.2016.00069, 2016b } \\
\text {-Thyboron: monitoring of well water and geologic modeling to assess saltwater contamination of groundwater } \\
\text {-Thyboron: storm surge flooding makes sewage system non-operational } \\
\text {-leveling surveys to assess uplift and tilting of Thyboron. }\end{array}$ \\
\hline Staneva et al (2016a) & $\begin{array}{l}\text { Staneva J, K Wahle, H Guenther, E Stanev, Coupling of wave and circulation models in coastal-ocean predicting } \\
\text { systems: a case study for the German Bight, Ocean Sci., 12, 797-806, 2016a. } \\
\text {-Xaver: 'Besides extreme high water levels along the coasts, extreme sea state conditions have been observed } \\
\text { causing serious erosion of dunes and sand displacement on the barrier islands' }\end{array}$ \\
\hline Staneva et al (2016) & $\begin{array}{l}\text { Staneva J, K Wahle, W Koch, A Behrens, L Fenoglio-Marc, EV Stanev, Coastal flooding: impact of waves on } \\
\text { storm surge during extremes - a case study for the German Bight, Nat. Hazards Earth Syst. Sci., 16, 2373-2389, } \\
2016 \\
-' \text {,... serious damage to the southern North Sea coastal areas' }\end{array}$ \\
\hline Brooks et al (2017) & $\begin{array}{l}\text { Brooks, SM, T Spencer, EK Christie, Storm impacts and shoreline recovery: Mechanisms and controls in the } \\
\text { southern North Sea, Geomorphology, 283, 48-60, } 2017 \text {. } \\
\text { *-FIG6. End point rate (m/y) for the barrier coast for the storm periods 2006-2007 } \\
\text { (blue), 2007-2008 (pink), and 2013-2014 (orage) for } \\
\text { (a) Brancaster Bay (14m), (b) Scolt Head Island (18m), (c) Holkham Bay (16m). } \\
\text { Also shown are the cross shore profile locations. } \\
\text { (d) end point rate (m/a) for the summer period } 2008 \text { to summer 2013, } \\
\text { a period of no storm activity along the barrier for Brancaster Bay (blue), } \\
\text { Scolt Head Island (red) and Holkham Bay (green). }\end{array}$ \\
\hline Jensen et al (2017) & $\begin{array}{l}\text { Jensen J, S Niehuser, A Arns, S Dangendorf, Sensor- und risikobasiertes Fruhwarn-system fuer Seedeiche } \\
\text { (EarlyDike), AP1 - Sturmflutmonitoring und Sturmflutssimulator - Fachbericht 2016, Siegen, April } 2017 \\
\text {-FIG5.1. Map of coastline dikes along German North Sea coast with heights; open coastline dikes between 8- } \\
\text { 10m }\end{array}$ \\
\hline $\begin{array}{lll}\text { World } & \text { Bank } & \text { Group } \\
(2017) & & \end{array}$ & $\begin{array}{l}\text { World Bank Group, Coastal Protection on the West Coast of Jutland, West Africa Coastal Areas Management } \\
\text { Program, Case Study } 02 \text { [PDF document date stamp: } 14 / 11 / 2017 \text { ] } \\
\text {-background information on heightened erosion and sand nourishment counter measures on Danish west coast } \\
\text {-defining regional storm took place Nov1981 with }>10 \mathrm{~m} \text { of coastline retreat } \\
\text {-Danish west coast only region with government supported coastal protection }\end{array}$ \\
\hline $\begin{array}{l}\text { Dreier and Froehle } \\
(2018)\end{array}$ & $\begin{array}{l}\text { Dreier, Norman and Peter Froehle, Operational wave forecast in the German Bight as part of a sensor- and risk } \\
\text { based early warning system, In: J-S Shim, I Chun, HS Lim (ed), Proceedings from the International Coastal } \\
\text { Symposium (ICS) } 2018 \text { (Busan, Republic of Korea), Journal of Coastal Research, Special Issue No. 85, 1161- } \\
1165,2018 \\
\text {-wave runup investigation at Untjehoern }\end{array}$ \\
\hline $\begin{array}{l}\text { Kystdirektoratet } \\
(2018)\end{array}$ & $\begin{array}{l}\text { Kystdirektoratet, Shoreface nourishment effects. An analysis of the } 2011 \text { nourishment performed at Skodbjerge. } \\
\text { Kystdirektoratet, Hojbovej } 1,7620 \text { Lemvig, Dec } 2018 \text { [pdf document properties: title=Shoreface nourishment } \\
\text { effects_Skodbjerge_20.12.2018, datestamp=20/12/2018] } \\
\text {-largest coastal erosion volume loss in winter 2013-2014 with Storm Bodil as the most serious storm. }\end{array}$ \\
\hline $\mathrm{Li}(2018)$ & $\begin{array}{l}\text { Li, H., The Ameland Inlet during the Sinterklaas Storm: the role of flooding of watersheds, 2DH model study in } \\
\text { Delft3D-FLOW, M.Sc. Thesis, Utrecht University, 01May2018 } \\
\text {-modelling study of flow Dutch Waddensee at Ameland and sediment trasnport }\end{array}$ \\
\hline
\end{tabular}




\begin{tabular}{|c|c|}
\hline & no wave, water level, or weather information presented \\
\hline $\begin{array}{l}\text { North Norfolk District } \\
\text { Council Coastal Team } \\
(2018)\end{array}$ & $\begin{array}{l}\text { North Norfolk District Council Coastal Team, Refurbishment of sea walls and groynes 2013-2015; Cromer Coast } \\
\text { Protection, (picture of plaque on wall taken 27Dec2018) www.northnorfolk.org/coastal } \\
\text {-some damage to Cromer sea defences; there had not been refurbishment for some years } \\
\text {-FIG6. [PHOTO] Photo damaged sea wall Cromer after Dec2013 storm Xaver }\end{array}$ \\
\hline $\begin{array}{l}\text { Giannopoulos et al } \\
(2019)\end{array}$ & $\begin{array}{l}\text { Giannopoulos G, L Peake, B Reid, J Andrews, A Grant, I Lorenzoni, M Goulden, J Waters, T Dolphin, J } \\
\text { Bremner, TJ Tolhurst, Environmental and social impacts of the } 2013 \text { storm surge on the North Norfolk coast, } \\
\text { powerpoint presentation date stamp 15May2019, unknown conference } \\
\text {-natural and manmade defences breached; coastal ecosys flooded } \\
\text {-significance of seawater flooding and soil salinization investigated } \\
\text {-dune overtopping and rollback at Blakeney and Cley }\end{array}$ \\
\hline $\begin{array}{l}\text { North Norfolk District } \\
\text { Council Coastal Team } \\
(2019)\end{array}$ & $\begin{array}{l}\text { North Norfolk District Council Coastal Team, Sheringham Sea Defences; Repair and Recovery from the } \\
\text { December } 2013 \text { storm surge, www.northnorfolk.org/coastal, photo of plaque on wall taken 30Dec2019 } \\
\text {-serious damage to section of Sheringham sea defence wall }\end{array}$ \\
\hline Andrews (2020) & $\begin{array}{l}\text { Andrews, JE, Spit extension and barrier rollover at Blakeney Point and Salthouse: historic map and field } \\
\text { observations, Bull. geol. Soc. Norfolk, 69, 35-63, } 2020 \\
\text {-mean landward shoreline movement: } \\
\text { Brancaster Bay } \quad 4.39 \mathrm{pm} 0.22 \mathrm{~m} \\
\text { Scolt Head Island } \quad 4.81 \mathrm{pm} 0.24 \mathrm{~m} \\
\text { Holkham Bay } \quad 7.36 \mathrm{pm} 0.97 \mathrm{~m}\end{array}$ \\
\hline $\begin{array}{l}\text { JBA Risk } \\
\text { Management (2020) }\end{array}$ & $\begin{array}{l}\text { JBA Risk Management, Storm Xaver 2013. Event Commentary, 2020. [PDF document properties: } \\
\text { author=Cameron Whitwham] } \\
\text {-several houses at Hemby fell into sea because of excessive cliff erosion. }\end{array}$ \\
\hline WIKI (20200124) & $\begin{array}{l}\text { WIKI, Cyclone Xaver, https://en.wikipedia.org/wiki/Cyclone_Xaver accessed 24Jan2020 } \\
\text {-Happisburgh, Hemsby; holiday homes Norlev strand Denmark undermined }\end{array}$ \\
\hline Wikipedia (20200502) & $\begin{array}{l}\text { Wikipedia, Orkan Xaver, https://de.wikipedia.org/wiki/Orkan_Xaver (accessed } 2 \text { May 2020) } \\
\text {-FIG6. [PHOTO] Coast damage at Hemsby, Norfolk } \\
\text {-25m of dune retreat at Hammersee/Juist } \\
\text {-significant dune damage Langeoog, Spiekeroog, Wangerooge } \\
\text {-Nordfriesischen Inseln: land loss at Sylt, Foehr, Amrum; most Halligen flooded; dike break on Keitum/Sylt; } \\
\text { damaged dune landscape on } 23 \mathrm{~m} \text { of Sylt coast; 20m cliff retreat at Hornumer Odde/Sylt; Duene next to } \\
\text { Helgoland had massive dune loss and water in-breaks }\end{array}$ \\
\hline
\end{tabular}

Table S35. Power interruptions (arranged by year and then alphabetically)

\begin{tabular}{|c|c|}
\hline Source & Full Reference and Notes \\
\hline $\begin{array}{l}\text { Air } \quad \text { Worldwide } \\
(20131212)\end{array}$ & $\begin{array}{l}\text { Air Worldwide, Press Release, Boston, 12Dec2013. https://www.air-worldwide.com/In-the-News/AIR- } \\
\text { Estimates-Losses-from-European-Windstorm-Xaver-at-Between-EUR-700-Million-and-EUR-1-4-Billion/ } \\
\text { (accessed 02Jan1990) } \\
\text {-power disrupted to } 100000 \text { homes Scotland \& } 6500 \text { in N Ireland }\end{array}$ \\
\hline BBC (20131205) & $\begin{array}{l}\text { BBC, In pictures: Winter storm hits UK, } 5 \text { December 2013, https://www.bbc.com/news/uk-scotland-25231224 } \\
\text {-many homes in northern Ireland left without power }\end{array}$ \\
\hline BBC (20131206) & $\begin{array}{l}\text { BBC20131206, Deadly storm and tidal surge batter northern Europe, BBC } 6 \text { Dec2013 (report by Anna Holligan) } \\
\text {-Poland: 400,000 homes without power due to severed lines } \\
\text {-Sweden and Norway: thousands of homes without power } \\
\text {-Germany, Hamburg: 1000s of homes without electricity }\end{array}$ \\
\hline Bloomberg (2013) & $\begin{array}{l}\text { Bloomberg, Hamburg has worst flood in } 37 \text { year amid European storms, Nicholas Brautlecht, 6Dec2013, } \\
\text { 02:58PM GMT https://www.bloomberg.com/news/articles/2013-12-06/hamburg-has-worst-flood-in-37-years-as- } \\
\text { storms-rage-across-europe } \\
\text {-Poland: } 400,000 \text { households without power }\end{array}$ \\
\hline $\begin{array}{l}\text { Daily } \\
(20131206)\end{array}$ & $\begin{array}{l}\text { Daily Mail, Huge storm strikes Europe causing death and destruction and leading to cancellation of hundreds of } \\
\text { flights, 6Dec2013. (correspondent: Nick Enoch), https://www.dailymail.co.uk/news/article-2519517/Huge-storm- } \\
\text { strikes-Europe-causing-death-destruction-leading-cancellation-hundreds-flights.html } \\
\text {-10000s without power Sweden }\end{array}$ \\
\hline $\begin{array}{l}\text { Gccapitalideas } \\
(20131209)\end{array}$ & $\begin{array}{l}\text { gccapitalideas, Windstorm Xaver, 9Dec2013, https://www.gccapitalideas.com/2013/12/09/windstorm-xaver/ } \\
\text {-northern Europe: } 500,000 \text { homes lost power at height of storm } \\
\text {-UK: } 130,000 \text { homes and businesses without power } \\
\text {-Poland: } 400,000 \text { homes without electricity } \\
\text {-Sweden: thousands homes without power }\end{array}$ \\
\hline GP (20131206) & $\begin{array}{l}\text { GP, Fortsatt risk for halka, 6Dec2013 (correspondent: D Henriksson, K Vikingsson, P Sydvik, TA Akerblom) } \\
\text { http://www.gp.se/nyheter/goteborg/1.2201325-fortsatt-risk-for-halka (accessed 1May2020) } \\
\text { storm caused big problems for electricity network } \\
\text {-28000 customers in Goteborg without power night to Friday } \\
\text {-Fjarrvarmeproduktionen? came back again Friday morning } \\
\text {-Torslanda, Tuve-Save, Gunnard: } 800 \text { customers with power loss Friday } \\
\text {-Goteborg Energi report at 23:00 that most have power restored } \\
\text {-few 10s of customers still without power in Torslands }\end{array}$ \\
\hline $\begin{array}{ll}\text { Kristeligt } & \text { Dagblad } \\
(20131205) & \end{array}$ & $\begin{array}{l}\text { Kristeligt Dagblad, Stormen blaeser Skotland omkuld: Doodsfald og nebrud, (contributor: Ritzau), 05Dec2013 } \\
\text { 12:50 https://www.kristeligt-dagblad.dk/udland/stormen-b1\%C3\%A6ser-skotland-omkuld-d\%C3\%B8dsfald- } \\
\text { og-nedbrud } \\
\text {-at least } 25000 \text { households without power in Scotland and Northern Ireland }\end{array}$ \\
\hline Kunz et al (20131206) & $\begin{array}{l}\text { Kunz M, B Muehr, K Schroeter, T Bessel, S Moehrle, T Muenzberg, S Brink, H-M Schmidt, Winterstorm Xaver - } \\
\text { Report. 06Dec2013 - Report No.1, Situation Report - 19:00CET, CEDIM Forensic Disaster Analysis Group } \\
\text { (FDA), Center for Disaster Management and Risk Reduction Technology. }\end{array}$ \\
\hline
\end{tabular}




\begin{tabular}{|c|c|}
\hline & 000 customers affected by power outages grid area of Wemag \\
\hline $\begin{array}{l}\text { Spiegel International } \\
(20131206)\end{array}$ & $\begin{array}{l}\text { Spiegel International, Winter storm 'Xaver' batters } \text { northern Europe,6 Dec 2013, 12:56 } \\
\text { https://www.spiegel.de/international/europe/tidal-surge-winter-storm-xaver-batters-northern-europe-a- } \\
\text { 937576.html\# } \\
\text {-power loss to } 100000 \text { households in Poland }\end{array}$ \\
\hline $\begin{array}{l}\text { TheJournal } \\
\text { (20131205) }\end{array}$ & $\begin{array}{l}\text { TheJournal, Homes remain without electricity after high winds batter power lines, 05Dec2013 08:20PM, } \\
\text { https://www.thejournal.ie/storm-ireland-1207783-Dec2013/ } \\
\text {-Northern Ireland, } \\
\text {-Donegal, Ballybofey, Buncrana, border near Derry, Meath, Dublin, Trim, Dublin coastal area Malahide \& East } \\
\text { Wall }\end{array}$ \\
\hline $\begin{array}{l}\text { Unwetterzentrale } \\
(201312)\end{array}$ & $\begin{array}{l}\text { Unwetterzentrale, Orkantief XAVER - ein weiterer schwerer Wintersturm der letzten Jahrzehnte, Thomas Savert } \\
\text { and Stefan Laps, Dec. } 2013 \mathrm{http} / / / \text { www.unwetterzentrale.de/uwz/928.html } \\
\text {-tens of thousands of houseas in Poland without power }\end{array}$ \\
\hline ABPmer (2014) & $\begin{array}{l}\text { ABP mer, Ensuring Flood Resilience. An overview of the 5/6 December 2013, Associated British Ports, Marine } \\
\text { Environmental Research, July, } 2014 \\
\text {-storm caused loss of power to more than 100,000 homes }\end{array}$ \\
\hline Dunbar et al (2014) & $\begin{array}{l}\text { Dunbar I, N Phipps, M Szonyi, Risk Nexus. After the storm: how the UK's flood defences performed during the } \\
\text { surge following Xaver, Flood resilience review 09.14, Zurich Insurance Company Ltd., Mythenquai 2, 8002, } \\
\text { Zurich, Switzerland [document properties: date 28Aug2014; author= Zurich] } \\
\text {-10 000s UK \& } 100 \text { 000's Europe left without power } \\
\text {-normal public activities came to standstill }\end{array}$ \\
\hline RMS (2014) & $\begin{array}{l}\text { RMS, 2013-2014 Winter Storms in Europe. An Insurance and Catastrophe Modeling Perspective. RMS White } \\
\text { Paper. [PDF TIMESTAMP 11Mar2014] } \\
- \text {-...downing power lines ...' }\end{array}$ \\
\hline SMHI (20140703) & $\begin{array}{cccccccc}\text { SMHI, } & \text { Simone, } & \text { Hilde, } \\
\text { https://www.smhi.se/kunskapsbanken/meteorologi/simone-hilde-sven-och-ivar-okt-dec-2013-1.76183 } & \text { Sven } & \text { och } & \text { Ivar } & \text { okt-dec } & 2013, & & \text { Jul } \\
\text {-50 000 households without power }\end{array}$ \\
\hline Sibley et al. (2015) & $\begin{array}{l}\text { Sibley A, D Cox, H Titley, Coastal flooding in England and Wales from Atlantic and North Sea storms during the } \\
\text { 2013/2014 winter, Weather, 70, 62-70, } 2015 \\
\text { - } \ldots \text { with } 200 \text { properties affected in Whitby and power outages to the town making recovery difficult' }\end{array}$ \\
\hline Wadey et al (2015a) & $\begin{array}{l}\text { Wadey MP, ID Haigh, RJ Nichols, JM Brown, K Horsburgh, B Carroll, SL Gallop, T Mason, E Bradshaw, A } \\
\text { comparison of the } 31 \text { January-1 February } 1953 \text { and 5-6 December } 2013 \text { coastal flood events around the UK, UK } \\
\text { Frontiers in Marine Science, 2, 84, 2015a. } \\
\text {-Electricity substation flooded in Middlesbrough }\end{array}$ \\
\hline Wadey et al (2015b) & $\begin{array}{l}\text { Wadey MP, JM Brown, ID Haigh, T Dolphin, P Wisse, Assessment and comparison of extreme sea levels and } \\
\text { waves during the 2013/2014 storm season in two UK coastal regions, Nat. Hazards Earth Syst. Sci. Discuss., } \\
\text { 3, 2665-2708, 2015b. } \\
\text {-listing of nuclear reactors Sizewell } \\
\text {-dune damage in Sizewell area worse in } 1953 \text { compared with } 2013\end{array}$ \\
\hline Ribeiro et al (2017) & $\begin{array}{l}\text { Ribeiro R, R Rudge, D Rucinska, Analysis of physical factors of the windstorm Xaver in Poland: post-hazard } \\
\text { review, Weather, 72, 2017, pp.378-382 } \\
\text {-downed electricity lines left many people without power }\end{array}$ \\
\hline Rucinska (2019) & $\begin{array}{l}\text { Rucinska D, Describing Storm Xaver in disaster terms, International Journal of Disaster Reduction, 34, 147-153, } \\
\quad 2019 \\
\text {-Poland: blackout in 400,000 homes affecting } 1.11 \text { million people } \\
\text {-people without power northern Germany } \\
-10.000 \text { 's without power in UK }\end{array}$ \\
\hline Wikipedia (20191002) & $\begin{array}{l}\text { Wikipedia, Stormen Bodil, https://da.wikipedia.org/wiki/Stormen_Bodil\#cite_note-39 (accessed 02Oct2019) } \\
\text {-Scotland: } 20000 \text { houses without power } \\
\text {-Northern Ireland: } 6000 \text { houses without poer }\end{array}$ \\
\hline $\begin{array}{l}\text { JBA Risk Managment } \\
(2020)\end{array}$ & $\begin{array}{l}\text { JBA Risk Management, Storm Xaver 2013. Event Commentary, 2020. [PDF document properties: } \\
\text { author=Cameron Whitwham] } \\
-130.000 \text { homes/businesses without power }\end{array}$ \\
\hline WIKI (20200124) & $\begin{array}{l}\text { WIKI, Cyclone Xaver, https://en.wikipedia.org/wiki/Cyclone_Xaver accessed 24Jan2020 } \\
\text {-Scotland: } 20000 \text { houses } \\
\text {-N Ireland: } 6500 \text { houses } \\
\text {-N England: } 20000 \text { homes without power across Cumbria and Teeside; } 135000 \text { properties across Northeast, } \\
\text { Yorkshire, North Lincolnshire had power disruptions } \\
\text {-Sweden: } 50000 \text { homes without power } \\
\text {-Poland: } 400000 \text { households with blackout } \\
\text {-power outage Middlesbrough } \\
\text {-Whitby: electrical substation short out }\end{array}$ \\
\hline Wikipedia (20200429) & $\begin{array}{l}\text { Wikipedia, Stormen Sven, https://sv.wikipedia.org/wiki/Stormen_Sven\#cite_note-15, accessed 29Apr2020 } \\
\text {-E.ON power company engaged } 4 \text { helicopters and } 200 \text { extra staff } \\
\text {-most of } 1600 \text { people working at Ringhals Nuclear Power station sent home 6Dec2013 }\end{array}$ \\
\hline Wikipedia (20200502) & $\begin{array}{l}\text { Wikipedia, Orkan Xaver, https://de.wikipedia.org/wiki/Orkan_Xaver (accessed } 2 \text { May 2020) } \\
\text {-power cuts to } 20000 \text { houses Scotland and } 6000 \text { houses Ireland }\end{array}$ \\
\hline
\end{tabular}

Table S36. List bridge closures, cancelled ferry crossings, port closures, airport cancel, rail interruptions, traffic accidents (arranged by year and then alphabetically)

\begin{tabular}{|l|llllll|}
\hline Source & Full Reference and Notes & & & & \\
\hline $\begin{array}{l}\text { Aftenposten } \\
(20131206)\end{array}$ & $\begin{array}{c}\text { Aftenposten, Varsler full storm I Sor-Norge, 5Dec2013 15:03 (correspondent } \\
\text { https://www.aftenposten.no/norge/i/bKEjl/varsler-full-storm-i-soer-norge }\end{array}$ & Annar & Holm) \\
\hline
\end{tabular}




\begin{tabular}{|c|c|}
\hline & $\begin{array}{l}\text {-cancelled flights in Avninor network in southern Norway } \\
\text {-Bodo airport closed due to heavy snowfall } \\
\text {-closed mountain pass roads souther Norway } \\
\text {-cancelled ferries Norway west coast to Denmark } \\
\text {-cancelled ferry: Larvik/Kristiansand and Hirtshals } \\
\text {-cancelled ferry: Sandefjord-Stromstad } \\
\text {-cancelled ferry: Bergen-Stavanger-Hirtshas-Langesund } \\
\text {-cancelled ferry: Hirtshals-Sorlandskysten } \\
\text {-Basto gerry normal } \\
\text {-no cancellation information on ferry Oslo-Kie } \\
\text {-no indication of cancelled ferry Oslo-Kiel }\end{array}$ \\
\hline BBC (20131205) & $\begin{array}{l}\text { BBC, In pictures: Winter storm hits UK, } 5 \text { December 2013, https://www.bbc.com/news/uk-scotland-25231224 } \\
\text { FIG4. [PHOTO] Earlier in West Lothian lorry driver dies when vehicle blown over } \\
\text { on to two cars (PA) } \\
\text { FIG5. [PHOTO] High winds brought down this lorry near Hamilton (PA). } \\
\text { FIG6. [PHOTO] Many roads have been closed across Scotland due to fallen trees (AP) } \\
\text { FIG7. [PHOTO] This HGV overturned and came to rest in a precarious position on the } \\
\text { M90 Friarton Bridge near Perth (PA) }\end{array}$ \\
\hline BBC (20131206) & $\begin{array}{l}\text { BBC20131206, Deadly storm and tidal surge batter northern Europe, BBC } 6 \text { Dec2013 (report by Anna Holligan) } \\
\text {-dozens of flights cancelled at Berlin Tegel, Copenhagen \& smaller airports } \\
\text {-many rail and ferry services cut in Germany and Scandinavia } \\
\text {-Sweden all rail services cancelled in Skane } \\
\text {-planes grounded at Sturup and Gothenburg's Landvetter airport } \\
\text {-heavy snow affected roads } \\
\text {-Denmark: } 1000 \text { people spent night in Copenhagen airport; Oresund bridge connecting Copenhagen to Denmark } \\
\text { closed on night of } 5 \text { Dec } \\
\text {-Hamburg port closed overnight; handles huge amount of European trade } \\
\text {-Netherlands Schipol airport: dozens flights cancelled 5Dec, airport okay on 6Dec } \\
\text {-Germany: many rail services stopped; fallen trees on railway lines }\end{array}$ \\
\hline Bloomberg (2013) & $\begin{array}{l}\text { Bloomberg, Hamburg has worst flood in } 37 \text { year amid European storms, Nicholas Brautlecht, 6Dec2013, } \\
\text { 02:58PM GMT https://www.bloomberg.com/news/articles/2013-12-06/hamburg-has-worst-flood-in-37-years-as- } \\
\text { storms-rage-across-europe } \\
\text {-ship traffic at Hamburg port halted overnight } \\
\text {-Hamburg airport cancelled } 100 \text { of } 400 \text { take-offs \& landings } \\
\text {-DB halted long distance trains via Schleswig-Holstein and cross border Denmark traffic } \\
\text {-DB stopped Hannover-Bremen line } \\
\text {-Scotland rail net work paralyzed 5Dec2013 } \\
\text {-extreme flooding caused repeated closing locks at both ends Kiel Canal, world's busiest artificial waterway } \\
\text {-commuter traffic Berlin \& Potsdam interrupted by damage along tracks } \\
\text {-Poland LOT airline some cancelled flights }\end{array}$ \\
\hline $\begin{array}{l}\text { Daily } \\
(20131206)\end{array}$ & $\begin{array}{l}\text { Daily Mail, Huge storm strikes Europe causing death and destruction and leading to cancellation of hundreds of } \\
\text { flights, 6Dec2013. (correspondent: Nick Enoch), https://www.dailymail.co.uk/news/article-2519517/Huge-storm- } \\
\text { strikes-Europe-causing-death-destruction-leading-cancellation-hundreds-flights.html } \\
\text {-Copenhagen airport closed overnight; } 1000 \text { people spend night in airport } \\
\text {-LOT airline Poland cancels some international and domestic flights } \\
\text {-traffic accidents kill } 3 \text { in Poland and } 1 \text { in Denmark by wind gusts }\end{array}$ \\
\hline $\begin{array}{l}\text { Deutschlander et al } \\
(2013)\end{array}$ & $\begin{array}{l}\text { Deutschlander T, K Frierich, S Haeseler, C Lefebvre, Orkantief XAVER ueber Nordeuropea von } 5 . \text { bis } 7 . \\
\text { Dezember 2013, Deutscher Wetterdienst DWD, Stand 30. Dezember 2013,19pp. } \\
\text {-schools and Weihnachtsmarkten in northern Germany closed } \\
\text {-cancelled flights } \\
\text {-interruptions of ship and train traffic. }\end{array}$ \\
\hline DW (20131205) & $\begin{array}{l}\text { DW, Storm Xaver pummels Europe, flood surge feared, 05/12/2013 } \\
\text {-Oresund Bridge linking Denmark and Sweden closed } \\
\text {-transport services stopped ahead of storm: flight, ferry, rail }\end{array}$ \\
\hline $\begin{array}{l}\text { Gccapitalideas } \\
(20131209)\end{array}$ & $\begin{array}{l}\text { gccapitalideas, Windstorm Xaver, 9Dec2013, https://www.gccapitalideas.com/2013/12/09/windstorm-xaver/ } \\
\text {-UK: all forms of transportation severely disrupted due to downed trees } \\
\text {-Germany: disruption rail traffic across northern Germany; dozens of flight cancellations Hamburg, Duesseldorf, } \\
\text { Cologne } \\
\text {-Netherlands: widespread travel disruption } \\
\text {-Denmark: Copenhagen airport closed }\end{array}$ \\
\hline GP (20131206) & $\begin{array}{l}\text { GP, Fortsatt risk for halka, 6Dec2013 (correspondent: D Henriksson, K Vikingsson, P Sydvik, TA Akerblom) } \\
\text { http://www.gp.se/nyheter/goteborg/1.2201325-fortsatt-risk-for-halka (accessed 1May2020) } \\
\text {-trains stopped west Goteland; replacement bus services } \\
\text {-road blockages mostly by fallen trees } \\
\text { - ferry to Knippla and Hyppeln in north Skargarden stopped for carrying cars because of high water levels } \\
\text {-flight cancellations and delays at Landvetter and Arlanda }\end{array}$ \\
\hline $\begin{array}{ll}\text { Kristeligt } & \text { Dagblad } \\
(20131205) & \end{array}$ & $\begin{array}{l}\text { Kristeligt Dagblad, Stormen blaeser Skotland omkuld: Doodsfald og nebrud, (contributor: Ritzau), } 05 \text { Dec2013 } \\
\text { 12:50 https://www.kristeligt-dagblad.dk/udland/stormen-b1\%C3\%A6ser-skotland-omkuld-d\%C3\%B8dsfald- } \\
\text { og-nedbrud } \\
\text {-cancelled departures from airports at Glasgow, Edinburgh, Aberdeen } \\
\text {-trains cancelled in Scotland } \\
\text {-police warns driver against going on roads }\end{array}$ \\
\hline Kunz (20131206) & $\begin{array}{l}\text { Kunz M, B Muehr, K Schroeter, T Bessel, S Moehrle, T Muenzberg, S Brink, H-M Schmidt, Winterstorm Xaver - } \\
\text { Report. 06Dec2013 - Report No.1, Situation Report - 19:00CET, CEDIM Forensic Disaster Analysis Group } \\
\text { (FDA), Center for Disaster Management and Risk Reduction Technology. }\end{array}$ \\
\hline
\end{tabular}




\begin{tabular}{|c|c|}
\hline & $\begin{array}{l}\text {-Hamburg port closed night 5-6Dec2013 } \\
\text {-St Pauli Elbtunnel closed } \\
\text {-locks in Kiel Canal at Holtenau and Brunsbuettel closed } \\
\text {-ferries cancelled in Kiel } \\
\text {-German-Danish ferry Scandlines stopped serice between Rostock and Gedser until 6Dec20131115 } \\
\text {-ferry traffic to East and North Frisian islands nearly shut down 5-6Dec2013 } \\
\text {-ferry companies operating trips to other countries in North and Baltic Sea affected (e.g. Stena) } \\
\text {-Hamburg and Bremen airport: 50\% cancellations 5Dec2013; 20\% cancellations 6Dec2013 } \\
\text {-Hannover, Duesseldorf, Koeln airport: minor impact } \\
\text {-DB cancelled 20 intercity trains night 5-6Dec2013 and 6-7Dec2013 } \\
\text {-cancellation of Schleswig-Holstein trains from afternoon 5Dec2013 } \\
\text {-no severe rail infrastructure damage }\end{array}$ \\
\hline Rtv Oost (20131205) & $\begin{array}{l}\text { Oost, Trainverkeer ten noorden van } \quad \text { Zwolle } \quad \text { stilgelegt vanwege } \text { storm 5Dec2013 } 14: 02 \text {, } \\
\text { https://www.rtvoost.nl/nieuws/177568/Treinverkeer-ten-noorden-van-Zwolle-stilgelegd-vanwege-storm } \\
\text { train traffic shurt down from 14:00 5Dec2013 from Zwolle to Lelystad, Leeuwarden, Groningen, Emmen }\end{array}$ \\
\hline $\begin{array}{l}\text { Spiegel International } \\
(20131206)\end{array}$ & $\begin{array}{l}\text { Spiegel International, Winter storm 'Xaver' batters northern Europe,6 Dec 2013, 12:56 } \\
\text { https://www.spiegel.de/international/europe/tidal-surge-winter-storm-xaver-batters-northern-europe-a- } \\
\text { 937576.html\# } \\
\text { FIG11.The UK, Germany, Netherlands, Scandinavia most heavily affected by the severe } \\
\text { storm, but cancelled flights and tranins caused problems across Europe. } \\
\text { By Thursday night, officials reported three storm-related deaths in the } \\
\text { UK and Denmark. Here, planes await takeoff at the Hamburg airport on } \\
\text { Thursday (DPA) } \\
\text { FIG15.Snow from Xaver gnarled traffic near Olpe in the state of Nord Rhine Westphalia } \\
\text { on Friday morning (DPA) } \\
\end{array}$ \\
\hline $\begin{array}{l}\text { TheJournal } \\
\text { (20131205) }\end{array}$ & $\begin{array}{l}\text { TheJournal, Homes remain without electricity after high winds batter power lines, 05Dec2013 08:20PM, } \\
\text { https://www.thejournal.ie/storm-ireland-1207783-Dec2013/ } \\
\text {-Wexford-Dublin route closed by dangerous trees }\end{array}$ \\
\hline The Local (20131205) & 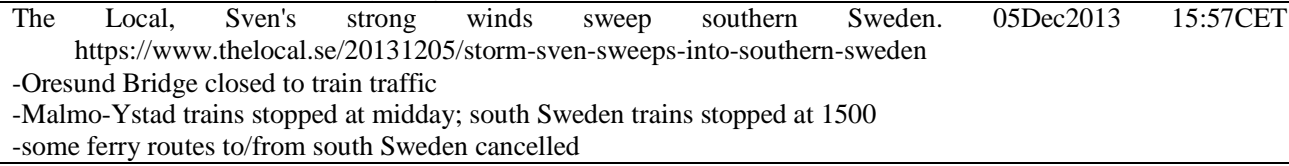 \\
\hline ABPmer (2014) & $\begin{array}{l}\text { ABPmer, Ensuring Flood Resilience. An overview of the 5/6 December 2013, Associated British Ports, Marine } \\
\text { Environmental Research, July, } 2014 \\
\text {-storm caused shutdown of Scotland's rail network } \\
\text {-Immingham: 'storm caused damage to critical infrastructure, assets and disrupted port operations' } \\
\text {-Lowestoft: 'supply damage/interruption to the Bascule Bridge, which in turn was non-operational for } \\
\text { approximately } 4 \text { days' }\end{array}$ \\
\hline Leiding et al (2014) & $\begin{array}{l}\text { Leiding T, B Tinz, G Rosenhagen, C Lefevre, S Haeseler, S Hagemann, I Bastigkeit, D Stein, P Schwenk, S } \\
\text { Mueller, O Outzen, K Herklotz, F Kinder, T Neumann, Meteorological and Oceanographic Conditions at the } \\
\text { FINO platforms during the severe storms Christian and Xaver, DEWI Magazin, No.44, p16-25, } 2014 . \\
\text {-shipping, rail, air traffic shut down }\end{array}$ \\
\hline Axer et al (2015) & $\begin{array}{l}\text { Axer T, T Bistry, M Klawa, M Mueller, M Suesser, Deutsche Ruck Sturm dokumentation } 2013 \text { Deutschland, } \\
\text { 2013, Deutsche Rueckversicherung Aktiengesellschaft, Hansaallee 177, 40549 } \begin{array}{c}\text { Duesseldorf, } \\
\text { www.deutscherueck.de [pdf document information: author=filiz; date stamp=07Aug2015] }\end{array} \\
\text {-all islands of Niedersachsen and Schleswig Holstein cut off from ferry traffic } \\
\end{array}$ \\
\hline Sibley et al. (2015) & $\begin{array}{l}\text { Sibley A, D Cox, H Titley, Coastal flooding in England and Wales from Atlantic and North Sea storms during the } \\
2013 / 2014 \text { winter, Weather, } 70,62-70,2015 \\
\text {-'On the North Sea coast pats of Newcastle's quayside were underwater at high tide as the Tyne estuary } \\
\text { overflowed' }\end{array}$ \\
\hline Wadey et al. (2015a) & $\begin{array}{l}\text { Wadey MP, ID Haigh, RJ Nichols, JM Brown, K Horsburgh, B Carroll, SL Gallop, T Mason, E Bradshaw, A } \\
\text { comparison of the } 31 \text { January-1 February } 1953 \text { and 5-6 December } 2013 \text { coastal flood events around the UK, } \\
\text { UK Frontiers in Marine Science, 2, 84, 2015a. } \\
\text {-TABLE3. List of flooded roads and rails in } 1953 \text { and } 2013\end{array}$ \\
\hline Wadey et al (2015b) & $\begin{array}{l}\text { Wadey MP, JM Brown, ID Haigh, T Dolphin, P Wisse, Assessment and comparison of extreme sea levels and } \\
\text { waves during the 2013/2014 storm season in two UK coastal regions, Nat. Hazards Earth Syst. Sci. Discuss., } \\
3,2665-2708,2015 \mathrm{~b} \text {. } \\
\text {-Lowestoft train station flooded; lines Lowestoft-Norwich and Lowestoft-Ipswich cut } \\
\text {-Lowestoft-Ipswich line closed for } 11 \text { days }\end{array}$ \\
\hline Ribeiro et al (2017) & $\begin{array}{l}\text { Ribeiro R, R Rudge, D Rucinska, Analysis of physical factors of the windstorm Xaver in Poland: post-hazard } \\
\text { review, Weather, 72, 2017, pp.378-382 } \\
\text {-some flights cancelled in Poland }\end{array}$ \\
\hline Rucinska (2019) & $\begin{array}{l}\text { Rucinska D, Describing Storm Xaver in disaster terms, International Journal of Disaster Reduction, 34, 147-153, } \\
\quad 2019 \\
\text {-cancelled ferry services across English Channel from Netherlands } \\
\text {-Netherlands: } 42 \text { cancelled KLM flights from Schipol } \\
\text {-Germany: Hamburg flights cancelled, interrupted high-speed rail between Hamburg \& Germany }\end{array}$ \\
\hline Wikipedia (20191002) & $\begin{array}{l}\text { Wikipedia, Stormen Bodil, https://da.wikipedia.org/wiki/Stormen_Bodil\#cite_note-39 (accessed 02Oct2019) } \\
\text {-UK: Glasgow: Central train station evacuated 5Dec when flying debris came through roof } \\
\text {-UK: Scotland suspended all rail services later in the day; trampolines \& trees on track } \\
\text {-Denmark: 5Dec Banedenmark shut rail services Jylland 14:00, Fyn 16:00, Sjaelland 18:00 } \\
\text {-Denmark: bridges shut: Storebaeltsbroen, Vejlefjordbroen, nye Lillebaeltsbro, } \\
\text { gamle lillebaeltsbro, Svendborgsundbroen, Oresundsbroen, } \\
\text { Limfjordsbrown, Alssundbroen }\end{array}$ \\
\hline
\end{tabular}




\begin{tabular}{|l|l|}
\hline & -Denmark: Copenhagen airport closed for flights 18:30 \\
\hline WIKI (20200124) & $\begin{array}{l}\text { WIKI, Cyclone Xaver, https://en.wikipedia.org/wiki/Cyclone_Xaver accessed 24Jan2020 } \\
\text { rail: Scotrail, Glasgow Central Station, Skane Sweden, Denmark, Schleswig-Holstein, Suffolk } \\
\text { road/bridge:UK, Stavanger }\end{array}$ \\
& $\begin{array}{l}\text { ferry: Isle of Man Packet Company, Norway } \\
\text { flight: Easyjet Bristol-Edinburgh flight struck by lightning diverted to Newcastle } \\
\text { airport: Glasgow, Edinburgh, Aberdeen, Birmingham, Stavanger } \\
\text { Goteborg Landvetter, Malmo, Amsterdam Schipol, Hamburg, } \\
\text { Berlin Tegel, Denmark Billund, Aalborg, Copenhagen }\end{array}$ \\
\hline Wikipedia (20200429) & $\begin{array}{l}\text { Wikipedia, Stormen Sven, https://sv.wikipedia.org/wiki/Stormen_Sven\#cite_note-15, accessed 29Apr2020 } \\
\text {-Malmo Sturup airport closed from evening 5 Dec to lunchtime 6 Dec 2013 } \\
\text {-all train traffic in Sweden south of Kungbacka and Nasssjo stopped evening 5Dec and morning 6 Dec }\end{array}$ \\
\hline Wikipedia (20200502) & $\begin{array}{l}\text { Wikipedia, Orkan Xaver, https://de.wikipedia.org/wiki/Orkan_Xaver (accessed 2 May 2020) } \\
\text { keyword ferry: Juist, Sylt, Norderney, Wangerooge; Bornhol Sweden ferry suspended } \\
\text { keyword bridges: Rader Hochbrucke, Fehmarnsundebruecke; Oresund bridge } \\
\text { keyword airport: Hamburg airport } \\
\text { keyword train: DB suspends train services N Germany, Schone Sweden trains suspended, Scotrail }\end{array}$ \\
\hline
\end{tabular}

Table S37. Structural damage to wind farms and wind energy impacts (arranged by year and then alphabetically)

\begin{tabular}{|c|c|}
\hline Source & Full Reference and Notes \\
\hline Gray (2013) & $\begin{array}{l}\text { Gray, Tom, Into the Wind, The AWEA Blog, Ireland, U.K., Germany set new wind generation records, } \\
\text { https://www.aweablog.org/ireland-u-k-germany-set-new-wind-generation-records/, } 11 \mathrm{Dec} 2013 \\
\text {-record wind production Germany 06Dec2013 at } 26 \mathrm{GW} \text {; one third of demand } 100 \mathrm{GW} \\
\text {-Britain wind energy production record } 02 \mathrm{Dec} 2013 \text { at } 6053 \mathrm{MW} \text { or } 14 \% \text { of electricity on UK system } \\
\text {-7900MW of gas-fired stations shut down during storm } \\
\text {-UK plans to triple capacity by } 2020 \\
\text {-Ireland wind energy record } 8 \text { Nov2013 at } 11564 \mathrm{MW} \text {; enough for } 1 \text { million homes } \& 45 \% \text { of demand } \\
\text {-IWEA Kenneth Mathews: ...combatting climate change ... Ireland leading way in demonstrating potential of wind } \\
\text { energy }\end{array}$ \\
\hline IWR (20131206) & $\begin{array}{l}\text { IWR, Orkantief 'Xaver' bringt neuen Rekord - Deutsche Windkraftanlagen produzieren erstmals Strom mit ueber } \\
26000 \text { MW Leistung, 06Dec2013 09:31, https://www.iwr.de/news.php?id=25168 (accessed 07May2020) } \\
\text {-record wind energy production Germany during storm Xaver } \\
\text {-power output equivalent to } 26 \text { nuclear power plants } \\
\text {-previous day record } 28 \text { Oct2013 during Storm Christian } \\
\text {-highest month wind energy production Germany Nov } 2011\end{array}$ \\
\hline Kunz et al (20131206) & $\begin{array}{l}\text { Kunz M, B Muehr, K Schroeter, T Bessel, S Moehrle, T Muenzberg, S Brink, H-M Schmidt, Winterstorm Xaver - } \\
\text { Report. 06Dec2013 - Report No.1, Situation Report - 19:00CET, CEDIM Forensic Disaster Analysis Group } \\
\text { (FDA), Center for Disaster Management and Risk Reduction Technology. } \\
\text {-wind park Baltic } 1 \text { closed 5Dec2013 }\end{array}$ \\
\hline $\begin{array}{l}\text { National Wind Watch } \\
\text { (20131210a) }\end{array}$ & $\begin{array}{l}\text { National Wind Watch, 10Dec2013, Xaver zerstoert ein Windrad bei Vlatten, original source: Aachener Zeitung, } \\
\text { 6Dec2013 https://www.wind-watch.org/news/2013/12/10/xaver-zerstort-ein-windrad-bei-vlatten/ } \\
-1 \text { turbine in } 11 \text { turbine array cracked off at wind farm near Vlatten; between Vlatten and Hergarten } \\
\text {-wind speed in Kreis Dueren } 75 \mathrm{~km} / \mathrm{h}\end{array}$ \\
\hline $\begin{array}{l}\text { National Wind Watch } \\
(20131210 b)\end{array}$ & $\begin{array}{l}\text { National Wind Watch, Sturm 'Xaver': Windrad stuerzt auf Acker, 10Dec2013b, credit: von Manfred Reinnarth, } \\
\text { Koelnische Rundschau, 06/12/2013, https://www.wind-watch.org/news/2013/12/10/sturm-xaver-windrad- } \\
\text { sturzt-auf-acker/ } \\
\text {-Storm Xaver toppled large wind turbine neaer Vlatten; cracked off at } 25 \mathrm{~m} \text { near wleded joint } \\
\text {-wind park had } 8 \text { similar instations } \\
\text {-wind turbine } 4 \text { had been operating for } 13 \text { years } \\
\text {-accident happened 05/12/2013 09:00CET when communications were lost }\end{array}$ \\
\hline $\begin{array}{l}\text { National Wind Watch } \\
\text { (20131212) }\end{array}$ & $\begin{array}{l}\text { National Wind Watch, Xaver knickte Windrad um: fear of metal thieves, 12Dec2013 (Credit: 11/12/2013 - Iris } \\
\text { Klingelhoefer and Alexander Kuffner, express.de) https://www.wind-watch.org/news/2013/12/12/xaver- } \\
\text { knickte-windrad-um-angst-vor-metall-dieben/ } \\
\text {-wind farm had } 8 \text { turbines } \\
\text {-on 5Dec2013 21:00CET turbine } 4 \text { toppled by Storm Xaver } \\
\text {-wind turbine should have been secure against wind speeds of } 180 \mathrm{~km} / \mathrm{h} \text {; cracked turbine at } 25 \mathrm{~m}\end{array}$ \\
\hline $\begin{array}{l}\text { Nordbayern } \\
(20131207)\end{array}$ & $\begin{array}{l}\text { Nordbayern, Deining: 'Xaver' reisst Rotorblatt von Windrad ab. Windkraftanlage schleuderte Eisbrocken auf die } \\
\text { Strasse, 07/12/2013, 12:07. https://www.nordbayern.de/region/neumarkt/deining-xaver-reisst-rotorblatt-von- } \\
\text { windrad-ab-1.3326492 } \\
\text {-previously damaged blade thrown from turbine and landed } 150 \mathrm{~m} \text { to north } \\
\text {-fist-size ice blocks thrown from turbine onto road } 80 \mathrm{~m} \text { away }\end{array}$ \\
\hline FINO1 (20140108) & $\begin{array}{l}\text { FINO1, } 15 \text {-m wave damaged FINO1, 08Jan2014. http://www.fino1.de/meldungen/alle-meldungen/137-15-meter- } \\
\text { welle-beschaedigt-fino1 } \\
\text {-15m access deck had railing bent on north side and decking torn up on south corner } \\
\text {-access structure at } 17 \mathrm{~m} \text { smashed by swinging } 500 \mathrm{~kg} \text { counterweight } \\
\text {-two photos of damage }\end{array}$ \\
\hline Leiding et al (2014) & $\begin{array}{l}\text { Leiding T, B Tinz, G Rosenhagen, C Lefevre, S Haeseler, S Hagemann, I Bastigkeit, D Stein, P Schwenk, S } \\
\text { Mueller, O Outzen, K Herklotz, F Kinder, T Neumann, Meteorological and Oceanographic Conditions at the } \\
\text { FINO platforms during the severe storms Christian and Xaver, DEWI Magazin, No.44, p16-25, 2014. } \\
\text {-turbulence intensity exceeds category A turbines for several } 10 \text { minute intervals at FINO1, FINO2, FINO3 during } \\
\text { Storm Xaver. } \\
\text {-wind speed jump from } 23 \mathrm{~m} / \mathrm{s} \text { to } 37 \mathrm{~m} / \mathrm{s} \text { with change of wind speed from } 220-290 \mathrm{deg} \text { (70deg) at FINO3 } \\
\text {-implications for Dantysk wind farm near FINO3 } \\
\text {-long interval when wind speed }>25 \mathrm{~m} / \mathrm{s} \text { turbine cutoff }\end{array}$ \\
\hline
\end{tabular}




\begin{tabular}{|c|c|}
\hline Axer et al (2015) & $\begin{array}{l}\text { Axer T, T Bistry, M Klawa, M Mueller, M Suesser, Deutsche Ruck Sturm dokumentation } 2013 \text { Deutschland, } \\
\text { 2013, Deutsche Rueckversicherung Aktiengesellschaft, Hansaallee 177, 40549 Duesseldorf, } \\
\text { www.deutscherueck.de [pdf document information: author=filiz; date stamp=07Aug2015] } \\
\text {-Doehlen Kreis Oldenburg 20t nacelle torn from turbine } \\
\text {-Deining Landkreis Neumarkt, Oberpfalz: fallen wind blade from turbine }\end{array}$ \\
\hline Fischer et al. (2015) & $\begin{array}{l}\text { Fischer, JG, C Senet, A Schneehorst, O Outzen, S Schirmel, K Herklotz, Sea state measurements in Germanys } \\
\text { first offshore wind farm "alpha ventus", in the south-eastern parts of the North Sea, } 2015 \text { IEEE/OES Eleventh } \\
\text { Current, Waves and Turbulence Measurement (CWTM), } 2015 \text { [PDF document properties: datestamp: } \\
\text { 14/01/2015] } \\
\text {-PHOTO of damage to the } 15 \mathrm{~m} \text { working platform during Storm Xaver }\end{array}$ \\
\hline $\begin{array}{l}\text { Caithness Windfarm } \\
(20160105)\end{array}$ & $\begin{array}{l}\text { Caithness Windfarm, craigdr, Detailed accidents to } 31 \text { December 2015. Document time stamp 5Jan2016, 175pp } \\
\text {-Corkmore Wind Farm Donegal, Ireland: broken blade } \\
\text {-Northern Bavaria, Germany: blade torn off } \\
\text {-Doehlen, Oldenburg, Germany: nacelle torn off turbine; falls } 60 \mathrm{~m} \\
\text {-Vlatten Energy Park, Germany: wind turbine crashed in field } \\
\text {-Seascale School wind turbine, Cumbria, UK: blade shears off } \\
\text {-Burradale Wind Farm Shetland, UK: lightning caused fist-sized hole } \\
\text {-Guenter-Ehrhorn Weg in Dohren: wind turbin expladed } \\
\text {-Schaeskdorf, Lausitz, Germany: blade tip lost }\end{array}$ \\
\hline $\begin{array}{l}\text { Christakos et } \quad \text { al. } \\
(2016)\end{array}$ & $\begin{array}{l}\text { Christakos K, I Cheliotis, G Varlas, G-J Steeneveld, Offshore wind energy analysis of Cyclone Xaver over North } \\
\text { Europe, } 13 \text { th Deep Sea Offshore Wind R\&D Conference, EERA DeepWind'2016, 20-22 January } 2016 \text {, } \\
\text { Trondheim, Norway, Energy Procedia, } 94,37-44,2016 \text {. } \\
\text {-record wind energy production Germany from onshore \& offshore turbines leads to decrease spot prices } \\
\text {-Denmark turbines shut down because of extreme winds \& high power consumption leads to increase in spot } \\
\text { price. } \\
\text {-WRF modelling shows North Sea wind over } 25 \mathrm{~m} / \mathrm{s} \text { cut-off threshold for } 30 \mathrm{~h} / 84 \mathrm{~h} \text { of model integraton }\end{array}$ \\
\hline Wikipedia (20200502) & $\begin{array}{l}\text { Wikipedia, Orkan Xaver, https://de.wikipedia.org/wiki/Orkan_Xaver (accessed } 2 \text { May 2020) } \\
\text {-data from European Energy Exchange \& Fraunhofer Institute for Solar Energy Systems: } \\
\text { wind energy production Germany on 5Dec2013 new record value 26.3GW } \\
\text {-6Dec2013: } 563 \mathrm{GWh} \text { energy produced with average power 23.5GW }\end{array}$ \\
\hline
\end{tabular}

Table S38. Hydropower impacts (arranged by year and then alphabetically)

\begin{tabular}{|l|l|}
\hline Source & Full Reference and Notes \\
\hline Cheliotis et al (2016) & $\begin{array}{l}\text { Cheliotis, I, G. Varlas, K. Christakos, The impact of cylone Xaver on hydropower potential in Norway, } \\
\text { conference paper, September 2016 In: T Karaostas, A Bais, PT Nastos (ed), Perspectives on Atmospheric } \\
\text { Sciences, Springer Atmospheric Sciences, Springer, Cham, 2017 https://doi.org/10.1007/978-3-319-35095-0_25, } \\
\text { first online 10Sep2016 [pdf document properties: author=Konstantinos Christakos; datestamp: 07/09/2016] } \\
\text {-WRF model to quantify hydropower potential of rainfall }\end{array}$ \\
\hline Cheliotis et al (2017) & $\begin{array}{l}\text { Cheliotis, I, G. Varlas, K. Christakos, The impact of cylone Xaver on hydropower potential in Norway, } \\
\text { conference paper, September 2016 In: T Karaostas, A Bais, PT Nastos (ed), Perspectives on Atmospheric } \\
\text { Sciences, Springer Atmospheric Sciences, Springer, Cham, 2017, https://doi.org/10.1007/978-3-319-35095-0_25 } \\
\text {-WRF model to quantify hydropower potential during Storm Xaver } \\
\text {-Norwegian hydropower system represents 50\% of European reservoir capacity (Statkraft, 2009) }\end{array}$ \\
\hline
\end{tabular}

Table S39. Structural damage to buildings, piers, and cultural monuments (arranged by year and then alphabetically)

\begin{tabular}{|c|c|}
\hline Source & Full Reference and Notes \\
\hline BT (20131208) & $\begin{array}{l}\text { BT, Offer for Bodil: Koebte huset 15.august - nu er det vaek, 08Dec2013, 16:44, (contributor: Morten Eggert) } \\
\text { https://www.bt.dk/danmark/offer-for-bodil-koebte-huset-15.-august-nu-er-det-vaek } \\
\text {-summer house over cliff at Norlev Strand on Friday 06Dec2013 during Storm Bodil }\end{array}$ \\
\hline $\begin{array}{l}\text { Gccapitalideas } \\
(20131209)\end{array}$ & $\begin{array}{l}\text { gccapitalideas, Windstorm Xaver, 9Dec2013, https://www.gccapitalideas.com/2013/12/09/windstorm-xaver/ } \\
\text {-some reports of roof damage }\end{array}$ \\
\hline $\begin{array}{l}\text { National Wind Watch } \\
\text { (20131210a) }\end{array}$ & $\begin{array}{l}\text { National Wind Watch, 10Dec2013a, Xaver zerstoert ein Windrad bei Vlatten, original source: Aachener Zeitung, } \\
\text { 6Dec2013 https://www.wind-watch.org/news/2013/12/10/xaver-zerstort-ein-windrad-bei-vlatten/ } \\
\text {-damage to houses by falling trees and branches }\end{array}$ \\
\hline $\begin{array}{l}\text { Spiegel International } \\
(20131206)\end{array}$ & $\begin{array}{l}\text { Spiegel International, Winter storm 'Xaver' batters northern } \begin{array}{c}\text { Europe,6 } \\
\text { https://www.spiegel.de/international/europe/tidal-surge-winter-storm-xaver-batters-northern-europe-a- }\end{array} \\
\text { 937576.html\# } \\
\text { FIG5. In Rostock fire fighters secure parts of a roof blown off by } \\
\text { hurricane-force winds (DPA) }\end{array}$ \\
\hline $\begin{array}{l}\text { Sylter Rundschau } \\
(20131209)\end{array}$ & $\begin{array}{l}\text { Sylter Rundschau, Folgen des Orkans. Xaver 'knabberte' Sylt massiv an, 09Dec2013 06:00 (from Friederike } \\
\text { Reussner) https://www.shz.de/lokales/sylter-rundschau/xaver-knabberte-sylt-massiv-an-id5098481.html } \\
\text {-most of beach access stairs at Hoernumer Odde not usable } \\
\text {-ground floor damge to baeach restaurant Wonnemeyer the Wenningstedt }\end{array}$ \\
\hline $\begin{array}{l}\text { Unwetterzentrale } \\
(201312)\end{array}$ & $\begin{array}{l}\text { Unwetterzentrale, Orkantief XAVER - ein weiterer schwerer Wintersturm der letzten Jahrzehnte, Thomas Savert } \\
\text { and Stefan Laps, Dec. } 2013 \text { http://www.unwetterzentrale.de/uwz/928.html } \\
\text {-some roof damage } \\
\text {-Unterfeuer on southern part of Sylt destroyed }\end{array}$ \\
\hline Axer et al (2015) & $\begin{array}{l}\text { Axer T, T Bistry, M Klawa, M Mueller, M Suesser, Deutsche Ruck Sturm dokumentation } 2013 \text { Deutschland, } \\
\text { 2013, Deutsche Rueckversicherung Aktiengesellschaft, Hansaallee 177, 40549 Duesseldorf, } \\
\text { www.deutscherueck.de [pdf document information: author=filiz; date stamp=07Aug2015] } \\
\text {-numerous cases roof damage \& toppled trees north Germany } \\
\text {-Stralsund supermarket lost roof } 200000 \text { EUR } \\
\text {-clock Marienkirche damaged }\end{array}$ \\
\hline
\end{tabular}




\begin{tabular}{|l|l|}
\hline Sibley et al. (2015) & $\begin{array}{l}\text { Sibley A, D Cox, H Titley, Coastal flooding in England and Wales from Atlantic and North Sea storms during the } \\
\text { 2013/2014 winter, Weather, 70, 62-70, 2015. } \\
\text {-lifeboat station at Hemsby destroyed as coastal cliffs undermined }\end{array}$ \\
\hline Matelski (2016) & $\begin{array}{l}\text { Matelski, Birgit, Erfahrungen aus der Sturmflut Xaver von 5. und 6.12.2013 und dem Weihnachshochwasser 2014 } \\
\text { in Schleswig-Holstein, IWASA 2016 Tagungsbeitrag, (46. IWASA, 7-8 Januar 2016; Internationales } \\
\text { Wasserbau-Symposium Aachen. [pdf document properties: autor=sonja; datestamp: 26Apr2016] } \\
\text {-destroyed deckwork at Halligen }\end{array}$ \\
\hline Sorensen (2016) & $\begin{array}{l}\text { Sorensen CS, Water NOT wanted - Coastal floods and flooding protection in Denmark, In RA Herrmann \& J } \\
\text { Jensen (eds), Sicherung von Daemmen, Deichen und Stauanlagen: Handbuch fuer Theorie und Praxis (Vol V } \\
\text { pp3-21). Siegen: Universitaet Siegen, 2016 } \\
\text {-inner Danish seas: Xaver flooding caused houses to be uninhabitable for a long time } \\
\text {-inner Danish seas: medieval town centers 1m above the highest floods experienced }\end{array}$ \\
\hline Rucinska (2019) & $\begin{array}{l}\text { Rucinska D, Describing Storm Xaver in disaster terms, International Journal of Disaster Reduction, 34, 147-153, } \\
\text { 2019 } \\
\text {-Poland: Pomeranian Duke's Castle damaged }\end{array}$ \\
\hline Wikipedia (20191002) & $\begin{array}{l}\text { Wikipedia, Stormen Bodil, https://da.wikipedia.org/wiki/Stormen_Bodil\#cite_note-39 (accessed 02Oct2019) } \\
\text {-damage to Glasgow train station causes evacuation }\end{array}$ \\
\hline WIKI (20200124) & $\begin{array}{l}\text { WIKI, Cyclone Xaver, https://en.wikipedia.org/wiki/Cyclone_Xaver accessed 24Jan2020 } \\
\text {-Happisburgh, Hemsby; holiday homes Norlev strand Denmark undermined } \\
\text {-monument: 1634 stone Old Quay damaged at Whitehaven Cumbria } \\
\text {-monument: Blackpool North Pier severely damaged. } \\
\text {-monument: Cromer significant damage to pier }\end{array}$ \\
\hline Wikipedia (20200429) & $\begin{array}{l}\text { Wikipedia, Stormen Sven, https://sv.wikipedia.org/wiki/Stormen_Sven\#cite_note-15, accessed 29Apr2020 } \\
\text {-Landskrona kallbadhus destroyed by the storm }\end{array}$ \\
\hline
\end{tabular}

Table S40. Forest damage and tree falls (arranged by year and then alphabetically)

\begin{tabular}{|c|c|}
\hline Source & Full Reference and Notes \\
\hline BBC (20131205) & $\begin{array}{l}\text { BBC, In pictures: Winter storm hits UK, } 5 \text { December 2013, https://www.bbc.com/news/uk-scotland-25231224 } \\
\text {-FIG6. [PHOTO] Many roads have been closed across Scotland due to fallen trees (AP) }\end{array}$ \\
\hline $\begin{array}{l}\text { Gccapitalideas } \\
(20131209)\end{array}$ & $\begin{array}{l}\text { gccapitalideas, Windstorm Xaver, 9Dec2013, https://www.gccapitalideas.com/2013/12/09/windstorm-xaver/ } \\
\text {-UK: fallen trees interrupt transport networks }\end{array}$ \\
\hline GP (20131206) & $\begin{array}{l}\text { GP, Fortsatt risk for halka, 6Dec2013 (correspondent: D Henriksson, K Vikingsson, P Sydvik, TA Akerblom) } \\
\text { http://www.gp.se/nyheter/goteborg/1.2201325-fortsatt-risk-for-halka (accessed 1May2020) } \\
\text {-during night to Friday police received } 90 \text { reports of traffic blockages; mostly fallen trees }\end{array}$ \\
\hline $\begin{array}{ll}\text { Krissteligt } & \text { Dagblad } \\
(20131205) & \end{array}$ & $\begin{array}{l}\text { Kristeligt Dagblad, Stormen blaeser Skotland omkuld: Doodsfald og nebrud, (contributor: Ritzau), 05Dec2013 } \\
\text { 12:50 https://www.kristeligt-dagblad.dk/udland/stormen-bl\%C3\%A6ser-skotland-omkuld-d\%C3\%B8dsfald- } \\
\text { og-nedbrud } \\
\text {-trains in Scotland cancelled by trees fallen on rails }\end{array}$ \\
\hline Kunz et al (20131206) & $\begin{array}{l}\text { Kunz M, B Muehr, K Schroeter, T Bessel, S Moehrle, T Muenzberg, S Brink, H-M Schmidt, Winterstorm Xaver - } \\
\text { Report. 06Dec2013 - Report No.1, Situation Report - 19:00CET, CEDIM Forensic Disaster Analysis Group } \\
\text { (FDA), Center for Disaster Management and Risk Reduction Technology. } \\
\text {-trees and branches on roads and rail tracksin Mecklenburg-Vorpommern \& Schleswig-Holstein }\end{array}$ \\
\hline $\begin{array}{l}\text { National Wind Watch } \\
(20131210 \mathrm{~b})\end{array}$ & $\begin{array}{l}\text { National Wind Watch, Sturm 'Xaver': Windrad stuerzt auf Acker, 10Dec2013b, credit: von Manfred Reinnarth, } \\
\text { Koelnische Rundschau, 06/12/2013, https://www.wind-watch.org/news/2013/12/10/sturm-xaver-windrad- } \\
\text { sturzt-auf-acker/ } \\
\text {-reference to a few toppled trees in Kreis Euskirchen }\end{array}$ \\
\hline $\begin{array}{l}\text { Nordbayern } \\
(20131207)\end{array}$ & $\begin{array}{l}\text { Nordbayern, Deining: 'Xaver' reisst Rotorblatt von Windrad ab. Windkraftanlage schleuderte Eisbrocken auf die } \\
\text { Strasse, 07/12/2013, 12:07. https://www.nordbayern.de/region/neumarkt/deining-xaver-reisst-rotorblatt-von- } \\
\text { windrad-ab-1.3326492 } \\
\text {-a few trees blown over in northern Bavaria }\end{array}$ \\
\hline $\begin{array}{l}\text { Spiegel International } \\
(20131206)\end{array}$ & $\begin{array}{l}\text { Spiegel International, Winter storm 'Xaver' batters northern } \begin{array}{c}\text { Europe,6 } \\
\text { https://www.spiegel.de/international/europe/tidal-surge-winter-storm-xaver-batters-northern-europe-a- }\end{array} \\
\text { 937576.html\# } \\
\text { FIG12.An uptrooted tree in the village of Sankt Peter Ording on the North Sea } \\
\text { coast on Thursday (Reuters) }\end{array}$ \\
\hline The Local (20131205) & $\begin{array}{l}\text { The } \begin{array}{c}\text { Local, Sven's strong winds sweep southern Sweden. } \\
\text { https://www.thelocal.se/20131205/storm-sven-sweeps-into-southern-sweden }\end{array} \\
\text {-toppled trees cut power lines for trains }\end{array}$ \\
\hline $\begin{array}{l}\text { Unwetterzentrale } \\
(201312)\end{array}$ & $\begin{array}{l}\text { Unwetterzentrale, Orkantief XAVER - ein weiterer schwerer Wintersturm der letzten Jahrzehnte, Thomas Savert } \\
\text { and Stefan Laps, Dec. } 2013 \mathrm{http}: / / \text { www.unwetterzentrale.de/uwz/928.html } \\
\text {-vegetation damage; numerous trees down }\end{array}$ \\
\hline SMHI (20140703) & $\begin{array}{l}\text { SMHI, Simone, } \begin{array}{l}\text { Hilde, } \\
\text { https://www.smhi.se/kunskapsbanken/meteorologi/simone-hilde-sven-och-ivar-okt-dec-2013-1.76183 }\end{array} \\
\text {-0.8 million m3 lost timber }\end{array}$ \\
\hline Axer et al (2015) & $\begin{array}{l}\text { Axer T, T Bistry, M Klawa, M Mueller, M Suesser, Deutsche Ruck Sturm dokumentation } 2013 \text { Deutschland, } \\
\text { 2013, Deutsche Rueckversicherung Aktiengesellschaft, Hansaallee 177, 40549 Duesseldorf, } \\
\text { www.deutscherueck.de [pdf document information: author=filiz; date stamp=07Aug2015] } \\
\text { * -Mecklenburg-Vorpommern: more toppled trees in Xaver (4000 trees or 3mill EUR) compared with Schleswig- } \\
\text { Holstein ( } 80 \% \text { fewer) }\end{array}$ \\
\hline Wikipedia (20191002) & $\begin{array}{l}\text { Wikipedia, Stormen Bodil, https://da.wikipedia.org/wiki/Stormen_Bodil\#cite_note-39 (accessed 02Oct2019) } \\
\text {-Denmark: storm knocked down many trees in an area in Stenderup at Kolding } \\
\text {-Denmark: Bodil with Allan destroyed almost } 2500 \text { hectares of forest with over } 2 \text { million trees }\end{array}$ \\
\hline$(20200502$ & de.wikipedia.org/wiki/Orkan_Xaver (accessed 2 May 2020) \\
\hline
\end{tabular}


Table S41. General ship/rig emergency reports/offshore incidents/platform evacuations (arranged by year and then alphabetically)

\begin{tabular}{|c|c|}
\hline Source & Full Reference and Notes \\
\hline 24liveblog (2013) & $\begin{array}{l}\text { 24liveblog, Storm 5-6 december 2013, https://live.24liveblog.com/live/UYXaD } \\
\text {-cutter GO-20 grounded ; stowed to Stellendam arrival 0600M } \\
\text {-Burak Bayraktar engine problem 00:40M } \\
\text {-Balticborg cargo shift 09:10M } \\
\text {-Elka Athina motor problem 16:15M }\end{array}$ \\
\hline BBC (20131204) & $\begin{array}{l}\text { BBC, Workers off Talisman Sinopec oil platform Buchan Alpha over bad weather forecast, 04Dec2013 } \\
\text {-evacuation of Buchan Alpha } \sim 100 \mathrm{~km} \text { NE of Aberdeen from } 04 \text { Dec } 2013\end{array}$ \\
\hline BBC (20131206) & $\begin{array}{l}\text { BBC20131206, Deadly storm and tidal surge batter northern Europe, BBC } 6 \text { Dec2013 (report by Anna Holligan) } \\
-2 \text { sailors swept off ship in south Sweden }\end{array}$ \\
\hline Cargolaw (2013) & $\begin{array}{l}\text { Cargolaw } 2013 \text { [Tim Schwabedissen, 12Dec2013] } \\
\text {-Finnish Ferry Finnsky ( } 28002 \text { gt) stranded } \\
\text {-ship anchored offshore to ride out storm after leaving Lubeck Dec6 } \\
\text {-became caught in on anchor chain \& unable to proceed }\end{array}$ \\
\hline DW (20131205) & $\begin{array}{l}\text { DW, Storm Xaver pummels Europe, flood surge feared, 05/12/2013 } \\
\text {-production cut and staff evacuated from some platforms } \\
\text {-search for } 2 \text { sailors swept off ship off south coast of Sweden }\end{array}$ \\
\hline $\begin{array}{l}\text { Energy } \\
(20131204)\end{array}$ & $\begin{array}{l}\text { Energy Voice, Oil staff withdrawn from North Sea ahead of storm conditions, 04/12/2013 10:30AM, } \\
\text { https://www.energyvoice.com/other-news/healthandsafety/50140/oil-staff-withdrawn-north-sea-ahead-storm- } \\
\text { conditions/ } \\
\text {-evacuation of Buchan Alpha FPSO (Talisman Sinopec), Ekofisk (ConocoPhillips), Valhall (BP) } \\
\text {-no effect on production during preparations. }\end{array}$ \\
\hline Expressen (2013) & $\begin{array}{l}\text { Expressen, Flera doda i Sverige efter stormen Sven, 06Dec2013, 17:34CET? } \\
\text {-Thursday morning } 2 \text { sailors swept off Dutch cargo vessel Marietje Andrea off Ystad; search called off after } 6 \mathrm{~h}\end{array}$ \\
\hline Fleetmon (20131207) & $\begin{array}{l}\text { Fleetmon, Container ship Burak Bayraktar in trouble off Texel, Netherlands, (correspondent: Mikhail Voytenko, } \\
\text { 7Dec2013 06:31) https://www.fleetmon.com/maritime-news/2013/2810/container-ship-burak-bayraktar-trouble- } \\
\text { texel-nethe/ } \\
\text {-early morning 06Dec2013 container ship reported engine trouble to Dutch coast guard } \\
\text {-ship had to anchor to avoid grounding; anchor dragged } \\
\text {-latest reports: vessel is stable } 4 \mathrm{~nm} \text { W of Texel }\end{array}$ \\
\hline Fleetmon (20131208) & $\begin{array}{ccccccc}\text { Fleetmon, Burak } & \text { Bayraktar safe, } & \text { Netherlands, } & \text { (Mikhail } & \text { Voytenko, Dec08, } & 2013 & 05: 07) \\
\text { https://www.fleetmon.com/maritime-news/2013/2816/burak-bayraktar-safe-netherlands/ } & & \\
\text {-ship taken in tow by Tug Zeus away from coast; repaired by crew underway } & & & \end{array}$ \\
\hline $\begin{array}{l}\text { Gandreassen } \\
(20131204)\end{array}$ & $\begin{array}{l}\text { Gandreassen, Oil workers moved from Ekofisk, 4Dec2013. https://gandreassen.com/oil-workers-moved-from-ekofisk/ } \\
\text {-ConocoPhillips transferred } 157 \text { workers from Ekofisk } 1 \text { day in advance of the storm; not an evacuation } \\
\text {-BP is considering moving people from the field. }\end{array}$ \\
\hline $\begin{array}{l}\text { Gcapitalideas } \\
\text { (20131209) }\end{array}$ & $\begin{array}{l}\text { gccapitalideas, Windstorm Xaver, 9Dec2013, https://www.gccapitalideas.com/2013/12/09/windstorm-xaver/ } \\
\text {-'North Sea oil and gas producers inluding ConocoPhillips, Maersk Oil and Statoil cut production and evacuated staff } \\
\text { from some platforms' }\end{array}$ \\
\hline GVA (20131205) & $\begin{array}{l}\text { GVA, Vijf containers even op drift op de Schelde, 05/12/2013 20:57. https://www.gva.be/cnt/aid1500792/vijf- } \\
\text { containers-op-drift-op-de-schelde- } 2 \\
-4 \text { empty containers and } 1 \text { container with tapioca fallen from ship in Westerscheldt } 2000 \mathrm{MET} \\
\text {-Ladybug ship adrift \& hit Deurganckdok at } 1800 \mathrm{MET} \text {; recovered by two tug boats }\end{array}$ \\
\hline HBVL (20131205) & $\begin{array}{l}\text { HBVL, Vijf containers even op drift op de Schelde, 05Dec2013 20:57, https://www.hbvl.be/cnt/aid1500792/vijf- } \\
\quad \text { containers-op-drift-op-de-schelde-2 } \\
\text {-ship traffice between Deurganckdok and Vlissingen stopped by } 5 \text { drifting containers than fell from ship 05Dec2013 } \\
\text { 20:00 } \\
\text {-C Ladybug adrift 05Dec2013 18:00; recovered by } 2 \text { tugboats }\end{array}$ \\
\hline Reuters (20131205) & 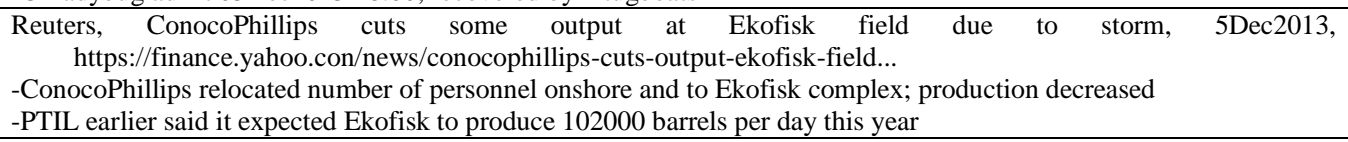 \\
\hline The Local (20131205) & $\begin{array}{ccccc}\text { The Local, Sven's strong winds sweep southern Sweden. } & \text { 05Dec2013 } & \text { 15:57CET } \\
\text { https://www.thelocal.se/20131205/storm-sven-sweeps-into-southern-sweden } & & \\
\text {-2 sailors fall off Dutch ship off Ystad } & & \\
\end{array}$ \\
\hline Upstream (20131204) & $\begin{array}{l}\text { Upstream, Talisman takes workers off Buchan Alpha, 4Dec2013 1710GMT (correspondent: Rob Watts) } \\
\text {-evacuation of Talisman Buchan Alpha pentagonal platform in northern North Sea in advance of storm. }\end{array}$ \\
\hline Upstream (20131205) & $\begin{array}{l}\text { Upstream, North Sea production curtailed over storm, 05Dec2013 (contributor: Bill Lehane and News Wires) } \\
\text {-producton reduced at } 2 \text { North Sea oil platforms as explorers continue to de-man installations } \\
\text {-waves up to } 10 \mathrm{~m} \text { expected before storm subsides late Thursday }\end{array}$ \\
\hline Upstream (20131206) & $\begin{array}{l}\text { Upstream, Buchan Alpha evacuated as North Sea storms loom, 6Dec2013 0000GMT (contributor Rob Watts) } \\
\text {-Talisman Sinopec Energy UK evac all workers off Buchan Alpha in UK Nsea ahead of severe storms }\end{array}$ \\
\hline Upstream (20131209) & $\begin{array}{l}\text { Upstream, Workers return to Buchan A, 09Dec2013 12:58GMT (contributor: Rob Watts) } \\
\text {-workers started returning to FPSO 08Dec2013 Sunday }\end{array}$ \\
\hline Axer et al (2015) & $\begin{array}{l}\text { Axer T, T Bistry, M Klawa, M Mueller, M Suesser, Deutsche Ruck Sturm dokumentation } 2013 \text { Deutschland, 2013, } \\
\text { Deutsche Rueckversicherung Aktiengesellschaft, Hansaallee 177, } 40549 \text { Duesseldorf, www.deutscherueck.de } \\
\text { [pdf document information: author=filiz; date stamp=07Aug2015] } \\
\text {-Finnlines freighter }(190 \mathrm{~m}) \text { headed from Warnemunde to Finnland; had to anchor for several days; trucks in ship }\end{array}$ \\
\hline Wadey et al (2015a) & $\begin{array}{l}\text { Wadey MP, ID Haigh, RJ Nichols, JM Brown, K Horsburgh, B Carroll, SL Gallop, T Mason, E Bradshaw, A } \\
\text { comparison of the } 31 \text { January-1 February } 1953 \text { and 5-6 December } 2013 \text { coastal flood events around the UK, UK }\end{array}$ \\
\hline
\end{tabular}




\begin{tabular}{|l|l|}
\hline & $\begin{array}{l}\text { Frontiers in Marine Science, 2, 84, 2015a. } \\
\text {-no maritime disasters for Storm Xaver as for 1953 storm }\end{array}$ \\
\hline Wadey et al (2015b) & $\begin{array}{c}\text { Wadey MP, JM Brown, ID Haigh, T Dolphin, P Wisse, Assessment and comparison of extreme sea levels and waves } \\
\text { during the 2013/2014 storm season in two UK coastal regions, Nat. Hazards Earth Syst. Sci. Discuss., 3, 2665- } \\
\text { 2708, 2015b. }\end{array}$ \\
& -Princess Victoria sank in Irish Sea during 1953 storm \\
\hline Wikipedia (20191002) & $\begin{array}{l}\text { Wikipedia, Stormen Bodil, https://da.wikipedia.org/wiki/Stormen_Bodil\#cite_note-39 (accessed 02Oct2019) } \\
\text {-British oil platform NE of Aberdeen evacuated 4Dec before storm }\end{array}$ \\
\hline WIKI (20200124) & $\begin{array}{l}\text { WIKI, Cyclone Xaver, https://en.wikipedia.org/wiki/Cyclone_Xaver accessed 24Jan2020 } \\
\text {-Buchan Alpha evacuated }\end{array}$ \\
\hline Wikipedia (20200429) & $\begin{array}{l}\text { Wikipedia, Stormen Sven, https://sv.wikipedia.org/wiki/Stormen_Sven\#cite_note-15, accessed 29Apr2020 } \\
\text {-2 men fell overboard from a freighter off Ystad and died }\end{array}$ \\
\hline Wikipedia (20200502) & $\begin{array}{l}\text { Wikipedia, Orkan Xaver, https://de.wikipedia.org/wiki/Orkan_Xaver (accessed 2 May 2020) } \\
\text {-2 sailors swept from ship off coast of Sweden; search unsuccessful }\end{array}$ \\
\hline
\end{tabular}

Table S42. Instrument failures during storm (arranged by year and then alphabetically)

\begin{tabular}{|c|c|}
\hline Source & Full Reference and Notes \\
\hline $\begin{array}{l}\text { Deutschlander et al } \\
(2013)\end{array}$ & $\begin{array}{l}\text { Deutschlander T, K Frierich, S Haeseler, C Lefebvre, Orkantief XAVER ueber Nordeuropea von } 5 \text {. bis } 7 . \\
\text { Dezember 2013, Deutscher Wetterdienst DWD, Stand 30. Dezember 2013, 19pp. } \\
\text {-partial malfunction of weather station at Hamburg St. Pauli; 5Dec2013 09:45-12:00UTC }\end{array}$ \\
\hline BSH (2013) & $\begin{array}{l}\text { BSH, Die Nordseesturmfluten von 5. und 6.12.2013, 3pp, prepared by Stockmann,K. (title: Sturmflut } \\
\text { 2011_bm1101; author stamp: bm1101; document time stamp: 12/102013 12:5136PM) } \\
\text {-Dagebuell tide gauge failed during evening high tide 6 Dec } 2013\end{array}$ \\
\hline $\begin{array}{l}\text { Deutschlander et al } \\
(2013)\end{array}$ & $\begin{array}{l}\text { Deutschlander T, K Frierich, S Haeseler, C Lefebvre, Orkantief XAVER ueber Nordeuropea von 5. bis } 7 . \\
\text { Dezember 2013, Deutscher Wetterdienst DWD, Stand 30. Dezember 2013, 19pp. } \\
\text {-partial malfunction of weather station at Hamburg St. Pauli; 5Dec2013 09:45-12:00UTC }\end{array}$ \\
\hline $\begin{array}{l}\text { McGarricle et al } \\
(2013)\end{array}$ & $\begin{array}{l}\text { McGarricle P (ed), UK coastal monitoring and forecasting: Annual report for } 2013 \text { for the UK National Tide } \\
\text { Gauge Network, NERC 100017897, } 2013 \text { [pdf document properties: author=pamcg; created=16Apr2014] } \\
\text {-Aberdeen: no record surge max? } \\
\text {-Cromer: no record surge max or water level max? } \\
\text {-Harwich: no record surge max or water level max? } \\
\text {-Sheerness: primary instrument offline; secondary instrument functional but no data presented } \\
\text {-Wick: no surge max }\end{array}$ \\
\hline $\begin{array}{l}\text { Oceanografisch } \\
\text { Meteorologisch } \\
\text { Station (2013) }\end{array}$ & $\begin{array}{l}\text { Oceanografisch Meteorologisch Station, Stormverslag 05-06 december 2013, 26pp, } 2013 \text { [pdf document } \\
\text { properties: author=Myriam Sys; datestamp=15Dec2013] } \\
\text {-large data gaps in Westhinder waverider series } \\
\text {-gap in Bol van Heist wave period at 05Dec2013 1300UTC }\end{array}$ \\
\hline SMHI (20131012) & $\begin{array}{l}\text { SMHI, Stormen Sven gav nya vattenstandsrekord i Oresund, https://www.smhi.se/nyhetsarkiv/stormen-sven-gav- } \\
\text { nya-vattenstandsrekord-i-oresund-1.34732, updated 20Mar2017; original datestamp 10Dec2013. } \\
\text {-tide gauge failure at Viken Sweden on 06Dec2013 14:00UTC }\end{array}$ \\
\hline ABPmer (2014) & $\begin{array}{l}\text { ABPmer, Ensuring Flood Resilience. An overview of the 5/6 December 2013, Associated British Ports, Marine } \\
\text { Environmental Research, July, } 2014 \\
\text {-Immingham tide gauge non-operational on } 06 \operatorname{Dec} 2013\end{array}$ \\
\hline Eriksen (2014) & $\begin{array}{l}\text { Eriksen J, Rekordvandstande i Isefjorden og Roskilde Fjord, Vejret, 138, 2 40-48, } 2014 \\
\text {-instrument irregularity Holbaek 7Dec2013 } \\
\text {-small decrease in water level graph of Holbaek 06:45CET 6Dec2013 }\end{array}$ \\
\hline Gautier et al (2014) & $\begin{array}{l}\text { Gautier C, A Camarena, J van Nieuwkoop, SWAN hindcasts Wadden Sea, December 2013. Tidal inlet of } \\
\text { Ameland and eastern Wadden Sea. Deltares, 2014, 197pp. Project 1209433-007, Reference 1209433-007- } \\
\text { HYE-0005 } \\
-5 \text { of } 32 \text { wave recorders had not data during storm Xaver } \\
\text {-data gaps in wind speed reports }\end{array}$ \\
\hline $\begin{array}{l}\text { Luecht and Peters } \\
(2014)\end{array}$ & $\begin{array}{l}\text { Luecht, Fabian and Ove Peters, Bericht ueber die Sturmflut vom 05.-0.6.12.2013 an der Westkueste Schleswig- } \\
\text { Holsteins, Landesbetrieb fuer Kuestenschutz, Nationalpark und Meeresschutz Schleswig-Holstein, Husum } \\
\text { 26Feb2014, 19pp. [pdf document properties: title=Lfd; Author=Thorsten Nommensen; datestamp: } \\
\text { 11Dec2018] } \\
\text {-no data for Dagebuell tide gauge during main storm tide }\end{array}$ \\
\hline RWS (2014b) & $\begin{array}{l}\text { RWS, Stormvloedrapport van } 5 \mathrm{t} / \mathrm{m} 7 \text { december (SR91) Sint-Nicolaasvloed 2013, Watermanagementcentrum } \\
\text { Nederland, Rijkswaterstaat, prepared by Ing. J. Kroos, } 19 \text { Mar 2014b, } 48 \text { pp } \\
\text {-Huibertgat anemometer not functional } \\
\text {-no data from Schiermonnikoog }\end{array}$ \\
\hline Leiding et al (2014) & $\begin{array}{l}\text { Leiding T, B Tinz, G Rosenhagen, C Lefevre, S Haeseler, S Hagemann, I Bastigkeit, D Stein, P Schwenk, S } \\
\text { Mueller, O Outzen, K Herklotz, F Kinder, T Neumann, Meteorological and Oceanographic Conditions at the } \\
\text { FINO platforms during the severe storms Christian and Xaver, DEWI Magazin, No.44, p16-25, 2014. } \\
\text {-data gaps in Hs and Hmax record for FINO1 FINO3 (6Dec2013 00:00-06:00 and 14:00-15:00) and FINO3 } \\
\text { (6Dec2013 05:00-06:00); unknown instrument }\end{array}$ \\
\hline $\begin{array}{l}\text { MIROS_ekofisk } \\
\text { (2014) }\end{array}$ & $\begin{array}{l}\text { MIROS, Monthly report, Ekofisk, December 2013, Doc. No. ND/1024/13/12, (prepared by SRS) 34pp, 9Jan2014 } \\
\text { [PDF document properties: author=Miros AS; datestamp=13Jan2014] } \\
\text {-low data capture rates epecially for th wave recorders }\end{array}$ \\
\hline $\begin{array}{l}\text { MIROS_heidrun } \\
\text { (2014) }\end{array}$ & $\begin{array}{l}\text { MIROS, Manedsrapport Heidrun, Desember 2013, Dok. Nr. ND/1010/13/12, } 21 \text { pp, 07Jan2014, carried out by } \\
\text { SRS, controlled by CNE, approved by OO [pdf properties: author=Miros AS; date stamp: 08/01/2014] } \\
\text {-data gaps in water level data during Storm Xaver }\end{array}$ \\
\hline $\begin{array}{l}\text { MIROS_heimdal } \\
\text { (2014) }\end{array}$ & $\begin{array}{l}\text { MIROS, Manedsrapport Heimdal, Desember 2013, Dok. Nr. ND/1047/13/12 (carried out be SRS, controlled by } \\
\text { CNE, approved by OO) [PDF document properties: author=Miros AS; datestamp=06Jan2014] }\end{array}$ \\
\hline
\end{tabular}




\begin{tabular}{|c|c|}
\hline & -all wave data missing above $\mathrm{Hs}=5 \mathrm{~m}$; almost no information during Storm Xave period \\
\hline MIROS_norne (2014) & $\begin{array}{l}\text { MIROS, Maanedsrapport Norne, Desember 2013, Dok. Nr. ND/1087/13/12, 21pp, carried out by SRS, controlled } \\
\text { by CNE, approved by OO [pdf properties: Author=Miros AS; datestamp: 06Jan2014] } \\
\text {-no data from waverider buoy during Dec } 2013\end{array}$ \\
\hline $\begin{array}{l}\text { MIROS_sleipner } \\
\text { (2014) }\end{array}$ & $\begin{array}{l}\text { MIROS, Manedsrapport Sleipner A, Desember 2013, Dok. Nr. ND/1017/13/12, 06Jan2014, prepared by SRS, } \\
\text { controlled by CNE, approved by OO. } \\
\text {-gaps in water level and wave data during Storm Xaver }\end{array}$ \\
\hline $\begin{array}{l}\text { Fenoglio-Marc et al } \\
(2015)\end{array}$ & $\begin{array}{l}\text { Fenoglio-Marc L, R Scharroo, A Annuziato, L Mendoza, M Becker, J Lillibridge, Cyclone Xaver seen by } \\
\text { geodetic observations, Geophys Research Letters, 42, 9925-9932, 2015 } \\
\text {-excessive scatter at sites: Emden, Wilhelmsahaven, Hoernum Sylt Island, Cuxhaven, Dagebuell }\end{array}$ \\
\hline Fischer et al. (2015) & $\begin{array}{l}\text { Fischer, JG, C Senet, A Schneehorst, O Outzen, S Schirmel, K Herklotz, Sea state measurements in Germanys } \\
\text { first offshore wind farm "alpha ventus", in the south-eastern parts of the North Sea, } 2015 \text { IEEE/OES Eleventh } \\
\text { Current, Waves and Turbulence Measurement (CWTM), } 2015 \text { [PDF document properties: datestamp: } \\
\text { 14/01/2015] } \\
\text {-gaps in RADAC Hs dara set near peak of storm Xaver }\end{array}$ \\
\hline Matelski et al (2015) & $\begin{array}{l}\text { Matelski, Birgit, Frerk Jensen, Peter Voss, Jorg Peters, Maria Blumel, Auswertung zur Hydrologie des Sturmtiefs } \\
\text { Xaver 05.12.2013 bis 06.12.2013, Statusbericht vom 01.10.2014, Landesbetrieb fuer Kuestenschutz, } \\
\text { Nationalpark und Meeresschutz, Schleswig-Holstein, Husum, 01.10.2014, 25pp. } \\
\text {-tabulated tide gauge station data indicates some instruments failures dduring the storm }\end{array}$ \\
\hline Spencer et al (2015) & $\begin{array}{l}\text { Spencer T, SM Brooks, BR Evans, JA Tempest, I Moeller, Southern North Sea storm surge event of Dec.5, 2013: } \\
\text { Water levels, waves, and coastal impacts, Earth Science Reviews, 146, 120-145, 2015 } \\
\text {-something wrong with water level record record at Wells next the Sea to prevent it from being matched to } \\
\text { astronomical tide model } \\
\text {-'surge associated wave action beneath Cromer Pier made the water level record at this location unusable for } \\
\text { subsequent analysis' } \\
\text {-'Sheerness tide gauge was offline at the time of the surge' } \\
\text {-Immingham figure shows tide gauge went down from afternoon } 6 \mathrm{Dec} 2013 \\
\text {-FIG7. time series of water levels and significant wave heights at Norfolk/Lincolnshire and Suffolk coast show } \\
\text { data gaps }\end{array}$ \\
\hline Brooks et al (2016) & $\begin{array}{l}\text { Brooks SM, T Spencer, A McIvor, I Moller, Reconstructing and understanding the impacts of storms and surges, } \\
\text { southern North Sea, Earth Surface Processes and Landforms, 41, 855-864, } 2016 . \\
\text {-problems with Cromer tide gauge due to wave effects at pier }\end{array}$ \\
\hline
\end{tabular}

Table S43. Model results and fields (arranged by year and then alphabetically)

\begin{tabular}{|c|c|}
\hline Source & Full Reference and Notes \\
\hline Cipollini et al (2014) & $\begin{array}{l}\text { Cipollini P, LJ West, HM Snaith, P Harwood, C Donlon, New altimetry products over shelf and coastal zone from } \\
\text { the eSurge processor, poster presentation, } 2014 \text { [document time stamp: 2014/10/22] } \\
\text {-DMI storm surge model to support Cryosat-2 altimeter strip map }\end{array}$ \\
\hline $\begin{array}{l}\text { Deutschlander et al } \\
(2013)\end{array}$ & $\begin{array}{l}\text { Deutschlander T, K Frierich, S Haeseler, C Lefebvre, Orkantief XAVER ueber Nordeuropea von } 5 . \text { bis } 7 . \\
\text { Dezember 2013, Deutscher Wetterdienst DWD, Stand 30. Dezember 2013, 19pp. }\end{array}$ \\
\hline $\begin{array}{l}\text { Gccapitalideas } \\
(20131209)\end{array}$ & $\begin{array}{l}\text { gccapitalideas, Windstorm Xaver, 9Dec2013, https://www.gccapitalideas.com/2013/12/09/windstorm-xaver/ } \\
\text {-map of gusts during storm }\end{array}$ \\
\hline KNMI (2013) & $\begin{array}{l}\text { KNMI, News report. De Zware storm van } 5 \text { december, } 06 \text { Dec 2013, https://www.knmi.nl/over-het- } \\
\text { knmi/nieuws/de-Zware-storm-van-5-december } \\
\text { FIG1. [MAP] Surface pressure model field } \sim 06 \text { Dec } 2013 \text {. In } 24 \mathrm{~h} \text { from } 4-5 \text { Dec } 2013 \\
\text { the central pressure deepened by } 41 \mathrm{hPa} \text {. The criterion for rapid cyclogenesis } \\
\text { is } 24 \mathrm{hPa} \text { in } 24 \mathrm{~h} \\
\text { FIG2. [MAP] Precipitation warning for } 5 \text { Dec } 20131700 \text { along gust line front over } \\
\text { De Bilt } \\
\text { FIG3. [MAP] showing large expanse of gust line }\end{array}$ \\
\hline Kunz et al (2013) & $\begin{array}{l}\text { Kunz M, B Muehr, K Schroeter, T Bessel, S Moehrle, T Muenzberg, S Brink, H-M Schmidt, Winterstorm Xaver - } \\
\text { Report. 06Dec2013 - Report No.1, Situation Report - 19:00CET, CEDIM Forensic Disaster Analysis Group } \\
\text { (FDA), Center for Disaster Management and Risk Reduction Technology. } \\
\text {-FIG1. Analysis of surface pressure and gust wind speed from Global Forecast System (GFS) } \\
\text { on 5Dec2013 07:00CET, 19:00 and 06Dec2013 07:00CET }\end{array}$ \\
\hline $\begin{array}{l}\text { Unwetterzentrale } \\
(201312)\end{array}$ & $\begin{array}{l}\text { Unwetterzentrale, Orkantief XAVER - ein weiterer schwerer Wintersturm der letzten Jahrzehnte, Thomas Savert } \\
\text { and Stefan Laps, Dec. } 2013 \mathrm{http} / / / \text { www.unwetterzentrale.de/uwz/928.html } \\
\text {-FIG4. [MAP] Animation of 10m model wspd together with surface pressure development from } \\
\text { Wednesday 04Dec2013 1900MEZ to Saturday 07Dec2013 1000MEZ } \\
\text { FIG8. [MAP] Forecast of pressure on Friday 29Nov2013 for 06Dec2013, 1900MEZ (GFS model) } \\
\text { FIG9. [MAP] Gust forecast of the European weather model from Mon 2Dec2013 for 5Dec 1900MEZ \& } \\
\text { 06Dec0000MEZ } \\
\text { FIG10.[MAP] Forecast of pressure division from 3Dec2013 for 5Dec2013 1900MEZ (ECMWF) } \\
\text { FIG11.[MAP] Prediction of peak gusts from the ECMWF model from 4Dec2013 0100MEZ for 5Dec2013 1300- } \\
\text { 1900MEZ } \\
\text { FIG13. Animation of } 850 \mathrm{hPa} \text { model average wspd (1500m) from 5Dec2013 0100MEZ to 7Dec2013 1900MEZ }\end{array}$ \\
\hline Eriksen (2013) & $\begin{array}{l}\text { Eriksen J, Rekordvandstande i Isefjorden og Roskilde Fjord, Vejret, 138, } 2 \text { 40-48, } 2014 \\
\text {-FIG14.[MAP] A GFS reanalysis for 6Dec2013 kl06UTC. The white line gives isobars. } \\
\text { A high pressure center lies west of British isles \& a power low P centre off } \\
\text { Sweden's east coast. }\end{array}$ \\
\hline Gautier et al (2014) & $\begin{array}{l}\text { Gautier C, A Camarena, J van Nieuwkoop, SWAN hindcasts Wadden Sea, December 2013. Tidal inlet of } \\
\text { Ameland and eastern Wadden Sea. Deltares, 2014, 197pp. Project 1209433-007, Reference 1209433-007- } \\
\text { HYE-0005 }\end{array}$ \\
\hline
\end{tabular}




\begin{tabular}{|c|c|}
\hline & -SWAN model results for Wadden Sea \\
\hline Hewson et al (2014) & $\begin{array}{l}\text { Hewson T, L Magnusson, O Breivik, F Prates, I Tsonevsky, HJW de Vries, Windstorms in northwest Europe in } \\
\text { late 2013, ECMWF Newsletter, No 139, pp 22-28, Spring } 2014 \text {. } \\
\text {-FIG5. Forecasts of 24h maximum wind between 00 and 24 UTC on 5Dec with } \\
\text { mean sea level pressure for 12UTC on 5Dec from data times of } \\
\text { (a) 00UTC on 3Dec and (b) 00UTC on 5Dec2013. } \\
\text { Crosses denote the remnant of a meso-vortex discusses in the text. } \\
\text { Panel (c) shows verifying data from observations. } \\
\text {-FIG6. Maximum gust forecasts from ENS represented as the EFI and SOT } \\
\text { for 00 to } 24 \text { UTC on 5Dec2013fro the data times (a) 00UTC on 30Nov, } \\
\text { (b) 00UTC on 2Dec and (c) 00UTC on 4Dec. } \\
\text { Panel (d) shows, for the same 24h period, maximum wind gust CDF's } \\
\text { for Torsminde in NW Denmark from } 14 \text { ENS runs. M-Clim (black line) } \\
\text { is the model climate }\end{array}$ \\
\hline $\begin{array}{l}\text { Knaack and Heyken } \\
(2014)\end{array}$ & $\begin{array}{l}\text { Knaack H and H Heyken, Xaver hatte sehr schwere Stumflut im Gepaeck, Jahresbericht 2013. Der Zukunft } \\
\text { verplichtet, NLWKN, Niedersaechsischer Landesbetrieb fuer Waserwirtschaft, Kuesten- und Naturschutz, } \\
\text { pp.8-9, document date stamp 24Apr2014. } \\
\text { FIG. [MAP] Differences between Hurricane Christian and Hurricane } \\
\text { Xaver in 2013. Christian came with strong winds from } \\
\text { the southwest along the coast. Xaver had winds from northwest } \\
\text { across the total length of the North Sea (modelled wind speeds over North Sea and northern Germany) }\end{array}$ \\
\hline Nossent et al (2014) & $\begin{array}{l}\text { Nossent J, L Boeckx, E Taverniers, M Deschamps, T Verwaest, F Mostaert, Sinterklaasstorm } 6 \text { december } 2013 . \\
\text { Beschrijving van de hydrometrische gebeurtenissen, Versie 4.0. WL Rapporten, 00-119, Waterbouwkundig } \\
\text { Laboratorium, Antwerpen, Belgie. October 2014, WL2014R00_119_5 [document date stamp: 15Oct2014; } \\
\text { author= Deschamps, Maarten] } \\
\text { * -FIG5. [TIMESERIES] Wind speed time series from Terneuzen; wspd max 2h later than expected } \\
\text { * -FIG6. [TIMESERIES] Wind speed time series from Hansweert; wspd max 2h later than expected } \\
\text {-FIG7. [TIMESERIES] Wind direction time series from Terneuzen } \\
\text {-FIG8. [TIMESERIES] Wind direction time series from Hansweert } \\
\text {-FIG9. [MAP] weather map 06Dec2015 0600UTC } \\
\text {-FIG10.[MAP] Model-derived wind fields at 06Dec2013 0100/0700/1300CET }\end{array}$ \\
\hline Pelt (2014) & $\begin{array}{l}\text { Pelt AS, BODIL's stormflod i de indre dansk farvande, Vejret, } 138,24-29,2014 \\
\text {-FIG6. Vejrkort fra 6Dec2013 00UTC from [8] showing height of the 500hPa surface } \\
\text { in dm, together with the surface pressure in hPa with 5hPa intervals. } \\
\text { Bodil sends a powerful windfield over Denmark from the NW which leads to } \\
\text { high water levels. }\end{array}$ \\
\hline RWS (2014a) & 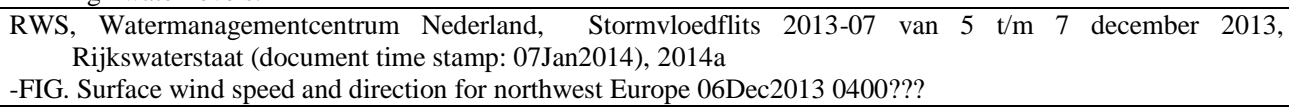 \\
\hline RWS (2014b) & $\begin{array}{l}\text { RWS, Stormvloedrapport van } 5 \mathrm{t} / \mathrm{m} 7 \text { december (SR91) Sint-Nicolaasvloed } 2013 \text {, Watermanagementcentrum } \\
\text { Nederland, Rijkswaterstaat, prepared by Ing. J. Kroos, } 19 \text { Mar 2014b, } 48 \text { pp } \\
\text { FIG_A4. [MAP] North Sea wind speed, direction, surface atmospheric pressure: } \\
\text { 05Dec 0600UTC, 05Dec 1200UTC, 05Dec 1800UTC, 06Dec 0000UTC, } \\
\text { 06Dec 0600UTC, 06Dec 1200UTC, 06Dec 1800UTC, 07Dec 0000UTC }\end{array}$ \\
\hline SMHI (20140703) & 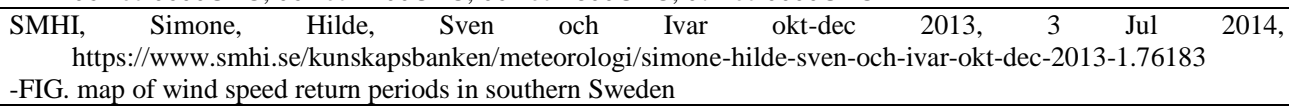 \\
\hline Stanev et al (2014) & $\begin{array}{l}\text { Stanev E, J Staneva, S Grayek, J Schulz-Stellenfleth, S. Grashorn, A Behrens, Numerical modelling and data } \\
\text { assimilation, COSYNA Progress Report 2013, pp.46-51, Helmholtz-Zentrum Geesthacht, June } 2014 . \\
\text {-FIG5. Sea surface elevation (SLE) difference between coupled wave circulation } \\
\text { model (WAM-GETM) and circulation model only (GETM) for the German Bight } \\
\text { on 3Dec2013 (top) and during Storm Xaver on 6Dec2013 (bottom). }\end{array}$ \\
\hline Staneva et al (2014) & $\begin{array}{l}\text { Staneva J, K Wahle, E Stanev, Response of the German Bight Hydro and Sediment Dynamics to Wave, Tidal and } \\
\text { Atmospheric Forcing, 3rd GODAE OceanView Coastal Oceans and Shelf Seas Task Team (COSS-TT) } \\
\text { International Coordination Workshop, 21-24 January 2014, Rncon Beach Resort, Puerto Rico, 36pp } \\
\text {-FIG. [MAP] DWD weather map showing clouds and peak gusts } \\
\text {-FIG. [TIMESERIES] Time series of tide gauge data for } 4 \text { location German Bight 3-9Dec2013 } \\
\text {-FIG. [TIMESERIES] modelled signficant wave height German Bight 3-9Dec2013 }\end{array}$ \\
\hline Axer et al (2015) & $\begin{array}{l}\text { Axer T, T Bistry, M Klawa, M Mueller, M Suesser, Deutsche Ruck Sturm dokumentation } 2013 \text { Deutschland, } \\
\text { 2013, Deutsche Rueckversicherung Aktiengesellschaft, Hansaallee 177, 40549 } \\
\text { www.deutscherueck.de [pdf document information: author=filiz; date stamp=07Aug2015] } \\
\text {-FIG_p51. [MAP] hurricane gust field Hurricane Xaver 05/12/2013 }\end{array}$ \\
\hline Cipollini et al (2015) & $\begin{array}{l}\text { Cipollini P, J Benveniste, H Bonekamp, L Miller, M Picot, P Ted Strub, D Vandemark, S Vignudelli, Recovering } \\
\text { more and better data from altimetry in the coastal zone: a community effort, Pilot ARCOM workshop Lisbon, } \\
\text { 1/2 Sept } 2015 \\
\text {-FIG. [MAP] DMI model of storm Xaver sea level Friday 06Dec2013 23:05 (Madsen etal) }\end{array}$ \\
\hline $\begin{array}{l}\text { Fenoglio-Marc et al } \\
(2015)\end{array}$ & $\begin{array}{l}\text { Fenoglio-Marc L, R Scharroo, A Annuziato, L Mendoza, M Becker, J Lillibridge, Cyclone Xaver seen by } \\
\text { geodetic observations, Geophys Research Letters, 42, 9925-9932, } 2015 \\
\text {-[MAP] North Sea storm surge elevations for BSHmod and HyFlux2 }\end{array}$ \\
\hline NLWKN (20151210) & $\begin{array}{l}\text { NLWKN20151210, Sturmflutwarndienst der Betriebstelle Norden-Norderney, NLWKN Niedersachsischer } \\
\text { Landesbetrieb fuer Wasserwirtschaft, Kuesten- und Naturschutz, Niedersachsen, document date stamp } \\
\text { 10Dec2015. } \\
\text {-[MAP] modelled forecast wspd \& wdir for 06Dec2013 0100MEZ (12h forecast). }\end{array}$ \\
\hline Patzer (2015) & Patzer, Marianne, Storm surge forecasting at DMI and perspectives on teh use of Earth Observations, ESA eSurge \\
\hline
\end{tabular}




\begin{tabular}{|c|c|}
\hline & $\begin{array}{l}\text { Sympositum, Deltares, the Netherlands, (powerpoint presentation) January } 21,2015 \\
\text {-FIG. [MAP] map of storm surge water level, altimeter strip water level \& } 3 \text { Zeeland tide gauges } \\
\text { 07Dec2013 00:00 }\end{array}$ \\
\hline Wadey et al (2015a) & $\begin{array}{l}\text { Wadey MP, ID Haigh, RJ Nichols, JM Brown, K Horsburgh, B Carroll, SL Gallop, T Mason, E Bradshaw, A } \\
\text { comparison of the } 31 \text { January-1 February } 1953 \text { and 5-6 December } 2013 \text { coastal flood events around the UK, UK } \\
\text { Frontiers in Marine Science, 2, 84, 2015a. } \\
\text {-FIG2. Surface wind and pressure fields in NW Europe from 04Dec2013 1200UTC to 06Dec2013 0000UTC }\end{array}$ \\
\hline Wadey et al (2015b) & $\begin{array}{l}\text { Wadey MP, JM Brown, ID Haigh, T Dolphin, P Wisse, Assessment and comparison of extreme sea levels and } \\
\text { waves during the 2013/2014 storm season in two UK coastal regions, Nat. Hazards Earth Syst. Sci. Discuss., } \\
\text { 3, 2665-2708, 2015b. } \\
\text {-FIG7. [MAP] Time series of pressure and winds during 4-6Dec2013. } \\
\text { The locations of the Liverpool and Lowestoft tide gauges are shown. } \\
\text { Plotted from gridded surface wind and pressure data from the NCEP/NCAR } \\
\text { re-analysis (Kalnay etal, 1996) }\end{array}$ \\
\hline Cheliotis et al. (2016) & $\begin{array}{l}\text { Cheliotis, I, G. Varlas, K. Christakos, The impact of cylone Xaver on hydropower potential in Norway, } \\
\text { conference paper, September } 2016 \text { In: T Karaostas, A Bais, PT Nastos (ed), Perspectives on Atmospheric } \\
\text { Sciences, Springer Atmospheric Sciences, Springer, Cham, } 2017 \text { https://doi.org/10.1007/978-3-319-35095- } \\
0 \_25 \text {, first online 10Sep2016 [pdf document properties: author=Konstantinos Christakos; datestamp: } \\
07 / 09 / 2016 \text { ] } \\
\text {-WRF model assessment of Norway precipitation during Storm Xaver }\end{array}$ \\
\hline Christakos et al (2016) & $\begin{array}{l}\text { Christakos K, I Cheliotis, G Varlas, G-J Steeneveld, Offshore wind energy analysis of Cyclone Xaver over North } \\
\text { Europe, 13th Deep Sea Offshore Wind R\&D Conference, EERA DeepWind'2016, 20-22 January 2016, } \\
\text { Trondheim, Norway, Energy Procedia, } 94,37-44,2016 \text {. } \\
\text { FIG5. Sum of hours for wind speed (a) within the range } 11-25 \mathrm{~m} / \mathrm{s} \text {, (b) exceeding } 25 \mathrm{~m} / \mathrm{s} \\
\text { for the period 4Dec2013 0000UTC to 7Dec2013 at } 1200 \mathrm{UTC} \text { at simulated by WRF model. } \\
\text { FIG6. Avg wind power density (W/m2) for the period 5Dec2013 0600-1200UTC at } \\
\text { (a) } 100 \mathrm{~m} \text { and (b) } 200 \mathrm{~m}\end{array}$ \\
\hline 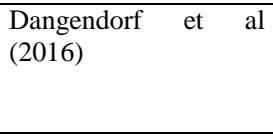 & $\begin{array}{l}\text { Dangendorf S, A Arns JG Pinto, P Ludwig, J Jensen, The exceptional influence of storm 'Xaver' on design water } \\
\text { levels in the German Bight, Environmental Research Letters, 11, 2016, 054001 } \\
\text {-FIG3. mean 10m wind speed 04Dec2013 1800UTC to 07Dec2013 1200UTC for Europe \& NE Atlantic Ocean } \\
\text {-FIG4. wind speed 06Dec2013 0200UTC from CCLM model over Europe and NE Europe }\end{array}$ \\
\hline ECMWF (20160316) & $\begin{array}{llccc}\text { ECMWF (20160316), 201312 - Windstorm }-\quad \text { Xaver/Bodil/Sven, } & \text { North-western } & \text { Europe } \\
\text { https://confluence.ecmwf.int/pages/viewpage.action?pageId=28315390 } & & \\
\text {-FIG1. [MAP] The figures above show analyses of MSLP and Eady index from } & & \\
\text { 04Dec 0000UTC to 06Dec 0000UTC every 12h. The cyclone developed } & \\
\text { south of Iceland after 1200UTC on 4Dec in a confluent flow of high } & \\
\text { vorticity. } & \\
\end{array}$ \\
\hline Staneva et al (2016a) & $\begin{array}{l}\text { Staneva J, K Wahle, H Guenther, E Stanev, Coupling of wave and circulation models in coastal-ocean predicting } \\
\text { systems: a case study for the German Bight, Ocean Sci., 12, 797-806, 2016a. } \\
\text {-FIG5. [TIMESERIES] Time series of sea level elevation (SLE) in (m) at } \\
\text { four coast stations of the German Bight (ST1-ST4). Black line } \\
\text { is tide gauge observations, red is coupled wave-circulation model } \\
\text { (WAM-GETM) \& green is circulation-only model (GETM), Storm Xaver } 2013 \\
\text {-FIG6. [MAP] Sea level elevation (SLE) difference (cm) between the } \\
\text { coupled wave circulation model (WAM-GETM) and circulation only } \\
\text { model (GETM) for the German Bight on 3Dec2013 at 0100UTC and } \\
\text { during Storm Xaver 6Dec2013 at 0100UTC. }\end{array}$ \\
\hline Staneva et al (2016) & $\begin{array}{l}\text { Staneva J, K Wahle, W Koch, A Behrens, L Fenoglio-Marc, EV Stanev, Coastal flooding: impact of waves on } \\
\text { storm surge during extremes - a case study for the German Bight, Nat. Hazards Earth Syst. Sci., 16, 2373- } \\
\text { 2389, } 2016 \\
\text {-FIG3. Meteorological situation during Xaver: } \\
\text { (a) DWD 10min wind speed \& direction Dec5 1800UTC, (b) sea level pressure on 05Dec2013 1800UTC; } \\
\text { (c) DWD 10min wind speed \& direction 06Dec2013 0300UTC, (d) sea level pressure 06Dec2013 } \\
\text { 0300UTC; } \\
\text { (e) DWD 10min wind speed \& direction 06Dec2013 0700UTC, (f) sea level pressure 06Dec2013 0700UTC } \\
\text { [wind jet S coast Norway] } \\
\text {-FIG11. Maximum surge over } 4 \text { different tidal periods T1-T4 }\end{array}$ \\
\hline Staneva et al (2017b) & $\begin{array}{l}\text { Staneva J, C Schrum, A Behrens, S Grayek, H Ho-Hagemann, V Alan, O Breivik, J-R. Bidlot, A North Sea-Baltic } \\
\text { Sea Regional Coupled Models: Atmosphere, wind waves and ocean, in Proceedings of the Eigth EuroGOOS } \\
\text { International Conference (Operational Oceanography. Serving Sustainable Marine Development), 3-5 October } \\
\text { 2017, Bergen, Norway, 2017b. } \\
\text {-FIG5. (left) Maximum surge difference in m during storm Xaver between } \\
\text { coupled and NEMO model, }\end{array}$ \\
\hline Cheliotis (2017) & $\begin{array}{l}\text { Cheliotis, I, G. Varlas, K. Christakos, The impact of cylone Xaver on hydropower potential in Norway, } \\
\text { conference paper, September } 2016 \text { In: T Karaostas, A Bais, PT Nastos (ed), Perspectives on Atmospheric } \\
\text { Sciences, Springer Atmospheric Sciences, Springer, Cham, 2017, https://doi.org/10.1007/978-3-319-35095-0_25 } \\
\text { FIG2. [MAP] (a) Domain of the simulation with orography (m) along with the mean sea level pressure track of } \\
\text { cyclone Xaver as simulated by the WRF model } \\
\text { (b) Accumulated precipitation (mm) simulated by WRF for the period 4Dec 06:00UTC to } 7 \text { Dec 06:00UTC } \\
\text { with particular observations. }\end{array}$ \\
\hline Jensen et al (2017) & $\begin{array}{l}\text { Jensen J, S Niehuser, A Arns, S Dangendorf, Sensor- und risikobasiertes Fruhwarn-system fuer Seedeiche } \\
\text { (EarlyDike), AP1 - Sturmflutmonitoring und Sturmflutssimulator - Fachbericht 2016, Siegen, April } 2017 \\
\text {-FIG1.1. Map of surface wind and pressure field to drive wave model at 06Dec2013 00:00 }\end{array}$ \\
\hline Staneva et al (2017) & Staneva J, H Guenther, O Krueger, C Schrumm, V Alari, O Breivik, J-R Bidlot, K Mogensen, Impact of wind \\
\hline
\end{tabular}




\begin{tabular}{|c|c|}
\hline & $\begin{array}{l}\text { waves on the air-sea momentum fluxes for different wind and sea state conditions and oceanic responses, 1st } \\
\text { International Workshop on waves, storm surges and coastal hazards, Liverpool, UK 10-15Sep2017 [pdf document } \\
\text { properties: title=Anlass; author=Patrick Kalb-Anlass, datestamp=20/09/2017] } \\
\text {-FIG. [MAP] model field of significant wave height 04Dec2013 21:00 } \\
\text {-FIG. [MAP] model field of 10m wind speed 03Dec2013 11:00 } \\
\text {-FIG. [MAP] Maps comparing wave processes during Storm Xaver 5-6Dec2013 versus Oct-Dec2013 average }\end{array}$ \\
\hline Wahle et al (2017) & $\begin{array}{l}\text { Wahle K, J Staneva, W Koch, L Fenoglio-Marc, HTM Ho-Hagemann, EV Stanev, An atmosphere-wave regional } \\
\text { coupled model: improving prediction of wave heights in the southern North Sea, Ocean Sci., 13, 289-301, } \\
\text { 2017.(doi:10.5194/os-13-289-2017). } \\
\text {-model can not capture small scale features in altimeter-derived signficant wave height } \\
\text {-FIG9. [MAP] Significant wave height in the North Sea and German Bight at the peak } \\
\text { of storm Xaver (6Dec2013) } \\
\text {-FIG10.[MAP] Average difference and rms difference of WAM modelled SWH and } \\
\text { COSMO modelled wind speed when comparing one-way minus two way coupled } \\
\text { modelling results. Differences are calculated as averages over the } \\
\text { whole 3-month period. } \\
\text {-FIG11.[MAP] COSMO pressure (Pa) at mean sea level height in the North Sea during } \\
\text { storm Xaver and (b) mean sea level pressure difference when comparing } \\
\text { one-way minus two-way coupled modelling. }\end{array}$ \\
\hline Fery et al (2018) & $\begin{array}{l}\text { Fery, Natascha, Birger Tinz, Lydia Gates, Reproduction of storms over the North Sea and the Baltic with the } \\
\text { regional analysis COSMO-REA6 ISPR 2018, 17-19July2018, Bonn [pdf document properties: } \\
\text { datestamp }=16 / 07 / 2018 \text { ] } \\
\text {-omparison of wind speed and sea level pressure for COSMO-REA6, ERA5, ERA-Interim, 20CRv2c }\end{array}$ \\
\hline Niehuser et al (2018) & $\begin{array}{l}\text { Niehuser S, S Dangendorf, A Arns, J Jensen, A high resolution storm surge forecast for the German Bight, } \\
\text { Conference: 9th Chinese-German Joint Symposium on Coastal and Ocean Engineering, Tainan, Taiwan, } 2018 \\
\text {-Fig 12. [MAP] maps of vectors sowing wisnd speed ad direction at } 12 \mathrm{~h} \text { intervals 5-7 Dec } 2013 \text {. }\end{array}$ \\
\hline
\end{tabular}

Table S44. Climatological background of storm; unusual preceding weather events (arranged by year and then alphabetically)

\begin{tabular}{|c|c|}
\hline Source & Full Reference and Notes \\
\hline BODC (201311205) & $\begin{array}{l}\text { BODC, Storm surge and coastal flooding, } \begin{array}{l}\text { News and } \\
\text { https://www.bodc.ac.uk/about/news and_events/storm_surge and_coastal_flooding.html }\end{array} \\
\text {-UK Coastal Monitoring and Forecast Service: frequency of severe and extreme events predicted to increase in future } \\
\text { due to onset of global climate change }\end{array}$ \\
\hline Met Eireann (2013) & $\begin{array}{l}\text { Met Eireann, Monthly Weather Bulletin } 2013 \text { issues, Met Eireann, Glasnevin Hill, Dublin } 9 . \\
\text { * -warmest temperature measured in the northern hemisphere and world during April: } \\
47.0 \text { at Matam, Senegal on April 16. Seychelles International Airport had } \\
\text { record } 34.8 \mathrm{C} \text { on April } 10 \text {. } \\
\text { * -coldest temperature in the southern hemisphere and world -76.3C Dome Fuji, } \\
\text { Antarctica April } 3 \\
\text { * -after } 6 \text { days in early July with airt>40C, Portugal officially entered a heatwave } \\
\text { Rising temperatures led to heat health warnings for southern England \& } \\
\text { the Midlands for the UKs first prolonged heatwave since } 2006 \text { on } 17 \mathrm{July} \text {. } \\
\text { The UK recorded its hotest day since July } 2006 \text { with } 33.5 \mathrm{C} \text { at Heathrow \& } \\
\text { Northolt west London on } 22 \text { July } \\
\text { *-Powerful thunderstorms swept through parts of central \& western Europe } \\
\text { between } 4-7 \text { Aug causing widespread damage \& state of emergency in the } \\
\text { Czech Republic. } \\
\text { * -Excessive rainfall led to major flooding across eastern Romania } 11-15 \\
\text { killing at least } 9 \text { people. } \\
\text { * -Nov } 8 \text { one of strongest tropical cyclones ever recorded made landfall central } \\
\text { Philippines: Super Typhoon Haiyin } 8 \text { Nov2013. Category } 5 \text { with max sustained wind } 315 \mathrm{kph} \text {. } \\
\text { Storm surge } 6 \mathrm{~m} \text {. } 8000 \text { people killed or missing. }\end{array}$ \\
\hline NLWKN (20131203) & $\begin{array}{l}\text { NLWKN, Sturmflutgefahr an der Kueste und auf den Inseln, Zwei meter ueber dem normalen Hochwasser in der } \\
\text { Nacht zum Freitag moeglich//pressinformation von } 29 \text { November } 2013 \text {, (written by Herma Heyken, Pressesprecherin) } \\
\text {,03/12/2013 } \\
\text { https://www.nlwkn.niedersachsen.de/startseite/aktuelles/presse und offentlichkeitsarbeit/pressemitteilungen/sturmflut } \\
\text { gefahr-an-der-kueste-und-auf-den-inseln-120187.html } \\
\text {-NLWKN registered } 17 \text { storm surges in } 2 \text { month period from end Nov } 2011 \text { to end Jan 2012, then } 12 \text { month quiet } \\
\text { period, then Hurrican Lennart Jan 2013, last great storm surge before Storm Xaver } \\
\text {-low storm surge activity 1950'-1960s; high activity } 1970 \text { s-1980s and } 2006-2008\end{array}$ \\
\hline AON Benfield (2014) & $\begin{array}{l}\text { AON Benfield, Impact forecasting. December } 2013 \text { Global Catastrophe Recap, 2014. [document properties: abrandt; } \\
\text { date stamp: 10Jan2014] } \\
\text {-patterns of severe weather across globe } \\
\text {-Storm Xaver: } 15 \text { dead, } 800 \text { mill EUR insured loss; total loss higher } \\
\text {-hurricane-force wind, heavy rain, thunderstorms, highest recorded coastal tide } \\
\text {-damage heaviest Scotland, Germany, Netherlands, Belgium, Scandinavia, Poland } \\
\text {-Storm Dirk: } 6 \text { dead; insured loss } 360 \text { mill EUR } \\
\text {-main damage UK, France, Spain } \\
\text {-winter storm US early Dec; } 18 \text { dead; southern plains to New England; loss 100mill USD }\end{array}$ \\
\hline
\end{tabular}




\begin{tabular}{|c|c|}
\hline & $\begin{array}{l}\text {-another winter storm central \& E US \& E Canada killed } 29 \text { people } \\
\text {-one strongest winter storms in years Middle East; } 10 \text { deaths } \\
\text {-some of worst flooding 90years affected SE Brazil killing } 48 \text { people; } 1.4 \text { bill USD } \\
\text {-heavy snow \& rain China; no fatalities; } 90000 \text { homeless from collapsed roofs; 410mill USD } \\
\text {-eastern Malaysia: seasonal monsoon rains caused floods } \\
\text {-floods Cuba, Leeward Islands, Indonesia } \\
\text {-Cyclone Christine made landfall W Australia }\end{array}$ \\
\hline Eden (201402) & $\begin{array}{l}\text { Eden, Phillip, Weather Log December 2013, Weather, Feb 2014, pp.i-iv } \\
\text {-in last } 100 \text { years there were } 10 \text { warmer winters }\end{array}$ \\
\hline Goennert et al (2014) & $\begin{array}{l}\text { Goennert G, O Mueller, M Schaper, K Sossidi, Die Sturmflut nach dem Tief Xaver von 5. bis 7. Dezember } 2013 . \\
\text { Berichte des Landesbetriebes Strassen, Bruecken und Gewaesser (LSBG), Freie und Hansestadt Hamburg, Nr. } \\
\text { 16/2014, 26pp. } \\
\text {-mention of flooding on Eble and Danube in June } 2013\end{array}$ \\
\hline $\begin{array}{l}\text { Knaack and Heyken } \\
(2014)\end{array}$ & $\begin{array}{l}\text { Knaack H and H Heyken, Xaver hatte sehr schwere Stumflut im Gepaeck, Jahresbericht 2013. Der Zukunft } \\
\text { verplichtet, NLWKN, Niedersaechsischer Landesbetrieb fuer Waserwirtschaft, Kuesten- und Naturschutz, pp.8-9, } \\
\text { document date stamp 24Apr2014. } \\
\text {-winter 2012-2013 was quiet with only one light storm surge at end of January } 2013\end{array}$ \\
\hline RMS (2014) & $\begin{array}{l}\text { RMS, 2013-2014 Winter Storms in Europe. An Insurance and Catastrophe Modeling Perspective. RMS White Paper. } \\
\text { [PDF TIMESTAMP 11Mar2014] } \\
\text {-very cold polar air over Canada \& warmer SST in subtropical west Atlantic caused stronger jet stream E seabd } \\
\text {-northerly deflection of North Pacific Jet } \\
\text {-jet stream strengthened by } 30 \% \text { greater than normal } \\
\text {-series of storms channelled across North Atlantic but most had nadir pressure west of UK and therefore gave lots of } \\
\text { precipitation instead of strong winds. Storm Xaver was exception with pressure nadir east of UK }\end{array}$ \\
\hline Slingo et al (2014) & $\begin{array}{l}\text { Slingo J, S Belcher, A Scaife, M McCarthy, A Saulter, K McBeath, A Jenkins, C Huntingford, T Marsh, J Hannaford, } \\
\text { S Parry, The Recent Storms and Floods in the UK, 29pp, Met Office, Fitzroy Road, Exeter, Devon, EX1 3PB, } \\
\text { UK, February, } 2014 \text { [pdf document properties: author=huw.lewis; date stamp=11Feb2014] } \\
\text {-unusual winter UK storms from strengthened jet stream in fixed position over Pacific and North Atlantic } \\
\text {-unusual cold conditions over Canada and US } \\
\text {-moisture convergence area in southwest US } \\
\text {-storms over Indonesia and warm conditions in western Pacific } \\
\text {-El Nino not a contributor to UK storm pattern } \\
\text {-North Atlantic storm track deflected to more southern latitudes; storms carry more moisture and severe sea state to } \\
\text { southwest Ireland and UK. }\end{array}$ \\
\hline Axer et al (2015) & $\begin{array}{l}\text { Axer T, T Bistry, M Klawa, M Mueller, M Suesser, Deutsche Ruck Sturm dokumentation } 2013 \text { Deutschland, 2013, } \\
\text { Deutsche Rueckversicherung Aktiengesellschaft, Hansaallee 177, } 40549 \text { Duesseldorf, www.deutscherueck.de } \\
\quad \text { [pdf document information: author=filiz; date stamp=07Aug2015] } \\
\text {-unusual flooding in Germany and central Europe in June: Danube, Elbe, Saale } \\
\text {-unusual thunderstorms and hail damage in August } \\
\text {-heat wave in Europe in July } \\
\text {-world wide temperature rank } 6 \text { of record since 1850; same as } 2007 \\
\text {-for record since } 2000,2013 \text { global temperatures were 13th of } 14\end{array}$ \\
\hline $\begin{array}{l}\text { Haigh and Bradshaw } \\
\text { (2015) }\end{array}$ & $\begin{array}{l}\text { Haigh I and E Bradshaw, A century of UK coastal flooding, Planet Earth, Winter 2015, (pdf document properties: } \\
\text { 13Jan2016) } \\
\text {-cluster of } 7 \text { severe surge events during winter } 2013 / 2014 \text { of } 96 \text { events in database } \\
\text {-Super typhoon Haiyan in Philippines Nov } 2013\end{array}$ \\
\hline $\begin{array}{l}\text { Kendon and McCarthy } \\
\text { (2015) }\end{array}$ & $\begin{array}{l}\text { Kendon M and M McCarthy, The UK's wet and stormy winter of 2013/2014, Weather, 70, 40-47, } 2015 \\
\text {-UK wet \& stormy conditions linked to low temperatures North America } \\
\text {-persistence \& unusual strength of jet stream } \\
\text {-high SST and westard displacement of precipitation in tropical Pacific }\end{array}$ \\
\hline Wikipedia (20200502) & $\begin{array}{l}\text { Wikipedia, Orkan Xaver, https://de.wikipedia.org/wiki/Orkan_Xaver (accessed } 2 \text { May 2020) } \\
\text {-Storm Xaver followed more powerful Storm Christian on } 28 \text { Oct } 2013 \\
\text {-large scale weather pattern that also caused low temperatures in North America } \\
\text {-pattern of unusual weather lasted until end of December }\end{array}$ \\
\hline
\end{tabular}

Table S45. Xaver surge soon after spring tide; phase of surge and tide (arranged by year and then alphabetically)

\begin{tabular}{|l|l|}
\hline Source & Full Reference and Notes \\
\hline GVA (20131205) & $\begin{array}{l}\text { GVA, Vijf containers even op drift op de Schelde, 05/12/2013 20:57. https://www.gva.be/cnt/aid1500792/vijf- } \\
\text { containers-op-drift-op-de-schelde-2 } \\
\text {-coincidence of storm and spring tide causes problems at Antwerp }\end{array}$ \\
\hline $\begin{array}{l}\text { Unwetterzentrale } \\
(201312)\end{array}$ & $\begin{array}{c}\text { Unwetterzentrale, Orkantief XAVER - ein weiterer schwerer Wintersturm der letzten Jahrzehnte, Thomas Savert } \\
\text { and Stefan Laps, Dec. 2013 http://www.unwetterzentrale.de/uwz/928.html } \\
\text {-new moon on 3Dec 2.5days before storm }\end{array}$ \\
\hline Hewson et al (2014) & $\begin{array}{l}\text { Hewson T, L Magnusson, O Breivik, F Prates, I Tsonevsky, HJW de Vries, Windstorms in northwest Europe in } \\
\text { late 2013, ECMWF Newsletter, No 139, pp 22-28, Spring 2014. } \\
\text {-'Evidently the peak of the storm surge coincided quite closely with the fortnightly spring tide which will occu } \\
\text { two of three days after the moon is new or full' }\end{array}$ \\
\hline Nossent et al (2014) & $\begin{array}{l}\text { Nossent J, L Boeckx, E Taverniers, M Deschamps, T Verwaest, F Mostaert, Sinterklaasstorm 6 december 2013. } \\
\text { Beschrijving van de hydrometrische gebeurtenissen, Versie 4.0. WL Rapporten, 00-119, Waterbouwkundig } \\
\text { Laboratorium, Antwerpen, Belgie. October 2014, WL2014R00_119_5 [document date stamp: 15Oct2014; } \\
\text { author= Deschamps, Maarten] } \\
\text {-listing of high and low water levels at Antwerp showing spring tide during storm } \\
\text {-spring tides about 2.5days after full and new moon; new moon on 2-3Dec2013 }\end{array}$ \\
\hline Axer T, T Bistry, M Klawa, M Mueller, M Suesser, Deutsche Ruck Sturm dokumentation 2013 Deutschland, \\
\hline Axer et al (2015)
\end{tabular}




\begin{tabular}{|l|l|}
\hline & $\begin{array}{c}2013, \quad \text { Deutsche Rueckversicherung Aktiengesellschaft, Hansaallee 177, 40549 Duesseldorf, } \\
\text { www.deutscherueck.de [pdf document information: author=filiz; date stamp=07Aug2015] } \\
\text {-coincidence of the storm surge with spring tide, 3 days after new/full moon }\end{array}$ \\
\hline Spencer et al (2015) & $\begin{array}{l}\text { Spencer T, SM Brooks, BR Evans, JA Tempest, I Moeller, Southern North Sea storm surge event of Dec.5, 2013: } \\
\text { Water levels, waves, and coastal impacts, Earth Science Reviews, 146, 120-145, 2015. } \\
\text {-surge peak and tidal maximum are not usually together in the UK } \\
\text {-Xaver was anomaly: surge peak was shortly before tidal maximum }\end{array}$ \\
\hline Wahle et al (2017) & $\begin{array}{l}\text { Wahle K, J Staneva, W Koch, L Fenoglio-Marc, HTM Ho-Hagemann, EV Stanev, An atmosphere-wave regional } \\
\text { coupled model: improving prediction of wave heights in the southern North Sea, Ocean Sci., 13, 289-301, } \\
\text { 2017.(doi:10.5194/os-13-289-2017). } \\
\text {-Xaver arrival coincided with time of high tide }\end{array}$ \\
\hline $\begin{array}{l}\text { Wikipedia, Orkan Xaver, https://de.wikipedia.org/wiki/Orkan_Xaver (accessed 2 May 2020) } \\
\text {-storm surge several days after new moon spring tide on 3Dec2013 }\end{array}$ \\
\hline
\end{tabular}

Table S46. Tide analysis (arranged by year and then alphabetically)

\begin{tabular}{|c|c|}
\hline Source & Full Reference and Notes \\
\hline Gonnert et al (2004) & $\begin{array}{l}\text { Gonnert G, K Isert, H Giese A Pluess, Charakterisierung der Tidekurve, Die Kueste, 68, 99-141, } 2004 \\
\text {-includes M4 and M6 tides in partial tide analysis } \\
\text {-tide component amplitudes Helgoland: O1(8-9cm); K1 (10-11cm); M2 (dominant); M4 (7-10cm); M6(1-5cm) }\end{array}$ \\
\hline Gonnert et al (2009) & $\begin{array}{l}\text { Gonnert G, J Jensen, H von Storch, S Thumm, T Wahl, R Weisse, Der Meeresspiegelansteig. Ursachen, } \\
\text { Tendezen, und Risikobwertung, Die Kueste, 76, FAK (2009), 225-256. } \\
\text {-Normal Null reference level 0m appears about 1945 } \\
\text {-MThw calculated over annual data segments } \\
\text {-FIG6. Time series of local avg MSL (annual average) for tide gauge Cuxhaven with smooth fitting function. } \\
\text { Note sea level given in cmNN with zero-crossing at } 1945 . \\
\text { acceleration of curve since } 1970 \text { s } \\
\text {-FIG7. Time series of avg high tide MThw for tide gauge Cuxhaven using annual avg (Gonnert et al 2007) }\end{array}$ \\
\hline Goennert et al (2012) & $\begin{array}{l}\text { Goennert G, B Gerkensmeier, J-M Mueller, Ermittlung des Sturmflutbemesungswasserstandes fuer den } \\
\text { oeffentlichen Hochwasserschutz in Hamburg, Berichte des Landesbetriebes Strassen, Bruecken und } \\
\text { Gewaesser Nr 12/2012. } \\
\text {-explanation of surge residual difference calculated using average tidal cycle versus astronomical tide. (FIG23) }\end{array}$ \\
\hline $\begin{array}{l}\text { Deutschlander et al } \\
\text { (2013) }\end{array}$ & $\begin{array}{l}\text { Deutschlander T, K Frierich, S Haeseler, C Lefebvre, Orkantief XAVER ueber Nordeuropea von 5. bis } 7 . \\
\text { Dezember 2013, Deutscher Wetterdienst DWD, Stand 30. Dezember 2013, 19pp. } \\
\text {-MThw and MTnw indicated on diagrams for Husum, Norderney-Riffgat, Flensburg, Warnemuende, and } \\
\text { Hamburg St. Pauli }\end{array}$ \\
\hline Jensen et al. (2013) & $\begin{array}{l}\text { Jensen J, S Niehuser, A Arns, S Dangendorf, Sensor- und risikobasiertes Fruhwarn-system fuer Seedeiche } \\
\text { (EarlyDike), AP1 - Sturmflutmonitoring und Sturmflutssimulator - Fachbericht 2016, Siegen, April } 2017 \\
\text {-tide components included in DTU10 model: M2, S2, N2 K2, K1, O1, P1, Q1, S1, M4 (shallow water tide) }\end{array}$ \\
\hline McGarricle et al (2013) & $\begin{array}{l}\text { McGarricle P (ed), UK coastal monitoring and forecasting: Annual report for } 2013 \text { for the UK National Tide } \\
\text { Gauge Network, NERC } 100017897,2013 \text { [pdf document properties: author=pamcg; created=16Apr2014] } \\
\text {-Doodson X0 filter used for sea level calculation } \\
\text {-check of temporal drift of tidal components: M2,S2,N2,K1,O1 and Z0 }\end{array}$ \\
\hline Stanev et al (2014) & $\begin{array}{l}\text { Stanev E, J Staneva, S Grayek, J Schulz-Stellenfleth, S. Grashorn, A Behrens, Numerical modelling and data } \\
\text { assimilation, COSYNA Progress Report 2013, pp.46-51, Helmholtz-Zentrum Geesthacht, June } 2014 . \\
\text {-tidal components included in NEMO model: M2, S2, N2, K2, K1, O1, P1, Q1, M4 } \\
\text {-inidividual coefficients from harmonic analysis provided by the OSU Tidal Inversion Software (OTIS) }\end{array}$ \\
\hline Axer et al (2015) & $\begin{array}{l}\text { Axer T, T Bistry, M Klawa, M Mueller, M Suesser, Deutsche Ruck Sturm dokumentation } 2013 \text { Deutschland, } \\
\text { 2013, Deutsche Rueckversicherung Aktiengesellschaft, Hansaallee 177, 40549 Duesseldorf, } \\
\text { www.deutscherueck.de [pdf document information: author=filiz; date stamp=07Aug2015] } \\
\text {-absolute water levels not good because of river dredging and sea level rise; height over average high tide better }\end{array}$ \\
\hline Fenoglio-Marc (2015) & $\begin{array}{l}\text { Fenoglio-Marc L, R Scharroo, A Annuziato, L Mendoza, M Becker, J Lillibridge, Cyclone Xaver seen by } \\
\text { geodetic observations, Geophys Research Letters, 42, 9925-9932, 2015 } \\
\text {-global ocean tide model GOT4.8 (Ray et al 2011) } \\
\text {-time series stations detides with Matlab software T_TIDE (Pawlowicz et al 2002) }\end{array}$ \\
\hline Gourgue et al (2015) & $\begin{array}{l}\text { Gourgue O, BB Sishah, J Vanlede, H Komijani, M Chen, Modelling tides and storm surges on the European } \\
\text { continental shelf, 22nd Telemac \& Mascaret User Club, STFC Daresbury Laboratory, UK, 13-16 Oct } 2015 . \\
\text { [PDF document properties: datestamp: 05/10/2015] } \\
\text {-OSU Tidal Data Inversion Products; } \\
\text {-OTIS Atlantic Ocean tidal solution; amplitude and phase of } 11 \text { harmonic constituents } \\
\text {-OSU Topex/Poseidon Global Inversion Solution (TPXO): amplitude \& phase of } 13 \text { harmonic constituents }\end{array}$ \\
\hline Sibley et al. (2015) & $\begin{array}{l}\text { Sibley A, D Cox, H Titley, Coastal flooding in England and Wales from Atlantic and North Sea storms during } \\
\text { the 2013/2014 winter, Weather, 70, 62-70, } 2015 \\
\text {-FIG2. Met Office North Sea storm surge extended forecast model predictions for Lowestoft and North Shields } \\
\text { from 01Dec2013 0600UTC and 05Dec2013 0000UTC. Forecast 01Dec } 2013 \text { shows extreme surge possibility } \\
\text { but } 12 \mathrm{~h} \text { later than it actually occurred. Forecast 05Dec } 2013 \text { gives quantitative flood levels } 18 \mathrm{~h} \text { ahead. }\end{array}$ \\
\hline Wadey et al (2015) & $\begin{array}{l}\text { Wadey MP, ID Haigh, RJ Nichols, JM Brown, K Horsburgh, B Carroll, SL Gallop, T Mason, E Bradshaw, A } \\
\text { comparison of the } 31 \text { January-1 February } 1953 \text { and 5-6 December } 2013 \text { coastal flood events around the UK, } \\
\text { UK Frontiers in Marine Science, 2, 84, } 2015 \text {. } \\
\text {-'Where high frequency time series longer than a month of data are available, sea levels were separated into the } \\
\text { main component parts of tide, non-tidal residual (usually mostly surge) and MSL (Pugh, , 1987).' } \\
\text {-'For most of the } 1953 \mathrm{HW} \text { recordings, there is not enough data available for a comparative tidal analysis. This } \\
\text { is because the data is a single HW value, or in the case of Rossiter (1954), a 7-day time series' }\end{array}$ \\
\hline
\end{tabular}


Table S47. Data filtering and discretization issues (arranged by year and then alphabetically)

\begin{tabular}{|l|l|}
\hline Source & Full Reference and Notes \\
\hline $\begin{array}{l}\text { Oceanografisch } \\
\text { Meteorologisch Station }\end{array}$ & $\begin{array}{c}\text { Oceanografisch Meteorologisch Station, Stormverslag 05-06 december 2013, 26pp, 2013 [pdf document } \\
\text { properties: author=Myriam Sys; datestamp=15Dec2013] } \\
\text {-comment on differences between 5-min data, 1-min data and highest manual measurement }\end{array}$ \\
\hline RWS (2014b) & $\begin{array}{c}\text { RWS, Stormvloedrapport van 5 t/m 7 december (SR91) Sint-Nicolaasvloed 2013, Watermanagementcentrum } \\
\text { Nederland, Rijkswaterstaat, prepared by Ing. J. Kroos, 19 Mar 2014b, 48 pp } \\
\text {-global average of significant wave height and period: running average three 20 minute data segments }\end{array}$ \\
\hline Spencer et al (2015) & $\begin{array}{l}\text { Spencer T, SM Brooks, BR Evans, JA Tempest, I Moeller, Southern North Sea storm surge event of Dec.5, 2013: } \\
\text { Water levels, waves, and coastal impacts, Earth Science Reviews, 146, 120-145, 2015 } \\
\text {-UK water level data stored at 15min intervals to overcome seiche and wave effects }\end{array}$ \\
\hline Wadey et al (2015b) & $\begin{array}{l}\text { Wadey MP, JM Brown, ID Haigh, T Dolphin, P Wisse, Assessment and comparison of extreme sea levels and } \\
\text { waves during the 2013/2014 storm season in two UK coastal regions, Nat. Hazards Earth Syst. Sci. Discuss., } \\
\text { 3, 2665-2708, 2015b. } \\
\text {-BODC 15min sampling interval chosen to remove seiches and waves }\end{array}$ \\
\hline Thompson et al (2020) & $\begin{array}{c}\text { Thompson F, E Renzl, A Sibley, DR Tappin, UK meteotsunamis: a revision and update on events and their } \\
\text { frequency, Weather, 2000. } \\
\text {-problems with characterizing meteotsunamis in UK data sets because of 15 min discretization interval } \\
\text {-European data sets better to work with because they have 1 minute resolution }\end{array}$ \\
\hline
\end{tabular}

Table S48. Difficulties in modelling Storm Xaver water levels and surge (arranged by year and then alphabetically)

\begin{tabular}{|c|c|}
\hline Source & Full Reference and Notes \\
\hline Gautier et al (2014) & $\begin{array}{l}\text { Gautier C, A Camarena, J van Nieuwkoop, SWAN hindcasts Wadden Sea, December 2013. Tidal inlet of } \\
\text { Ameland and eastern Wadden Sea. Deltares, 2014, 197pp. Project 1209433-007, Reference 1209433-007-HYE- } \\
0005 \\
\text {-observation overshoot of model predictions mostly below } 0.5 \mathrm{~m} \\
\text {-Diflzijl had 1m observation overshoot at the height of the storm surge }\end{array}$ \\
\hline Kristandt et al (2014) & $\begin{array}{l}\text { Kristandt, J., B. Brecht, H. Frank, H. Knaack, Optimization of empirical storm surge forecast-modeling of high } \\
\text { resolution wind fields, Die Küste, } 81,301-348,2014 \\
\text {-new empirical surge level prameterizations give modelled water levels good to } 20 \mathrm{~cm} \text { of observations. }\end{array}$ \\
\hline Nossent et al (2014) & $\begin{array}{l}\text { Nossent J, L Boeckx, E Taverniers, M Deschamps, T Verwaest, F Mostaert, Sinterklaasstorm } 6 \text { december } 2013 . \\
\text { Beschrijving van de hydrometrische gebeurtenissen, Versie 4.0. WL Rapporten, 00-119, Waterbouwkundig } \\
\text { Laboratorium, Antwerpen, Belgie. October 2014, WL2014R00_119_5 [document date stamp: 15Oct2014; } \\
\text { author= Deschamps, Maarten] } \\
\text {-2 hour difference between measured and modelled wind speeds at Hansweert and Terneuzen }\end{array}$ \\
\hline Zijl et al (2015) & $\begin{array}{l}\text { F Zijl, J Sumihar, M Verlaan, Application of data assimilation for improved operational water level forecasting } \\
\text { on the northwest European shelf and North Sea, Ocean Dynamics, 65, 1699-1716, } 2015 \\
\text {-model predicted surge level 35cm under true level for Hoek of Holland }\end{array}$ \\
\hline Staneva et al (2016a) & $\begin{array}{l}\text { Staneva J, K Wahle, H Guenther, E Stanev, Coupling of wave and circulation models in coastal-ocean predicting } \\
\text { systems: a case study for the German Bight, Ocean Sci., 12, 797-806, 2016a. } \\
\text {-difference in water levels between coupled wave-circulation model and wave only model } 30 \mathrm{~cm} \text { along German } \\
\text { Bight coast and }>50 \mathrm{~cm} \text { for Waddensee. }\end{array}$ \\
\hline Staneva et al (2016) & $\begin{array}{l}\text { Staneva J, K Wahle, W Koch, A Behrens, L Fenoglio-Marc, EV Stanev, Coastal flooding: impact of waves on } \\
\text { storm surge during extremes - a case study for the German Bight, Nat. Hazards Earth Syst. Sci., 16, 2373- } \\
\text { 2389, } 2016 \\
\text {-smallest RMSE error } 0.15 \mathrm{~m} \text { between best model run and set of BODC tide gauge stations } \\
\text {-smallest bias of }-0.10 \mathrm{~m} \text { between best model run and set of BODC tide gauge stations }\end{array}$ \\
\hline Staneva et al (2017b) & $\begin{array}{l}\text { Staneva J, C Schrum, A Behrens, S Grayek, H Ho-Hagemann, V Alan, O Breivik, J-R. Bidlot, A North Sea-Baltic } \\
\text { Sea Regional Coupled Models: Atmosphere, wind waves and ocean, in Proceedings of the Eigth EuroGOOS } \\
\text { International Conference (Operational Oceanography. Serving Sustainable Marine Development), 3-5 } \\
\text { October 2017, Bergen, Norway, 2017b. } \\
\text {-ordinary surge model underpredicts actual surge } \\
\text {-0.48m difference between surge model with and without waves }\end{array}$ \\
\hline Wahle et al (2017) & $\begin{array}{l}\text { Wahle K, J Staneva, W Koch, L Fenoglio-Marc, HTM Ho-Hagemann, EV Stanev, An atmosphere-wave regional } \\
\text { coupled model: improving prediction of wave heights in the southern North Sea, Ocean Sci., 13, 289-301, } \\
\text { 2017.(doi:10.5194/os-13-289-2017). } \\
\text {-model can not capture small scale features in altimeter-derived significant wave height }\end{array}$ \\
\hline Niehuser et al (2018) & $\begin{array}{l}\text { Niehuser S, S Dangendorf, A Arns, J Jensen, A high resolution storm surge forecast for the German Bight, } \\
\text { Conference: } 9 \text { th Chinese-German Joint Symposium on Coastal and Ocean Engineering, Tainan, Taiwan, } 2018 \\
\text {-RMSE at stations range from } 0.13 \mathrm{~m} \text { ( for Helgoland) to } 0.20 \mathrm{~m} \text { (for Husum) } \\
\text {-maximum surge model prediction differnces: } 78 \mathrm{~cm} \text { for Wyk, } 90 \mathrm{~cm} \text { for Husum \& Emden, } 27 \mathrm{~cm} \text { for Norderney }\end{array}$ \\
\hline Wikipedia (20191002) & $\begin{array}{l}\text { Wikipedia, Stormen Bodil, https://da.wikipedia.org/wiki/Stormen_Bodil\#cite_note-39 (accessed 02Oct2019) } \\
\text {-Calais \& Boulogne-sur-Mer large differences between expected and meas water levels }\end{array}$ \\
\hline
\end{tabular}

Table S49. Future sea level rise and flooding effects (arranged by year and then alphabetically) 


\begin{tabular}{|c|c|}
\hline Source & Full Reference and Notes \\
\hline Goennert et al (2012) & $\begin{array}{l}\text { Goennert G, B Gerkensmeier, J-M Mueller, Ermittlung des Sturmflutbemesungswasserstandes fuer den } \\
\text { oeffentlichen Hochwasserschutz in Hamburg, Berichte des Landesbetriebes Strassen, Bruecken und Gewaesser } \\
\mathrm{Nr} 12 / 2012 \text {. } \\
\text {-large land subsidence in German Bight area (sea level rise about twice as great as UK) }\end{array}$ \\
\hline $\begin{array}{l}\text { The Lowestoft Journal } \\
\text { (20131214) }\end{array}$ & $\begin{array}{l}\text { The Lowestoft Journal, Suffolk MP hits out at environment secretary in statement about the floods, 14Dec2013, } \\
\text { correspondent: Annabelle Dickson. } \\
\text {-MP Peter Aldous says that North Sea storm surges will be more frequent with future sea level rise }\end{array}$ \\
\hline ABPmer (2014) & $\begin{array}{l}\text { ABPmer, Ensuring Flood Resilience. An overview of the 5/6 December 2013, Associated British Ports, Marine } \\
\text { Environmental Research, July, } 2014 \\
\text {-UK Climate Projections Report (2009): 'climate change is expected to increase the likelihood of flooding in the } \\
\text { future through mean sea level rise and higher river flows' } \\
\text {-IPCC2013: 'sea level rise may accelerate coastal erosion and the deterioration of coastal flood defences...' }\end{array}$ \\
\hline Dunbar et al (2014) & $\begin{array}{l}\text { Dunbar I, N Phipps, M Szonyi, Risk Nexus. After the storm: how the UK's flood defences performed during the } \\
\text { surge following Xaver, Flood resilience review 09.14, Zurich Insurance Company Ltd., Mythenquai 2, 8002, } \\
\text { Zurich, Switzerland [document properties: date 28Aug2014; author= Zurich] } \\
\text {-sea level rise taken into account for assessing } 1 \text { in 1000y standard for Thames Barrier }\end{array}$ \\
\hline Slingo et al (2014) & $\begin{array}{l}\text { Slingo J, S Belcher, A Scaife, M McCarthy, A Saulter, K McBeath, A Jenkins, C Huntingford, T Marsh, J } \\
\text { Hannaford, S Parry, The Recent Storms and Floods in the UK, 29pp, Met Office, Fitzroy Road, Exeter, } \\
\text { Devon, EX1 3PB, UK, February, } 2014 \text { [pdf document properties: author=huw.lewis; date stamp=11Feb2014] } \\
\text {-sea level along English Channel has risen by } 12 \mathrm{~cm} \text { during 20th century; further increases } 11-16 \mathrm{~cm} \text { expected in } \\
\text { period } 1990-2030 \\
\text {-'clearly sea level rise from whatever source has to be factored into discussions about resilience to coastal and } \\
\text { river inundations' }\end{array}$ \\
\hline Sibley et al. (2015) & $\begin{array}{l}\text { Sibley A, D Cox, H Titley, Coastal flooding in England and Wales from Atlantic and North Sea storms during the } \\
\text { 2013/2014 winter, Weather, 70, 62-70, } 2015 \\
\text {-'the return period for the } 1953 \text { surge component at Lowestoft is estimated to be around } 50 \text { years, although rising } \\
\text { sea level will likely reduce the return period in coming decades' } \\
\text {-'Between } 1920 \text { and } 2012 \text { the Newlyn annual mean sea level rose by } 15.2 \mathrm{~cm} \text {, and by } 20.6 \mathrm{~cm} \text { at North Shields' } \\
\text {-Julia Slingo: 'it is uncertain whether these coastal flood events can be attributed directly to climate change' } \\
\text { (Slingo, 2014) }\end{array}$ \\
\hline $\begin{array}{l}\text { Haigh and Bradshaw } \\
(2015)\end{array}$ & $\begin{array}{l}\text { Haigh I and E Bradshaw, A century of UK coastal flooding, Planet Earth, Winter 2015, (pdf document properties: } \\
\text { 13Jan2016) } \\
-6 \text {. risks that will increase over the coming century as sea levels rise and coastal populations continue to grow' }\end{array}$ \\
\hline Spencer et al (2015) & $\begin{array}{l}\text { Spencer T, SM Brooks, BR Evans, JA Tempest, I Moeller, Southern North Sea storm surge event of Dec.5, 2013: } \\
\text { Water levels, waves, and coastal impacts, Earth Science Reviews, 146, 120-145, } 2015 \\
\text {-"for future planning, adaptive coastal management strategies need to cope with the progressive acceleration in } \\
\text { sea level rise as well as less predicatable impacts of large storms or phases of enhanced storminess' }\end{array}$ \\
\hline Wadey et al (2015b) & $\begin{array}{l}\text { Wadey MP, JM Brown, ID Haigh, T Dolphin, P Wisse, Assessment and comparison of extreme sea levels and } \\
\text { waves during the 2013/2014 storm season in two UK coastal regions, Nat. Hazards Earth Syst. Sci. Discuss., } \\
3,2665-2708,2015 \mathrm{~b} \text {. } \\
\text {-corrections of sea level to } 2008 \text { in calculation of surge return period } \\
\text {-sea level rise global } 1.8 \mathrm{~mm} / \text { year; also for stations Llandudno, Liverpool, Heysham, Lowestoft; Newlyn } \\
\text {-satellite derived sea level rise } 3 \mathrm{~mm} / \text { year from 1990s }\end{array}$ \\
\hline Brooks et al (2016) & $\begin{array}{l}\text { Brooks SM, T Spencer, A McIvor, I Moller, Reconstructing and understanding the impacts of storms and surges, } \\
\text { southern North Sea, Earth Surface Processes and Landforms, } 41,855-864,2016 . \\
\text {-data from Wahl et al (2013) indicates recent acceleration of sea levl rise at Lowestoft; signficant increase in } \\
\text { coastline retreat through remainder of } 21 \text { st century } \\
\text {-Horsburgh and Lowe (2013): future climate change will not lead to higher storm extremes; only increase in still } \\
\text { water levels to take into account }\end{array}$ \\
\hline $\begin{array}{l}\text { Dangendorf et } \quad \text { al } \\
(2016)\end{array}$ & $\begin{array}{l}\text { Dangendorf S, A Arns JG Pinto, P Ludwig, J Jensen, The exceptional influence of storm 'Xaver' on design water } \\
\text { levels in the German Bight, Environmental Research Letters, 11, 2016, 054001 } \\
\text {-increase in design water level for stations in Lower Saxony greater than 20th century sea level rise } \\
\text {-NOTE: subtraction of } 1 \text { year running sea level average to remove long-term trends. }\end{array}$ \\
\hline Gerber et al (2016) & $\begin{array}{l}\text { Gerber M, A Ganske, S Mueller-Navarra, G Rosenhagen, Categorization of meteorological conditions for storm } \\
\text { tide episodes in the German Bight, Meteorologische Zeitschrift, 25, 447-462, } 2016 . \\
\text {-sea lel rise Germany Bight 1949-2012 15-20cm; almost twice as fast as UK number }\end{array}$ \\
\hline Sorensen et al (2016b) & $\begin{array}{l}\text { Sorensen C, NH Broge, MR Molgaard, CS Schow, PThomsen, K Vognsen, P Knudsen, Assessing future flood } \\
\text { hazards for adaptation planning in a northern European Coastal Community, Frontiers in Marine Science, } \\
\text { 3:69, doi:10.3389/fmars.2016.00069, 2016b } \\
\text {-future sea level rise and flooding threat to Thyboron in Denmark } \\
\text {-map of glacial isostatic uplift for Denmark } \\
\text {-long term tide gauge records for Thyboron show sea level rise effects }\end{array}$ \\
\hline Niehuser et al (2018) & $\begin{array}{l}\text { Niehuser S, S Dangendorf, A Arns, J Jensen, A high resolution storm surge forecast for the German Bight, } \\
\text { Conference: 9th Chinese-German Joint Symposium on Coastal and Ocean Engineering, Tainan, Taiwan, } 2018 \\
\text {-'With ongoing sea level rise, an accumulation of such events can be expected by the end of the 21st century' }\end{array}$ \\
\hline WIKI (20200124) & $\begin{array}{l}\text { WIKI, Cyclone Xaver, https://en.wikipedia.org/wiki/Cyclone_Xaver accessed 24Jan2020 } \\
\text {-Peter Aldous (MP Waveney) house of Commons debate: 'It is wrong to dismiss these floods as a one in } 500 \text {-year } \\
\text { occurrence. There were floods six years ago. I think with rising sea levels these are going to be a thing of } \\
\text { the future and we do need to be looking at protecting the most vulnerable areas.' }\end{array}$ \\
\hline
\end{tabular}

Table S50. Storm event as manifestation of climate change (arranged by year and then alphabetically)

\begin{tabular}{|l|l|}
\hline Source & Full Reference and Notes \\
\hline Kunz et al (20131206) & Kunz M, B Muehr, K Schroeter, T Bessel, S Moehrle, T Muenzberg, S Brink, H-M Schmidt, Winterstorm Xaver \\
\hline
\end{tabular}




\begin{tabular}{|c|c|}
\hline & $\begin{array}{l}\text { - Report. 06Dec2013 - Report No.1, Situation Report - 19:00CET, CEDIM Forensic Disaster Analysis } \\
\text { Group (FDA), Center for Disaster Management and Risk Reduction Technology. } \\
\text {-'single events such as Xaver cannot be attributed to any changes in the local or regional climate' } \\
\text {-'according to statistical analysis from regional climate models, extreme wind speeds increas in the future over } \\
\text { several European regons such as Northern Germany'... southern Germany with decrease in storm speeds }\end{array}$ \\
\hline Slingo et al (2014) & $\begin{array}{l}\text { Slingo J, S Belcher, A Scaife, M McCarthy, A Saulter, K McBeath, A Jenkins, C Huntingford, T Marsh, J } \\
\text { Hannaford, S Parry, The Recent Storms and Floods in the UK, 29pp, Met Office, Fitzroy Road, Exeter, } \\
\text { Devon, EX1 3PB, UK, February, } 2014 \text { [pdf document properties: author=huw.lewis; date stamp=11Feb2014] } \\
\text {-intensity of recent storms unusual but not necessarily unprecedented } \\
\text {-continued run of deep depressions Dec-Jan-Feb is not common } \\
\text {-'it raises the possibility that disruption of our usual weather patterns } \\
\text { may be how climate change may manifest itself' } \\
\text {-heavy rain events becoming more freq: } 125 \mathrm{~d} \text { rain event fro } 1960 \text { s becomes } 85 \mathrm{~d} \text { rain event now } \\
\text {-'it is not possible yet to give a definitive answer on whether climate change } \\
\text { has been a contributor or not' }\end{array}$ \\
\hline Thorne (2014) & $\begin{array}{l}\text { Thorne, Colin, Geographies of UK flooding in 2013/4, The Geographical Journal, 180, 297-309, } 2014 . \\
\text {-'A causal link to climate change cannot be proved for any of the floods of 2013/13, } \\
\text { but they (and the jet stream pattern and position that generated them) do look like } \\
\text { those predicted in a warming world' } \\
\text {-'As yet, there is no definitive answer on the possible contribution of climate change } \\
\text { to the recent storminess, rainfall amounts and the consequent flooding. } \\
\text { This is partly due to the highly variable nature of UK weather and climate' } \\
\text {-couple of decades needed to clarify whether floods 2013/14 from extreme whether or } \\
\text { start of climate change pattern } \\
\text {-'Although no individual storm can be regards as exceptional, the clustering and persistence of } \\
\text { of storms is highly unusual' }\end{array}$ \\
\hline $\begin{array}{l}\text { Kendon and McCarthy } \\
\text { (2015) }\end{array}$ & $\begin{array}{l}\text { Kendon M and M McCarthy, The UK's wet and stormy winter of 2013/2014, Weather, 70, 40-47, } 2015 \\
\text {-question if UK experiencing more record breaking weather than in the past; } \\
\text { highly variable nature of UK's climate }\end{array}$ \\
\hline Sibley et al (2015) & $\begin{array}{l}\text { Sibley A, D Cox, H Titley, Coastal flooding in England and Wales from Atlantic and North Sea storms during the } \\
\text { 2013/2014 winter, Weather, } 70,62-70,2015 \\
\text {-Julia Slingo: 'it is uncertain whether these coastal flood events can be attributed directly to climate change' } \\
\text { (Slingo, 2014) }\end{array}$ \\
\hline Sorensen (2016) & $\begin{array}{l}\text { Sorensen CS, Water NOT wanted - Coastal floods and flooding protection in Denmark, In RA Herrmann \& J } \\
\text { Jensen (eds), Sicherung von Daemmen, Deichen und Stauanlagen: Handbuch fuer Theorie und Praxis (Vol } \\
\text { V pp3-21). Siegen: Universitaet Siegen, } 2016 \\
\text {-'different to the } 2006 \text { event the floods were perceived more as a consequence of climate change } \\
\text { than being due to an extreme event' }\end{array}$ \\
\hline WIKI (20200124) & $\begin{array}{l}\text { WIKI, Cyclone Xaver, https://en.wikipedia.org/wiki/Cyclone_Xaver accessed 24Jan2020 } \\
\text {-Peter Aldous (MP Waveney) house of Commons debate: 'It is wrong to dismiss these floods as a one in } 500 \text {-year } \\
\text { occurrence. There were floods six years ago. I think with rising sea levels these are going to be a thing of } \\
\text { the future and we do need to be looking at protecting the most vulnerable areas.' }\end{array}$ \\
\hline Wikipedia (20200502) & $\begin{array}{l}\text { Wikipedia, Orkan Xaver, https://de.wikipedia.org/wiki/Orkan_Xaver (accessed } 2 \text { May 2020) } \\
\text {-much media attention on Storm Xaver because of public awareness of climate change and weather extremes } \\
\text {-repeated extreme storm reports helped avert damage in Cuxhaven. }\end{array}$ \\
\hline
\end{tabular}

Table S51. Baltic Sea events (arranged by year and then alphabetically)

\begin{tabular}{|c|c|}
\hline Source & Full Reference and Notes \\
\hline BBC (20131206) & $\begin{array}{l}\text { BBC20131206, Deadly storm and tidal surge batter northern Europe, BBC } 6 \text { Dec2013 (report by Anna Holligan) } \\
\text {-2 sailors swept off ship on south coast Sweden } \\
\text {-Poland: } 400000 \text { homes without power; } 3 \text { fatalies due to tree falling on car. }\end{array}$ \\
\hline Daily Mail (20131206) & $\begin{array}{l}\text { Daily Mail, Huge storm strikes Europe causing death and destruction and leading to cancellation of hundreds of } \\
\text { flights, 6Dec2013. (correspondent: Nick Enoch), https://www.dailymail.co.uk/news/article-2519517/Huge-storm- } \\
\text { strikes-Europe-causing-death-destruction-leading-cancellation-hundreds-flights.html } \\
\text {-gusts cause tfatal traffic accident in N Poland; Poland LOT airline cancels flights. }\end{array}$ \\
\hline $\begin{array}{l}\begin{array}{l}\text { Deutschlander et } \\
\text { (2013) }\end{array} \\
\text { lal }\end{array}$ & $\begin{array}{l}\text { Deutschlander T, K Frierich, S Haeseler, C Lefebvre, Orkantief XAVER ueber Nordeuropea von 5. bis } 7 . \\
\text { Dezember 2013, Deutscher Wetterdienst DWD, Stand 30. Dezember 2013, 19pp. } \\
\text {-water level time series for Flensburg and Warnemuende } \\
\text {-model field of significant wave height for Baltic Sea } \\
\text {-summary of met-ocean data for Poland }\end{array}$ \\
\hline Kunz (20131206) & $\begin{array}{l}\text { Kunz M, B Muehr, K Schroeter, T Bessel, S Moehrle, T Muenzberg, S Brink, H-M Schmidt, Winterstorm Xaver } \\
\text { - Report. 06Dec2013 - Report No.1, Situation Report - 19:00CET, CEDIM Forensic Disaster Analysis } \\
\text { Group (FDA), Center for Disaster Management and Risk Reduction Technology. } \\
\text {-event description on German Baltic Sea coast }\end{array}$ \\
\hline SMHI (20131210) & $\begin{array}{l}\text { SMHI, Stormen Sven gav nya vattenstandsrekord i Oresund, https://www.smhi.se/nyhetsarkiv/stormen-sven-gav- } \\
\text { nya-vattenstandsrekord-i-oresund-1.34732, updated 20Mar2017; original datestamp 10Dec2013. } \\
\text {-water level extremes around coast of Sweden from Stockholm to Norway } \\
\text {-extreme minimum water levels on Baltic Sea coast }\end{array}$ \\
\hline $\begin{array}{l}\text { Unwetterzentrale } \\
(201312)\end{array}$ & $\begin{array}{l}\text { Unwetterzentrale, Orkantief XAVER - ein weiterer schwerer Wintersturm der letzten Jahrzehnte, Thomas Savert } \\
\text { and Stefan Laps, Dec. } 2013 \text { http://www.unwetterzentrale.de/uwz/928.html } \\
\text {-vegetation damage; numerous trees down } \\
\text {-gusts and snowfall accumulations for the German Baltic Sea region. }\end{array}$ \\
\hline Leiding et al (2014) & $\begin{array}{l}\text { Leiding T, B Tinz, G Rosenhagen, C Lefevre, S Haeseler, S Hagemann, I Bastigkeit, D Stein, P Schwenk, S } \\
\text { Mueller, O Outzen, K Herklotz, F Kinder, T Neumann, Meteorological and Oceanographic Conditions at the }\end{array}$ \\
\hline
\end{tabular}




\begin{tabular}{|c|c|}
\hline & $\begin{array}{l}\text { FINO platforms during the severe storms Christian and Xaver, DEWI Magazin, No.44, p16-25, } 2014 . \\
\text {-meteorological conditions at FINO1. }\end{array}$ \\
\hline Pelt (2014) & $\begin{array}{l}\text { Pelt AS, BODIL's stormflod i de indre dansk farvande, Vejret, 138, 24-29, } 2014 \\
\text {-unusually low water levels south of Danish Belt during Storm Bodil; opposite of Storm Britta }\end{array}$ \\
\hline SMHI (20140703) & $\begin{array}{l}\text { SMHI, Simone, } \quad \text { Hilde, } \quad \text { Sven } \quad \text { och } \quad \begin{array}{l}\text { Ivar } \\
\text { https://www.smhi.se/kunskapsbanken/meteorologi/simone-hilde-sven-och-ivar-okt-dec-2013-1.76183 }\end{array} \\
\text {-minimum water levels at Skanor } \\
\text {-map of wind speed return periods also for Baltic region }\end{array}$ \\
\hline Axer et al (2015) & $\begin{array}{l}\text { Axer T, T Bistry, M Klawa, M Mueller, M Suesser, Deutsche Ruck Sturm dokumentation 2013 Deutschland, } \\
\text { 2013, Deutsche Rueckversicherung Aktiengesellschaft, Hansaallee 177, 40549 Duesseldorf, } \\
\text { www.deutscherueck.de [pdf document information: author=filiz; date stamp=07Aug2015] } \\
\text {-high sea state along Baltic coast } \\
\text {-cargo ferry to Finnland disabled offshore } \\
\text {-alot of fallen trees from wind gusts in Mecklenburg-Vorpommern } \\
\text {-alot of emergency call-outs Berlin }\end{array}$ \\
\hline Gierlevsen et al (2015) & $\begin{array}{l}\text { Gierlevsen T, H Lauridsen, F Langhans, J Bejdic, Met-ocean and wind resource related studies for nearshore } \\
\text { windfarms in Denmark, seminar at the Danish Energy Agency, } 27 \text { February } 2015 . \\
\text {-Bornholm airport wind speed } \\
\text {-Arkona wind speed and direction }\end{array}$ \\
\hline Christakos et al (2016) & $\begin{array}{l}\text { Christakos K, I Cheliotis, G Varlas, G-J Steeneveld, Offshore wind energy analysis of Cyclone Xaver over North } \\
\text { Europe, 13th Deep Sea Offshore Wind R\&D Conference, EERA DeepWind'2016, 20-22 January 2016, } \\
\text { Trondheim, Norway, Energy Procedia, 94, 37-44, 2016. } \\
\text {-WRF model study shows wind speed over Baltic in optimal range } 11-25 \mathrm{~m} / \mathrm{s} \text { for } 70 \mathrm{~h} \text { during } 84 \mathrm{~h} \text { integration. }\end{array}$ \\
\hline Sorensen (2016) & $\begin{array}{l}\text { Sorensen CS, Water NOT wanted - Coastal floods and flooding protection in Denmark, In RA Herrmann \& J } \\
\text { Jensen (eds), Sicherung von Daemmen, Deichen und Stauanlagen: Handbuch fuer Theorie und Praxis (Vol } \\
\text { V pp3-21). Siegen: Universitaet Siegen, } 2016 \\
\text {-inner Danish seas: flooding of houses; highest ever water levels at Hornbaek \& Copenhagen (series 1890-2015) }\end{array}$ \\
\hline Ribeiro et al (2017) & $\begin{array}{l}\text { Ribeiro R, R Rudge, D Rucinska, Analysis of physical factors of the windstorm Xaver in Poland: post-hazard } \\
\text { review, Weather, 72, 2017, pp.378-382 } \\
\text {-impacts of storm Xaver in Poland }\end{array}$ \\
\hline Ditlevsen et al. (2018) & $\begin{array}{l}\text { Ditlevsen C, MM Ramos, C Sorensen, UR Ciocan, T Pionkowitz, Hojvandsstatistikker 2017, Miljo- og } \\
\text { Foedevaremnisteriet, Kystdirektoratet, Lemvig, Februar, } 2018 \\
\text {-ranked tide gauge storm levels for both the North Sea and Baltic }\end{array}$ \\
\hline Rucinska (2019) & $\begin{array}{l}\text { Rucinska D, Describing Storm Xaver in disaster terms, International Journal of Disaster Reduction, 34, 147-153, } \\
2019 \\
\text {-Poland: } 4 \text { fatalities, blackout in 400,000 homes affecting } 1.11 \text { million people }\end{array}$ \\
\hline WIKI (20200124) & $\begin{array}{l}\text { WIKI, Cyclone Xaver, https://en.wikipedia.org/wiki/Cyclone_Xaver accessed 24Jan2020 } \\
\text {-power outage Poland }\end{array}$ \\
\hline Wikipedia (20200502) & $\begin{array}{l}\text { Wikipedia, Orkan Xaver, https://de.wikipedia.org/wiki/Orkan_Xaver (accessed } 2 \text { May 2020) } \\
\text {-fatal traffic accident associated with tree fall in Poraj, Poland } \\
\text {-offshore winds cause low water levels in Flensburgfoerde and Schleimuendung } \\
\text {-Badewanneeffekt caused water levels to increase to }+140 \mathrm{~cm} \text { on evening 6Dec2013 } \\
\text {-PHOTO of extreme low water levels in Ekernfoerde }\end{array}$ \\
\hline
\end{tabular}

Table S52. Aftermath: new defenses; new design criteria; assessment of sea level rise (arranged by year and then alphabetically)

\begin{tabular}{|c|c|}
\hline Source & dll Reference and Notes \\
\hline $\begin{array}{l}\text { The Lowestoft Journal } \\
\text { (20131214) }\end{array}$ & $\begin{array}{l}\text { The Lowestoft Journal, Suffolk MP hits out at environment secretary in statement about the floods, 14Dec2013, } \\
\text { correspondent: Annabelle Dickson. } \\
\text {-UK MP Peter Aldous and the return period definition }\end{array}$ \\
\hline $\begin{array}{l}\text { Sueddeutsche Zeitung } \\
\text { (20131215) }\end{array}$ & $\begin{array}{l}\text { Sueddeutsche Zeitung, } \quad \text { Xaver holte sich } \begin{array}{c}\text { Land; } \\
\text { https://www.sueddeutsche.de/panorama/sturmschaeden-auf-sylt-xaver-holte-sich-land-1.1844100 }\end{array} \\
\text {-budget for reconstructing coast defenses at Sylt not settled }\end{array}$ \\
\hline $\begin{array}{l}\text { Sylter Rundschau } \\
(20131209)\end{array}$ & $\begin{array}{l}\text { Sylter Rundschau, Folgen des Orkans. Xaver 'knabberte' Sylt massiv an, 09Dec2013 06:00 (from Friederike } \\
\text { Reussner) https://www.shz.de/lokales/sylter-rundschau/xaver-knabberte-sylt-massiv-an-id5098481.html } \\
\text {-Sylt west coast dune loss during Xaver will be partially replaced by natural wind action } \\
\text {-bad erosion damge at Hoernum Odde will not recover by wind action } \\
\text {-provisional repair to concrete place armour at List Moevenberg Dike. }\end{array}$ \\
\hline $\begin{array}{l}\text { CH2MHill Halcrow } \\
\text { (2014) }\end{array}$ & $\begin{array}{l}\text { CH2MHill Halcrow, Cell } 1 \text { Regional Coastal Monitoring Programme, Wave Data Analysis Report 2: 2013-2014, } \\
\text { Final Report, March } 2014 \text { [document properties: author=Andy.Parson@ @ ch2m.com; datestamp; 04/04/2014] } \\
\text {-'it is recommended that extreme water level statistics are now revised to take } \\
\text { the event into account for future predictions' }\end{array}$ \\
\hline $\begin{array}{l}\text { Knaack and Heyken } \\
\text { (2014) }\end{array}$ & $\begin{array}{l}\text { Knaack H and H Heyken, Xaver hatte sehr schwere Stumflut im Gepaeck, Jahresbericht 2013. Der Zukunft } \\
\text { verplichtet, NLWKN, Niedersaechsischer Landesbetrieb fuer Waserwirtschaft, Kuesten- und Naturschutz, } \\
\text { pp.8-9, document date stamp 24Apr2014. } \\
\text { *-response: summer 2014: 2.5million Euro \& } 180000 \mathrm{~m} 3 \text { sand for strengthening dune collapses after Xaver }\end{array}$ \\
\hline NOZ (20140129) & $\begin{array}{l}\text { NOZ Nach Xaver: 9,4 Millionen Euro fuer Juist, Spiekeroog and Wangerooge, 29Jan2014 } \\
\text { https://www.noz.de/deutschland-welt/niedersachsen/artikel/446888/nach-xaver-9-4-millionen-euro-fur- } \\
\text { juist-spiekeroog-und-wangerooge-1 } \\
\text {-Ostfriesen island dunes to be strengthened by additional sand summer } 2014 \\
\text {-exact sand amounts for Juist, Wangeroog, Spiekeroog } \\
\text {-sand-catching fence to be rebuilt }\end{array}$ \\
\hline Thorne (2014) & $\begin{array}{l}\text { Thorne, Colin, Geographies of UK flooding in 2013/4, The Geographical Journal, 180, 297-309, } 2014 \text {. } \\
\text {-map of damaged of REngland coastal defences that had to be repaired rapidly or after winter 2013/2014 }\end{array}$ \\
\hline
\end{tabular}




\begin{tabular}{|c|c|}
\hline $\begin{array}{l}\text { Vanmassenhove } \\
\text { (2015) }\end{array}$ & $\begin{array}{l}\text { Vanmassenhove, Niels, Storm surge measures ports Flemish coast, Blankenberge, Tuesday February 3rd, } \\
\text { Maritieme Sientverlening en Kust, Coastal Division, Flanders Hydraulic Research.[document properties: } \\
\text { title=Geintegreerd Kustveiligheidsplan; author=Maarten; datestamp=27/02/2015] } \\
\text {-photos of new surge wall protection Ostend } \\
\text {-schematics of proposed quayside protection Zeebrugge } \\
\text {-map of coastal protection strategies Belgian coast: storm return wall/barrier, stilling wave basin, beach } \\
\text { nourishment, dune nourishment. }\end{array}$ \\
\hline Wadey et al (2015b) & $\begin{array}{l}\text { Wadey MP, JM Brown, ID Haigh, T Dolphin, P Wisse, Assessment and comparison of extreme sea levels and } \\
\text { waves during the 2013/2014 storm season in two UK coastal regions, Nat. Hazards Earth Syst. Sci. Discuss., } \\
\text { 3, 2665-2708, 2015b. } \\
\text {-new } 30 \text { million GBP from Suffolk County Council for major flood defence program Lowestoft }\end{array}$ \\
\hline $\begin{array}{l}\text { Dangendorf et al } \\
(2016)\end{array}$ & $\begin{array}{l}\text { Dangendorf S, A Arns JG Pinto, P Ludwig, J Jensen, The exceptional influence of storm 'Xaver' on design water } \\
\text { levels in the German Bight, Environmental Research Letters, 11, 2016, } 054001 \\
\text {-Storm Xaver modified existing design criteria }\end{array}$ \\
\hline Soresent et al (2016a) & $\begin{array}{l}\text { Sorensen CS, NK Dronen, P Knudsen, J Jensen, P Sorensen, An extreme event as a games changer in coastal } \\
\text { zone management, Journal of Coastal Research, (Special Issue, No 75), 700-704, 2016. Proceedings of the } \\
\text { 14th International Coastal Symposium (Sydney, Australia) ed by A Vila-Concejo, E Bruce, DM Kennedy, RJ } \\
\text { McCarroll } \\
\text {-upward revision of 100y water level estimate including Storm Xaver event }\end{array}$ \\
\hline Sorensen et al (2016b) & $\begin{array}{l}\text { Sorensen C, NH Broge, MR Molgaard, CS Schow, PThomsen, K Vognsen, P Knudsen, Assessing future flood } \\
\text { hazards for adaptation planning in a northern European Coastal Community, Frontiers in Marine Science, } \\
\text { 3:69, doi:10.3389/fmars.2016.00069, } 2016 \mathrm{~b} \\
\text {-between } 1958-2005 \text { stat extreme water return period increased from } 1.73 \text { to } 1.99 \mathrm{~m} \text { DVR90 } \\
\text {-Thyboron harbour is flooded at } 1.80 \mathrm{~m} \text { threshold }\end{array}$ \\
\hline Jee (2017) & $\begin{array}{l}\begin{array}{l}\text { Jee, Andrew, EA/13/2 Appendix } \\
\begin{array}{l}\text { author=Andrew A summary of flooding events in Boston. [pdf document properties: } \\
\text { agency.gov.uk/engagement/bostonbarriertwao/ }\end{array} \\
\text { stamp=17Mar2017] }\end{array} \\
\text {-supporting document for construction of Boston surge defence barrier }\end{array}$ \\
\hline $\begin{array}{l}\text { North Norfolk District } \\
\text { Council Coastal Team } \\
(2018)\end{array}$ & $\begin{array}{l}\text { North Norfolk District Council Coastal Team, Refurbishment of sea walls and groynes 2013-2015; Cromer Coast } \\
\text { Protection, (picture of plaque on wall taken 27Dec2018) www.northnorfolk.org/coastal } \\
-8 \text { million GBP project for refurbishment of Cromer sea defences winter 2013-2014 and 2014-2015; no work in } \\
\text { summer or holidays. }\end{array}$ \\
\hline Ulm et al (2018) & $\begin{array}{l}\text { Ulm, Marius, Arne Arns, Juergen Jensen, Assessing consequences of extreme events for the German Bight, 36th } \\
\text { International Conference on Coastal Engineering } 2018 \text { - Coastal Protection and Risk - ID } 1527 \\
\text {-Black Swan risk } \\
\text {-Project Extremeness } \\
\text {-assessment of highest possible storm tide. }\end{array}$ \\
\hline BAM (2019) & $\begin{array}{l}\text { BAM, Safe arrival of Boston Barrier for tidal flood alleviation scheme, 15Nov2019 11:51, BAM Nuttall Ltd, } \\
\text { https://www.bam.com/en/press/press-releases/2019/11/safe-arrival-of-boston-barrier-for-tidal-flood- } \\
\text { alleviation-scheme } \\
\text {-new Boston Barrier to provide level of flood protection second only to London } \\
\text {-new barrier intended to prevent repeat of flooding during Storm Xaver Dec } 2013\end{array}$ \\
\hline $\begin{array}{l}\text { North Norfolk District } \\
\text { Council Coastal Team } \\
(2019)\end{array}$ & $\begin{array}{l}\text { North Norfolk District Council Coastal Team, Sheringham Sea Defences; Repair and Recovery from the } \\
\text { December } 2013 \text { storm surge, www.northnorfolk.org/coastal, photo of plaque on wall taken 30Dec2019 } \\
\text {-repair of Sheringham sea defences by engineering firms Mott MacDonald and BAM Nuttall } \\
\text {-Council to do reports to sea defences on section of coast between Weybourne and Happisburgh }\end{array}$ \\
\hline
\end{tabular}

Table S53. Worst case storm surge situation (arranged by year and then alphabetically)

\begin{tabular}{|c|c|}
\hline Source & Full Reference and Notes \\
\hline $\begin{array}{l}\text { Cruz and Krausmann } \\
(2008)\end{array}$ & $\begin{array}{l}\text { Cruz AM and E Krausmann, Damage to offshore oil and gas facilities following hurricanes Katrina and Rita: An } \\
\text { overview, Journal of Loss Prevention in the Process Industries, 21, 620-626, } 2008 . \\
\text {-Katrina and Rita worst hurricanes for offshore platform damage in US history }\end{array}$ \\
\hline Diamond (2012) & $\begin{array}{l}\text { Diamond KE, Extreme weather impacts on offshore wind turbines: Lessons learned, Natural Resources and } \\
\text { Environment, 27, fall, pp.1-5, } 2012 \text {. } \\
\text {-climate change and black swan event }\end{array}$ \\
\hline $\begin{array}{l}\text { Oceanografisch } \\
\text { Meteorologisch Station } \\
(2013)\end{array}$ & $\begin{array}{l}\text { Oceanografisch Meteorologisch Station, Stormverslag 05-06 december 2013, 26pp, } 2013 \text { [pdf document } \\
\text { properties: author=Myriam Sys; datestamp=15Dec2013] } \\
\text {-worst case storm; Xaver pressure could have been higher with wind speeds more northerly }\end{array}$ \\
\hline $\begin{array}{l}\text { Van Rooijen and Oost } \\
(2014)\end{array}$ & $\begin{array}{l}\text { van Rooijen A, A Oost, Memo: Regionale advisering Ameland Noordwest, Deltares, 1209381-008-ZKS-0008, } \\
\text { 43pp, 18Dec2014 [PDF document properties: titl=Regional advisering: Ameland NW; author=Arnold van } \\
\text { Rooijen; keywords: } 1209381-008-Z K S-0008 \text {; date stamp: } 18 / 12 / 2014] \\
\text {-Xbeach model study of how Ameland protection dunes would be completely eroded for a 50y storm or worse }\end{array}$ \\
\hline Fery et al (2018) & $\begin{array}{l}\text { Fery, Natascha, Birger Tinz, Lydia Gates, Reproduction of storms over the North Sea and the Baltic with the } \\
\text { regional analysis COSMO-REA6 ISPR 2018, 17-19July2018, Bonn [pdf document properties: } \\
\text { datestamp }=16 / 07 / 2018 \text { ] } \\
\text {-comparison of the reanalysis model fields for the } 5 \text { North Sea storms as part of the Extremeness Project }\end{array}$ \\
\hline Ulm et al. (2018) & $\begin{array}{l}\text { Ulm, Marius, Arne Arns, Juergen Jensen, Assessing consequences of extreme events for the German Bight, 36th } \\
\text { International Conference on Coastal Engineering } 2018 \text { - Coastal Protection and Risk - ID } 1527 \\
\text {-Project Extremeness }\end{array}$ \\
\hline
\end{tabular}

Table S54. Damage costs; insurance losses (arranged by year and then alphabetically)

\begin{tabular}{|l|l|l|}
\hline Source & Full Reference and Notes & \\
\hline Air & Worldwide & Air Worldwide, Press Release, Boston, 12Dec2013. https://www.air-worldwide.com/In-the-News/AIR- \\
\hline
\end{tabular}




\begin{tabular}{|c|c|}
\hline$(20201212)$ & $\begin{array}{l}\text { Estimates-Losses-from-European-Windstorm-Xaver-at-Between-EUR-700-Million-and-EUR-1-4-Billion/ } \\
\text { (accessed 02Jan2020) } \\
\text {-Air worldwide est insured lossed from Storm Xaver 0.7-1.4 billion EUR } \\
\text {-most losses Denmark, Germany, UK } \\
\text {-some losses Netherlands, Belgium, Sweden, Norway } \\
\text {-flood and coastal storm surge losses will like be significant } \\
\text {-AIR's insured losses include: } \\
\text {-wind damage to onshore property; residential \& commercial } \\
\text {-losses to insured forestry in Norway \& Sweden } \\
\text {-AIR's insured losses do not include: } \\
\text {-losses from coastal surge \& inland flooding } \\
\text {-business interruption expenses } \\
\text {-UK impacted worst by Xaver }\end{array}$ \\
\hline AON Benfield (2014) & $\begin{array}{l}\text { AON Benfield, Impact forecasting. December } 2013 \text { Global Catastrophe Recap, 2014. [document properties: } \\
\text { abrandt; date stamp: 10Jan2014] } \\
\text {-Storm Xaver: } 15 \text { dead, 800mill EUR insured loss; total loss higher }\end{array}$ \\
\hline $\begin{array}{l}\text { Luecht and Peters } \\
(2014)\end{array}$ & $\begin{array}{l}\text { Luecht, Fabian and Ove Peters, Bericht ueber die Sturmflut vom 05.-0.6.12.2013 an der Westkueste Schleswig- } \\
\text { Holsteins, Landesbetrieb fuer Kuestenschutz, Nationalpark und Meeresschutz Schleswig-Holstein, Husum } \\
\text { 26Feb2014, 19pp. [pdf document properties: title=Lfd; Author=Thorsten Nommensen; datestamp: } \\
\text { 11Dec2018] } \\
\text {-itemized cost of repairs and cleanup for Schleswig-Holstein }\end{array}$ \\
\hline Thorne (2014) & $\begin{array}{l}\text { Thorne, Colin, Geographies of UK flooding in 2013/4, The Geographical Journal, 180, 297-309, } 2014 . \\
\text {-cost of all UK flooding winter 2013/2014 } 290 \text { million GBP }\end{array}$ \\
\hline Axer et al (2015) & $\begin{array}{l}\text { Axer T, T Bistry, M Klawa, M Mueller, M Suesser, Deutsche Ruck Sturm dokumentation } 2013 \text { Deutschland, } \\
\text { 2013, Deutsche Rueckversicherung Aktiengesellschaft, Hansaallee 177, } 40549 \text { Duesseldorf, } \\
\text { www.deutscherueck.de [pdf document information: author=filiz; date stamp=07Aug2015] } \\
\text {-insured storm damage in Germany less than Hurricane Christian; estimated at 100-200 million EU } \\
\text {-insured damage across Europe } 759 \text { million EUR }\end{array}$ \\
\hline Matelski (2016) & $\begin{array}{l}\text { Matelski, Birgit, Erfahrungen aus der Sturmflut Xaver von 5. und 6.12.2013 und dem Weihnachshochwasser } \\
2014 \text { in Schleswig-Holstein, IWASA } 2016 \text { Tagungsbeitrag, (46. IWASA, 7-8 Januar 2016; Internationales } \\
\text { Wasserbau-Symposium Aachen. [pdf document properties: autor=sonja; datestamp: 26Apr2016] } \\
\text {-3.4 million EUR damage cost for Schleswig-Holstein: clearing Treibsel, fising broken deckwork, wave break } \\
\text { lengthening }\end{array}$ \\
\hline DEMA (2018) & $\begin{array}{l}\text { Danish Emergency Management Agency DEMA, National Risk Profile for Denmark, April } 2018 \\
\text {-Denmark: } 900 \text { million DKK costs }\end{array}$ \\
\hline Wikipedia (20191002) & $\begin{array}{l}\text { Wikipedia, Stormen Bodil, https://da.wikipedia.org/wiki/Stormen_Bodil\#cite_note-39 (accessed 02Oct2019) } \\
\text {-Denmark damage evaluated at } 11 \text { million } \mathrm{Kr}\end{array}$ \\
\hline $\begin{array}{l}\text { JBA Risk Management } \\
(2020)\end{array}$ & $\begin{array}{l}\text { JBA Risk Management, Storm Xaver 2013. Event Commentary, 2020. [PDF document properties: } \\
\text { author=Cameron Whitwham] } \\
\text {-Economics and business } \\
\text {-Europe total loss }=1.22-1.65 \text { bill GBP (Zurich 2014) } \\
\text {-Europe total insured loss }=0.662 \text { billion GBP (PERILS 2017) } \\
\text {-UK total loss }=0.250 \text { billion GBP } \\
\text {-UK insured loss }=0.1 \text { billion GBP } \\
\text {-1000 business flooded in UK } \\
\text { *-flood defences avoided an estimated } 32 \text { billion in cost (Zurich 2014) } \\
\text {-Social, Housing, Other } \\
\text {-1400 houses flooded in UK; } 4200 \text { people affected (Zurich 2014) } \\
\text {-130000 homes/businesses without power (GC Capital Ideas 2013) } \\
\text {-fatalities: } 15 \text { in Europe; } 2 \text { in UK } \\
\text {-up } 800,000 \text { homes protected from flood defence systems in place } \\
\text {-several houses collapsed into the sea at Hemsby because of excessive cliff erosion } \\
\text {-6.8km2 agricultural land flooded }\end{array}$ \\
\hline $\begin{array}{l}\text { Surgewatch } \\
(20200304)\end{array}$ & $\begin{array}{l}\text { Surgewatch, Storm event 6th December 2013, https://www.surgewatch.org/events/1/ (last accessed 04Mar2020) } \\
\text {-insured losses 1.4-1.9 billion EUR, including wind \& surge flooding }\end{array}$ \\
\hline Wikipedia (20200502) & $\begin{array}{l}\text { Wikipedia, Orkan Xaver, https://de.wikipedia.org/wiki/Orkan_Xaver (accessed } 2 \text { May 2020) } \\
\text {-insured damage 0.7-1.4 billion EUR (Air Worldwide) } \\
\text {-100-200 million EUR insured damage Germany (Aon Benfield) }\end{array}$ \\
\hline
\end{tabular}

Table S55. Online data sets (arranged by year and then alphabetically)

\begin{tabular}{|l|l|}
\hline Source & Full Reference and Notes \\
\hline Wahle et al (2017) & Wahle K, J Staneva, W Koch, L Fenoglio-Marc, HTM Ho-Hagemann, EV Stanev, An atmosphere-wave regional \\
& coupled model: improving prediction of wave heights in the southern North Sea, Ocean Sci., 13, 289-301, \\
& 2017.(doi:10.5194/os-13-289-2017). \\
& -SARAL/AltiKa data: http://aviso.altimetry.fr, \\
& -RADS data: http://rads.tudelft.nl, GPOD data: https://gpod.eo.esa.in \\
& -WAM: http://mywave.github.io/WAM \\
& -COSMO: http://www2.cosmo-model.org/, \\
& -Bathymetry: http://www.emodnet-hydrography.eu/ \\
& -In-situ data used for validation: MARNET data http://www.bsh.de/de/Meeresdaten/Beobachtungen/MARNET- \\
Messnetz/index.jsp
\end{tabular}


BSH mooring data: http://www.bsh.de/en/Marine_data/Observations/Marine_physical_data/moorings.jsp

-Model output of the pre-operational models: http://codm.hzg.de/codm/

Table S56. Context and background information where Storm Xaver not mentioned (arranged by year and then alphabetically)

\begin{tabular}{|c|c|}
\hline Source & Full Reference and Notes \\
\hline Horner (1979) & $\begin{array}{l}\text { Horner RW, The Thames Barrier Project, The Geographical Journal, 145, 242-253, } 1979 . \\
-10000 \text { year return period design standard for Thames Barrier }\end{array}$ \\
\hline $\begin{array}{l}\text { Sanders and Gyakum } \\
(1980)\end{array}$ & $\begin{array}{l}\text { Sanders F and JR Gyakum, Synoptic-dynamic climatology of the 'Bomb', MWR, 108, 1589-1606, } 1980 \\
\text {-explamation of meteorological bomb }\end{array}$ \\
\hline Stull (1988) & Stull, R.B.: An Introduction to Boundary Layer Meteorology, Kluwer Academic Publishers, Dordrecht, 1988. \\
\hline $\begin{array}{l}\text { Winther-Jensen and } \\
\text { Jorgensen (1999) }\end{array}$ & $\begin{array}{l}\text { Winther-Jensen, M and ER Jorgensen, When real life wind speed exceeds design wind assumptions, } 1999 \\
\text { European Wind Energy Conference, 1-5 March 1999, Nice, France pp.220-223. } \\
\text {-extensive damage to } 3 \text { large wind farms near Porbandar during Gujarat cyclone of June } 1998 \\
\text {-recurrence interval of extreme events and design guidelines } \\
\text {-accidental loading } \\
\text {-free-wheeling and turbine tower collapse } \\
\text {-loading depends on quadratic of wind speed }\end{array}$ \\
\hline $\begin{array}{l}\text { Mueller-Navarra et al } \\
(2003)\end{array}$ & $\begin{array}{l}\text { Mueller-Navarra SH, W Lang, S Dick, KC Soetje, Ueber de Verfahren der Wasserstands- und } \\
\text { Stormflutvorhersage. Hydrodynamisch-numerische Modelle der Nord- und Ostsee und ein empirisch-statistisches } \\
\text { Verfahren fuer die Deutsche Bucht, promet. Jahrg. 29, Nr 1-4, 117-124, June } 2003 \\
\text {-explanation of German tide gauge analysis procedures and water level reporting conventions. }\end{array}$ \\
\hline Ishihara et al (2005) & $\begin{array}{l}\text { Ishihara T, Tamaguchi, A, Takahara K, Mekaru T, Matsuura S, An analysis of damaged wind turbines by } \\
\text { Typhoon Maemi in 2003, The sixth Asia-Pacific Conference on Wind Engineering (APCWE-VI), Seoul, Korea, } \\
\text { September 12-14, 2005 } \\
\text {-wind farm damage due to typhoon }\end{array}$ \\
\hline $\begin{array}{l}\text { Rosenthal and Lehner } \\
(2007)\end{array}$ & $\begin{array}{l}\text { Rosenthal, W. and S. Lehner, Individual wave height from SAR, Proc. 'Envisat Symposium 2007', Montreux, } \\
\begin{array}{l}\text { Switzerland, } \quad 23-27 \quad \text { April } 2007 \\
\text { https://www.semanticscholar.org/paper/INDIVIDUAL-WAVE-HEIGHT-FROM-SAR-Rosenthal- }\end{array} \\
\text { Lehner/affabe337b05276112fca662375ec8a8b4bba4a6 } \\
\text {-return periods for extreme indivual waves }>20 \mathrm{~m} \text { predicted to be } 40 \mathrm{y} \text { from Rayleigh distribution; 11y time } \\
\text { separation between Alfried Krupp accident } 1 \text { Jan } 1995 \text { and FINO1 wave 1Nov2006 means return period for } \\
\text { extreme waves in German Bight should be reconsidered. }\end{array}$ \\
\hline Lenton et al (2008) & $\begin{array}{l}\text { Lenton TM, H Held, E Kiegler, JW Hall, W Lucht, S Rahmstorf, HJ Schellnhuber, Tipping elements in the } \\
\text { Earth's climate system, PNAS, 105, 1786-1793, } 2008 \text { www.pnas.org/cgi/doi/10.1073/pnas.0705414105 }\end{array}$ \\
\hline $\begin{array}{l}\text { Wilhelmi and } \\
\text { Barjenbruch (2008) }\end{array}$ & $\begin{array}{l}\text { Wilhelmi J and U Barjenbruch, 2008, Application of radar gauges to measure the water level and the state of the } \\
\text { sea Proceedings of } 31 \text { st international conference on coastal engineering, Hamburg, Germany } \\
\text {-background information on the BAFG radar water level gauges at Borkum Suedstrand, Leuchtturm Alte Weser, } \\
\text { and FINO1 }\end{array}$ \\
\hline De Jong (2012) & $\begin{array}{l}\text { de Jong, Matthijs S, Developing a parametric model for storm to determine the extreme surge level at the Dutch } \\
\text { coast, Delft University of Technology, June, } 2012 \\
-10000 \text { year return period design standard for Netherlands coastal defence from 1960s Delta Committee report }\end{array}$ \\
\hline Diamond (2012) & $\begin{array}{l}\text { Diamond KE, Extreme weather impacts on offshore wind turbines: Lessons learned, Natural Resources and } \\
\text { Environment, 27, fall, pp.1-5, } 2012 \text {. } \\
\text {-failed grouting connection on } 80 \% \text { offshore wind turbines } \\
\text {-shifting seabed and sand waves; exposed cables } \\
\text {-black-swan hurricane with climate change } \\
\text {-cases of blade throw for onshore turbines } \\
\text {-offshore turbines only designed to withstand Category } 1 \text { hurricanes; economics of replacement }\end{array}$ \\
\hline Goennert et al (2012) & $\begin{array}{l}\text { Goennert G, B Gerkensmeier, J-M Mueller, Ermittlung des Sturmflutbemesungswasserstandes fuer den } \\
\text { oeffentlichen Hochwasserschutz in Hamburg, Berichte des Landesbetriebes Strassen, Bruecken und Gewaesser } \\
\text { Nr 12/2012. } \\
\text {-storm surge flooding protection level around North Sea } \\
\text {-background for MThw skew surge convention in Germany } \\
\text {-list of past strom trajectories giveing storm surge floods in the German Bight } \\
\text {-storm trajectory types: Scandinavian type, Skagerrak type, Jutland type. } \\
\text {-North Sea tide phase map with amphidromic points shown } \\
\text {-importance of extermal surge for storm surge levels in German Bight } \\
\text {-large land subsidence in German Bight area }\end{array}$ \\
\hline Rose et al (2012) & $\begin{array}{l}\text { Rose S, P Jaramillo, MJ Small, I Grossmann, J Apt, Quantifying the hurricane risk to the offshore wind turbines, } \\
\text { PNAS, 109, 1-6, 2012. } \\
\text {-probabilistic model of turbine buckling for offshore turbines on US Atlantic coast and Gulf of Mexico } \\
\text {-Hurricane Katrina (2006) } \\
\text {-wind farm Okinawa Japan during Typoon Maemi } \\
\text {-wind farm China during Typhoon Dujuan }\end{array}$ \\
\hline Li et al (2013) & $\begin{array}{l}\text { Li Z-q, Chen S-j, Ma H, Feng T, Design defect of wind turbine operating in typhoon activity zone, Engineering } \\
\text { Failure Analysis, 27, 165-172, } 2013 \text {. } \\
\text {-Typhoon Saomai 10Aug2006 damaged } 17 \text { turbines including } 6 \text { cases of turbine collapse } \\
\text {-most tower collapses due to failure at base of tower } \\
\text {-important to have secondary power backup to maintain yawing system. } \\
\text {-twisting resonance and blade failure }\end{array}$ \\
\hline $\begin{array}{l}\text { 4Coffshore } \\
(20151203)\end{array}$ & $\begin{array}{l}\text { 4Coffshore, Samso turbine collapse, Dec.03, } 2015 \\
\text {-nacelle breaks off turbine } 7 \text { of } 9 \text { turbine array at Paludans Flak offshore wind farm 28Nov2015 0940Z }\end{array}$ \\
\hline $\begin{array}{l}\text { 4Coffshore } \\
(20151215)\end{array}$ & $\begin{array}{l}\text { o turbine collapse due to welding crack, Dec.15, } 2015 \\
\text { bine accident due to welding crack }\end{array}$ \\
\hline
\end{tabular}




\begin{tabular}{|l|l|}
\hline Chen and Xu (2016) & $\begin{array}{l}\text { Chen X and J-Z Xu, Structural failure analysis of wind turbines impacted by super typhoon Usagi, Engineering } \\
\text { Failure Analysis, 60,391-404, 2016 } \\
\text {-China onshore wind farm damage during Super typhoon Usagi 16Sep2013 }\end{array}$ \\
\hline Gosselin (2018) & $\begin{array}{l}\text { Gosselin, P., Massive damage ... Large Scale Engineering debacle threatens as North Sea wind turbine breaks } \\
\text { apart, 27April2018 https://notrickszone.com/2018/04/27/massive-damage-large-scale-engineering-debacle- } \\
\text { threatens-as-north-sea-wind-turbine-breaks-apart/ } \\
\text {-turbine housing breaks off Adwen 5MW turbine at Alpha Ventus } \\
\text {-Adwen's 126 5MW turbine fleet suspends operations } \\
\text {-connecting bolts fatigue failure/corrosion or undersized } \\
\text {-possible start of problems at North Sea offshore wind farms }\end{array}$ \\
\hline $\begin{array}{l}\text { Wind Action } \\
\text { (20180425) }\end{array}$ & $\begin{array}{l}\text { Wind Action, Massive damage in the wind farm - cause unclear, NDR.de, Christina Gerlach, 25Apr2018 } \\
\text { htt//www.windaction.org/posts/48258-massive-damage-in-the-wind-farm-cause-unclear\#.XsD_dDl7nIU } \\
\text {-possible corrosion damage at AV07 turbine leads to nacelle cover in early April 2018 } \\
\text {-prohibited access to same turbine type at Alpha Ventus and 2 other Geman Bight wind farms }\end{array}$ \\
\hline $\begin{array}{l}\text { Buchana } \\
\text { McSharry (2019) and }\end{array}$ & $\begin{array}{l}\text { Buna P, PR McSharry, Windstorm risk assessment for offshore wind farms in the North Sea, Wind Energy, } \\
\text {-calculation of number of buckled North Sea wind turbine towers in each offshore wind farm over a 20 year } \\
\text { period from extreme European windstorms database. }\end{array}$ \\
\hline
\end{tabular}

Table S57. Climatological background for Storm Britta in Nov 2006; Storm Xaver not mentioned (arranged alphabetically)

\begin{tabular}{|c|c|}
\hline Source & Full Reference and Notes \\
\hline Cappelen (2007a) & $\begin{array}{l}\text { Cappelen, J., Vejret I } 2006 \text { - I Denmark, I Nuuk pa Gronland og I Torshavn pa faeroerne, Vejret, 110, 13-17, } \\
\text { 2007a. } \\
\text {-description of record warm autumn for Denmark, Torshavn \& Greenland } \\
\text {-global \& northern hemisphere climate very warm } 2006\end{array}$ \\
\hline Cappelen (2007b) & $\begin{array}{l}\text { Cappelen, John, Teknisk rapport 07-01, Danmarks klima } 2006 \text { med Torshavn, Faeroerne og Nuuk, Groenland - } \\
\text { with English translations, DMI, Transport- og Energiministeriet, www.dmi.dk/dmi/tr07-01, Kobenhavn, } 2007 \mathrm{~b} . \\
\text {-record breaking warm year Denmark avg=9,4C; } 0.1 \mathrm{C} \text { higher than previous record } 1990 \\
\text {-record monthly temperatures Denmark July-September } \\
\text {-annual temperature } 0.42 \mathrm{C} \text { above normal; } 5^{\text {th }} \text { warmest on record } \\
\text {-Torshavn warmest September on record from advection warm air from south } \\
\text {-Torshavn } 3^{\text {rd }} \text { warmest year on record after } 2003,1993\end{array}$ \\
\hline Eden (2006) & $\begin{array}{l}\text { Eden, P., Weather log. Consistently warm especially by night. Some heavy falls of rain. Weather Magazine, 61, i- } \\
\text { iv, } 2006 \\
-3^{\text {rd }} \text { warmest Oct on record after } 2001 \& 2005 \\
- \text { Scotland with } 2-3 \text { times more rain than avg }\end{array}$ \\
\hline Eden (2007) & $\begin{array}{l}\text { Eden, P., Weather log, November, 2006, Weather Magazine, 62, i-iv, } 2007 . \\
\text {-weather log for Nov. } 2006 \\
\text {-new temperature record autumn quarter, CET=12.6C, } 0.8 \mathrm{C} \text { over previous records } 1730,1731 \\
\text {-new sunshine records in central \& eastern England }\end{array}$ \\
\hline NCIC (2007) & $\begin{array}{l}\text { NCIC, National Climate Information Centre, UK annual weather summary, Weather, 62, p.42, } 2007 . \\
\text {-temperature record in UK: warmest year of series since record start } 1914 \\
\text {-Scotland had } 6^{\text {th }} \text { wettest year; Cornwall had } 8^{\text {th }} \text { driest }\end{array}$ \\
\hline Nielsen (2007) & $\begin{array}{l}\text { Nielsen, Niels Woetmann, Om stormflod og eftersarsvejr I Danmark anno 2006, Vejret, 110, 24-33, } 2007 . \\
\text {-warmest September-November on record }\end{array}$ \\
\hline Paskal (2009) & $\begin{array}{l}\text { Paskal, C., The vulnerability of energy infrastructure to environmental change, Energy, environment and resource } \\
\text { governance, Apr. 2009, EERG BP 2009/11 } \\
\text {-The vulnerability of energy infrastructure to environmental change } \\
\text {-hot European summer 2006; France, Spain, Germany had to shut down nuclear plants because of heat \& water } \\
\text { problems } \\
\text {-clarification that } 2006 \text { summer not as hot at } 2003\end{array}$ \\
\hline Rosenorn (2007) & $\begin{array}{l}\text { Rosenorn, Stig, Efterarsvejret } 2006 \text { (SON autumn quarter summary), Vejret, 110, pp.20-23, } 2007 \\
\text {-Sept } 2006 \text { same as previous warm record 1999; temperature higher than normal July \& Aug temperature } \\
\text {-Oct } 2006 \text { highest temperature } 12.2 \mathrm{C} \text { on record; no frost; previous record Oct } 2001 \text { with } 12.0 \mathrm{C} \\
\text {-Nov } 20060.4 \mathrm{C} \text { higher than previous } 1938 \text { record } 7.7 \mathrm{C} \\
\text {-storm on Nov1-2 only two cold days; wind blast at Gillileje described by Leif Rasmussen } 26 \mathrm{~m} / \mathrm{s} 20 \mathrm{~min} \text { avg }\end{array}$ \\
\hline $\begin{array}{l}\text { Spiegel Online (Nov.2, } \\
\text { 2006c) }\end{array}$ & $\begin{array}{l}\text { Spiegel Online, Storm bashes northern Europe, one dead as freighter sinks in Baltic Storm, 02/11/2006c, 13:00. } \\
\text {-Storm bashes northern Europe, one dead as freighter sinks in storm } \\
\text {-comparison Britta to Katrina hurricane } \\
\text {-global warming leads to extreme weather } \\
\text {-Britta came directly after second warmest October in Germany records (since 1901) } \\
\text {-Erich Roeckner, MPI-Meteorology, world climate will change more rapidly than ever before in next 100y }\end{array}$ \\
\hline
\end{tabular}

Table S58. Errors/typos in source reports for Storm Xaver (arranged by year and then alphabetically)

\begin{tabular}{|l|l|}
\hline Source & Full Reference and Notes \\
\hline $\begin{array}{l}\text { Air Worldwide } \\
(201212)\end{array}$ & $\begin{array}{l}\text { Air Worldwide, Press Release, Boston, 12Dec2013. https://www.air-worldwide.com/In-the-News/AIR- } \\
\text { Estimates-Losses-from-European-Windstorm-Xaver-at-Between-EUR-700-Million-and-EUR-1-4-Billion/ } \\
\text { (accessed 02Jan2020) } \\
\text {-maximum water level Hamburg Germany reported to be 4m above sea level when it should be 4m MTHW } \\
\text {-streets along Elbe in Hamburg reported to be under 6m of water; the maximum water level was about 6m above } \\
\text { sea level with about 1m of water on the streets }\end{array}$ \\
\hline Luecht and Peters & Luecht, Fabian and Ove Peters, Bericht ueber die Sturmflut vom 05.-0.6.12.2013 an der Westkueste Schleswig- \\
\hline
\end{tabular}




\begin{tabular}{|c|c|}
\hline$(2014)$ & $\begin{array}{l}\text { Holsteins, Landesbetrieb fuer Kuestenschutz, Nationalpark und Meeresschutz Schleswig-Holstein, Husum } \\
\text { 26Feb2014, 19pp. [pdf document properties: title=Lfd; Author=Thorsten Nommensen; datestamp: } \\
\text { 11Dec2018] } \\
\text {-typo on p.10, section 3.6.4. year of storm Xaver erroneously reported as } 2012 \text { instead of } 2013\end{array}$ \\
\hline Fischer et al. (2015) & $\begin{array}{l}\text { Fischer, JG, C Senet, A Schneehorst, O Outzen, S Schirmel, K Herklotz, Sea state measurements in Germanys } \\
\text { first offshore wind farm "alpha ventus", in the south-eastern parts of the North Sea, 2015 IEEE/OES } \\
\text { Eleventh Current, Waves and Turbulence Measurement (CWTM), } 2015 \text { [PDF document properties: } \\
\text { datestamp: } 14 / 01 / 2015 \text { ] } \\
\text {-data table showing height range of waverider measurements at }+/-20 \mathrm{~m} \text {; limitation only for telemetered data } \\
\text {-unclear if ADCP should have upper limits to wave period measurements }\end{array}$ \\
\hline & sed in manuscript (arranged by year and then alphabetically) \\
\hline Abbreviation & Full name \\
\hline BAFG & Bundesanstalt für Gewässerkunde \\
\hline $\mathrm{BSH}$ & Bundesamt für Seeschifffahrt und Hydrolographie \\
\hline CEFAS & Centre for Environment, Fisheries and Aquaculture Science \\
\hline GESLA & Global Extreme Sea Level Analysis \\
\hline IEC & International Electrotechnical Commission \\
\hline IOC & Intergovernmental Oceanographic Commission \\
\hline KNRM & Koninklijke Nederlandse Redding Maatschappij \\
\hline NCDC & National Climate Data Center \\
\hline RMS & Risk Management Solutions \\
\hline RWS & Rijkswaterstaat \\
\hline $\mathrm{UK}$ & United Kingdom \\
\hline
\end{tabular}

Table S60. People contacted for information about Storm Xaver (arranged by year and then alphabetically)

\begin{tabular}{|c|c|}
\hline Name & Affilation \\
\hline Beswick, Mark & Met Office National Meteorological Archive \\
\hline Bluemel, Maria & Landesbetrieb für Küstenschutz, Nationalpark und Meereschutz Schleswig-Holstein (LKN-SH) \\
\hline Dhoop, Thomas & Channel Coast Observatory (CCO \\
\hline Eecen, Peter J. & ECN \\
\hline Egset, Cathrine Netland & MIROS \\
\hline Fijnaut, Charlotte & Koninklijk Nederlands Meteorologisch Instituut (KNMI) \\
\hline Frederiksen, Bjørn & Kustdirektoratet (KDI) \\
\hline Gates, Lydia & Deutscher Wetterdienst (DWD) \\
\hline Granneman, Edwin & Kustwacht, Netherlands Coastguard \\
\hline Haigh, Ivan & National Oceanography Centre, University of Southampton \\
\hline Horsburgh, Kevin J. & National Oceanography Centre, Liverpool \\
\hline Huess, Vibeke & Danish Meteorological Institute (DMI) \\
\hline McKenny, Collette & JBA Risk Management Limited \\
\hline Murphy, Aidan & Met Éireann \\
\hline Pearce, Chris & Associated British Ports Marine Environmental Research (APB MER) \\
\hline Schaap, Wendy & Noordzeewind \\
\hline Skaland, Reidun Gangst $\varnothing$ & Met.no \\
\hline Snaith, Helen & British Oceanographic Data Centre (BODC) \\
\hline Spencer, Tom & University of Cambridge \\
\hline Stoker, Eric & Datawell BV \\
\hline Van Hoorne, Bart & Intergovernmental Oceanographic Commission (IOC) \\
\hline Van Vliet, Gerda & Koninklijke Nederlandse Redding Maatschappij (KNRM) \\
\hline Wilhelmi, Jens & Bundesanstalt für Gewässerkunde (BAFG) \\
\hline Zijderveld, Annette & Rijkswaterstaat (RWS) \\
\hline
\end{tabular}

\section{References:}

24liveblog, Storm 5-6 december 2013, https://live.24liveblog.com/live/UYXaD

4Coffshore, Samso turbine collapse, Dec.03, 2015

4Coffshore, Samso turbine collapse due to welding crack, Dec.15, 2015

ABPmer, Ensuring Flood Resilience. An overview of the 5/6 December 2013, Associated British Ports, Marine Environmental Research, July, 2014

Aftenposten, Varsler full storm I Sor-Norge, 5Dec2013 15:03 (correspondent Per Annar Holm) https://www.aftenposten.no/norge/i/bKEj1/varsler-full-storm-i-soer-norge

Air Worldwide, Press Release, Boston, 12Dec2013. https://www.air-worldwide.com/In-the-News/AIR-Estimates-Losses-from-EuropeanWindstorm-Xaver-at-Between-EUR-700-Million-and-EUR-1-4-Billion/ (accessed 02Jan2020)

Andrews, JE, Spit extension and barrier rollover at Blakeney Point and Salthouse: historic map and field observations, Bull. geol. Soc. Norfolk, 69, 35-63, 2020

AON Benfield, Impact forecasting. December 2013 Global Catastrophe Recap, 2014. [document properties: abrandt; date stamp: 10Jan2014].

Axer T, T Bistry, M Klawa, M Mueller, M Suesser, Deutsche Ruck Sturm dokumentation 2013 Deutschland, 2013 , Deutsche Rueckversicherung Aktiengesellschaft, Hansaallee 177, 40549 Duesseldorf, www.deutscherueck.de [pdf document information: author=filiz; date stamp=07Aug2015] 
Badewien T., Long-term observatory @ Pile Spiekeroog ICBM,COSYNA Progress Report 2013, p24. Helmholtz-Zentrum Geesthacht, June 2014

BAM, Safe arrival of Boston Barrier for tidal flood alleviation scheme, 15Nov2019 11:51, BAM Nuttall Ltd, https://www.bam.com/en/press/press-releases/2019/11/safe-arrival-of-boston-barrier-for-tidal-flood-alleviation-scheme

Bancroft, George P, Marine Weather Review - North Atlantic Area, September through December, Mariners Weather Log, volume 58, No.1, April 2014, pp.33-40

BBC, Workers off Talisman Sinopec oil platform Buchan Alpha over bad weather forecast,04Dec2013

BBC, In pictures: Winter storm hits UK, 5 December 2013, https://www.bbc.com/news/uk-scotland-25231224

BBC, Deadly storm and tidal surge batter northern Europe, BBC 6 Dec2013 (report by Anna Holligan)

BFG, Orkan Xaver: BfG beobachtet extreme Wellen in der Nordsee (06.12.2013)

Blasi C, S Mai, J Wilhelmi, T Zenz, U Barjenbruch, A powerful method of measuring sea wave spectra and their direction, ICHE 2014, Hamburg - Lehfeldt and Kopmann (eds), Bundesanstalt fuer Wasserbau, 2014. ISBN 978-3-939230-32-8

Bloomberg, Hamburg has worst flood in 37 year amid European storms, Nicholas Brautlecht, 6Dec2013, 02:58PM GMT https://www.bloomberg.com/news/articles/2013-12-06/hamburg-has-worst-flood-in-37-years-as-storms-rage-across-europe

BODC, Storm surge and coastal flooding, News and https://www.bodc.ac.uk/about/news and events/storm surge and coastal flooding.html

Brooks SM, T Spencer, A McIvor, I Moller, Reconstructing and understanding the impacts of storms and surges, southern North Sea, Earth Surface Processes and Landforms, 41, 855-864, 2016.

Brooks, SM, T Spencer, EK Christie, Storm impacts and shoreline recovery: Mechanisms and controls in the southern North Sea, Geomorphology, 283, 48-60, 2017.

BSH, Die Nordseesturmfluten von 5. und 6.12.2013, 3pp, prepared by Stockmann,K. (title: Sturmflut 2011_bm1101; author stamp: bm1101; document time stamp: 12/102013 12:5136PM)

BT, Offer for Bodil: Koebte huset 15.august - nu er det vaek, 08Dec2013, 16:44, (contributor: Morten Eggert) https://www.bt.dk/danmark/offer-for-bodil-koebte-huset-15.-august-nu-er-det-vaek

Buchana P, PR McSharry, Windstorm risk assessment for offshore wind farms in the North Sea, Wind Energy, 22, 1219-1229, 2019

Caithness Windfarm, craigdr, Detailed accidents to 31 December 2015. Document time stamp 5Jan2016, 175pp

Cappelen, J., Vejret I 2006 - I Denmark, I Nuuk pa Gronland og I Torshavn pa faeroerne, Vejret, 110, 13-17, 2007a.

Cappelen, J., Teknisk rapport 07-01, Danmarks klima 2006 med Torshavn, Faeroerne og Nuuk, Groenland - with English translations, DMI, Transport- og Energiministeriet, www.dmi.dk/dmi/tr07-01, Kobenhavn, 2007b.

Cargolaw 2013 [Tim Schwabedissen, 12Dec2013]

Carrion Aretxabala, BI, Morphological impact of the Sinterklaas storm at Het Zwin. Numerical modelling with Xbeach, M.Sc. Civil Engineering, Delft University of Technology, 2015.

CH2MHill Halcrow, Cell 1 Regional Coastal Monitoring Programme, Wave Data Analysis Report 2: 2013-2014, Final Report, March 2014 [document properties: author=Andy.Parson@ch2m.com; datestamp; 04/04/2014]

Cheliotis, I, G. Varlas, K. Christakos, The impact of cylone Xaver on hydropower potential in Norway, conference paper, September 2016 In: T Karaostas, A Bais, PT Nastos (ed), Perspectives on Atmospheric Sciences, Springer Atmospheric Sciences, Springer, Cham, 2017 https://doi.org/10.1007/978-3-319-35095-0_25, first online 10Sep2016 [pdf document properties: author=Konstantinos Christakos; datestamp: 07/09/2016]

Cheliotis, I, G. Varlas, K. Christakos, The impact of cylone Xaver on hydropower potential in Norway, conference paper, September 2016 In: T Karaostas, A Bais, PT Nastos (ed), Perspectives on Atmospheric Sciences, Springer Atmospheric Sciences, Springer, Cham, 2017, https://doi.org/10.1007/978-3-319-35095-0_25

Chen $\mathrm{X}$ and $\mathrm{J}-\mathrm{Z} \mathrm{Xu}$, Structural failure analysis of wind turbines impacted by super typhoon Usagi, Engineering Failure Analysis, 60 , 391 404, 2016

Cipollini P, LJ West, HM Snaith, P Harwood, C Donlon, New altimetry products over shelf and coastal zone from the eSurge processor, poster presentation, 2014 [document time stamp: 2014/10/22]

Cipollini P, J Benveniste, H Bonekamp, L Miller, M Picot, P Ted Strub, D Vandemark, S Vignudelli, Recovering more and better data from altimetry in the coastal zone: a community effort, Pilot ARCOM workshop Lisbon, 1/2 Sept 2015

Christakos K, I Cheliotis, G Varlas, G-J Steeneveld, Offshore wind energy analysis of Cyclone Xaver over North Europe, 13th Deep Sea Offshore Wind R\&D Conference, EERA DeepWind'2016, 20-22 January 2016, Trondheim, Norway, Energy Procedia, 94, 37-44, 2016.

Cruz AM and E Krausmann, Damage to offshore oil and gas facilities following hurricanes Katrina and Rita: An overview, Journal of Loss Prevention in the Process Industries, 21, 620-626, 2008.

Daily Mail, Huge storm strikes Europe causing death and destruction and leading to cancellation of hundreds of flights, 6 Dec2013. (correspondent: Nick Enoch), https://www.dailymail.co.uk/news/article-2519517/Huge-storm-strikes-Europe-causing-deathdestruction-leading-cancellation-hundreds-flights.html

Dan, Sebastian, Anne-Lise Montreuil, Rosalia Delgado, Tomas van Oyen, Large storm impact on a beach under sand nourishments, The Proceedings of the Coastal Sediments 2015, edited by Ping Wang, Julie D Rosati, and Jun Cheng, Coastal Sediments 2015, San Diego, USA, 11-15May2015

Dangendorf S, A Arns JG Pinto, P Ludwig, J Jensen, The exceptional influence of storm 'Xaver' on design water levels in the German Bight, Environmental Research Letters, 11, 2016, 054001

Danish Emergency Management Agency DEMA, National Risk Profile for Denmark, April 2018

Deutschlander T, K Frierich, S Haeseler, C Lefebvre, Orkantief XAVER ueber Nordeuropea von 5. bis 7. Dezember 2013, Deutscher Wetterdienst DWD, Stand 30. Dezember 2013, 19pp.

Diamond KE, Extreme weather impacts on offshore wind turbines: Lessons learned, Natural Resources and Environment, 27, fall, pp.1-5, 2012.

Dissanayake P and H Karunarathna, Effect of storm clustering on beach/dune erosion, E-proceedings of the 36th IAHR World Congress, 28June-3July, 2015, The Hague, The Netherlands

Dissanayake P, J Brown, H Karunarathna, Impacts of storm chronology on the morphological changes of the Formby beach and dune system, UK, Nat. Hazards Earth Syst. Sci., 15,1533-1543, 2015.

Dissanayake P, J Brown, P. Wisse, H Karunarathna, Comparison of storm cluster vs isolated event impacts on beach.dune morphodynamics, Estuarine, Coastal, and Shelf Science, 164, 301-312, 2015b.

Ditlevsen C, MM Ramos, C Sorensen, UR Ciocan, T Pionkowitz, Hojvandsstatistikker 2017, Miljo- og Foedevaremnisteriet, Kystdirektoratet, Lemvig, Februar, 2018 
Dreier, Norman and Peter Froehle, Operational wave forecast in the German Bight as part of a sensor- and risk based early warning system, In: J-S Shim, I Chun, HS Lim (ed), Proceedings from the International Coastal Symposium (ICS) 2018 (Busan, Republic of Korea), Journal of Coastal Research, Special Issue No. 85, 1161-1165, 2018

Dunbar I, N Phipps, M Szonyi, Risk Nexus. After the storm: how the UK's flood defences performed during the surge following Xaver, Flood resilience review 09.14, Zurich Insurance Company Ltd., Mythenquai 2, 8002, Zurich, Switzerland [document properties: date 28Aug2014; author= Zurich]

DW, Storm Xaver pummels Europe, flood surge feared, 05/12/2013

ECMWF (20160316), 201312 - Windstorm - Xaver/Bodil/Sven, North-western Europe, prepared by Linus Magnusson, last modified 10 Mar 2016, https://confluence.ecmwf.int/pages/viewpage.action?pageId=28315390

Eden, P., Weather log. Consistently warm especially by night. Some heavy falls of rain. Weather Magazine, 61, i-iv, 2006

Eden, P., Weather log, November, 2006, Weather Magazine, 62, i-iv, 2007.

Eden, Phillip, Weather Log December 2013, Weather, Feb 2014, pp.i-iv

Energy Voice, Oil staff withdrawn from North Sea ahead of storm conditions, 04/12/2013 10:30AM, https://www.energyvoice.com/other news/healthandsafety/50140/oil-staff-withdrawn-north-sea-ahead-storm-conditions/

Environment Agency, Thames Barrier Project Pack 2018, January, 2018

Eriksen J, Rekordvandstande i Isefjorden og Roskilde Fjord, Vejret, 138, 2 40-48, 2014

Evans, Sun Yan, EA/2/2 Appendix 1, History of flooding sources, Boston Barrier Transport \& Works Act order application, Public Inquiry documents, [pdf document properties: author=BDB; datestamp=16Mar2017] https://consult.environmentagency.gov.uk/engagement/bostonbarriertwao/results/appendix-1---history-of-flooding-sources.pdf

Expressen, Flera doda i Sverige efter stormen Sven, 06Dec2013, 17:34CET?

Fenoglio-Marc L, R Scharroo, A Annuziato, L Mendoza, M Becker, J Lillibridge, Cyclone Xaver seen by geodetic observations, Geophys Research Letters, 42, 9925-9932, 2015

Fenoglio-Marc L, R Scharroo, A Annuziato, L Mendoza, M Becker, J Lillibridge, Cyclone Xaver seen by geodetic observations, SUPPORTING MATERIAL, Geophys Research Letters, 42, 9925-9932, 2015

FINO1, 15-m wave damaged FINO1, 08Jan2014. http://www.fino1.de/meldungen/alle-meldungen/137-15-meter-welle-beschaedigt-fino1

Fischer, JG, C Senet, A Schneehorst, O Outzen, S Schirmel, K Herklotz, Sea state measurements in Germanys first offshore wind farm "alpha ventus", in the south-eastern parts of the North Sea, 2015 IEEE/OES Eleventh Current, Waves and Turbulence Measurement (CWTM), 2015 [PDF document properties: datestamp: 14/01/2015]

Fleetmon, Container ship Burak Bayraktar in trouble off Texel, Netherlands, (correspondent: Mikhail Voytenko, 7Dec2013 06:31) https://www.fleetmon.com/maritime-news/2013/2810/container-ship-burak-bayraktar-trouble-texel-nethe/

Fleetmon, Burak Bayraktar safe, Netherlands, (Mikhail Voytenko, Dec08, 2013 05:07) https://www.fleetmon.com/maritimenews/2013/2816/burak-bayraktar-safe-netherlands/

Fery, Natascha, Birger Tinz, Lydia Gates, Reproduction of storms over the North Sea and the Baltic with the regional analysis COSMOREA6 ISPR 2018, 17-19July2018, Bonn [pdf document properties: datestamp=16/07/2018]

FUGRO GEOS Ltd, Ormen Lange Monthly reports of wavescan data: December. Reporting period: 1 December 2013 to 31 December 2013, Report Number: C70101/8177/R0, Issue date: 23 January 2014, prepared by Heather Holt, Checked by Donald Brockie, approved by Mark Jones

Gandreassen, Oil workers moved from Ekofisk, 4Dec2013. https://gandreassen.com/oil-workers-moved-from-ekofisk/

Gautier C, A Camarena, J van Nieuwkoop, SWAN hindcasts Wadden Sea, December 2013. Tidal inlet of Ameland and eastern Wadden Sea. Deltares, 2014, 197pp. Project 1209433-007, Reference 1209433-007-HYE-0005

Gerber M, A Ganske, S Mueller-Navarra, G Rosenhagen, Categorization of meteorological conditions for storm tide episodes in the German Bight, Meteorologische Zeitschrift, 25, 447-462, 2016.

Giannopoulos G, L Peake, B Reid, J Andrews, A Grant, I Lorenzoni, M Goulden, J Waters, T Dolphin, J Bremner, TJ Tolhurst, Environmental and social impacts of the 2013 storm surge on the North Norfolk coast, powerpoint presentation date stamp 15May2019, unknown conference

Gierlevsen T, H Lauridsen, F Langhans, J Bejdic, Met-ocean and wind resource related studies for nearshore windfarms in Denmark, seminar at the Danish Energy Agency, 27 February 2015.

Goennert G, SK Dube, T Murthy, W Siefert (2001): 7. Storm surges generated by extratropical cyclones - case studies. In: Die Kueste 63 Sonderheft. Heide, Holstein: Boyens. pp 455-546

Gonnert G, K Isert, H Giese A Pluess, Charakterisierung der Tidekurve, Die Kueste, 68, 99-141, 2004

Gonnert G, J Jensen, H von Storch, S Thumm, T Wahl, R Weisse, Der Meeresspiegelansteig. Ursachen, Tendezen, und Risikobwertung, Die Kueste, 76, FAK (2009), 225-256.

Goennert G, B Gerkensmeier, J-M Mueller, Ermittlung des Sturmflutbemesungswasserstandes fuer den oeffentlichen Hochwasserschutz in Hamburg, Berichte des Landesbetriebes Strassen, Bruecken und Gewaesser Nr 12/2012.

Goennert G, O Mueller, M Schaper, K Sossidi, Die Sturmflut nach dem Tief Xaver von 5. bis 7. Dezember 2013. Berichte des Landesbetriebes Strassen, Bruecken und Gewaesser (LSBG), Freie und Hansestadt Hamburg, Nr. 16/2014, 26pp.

Gosselin, P., Massive damage ... Large Scale Engineering debacle threatens as North Sea wind turbine breaks apart, 27April2018 https://notrickszone.com/2018/04/27/massive-damage-large-scale-engineering-debacle-threatens-as-north-sea-wind-turbine-breaksapart/

Gourgue O, BB Sishah, J Vanlede, H Komijani, M Chen, Modelling tides and storm surges on the European continental shelf, 22nd Telemac \& Mascaret User Club, STFC Daresbury Laboratory, UK, 13-16 Oct 2015. [PDF document properties: datestamp: 05/10/2015]

GP, Fortsatt risk for halka, 6Dec2013 (correspondent: D Henriksson, K Vikingsson, P Sydvik, TA Akerblom) http://www.gp.se/nyheter/goteborg/1.2201325-fortsatt-risk-for-halka (accessed 1May2020)

Gray, Tom, Into the Wind, The AWEA Blog, Ireland, U.K., Germany set new wind generation records, https://www.aweablog.org/irelandu-k-germany-set-new-wind-generation-records/, 11Dec2013

GVA, Vijf containers even op drift op de Schelde, 05/12/2013 20:57. https://www.gva.be/cnt/aid1500792/vijf-containers-op-drift-op-deschelde-2

Haigh I and E Bradshaw, A century of UK coastal flooding, Planet Earth, Winter 2015, (pdf document properties: 13Jan2016)

HBVL, Vijf containers even op drift op de Schelde, 05Dec2013 20:57, https:/www.hbvl.be/cnt/aid1500792/vijf-containers-op-drift-op-deschelde-2

Hewson T, L Magnusson, O Breivik, F Prates, I Tsonevsky, HJW de Vries, Windstorms in northwest Europe in late 2013, ECMWF Newsletter, No 139, pp 22-28, Spring 2014.

Horner RW, The Thames Barrier Project, The Geographical Journal, 145, 242-253, 1979.

Ishihara T, Tamaguchi, A, Takahara K, Mekaru T, Matsuura S, An analysis of damaged wind turbines by Typhoon Maemi in 2003, The sixth Asia-Pacific Conference on Wind Engineering (APCWE-VI), Seoul, Korea, September 12-14, 2005 
IWR, Orkantief 'Xaver' bringt neuen Rekord - Deutsche Windkraftanlagen produzieren erstmals Strom mit ueber 26000 MW Leistung, 06Dec2013 09:31, https://www.iwr.de/news.php?id=25168 (accessed 07May2020)

JBA Risk Management, Storm Xaver 2013. Event Commentary, 2020. [PDF document properties: author=Cameron Whitwham]

Jee, Andrew, EA/13/2 Appendix 1. A summary of flooding events in Boston. [pdf document properties: author=Andrew Jee; Date stamp=17Mar2017] https://consult.environment-agency.gov.uk/engagement/bostonbarriertwao/

Jensen, J., A. Arns, T. Wahl, Yet another 100yr storm surge event: the role of individual storm surges on design water levels, Journal of Marine Science and Technology, 23, 882-887, 2015.

Jensen J, S Niehuser, A Arns, S Dangendorf, Sensor- und risikobasiertes Fruhwarn-system fuer Seedeiche (EarlyDike), AP1 Sturmflutmonitoring und Sturmflutssimulator - Fachbericht 2016, Siegen, April 2017

de Jong, Matthijs S, Developing a parametric model for storm to determine the extreme surge level at the Dutch coast, Delft University of Technology, June, 2012

Kendon M and M McCarthy, The UK's wet and stormy winter of 2013/2014, Weather, 70, 40-47, 2015

Knaack H and H Heyken, Xaver hatte sehr schwere Stumflut im Gepaeck, Jahresbericht 2013. Der Zukunft verplichtet, NLWKN, Niedersaechsischer Landesbetrieb fuer Waserwirtschaft, Kuesten- und Naturschutz, pp.8-9, document date stamp 24 Apr2014.

KNMI, News report. De Zware storm van 5 december, 06 Dec 2013, https://www.knmi.nl/over-het-knmi/nieuws/de-zware-storm-van-5december

Kristandt, J., B. Brecht, H. Frank, H. Knaack, Optimization of empirical storm surge forecast-modeling of high resolution wind fields, Die Kuste, 81, 301-348, 2014

Kristeligt Dagblad, Stormen blaeser Skotland omkuld: Doodsfald og nebrud, (contributor: Ritzau), 05Dec2013 12:50 https://www.kristeligtdagblad.dk/udland/stormen-bl\%C3\%A6ser-skotland-omkuld-d\%C3\%B8dsfald-og-nedbrud

Kunz M, B Muehr, K Schroeter, T Bessel, S Moehrle, T Muenzberg, S Brink, H-M Schmidt, Winterstorm Xaver - Report. 06Dec2013 Report No.1, Situation Report - 19:00CET, CEDIM Forensic Disaster Analysis Group (FDA), Center for Disaster Management and Risk Reduction Technology.

Kystdirektoratet, Shoreface nourishment effects. An analysis of the 2011 nourishment performed at Skodbjerge. Kystdirektoratet, Hojbovej 1, 7620 Lemvig, Dec 2018 [pdf document properties: title=Shoreface nourishment effects_Skodbjerge_20.12.2018, datestamp=20/12/2018]

Leiding T, B Tinz, G Rosenhagen, C Lefevre, S Haeseler, S Hagemann, I Bastigkeit, D Stein, P Schwenk, S Mueller, O Outzen, K Herklotz, F Kinder, T Neumann, Meteorological and Oceanographic Conditions at the FINO platforms during the severe storms Christian and Xaver, DEWI Magazin, No.44, p16-25, 2014.

Lenton TM, H Held, E Kiegler, JW Hall, W Lucht, S Rahmstorf, HJ Schellnhuber, Tipping elements in the Earth's climate system, PNAS, 105, 1786-1793, 2008 www.pnas.org/cgi/doi/10.1073/pnas.0705414105.

Li Z-q, Chen S-j, Ma H, Feng T, Design defect of wind turbine operating in typhoon activity zone, Engineering Failure Analysis, 27, 165$172,2013$.

Li, H., The Ameland Inlet during the Sinterklaas Storm: the role of flooding of watersheds, 2DH model study in Delft3D-FLOW, M.Sc. Thesis, Utrecht University, 01May2018

Luecht, Fabian and Ove Peters, Bericht ueber die Sturmflut vom 05.-0.6.12.2013 an der Westkueste Schleswig-Holsteins, Landesbetrieb fuer Kuestenschutz, Nationalpark und Meeresschutz Schleswig-Holstein, Husum 26Feb2014, 19pp. [pdf document properties: title=Lfd; Author=Thorsten Nommensen; datestamp: 11Dec2018]

Liverpool Echo, Formby sand dunes hit by stormy seas, 11Dec2013, (correspondent: Eleanor Barlow) https://www.liverpoolecho.co.uk/news/liverpool-news/formby-sand-dunes-hit-stormy-6397344, accessed 19Apr2020

The Lowestoft Journal, "There is a strong sense Parliament has not yet considered properly this narrowly averted national crisis" - MP secures Commons debate on flood, 12Dec2013, correspondent: Martin George

The Lowestoft Journal, Suffolk MP hits out at environment secretary in statement about the floods, 14Dec2013, correspondent: Annabelle Dickson.

Mai, S., Sea state at the research platform FINO1 during the winter storm 'Xaver', BFG, Bundesamt fuer Gewasserkunde, document properties: author=Mai, date $=16$ May2014

Mai, S, J Wilhelmi, T Zenz, U Barjenbruch, Orkan 'Xaver' - Seegangsstatistik an den Stationen FINO1 und Borkum-Suedstrand, 19. KFKISeminar, Bremerhaven 11.11.2014 (presentation slides)

Mai S and U Barjenbruch, Water level measurements with radar gauges at the German North Sea coast, [PDF document properties: author=IOC; subject: IOC/2016/MG/14 vol.5; datestamp: 18/04/2017]

Matelski, Birgit, Frerk Jensen, Peter Voss, Jorg Peters, Maria Blumel, Auswertung zur Hydrologie des Sturmtiefs Xaver 05.12.2013 bis 06.12.2013, Statusbericht vom 01.10.2014, Landesbetrieb fuer Kuestenschutz, Nationalpark und Meeresschutz, Schleswig-Holstein, Husum, 01.10.2014, 25pp.

Matelski, Birgit, Erfahrungen aus der Sturmflut Xaver von 5. und 6.12.2013 und dem Weihnachshochwasser 2014 in Schleswig-Holstein, IWASA 2016 Tagungsbeitrag, (46. IWASA, 7-8 Januar 2016; Internationales Wasserbau-Symposium Aachen. [pdf document properties: autor=sonja; datestamp: 26Apr2016]

Mawdsley RJ and ID Haigh, Spatial and temporal variability and long-term trends in skew surges globally, Frontiers in Marine Science, 2016, doi: 10.3389/fmars.2016.00029

McGarricle P (ed), UK coastal monitoring and forecasting: Annual report for 2013 for the UK National Tide Gauge Network, NERC 100017897, 2013 [pdf document properties: author=pamcg; created=16Apr2014]

Met Eireann, Monthly Weather Bulletin 2013 issues, Met Eireann, Glasnevin Hill, Dublin 9.

Mills, Ian, Remko Scharoo, Luciana Fenoglio, Xaver affected much of northern Europe on 5 and 6 December and caused worst storm surge for decades in the North Sea. https://www.eumetsat.int/website/home/News/DAT_2087062.html (last accessed: 19Nov2019, 28Jun2020)

MIROS, Monthly Report, Draugen, December 2013, Doc. No. ND/1022/13/12 (prepared by SRS, checked by OO, approved by CNE) [pdf document properties: author=MIROS AS; datestamp=30Jan2014]

MIROS, Monthly report, Ekofisk, December 2013, Doc. No. ND/1024/13/12, (prepared by SRS) 34pp, 9Jan2014 [PDF document properties: author=Miros AS; datestamp=13Jan2014]

MIROS, Manedsrapport Gullfaks C, Desember 2013, Dok. Nr ND/1013/13/12, carried out by SRS, controlled by CNE, approved by CO [pdf document properties: author=MIROS AS; date stamp: 13Jan2014]

MIROS, Manedsrapport Heidrun, Desember 2013, Dok. Nr. ND/1010/13/12, 21 pp, 07Jan2014, carried out by SRS, controlled by CNE, approved by OO [pdf properties: author=Miros AS; date stamp: 08/01/2014]

MIROS, Manedsrapport Heimdal, Desember 2013, Dok. Nr. ND/1047/13/12 (carried out be SRS, controlled by CNE, approved by OO) [PDF document properties: author=Miros AS; datestamp=06Jan2014] 
MIROS, Maanedsrapport Norne, Desember 2013, Dok. Nr. ND/1087/13/12, 21pp, carried out by SRS, controlled by CNE, approved by OO [pdf properties: Author=Miros AS; datestamp: 06Jan2014]

MIROS, Manedsrapport, Troll A, Desember 2013, Doc. No. ND/1012/13/12, (prepared by SRS) 21pp, 7Jan2014 [PDF document properties: author=Miros AS; datestamp=10Jan2014]

Mueller-Navarra SH, W Lang, S Dick, KC Soetje, Ueber de Verfahren der Wasserstands- und Stormflutvorhersage. Hydrodynamischnumerische Modelle der Nord- und Ostsee und ein empirisch-statistisches Verfahren fuer die Deutsche Bucht, promet. Jahrg. 29, Nr 14, 117-124, June 2003

National Wind Watch, 10Dec2013, Xaver zerstoert ein Windrad bei Vlatten, original source: Aachener Zeitung, 6Dec2013 https://www.wind-watch.org/news/2013/12/10/xaver-zerstort-ein-windrad-bei-vlatten/

National Wind Watch, Sturm 'Xaver': Windrad stuerzt auf Acker, 10Dec2013b, credit: von Manfred Reinnarth, Koelnische Rundschau, 06/12/2013, https://www.wind-watch.org/news/2013/12/10/sturm-xaver-windrad-sturzt-auf-acker/

National Wind Watch, Sturm 'Xaver': Windrad stuerzt auf Acker, 10Dec2013b, credit: von Manfred Reinnarth, Koelnische Rundschau, 06/12/2013, https://www.wind-watch.org/news/2013/12/10/sturm-xaver-windrad-sturzt-auf-acker/

NCIC, National Climate Information Centre, UK annual weather summary, Weather, 62, p.42, 2007.

Nederhoff K, E Elias, T Vermaas, Erosie op Ameland Noordwest. Modelstudie: simulaties met Delft3D en XBeach, Deltares, 117pp, July, 2016

Niehuser S, S Dangendorf, A Arns, J Jensen, A high resolution storm surge forecast for the German Bight, Conference: 9th ChineseGerman Joint Symposium on Coastal and Ocean Engineering, Tainan, Taiwan, 2018

Nielsen, Niels Woetmann, Om stormflod og eftersarsvejr I Danmark anno 2006, Vejret, 110, 24-33, 2007.

NLWKN, Schwere Sturmflut gut Ueberstanden, Wasserstaende zwischen Zweieinhale und Knapp vier metern registriert // presseinformation von 6. Dezember 2013, (Ansprechpartnerin: Herma Heyken), 06/12/2013.

NLWKN20151210, Sturmflutwarndienst der Betriebstelle Norden-Norderney, NLWKN Niedersachsischer Landesbetrieb fuer Wasserwirtschaft, Kuesten- und Naturschutz, Niedersachsen, document date stamp 10Dec2015.

Nordbayern, Deining: 'Xaver' reisst Rotorblatt von Windrad ab. Windkraftanlage schleuderte Eisbrocken auf die Strasse, 07/12/2013, 12:07. https://www.nordbayern.de/region/neumarkt/deining-xaver-reisst-rotorblatt-von-windrad-ab-1.3326492

North Norfolk District Council Coastal Team, Refurbishment of sea walls and groynes 2013-2015; Cromer Coast Protection, (picture of plaque on wall taken 27Dec2018) www.northnorfolk.org/coastal

North Norfolk District Council Coastal Team, Sheringham Sea Defences; Repair and Recovery from the December 2013 storm surge, www.northnorfolk.org/coastal, photo of plaque on wall taken 30Dec2019

NOS, Hoogste waterstand sinds 1953, 06/12/2013, 09:52, https://nos.nl/artikel/583218-hoogste-waterstand-sinds-1953.html

Nossent J, L Boeckx, E Taverniers, M Deschamps, T Verwaest, F Mostaert, Sinterklaasstorm 6 december 2013. Beschrijving van de hydrometrische gebeurtenissen, Versie 4.0. WL Rapporten, 00-119, Waterbouwkundig Laboratorium, Antwerpen, Belgie. October 2014, WL2014R00 1195 [document date stamp: 15Oct2014; author= Deschamps, Maarten]

NOZ Nach Xaver: 9,4 Millionen Euro fuer Juist, Spiekeroog and Wangerooge, 29Jan2014 https://www.noz.de/deutschlandwelt/niedersachsen/artikel/446888/nach-xaver-9-4-millionen-euro-fur-juist-spiekeroog-und-wangerooge-1

Oceanografisch Meteorologisch Station, Stormverslag 05-06 december 2013, 26pp, 2013 [pdf document properties: author=Myriam Sys; datestamp=15Dec2013]

Paskal, C., The vulnerability of energy infrastructure to environmental change, Energy, environment and resource governance, Apr. 2009, EERG BP 2009/11

Patzer, Marianne, Storm surge forecasting at DMI and perspectives on teh use of Earth Observations, ESA eSurge Sympositum, Deltares, the Netherlands, (powerpoint presentation) January 21, 2015

Pelt AS, BODIL's stormflod i de indre dansk farvande, Vejret, 138, 24-29, 2014

Reuters, ConocoPhillips cuts some output at Ekofisk field due to storm, 5Dec2013, https://finance.yahoo.con/news/conocophillips-cutsoutput-ekofisk-field...

Ribeiro R, R Rudge, D Rucinska, Analysis of physical factors of the windstorm Xaver in Poland: post-hazard review, Weather, 72, 2017, pp. $378-382$

RMS, 2013-2014 Winter Storms in Europe. An Insurance and Catastrophe Modeling Perspective. RMS White Paper. [PDF TIMESTAMP 11Mar2014]

Rose S, P Jaramillo, MJ Small, I Grossmann, J Apt, Quantifying the hurricane risk to the offshore wind turbines, PNAS, 109, 1-6, 2012.

Rosenorn, S,, Efterarsvejret 2006 (SON autumn quarter summary), Vejret, 110, pp.20-23, 2007.

Rosenthal, W. and S. Lehner, Individual wave height from SAR, Proc. 'Envisat Symposium 2007', Montreux, Switzerland, $23-27$ April 2007 (ESA SF-636, July 2007) https://www.semanticscholar.org/paper/INDIVIDUAL-WAVE-HEIGHT-FROM-SAR-RosenthalLehner/affabe337b05276112fca662375ec8a8b4bba4a6

rtv Oost, Trainverkeer ten noorden van Zwolle stilgelegt vanwege storm 5Dec2013 14:02, https://www.rtvoost.nl/nieuws/177568/Treinverkeer-ten-noorden-van-Zwolle-stilgelegd-vanwege-storm

Rucinska D, Describing Storm Xaver in disaster terms, International Journal of Disaster Reduction, 34, 147-153, 2019

RWS, Watermanagementcentrum Nederland, Stormvloedflits 2013-07 van $5 \mathrm{t} / \mathrm{m} 7$ december 2013, Rijkswaterstaat (document time stamp: 07Jan2014), 2014a

RWS, Stormvloedrapport van 5 t/m 7 december (SR91) Sint-Nicolaasvloed 2013, Watermanagementcentrum Nederland, Rijkswaterstaat, prepared by Ing. J. Kroos, 19 Mar 2014b, 48 pp

Sanders F and JR Gyakum, Synoptic-dynamic climatology of the 'Bomb', MWR, 108, 1589-1606, 1980

Schenk, L and S Mueller-Navarra. 3.4.4. Windstaustatistiken und Haufigkeit von Sturmfluten 2012-2015 https://www.bsh.de/DE/PUBLIKATIONEN/Nordseezustand_Aktuell/_Anlagen/Downloads/3_4_4_Windstatistiken.pdf?_blob=public ationFile \&v=2 [pdf document properties: author=Ludwig Schenk; datestamp=14Feb2019]

Sibley A, D Cox, H Titley, Coastal flooding in England and Wales from Atlantic and North Sea storms during the 2013/2014 winter, Weather, 70, 62-70, 2015

Slingo J, S Belcher, A Scaife, M McCarthy, A Saulter, K McBeath, A Jenkins, C Huntingford, T Marsh, J Hannaford, S Parry, The Recent Storms and Floods in the UK, 29pp, Met Office, Fitzroy Road, Exeter, Devon, EX1 3PB, UK, February, 2014 [pdf document properties: author=huw.lewis; date stamp=11Feb2014]

SMHI, Stormen Sven gav nya vattenstandsrekord i Oresund, https://www.smhi.se/nyhetsarkiv/stormen-sven-gav-nya-vattenstandsrekord-ioresund-1.34732, updated 20Mar2017; original datestamp 10Dec2013.

SMHI, Simone, Hilde, Sven och Ivar okt-dec 2013, 3 Jul 2014, https://www.smhi.se/kunskapsbanken/meteorologi/simone-hilde-sven-ochivar-okt-dec-2013-1.76183

SMHI, Egon - saesongens foersta ordentliga storm, 12Jan2015. https://www.smhi.se/nyhetsarkiv/egon-sasongens-forsta-ordentliga-storm1.83408 
Sorensen CS, Water NOT wanted - Coastal floods and flooding protection in Denmark, In RA Herrmann \& J Jensen (eds), Sicherung von Daemmen, Deichen und Stauanlagen: Handbuch fuer Theorie und Praxis (Vol V pp3-21). Siegen: Universitaet Siegen, 2016

Sorensen CS, NK Dronen, P Knudsen, J Jensen, P Sorensen, An extreme event as a games changer in coastal zone management, Journal of Coastal Research, (Special Issue, No 75), 700-704, 2016. Proceedings of the 14th International Coastal Symposium (Sydney, Australia) ed by A Vila-Concejo, E Bruce, DM Kennedy, RJ McCarroll, 2016a

Sorensen C, NH Broge, MR Molgaard, CS Schow, PThomsen, K Vognsen, P Knudsen, Assessing future flood hazards for adaptation planning in a northern European Coastal Community, Frontiers in Marine Science, 3:69, doi:10.3389/fmars.2016.00069, 2016b.

Spencer, T, S.M. Brooks, I. Moller, B.R. Evans, Where local matters: Impacts of a major North Sea storm surge, EOS, 95, 269-270, 29July 2014

Spencer T, SM Brooks, BR Evans, JA Tempest, I Moeller, Southern North Sea storm surge event of Dec.5, 2013: Water levels, waves, and coastal impacts, Earth Science Reviews, 146, 120-145, 2015.

Spiegel International, Winter storm 'Xaver' batters northern Europe,6 Dec 2013, 12:56 https://www.spiegel.de/international/europe/tidalsurge-winter-storm-xaver-batters-northern-europe-a-937576.html\#

Spiegel Online, Storm bashes northern Europe, one dead as freighter sinks in Baltic Storm, 02/11/2006c, 13:00.

Sueddeutsche Zeitung, Xaver holte sich Land; Sturmschaden auf Sylt, 15Dec201315:36 https://www.sueddeutsche.de/panorama/sturmschaeden-auf-sylt-xaver-holte-sich-land-1.1844100

Surgewatch, Storm event 6th December 2013, https://www.surgewatch.org/events/1/ (last accessed 04Mar2020)

Stanev E, J Staneva, S Grayek, J Schulz-Stellenfleth, S. Grashorn, A Behrens, Numerical modelling and data assimilation, COSYNA Progress Report 2013, pp.46-51, Helmholtz-Zentrum Geesthacht, June 2014.

Staneva J, K Wahle, E Stanev, Response of the German Bight Hydro and Sediment Dynamics to Wave, Tidal and Atmospheric Forcing, 3rd GODAE OceanView Coastal Oceans and Shelf Seas Task Team (COSS-TT) International Coordination Workshop, 21-24 January 2014, Rncon Beach Resort, Puerto Rico, 36pp

Staneva J, K Wahle, W Koch, A Behrens, L Fenoglio-Marc, EV Stanev, Coastal flooding: impact of waves on storm surge during extremes a case study for the German Bight, Nat. Hazards Earth Syst. Sci., 16, 2373-2389, 2016

Staneva J, K Wahle, H Guenther, E Stanev, Coupling of wave and circulation models in coastal-ocean predicting systems: a case study for the German Bight, Ocean Sci., 12, 797-806, 2016a.

Staneva J, H Guenther, O Krueger, C Schrumm, V Alari, O Breivik, J-R Bidlot, K Mogensen, Impact of wind waves on the air-sea momentum fluxes for different wind and sea state conditions and oceanic responses, 1st International Workshop on waves, storm surges and coastal hazards, Liverpool, UK 10-15Sep2017 [pdf document properties: title=Anlass; author=Patrick Kalb-Anlass, datestamp=20/09/2017]

Staneva J, C Schrum, A Behrens, S Grayek, H Ho-Hagemann, V Alan, O Breivik, J-R. Bidlot, A North Sea-Baltic Sea Regional Coupled Models: Atmosphere, wind waves and ocean, in Proceedings of the Eigth EuroGOOS International Conference (Operational Oceanography. Serving Sustainable Marine Development), 3-5 October 2017, Bergen, Norway, $2017 \mathrm{~b}$.

Stull, R.B.: An Introduction to Boundary Layer Meteorology, Kluwer Academic Publishers, Dordrecht, 1988.

Sueddeutsche Zeitung, Xaver holte sich Land; Sturmschaden auf Sylt, 15Dec201315:36 https://www.sueddeutsche.de/panorama/sturmschaeden-auf-sylt-xaver-holte-sich-land-1.1844100

Sylter Rundschau, Folgen des Orkans. Xaver 'knabberte' Sylt massiv an, 09Dec2013 06:00 (from Friederike Reussner) https://www.shz.de/lokales/sylter-rundschau/xaver-knabberte-sylt-massiv-an-id5098481.html

TheJournal, Homes remain without electricity after high winds batter power lines, 05Dec2013 08:20PM, https://www.thejournal.ie/stormireland-1207783-Dec2013/

The Local, Sven's strong winds sweep southern Sweden. 05Dec2013 15:57CET https://www.thelocal.se/20131205/storm-sven-sweeps-intosouthern-sweden

Thompson F, E Renzl, A Sibley, DR Tappin, UK meteotsunamis: a revision and update on events and their frequency, Weather, 2020

Thorne, Colin, Geographies of UK flooding in 2013/4, The Geographical Journal, 180, 297-309, 2014.

Ulm, Marius, Arne Arns, Juergen Jensen, Assessing consequences of extreme events for the German Bight, 36th International Conference on Coastal Engineering 2018 - Coastal Protection and Risk - ID 1527

Unwetterzentrale, Orkantief XAVER - ein weiterer schwerer Wintersturm der letzten Jahrzehnte, Thomas Savert and Stefan Laps, Dec. $2013 \mathrm{http}: / /$ www.unwetterzentrale.de/uwz/928.html

Upstream, Talisman takes workers off Buchan Alpha, 4Dec2013 1710GMT (correspondent: Rob Watts)

Upstream, North Sea production curtailed over storm, 05Dec2013 (contributor: Bill Lehane and News Wires)

Upstream, Buchan Alpha evacuated as North Sea storms loom, 6Dec2013 0000GMT (contributor Rob Watts)

Upstream, Workers return to Buchan A, 09Dec2013 12:58GMT (contributor: Rob Watts)

van Dorland, R, Zware storm op 5 december, Zenit, p43, Januari 2014

van Rooijen A, A Oost, Memo: Regionale advisering Ameland Noordwest, Deltares, 1209381-008-ZKS-0008, 43pp, 18Dec2014 [PDF document properties: titl=Regional advisering: Ameland NW; author=Arnold van Rooijen; keywords: 1209381-008-ZKS-0008; date stamp: $18 / 12 / 2014]$

Vanmassenhove, Niels, Storm surge measures ports Flemish coast, Blankenberge, Tuesday February 3rd, Maritieme Sientverlening en Kust, Coastal Division, Flanders Hydraulic Research.[document properties: title=Geintegreerd Kustveiligheidsplan; author=Maarten; datestamp=27/02/2015]

Wadey MP, ID Haigh, RJ Nichols, JM Brown, K Horsburgh, B Carroll, SL Gallop, T Mason, E Bradshaw, A comparison of the 31 January1 February 1953 and 5-6 December 2013 coastal flood events around the UK, UK Frontiers in Marine Science, 2, 84, 2015a.

Wadey MP, JM Brown, ID Haigh, T Dolphin, P Wisse, Assessment and comparison of extreme sea levels and waves during the 2013/2014 storm season in two UK coastal regions, Nat. Hazards Earth Syst. Sci. Discuss., 3, 2665-2708, 2015 b.

Wahle K, J Staneva, W Koch, L Fenoglio-Marc, HTM Ho-Hagemann, EV Stanev, An atmosphere-wave regional coupled model: improving prediction of wave heights in the southern North Sea, Ocean Sci., 13, 289-301, 2017.(doi:10.5194/os-13-289-2017).

Wikipedia, Stormen Bodil, https://da.wikipedia.org/wiki/Stormen_Bodil\#cite_note-39 (accessed 02Oct2019)

Wikipedia, Cyclone Xaver, https://en.wikipedia.org/wiki/Cyclone_Xaver accessed 24Jan2020

Wikipedia, Stormen Sven, https://sv.wikipedia.org/wiki/Stormen_Sven\#cite_note-15, accessed 29Apr2020

Wikipedia, Orkan Xaver, https://de.wikipedia.org/wiki/Orkan_Xaver (accessed 2 May 2020)

Wilhelmi J and U Barjenbruch, 2008, Application of radar gauges to measure the water level and the state of the sea Proceedings of 31 st international conference on coastal engineering, Hamburg, Germany

Wind Action, Massive damage in the wind farm - cause unclear, NDR.de, Christina Gerlach, 25Apr2018 http://www.windaction.org/posts/48258-massive-damage-in-the-wind-farm-cause-unclear\#.XsD_dD17nIU

Winther-Jensen, M and ER Jorgensen, When real life wind speed exceeds design wind assumptions, 1999 European Wind Energy Conference, 1-5 March 1999, Nice, France pp.220-223. 
World Bank Group, Coastal Protection on the West Coast of Jutland, West Africa Coastal Areas Management Program, Case Study 02 [PDF document date stamp: 14/11/2017]

Zijl F, J Sumihar, M Verlaan, Application of data assimilation for improved operational water level forecasting on the northwest European shelf and North Sea, Ocean Dynamics, 65, 1699-1716, 2015 


\section{SECTION IV. Selected referee comments and author responses}

Anthony James Kettle, Storm Xaver over Europe in December 2013: Overview of energy impacts and North Sea events

\section{REFEREE 1:}

\section{Referee 1 comment}

The study portraits the Xaver storm in December 2013 on its impact on a series of societal and energy infrastructure. This paper collects very interesting information on the damage of this severe storm, which will help raising attention to both scientific and political panels.

Author response:

Thanks for the positive overview

Referee 1 comment:

1. Line 58, 'rapid rotation of extreme winds' - extreme wind in offshore wind application means something special. I think what you mean here are tropical strong winds.

Author response:

The accident reports from south and east Asia comment that the wind turbines collapsed during the tropical hurricane force winds but also clarified that the rapid rotation of the wind field was an important factor in turbine tower collapse. When the turbine is misaligned with the wind, the blades present a greater crosssectional area with greater wind loading on the structure. In the North Sea area, Argyriadis et al. (2005) analyze storm data from FINO1 and also note the importance of rapid wind direction changes during storm conditions.

Referee 1 comment:

2. Line 64: what happened exactly to those wind farms?

Author response:

For the Sams $\varnothing$ wind farm, one of turbines lost its nacelle and blade assembly, which fell from the top of the tower into the sea. For the Alpha Ventus case, one turbine lost its nacelle cover. Media reports were making a big deal of this as it was long after the period when start up problems would have been addressed. The fault should not have happened so long into its operating lifetime. There was media speculation that the environmental forces were misjudged at the offshore site (turbulence or corrosion). I have not found a technical report of either accident to give more information.

Referee 1 comment:

4. Line 78: Difficult to see what content the reference is for from this sentence - need a rephrase

Author response:

The sentence makes reference to the fact that Storm Xaver belonged to the class of "super-bomb" storms of explosive cyclogenesis that was characterized in an earlier meteorological study by Sanders and Gyakum (1980). The concept would help to frame an intercomparison of severe North Sea winter storms for meteorologists. However, it does not help to understand energy and societal infrastructure damage during Storm Xaver, and the sentence has been removed.

Referee 1 comment

5. Line 86: What's special about the surge event of 1953? You explained at the end of the paper, maybe it should be here 
Author response:

I have expanded to sentence to explain why the 1953 event was important.

\section{REFEREE 2}

\section{Referee 2 comment}

It is a good paper on storm Xaver in 2013. In a first step, you describe impacts on societal and energy infrastructure. Then, you use tide gauge records to reconstruct the progression of the storm and calculate the skew surge for every of your 77 stations. In a next step you analyse the spectra of the water level data by separating them into three different frequency components.

Author response:

Thanks for the positive comments.

Referee 2 comment

(1) The abstract is clearly written, but the added value of the paper is not obvious. Please elaborate on the research question of the paper more clearly and state the most important results of the research conducted.

Author response:

Similar to other review papers, the contribution brings together and compares information from different sources to identify the important meteorological impacts on infrastructure. The synthesis of information from the different sources allows the storm events to be related in time and space, and it also highlights knowledge gaps.

The impact of large waves offshore is an important knowledge gap. A lot of energy infrastructure is located offshore, so that met-ocean conditions during the storm are important. Some of the offshore incidents and measurements indicate the presence of large infragravity waves.

\section{Referee 2 comment}

(2) Lines 70-73: Please elaborate on the description in the research question of this study. Are there no prior investigations on this storm?

Author response:

The scientific objective of the contribution is to present a literature review that catalogues storm infrastructure impacts, and gives information to relate the storm events in time and location. The study is partly motivated by the occurrence of a large wave at the FINO1 platform during Storm Britta on 31 Oct.-1 Nov. 2006. The platform was damaged four times by large waves in 2006, 2007, 2009, and 2013.

There are previous investigations on this storm, and these are presented in the literature survey. However, they tend to focus on particular subject areas (e.g., coastal erosion in eastern England or water levels along the coast of the Netherlands) or geographic areas (e.g., meteorological conditions over Germany). The value of this review is that it assembles the information from different types of reports.

\section{Referee 2 comment}

(3) Line 150: To compare the data in spectral analysis, they must have the same time resolution

Author response:

To compare the data in spectral analysis, the importance of the time resolution depends on the characteristic periods being compared. For example, if one were comparing the diurnal or semi-diurnal tidal reconstructions among the group of tide gauge stations, then it makes little difference if the data are presented with a one hour 
or one minute time discretization. The same is also true comparing the long period time series reconstructions created using the spectral cutoff threshold of 0.2 days.

However, if one is looking for harbour seiches or meteotsunamis in the short period time series reconstructions, then it does make difference whether the data are presented with the discretization of 1 minute, 10 minutes, or 1 hour. The characteristic period of harbour seiches is on the order of minutes. This would appear as noise in records of 10 or 15 minute discretization, possibly with aliasing effects. The impact of different time discretizations is not so important in the present analysis as most of the records going into the spectral analysis have time steps of 10 or 15 minutes.

Before computer analysis, the old discretization standard was 1 hour to give manageable amount of data to remove diurnal and semidiurnal tide and assess the nontidal residual (surge). With the advent of computer analysis, it became possible work with data sets of shorter time discretizations, and the UK (for example) switched to a 15 minute standard. With further computer developments and recent interest in meteo-tsunamis, there has been a trend to using 1 minute data discretizations. Thompson et al. (2020) have highlighted the deficiency of the UK tide gauge data discretization in light of European norms and user requirements.

\section{Referee 2 comment}

(4) In the conclusions you do not refer satisfactorily to your own results. Please elaborate on this rather than giving an overview of other studies.

Author response:

I have rearranged the information in the conclusion and included a passage that summarizes the results from the tide gauge analysis. There is some evidence for an important role of large waves in causing offshore accidents and possibly some harbour flooding. The issue has been highlighted by Pleskachevsky et al. (2012).

Other important issues that came out of the literature survey were that the statistics of the return period of extreme surge levels was changing, and that there was a fear that an atmospheric circulation tipping point had been passed.

\section{Referee 2 comment}

Line 66: Please give reference to the case studies

Author response:

The only information about these North Sea wind turbine incidents were descriptions in the media reports, which are already cited in the manuscript. There was speculation in the media reports about the possible causes of the accidents, but no follow-on case study reports have been located. I emailed the wind farm operators for more information on the accidents, but I have received no response.

\section{Referee 2 comment}

Lines 190-197: I am not sure I got the statement right. Can the magnitude of the oscillation not also be due to the fact that the 14-day period is considered, which takes into account both storm conditions and normal water levels?

Author response:

The referee's question relates to whether the features of the short period oscillations shown in Fig. 4d would change significantly if another time interval instead of 14 days were chosen for the discrete Fourier transform analysis. The characteristics or magnitudes of the short period oscillations over the two day period of the storm would not change significantly whether the analysis period chosen was 28 days or 7 days. The discrete Fourier transform does a very good job of isolating the short period component of the original time series from long period component and the diurnal/semidiurnal tides.

\section{Referee 2 comment}


Lines 207-209: You mention that "there are large differences in the skew surge values around the North Sea, with stations in the German Bight and northern Netherlands approaching $4 \mathrm{~m}$ in some instances." Do you have an explanation for this?

Author response:

There are two main factors influencing the height of the surge around the North Sea. The first is the action of the strong northwest wind pushing water from north to south so that water levels along the coast of the Netherlands and Germany would be expected to have much higher surge levels than Scotland. The second the factor is that the external surge travels as a Kelvin wave similar to the semidiurnal tidal wave and would be expected to have similar amplitude characteristics as the tides around the North Sea. The tidal range in the German Bight is larger than in Scotland.

Referee 2 comment

Figure 3: Please elaborate on the calculation of the noise level in the methods

Author response:

The noise level labelling was removed from the spectral graph. Following Stull (1988), the spectrum is calculated without uncertainties and is treated as a mathematical tool to move between the time series and spectral domains without loss of information.

References for the referee comments section

Argyriadis, K., Fischer, G., Frohbose, P., Kindler, D., and Reher, F.: Forschungsplattform FINO1 - einige Messergebnisse, Tagungsband der 4. Tagung "Offshore-Windenergie" am 14/15 Juni 2005 in Hamburg, Germanischer Lloyd WindEnergie GmbH, Hamburg, 2005.

Sanders, F. and Gyakum, J.R.: Synoptic-dynamic climatology of the 'Bomb', MWR, 108, 1589-1606, 1980.

Thompson, F., Renzl, E., Sibley, A., and Tappin, D.R., UK meteotsunamis: a revision and update on events and their frequency, Weather, 2020. 